\title{
Long-term Acoustic Assessment of Bats at Coal Mines across Southcentral Montana and Management Recommendations for Bats
}

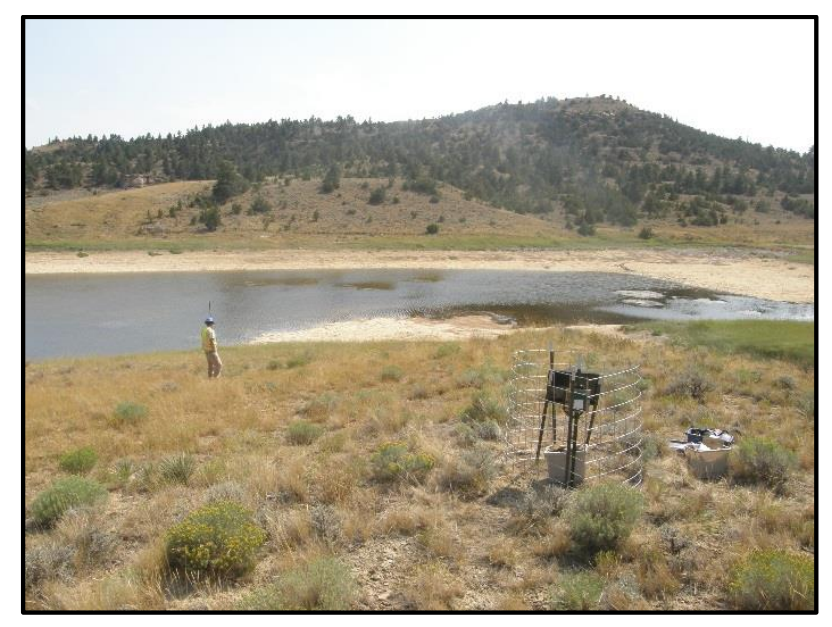

Prepared for:

Montana Department of Environmental Quality

Air, Energy, \& Mining Division, Coal Section

Prepared by:

Dan Bachen, Alexis McEwan, Braden Burkholder, Scott Blum, and Bryce Maxell Montana Natural Heritage Program

A cooperative program of the Montana State Library and the University of Montana March 2018 



\title{
Long-term Acoustic Assessment of Bats at Coal Mines across Southcentral Montana and Management Recommendations for Bats
}

\author{
Prepared for: \\ Montana Department of Environmental Quality \\ Air, Energy, \& Mining Division, CoalSection \\ 1218 E Sixth Ave, Helena, MT59620-0901
}

Agreement Numbers:

$13-475 ; 14-641 ; 15-536 ; 16-536$

Prepared by:

Dan Bachen, Alexis McEwan, Braden Burkholder, Scott Blum, and Bryce Maxell
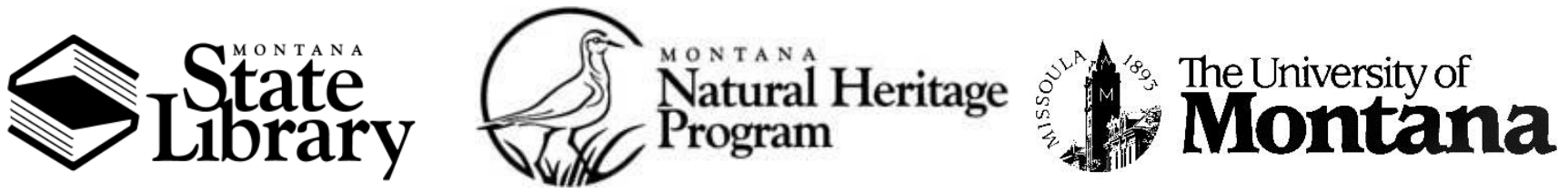

(C) 2018 Montana Natural Heritage Program

P.O. Box $201800 \bullet 1515$ East Sixth Avenue • Helena, MT 59620-1800 • 406-444-3290 
This document should be cited as follows:

Bachen, D.A., A. McEwan, B. Burkholder, S. Blum, and B. Maxell. 2018. Long-term a coustic assessment of bats at coal mines across southcentral Montana and management recommendations for bats. Report to Montana Department of Environmental Quality. Montana Natural Heritage Program, Helena, Montana. 169 pp. plus appendices. 


\section{EXECUTIVE SUMMARY}

Montana's bat populations face a wide array of conservation challenges, including loss of roos ting sites, elimination of prey species, collision or drowning hazards at sites where they forage, drink, and mate, and a lack of bas eline information on distribution and habitat use that is available to res ource managers. In recent years, concerns have focused on fatalities at wind turbine facilities and those resulting from White-nose Syndrome (WNS). WNS has killed an estimated 5.7 to 6.7 million bats in eastern North America and 600,000 to 888,000 bats are estimated to have been killed at wind energy facilities a cross the United States in 2012 alone. These and other sources of mortality may be having significant impacts on bat populations because bats are long-lived and have only one or two young per year. Given thes e concerns, detectors were installed at nine sites in proximity to active, inactive, or remediated coal mines or proposed project areas a cross Southcentral Montana. These detectors are part of a regional network deployed over multiple years to document activity patterns of bats across Montana, and portions of northern Idaho, and the western Dakotas.

The overarching objectives of this project were to gather multiple years of year-round baseline information on: (1) bat s pecies composition and activity levels; (2) timing of species immergence to and emergence from hibernacula for nonmigratory bat species; (3) timing of migrations by tree roos ting migratory s pecies that have been documented as having the highest levels of mortality from collisions with wind turbines; and (4) correlates of bat activity such as wind speed, temperature, precipitation, barometric pressure, and moon illumination.

We recorded bat echolocation calls from sunset to s unrise nightly using a SM2Bat+ detector/recorder at each site. At the West Decker, Spring Creek, and Big Sky mines we placed a single detector/recorder unit in proximity to a waterbody. At the Abs aloka, and Ros ebud Mines we placed detectors a way from water in habitat representative of the surrounding area. We placed and additional detector at the Rosebud Mine in proximity to a waterbody. Two detectors were deployed at waterbodies at the Signal Peak Mine.

Additionally, we placed a single detector at the Otter Creek Coal Tract next to a water source. Six detectors were deployed in 2012 and three more in 2013. Six detectors were deployed for between three years and five years. Three detectors at the Signal Peak or Big Sky mines a re still in operation. Across allsites, 1,539,851 call sequences were recorded through spring of 2017, with $24.5 \%$ auto-identified to species by Sonobat 4.2.1 or Kaleidoscope Pro 4.1 software.

Across all detectors and mine sites, 12 species (range of seven to ten species per site) were definitivelyconfirmed by hand review using the bat call characteristic identification guidelines in Montana's Bat and White-Nose Syndrome Surveillance Plan and Protocols (Maxell 2015): Pallid Bat (Antrozous pallidus), Townsend's Bigeared Bat (Corynorhinustownsendii), Big Brown Bat (Eptesicus fuscus), Spotted Bat (Euderma maculatum), Hoary Bat (Lasiuruscinereus), Silver-haired Bat (Lasionycterisnoctivagans), Eastern Red Bat (Lasiurus borealis), Wes tern Small-footed Myotis (Myotis ciliolabrum), Longeared Myotis (Myotisevotis), Little Brown Myotis (Myotis lucifugus), Long-legged Myotis (Myotis volans). The West Decker and Absaloka mines had the least diversity (six species), while ten species were detected at the Signal Peak Mine, Big Sky Mine, and Otter Creek Coal Tract. Signal Peak detectors a lso consistently recorded Spotted Bats. Surprisingly, Eastern Red Bats were confirmed at all mine sites which indicates that they are more common across this region during fall migration than previously thought. 
Activity of all species was greatest between April and late September at all sites. While all detectors recorded some level of activity during the inactive or hibernation season (November through March), Signal Peak and Big Sky mines had consistently high levels of activity throughout the winter. This likely indicates proximity to hibernacula in cracks or crevices in the surrounding rock outcrops, or similar habitat. At both mines, Big Brown Bat and Western Small-footed Myotis were confirmed to be active through the fall, winter, and spring. Additionally, at the Big Sky Mine Silver-haired Bats were also detected during the inactive season.

While we did not increase the number of species known to be in the region, us e of acoustic detectors was an effective method for gaining a more complete understanding of species presence throughout the year. Across all sites, we documented 170 detections of species within months (species/months), which represented an a verage increase per mine of 24.3 species/months. The largest increase in information was at the Big Sky Mine, with an increase of 38 species/months. At the Big Sky, Ros ebud, and Signal Peak mines, the Eastern Red Bat pres ence was documented for the first time. Patterns of recorded bat activity were consistent with the regional network of acoustic detectors. Apart from the Signal Peakand Big Sky mines, activity was very limited between November and February. However, at least some bat activity was documented every month in at least one of the study years across all detectors. Average nightly bat passes began to increase each year in mid to late April, reached a maximum between June and September after young became flighted and during migration and swarming, and were greatly reduced again by mid-October.

During the active season (April to October), bats were active near the detectors throughout the night. However, in the early and late season we detected a major pulse of activity in the firs $t$ hour after sunset and most activity occurred during the first two to three hours after sunset. In the middle of the summer, an additional peak in activity between midnight and dawn was obs erved. This increased activity may be the result of an additional foraging bout in res ponse to warmer temperatures and increased availability of prey.

Across the entire acoustic network, patterns between bat activity and landscape va riables, such as ruggedness, the presence or absence of trees, and type of water body, were evident. In rugged landscapes, or a reas with high densities of rock outcrops and cliffs available to roosting bats, activity was significa ntly higher tha $n$ in non-rugged landscapes, such as pra irie or grassland cover types. The presence of trees in rugged landscapes did not a ppear to influence bat activity across the network. However, detectors in non-rugged landscapes recorded more activity when trees were present. Detectors nearlarge and small lentic waterbodies recorded greater activity than detectors near lotic waterbodies or detectors placed a way from water. These results suggest that rock outcrop or tree roos ts as well as reservoirs are important landscape features to bats.

Nightly average bat pass temperatures recorded a cross all detectors ranged from -1.3 to $31.8^{\circ} \mathrm{C}$ during the active season and -2.3 to $14.0^{\circ} \mathrm{C}$ during the inactive season. Throughout the study, maximum background and bat pass temperatures recorded at the detectors closely approximated one a nother. However, average and minimum bat pass temperatures recorded at the detector were consistently much higher than average and minimum background temperatures recorded at the nearest weather station. Monthly average pat bass temperatures ranged from -1.3 to $13.3^{\circ} \mathrm{C}$ higher and monthly minimums pass temperatures ranged from -1 to $26.4^{\circ} \mathrm{C}$ higher. Similarly, the distribution of temperatures recorded at the closest available weather stations associated with bat passes 
were significantly higher than the distribution of background temperatures. Thus, bats consistently restricted their activity to wa rmer periods from the range of background temperatures that were available to them. This same pattern held across these sites and the entire detector network with more than $99 \%$ of bat activity restricted to temperatures above freezing and $97 \%$ of bat activity restricted to temperatures above $5^{\circ} \mathrm{C}$.

Across ourstudy a rea and the entire network bats were more active at lower wind speeds. Detectors places at the mine sites generally recorded more activity at wind speeds of between one to four meters per second than would be expected if activity was randomly distributed across all available wind speeds. Almost all activity was recorded at or below five meters persecond. Across the entire detector network, bat activity was greater than expected at random for wind speeds at one to three meters per second. Wind speeds less than three meters per second accounted for $72 \%$ of bat passes and wind speeds less than seven meters per second accounted for $97 \%$ of bat passes.

Except for West Decker Mine, increased bat activity was associated with stable or falling barometric pressure. This is similar to the pattern observed across the regional network. Approximately $73 \%$ of bat activity was associated with little to no change (negative one to one millibars) in hourly ba rometric pressure. However, bat activity was greater than expected during negative hourly changes (negative one to negative three millibars) and is less than expected with neutral or positive hourly changes (one to two millibars) than ifit were randomly distributed a cross background press ure change classes.

Within the study a rea precipitation did not appear to affect recorded bat activity. Decreased activity during precipitation events is frequently obs erved during mist netting efforts. As we would expect the same behavior by animals in the vicinity of the acoustic detectors, this difference may be driven by the nature of precipitation events in the area and the distance between weather stations and detectors. During the active season, thunderstorms a re common and precipitation can be local. The dis tance between detectors and weather stations ranges from $7.9 \mathrm{~km}$ for the Busse Water Res ervoir detector at the Signal Peak Mine, to $41.2 \mathrm{~km}$ for the Absaloka Mine, and averages $26.9 \mathrm{~km}$ a cross all sites and stations. Given that bats are capable of flight within minutes after the passage of a storm front and precipitation was aggregated by hour, our a nalysis of thes e data may not be adequate to detect a relationship between activity and precipitation.

Bas ed on activity recorded at project sites, bats appeared to become more active during periods of low lunarillumination (Figure 22). During periods of low light when the moon was below the horizon or was new o close to new, bats were more active than would be expected if activity were random. This pattern was similar across the regional network of bat detectors, with a few minor exceptions (Figure 23).

Activity decreased as moon phase became brighter and above the horizon. This pattern was documented across the network, except at sites in canyons or in proximity to terrain that blocked moonlight. At these sites activity often increased during periods of high lunar illumination. This may indicate that animals are selecting areas that provide refuge from illumination during bright periods.

Identification of individual species activity patterns was hindered by relatively low, and potentially inconsistent, rates of autoidentification of call sequences to s pecies (Table 4, Maxell 2015). Big Brown Bat, Spotted Bat, Hoary Bat, Silver-haired Bat, Western Smallfooted Myotis, Long-eared Myotis, and Little Brown Myotis had relatively high rates of confirmation of monthly presence and enough calls auto-identified, to examine trends (Table 5). However, activity patterns for all species 
from auto-identified call sequences should still be regarded as speculative due to a va riety of iss ues that might cause auto-identifications to be inaccurate and/or inconsistent (Maxell 2015).

Measures of overall bat activity near the detector, hand confirmed presence of individual species by month, and hand confirmed minimum temperatures associated with bat passes ofindividual s pecies are all stable metrics upon which management recommendations can be made. However, patterns of activity of individual species resulting from a utoma ted a nalyses should be used with a great deal of caution due to low rates of pecies assignment and low or uncerta in rates of accuracy of those assignments. Furthermore, it s hould be noted that bat activity measured during this study was made by a microphone on a nine to ten-foot mast and may not have a dequately sampled the activity of high flying bats such as the Hoary Bat and Silver-haired Bat, which together with the Eastern Red Bat are the three species that have suffered approximately $75 \%$ of the documented mortalities associated with wind turbines across North America (Kunzet al. 2007). Thus, the following management recommendations avoid use of activity patterns of individual species as determined by automated a nalyses and instead rely on results of hand confirmed analyses, general patterns of bat activity that were recorded at the study site, and results of published studies of wind turbine impacts on bat species.

General management recommendations for species observed at project sites include:

(1) protect potential natural roost sites by conserving large diameter trees (es pecially snags with loose bark), rock outcrops, cliff crevices, and caves (Appendix C).
(2) maintain accessibility for underground mine entrances that bats may be using as summer or winter roosts. Install bat friendly gates if closure is required.

(3) When removing bat colonies from buildings or other structures follow current best practices, including waiting until the late fall and winter to seal entry points and placing bat houses to compensate for elimination of the roost.

(4) Reduce structural complexity of vegetation (e.g., short stature grasslands) and availability of standing waters in proximity to wind turbines or other human structures that might represent a threat to bats or where bats are undesired.

(5) In safe environments, maintain loticor lentic waterbodies to provide habitat for foraging and drinking.

(6) If wind turbines a re installed in the region, set turbine cut-in speeds to $>6.0 \mathrm{~m} / \mathrm{sec}$ between April and October-especially important in July during peak bat activity when young a re newly flighted, and August, September, and October when migratory species are passing through and local bats are swarming and breeding. Feather wind turbine blades, making them parallel to wind direction, when wind speeds a re $<6 \mathrm{~m} / \mathrm{sec}$ to reduce ris $\mathrm{k}$ of ba rotrauma during times of relatively high bat activity.

(7) Report dead bats of any s pecies found in the winter or spring to Montana Fish Wildlife and Parks or Montana Natural Heritage Program pers onnel. Animals found dead during these seasons may have contracted WNS and should be testedas part of Montana's Passive WNS surveillance protocol. 


\section{ACKNOWLEDGEMENTS}

This project was conducted with grants from the Montana Department of Environmental Quality Air, Energy, \& Mining Division, CoalSection, and would not have been possible without the support of this agency and its staff. We extend considerable thanks to Chris Yde for facilitating this work, providing feedback on project implementation, and recognizing the importance of gathering year-round baseline information on bat activity in the region. We would also like to thank Mike Glenn for his as sistance in detector placement, maintaining detector sites and collecting data, and As hley Eichhorn for assistance with grants administration. Staffat Wildlife Acoustics assisted with questions regarding the SM2Bat+ ultra sonic detector/recorders and the SMX-US and SMX-U1 microphones and WAC to WAV and Kaleidoscope Pro software. Joe Szewczak provided Sonobat 4.1 software, feedback on its use, and the 2011 Humboldt State University Bat Lab's echolocation call characteristic s ummaries for wes tern and eas tern U.S. bats that we used to develop the call characteristic summary for Montana bats. John Horel with the MesoWest Res earch Group as sisted with acquisition of weather station data through the MesoWest a pplication programming interface. At the Montana Natural Heritage Program, Darlene Patzer ass isted with grant a dministration, Shannon Hilty assisted with hand review of bat calls, and Dave Ratz ass isted with downloading of weather station data from the Mes owest a pplication programming interface.

This project was supported by agreements between the Division Montana Department of Environmental Quality, and the Montana Natural Heritage Program, a cooperative program of the Montana State Library and the University of Montana (13-475; 14-641; 15-536; 16-536). 


\section{TABLE OF CONTENTS}

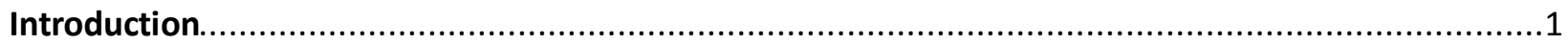

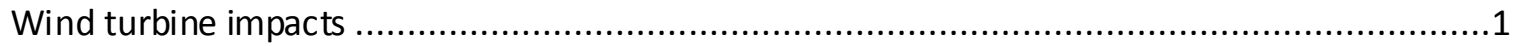

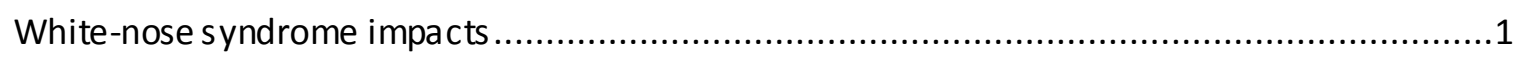

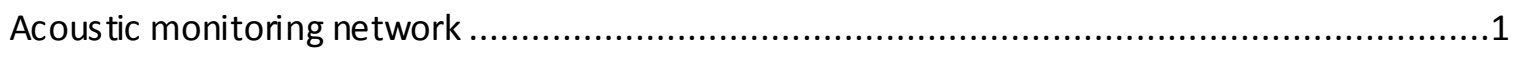

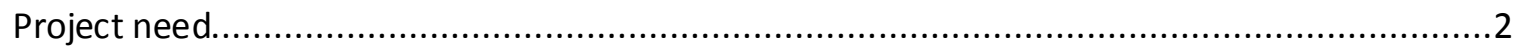

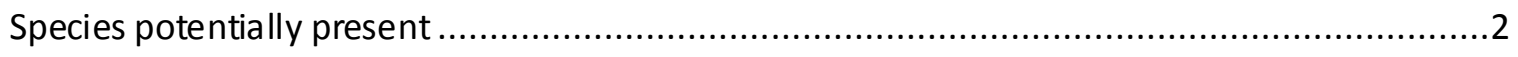

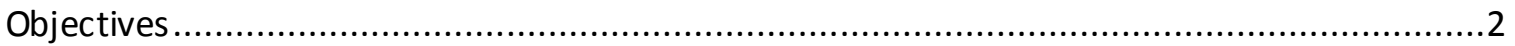

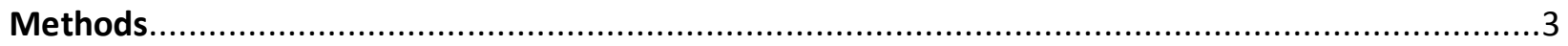

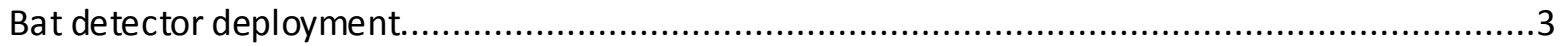

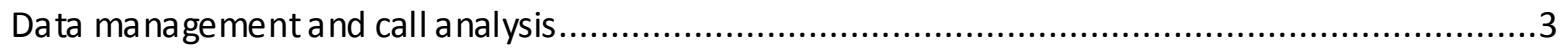

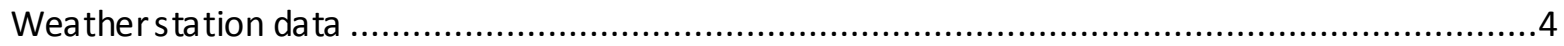

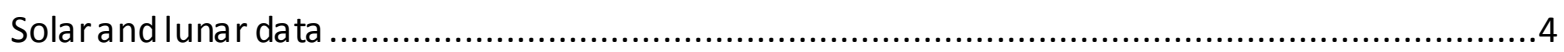

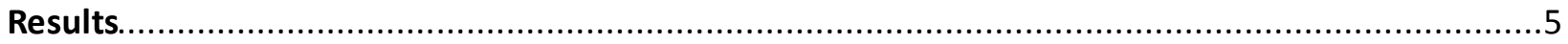

Total volume of bat passes and auto-identification rates ....................................................

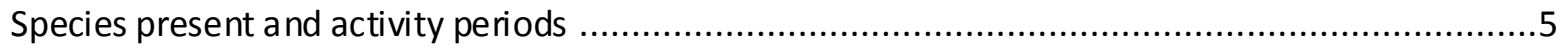

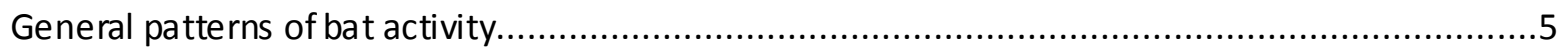

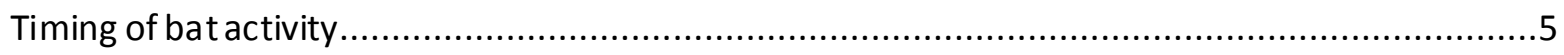

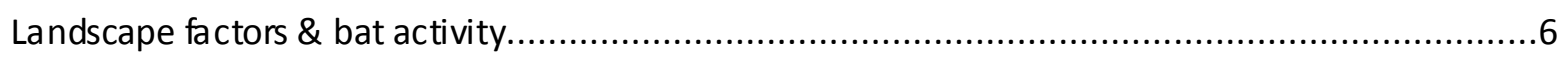

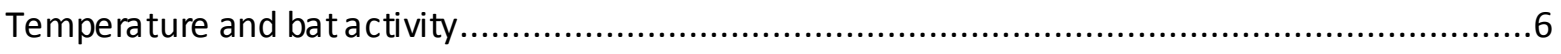

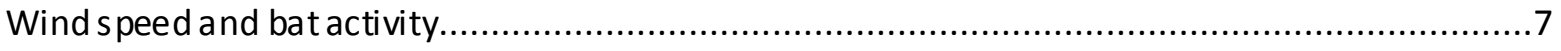

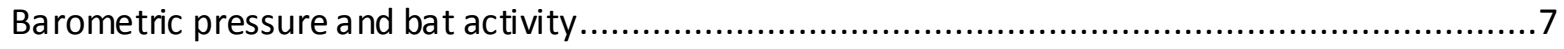

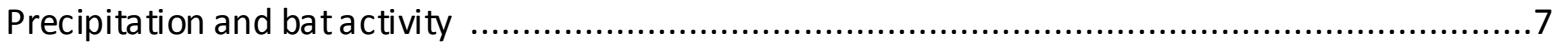

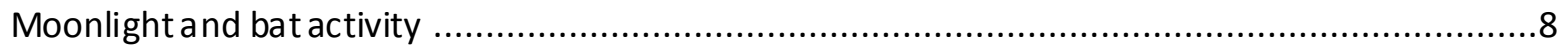

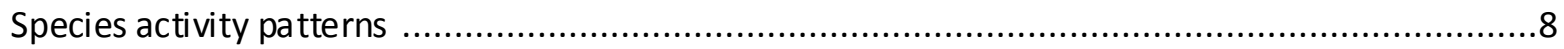

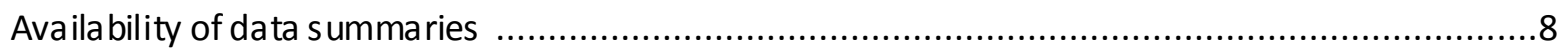

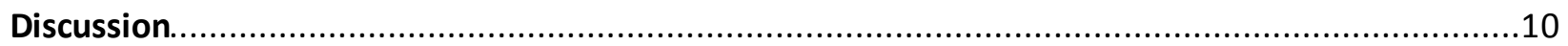

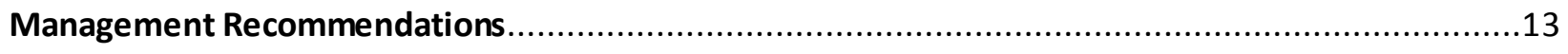

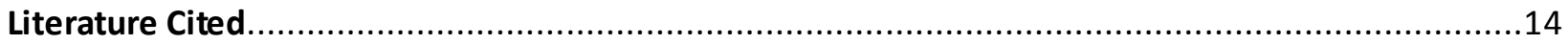




\section{LIST OF TABLES}

Table 1. Montana bat species, conservation status, and known or potential concerns from WNS and wind turbine facilities.

Table 2. Bat species documented and potentially present at or within $50 \mathrm{~km}$ of: West Decker Mine (a), Spring Creek Mine (b), Otter Creek Coal Tract (c), Big Sky Mine (d), Absaloka Mine (e), Rosebud Mine (f), Signal Peak Mine (g)

Table 3. Deployment history for SM2 Bat+ detector/ recorders deployed at coal mines ................25

Table 4. Proximity of weather stations to detector locations for all sites and data types ...............26

Table 5. Detector status as measured by percent of calls a uto-identified to s pecies for: West Decker Mine (a), Spring Creek Mine (b), Otter Creek Coal Tract (c), Big Sky Mine (d), Absaloka Mine (e), Rosebud Mine Area C pond (f), Rosebud Mine Area F pond 7 (g), Signal Peak Mine Reservoir 1 (h), Signal Peak Mine Busse Water Reservoir (i) .

Table 6. Monthly rates of hand confirmation from automated a nalysis results across all mine sites and detectors

Table 7. Species definitively detected by month each year of the study at: West Decker Mine (a), Spring Creek Mine (b), Otter Creek Coal Tract (c), Big Sky Mine (d), Absaloka Mine (e), Rosebud Mine (f), Signal Peak Mine (g)

Table 8. Species definitively detected by month across the acoustic detector network and at project detectors

Table 9. Bat Passes summarized by month across all species at: West Decker Mine (a), Spring Creek Mine (b), Otter Creek Coal Tract (c), Big Sky Mine (d), Absaloka Mine (e), Rosebud Mine Area C pond (f), Rosebud Mine Area F pond 7 (g), Signal PeakMine Res ervoir 1 (h), Signal Peak Mine Busse Water Reservoir (i)

Table 10. Nightly background and bat pass temperatures summarized by month at: West Decker Mine (a), Spring Creek Mine (b), Otter Creek Coal Tract (c), Big Sky Mine (d), Absaloka Mine (e), Rosebud Mine Area C pond (f), Rosebud Mine Area F pond 7 (g), Signal Peak Mine Reservoir 1 (h), Signal Peak Mine Busse Water Reservoir (i)

Table 11. Monthly minimum bat pass temperatures $\left({ }^{\circ} \mathrm{C}\right)$ recorded for individual species hand confirmed as definitively present at: West Decker Mine (a), Spring Creek Mine (b), Otter Creek Coal Tract (c), Big Sky Mine (d), Abs aloka Mine (e), Ros ebud Mine Area C pond (f), Rosebud Mine Area F pond 7 (g), Signal Peak Mine Reservoir 1 (h), Signal Peak Mine Busse Water Reservoir (i)

Table 12. Minimum bat pass temperatures recorded for definitive call sequences of s pecies across the detector network compared to mine sites

\section{LIST OF FIGURES}

Figure 1. Network of long term ultras onic acous tic detectors 
Figure 2. Landscape level overview of detector locations: Spring Creek and West decker mines, and Otter Creek Coal Tract (a); Big Sky, Absaloka, and Rosebud mines (b); Signal Peak Mine (c).95

Figure 3. Local scale site overview photos for the: (a) West Decker Coal Mine, (b)Spring Creek Mine, (c) Otter Creek Coal Tract, (d) Big Sky Mine, (e) Absaloka Mine, (f) Rosebud Mine, (g) Signal Peak Mine .98

Figure 4. Site photos of detector placements at: (a) West Decker Coal Mine, (b) Spring Creek Mine, (c) Otter Creek Coal Tract, (d) Big Sky Mine, (e) Absaloka Mine, (f) Ros ebud Mine, (g) Signal Peak Mine. 105

Figure 5. Percent of call s equences auto-identified to s pecies each month for: (a) West Decker Coal Mine, (b) Spring Creek Mine, (c) Otter Creek Coal Tract, (d) Big Sky Mine, (e) Absaloka Mine, (f) Rosebud Mine, (g) Signal Peak Mine 110

Figure 6. Average and maximum counts of bat pas ses per night by month for: (a) West DeckerCoal Mine, (b) Spring Creek Mine, (c) Otter Creek Coal Tract, (d) Big Sky Mine, (e) Absaloka Mine, (f) Rosebud Mine, (g) Signal Peak Mine .117

Figure 7. Average number of bat passes per night by week for the active and inactive season at: (a) West Decker Coal Mine, (b)Spring Creek Mine, (c) Otter Creek Coal Tract, (d) Big Sky Mine, (e) Absaloka Mine, (f) Ros ebud Mine, (g) Signal Peak Mine

Figure 8. Average number of bat passes per night by week a cross the detector network for active season (a) and inactive season (b).

Figure 9. Total number of bat passes per night by week across the detector network across all years for active seas on (a) a nd inactive season (b).....

Figure 10. Average number of bat passes each hour after sunset across allyears during active (a) and inactive season (b).

Figure 11. Average number of bat passes pernight by week across the detector network and across all years for active season (a) and inactive season (b) in rugged and non-rugged landscapes with and without trees

Figure 12. Average number of bat passes per night by week across the detector network and across all years for active season (a) and inactive seas on (b) at different water body types

Figure 13. Average nightly background and bat pass temperatures by month at: (a) West Decker Coal Mine, (b) Spring Creek Mine, (c) Otter Creek Coal Tract, (d) Big Sky Mine, (e) Absaloka Mine, (f) Rosebud Mine, (g) Signal Peak Mine..... .136

Figure 14. Percent of nightly hours with average background tempera tures and average temperatures as sociated with bat passes for the clos est weather station at: (a) West Decker Coal Mine, (b) Spring Creek Mine, (c) Otter Creek Coal Tract, (d) Big Sky Mine, (e) Abs aloka Mine, (f) Rosebud Mine, (g) Signal Peak Mine

Figure 15. Percent of nightly hours with average background temperatures and average temperatures associated with bat passes across the regional network of detectors

Figure 16. Percent of hours with average background wind speeds and average wind speeds associated with bat passes at the closest associated weather station at: (a) West Decker Coal Mine, (b) Spring Creek Mine, (c) Otter Creek Coal Tract, (d) Big Sky Mine, (e) Absaloka Mine, (f) Rosebud Mine, (g) Signal Peak Mine 
Figure 17. Percent of hours with average background wind speeds and average wind speeds associated with bat passes across the regional network of detectors

Figure 18. Percent of hours with background barometric pressure changes and ba rometric pressure changes associated with bat passes at the closest associated weather station at: (a) West Decker Coal Mine, (b) Spring Creek Mine, (c) Otter Creek Coal Tract, (d) Big Sky Mine, (e) Absaloka Mine, (f) Rosebud Mine 149

Figure 19. Percent of hours with background ba rometric pressure changes and ba rometric pressure changes associated with bat passes across the regional network of detectors

Figure 20. Percent of background hours (blue) and hours with bat passes with and without precipitation at the closest weather station to: (a) West Decker Coal Mine, (b)Spring Creek Mine, (c) Otter Creek Coal Tract, (d) Big Sky Mine, (e) Absaloka Mine, (f) Ros ebud Mine ..153

Figure 21. Percent of background hours and hours with bat passes with and without precipitation across the regional network of detectors

Figure 22. Percent of background hours and hours with bat passes at various moon illumination categories with the moon above and below the horizon at: (a) West Decker Coal Mine, (b) Spring Creek Mine, (c) Otter Creek Coal Tract, (d) Big Sky Mine, (e) Absaloka Mine, (f) Ros ebud Mine, (g) Signal Peak Mines

Figure 23. Percent of background hours and hours with bat passes associated with va rious moon illumination categories (with the moon below or above the horizon across the regional network of detectors

Figure 24. Average number of nightly bat passes each week auto-identified by species at: (a) West Decker Coal Mine, (b) Spring Creek Mine, (c) Otter Creek Coal Tract, (d) Big Sky Mine, (e) Absaloka Mine, (f) Rosebud Mine, (g) Signal Peak Mine

\section{APPENDICES}

Appendix A. References on wind turbine and other human structure collision impacts on bats

Appendix B. Bat Pass temperatures summarized by s pecies and month for all project detectors ....B1-44

Appendix C. Overview of roosting habitat and home range/foraging distance documented for Montana batspecies. 


\section{INTRODUCTION}

Populations of bats within Montana facea wide a rray of threats to their persistence, including loss of roosting sites, elimination of prey species, collision or drowning hazards at sites where they forage, drink, and mate, and a lack of baseline information on distribution and habitat use that is available to resource managers. In recent years, concerns have focused on fatalities at wind turbine facilities and those resulting from White-nose Syndrome (WNS) (Table 1). The large increases in mortality posed by these threats a re especially significant to bat populations because bats are long-lived and have only one or two young per year (Barclay and Harder 2003).

\section{WIND TURBINE IMPACTS}

Bat fatalities a re prevalent at wind energy facilities a cross the United States with 600,000 to 888,000 fatalities estimated in 2012 alone (Hayes 2013, Smallwood 2013). The widespread nature of these fatalities coupled with low fecundities of bats raises concerns that wind turbines may be having significant impacts on bat populations (Barclay and Harder 2003, Kunz et al. 2007, Arnett et al. 2008). Of North America's 45 bat species, 11 have had documented mortalities from wind turbines. Five of these species occur in the project area for at least a portion of the year (Tables 1 \& 2; Kunz et al. 2007, Arnett et al. 2008). Across all species, mortality rates have been highest $(\geq$ $75 \%$ of mortalities) in tree roos ting migratory species such as the Eastern Red Bat (Lasiurus borealis), Hoary Bat (Lasiuruscinereus) and Silver-haired Bat (Lasionycterisnoctivagans) (Kunz et al. 2007, Arnett et al. 2008, Arnett et al. 2011). Thus, if wind turbines were to be installed in the region, most mortalities would be expected to be associated with Hoary Bats and Silver-haired Bats during migratory or mating events (Cryan 2008). However, resident bats may also be impacted (Poulton and
Erickson 2010) and impacts may occur even during the winter (Lausen and Barclay 2006).

\section{WHITE-NOSE SYNDROME IMPACTS}

Since 2006, White-Nose Syndrome (WNS), resulting from the cold a dapted fungus Pseudogymnoascus destructans (PD), has killed an es timated 5.7 to 6.7 million bats in eastern North America (Blehert et al. 2008, Lorch et al. 2011, USFWS News Release January 17, 2012, Minnis and Lindner 2013). As a res ult, the extinction of Little Brown Myotis (Myotis lucifugus) is predicted in eastern North America by 2026 (Frick et al. 2010), Little Brown Myotis, Northern Myotis (M. septentrionalis), and Tricolored Bat (Perimyotissubflavus) were emergency listed as Endangered under Canada's Species at Risk Act (COSEWIC 2012), Little Brown Myotis has been petitioned for emergency listing under the United States Endangered Species Act (Kunzand Reichard 2010), and Northern Myotis has been listed as Threatened under the United States Endangered Species Act across its range, including nine eastern Montana counties (USFWS 2015). P. destructans has progressed westward to states along the Mis sissippi River corridor as well as the Province of Ontario, Canada and, recently was detected in Washington State (WDFW, USFWS, and USGS 2016). It has caused WNS in at least four species documented in Montana, has been detected in other s pecies that may serve as local or regional vectors. Many western species have not yet been exposed to Pd, but as they a re closely related to susceptible impacts of the disease are possible (Table 1, Blehert et al. 2011).

\section{ACOUstic MONITORING NETWORK}

Starting in the fall of 2011, va rious federal, state, and tribal partners began deploying SM2Bat, SM2Bat+, and SM3Bat ultrasonic detector/recorders to gatheryear-round baseline information on bat activity in va rious 
localities across Montana. During 2012, individual efforts began to coalesce into a regional network of detectors to address most bat species known to occur in Montana (Table 1, Figure 1, Maxell 2015). Most recordings from this array are being processed, analyzed, and archived at the Montana Natural Heritage Program.

\section{Project Need}

Although previous projects to determine what species of bat are present in the region have been conducted, data on presence throughout the year are lacking. Previous surveys using acoustic detectors and mist nets in southcentral Montana were limited to single night s ampling between late June and ea rly September. Roost searches were conducted between March and October in some areas, and contributed additional active season detections for some species. However, the scope of previous projects was limited both in geogra phic scope and project duration. Therefore, our understanding of distribution, occupancy, and phenology for many of the species is limited. One notable exception is the intensive surveys of caves and water sources in the Pryor Mountains conducted in the late 1980's and early 1990's as part of a University of Montana Masters project and subsequent surveys by Montana Natural Heritage Program personnel (Worthington and Ross 1990; Worthington 1991). Through these surveys and other efforts, this mountain range has some of the most extensive data on bats in the state, but due to the unique ecosystems and geographic fea tures of this area, it is unlikely that inferences about the natural history of the region's bats species can be applied to the largergeographic area including the mine sites to the north and east. Thus, southcentral and southeast Montana lack bas eline data on year-round patterns of bat activity that could be us ed to inform resource management plans or individual projects.

\section{Species Potentially Present}

Of the 15 species of bat known to occur within Montana, 13 have been confirmed to be pres ent within southcentral and southeastern Montana: Townsend's Big-eared Bat (Corynorhinustownsendii), Big Brown Bat (Eptesicus fuscus), Spotted Bat (Euderma maculatum), Hoary Bat, Eastern Red Bat, Silverhaired Bat, Pallid Bat (Antrozouspallidus), California Myotis (Myotiscalifornicus), Western Small-footed Myotis (Myotis ciliolabrum), Longeared Myotis (Myotisevotis), Little Brown Myotis, Fringed Myotis (Myotis thysanodes), and Long-legged Myotis (Myotisvolans) (Table 2, MTNHP 2017). Within $50 \mathrm{~km}$ of the mine sites, all species except for the California Myotis have been documented on at least one occasion during the active season. It is possible that the California Myotis may be present within the a rea of focus, but a detection would represent a significant eastward expansion of this species known range (MTNHP 2017). Within $50 \mathrm{~km}$ of each individual mine the number of observed species is lower. The number of previously documented species at the West Decker Mine was 11, ten at the Spring Creek Mine, 12 at the Otter Creek Coal Tract, eight at the Big Sky and Absaloka mines, seven at the Ros ebud Mine, and ten at the Signal Peak Mine.

\section{OBJeCtives}

The major goals of this project were to: (1) gather baseline information on bat species composition and activity levels at coal mines year-round for two-three years; (2) identify timing of species immergence to and emergence from hibernacula for non-migratory bat species; (3) identify timing of migrations by tree roosting migratory s pecies; and (4) identify relationships between bat activity and wind speed, temperature, precipitation, barometric pressure, and moon illumination. Furthermore, placement at active, proposed, and remediated coal mines may allow us to explore trends in activity that may be influenced by management of these sites. 


\section{MethodS}

\section{Bat Detector Deployment}

Across Southcentral Montana nine detectors were deployed at seven areas with past, current, or propos ed mining activity: West Decker (one), Spring Creek (one), Big Sky (one), Abs aloka (one), Rosebud (two), and Signal Peak Mines (two), and one propos ed minesite, the Otter Creek Coal Tract (Figures 1, 2, 3). Of these, six detectors were deployed in the late summer or early fall of 2012. The remaining three were deployed in 2013 (Table 3). The detector deployed at the Ros ebud minewas deployed in 2012 but moved to a different site within the mine in 2013, while the Signal Peak Mine has had two detectors deployed at different sites for the duration of the project. At six of the sites, a coustic surveys were conducted for three to four years and detectors were decommissioned in 2015 or 2016 . Both detectors at the Signal Peak, and the detector at the Big Sky mine are still in operation.

To determine the best placement, each area was assessed for a location with: (1) open water for as much of the year as possible; (2) rock outcrops and trees that might be us ed as roosts by bats; (3) southern solar exposure that would allow a solar panel to charge a battery even during the winter; (4) year-round accessibility; and (5) a low likelihood of vandalism (Figure 4). At all sites, a Song Meter SM2Bat+ detector/recorder with an SMX-US microphone (Wildlife Acous tics Inc., Maynard, MA) was deployed. The microphones at all operational sites in 2015 were upgra ded to SMX-U1 microphones (Wildlife Acoustics Inc., Maynard, MA). The SM2Bat+detector/recorder was deployed, monitored, and maintained with the equipment, supplies, settings, and protocols listed in Montana's Bat and White-Nose Syndrome Surveillance Plan and Protocols 20122016 (Maxell 2015).

Many aspects of the equipment and site selections influence the detection of a bat echolocation call and the quality of the resulting recording. These include sensitivity of the individual microphone, temperature, humidity, wind speed, and frequency, a mplitude, distance, and directionality of echolocation calls emitted by bats (Parsons and Szewczak 2009, Agranat 2014). The energy of sounds spreading in all directions diminishes by one fourth for every doubling of distance because the surface area of a sphere is related to the square ofits radius. Furthermore, higher frequency sounds are diminished over shorter dis tances because of atmos pheric absorption (Parsons and Szewczak 2009, Agranat 2014). Tes ting of the SMX-US microphone used through June 2015 in this study indicates that bats emitting frequencies in the range of $20 \mathrm{kHz}$ should be detected at distances of 24 to 33 meters from the microphone while those emitting frequencies in the range of $40 \mathrm{kHz}$ should be detected at distances of 18 to 22 meters (Agranat 2014). These distances are the radii of the relevant spheres of detection around microphones when they a re at full sensitivity. However, we know that sensitivity varied over time by an unknown magnitude because some precipitation and freezing events permanently reduced the sensitivity (Table 3). In 2015 the microphones at eight detectors were upgraded to the SMX-U1 microphone, which increased the quality of recorded calls and reduced the effect of adverse weather on microphone sensitivity over time. Due to this change in hardware, comparisons between data collected before and after June 2015 should be made with caution as the different models of microphone may affect the number of calls and species detected.

\section{Data Management \& Call Analyses}

Acous tic file recordings, in both original WAC and processed WAV formats, a re stored in the Montana Bat Call Library which is housed on a series of 15-20 Terabyte Drobo 5D and 5N 
storage arrays at the Montana State Library as well as a secondary offsite location to protect against catastrophic loss. Acoustic a nalysis results, temperature files, weather station da ta, and solar and lunar da ta were all processed and combined within SQL database tables in accordance with the general work flow pattern for data management and analysis ou tlined in the text and in Appendices 8-10 of Maxell (2015). Bat call sequences were a nalyzed with the goal of definitively identifying individual species presence by month and individual species' minimum temperatures of activity in accordance with the Echolocation Call Characteristics of Montana Bats and Montana Bat Call Identification materials in Appendices 6 and 7 of Montana's Bat and White-Nose Syndrome Surveillance Plan and Protocols 20122016 (Maxell 2015).

\section{Weather Station Data}

Weather station data were downloaded using the Mesowest application programming interface as outlined in Appendix 9 of Maxell (2015). Temperature, wind speed, solar, and precipitation data were downloaded from weather stations across the regions. Distance from the detector to the station varied by site and data type (Table 4). All data from weather stations were averaged by hour and associated with all call sequences recorded within this hour bin for use in our analyses.

\section{SOLAR AND LUNAR DATA}

Solar and lunar data were calculated for all hours of detector deployment using the Python package ephem (3.7.6.0), which uses well established numeric routines to produce high precision astronomy computations (see Appendix 10 of Maxell 2015). The underlying code produces results nearly id entical to data available from the U.S. Naval Obs ervatory (Astronomical Applications Department). Precise times for sunrise, sunset, moonrise, moonset, and percent illumination at the detector were calculated based on latitude, longitude, and date. It should be noted that local topography is not incorporated into any of thes e calculations. Therefore, the exact timing of these events on the ground may differ slightly from those produced by this model, but should typically be within a few minutes unless local terrain differs greatly from the modeled horizon (e.g. if the site is at the bottom ofa canyon). 


\section{RESULTS}

Total Volume of Bat Passes and AutoIDENTIFICATION RATES

Between August 2012 and March 2017, a total of $1,539,851$ bat call sequences were recorded across all detectors, with $24.5 \%$ (average range $16.1 \%$ to $32.4 \%$ a cross detector sites) autoidentified to species by Sonobat 4.2.1 or Kaleidoscope Pro 4.1 software. Individual detectors varied in both call sequences and auto identification rates (Table 5). Overall rates of a uto-identification were very similar to the regional network average of $25.5 \%$ for many months of the study (Table 6, Figure 5).

\section{Species Present \& Activity Periods}

Across all sites and detector deployments we confirmed the presence of presence of 12 species of bat. The diversity of species across mines a veraged $8.5 \mathrm{~s}$ pecies/detector site (Table7). The Absaloka Mine and West Decker mines had the fewest confirmed species (seven) while the detector placed at Reservoir 1 at the Signal Peak Mine, Big Sky Mine, and Otter Creek Coal Tract had the highest diversity with ten species confirmed at each. Six species were confirmed across every mine site and detector placement: Big Brown Bat, Hoary Bat, Silverhaired Bat, Western Small-footed Myotis, Longeared Myotis, and Little Brown Bat. Pallid Bat was confirmed at the Big Sky Mine and Otter Creek Coal Tract. Spotted Bat was detected at the Big Sky Mine, and both detectors at the Signal Peak Mine. Townsend's Big Eared Bat was detected at Otter Creek and both Signal Peak Mine detectors. The Long-legged Myotis was confirmed at the Big Sky Mine, Otter Creek Coal Tract, both Rosebud Mine Detectors, Signal Peak Mine, and Spring Creek Mine. Fringed Myotis was confirmed only at the Spring Creek Mine.

During the active season, sixs pecies were detected across all sites: Big Brown Bat, Hoary Bat, Silver-haired Bat, Western Small-footed
Myotis, Long-eared Myotis, and Little Brown Myotis. During the fall migration period (JulyOctober), Eastern Red Bats were detected at all detectors except Ros ebud Mine Area F Pond 7. Additionally, Eastern Red Bats were detected during spring migration at the Spring Creek Mine.

Five additional species were detected during the active season. Townsend's Big-eared Bat was detected across the active s eason at both the Otter Creek and Signal Peak Mine detectors. This species was also detected in September at the Signal Peak Mine Busse Water Reservoir detector. Spotted bats were detected in June at the Big Sky Mine detector, and between April and September and October at both Signal Peak detectors. Pallid Bats were detected in April and May at the Big Sky Mine, and in June at the Otter Creek Site. Long-Legged Myotis was confirmed in single months during the active season at the Big Sky Mine, Otter Creek, and both Rosebud and Signal Peak Mine detectors. Fringed Myotis was detected in July at the Spring Creek Mine.

Three species were recorded over the winter. Big Brown Bats were active throughout the hibernation season at Big Sky Mine detector, and both Signal PeakMine detectors. At the Big Sky Mine, Silver-haired Bats were active across the winter. Additionally, Western Small-footed Myotis was detected throughout the winter at the Signal Peak Busse Water Reservoir detector.

\section{General Patterns of Bat Activity}

Patterns of activity recorded a cross all mines were generally consistent with the overall activity patterns a cross the regional network of acoustic detectors (Table 8). Between November and February, activity was limited at most sites. Detectors at Big Sky and Signal Peak mines recorded relatively high and consistent activity throughout the winter (Table 9, Figures $6,7)$, which differs significantly from other 
project detectors and the network as a whole (Figures 8, 9). It is likely that these detectors were in proximity to hibernacula used by several species. Across all detectors, average nightly passes began to increase in mid to late April, peaking in late May through early June. After the summer, activity began to decline in September, reaching typical winter levels in October. No detectors or mine sites had higher than expected a ctivity in the fall typical of a swarming site.

\section{TIMING OF BAT ACTIVITY}

Across all detectors some level of bat activity was evident throughout the night during the active season (April through October). In the spring activity was generally highest early in the evening, then decreased through dawn (Figure 10). As the season progressed, activity began to peak within a few hours after sunset and again within a few hours of sunrise, which is likely the result of multiple bouts of foraging by some species. In the late summer and early fall, activity returned a single peak in activity similar to spring.

\section{LANDSCAPE FACTORS \& BAT ACTIVITY}

Across the entire acoustic network, patterns were evident between bat activity and landscape variables, such as ruggedness, the presence or absence of trees, and water body type (Figures $11 \& 12$ ). Bat activity was significantly higher during both the active and inactive seasons in rugged landscapes, a reas with high densities of rock outcrops and cliffs available to roosting bats, as compa red with non-rugged landscapes such as prairie or grassland habitats (Figure 11). The presence or abs ence of trees in rugged landscapes did not appear to influence bat activity a cross the network (Figure 11). However, non-rugged landscapes had much higher bat activity levels when trees were present and non-rugged lands capes without trees lacked any bat activity from November through March (Figure 11). Trees provide both roos ting and foraging habitat, and this pattern indicates that they are an important feature to bats in non-rugged landscapes.

During the active season, there was greater activity at detectors near la rge and small lentic waterbodies than at detectors near lotic waterbodies or those placed away from water (Figure 12a). This suggests that standing water bodies, especially large ones, provide bats with important drinking and foraging habitat. However, small and large rivers are also important for providing drinking opportunities for bats during the colder months of November through March (Figure 12b).

Ruggedness, tree cover, and water vary across mine sites and detector placements. Abs aloka and Rosebud mines had the only detector placements in upland sites, all others were placed in proximity to lentic or lotic waterbodies. Except for the Rosebud and Signal Peak mines, all detector placements had either deciduous or conifer trees nearby. Apart from the Otter Creek Coal Track, and West Decker and Abs aloka mines, all sites were considered rugged.

\section{TEMPERATURE \& BAT ACTIVITY}

Nightly average bat pass temperatures recorded across all detectors ranged from -1.3 to $31.8^{\circ} \mathrm{C}$ during the active season and -2.3 to $14.0^{\circ} \mathrm{C}$ during the inactive season (Table 9). Throughout the study, maximum background and bat pass temperatures recorded at the detectors closely a pproximated one a nother (Table 10). However, average and minimum bat pass temperatures recorded at the detector were cons istently much higher than average and minimum background temperatures recorded at the nearest weather station. Monthly average pat bass temperatures ranged from -1.3 to $13.3^{\circ} \mathrm{C}$ higher than average temperatures and monthly minimums pass temperatures ranged from -1 to $26.4^{\circ} \mathrm{C}$ higher than recorded minimums (Table 11, Figure 13). Similarly, the distribution of temperatures 
recorded at the closest a vailable weather stations associated with bat passes were significantly higher than the distribution of background temperatures (Figure 14). Thus, bats consistently restricted their activityto warmer periods from the range of background temperatures that were available to them. This same pattern held across these sites and the entire detector network with more than $99 \%$ of bat activity res tricted to temperatures above freezing and $97 \%$ of bat a ctivity restricted to temperatures above $5^{\circ} \mathrm{C}$ (Figure 15).

Monthly minimum bat pass temperatures confirmed for individual species ranged from 0.8 to $15^{\circ} \mathrm{C}$ for the Pallid Bat, 6.7 to $25.7^{\circ} \mathrm{C}$ for Townsend's Big-eared Bat, -1.3 to $28.4^{\circ} \mathrm{C}$ for Big Brown Bat, 8.9 to $23.9^{\circ} \mathrm{C}$ for Spotted Bat, 1.6 to $29.3^{\circ} \mathrm{C}$ for the Eastern Red Bat, -0.1 to $25.9^{\circ} \mathrm{C}$ for Hoary Bat, -1.8 to $27.9^{\circ} \mathrm{C}$ for Silver-haired Bat, -1 to $26.7^{\circ} \mathrm{C}$ for Western Small-footed Bat, 0.3 to $26.5^{\circ} \mathrm{C}$ for Long-eared Myotis, -0.5 to $26.2^{\circ} \mathrm{C}$ for Little Brown Myotis, 19.4 for Fringed Myotis (one detection), and 0.6 to $22.4^{\circ} \mathrm{C}$ for Long-legged Myotis (Tables 11 \& 12, Appendix $B)$. Across the mine sites minimum temperatures for many were up to $11.8^{\circ} \mathrm{C}$ greater than the minimums across the network (Table 11, Appendix B). At the Absaloka mine the coldest minimum temperature was recorded for the Eastern Red Bat across the network.

\section{WIND SPEED \& BAT ACTIVITY}

Across allsites, bats were generally more active at wind speeds of between one and four meters per second than would be expected ifbat activity was randomly distributed a cross all available wind speeds. Furthermore, a lmost all activity occurred at or below five meters per second (Figure 16). Across the entire detector network, bat activity was greater than expected at random for wind speeds at one to three meters per second (Figure 17). Wind s peeds less than three meters per second accounted for $72 \%$ of bat passes and wind speeds less than seven meters per second accounted for $97 \%$ of bat passes (Figure 17). Given the possible difference between wind speeds at bat detectors and weather stations, it seems likely that bats restrict their flight to even lower wind speeds than the associations in Figures 16 \& 17 indicate.

\section{Barometric Pressure \& Activity}

For all sites except the West Decker Mine, increased bat activity was associated negative pressure change classes down to negative three millibars of change per hour. Less activity than expected was recorded during neutral or positive changes up to one to two millibars per hour. This same pattern is evident across the detector network (Figure 18). Approximately $73 \%$ of bat activity a cross the network was associated with little to no change (negative one to one millibars) in hourly ba rometric pressure. However, bat activity was greater than expected during negative hourly changes (negative one to negative three millibars) and is less than expected with neutral or pos itive hourly changes (one to two millibars) than if activity were randomly distributed across background pressure change classes (Figure 19). At West Decker, increased bat activity was associated with stable or slightly increasing pressure. The closest weather station to this detector that can provide pressure data is in Sheridan, Wyoming, $32 \mathrm{~km}$ to the south. Although it is possible that bats at the West Decker site behave differently, it is more likely that the press ure measured at the weather station may not accurately reflect conditions at the detector.

\section{Precipitation \& Bat Activity}

Across allsites and detectors, we found little correlation with bat activity and precipitation measured at proximal weather stations (Figure 20). Decreases in activity in res ponse to precipitation events a re frequently observed during mist netting efforts. The discrepancy between the data and observed behavior may 
be driven by the nature of precipitation events in the area and the distance between weather stations and detectors. During the active season, thunderstorms are common and precipitation can be local. The distance between detectors and weather stations ranges from $7.9 \mathrm{~km}$ for the Busse Water Reservoir detector at the Signal Peak Mine, to $41.2 \mathrm{~km}$ for the Absaloka Mine, and averages $26.9 \mathrm{~km}$ across all sites and stations. Given that bats are capable offlight within minutes after the pas sage of a storm front and precipitation was coded in hourly bins, timing of recorded precipitation maynot accurately reflect conditions at detector sites.

Across the acoustic detector network, bat activity was slightly higher (less than $1 \%$ ) during hours without precipitation and slightly lower (less than 1\%) during hours with precipitation than would be expected if bat activity was randomly distributed a cross available time (Figure 21). This lack of correlation between precipitation and activity is probably due to similar issues in qua ntifying precipitation at the detector. Given these issues patterns of bat activity relative to recorded precipitation events at weather stations may not be all that meaningful across the network.

\section{MOONLIGHT \& BAT ACTIVITY}

Bas ed on activity recorded at project sites, bats appeared to become more active during periods of low lunar illumination (Figure 22). During periods oflow light when the moon was below the horizon or was new o close to new, bats were more active than would be expected if activity were random. This pattern was similar across the regional network of bat detectors, with a few minor exceptions (Figure 23). Activity decreased as moon phase became brighter and above the horizon. This pattern was documented across the network, except at sites in canyons or in proximity to terrain that blocked moonlight. At these sites activity often increased during periods of high lunar illumination. This may indicate that a nimals are selecting areas that provide refuge from illumination during bright periods.

\section{Species Activity Patterns}

Identification ofindividual species activity patterns was hindered by relatively low, and potentially inconsistent, rates of autoidentification of call sequences to species (Table 5, Maxell 2015). Big Brown Bat, Spotted Bat, Hoary Bat, Silver-haired Bat, Western Smallfooted Myotis, Long-eared Myotis, and Little Brown Myotis had relatively high rates of confirmed monthly presence and enough calls a uto-identified to exa mine trends (Table 6). Call sequences of known species identity in the Montana Bat Call Library have also had relatively high accuracy rates $>50 \%$ correct a uto-identification rates) for these species. However, activity patterns for these s pecies from auto-identified call s equences should still be regarded as speculative due to a variety of iss ues that might cause auto-identifications to be inaccurateand/orinconsistent (Maxell 2015).

Upgra ding the microphones in June of 2015 had a significant effect on the ability of the softwa re to identify calls to species. At detectors deployed with both the SMX-US and SMX-U1 microphones, an increase in the number of call sequences identified to s pecies is clearly visible in the months following installation (Figure 24). Across all detectors, the use of the SMX-U1 microphone was correlated with a $10-20 \%$ increase in auto identification rates. Given thes e differences, comparisons of activity patterns for species from auto-identified call sequences through time should be further regarded with caution.

\section{AVAILABILITY OF DATA SUMmaries}

Current ta bular and chart data summaries for bat activity patterns in association with time, weather, and other correlates for detectors across the regional network of ultrasonic acoustic monitoring stations a re availableby request from the Montana Natural Heritage 
Program through an Excel workbook. Pivot tables and charts in topical worksheets in this workbook can be filtered to produce the latest data summaries for one or more sites, time periods, and species. As confirmations of individual species monthly presence and minimum temperatures of activity a re made, this information is a dded to the animal point obs ervation database at the Montana Natural Heritage Program and is available to agency biologists and resource managers for regional and project-level planning online in the context of a variety ofmap information through the MapViewer web application http://mtnhp.org/mapviewer 


\section{Discussion}

Across mine sites, we confirmed several uncommon and noteworthy species. At both Signal Peak detectors, Spotted Bats were frequently recorded from April to September and October. This species was once considered one of the rarest mammals in North America (Foresman 2012), and consistent detections of this species a re rare across the state-wide detector network. The species is currently thought to roost primarily in la rge cliff habitat (e.g. Big Horn Canyon, Chambers et al. 2011), however the closest la rge cliffs to the detectors are on the Yellowstone River. While Spotted Bats have been documented traveling greater distances from roosts to foraging a reas (e.g. Rabe et al. 1998), calls were recorded early in the evening which indic ates that at least some individuals must be roos ting in the local area. Small sandstone outcrops are abundant across the Bull Mountains and use of thes efeatures as roosts a re likely, but the use of other features cannot be ruled out. Iffuture work identifies what local features are used, it will improve our understanding of the life history of this species within the northern extent of its range.

Eastern Red Bats were consistently detected during their migration period at every mine site and all but one detector. Prior long-term acoustic monitoring a cross the state, this species was considered rare and only nine individuals had been observed (MTNHP Point Observation Database 2018). The data generated from this and other projects has shown that Eastern Red Bats a re common in late summer and fall as they presumably migrate south through Montana. Given that all captured individuals were female, mortality of individuals migrating through the state may negativelyimpact this species across its range.

Although Silver-haired Bats a re a common species, their consistent detection throughout the winter at the Big Sky Mine site is noteworthy. This species is predominately a tree roosting bat, although it has been recorded using s tructures, caves and mines outside of Montana (reviewed in Maxell 2015). To date we have not detected this species during cave or mine surveys across the state in either the active season or over winter. The most likely hibernacula in proximity to this detector are a butte $300 \mathrm{~m}$ to the east that has sandstone outcrops and cliffs, or other smaller sandstone outcrops in the fores ted area to the west of the reservoir. This site is unique a mong network sites with winter detections of this species as the potential hibernacula in proximity are limited. Although we cannot be certain what features the animals are roosting in, rock outcrops are likely and could be confirmed with roost surveys.

Southcentral and southeastern Montana have a relatively diverse community of bat species. Of the $15 \mathrm{~s}$ pecies found across the state, 13 are found within this geographic area, and 12 of thes e were detected across all sites. Although sites averaged 8.5 species, not all species pres ent a re consistently detected using acoustic methods, even a cross years of recording. Quiet species like Townsend's Big-eared Bat may not trigger the detector and species like the Longlegged Myotis have calls similar to other Myotis and confirmation of species is impossible for most s equences. Species like Spotted Bat and Pallid Bat are on the northern edge of their range and may be locally common but ra re across the landscape. Fringed Myotis has been not been consistently detected across its rage within eastern Montana and since it can be identified using acoustic methods, its absence from all but one site is probably due to its rarity.

Across all years, bat activity a ppeared stable at all sites regardless of previous history of mining or otheranthropogenic uses. The most 
noticeable difference in activity between years is caused by the transition from the older SMXUX and newer SMX-U1 microphones in 2014 (Figures 6 \& 7). Aside from this, the increase or decrease in subsequent years at any site does not appear to exceed the variation found across years. If previous, current, or proposed mining activities at a given site or a rea were having a detectable impact on abundance of detected species, we would expect to see declines in the a mount of activity recorded a cross all species over time or within auto identified s pecies groups. This trend was not apparent at any site or within any of the auto identified species trends.

Species presence and activity metrics recorded at thes e sites will serve as robust baseline that can be us ed to assess the status of populations at sites into the future. This is particularly important due to the imminent threats to bat species posed by White-Nose Syndromeand wind energy development.

To-date, the presence of $\mathrm{Pd}$ and ass ociated WNS have not been detected in Montana. However, detections of Pd and WNS in Was hington in 2015 (Lorch et al. 2016), and the continued spread westward into the Great Plains have increased the urgency for establishing baseline metrics to assess future impacts on resident bats. Of the 12 species detected within the project a rea, two have been shown to develop WNS when exposed to $\mathrm{Pd}$. These species are the Big Brown Bat, and Little Brown Myotis. Additionally, the Silverhaired Bat, Eastern Red Bat, and Townsend's Big-eared Bat have been shown to carry Pd, but not exhibit symptoms ofWNS (Table 1 ). The remaining Myotis s pecies have not been shown to carry Pd or develop WNS. Rather than indicating immunity, the lack of detections of $\mathrm{Pd}$ positive individuals or WNS is likely a result of their western distribution that does not overlap affected areas. As many other Myotis species areimpacted by WNS, it is probably best to consider these species as susceptible until proven otherwise.

Tree roos ting species such as the Hoary Bat, Eastern Red Bat, and Silver-haired Bat a re not known to be susceptible to WNS, but suffer mortality at wind farms. These species often fly near turbines, and suffer barotra uma when in close proximity to the turbine blades. Due to thes e species low reproductive rate and long life, unmitigated wind energy development may cause precipitous declines of these species over the next 50 years (Frick et al. 2017). Wind energy has not been developed on any of the sites, but this threat may have indirect impacts on bats using this site due to mortality during migration or decreased regional populations. If development of wind energy is considered within the local area, mitigation measures should be implemented to reduce potential impacts on resident and migratory s pecies.

High levels of winter activity at the Signal Peak and Big Sky mines indicates proximity to hibernacula. Species identified as active during these times include: Big Brown, Western Smallfooted Myotis, Little Brown Myotis, Long-eared Myotis, and Silver-haired Bat. All have been known to use cracks and crevices in rock outcrops and badlands as winter hibernacula (see Appendix C for information on hibernacula preference). Because no known caves are in proximity to these sites, it is likely that rock outcrops or otherfeatures including abandoned mines a re providing overwintering habitat. If mining activities alter rock outcrops or badlands, roost searches in both the active and hibernation seasons should be conducted to determine the potential impact of these activities to bats and acoustic monitoring should continue to assess impacts on activity over the duration of the project.

Bats also may use both active, inactive, and abandoned mines as roost and hibernacula. If an adit is being considered for closure, first determine its workings a re being used by bats. Bats may move between roosts over a season, 
so multiple surveys a re often necessary to determine if the mine is used as an active season roost. If conditions all ow safe entry into the mine, a winter survey should be conducted between late December and April to determine if it is used as a hibernacul um. If pos sible, mines with bat us e should be decommissioned with a bat friendly gate. See Sherwin et al. 2009 for a guidance on determining bat use and implementation of bat friendly closures.

Across the acoustic network and within the study a rea, detectors placed near water recorded high levels of activity. As open water is essential for bats, ensure that waterbodies remain accessible, free from clutter, and regularly contain water throughout the active season. As many of the mine sites are in relatively xeric environments, each individual waterbody is valuable to s upporting local populations. Bats drink on the wing, and while smaller species (e.g. Myotis bats) may be able to exploit small a reas of standing water, larger less maneuverable species such as Hoary and Spotted Bats need clear flight paths and large areas of open water to drink.

While we did not include the Northern Myotis (Myotis septentrionalis) as a potential species, the US FWS has designated Powder River County a long with eight other counties in eas tern Montana as within the range of this species. Therefore, the Northern Myotis could possibly be present at Otter Creek Coal Tract. Within Wyoming, and the Dakotas, this species has been found in both coniferous uplands and ripa rian hardwood fores ts (Miller and Allen 1928, Tigner and Aney 1993, Tigner and Aney 1994, Worthington and Bogan 1993, Tigner and Stukel 2003, Griscom and Keinath 2011, Peurach 2017). In Montana the species has been found at five sites along the Missouri River at or downs tream from Culbertson (MT NHP point obs ervation data, 2018). Bat surveys of hardwood and conifer forest habitats across the nine counties including Powder River have been conducted either to document species diversity or specifically to detect Northern Myotis. Within Powder River County 21 sites have been surveyed with mist nets, and 156 s ites have had been surveyed with acoustic detectors for at least one night. Across these surveys no Northern Myotis have been captured or recorded. Targeted surveys within the island buttes of Carter County have produced similar results as have the surveys of ripa rian forest along the Little Missouri, Tongue, and Powder Rivers within Montana. Although it is difficult to definitivelydetermine true absence ofa species, given the number of surveys conducted in Powder River County that have failed to detect this species, its presence appears unlikely. 


\section{ManAgement ReCOMmendations}

Measures of overall bat activity near the detector, hand confirmed presence of individual species by month, and hand confirmed minimum temperatures associated with bat pas ses of individual s pecies are all stable metrics upon which management recommendations can be made. However, patterns of activity of individual species resulting from automated analyses should be used with a great deal of caution due to low rates of pecies assignment and low or uncertain rates of accuracy of those assignments. Furthermore, it s hould be noted that bat activity measured during this s tudy was made by a microphone on a nine to ten-foot mast and may not have a dequately sampled the activity of high flying bats such as the Hoa ry Bat and Silver-haired Bat, which together with the Eastern Red Bat are the three species that have suffered approximately $75 \%$ of the documented mortalities associated with wind turbines across North America (Kunzet al. 2007). Thus, the following management recommendations avoid use of activity patterns of individual species as determined by a utomated a nalyses and instead rely on results of hand confirmed analyses, general patterns of bat activity that were recorded at the study site, and results of published studies of wind turbine impacts on bat species.

General management recommendations for species observed at project sites include:

(1) protect potential natural roost sites by conserving large diameter trees (especially snags with loose bark), rock outcrops, cliff crevices, and caves (Appendix C).

(2) maintain accessibility for underground mine entrances that bats may be using as summer or winter roosts. Install bat friendly gates if closure is required.
(3) When removing bat colonies from buildings or other structures follow current best practices, including waiting until the late fall and winter to seal entry points and placing bat houses to compensate for elimination of the roost.

(4) Reduce structural complexity of vegetation (e.g., short stature grasslands) and availability of standing waters in proximity to wind turbines or other human structures that might represent a threat to bats or where bats are undesired.

(5) In s afe environments, maintain lotic or lentic waterbodies to provide habitat for foraging and drinking.

(6) If wind turbines are installed in the region, set turbine cut-in speeds to $>6.0 \mathrm{~m} / \mathrm{sec}$ between April and October-especially important in July during peak bat activity when young are newly flighted, and August, September, and October when migratory species are passing through and local bats are swarming and breeding. Feather wind turbine blades, making them parallel to wind direction, when wind speeds a re $<6 \mathrm{~m} / \mathrm{sec}$ to reduce risk of ba rotra uma during times of relatively high bat activity.

(7) Report dead bats of any species found in the winter or spring to Montana Fish Wildlife and Parks or Montana Natural Heritage Program pers onnel. Animals found dead during these seasons may have contracted WNS and should be testedas part of Montana's Passive WNS surveillance protocol. 


\section{Literature Cited}

Agnarsson I, C.M. Zambrana-Torrelio, N.P. FloresSaldana, and L.J. May-Collado. 2011. A timecalibrated species-level phylogeny of bats (Chiroptera, Mammalia). PLOS Currents Tree of Life. 2011 Feb 4. Edition 1. doi: 10.1371/currents. RRN1212.

Agranat, I. 2014. Detecting bats with ultrasonic microphones: understanding the effects of microphone variance and placement on detection rates. Unpublished white paper. Wildlife Acoustics, Maynard, MA. 14 pp.

[AWEA] American Wind Energy Association. 2015. Wind energy industry a nnounces new voluntary practices to reduce overall impacts on bats by 30 percent. American Wind Energy Association Press Release. September 3, 2015. Accessed at: http://www.awea.org/MediaCenter/pressrele ase.aspx? ItemNumber $=7833$

Arnett, E.B., W.K. Brown, W.P. Erickson, J.K. Fiedler, B.L. Ha milton, T.H. Henry, A. Jain, G.D. Johns on, J. Kerns, R.R. Koford, C.P. Nicholson, T.J. O'Connell, M.D. Piorkowski, and R.D. Tankersley, Jr. 2008. Patterns of bat fatalities at wind energy facilities in North America. Journal of Wildlife Management 72(1):61-78.

Arnett, E.B., M.M.P. Huso, M.R. Schirmacher, and J.P. Hayes. 2011. Altering turbine speed reduces bat mortalityat wind-energy facilities. Frontiers in Ecology and the Environment 9(4):209-214.

Baerwald, E.F., J. Edworthy, M. Holder, and R.M.R. Barclay. 2009. Alarge-scale mitigation experiment to reduce bat fatalities at wind energy facilities. Journal of Wildlife Management 73(7):1077-1081.
Barclay, R.M. and L.D. Harder. 2003. Life histories of bats: life in the slow lane. Pp. 209-256 In: T.H. Kunz and M.B. Fenton (eds.) Bat Ecology. Chicago: University of Chicago Press. 779p.

Bernard, R.F., J.T. Foster, E.V. Willcox, K.L. Parise, and G.F. McCracken. 2015. Molecular detection of the caus ative agent of Whitenose Syndrome on Rafinesque's big-eared bats (Corynorhinusrafinesquii) and two species of migratory bats in the southeastern USA. Journal of Wildlife Diseases 51(2):519-522.

Blehert, D.S., A.C. Hicks, M. Behr, C.U. Meteyer, B.M. Berlowski-Zier, E.L. Buckles, J.T.H. Coleman, S.R. Darling, A. Gargas, R. Niver, J.C. Okoniewski, R.J. Rudd, and W.B. Stone. 2008. Bat white-nose syndrome: an emerging fungal pathogen? Science 323: 227. DOI: $10.1126 /$ science. 1163874

Blehert, D.S., J.M. Lorch, A.E. Ballmann, P.M. Cryan, and C.U. Meteyer. 2011. Bat white-nose syndrome in North America. Microbe Magazine 6:267-273.

Chambers, C.L., M.J. Herder, K. Yasuda, D.G. Mikesic, S.M. Dewhurst, W.M. Masters, and d. Vleck. 2011. Roosts and home ranges of spotted bats (Euderma maculatum) in northern Arizona. Cana dian journal of zoology 89(12): 1256-1267.

[COSEWIC] Committee on the Status of Endangered Wildlife in Canada. 3 February 2012. Emergency assessment concludes that three bat s pecies a re endange red in Canada. http://www.cosewic.gc.ca/eng/sct7/Bat_Emer gency_Assessment_Press_Release_e.cfm

Cryan, P.M. 2008. Mating behavior as a possible caus e of bat fatalities at wind turbines. Journal of Wildlife Management 72(3): 845-849.

Foresman, K.R. 2012. Mammals of Montana. Second edition. Mountain Press Publishing, Mis soula, Montana. 429 pp.

Frank, C.L., A. Michalski, A.A. McDonough, M. Rahimian, R.J. Rudd, and C. Herzog. 2014. The resistance of a North American bat species 
(Eptesicus fuscus) to White-Nose Syndrome (WNS). Plos One 9:e113958.

DOI:10.1371/2Fjournal.pone.0113958.

Frick W.F., J.F. Pollock, A.C. Hicks, K.E. Langwig, D.S. Reynolds, G.G. Turner, C.M. Butchkoski, and T.H. Kunz. 2010. An emerging disease causes regional population collapse of a common North American bat s pecies. Science 329:679-682. DOI:10.1126/science.1188594.

Frick, W.F., E.F. Baerwald, J.F. Pollock, R.M.R. Barclay, J.A. Szymanski, T.J. Weller, A.L. Russell, S.C. Loeb, R.A. Medellin, and L.P. McGuire. 2017. Fatalities at wind turbines may threaten population viability of a migratory bat. Biological Conservation 209: 172-177.

Griscom, H.R. and D.A. Keinath. 2011. Inventory and status of bats at Devil's Tower National Monument. Report prepared for the USDI National Park Service by the Wyoming Natural Diversity Database. University of Wyoming, Laramie, WY. 34 pp.

Hayes, M. 2013. Bats killed in large numbers at United States wind energy facilities. BioScience 63(12):975-979.

Johns on, G.D., M.K. Perlik, W.P. Erickson, and M.D. Strickland. 2004. Bat activity, composition, and collision mortality at large wind plant in Minnesota. Wildlife Society Bulletin 32(4):1278-1288.

Johns on J.S., D.M. Reeder DM, J.W. McMichael III, M.B. Meierhofer, D.W.F. Stern, S.S. Lumadue, L.E. Sigler, H.D. Winters, M.E. Vodzak, A. Kurta, J.A. Kath, and K.A. Field. 2014. Host, pathogen, and environmental characteristics predict white-nose syndrome mortality in captive little brown myotis (Myotis/ucifugus). PLoS ONE 9(11): e112502.

DOI:10.1371/journal.pone.0112502

Kunz, T.H., E.B. Arnett, W.P. Erickson, A.R. Hoar, G.D. Johnson, R.P. Larkin, M.D. Strickland, R.W. Thresher, and M.D. Tuttle. 2007. Ecological impacts of wind energy development on bats: questions, research needs, and hypotheses. Frontiers in Ecology and the Environment 5(6):315-324.
Kunz, T.H. and J.D. Reichard. 2010. Status review of the Little Brown Myotis (Myotis/ucifugus) and determination that immediate listing under the Endangered Species Act is scientifically and legally warranted. $30 \mathrm{pp}$.

Langwig, K.E., W.F. Frick, J.T. Bried, A.C. Hicks, T.H. Kunz, and A.M. Kilpatrick. 2012. Ecology Letters 15:1050-1057. DOI: 10.1111/j.14610248.2012.01829.x

Langwig, K.E., W.F. Frick, R. Reynolds, K.L. Parise, K.P. Drees, J.R. Hoyt, T.L. Cheng, T.H. Kunz, J.T. Foster, and A.M. Kilpatrick. 2014. Host and pathogen ecology drive the seasonal dynamics of a fungal disease, white-nose syndrome. Proceedings Royal Society B 282: 20142335. DOI: $10.1098 / \mathrm{rs} \mathrm{pb} .2014 .2335$

Lausen, C.L. and R.M.R. Barclay. 2006. Winter bat activity in the Canadian prairies. Canadian Journal of Zoology 84:1079-1086.

Lorch J.M., C.U. Meteyer, M.J. Behr, J.G. Boyles, P.M. Cryan, A.C. Hicks, A.E. Ballmann, J.T.H. Coleman, D.N. Redell, D.M. Reeder, and D.S. Blehert. 2011. Experimental infection of bats with Geomyces destructans causes white-nose syndrome. Nature 480:376-378. DOI:10.1038/nature10590.

Lorch, J.M., J.M. Palmer, D. L. Lindner, A.E. Ballmann, K.G. George, K. Griffin, S. Knowles. 2016. First detection of bat white-nose syndrome in western North America. mSphere 1(4):00148-16.

Maxell, B.A. Coordinator. 2015. Montana Bat and White-Nose Syndrome Surveillance Plan and Protocols 2012-2016. Montana Natural Heritage Program. Helena, MT. 185 pp.

Miller, G. and G. Allen. 1928. The American bats of the genera Myotis and Pizonyx. Bulletin of the United States National Museum 144: 1218.

Minnis, A.M. and D.L. Lindner. 2013. Phylogenetic evaluation of Geomyces and allies reveals no close relatives of Pseudogymnoascus destructans, comb. nov., in hibernacula of eastern North America. Fungal Biology 117(9):638-649. 
[MTNHP] Montana Natural Heritage Program. 2017. Animal point observation da ta base. Montana Natural Heritage Program. Helena, MT. Accessed June 2017.

Parsons, S. and J.M. Szewczak. 2009. Detecting, recording, and a nalyzing the vocalizations of bats. Pp. 91-111 In: Kunz, T.H. and S. Parsons. Ecological and behavioral methods for the study of bats. $2^{\text {nd }}$ edition. Johns Hopkins University Press. Baltimore, MD.

Peurach, S. Confirmation and coll ection details of Myotis septentrionalis specimens housed at the National Mus eum of Natural History. 25 January 2017.

Poulton, V. and W. Erickson. 2010. Postconstruction bat and bird fa ta lity study Judith Gap Wind Farm Wheatland County, Montana. Final Report. Results from June-October 2009 study and comparison with 2006-2007 study. Western Ecosystems Technology, Inc. 2003 Central Avenue, Cheyenne, WY. 35 p.

Rabe, M.J., M.S. Siders, C.R. Miller, and T.K. Snow. 1998. Long foraging distance for a s potted bat (Euderma maculatum) in northern Arizona. The Southwestern Naturalist 43(2): 266-269.

Schuster, E., L. Bulling, and J. Koppel. 2015. Consolidating the state of knowledge: a synoptical review of wind energy's wildlife effects. Environmental Management 56:300331.

Sherwin, R.E., J.S. Altenbach, and D.L. Waldien. 2009. Managing abandoned mines for bats. Bat Cons ervation International. 103 pp.

Smallwood, K.S. 2013. Comparing bird and bat fatality-rate estimates a mong North American wind-energy projects. Wildlife Society Bulletin 37(1):19-33.

Tigner, J. and W.C. Aney. 1993. Report of the northern Black Hills bat survey. Spearfish/Nemo Ranger District, Black Hills National Forest. Unpublished Report. 16 pp.

Tigner, J. and W.C. Aney. 1994. Report of Black Hills bat survey. Spearfish/Nemo Ranger District, Black Hills National Forest. Unpublis hed Report. 19 pp.
Tigner, J. and E. D. Stukel. 2003. Bats of the Black Hills: a description of status and conservation needs. South Dakota Department of Game, Fish, and Parks Wildlife Division Report 2003$05.94 \mathrm{p}$.

U.S. Fish and Wildlife Service. 2012. North American bat death toll exceeds 5.5 million from white-nose syndrome. News Release. January 17, 2012.

U.S. Fish and Wildlife Service. 2014. Bats affected by WNS. Accessed 22 December 2014. https://www.whitenosesyndrome.org/about/ bats-affected-wns

U.S. Fish and Wildlife Service. 2015. Endangered and threatened wildlife and plants; threatened species status for the Northern Long-eared Bat with 4(d) rule; final rule and interim rule. Federal Register 80(63):17974-18033.

Warnecke L., J.M. Turner, T.K. Bollinger, J.M. Lorch, V. Mis ra, P.M. Cryan, G. Wibbelt, D.S. Blehert, and C.K.R. Willis. 2012. Inoculation of bats with European Geomyces destructans supports the novel pathogen hypothesis for the origin of white-nose syndrome. Proceedings of the National Academy of Sciences 109:6999-7003. DOI:10.1073/pnas.1200374109

Worthington, D.J. and H.N. Ross. 1990. Abundance and distribution of bats in the Pryor Mountains of south central Montana. Montana Natural Heritage Program, Helena, MT. 20 pp.

Worthington, D.J. 1991. Abundance and distribution of bats in the Pryor Mountains of south central Montana and north eastern Wyoming. Montana Natural Heritage Program, Helena, MT. 23 pp.

Worthington, D.J. and M.A. Bogan. 1993. Cave entrance modification and potential impact on bat populations at Jewel Cave National Monument, South Dakota. Unpublished Report. 10 pp. 
Table 1. Montana bat species, conservation status, and known or potential concerns from WNS and wind turbine facilities.

\begin{tabular}{|c|c|c|c|}
\hline Species & Conservation Status & $\begin{array}{l}\text { Species known to be affected by } \\
\text { White-Nose Syndrome / P. destructans }\end{array}$ & $\begin{array}{l}\text { Species known to be subject } \\
\text { to mortality at wind turbines }\end{array}$ \\
\hline $\begin{array}{c}\text { Pallid Bat } \\
\text { (Antrozous pallidus) }=\text { ANPA }\end{array}$ & $\begin{array}{l}\text { G4 S3, MT SOC, BLM } \\
\text { Sensitive, USFS Sensitive }\end{array}$ & No connection known at this time. & $\begin{array}{l}\text { No mortalities documented in } \\
\text { literature. }\end{array}$ \\
\hline $\begin{array}{c}\text { Townsend's Big-eared Bat } \\
\text { (Corynorhinus townsendii) = СОТО }\end{array}$ & \begin{tabular}{|l} 
G4 S3, MT SOC, BLM \\
Sensitive, USFS Sensitive
\end{tabular} & $\begin{array}{l}\text { Detected, but no diagnostic sign of WNS (USFWS 2014). } \\
\text { Potential winter roost vector. }\end{array}$ & $\begin{array}{l}\text { No mortalities documented in } \\
\text { literature. }\end{array}$ \\
\hline $\begin{array}{c}\text { Big Brown Bat } \\
\text { (Eptesicus fuscus) = EPFU }\end{array}$ & G5 S4 & $\begin{array}{l}\text { Blehert et al. 2008, Langwig et al. 2012, 2014, Frank et al. } \\
2014 .\end{array}$ & $\begin{array}{l}\text { Johnson et al. 2004; Kunz et al. } \\
\text { 2007; Arnett et al. 2008, } 2011 .\end{array}$ \\
\hline $\begin{array}{c}\text { Spotted Bat } \\
(\text { Euderma maculatum })=\text { EUMA }\end{array}$ & \begin{tabular}{|l|} 
G4 S3, MT SOC, BLM \\
Sensitive, USFS Sensitive
\end{tabular} & No connection known at this time. & $\begin{array}{l}\text { No mortalities documented in } \\
\text { literature. }\end{array}$ \\
\hline $\begin{array}{c}\text { Silver-haired Bat } \\
\text { (Lasionycteris noctivagans) }=\text { LANO }\end{array}$ & G3G4, Potential MT SOC & $\begin{array}{l}\text { Detected, but no diagnostic sign of WNS (Bernard et al. 2015, } \\
\text { USFWS 2014). Potential regional migratory vector. }\end{array}$ & $\begin{array}{l}\text { Johnson et al. 2004; Kunz et al. } \\
\text { 2007; Arnett et al. 2008, 2011; } \\
\text { Baerwald et al. 2009; Poulton } \\
\text { and Erickson 2010. }\end{array}$ \\
\hline $\begin{array}{c}\text { Eastern Red Bat } \\
\text { (Lasiurus borealis) }=\text { LABO }\end{array}$ & $\begin{array}{l}\text { G3G4 SU, Potential MT } \\
\text { SOC }\end{array}$ & $\begin{array}{l}\text { Detected, but no diagnostic sign of WNS (Bernard et al. 2015, } \\
\text { USFWS 2014). Potential regional migratory vector. }\end{array}$ & $\begin{array}{l}\text { Kunz et al. 2007; Arnett et al. } \\
2008,2011 .\end{array}$ \\
\hline $\begin{array}{c}\text { Hoary Bat } \\
\text { (Lasiurus cinereus })=\mathrm{LACl}\end{array}$ & G3G4 S3, MT SOC & No connection known at this time. & $\begin{array}{l}\text { Johnson et al. 2004; Kunz et al. } \\
\text { 2007; Arnett et al. 2008, 2011; } \\
\text { Baerwald et al. 2009; Poulton } \\
\text { and Erickson 2010. }\end{array}$ \\
\hline $\begin{array}{c}\text { California Myotis } \\
\text { (Myotis californicus) }=\text { MYCA }\end{array}$ & G5 S4 & $\begin{array}{l}\text { Close relatedness to M. leibii indicates possible susceptibility } \\
\text { (Agnarsson et al. 2011, Langwig et al. 2012) }\end{array}$ & $\begin{array}{l}\text { No mortalities documented in } \\
\text { literature. }\end{array}$ \\
\hline $\begin{array}{l}\text { Western Small-footed Myotis } \\
(\text { Myotis ciliolabrum })=\mathrm{MYCl}\end{array}$ & G5 S4 & $\begin{array}{l}\text { Relatively close relatedness to M. lucifugus indicates possible } \\
\text { susceptibility (Frick et al. 2010, Agnarsson et al. 2011) }\end{array}$ & $\begin{array}{l}\text { No mortalities documented in } \\
\text { literature. }\end{array}$ \\
\hline $\begin{array}{c}\text { Long-eared Myotis } \\
\text { (Myotis evotis) }=\text { MYEV }\end{array}$ & G5 S4 & $\begin{array}{l}\text { Close relatedness to } M \text {. sodalis indicates possible } \\
\text { susceptibility (Agnarsson et al. 2011, Langwig et al. 2012) }\end{array}$ & Kunz et al. 2007 \\
\hline $\begin{array}{l}\text { Little Brown Myotis } \\
\text { (Myotis lucifugus) }=\text { MYLU }\end{array}$ & G3 S3, MT SOC & $\begin{array}{l}\text { Blehert et al. 2008, Frick et al. 2010, Lorch et al. 2011, } \\
\text { Warnecke et al. 2012, Johnson et al. 2014, Langwig et al. } \\
\text { 2012, } 2014 .\end{array}$ & $\begin{array}{l}\text { Johnson et al. 2004; Kunz et al. } \\
\text { 2007; Arnett et al. 2008, } 2011 .\end{array}$ \\
\hline $\begin{array}{c}\text { Northern Myotis } \\
\text { (Myotis septentrionalis) }=\text { MYSE }\end{array}$ & $\begin{array}{l}\text { G1G2 SU, BLM Special } \\
\text { Status, USFS Threatened, } \\
\text { USFWS Listed Threatened }\end{array}$ & Blehert et al. 2008, Langwig et al. 2012, 2014, USFWS 2015. & $\begin{array}{l}\text { Kunz et al. 2007; Arnett et al. } \\
2008\end{array}$ \\
\hline $\begin{array}{c}\text { Fringed Myotis } \\
\text { (Myotis thysanodes) }=\text { MYTH }\end{array}$ & $\begin{array}{l}\text { G4 S3, MT SOC, BLM } \\
\text { Sensitive }\end{array}$ & $\begin{array}{l}\text { Relatively close relatedness to M. lucifugus indicates possible } \\
\text { susceptibility (Frick et al. 2010, Agnarsson et al. 2011) }\end{array}$ & $\begin{array}{l}\text { No mortalities documented in } \\
\text { literature. }\end{array}$ \\
\hline $\begin{array}{c}\text { Long-legged Myotis } \\
\text { (Myotis volans) }=\text { MYVO }\end{array}$ & G4G5 S4 & $\begin{array}{l}\text { Close relatedness to } M \text {. sodalis indicates possible } \\
\text { susceptibility (Agnarsson et al. 2011, Langwig et al. 2012) }\end{array}$ & $\begin{array}{l}\text { No mortalities documented in } \\
\text { literature. }\end{array}$ \\
\hline $\begin{array}{c}\text { Yuma Myotis } \\
\text { (Myotis yumanensis) }=\text { MYYU }\end{array}$ & $\begin{array}{l}\text { G5 S3S4, Potential MT } \\
\text { SOC }\end{array}$ & Susceptible (USFWS 2017) & $\begin{array}{l}\text { No mortalities documented in } \\
\text { literature. }\end{array}$ \\
\hline
\end{tabular}

*Unidentified Myotis species mortalities have also been reported at the Judith Gap Wind Farm (Poulton and Erickson 2010). 
Table 2a. Bat species documented and potentially present at or within $50 \mathrm{~km}$ of the West Decker Mine ${ }^{1}$.

\begin{tabular}{|c|c|c|c|c|c|c|c|c|c|c|c|c|}
\hline Species & Jan & Feb & March & April & May & June & July & Aug & Sept & Oct & Nov & Dec \\
\hline $\begin{array}{c}\text { Pallid Bat } \\
\text { (Antrozous pallidus) }\end{array}$ & & & & & $C(1)$ & & & $C(1)$ & & & & \\
\hline $\begin{array}{l}\text { Townsend's Big-eared Bat } \\
\text { (Corynorhinus townsendii) }\end{array}$ & & & & & & & & C (3) & & & & \\
\hline $\begin{array}{c}\text { Big Brown Bat } \\
\text { (Eptesicus fuscus) }\end{array}$ & & & & & & & $C(3)$ & C (3) & & & & \\
\hline $\begin{array}{c}\text { Spotted Bat } \\
\text { (Euderma maculatum) }\end{array}$ & & & & & & & $A(3)$ & $A(2)$ & & & & \\
\hline $\begin{array}{c}\text { Hoary Bat } \\
\text { (Lasiurus cinereus) }\end{array}$ & & & & & & $A(1)$ & & $\begin{array}{l}A(1) \\
C(1)\end{array}$ & & & & \\
\hline $\begin{array}{c}\text { Silver-haired Bat } \\
\text { (Lasionycteris noctivagans) }\end{array}$ & & & & & & $A(1)$ & & $C(3)$ & & & & \\
\hline $\begin{array}{l}\text { Eastern Red Bat } \\
\text { (Lasiurus borealis) }\end{array}$ & & & & & & & & $\begin{array}{l}\text { A (1) } \\
C \text { (1) }\end{array}$ & & & & \\
\hline $\begin{array}{l}\text { Western Small-footed Myotis } \\
\text { (Myotis ciliolabrum) }\end{array}$ & & & & & & $\begin{array}{l}C(7) \\
\text { A (2) }\end{array}$ & $C(10)$ & $A(1)$ & $C(2)$ & & & \\
\hline $\begin{array}{l}\text { Long-eared Myotis } \\
\text { (Myotis evotis) }\end{array}$ & & & & & $C(1)$ & $C(3)$ & $C(9)$ & $C(3)$ & $C(1)$ & & & \\
\hline $\begin{array}{l}\text { Little Brown Myotis } \\
\text { (Myotis lucifugus) }\end{array}$ & & & & & & $C(1)$ & $C(1)$ & $C(2)$ & $C(1)$ & & & \\
\hline $\begin{array}{c}\text { Fringed Myotis } \\
\text { (Myotis thysanodes) }\end{array}$ & & & & & & & & & & & & \\
\hline $\begin{array}{l}\text { Long-legged Myotis } \\
\text { (Myotis volans) }\end{array}$ & & & & & & & $C(5)$ & $C(1)$ & & & & \\
\hline
\end{tabular}

${ }^{1}$ Number of records in the point observation database at the Montana Natural Heritage Program prior to this study (MTNHP 2017). A = acoustic record. C $=$ capture record. Records may include multiple individuals. 
Table 2b. Bat species documented and potentially present at or within $50 \mathrm{~km}$ of the Spring Creek Mine ${ }^{1}$.

\begin{tabular}{|c|c|c|c|c|c|c|c|c|c|c|c|c|}
\hline Species & Jan & Feb & March & April & May & June & July & Aug & Sept & Oct & Nov & Dec \\
\hline $\begin{array}{c}\text { Pallid Bat } \\
\text { (Antrozous pallidus) }\end{array}$ & & & & & $C(1)$ & & & $C(4)$ & & & & \\
\hline $\begin{array}{l}\text { Townsend's Big-eared Bat } \\
\text { (Corynorhinus townsendii) }\end{array}$ & & & & & & & & C (3) & $C(1)$ & & & \\
\hline $\begin{array}{c}\text { Big Brown Bat } \\
\text { (Eptesicus fuscus) }\end{array}$ & & & & & & & $C(7)$ & $C(2)$ & & & & \\
\hline \multicolumn{13}{|l|}{$\begin{array}{c}\text { Spotted Bat } \\
\text { (Euderma maculatum) }\end{array}$} \\
\hline $\begin{array}{c}\text { Hoary Bat } \\
\text { (Lasiurus cinereus) }\end{array}$ & & & & & & $A(1)$ & & $\begin{array}{l}C(1) \\
A(1)\end{array}$ & & & & \\
\hline $\begin{array}{c}\text { Silver-haired Bat } \\
\text { (Lasionycteris noctivagans) }\end{array}$ & & & & & & $A(1)$ & & $C(2)$ & & & & \\
\hline $\begin{array}{l}\text { Eastern Red Bat } \\
\text { (Lasiurus borealis) }\end{array}$ & & & & & & & & $\begin{array}{l}C(1) \\
A(1)\end{array}$ & & & & \\
\hline $\begin{array}{l}\text { Western Small-footed Myotis } \\
\text { (Myotis ciliolabrum) }\end{array}$ & & & & & & $\begin{array}{l}C(5) \\
A(2)\end{array}$ & $C(10)$ & $A(1)$ & $C(1)$ & & & \\
\hline $\begin{array}{l}\text { Long-eared Myotis } \\
\text { (Myotis evotis) }\end{array}$ & & & & & $C(1)$ & $C(3)$ & $C(7)$ & $C(4)$ & $C(1)$ & & & \\
\hline $\begin{array}{l}\text { Little Brown Myotis } \\
\text { (Myotis lucifugus) }\end{array}$ & & & & & & $C(1)$ & $C(1)$ & $C(2)$ & $C(1)$ & & & \\
\hline \multicolumn{13}{|l|}{$\begin{array}{c}\text { Fringed Myotis } \\
\text { (Myotis thysanodes) }\end{array}$} \\
\hline $\begin{array}{l}\text { Long-legged Myotis } \\
\text { (Myotis volans) }\end{array}$ & & & & & & & & $C(4)$ & $C(1)$ & & & \\
\hline
\end{tabular}

$1 \quad$ Number of records in the point observation database at the Montana Natural Heritage Program prior to this study (MTNHP 2017). A $=$ acoustic record. C $=$ capture record. Records may include multiple individuals. 
Table 2c. Bat species documented and potentially present at or within $50 \mathrm{~km}$ the Otter Creek Coal Tract ${ }^{1}$.

\begin{tabular}{|c|c|c|c|c|c|c|c|c|c|c|c|c|}
\hline Species & Jan & Feb & March & April & May & June & July & Aug & Sept & Oct & Nov & Dec \\
\hline $\begin{array}{c}\text { Pallid Bat } \\
\text { (Antrozous pallidus) }\end{array}$ & & & & & $C(2)$ & & $C(1)$ & $C(1)$ & & & & \\
\hline $\begin{array}{l}\text { Townsend's Big-eared Bat } \\
\text { (Corynorhinus townsendii) }\end{array}$ & & & & & $C(1)$ & $A(2)$ & & $\begin{array}{l}C(1) \\
A(1)\end{array}$ & $A(1)$ & $A(1)$ & & \\
\hline $\begin{array}{c}\text { Big Brown Bat } \\
\text { (Eptesicus fuscus) }\end{array}$ & & & & & $A(4)$ & $C(1)$ & $\begin{array}{l}C(4) \\
A(1)\end{array}$ & $\begin{array}{l}C(5) \\
A(3)\end{array}$ & $A(3)$ & & & \\
\hline $\begin{array}{c}\text { Spotted Bat } \\
\text { (Euderma maculatum) }\end{array}$ & & & & & & cted be & $\begin{array}{l}A(1) * \\
\text { een May }\end{array}$ & hd Septer & & & & \\
\hline $\begin{array}{c}\text { Hoary Bat } \\
\text { (Lasiurus cinereus) }\end{array}$ & & & & & $A(4)$ & $A(2)$ & $\begin{array}{l}C(3) \\
A(2)\end{array}$ & $\begin{array}{l}C(3) \\
A(7)\end{array}$ & $A(1)$ & & & \\
\hline $\begin{array}{c}\text { Silver-haired Bat } \\
\text { (Lasionycteris noctivagans) }\end{array}$ & & & & & $A(3)$ & $A(1)$ & $A(3)$ & $\begin{array}{c}C(5) \\
A(11)\end{array}$ & $A(2)$ & & & \\
\hline $\begin{array}{l}\text { Eastern Red Bat } \\
\text { (Lasiurus borealis) }\end{array}$ & & & & & & & & $\begin{array}{l}C(1) \\
A(2)\end{array}$ & & & & \\
\hline $\begin{array}{l}\text { Western Small-footed Myotis } \\
\text { (Myotis ciliolabrum) }\end{array}$ & & & & & $A(3)$ & $\begin{array}{l}\text { C (5) } \\
A(3)\end{array}$ & $\begin{array}{l}C(7) \\
A(3)\end{array}$ & $A(10)$ & $A(1)$ & & & \\
\hline $\begin{array}{l}\text { Long-eared Myotis } \\
\text { (Myotis evotis) }\end{array}$ & & & & $A(1)$ & $\begin{array}{l}C(1) \\
A(6)\end{array}$ & $\begin{array}{l}\text { C (8) } \\
A(1)\end{array}$ & $C(7)$ & $\begin{array}{l}C(4) \\
A(4)\end{array}$ & $A(1)$ & & & \\
\hline $\begin{array}{l}\text { Little Brown Myotis } \\
\text { (Myotis lucifugus) }\end{array}$ & & & & & $A(2)$ & $C(1)$ & $C(1)$ & $A(6)$ & & & & \\
\hline $\begin{array}{l}\text { Fringed Myotis } \\
\text { (Myotis thysanodes) }\end{array}$ & & & & $A(1)$ & $A(1)$ & & $C(2)$ & & & & & \\
\hline $\begin{array}{l}\text { Long-legged Myotis } \\
\text { (Myotis volans) }\end{array}$ & & & & & & & $C(1)$ & $C(4)$ & & & & \\
\hline
\end{tabular}

1 Number of records in the point observation database at the Montana Natural Heritage Program prior to this study (MTNHP 2017). A $=$ acoustic record. C $=$ capture record. Records may include multiple individuals. 
Table 2d. Bat species documented and potentially present at or within $50 \mathrm{~km}$ the Big Sky Mine ${ }^{1}$.

\begin{tabular}{|c|c|c|c|c|c|c|c|c|c|c|c|c|}
\hline Species & Jan & Feb & March & April & May & June & July & Aug & Sept & Oct & Nov & Dec \\
\hline $\begin{array}{c}\text { Pallid Bat } \\
\text { (Antrozous pallidus) }\end{array}$ & & & & & $C(1)$ & & & $C(1)$ & & & & \\
\hline \multicolumn{13}{|l|}{$\begin{array}{l}\text { Townsend's Big-eared Bat } \\
\text { (Corynorhinus townsendii) }\end{array}$} \\
\hline $\begin{array}{c}\text { Big Brown Bat } \\
\text { (Eptesicus fuscus) }\end{array}$ & & & & & & & & $C(2)$ & & & & \\
\hline \multicolumn{13}{|l|}{$\begin{array}{c}\text { Spotted Bat } \\
\text { (Euderma maculatum) }\end{array}$} \\
\hline $\begin{array}{c}\text { Hoary Bat } \\
\text { (Lasiurus cinereus) }\end{array}$ & & & & & & $A(2)$ & & & & & & \\
\hline $\begin{array}{c}\text { Silver-haired Bat } \\
\text { (Lasionycteris noctivagans) }\end{array}$ & & & & & & & & $\begin{array}{l}C(1) \\
A(4)\end{array}$ & & & & \\
\hline \multicolumn{13}{|l|}{$\begin{array}{l}\text { Eastern Red Bat } \\
\text { (Lasiurus borealis) }\end{array}$} \\
\hline $\begin{array}{l}\text { Western Small-footed Myotis } \\
\text { (Myotis ciliolabrum) }\end{array}$ & & & & & & & & $\begin{array}{l}C(1) \\
A(3)\end{array}$ & & & & \\
\hline $\begin{array}{l}\text { Long-eared Myotis } \\
\text { (Myotis evotis) }\end{array}$ & & & & & & & & $\begin{array}{l}C(2) \\
A(3)\end{array}$ & & & & \\
\hline $\begin{array}{l}\text { Little Brown Myotis } \\
\text { (Myotis lucifugus) }\end{array}$ & & & & & & & & $A(2)$ & & & & \\
\hline \multicolumn{13}{|l|}{$\begin{array}{c}\text { Fringed Myotis } \\
\text { (Myotis thysanodes) }\end{array}$} \\
\hline $\begin{array}{l}\text { Long-legged Myotis } \\
\text { (Myotis volans) }\end{array}$ & & & & & & & & $C(1)$ & & & & \\
\hline
\end{tabular}

$1 \quad$ Number of records in the point observation database at the Montana Natural Heritage Program prior to this study (MTNHP 2017). A $=$ acoustic record. $C=$ capture record. Records may include multiple individuals. 
Table 2e. Bat species documented and potentially present at or within $50 \mathrm{~km}$ the Absaloka Mine ${ }^{1}$.

\begin{tabular}{|c|c|c|c|c|c|c|c|c|c|c|c|c|}
\hline Species & Jan & Feb & March & April & May & June & July & Aug & Sept & Oct & Nov & Dec \\
\hline $\begin{array}{c}\text { Pallid Bat } \\
\text { (Antrozous pallidus) }\end{array}$ & & & & & & & & $C(1)$ & & & & \\
\hline \multicolumn{13}{|l|}{$\begin{array}{l}\text { Townsend's Big-eared Bat } \\
\text { (Corynorhinus townsendii) }\end{array}$} \\
\hline $\begin{array}{c}\text { Big Brown Bat } \\
\text { (Eptesicus fuscus) }\end{array}$ & & & & & & & C (1) & C (3) & & & & \\
\hline \multicolumn{13}{|l|}{$\begin{array}{c}\text { Spotted Bat } \\
\text { (Euderma maculatum) }\end{array}$} \\
\hline $\begin{array}{c}\text { Hoary Bat } \\
\text { (Lasiurus cinereus) }\end{array}$ & & & & & & $A(2)$ & & & & & & \\
\hline $\begin{array}{c}\text { Silver-haired Bat } \\
\text { (Lasionycteris noctivagans) }\end{array}$ & & & & & & & & $C(1)$ & & & & \\
\hline \multicolumn{13}{|l|}{$\begin{array}{l}\text { Eastern Red Bat } \\
\text { (Lasiurus borealis) }\end{array}$} \\
\hline $\begin{array}{l}\text { Western Small-footed Myotis } \\
\text { (Myotis ciliolabrum) }\end{array}$ & & & & & & & & $C(1)$ & & & & \\
\hline $\begin{array}{l}\text { Long-eared Myotis } \\
\text { (Myotis evotis) }\end{array}$ & & & & & & & & $C(2)$ & & & & \\
\hline $\begin{array}{l}\text { Little Brown Myotis } \\
\text { (Myotis lucifugus) }\end{array}$ & & & & & & & $C(1)$ & & & & & \\
\hline \multicolumn{13}{|l|}{$\begin{array}{c}\text { Fringed Myotis } \\
\text { (Myotis thysanodes) }\end{array}$} \\
\hline $\begin{array}{l}\text { Long-legged Myotis } \\
\text { (Myotis volans) }\end{array}$ & & & & & & & & $C(1)$ & & & & \\
\hline
\end{tabular}

$1 \quad$ Number of records in the point observation database at the Montana Natural Heritage Program prior to this study (MTNHP 2017). A $=$ acoustic record. $C=$ capture record. Records may include multiple individuals. 
Table 2f. Bat species documented and potentially present at or within $50 \mathrm{~km}$ the Rosebud Coal Mine ${ }^{1}$.

\begin{tabular}{|c|c|c|c|c|c|c|c|c|c|c|c|c|}
\hline Species & Jan & Feb & March & April & May & June & July & Aug & Sept & Oct & Nov & Dec \\
\hline $\begin{array}{c}\text { Pallid Bat } \\
\text { (Antrozous pallidus) }\end{array}$ & & & & & & & & $C(1)$ & & & & \\
\hline \multicolumn{13}{|l|}{$\begin{array}{l}\text { Townsend's Big-eared Bat } \\
\text { (Corynorhinus townsendii) }\end{array}$} \\
\hline $\begin{array}{c}\text { Big Brown Bat } \\
\text { (Eptesicus fuscus) }\end{array}$ & & & & & & $C(1)$ & & $C(2)$ & & & & \\
\hline \multicolumn{13}{|l|}{$\begin{array}{c}\text { Spotted Bat } \\
\text { (Euderma maculatum) }\end{array}$} \\
\hline $\begin{array}{c}\text { Hoary Bat } \\
\text { (Lasiurus cinereus) }\end{array}$ & & & & & & $A(2)$ & & $C(1)$ & & & & \\
\hline $\begin{array}{c}\text { Silver-haired Bat } \\
\text { (Lasionycteris noctivagans) }\end{array}$ & & & & & & & & $\begin{array}{l}\text { C (1) } \\
\text { A (3) }\end{array}$ & & & & \\
\hline \multicolumn{13}{|l|}{$\begin{array}{l}\text { Eastern Red Bat } \\
\text { (Lasiurus borealis) }\end{array}$} \\
\hline $\begin{array}{l}\text { Western Small-footed Myotis } \\
\text { (Myotis ciliolabrum) }\end{array}$ & & & & & & & & $\begin{array}{l}\text { C (1) } \\
A(1)\end{array}$ & & & & \\
\hline $\begin{array}{l}\text { Long-eared Myotis } \\
\text { (Myotis evotis) }\end{array}$ & & & & & & & & $\begin{array}{l}\text { C (3) } \\
\text { A (2) }\end{array}$ & & & & \\
\hline $\begin{array}{l}\text { Little Brown Myotis } \\
\text { (Myotis lucifugus) }\end{array}$ & & & & & & & & & $C(1)$ & & & \\
\hline \multicolumn{13}{|l|}{$\begin{array}{c}\text { Fringed Myotis } \\
\text { (Myotis thysanodes) }\end{array}$} \\
\hline $\begin{array}{l}\text { Long-legged Myotis } \\
\text { (Myotis volans) }\end{array}$ & & & & & & & & & & & & \\
\hline
\end{tabular}

$1 \quad$ Number of records in the point observation database at the Montana Natural Heritage Program prior to this study (MTNHP 2017). A = acoustic record. C $=$ capture record. Records may include multiple individuals. 
Table 2g. Bat species documented and potentially present at or within $50 \mathrm{~km}$ the Signal Peak Mine ${ }^{1}$.

\begin{tabular}{|c|c|c|c|c|c|c|c|c|c|c|c|c|}
\hline Species & Jan & Feb & March & April & May & June & July & Aug & Sept & Oct & Nov & Dec \\
\hline $\begin{array}{c}\text { Pallid Bat } \\
\text { (Antrozous pallidus) }\end{array}$ & & & & & & & $C(1)$ & & & & & \\
\hline $\begin{array}{l}\text { Townsend's Big-eared Bat } \\
\text { (Corynorhinus townsendii) }\end{array}$ & & & $C(1)$ & & & & $\begin{array}{l}C(2) \\
A(2)\end{array}$ & & $C(1)$ & & & \\
\hline $\begin{array}{l}\text { Big Brown Bat } \\
\text { (Eptesicus fuscus) }\end{array}$ & & & & & & $C(2)$ & $\begin{array}{c}C(15) \\
A(6)\end{array}$ & $\begin{array}{l}C(7) \\
A(3)\end{array}$ & $C(6)$ & & & \\
\hline $\begin{array}{c}\text { Spotted Bat } \\
\text { (Euderma maculatum) }\end{array}$ & & & & & & & $A(6)$ & $A(1)$ & $A(2)$ & & & \\
\hline $\begin{array}{c}\text { Hoary Bat } \\
\text { (Lasiurus cinereus) }\end{array}$ & & & & & & & $\begin{array}{c}C(5) \\
A(11)\end{array}$ & $\begin{array}{c}C(1) \\
A(11)\end{array}$ & & & & \\
\hline $\begin{array}{c}\text { Silver-haired Bat } \\
\text { (Lasionycteris noctivagans) }\end{array}$ & & & & & & $C(1)$ & $\begin{array}{l}C(6) \\
A(9)\end{array}$ & $\begin{array}{l}C(2) \\
A(8)\end{array}$ & $C(2)$ & & & \\
\hline \multicolumn{13}{|l|}{$\begin{array}{l}\text { Eastern Red Bat } \\
\text { (Lasiurus borealis) }\end{array}$} \\
\hline $\begin{array}{l}\text { Western Small-footed Myotis } \\
\text { (Myotis ciliolabrum) }\end{array}$ & & & $C(1)$ & & & & $\begin{array}{l}C(2) \\
A(5)\end{array}$ & $\begin{array}{l}C(2) \\
A(3)\end{array}$ & $C(3)$ & & & \\
\hline $\begin{array}{l}\text { Long-eared Myotis } \\
\text { (Myotis evotis) }\end{array}$ & & & & & & & $\begin{array}{l}C(6) \\
A(7)\end{array}$ & $\begin{array}{l}C(3) \\
A(2)\end{array}$ & C (4) & & & \\
\hline $\begin{array}{l}\text { Little Brown Myotis } \\
\text { (Myotis lucifugus) }\end{array}$ & & & & & & C (1) & $\begin{array}{c}C(10) \\
A(8)\end{array}$ & $\begin{array}{l}C(3) \\
A(5)\end{array}$ & $\begin{array}{l}C(2) \\
A(2)\end{array}$ & $C(1)$ & & \\
\hline \multicolumn{13}{|l|}{$\begin{array}{c}\text { Fringed Myotis } \\
\text { (Myotis thysanodes) }\end{array}$} \\
\hline $\begin{array}{l}\text { Long-legged Myotis } \\
\text { (Myotis volans) }\end{array}$ & & & & & & & $C(4)$ & $C(1)$ & $C(1)$ & & & \\
\hline
\end{tabular}

$1 \quad$ Number of records in the point observation database at the Montana Natural Heritage Program prior to this study (MTNHP 2017). A $=$ acoustic record. C $=$ capture record. Records may include multiple individuals. 
Table 3. Deployment history for SM2 Bat+ detector/ recorders deployed at coal mines.

\begin{tabular}{|c|c|c|c|}
\hline Mine & Site & $\begin{array}{l}\text { Installation } \\
\text { Date }\end{array}$ & $\begin{array}{c}\text { Decommission } \\
\text { Date }\end{array}$ \\
\hline $\begin{array}{c}\text { West Decker Coal } \\
\text { Mine }\end{array}$ & & August 2012 & July 2016 \\
\hline Spring Creek Mine & & August 2012 & December 2016 \\
\hline Otter Creek Coal Track & & October 2013 & June 2016 \\
\hline Big Sky Mine & & October 2013 & In operation \\
\hline Absaloka Mine & & August 2012 & June 2016 \\
\hline Rosebud Coal Mine & Area C Pond & October 2013 & June 2016 \\
\hline Rosebud Coal Mine & Area F Pond 7 & August 2012 & October 2013 \\
\hline Signal Peak Mine & Reservoir 1 & August 2012 & In operation \\
\hline Signal Peak Mine & $\begin{array}{l}\text { Busse Water } \\
\text { Reservoir }\end{array}$ & August 2012 & In operation \\
\hline
\end{tabular}


Table 4. Proximity of weather stations to detector locations for all sites and data types. Distance in kilometers between the station and detector is given in brackets after the station name. Note that some detectors used multiple stations over their deployment.

\begin{tabular}{|c|c|c|c|c|}
\hline Detector Site & Wind & Temperature & Pressure & Precipitation \\
\hline West Decker Mine & $\begin{array}{c}\text { CW7283 Sheridan } \\
(32.0)\end{array}$ & $\begin{array}{c}\text { CW7283 Sheridan (32.0) / } \\
\text { Tongue River Dam (8.0) }\end{array}$ & CW7283 Sheridan (32.0) & Wolf Mountain (38.0) \\
\hline Spring Creek Mine & Wolf Mountain (27.9) & $\begin{array}{c}\text { Tongue River Dam (14.6) / } \\
\text { Wolf Mountain (27.9) }\end{array}$ & CW7283 Sheridan (33.6) & Wolf Mountain (27.9) \\
\hline Otter Creek Mine & Fort Howes (20.8) & Fort Howes (20.8) & $\begin{array}{c}\text { Weston (94.5)/ Forsyth } \\
(94.5) \\
\end{array}$ & Fort Howes (20.8) \\
\hline Big Sky Mine & Badger Peak (22.4) & Badger Peak (22.4) & Forsyth (51.6) & Badger Peak (22.4) \\
\hline Absaloka Mine & Little Bighorn (41.2) & Little Bighorn (41.2) & Forsyth (59.6) & Little Bighorn (41.2) \\
\hline Rosebud Mine Area C Pond & Badger Peak (33.08) & Badger Peak (33.08) & Forsyth (45.4) & Badger Peak (33.08) \\
\hline Rosebud Mine Area F Pond 7 & Badger Peak (42.6) & Badger Peak (42.6) & Forsyth (49.7) & Badger Peak (42.6) \\
\hline Signal Peak Mine Reservoir 1 & $\begin{array}{l}\text { Bull Mountain US-87 } \\
\text { MP } 33.3(7.9)\end{array}$ & $\begin{array}{c}\text { Bull Mountain US-87 MP } \\
33.3(7.9)\end{array}$ & Roundup (29.7) & $\begin{array}{c}\text { Bull Mountain US-87 MP } \\
33.3(7.9)\end{array}$ \\
\hline $\begin{array}{l}\text { Signal Peak Mine Busse } \\
\text { Water Reservoir }\end{array}$ & $\begin{array}{l}\text { Bull Mountain US-87 } \\
\text { MP } 33.3(8.2)\end{array}$ & $\begin{array}{c}\text { Bull Mountain US-87 MP } \\
33.3(8.2)\end{array}$ & Roundup (25.7) & $\begin{array}{c}\text { Bull Mountain US-87 MP } \\
33.3(8.2)\end{array}$ \\
\hline
\end{tabular}


Table 5a. Detector status as measured by percent of calls auto-identified to species for West Decker Coal Mine

\begin{tabular}{|c|c|c|c|c|}
\hline Year & Month & Total No. of Calls & $\begin{array}{c}\text { No. Calls } \\
\text { Classified to } \\
\text { Species }\end{array}$ & $\begin{array}{c}\% \text { Auto-identified } \\
\text { to Species }\end{array}$ \\
\hline 2012 & August & 8968 & 1947 & $21.7 \%$ \\
\hline 2012 & September & 10927 & 2035 & $18.6 \%$ \\
\hline 2012 & October & 33 & 14 & $42.4 \%$ \\
\hline 2012 & November & 0 & 0 & - \\
\hline 2012 & December & 0 & 0 & - \\
\hline 2013 & January & 0 & 0 & - \\
\hline 2013 & February & 0 & 0 & - \\
\hline 2013 & March & 0 & 0 & - \\
\hline 2013 & April & 1065 & 3 & $0.3 \%$ \\
\hline 2013 & May & 1492 & 7 & $0.5 \%$ \\
\hline 2013 & June & 2538 & 3 & $0.1 \%$ \\
\hline 2013 & July & 1422 & 1 & $0.1 \%$ \\
\hline 2013 & August & - & - & - \\
\hline 2013 & September & - & - & - \\
\hline 2013 & October & 4 & 0 & $0.0 \%$ \\
\hline 2013 & November & 0 & 0 & - \\
\hline 2013 & December & 0 & 0 & - \\
\hline 2014 & January & 0 & 0 & - \\
\hline 2014 & February & 0 & 0 & - \\
\hline 2014 & March & 0 & 0 & - \\
\hline 2014 & April & 1475 & 3 & $0.2 \%$ \\
\hline 2014 & May & 5567 & 272 & $4.9 \%$ \\
\hline 2014 & June & 2497 & 159 & $6.4 \%$ \\
\hline 2014 & July & 1055 & 7 & $0.7 \%$ \\
\hline 2014 & August & 8140 & 1087 & $13.4 \%$ \\
\hline 2014 & September & 25643 & 2230 & $8.7 \%$ \\
\hline 2014 & October & 74 & 15 & $20.3 \%$ \\
\hline 2014 & November & 2 & 2 & $100.0 \%$ \\
\hline 2014 & December & 0 & 0 & - \\
\hline 2015 & January & 20 & 0 & $0.0 \%$ \\
\hline 2015 & February & 34 & 0 & $0.0 \%$ \\
\hline 2015 & March & 208 & 29 & $13.9 \%$ \\
\hline 2015 & April & 7665 & 620 & $8.1 \%$ \\
\hline 2015 & May & 21295 & 656 & $3.1 \%$ \\
\hline 2015 & June & 33221 & 12935 & $38.9 \%$ \\
\hline 2015 & July & 22230 & 10267 & $46.2 \%$ \\
\hline 2015 & August & 60491 & 20312 & $33.6 \%$ \\
\hline 2015 & September & 7376 & 3255 & $44.1 \%$ \\
\hline 2015 & October & 75 & 35 & $46.7 \%$ \\
\hline 2012 & November & 0 & 0 & - \\
\hline 2015 & December & $63.6 \%$ & 14 & 22 \\
\hline
\end{tabular}




\begin{tabular}{|c|c|c|c|c|}
\hline Year & Month & Total No. of Calls & $\begin{array}{c}\text { No. Calls } \\
\text { Classified to } \\
\text { Species }\end{array}$ & $\begin{array}{c}\text { \% Auto-identified } \\
\text { to Species }\end{array}$ \\
\hline 2016 & January & $100.0 \%$ & 1 & 1 \\
\hline
\end{tabular}

Table 5b. Detector status as measured by percent of calls auto-identified to species for Spring Creek Mine

\begin{tabular}{|c|c|c|c|c|}
\hline Year & Month & Total No. of Calls & $\begin{array}{c}\text { No. Calls } \\
\text { Classified to } \\
\text { Species }\end{array}$ & $\begin{array}{c}\% \text { Auto-identified } \\
\text { to Species }\end{array}$ \\
\hline 2012 & August & 11910 & 1587 & $13.3 \%$ \\
\hline 2012 & September & 2082 & 183 & $8.8 \%$ \\
\hline 2012 & October & 0 & 0 & - \\
\hline 2012 & November & 0 & 0 & - \\
\hline 2012 & December & 0 & 0 & - \\
\hline 2013 & January & 0 & 0 & - \\
\hline 2013 & February & 0 & 0 & - \\
\hline 2013 & March & 0 & 0 & - \\
\hline 2013 & April & 602 & 251 & $41.7 \%$ \\
\hline 2013 & May & 22668 & 2738 & $12.1 \%$ \\
\hline 2013 & June & 22665 & 2372 & $10.5 \%$ \\
\hline 2013 & July & 0 & 0 & - \\
\hline 2013 & August & 0 & 0 & - \\
\hline 2013 & September & 0 & 0 & - \\
\hline 2013 & October & 49 & 9 & $18.4 \%$ \\
\hline 2013 & November & 17 & 1 & $5.9 \%$ \\
\hline 2013 & December & 6 & 0 & $0.0 \%$ \\
\hline 2014 & January & 0 & 0 & - \\
\hline 2014 & February & 0 & 0 & - \\
\hline 2014 & March & 1 & 0 & $0.0 \%$ \\
\hline 2014 & April & 238 & 2 & $0.8 \%$ \\
\hline 2014 & May & 3380 & 82 & $2.4 \%$ \\
\hline 2014 & June & 24774 & 537 & $2.2 \%$ \\
\hline 2014 & July & 26885 & 488 & $1.8 \%$ \\
\hline 2014 & August & 15343 & 327 & $2.1 \%$ \\
\hline 2014 & September & 364 & 11 & $3.0 \%$ \\
\hline 2014 & October & 0 & 0 & - \\
\hline 2014 & November & 23 & 7 & $30.4 \%$ \\
\hline 2014 & December & 15 & 5 & $33.3 \%$ \\
\hline 2015 & January & 1 & 0 & $0.0 \%$ \\
\hline 2015 & February & 0 & 0 & - \\
\hline 2015 & March & 5 & 0 & $0.0 \%$ \\
\hline 2015 & April & 92 & 9 & $9.8 \%$ \\
\hline 2015 & May & 677 & 94 & $13.9 \%$ \\
\hline 2015 & June & 5812 & 3561 & $61.3 \%$ \\
\hline 2015 & July & 7785 & 4442 & $57.1 \%$ \\
\hline
\end{tabular}




\begin{tabular}{|c|c|c|c|c|}
\hline Year & Month & Total No. of Calls & $\begin{array}{c}\text { No. Calls } \\
\text { Classified to } \\
\text { Species }\end{array}$ & $\begin{array}{c}\text { \% Auto-identified } \\
\text { to Species }\end{array}$ \\
\hline 2015 & August & 8690 & 4391 & $50.5 \%$ \\
\hline 2015 & September & 3990 & 1669 & $41.8 \%$ \\
\hline 2015 & October & 168 & 47 & $28.0 \%$ \\
\hline 2012 & November & 49 & 25 & $51.0 \%$ \\
\hline 2015 & December & 49 & 29 & $59.2 \%$ \\
\hline 2016 & January & 40 & 28 & $70.0 \%$ \\
\hline 2016 & February & 46 & 21 & $45.7 \%$ \\
\hline 2016 & March & 18 & 11 & $61.1 \%$ \\
\hline 2016 & April & 19 & 12 & $63.2 \%$ \\
\hline
\end{tabular}

Table 5c. Detector status as measured by percent of calls auto-identified to species for Otter Creek Coal Tract

\begin{tabular}{|c|c|c|c|c|}
\hline Year & Month & Total No. of Calls & $\begin{array}{c}\text { No. Calls } \\
\text { Classified to } \\
\text { Species }\end{array}$ & $\begin{array}{c}\% \text { Auto-identified } \\
\text { to Species }\end{array}$ \\
\hline 2013 & October & 14 & 2 & $14.3 \%$ \\
\hline 2013 & November & 0 & 0 & - \\
\hline 2013 & December & 0 & 0 & - \\
\hline 2014 & January & 0 & 0 & - \\
\hline 2014 & February & 0 & 0 & - \\
\hline 2014 & March & 3 & 0 & $0.0 \%$ \\
\hline 2014 & April & 0 & 0 & - \\
\hline 2014 & May & 17936 & 648 & $3.6 \%$ \\
\hline 2014 & June & 20205 & 1988 & $9.8 \%$ \\
\hline 2014 & July & 13931 & 2613 & $18.8 \%$ \\
\hline 2014 & August & 6988 & 629 & $9.0 \%$ \\
\hline 2014 & September & 2678 & 175 & $6.5 \%$ \\
\hline 2014 & October & 0 & 0 & - \\
\hline 2014 & November & 2 & 0 & $0.0 \%$ \\
\hline 2014 & December & 3 & 0 & $0.0 \%$ \\
\hline 2015 & January & 0 & 0 & - \\
\hline 2015 & February & 2 & 1 & $50.0 \%$ \\
\hline 2015 & March & 115 & 16 & $13.9 \%$ \\
\hline 2015 & April & 5288 & 233 & $4.4 \%$ \\
\hline 2015 & May & 19601 & 2735 & $14.0 \%$ \\
\hline 2015 & June & 19435 & 7456 & $38.4 \%$ \\
\hline 2015 & July & 17220 & 6305 & $36.6 \%$ \\
\hline 2015 & August & 31738 & 8943 & $28.2 \%$ \\
\hline 2015 & September & 15397 & 3271 & $21.2 \%$ \\
\hline
\end{tabular}


Table 5d. Detector status as measured by percent of calls auto-identified to species for Big Sky Mine

\begin{tabular}{|c|c|c|c|c|}
\hline Year & Month & Total No. of Calls & $\begin{array}{c}\text { No. Calls } \\
\text { Classified to } \\
\text { Species }\end{array}$ & $\begin{array}{c}\% \text { Auto-identified } \\
\text { to Species }\end{array}$ \\
\hline 2013 & October & 1171 & 335 & $28.6 \%$ \\
\hline 2013 & November & 1970 & 585 & $29.7 \%$ \\
\hline 2013 & December & 411 & 176 & $42.8 \%$ \\
\hline 2014 & January & 596 & 240 & $40.3 \%$ \\
\hline 2014 & February & 199 & 88 & $44.2 \%$ \\
\hline 2014 & March & 748 & 262 & $35.0 \%$ \\
\hline 2014 & April & 5918 & 2667 & $45.1 \%$ \\
\hline 2014 & May & 732 & 161 & $22.0 \%$ \\
\hline 2014 & June & 0 & 0 & - \\
\hline 2014 & July & 598 & 63 & $10.5 \%$ \\
\hline 2014 & August & 3985 & 763 & $19.2 \%$ \\
\hline 2014 & September & 351 & 63 & $18.0 \%$ \\
\hline 2014 & October & 0 & 0 & - \\
\hline 2014 & November & 0 & 0 & - \\
\hline 2014 & December & 0 & 0 & - \\
\hline 2015 & January & 0 & 0 & - \\
\hline 2015 & February & 0 & 0 & - \\
\hline 2015 & March & 11508 & 4868 & $42.3 \%$ \\
\hline 2015 & April & 6384 & 3006 & $47.1 \%$ \\
\hline 2015 & May & 9267 & 2546 & $27.5 \%$ \\
\hline 2015 & June & 26664 & 16867 & $63.3 \%$ \\
\hline 2015 & July & 42725 & 24425 & $57.2 \%$ \\
\hline 2015 & August & 25093 & 13814 & $55.1 \%$ \\
\hline 2015 & September & 17829 & 10387 & $58.3 \%$ \\
\hline 2015 & October & 8034 & 4616 & $57.5 \%$ \\
\hline 2012 & November & 5764 & 3597 & $62.4 \%$ \\
\hline 2015 & December & 3211 & 2039 & $63.5 \%$ \\
\hline 2016 & January & 2356 & 1520 & $64.5 \%$ \\
\hline 2016 & February & 9810 & 6015 & $61.3 \%$ \\
\hline 2016 & March & 13322 & 8553 & $64.2 \%$ \\
\hline 2016 & April & 6286 & 4101 & $65.2 \%$ \\
\hline
\end{tabular}

Table 5e. Detector status as measured by percent of calls auto-identified to species for Absaloka Mine

\begin{tabular}{|c|c|c|c|c|}
\hline Year & Month & Total No. of Calls & $\begin{array}{c}\text { No. Calls } \\
\text { Classified to } \\
\text { Species }\end{array}$ & $\begin{array}{c}\text { \% Auto-identified } \\
\text { to Species }\end{array}$ \\
\hline 2012 & August & 4035 & 663 & $16.43 \%$ \\
\hline 2012 & September & 5528 & 845 & $15.3 \%$ \\
\hline 2012 & October & 30 & 7 & $23.3 \%$ \\
\hline 2012 & November & 0 & - & - \\
\hline 2012 & December & 0 & - & - \\
\hline
\end{tabular}




\begin{tabular}{|c|c|c|c|c|}
\hline Year & Month & Total No. of Calls & $\begin{array}{c}\text { No. Calls } \\
\text { Classified to } \\
\text { Species }\end{array}$ & $\begin{array}{c}\% \text { Auto-identified } \\
\text { to Species }\end{array}$ \\
\hline 2013 & January & 0 & - & - \\
\hline 2013 & February & 0 & - & - \\
\hline 2013 & March & 0 & - & - \\
\hline 2013 & April & 36 & 13 & $36.1 \%$ \\
\hline 2013 & May & 956 & 262 & $27.4 \%$ \\
\hline 2013 & June & 791 & 129 & $16.3 \%$ \\
\hline 2013 & July & 269 & 35 & $13.0 \%$ \\
\hline 2013 & August & 0 & - & - \\
\hline 2013 & September & 0 & - & - \\
\hline 2013 & October & 24 & 5 & $20.8 \%$ \\
\hline 2013 & November & 1 & 0 & $0.0 \%$ \\
\hline 2013 & December & 0 & - & - \\
\hline 2014 & January & 0 & - & - \\
\hline 2014 & February & 0 & - & - \\
\hline 2014 & March & 0 & - & - \\
\hline 2014 & April & 98 & 7 & $7.1 \%$ \\
\hline 2014 & May & 1476 & 553 & $37.5 \%$ \\
\hline 2014 & June & 1121 & 410 & $36.6 \%$ \\
\hline 2014 & July & 20 & 0 & $0.0 \%$ \\
\hline 2014 & August & 1337 & 541 & $40.5 \%$ \\
\hline 2014 & September & 1415 & 347 & $24.5 \%$ \\
\hline 2014 & October & 20 & 2 & $10.0 \%$ \\
\hline 2014 & November & 1 & 1 & $100.0 \%$ \\
\hline 2014 & December & 4 & 1 & $25.0 \%$ \\
\hline 2015 & January & 1 & 0 & $0.0 \%$ \\
\hline 2015 & February & 2 & 0 & $0.0 \%$ \\
\hline 2015 & March & 32 & 20 & $62.5 \%$ \\
\hline 2015 & April & 352 & 145 & $41.2 \%$ \\
\hline 2015 & May & 1962 & 728 & $37.1 \%$ \\
\hline 2015 & June & 9980 & 5797 & $58.1 \%$ \\
\hline 2015 & July & 8365 & 5248 & $62.7 \%$ \\
\hline 2015 & August & 674 & 51 & $7.6 \%$ \\
\hline 2015 & September & 23 & 2 & $8.7 \%$ \\
\hline
\end{tabular}

Table 5f. Detector status as measured by percent of calls auto-identified to species for Rosebud Mine $\mathrm{F}$ Pond 7

\begin{tabular}{|c|c|c|c|c|}
\hline Year & Month & Total No. of Calls & $\begin{array}{c}\text { No. Calls } \\
\text { Classified to } \\
\text { Species }\end{array}$ & $\begin{array}{c}\text { \% Auto-identified } \\
\text { to Species }\end{array}$ \\
\hline 2012 & August & 1133 & 274 & $24.2 \%$ \\
\hline 2012 & September & 1401 & 318 & $22.7 \%$ \\
\hline 2012 & October & 30 & 4 & $13.3 \%$ \\
\hline 2012 & November & 2 & 0 & $0.0 \%$ \\
\hline
\end{tabular}




\begin{tabular}{|c|c|c|c|c|}
\hline Year & Month & Total No. of Calls & $\begin{array}{c}\text { No. Calls } \\
\text { Classified to } \\
\text { Species }\end{array}$ & $\begin{array}{c}\text { \% Auto-identified } \\
\text { to Species }\end{array}$ \\
\hline 2012 & December & 0 & 0 & - \\
\hline 2013 & January & 0 & 0 & - \\
\hline 2013 & February & 0 & 0 & - \\
\hline 2013 & March & 0 & 0 & - \\
\hline 2013 & April & 0 & 0 & $0.0 \%$ \\
\hline 2013 & May & 39 & 0 & $0.0 \%$ \\
\hline 2013 & June & 218 & 0 & $0.3 \%$ \\
\hline 2013 & July & 295 & 1 & $1.9 \%$ \\
\hline 2013 & August & 317 & 6 & $0.0 \%$ \\
\hline 2013 & September & 60 & 1 & \\
\hline 2013 & October & 3 & 0 & \\
\hline
\end{tabular}

Table 5g. Detector status as measured by percent of calls auto-identified to species for Rosebud Mine Area $\mathrm{C}$ pond

\begin{tabular}{|c|c|c|c|c|}
\hline Year & Month & Total No. of Calls & $\begin{array}{c}\text { No. Calls } \\
\text { Classified to } \\
\text { Species }\end{array}$ & $\begin{array}{c}\% \text { Auto-identified } \\
\text { to Species }\end{array}$ \\
\hline 2013 & October & 8 & 4 & $50.0 \%$ \\
\hline 2013 & November & 2 & 1 & $50.0 \%$ \\
\hline 2013 & December & 0 & & \\
\hline 2014 & January & 0 & & \\
\hline 2014 & February & 3 & 2 & $66.7 \%$ \\
\hline 2014 & March & 0 & & \\
\hline 2014 & April & 326 & 105 & $32.2 \%$ \\
\hline 2014 & May & 2491 & 935 & $37.5 \%$ \\
\hline 2014 & June & 7223 & 1573 & $21.8 \%$ \\
\hline 2014 & July & 14728 & 3339 & $22.7 \%$ \\
\hline 2014 & August & 9654 & 2244 & $23.2 \%$ \\
\hline 2014 & September & 3146 & 450 & $14.3 \%$ \\
\hline 2014 & October & 96 & 37 & $38.5 \%$ \\
\hline 2014 & November & 4 & 1 & $25.0 \%$ \\
\hline 2014 & December & 4 & 1 & $25.0 \%$ \\
\hline 2015 & January & 0 & 0 & - \\
\hline 2015 & February & 0 & 0 & - \\
\hline 2015 & March & 70 & 24 & $34.3 \%$ \\
\hline 2015 & April & 117 & 50 & $42.7 \%$ \\
\hline 2015 & May & 2410 & 1117 & $46.4 \%$ \\
\hline 2015 & June & 24939 & 12034 & $48.3 \%$ \\
\hline 2015 & July & 32479 & 19038 & $58.6 \%$ \\
\hline 2015 & August & 16920 & 8244 & $48.7 \%$ \\
\hline 2015 & September & 4141 & 2151 & $51.9 \%$ \\
\hline 2015 & October & 65 & 34 & $52.3 \%$ \\
\hline 2012 & November & 9 & 1 & $11.1 \%$ \\
\hline
\end{tabular}




\begin{tabular}{|c|c|c|c|c|}
\hline Year & Month & Total No. of Calls & $\begin{array}{c}\text { No. Calls } \\
\text { Classified to } \\
\text { Species }\end{array}$ & $\begin{array}{c}\text { \% Auto-identified } \\
\text { to Species }\end{array}$ \\
\hline 2015 & December & 12 & 6 & $50.0 \%$ \\
\hline 2016 & January & 13 & 11 & $84.6 \%$ \\
\hline 2016 & February & - & - & - \\
\hline 2016 & March & - & - & $51.2 \%$ \\
\hline 2016 & April & 605 & 310 & $55.3 \%$ \\
\hline 2016 & May & 3732 & 2065 & - \\
\hline 2016 & June & - & - & - \\
\hline 2016 & July & - & - & \\
\hline
\end{tabular}

Table 5h. Detector status as measured by percent of calls auto-identified to species for Signal Peak Mine Reservoir 1

\begin{tabular}{|c|c|c|c|c|}
\hline Year & Month & Total No. of Calls & $\begin{array}{c}\text { No. Calls } \\
\text { Classified to } \\
\text { Species }\end{array}$ & $\begin{array}{c}\% \text { Auto-identified } \\
\text { to Species }\end{array}$ \\
\hline 2012 & August & 3553 & 380 & $10.7 \%$ \\
\hline 2012 & September & 7884 & 929 & $11.8 \%$ \\
\hline 2012 & October & 790 & 46 & $5.8 \%$ \\
\hline 2012 & November & 901 & 54 & $6.0 \%$ \\
\hline 2012 & December & 421 & 19 & $4.5 \%$ \\
\hline 2013 & January & 290 & 40 & $13.8 \%$ \\
\hline 2013 & February & 183 & 5 & $2.7 \%$ \\
\hline 2013 & March & 727 & 101 & $13.9 \%$ \\
\hline 2013 & April & 3266 & 693 & $21.2 \%$ \\
\hline 2013 & May & 5628 & 625 & $11.1 \%$ \\
\hline 2013 & June & 5998 & 719 & $12.0 \%$ \\
\hline 2013 & July & 5076 & 663 & $13.1 \%$ \\
\hline 2013 & August & 7609 & 1138 & $15.0 \%$ \\
\hline 2013 & September & 1527 & 262 & $17.2 \%$ \\
\hline 2013 & October & 406 & 64 & $15.8 \%$ \\
\hline 2013 & November & 447 & 41 & $9.2 \%$ \\
\hline 2013 & December & 67 & 7 & $10.5 \%$ \\
\hline 2014 & January & 31 & 5 & $16.1 \%$ \\
\hline 2014 & February & 5 & 0 & $0.0 \%$ \\
\hline 2014 & March & 0 & 0 & - \\
\hline 2014 & April & 164 & 28 & $17.1 \%$ \\
\hline 2014 & May & 4004 & 476 & $11.9 \%$ \\
\hline 2014 & June & 5792 & 1204 & $20.8 \%$ \\
\hline 2014 & July & 14738 & 3886 & $26.4 \%$ \\
\hline 2014 & August & 8517 & 2176 & $25.6 \%$ \\
\hline 2014 & September & 1519 & 243 & $16.0 \%$ \\
\hline 2014 & October & 286 & 88 & $30.8 \%$ \\
\hline 2014 & November & 297 & 52 & $17.5 \%$ \\
\hline 2014 & December & 163 & 7 & $4.3 \%$ \\
\hline
\end{tabular}




\begin{tabular}{|c|c|c|c|c|}
\hline Year & Month & Total No. of Calls & $\begin{array}{c}\text { No. Calls } \\
\text { Classified to } \\
\text { Species }\end{array}$ & $\begin{array}{c}\text { \% Auto-identified } \\
\text { to Species }\end{array}$ \\
\hline 2015 & January & 30 & 0 & $0.0 \%$ \\
\hline 2015 & February & 60 & 0 & $0.0 \%$ \\
\hline 2015 & March & 1682 & 160 & $9.5 \%$ \\
\hline 2015 & April & 1621 & 121 & $7.5 \%$ \\
\hline 2015 & May & 1449 & 187 & $12.9 \%$ \\
\hline 2015 & June & 12390 & 7906 & $63.8 \%$ \\
\hline 2015 & July & 24210 & 15560 & $64.3 \%$ \\
\hline 2015 & August & 14255 & 8582 & $60.2 \%$ \\
\hline 2015 & September & 9275 & 5193 & $56.0 \%$ \\
\hline 2015 & October & 1436 & 614 & $42.8 \%$ \\
\hline 2012 & November & 1355 & 507 & $37.4 \%$ \\
\hline 2015 & December & 1013 & 403 & $39.8 \%$ \\
\hline 2016 & January & 560 & 257 & $45.9 \%$ \\
\hline 2016 & February & 1082 & 394 & $36.4 \%$ \\
\hline 2016 & March & - & - & - \\
\hline 2016 & April & - & - & - \\
\hline 2016 & May & - & - & - \\
\hline 2016 & June & - & - & - \\
\hline 2016 & July & - & & \\
\hline
\end{tabular}

Table 5i. Detector status as measured by percent of calls auto-identified to species for Signal Peak Mine Busse Water Reservoir

\begin{tabular}{|c|c|c|c|c|}
\hline Year & Month & Total No. of Calls & $\begin{array}{c}\text { No. Calls } \\
\text { Classified to } \\
\text { Species }\end{array}$ & $\begin{array}{c}\% \text { Auto-identified } \\
\text { to Species }\end{array}$ \\
\hline 2012 & August & 13768 & 3542 & $25.7 \%$ \\
\hline 2012 & September & 23576 & 7578 & $32.1 \%$ \\
\hline 2012 & October & 257 & 71 & $27.6 \%$ \\
\hline 2012 & November & 57 & 6 & $10.5 \%$ \\
\hline 2012 & December & 74 & 19 & $25.7 \%$ \\
\hline 2013 & January & 7 & 0 & $0.0 \%$ \\
\hline 2013 & February & 0 & 0 & - \\
\hline 2013 & March & 0 & 0 & $17.6 \%$ \\
\hline 2013 & April & 1366 & 241 & $11.0 \%$ \\
\hline 2013 & May & 7956 & 874 & $20.4 \%$ \\
\hline 2013 & June & 30880 & 6313 & $24.6 \%$ \\
\hline 2013 & July & 41451 & 10202 & $16.1 \%$ \\
\hline 2013 & August & 12566 & 2019 & $8.8 \%$ \\
\hline 2013 & September & 3728 & 329 & $37.0 \%$ \\
\hline 2013 & October & 343 & 127 & $42.9 \%$ \\
\hline 2013 & November & 91 & 39 & $40.9 \%$ \\
\hline 2013 & December & 22 & 9 & $40.0 \%$ \\
\hline 2014 & January & 20 & 8 & \\
\hline
\end{tabular}




\begin{tabular}{|c|c|c|c|c|}
\hline Year & Month & Total No. of Calls & $\begin{array}{c}\text { No. Calls } \\
\text { Classified to } \\
\text { Species }\end{array}$ & $\begin{array}{c}\% \text { Auto-identifiec } \\
\text { to Species }\end{array}$ \\
\hline 2014 & February & 9 & 5 & $55.6 \%$ \\
\hline 2014 & March & 12 & 6 & $50.0 \%$ \\
\hline 2014 & April & 2204 & 817 & $37.1 \%$ \\
\hline 2014 & May & 15852 & 4774 & $30.1 \%$ \\
\hline 2014 & June & 30687 & 7836 & $25.5 \%$ \\
\hline 2014 & July & 43747 & 13386 & $30.6 \%$ \\
\hline 2014 & August & 22965 & 5784 & $25.2 \%$ \\
\hline 2014 & September & 4284 & 832 & $19.4 \%$ \\
\hline 2014 & October & 99 & 19 & $19.2 \%$ \\
\hline 2014 & November & 25 & 5 & $20.0 \%$ \\
\hline 2014 & December & 104 & 21 & $20.2 \%$ \\
\hline 2015 & January & 17 & 7 & $41.2 \%$ \\
\hline 2015 & February & 31 & 4 & $12.9 \%$ \\
\hline 2015 & March & 1054 & 332 & $31.5 \%$ \\
\hline 2015 & April & 2400 & 1102 & $45.9 \%$ \\
\hline 2015 & May & 10230 & 3206 & $31.3 \%$ \\
\hline 2015 & June & 24655 & 12617 & $51.2 \%$ \\
\hline 2015 & July & 68847 & 30916 & $44.9 \%$ \\
\hline 2015 & August & 27061 & 13803 & $51.0 \%$ \\
\hline 2015 & September & 28898 & 11953 & $41.4 \%$ \\
\hline 2015 & October & 394 & 256 & $65.0 \%$ \\
\hline 2012 & November & 255 & 138 & $54.1 \%$ \\
\hline 2015 & December & 413 & 244 & $59.1 \%$ \\
\hline 2016 & January & 372 & 231 & $62.1 \%$ \\
\hline 2016 & February & 218 & 111 & $50.9 \%$ \\
\hline 2016 & March & - & - & - \\
\hline 2016 & April & - & - & - \\
\hline 2016 & May & - & - & - \\
\hline 2016 & June & - & - & - \\
\hline 2016 & July & - & - & - \\
\hline
\end{tabular}


Table 6. Monthly rates of hand confirmation from automated analysis results across all mine sites and detectors

\begin{tabular}{|c|c|c|c|}
\hline Species & $\begin{array}{l}\text { No. months with } \\
\text { automated } \\
\text { identification of } \\
\text { species }\end{array}$ & $\begin{array}{l}\text { No. months with } \\
\text { hand confirmed } \\
\text { identification of } \\
\text { species }\end{array}$ & $\begin{array}{l}\text { Percent of months } \\
\text { automated } \\
\text { identification was } \\
\text { hand confirmed }\end{array}$ \\
\hline $\begin{array}{l}\text { Pallid Bat } \\
\text { (Antrozous pallidus) }\end{array}$ & 86 & 0 & $0.0 \%$ \\
\hline $\begin{array}{l}\text { Townsend's Big-eared Bat } \\
\text { (Corynorhinus townsendii) }^{1}\end{array}$ & 77 & 3 & $5.3 \%$ \\
\hline $\begin{array}{l}\text { Big Brown Bat } \\
\text { (Eptesicus fuscus) }\end{array}$ & 124 & 59 & $68.6 \%$ \\
\hline $\begin{array}{l}\text { Spotted Bat } \\
\text { (Euderma maculatum) }\end{array}$ & 19 & 17 & $100.0 \%$ \\
\hline $\begin{array}{l}\text { Hoary Bat } \\
\text { (Lasiurus cinereus) }\end{array}$ & 96 & 56 & $71.8 \%$ \\
\hline $\begin{array}{l}\text { Silver-haired Bat } \\
\text { (Lasionycterus noctivagans) }\end{array}$ & 128 & 70 & $71.2 \%$ \\
\hline $\begin{array}{l}\text { Eastern Red Bat } \\
\text { (Lasiurus borealis) }\end{array}$ & 135 & 10 & $10.5 \%$ \\
\hline $\begin{array}{l}\text { Western Small-footed Myotis } \\
\text { (Myotis ciliolabrum) }\end{array}$ & 142 & 66 & $64.7 \%$ \\
\hline $\begin{array}{l}\text { Long-eared Myotis } \\
\text { (Myotis evotis) }\end{array}$ & 76 & 60 & $87.0 \%$ \\
\hline $\begin{array}{l}\text { Little Brown Myotis } \\
\text { (Myotis lucifugus) }\end{array}$ & 105 & 56 & $73.7 \%$ \\
\hline $\begin{array}{l}\text { Fringed Myotis } \\
\text { (Myotis thysanodes) }\end{array}$ & 41 & 0 & $0.0 \%$ \\
\hline \multirow[t]{2}{*}{ 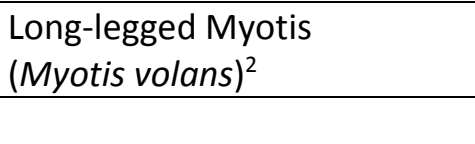 } & 71 & 3 & $6.0 \%$ \\
\hline & $\Sigma=1100$ & $\Sigma=400$ & $X=54.95 \%$ \\
\hline
\end{tabular}

1 Species is relatively quiet and often does not create fully definitive echolocation call recordings on bat detectors.

2 Long-legged Myotis calls can overlap with Western Small-footed Myotis, Long-eared Myotis, Little Brown Myotis, and Fringed Myotis calls and rarely have call characteristics recorded that allow them to be definitively identified as Long-legged Myotis (Maxell 2015). Several call sequences were auto-identified as Long-legged Myotis. However, these call sequences lacked the definitive characteristics necessary to confirm the species' presence. 
Table 7a. Species definitively detected by month each year of the study at the West Decker Coal Mine ${ }^{1}$

\begin{tabular}{|c|c|c|c|c|c|c|c|c|c|c|c|c|}
\hline Species & Jan & Feb & March & April & May & June & July & Aug & Sept & Oct & Nov & Dec \\
\hline \multicolumn{13}{|l|}{$\begin{array}{c}\text { Pallid Bat } \\
\text { (Antrozous pallidus) }\end{array}$} \\
\hline \multicolumn{13}{|l|}{ 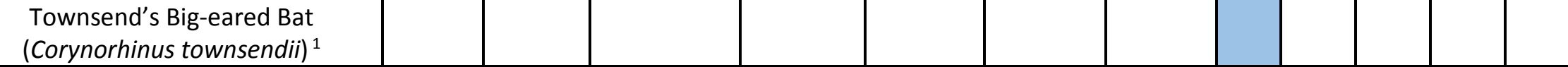 } \\
\hline $\begin{array}{c}\text { Big Brown Bat } \\
\text { (Eptesicus fuscus) }\end{array}$ & & & & $\begin{array}{l}2013 \\
2016\end{array}$ & $\begin{array}{l}2013 \\
2014\end{array}$ & $\begin{array}{l}2013 \\
2014\end{array}$ & 2013 & 2012 & $\begin{array}{l}2012 \\
2014\end{array}$ & & & \\
\hline \multicolumn{13}{|l|}{$\begin{array}{c}\text { Spotted Bat } \\
\text { (Euderma maculatum) }\end{array}$} \\
\hline $\begin{array}{c}\text { Hoary Bat } \\
\text { (Lasiurus cinereus) }\end{array}$ & & & & & $\begin{array}{l}2014 \\
2016\end{array}$ & $\begin{array}{l}2013 \\
2015 \\
2016 \\
\end{array}$ & $\begin{array}{l}2014 \\
2015 \\
2016 \\
\end{array}$ & $\begin{array}{l}2012 \\
2014 \\
2015 \\
\end{array}$ & $\begin{array}{l}2012 \\
2014 \\
2015 \\
\end{array}$ & & & \\
\hline $\begin{array}{c}\text { Silver-haired Bat } \\
\text { (Lasionycterus noctivagans) }\end{array}$ & & & & $\begin{array}{l}2013 \\
2016\end{array}$ & $\begin{array}{l}2013 \\
2014 \\
2016\end{array}$ & $\begin{array}{l}2014 \\
2015 \\
2016\end{array}$ & $\begin{array}{l}2015 \\
2016\end{array}$ & $\begin{array}{l}2012 \\
2014 \\
2015\end{array}$ & $\begin{array}{l}2012 \\
2014 \\
2015\end{array}$ & $\begin{array}{l}2012 \\
2014\end{array}$ & & \\
\hline $\begin{array}{l}\text { Eastern Red Bat } \\
\text { (Lasiurus borealis) }\end{array}$ & & & & & & & & 2012 & $\begin{array}{l}2014 \\
2015\end{array}$ & 2015 & & \\
\hline $\begin{array}{c}\text { Western Small-footed Myotis } \\
\text { (Myotis ciliolabrum) }\end{array}$ & & & & $\begin{array}{l}2013 \\
2014\end{array}$ & $\begin{array}{l}2013 \\
2014\end{array}$ & $\begin{array}{l}2013 \\
2014 \\
2015\end{array}$ & $\begin{array}{l}2013 \\
2014 \\
2015\end{array}$ & $\begin{array}{l}2012 \\
2014\end{array} \mid$ & $\begin{array}{l}2012 \\
2014\end{array}$ & 2015 & & \\
\hline $\begin{array}{l}\text { Long-eared Myotis } \\
\text { (Myotis evotis) }\end{array}$ & & & & 2013 & $\begin{array}{l}2013 \\
2014\end{array}$ & $\begin{array}{l}2013 \\
2014 \\
2015 \\
\end{array}$ & $\begin{array}{l}2015 \\
2016\end{array}$ & \begin{tabular}{|l|}
2012 \\
2014 \\
2015 \\
\end{tabular} & $\begin{array}{l}2012 \\
2014 \\
2015 \\
\end{array}$ & & & \\
\hline $\begin{array}{l}\text { Little Brown Myotis } \\
\text { (Myotis lucifugus) }\end{array}$ & & & & 2015 & $\begin{array}{l}2013 \\
2014 \\
2015 \\
\end{array}$ & $\begin{array}{l}2013 \\
2014 \\
2015 \\
\end{array}$ & $\begin{array}{l}2013 \\
2014 \\
2015 \\
\end{array}$ & $\begin{array}{l}2012 \\
2014 \\
2015 \\
\end{array}$ & $\begin{array}{l}2012 \\
2014 \\
2015 \\
\end{array}$ & 2012 & & \\
\hline \multicolumn{13}{|l|}{$\begin{array}{c}\text { Fringed Myotis } \\
\text { (Myotis thysanodes) }\end{array}$} \\
\hline $\begin{array}{l}\text { Long-legged Myotis } \\
\text { (Myotis volans) }^{3}\end{array}$ & & & & & & & & & & & & \\
\hline
\end{tabular}


${ }^{1}$ Blue cells of table indicate documentation of the species in the region during this month prior to this study. 
Table 7b. Species definitively detected by month each year of the study at the Spring Creek Mine

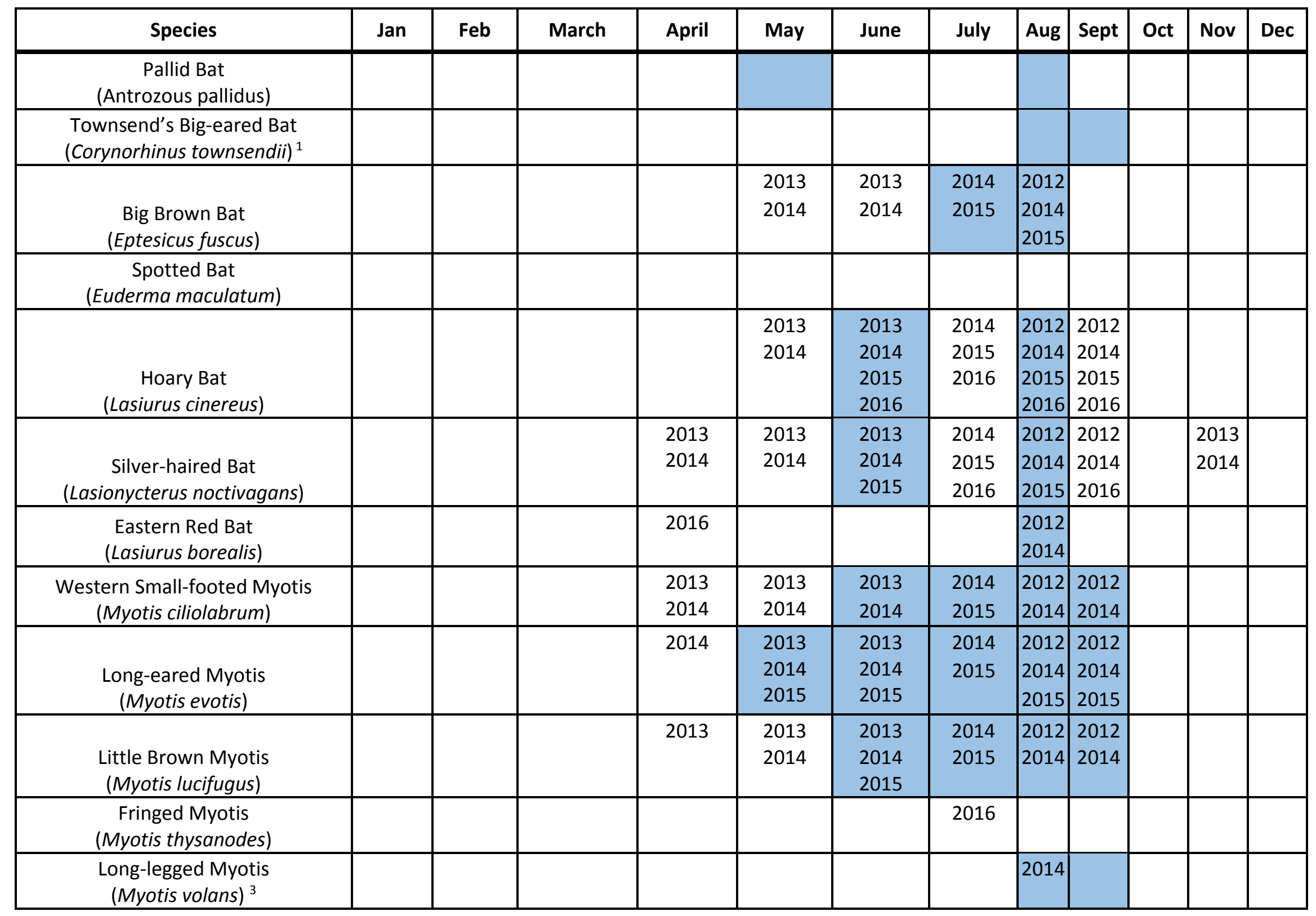


Table 7c. Species definitively detected by month each year of the study at the Otter Creek Coal Tract

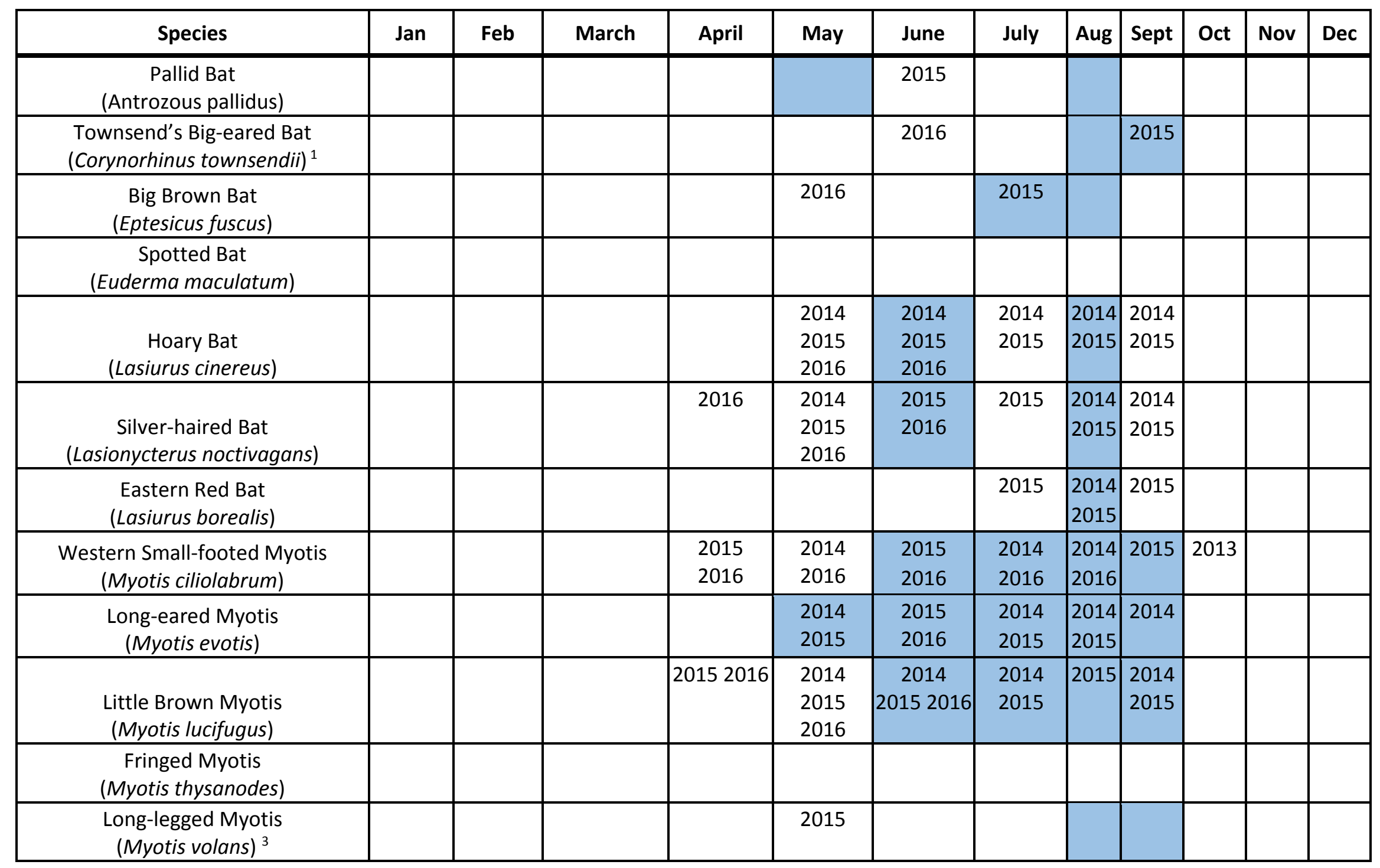


Table 7d. Species definitively detected by month each year of the study at the Big Sky Mine

\begin{tabular}{|c|c|c|c|c|c|c|c|c|c|c|c|c|}
\hline Species & Jan & Feb & March & April & May & June & July & Aug & Sept & Oct & Nov & Dec \\
\hline $\begin{array}{c}\text { Pallid Bat } \\
\text { (Antrozous pallidus) }\end{array}$ & & & & 2014 & 2016 & & & & & & & \\
\hline \multicolumn{13}{|l|}{$\begin{array}{l}\text { Townsend's Big-eared Bat } \\
\text { (Corynorhinus townsendii) }^{1}\end{array}$} \\
\hline $\begin{array}{c}\text { Big Brown Bat } \\
\text { (Eptesicus fuscus) }\end{array}$ & 2014 & 2014 & 2014 & 2014 & 2014 & 2015 & 2014 & 2014 & 2014 & 2013 & 2013 & \\
\hline $\begin{array}{c}\text { Spotted Bat } \\
\text { (Euderma maculatum) }\end{array}$ & & & & & & 2015 & & & & & & \\
\hline $\begin{array}{c}\text { Hoary Bat } \\
\text { (Lasiurus cinereus) }\end{array}$ & & & 2015 & 2015 & $\begin{array}{l}2015 \\
2016\end{array}$ & $\begin{array}{l}2015 \\
2016 \\
\end{array}$ & $\begin{array}{l}2015 \\
2016\end{array}$ & \begin{tabular}{|l|}
2015 \\
2016
\end{tabular} & $\begin{array}{l}2015 \\
2016\end{array}$ & 2015 & 2015 & \\
\hline $\begin{array}{c}\text { Silver-haired Bat } \\
\text { (Lasionycterus noctivagans) }\end{array}$ & 2014 & 2014 & $\begin{array}{l}2014 \\
2015 \\
2016 \\
\end{array}$ & $\begin{array}{l}2014 \\
2015 \\
2016 \\
\end{array}$ & $\begin{array}{l}2014 \\
2015 \\
2016 \\
\end{array}$ & 2015 & $\begin{array}{l}2015 \\
2016\end{array}$ & \begin{tabular}{|l|}
2014 \\
2015 \\
2016 \\
\end{tabular} & $\begin{array}{l}2015 \\
2016\end{array}$ & \begin{tabular}{l|}
2013 \\
2015 \\
2016 \\
\end{tabular} & $\begin{array}{l}2013 \\
2015\end{array}$ & $\begin{array}{l}2013 \\
2015\end{array}$ \\
\hline $\begin{array}{c}\text { Eastern Red Bat } \\
\text { (Lasiurus borealis) }\end{array}$ & & & & & & 2015 & & 2014 & & & & \\
\hline $\begin{array}{c}\text { Western Small-footed Myotis } \\
\text { (Myotis ciliolabrum) }\end{array}$ & $\begin{array}{l}2014 \\
2016\end{array}$ & $\begin{array}{l}2014 \\
2016\end{array}$ & $\begin{array}{l}2014 \\
2016\end{array}$ & $\begin{array}{l}2014 \\
2016 \\
\end{array}$ & $\begin{array}{l}2014 \\
2016\end{array}$ & $\begin{array}{l}2015 \\
2016 \\
\end{array}$ & $\begin{array}{l}2015 \\
2016\end{array}$ & $\begin{array}{l}2014 \\
2015\end{array}$ & $\begin{array}{l}2015 \\
2016 \\
\end{array}$ & $\begin{array}{l}2013 \\
2015\end{array}$ & $\begin{array}{l}2013 \\
2015\end{array}$ & 2015 \\
\hline $\begin{array}{l}\text { Long-eared Myotis } \\
\text { (Myotis evotis) }\end{array}$ & & & & & $\begin{array}{l}2015 \\
2016 \\
\end{array}$ & 2015 & $\begin{array}{l}2014 \\
2015 \\
\end{array}$ & \begin{tabular}{|l|}
2014 \\
2015 \\
\end{tabular} & $\begin{array}{l}2015 \\
2016 \\
\end{array}$ & & & \\
\hline $\begin{array}{l}\text { Little Brown Myotis } \\
\text { (Myotis lucifugus) }\end{array}$ & & & & $\begin{array}{l}2014 \\
2016\end{array}$ & $\begin{array}{l}2014 \\
2016\end{array}$ & $\begin{array}{l}2015 \\
2016\end{array}$ & $\begin{array}{l}2015 \\
2016\end{array}$ & \begin{tabular}{|l|}
2014 \\
2015 \\
2016 \\
\end{tabular} & $\begin{array}{l}2014 \\
2015 \\
2016 \\
\end{array}$ & & & \\
\hline $\begin{array}{c}\text { Fringed Myotis } \\
\text { (Myotis thysanodes) }\end{array}$ & & & & & & & & & & & & \\
\hline $\begin{array}{l}\text { Long-legged Myotis } \\
{\text { (Myotis volans })^{3}}^{3}\end{array}$ & & & & & & & & & & 2015 & & \\
\hline
\end{tabular}


Table 7e. Species definitively detected by month each year of the study at the Absaloka Mine

\begin{tabular}{|c|c|c|c|c|c|c|c|c|c|c|c|c|}
\hline Species & Jan & Feb & March & April & May & June & July & Aug & Sept & Oct & Nov & Dec \\
\hline \multicolumn{13}{|l|}{$\begin{array}{c}\text { Pallid Bat } \\
\text { (Antrozous pallidus) }\end{array}$} \\
\hline \multicolumn{13}{|l|}{$\begin{array}{l}\text { Townsend's Big-eared Bat } \\
\text { (Corynorhinus townsendii) }^{1}\end{array}$} \\
\hline $\begin{array}{c}\text { Big Brown Bat } \\
\text { (Eptesicus fuscus) }\end{array}$ & & & & & $\begin{array}{l}2013 \\
2014\end{array}$ & 2013 & 2013 & & 2012 & & & \\
\hline \multicolumn{13}{|l|}{$\begin{array}{c}\text { Spotted Bat } \\
\text { (Euderma maculatum) }\end{array}$} \\
\hline $\begin{array}{c}\text { Hoary Bat } \\
\text { (Lasiurus cinereus) }\end{array}$ & & & & 2015 & $\begin{array}{l}2013 \\
2014 \\
2015 \\
\end{array}$ & $\begin{array}{l}2013 \\
2014 \\
2015 \\
\end{array}$ & $\begin{array}{l}2014 \\
2015\end{array}$ & \begin{tabular}{|l|}
2012 \\
2014 \\
2015 \\
\end{tabular} & $\begin{array}{l}2012 \\
2014\end{array}$ & & & \\
\hline $\begin{array}{c}\text { Silver-haired Bat } \\
\text { (Lasionycterus noctivagans) }\end{array}$ & & & & 2014 & $\begin{array}{l}2013 \\
2014\end{array}$ & $\begin{array}{l}2013 \\
2015\end{array}$ & & $\begin{array}{l}2012 \\
2014\end{array}$ & $\begin{array}{l}2012 \\
2014\end{array}$ & 2012 & & \\
\hline $\begin{array}{l}\text { Eastern Red Bat } \\
\text { (Lasiurus borealis) }\end{array}$ & & & & & & & & 2012 & 2014 & & & \\
\hline $\begin{array}{l}\text { Western Small-footed Myotis } \\
\text { (Myotis ciliolabrum) }\end{array}$ & & & & & & $\begin{array}{l}2013 \\
2015\end{array}$ & 2015 & 2012 & & & & \\
\hline $\begin{array}{l}\text { Long-eared Myotis } \\
\text { (Myotis evotis) }\end{array}$ & & & & & $\begin{array}{l}2013 \\
2014\end{array}$ & $\begin{array}{l}2013 \\
2014 \\
2015\end{array}$ & 2015 & $\begin{array}{l}2012 \\
2014\end{array}$ & $\begin{array}{l}2012 \\
2014\end{array}$ & & & \\
\hline $\begin{array}{l}\text { Little Brown Myotis } \\
\text { (Myotis lucifugus) }\end{array}$ & & & & & & 2015 & 2015 & 2012 & 2012 & & & \\
\hline \multicolumn{13}{|l|}{$\begin{array}{c}\text { Fringed Myotis } \\
\text { (Myotis thysanodes) }\end{array}$} \\
\hline $\begin{array}{l}\text { Long-legged Myotis } \\
\text { (Myotis volans) }^{3}\end{array}$ & & & & & & & & & & & & \\
\hline
\end{tabular}


Table 7f. Species definitively detected by month each year of the study at the Rosebud Mine Area C and $\mathrm{F}$ pond sites

\begin{tabular}{|c|c|c|c|c|c|c|c|c|c|c|c|c|}
\hline Species & Jan & Feb & March & April & May & June & July & Aug & Sept & Oct & Nov & Dec \\
\hline $\begin{array}{c}\text { Pallid Bat } \\
\text { (Antrozous pallidus) }\end{array}$ & & & & & & & & & & & & \\
\hline $\begin{array}{l}\text { Townsend's Big-eared Bat } \\
\text { (Corynorhinus townsendii) }^{1}\end{array}$ & & & & & & & & & & & & \\
\hline $\begin{array}{c}\text { Big Brown Bat } \\
\text { (Eptesicus fuscus) }\end{array}$ & & & & 2014 & $\begin{array}{l}2013 \\
2014 \\
2016\end{array}$ & $\begin{array}{l}2013 \\
2014\end{array}$ & $\begin{array}{l}2013 \\
2014\end{array}$ & \begin{tabular}{|l|}
2012 \\
2013 \\
2014 \\
\end{tabular} & $\begin{array}{l}2012 \\
2014\end{array}$ & 2014 & 2012 & \\
\hline $\begin{array}{c}\text { Spotted Bat } \\
\text { (Euderma maculatum) }\end{array}$ & & & & & & & & & & & & \\
\hline $\begin{array}{c}\text { Hoary Bat } \\
\text { (Lasiurus cinereus) }\end{array}$ & & & & & $\begin{array}{l}2014 \\
2016\end{array}$ & $\begin{array}{l}2013 \\
2014 \\
2015\end{array}$ & $\begin{array}{l}2013 \\
2014 \\
2015\end{array}$ & $\begin{array}{l}2013 \\
2014 \\
2015\end{array}$ & $\begin{array}{l}2014 \\
2015\end{array}$ & & & \\
\hline $\begin{array}{c}\text { Silver-haired Bat } \\
\text { (Lasionycterus noctivagans) }\end{array}$ & & & & $\begin{array}{l}2014 \\
2016\end{array}$ & $\begin{array}{l}2013 \\
2014 \\
2015 \\
2016\end{array}$ & $\begin{array}{l}2013 \\
2014 \\
2015\end{array}$ & $\begin{array}{l}2013 \\
2014 \\
2015\end{array}$ & \begin{tabular}{|l|}
2012 \\
2013 \\
2014 \\
2015
\end{tabular} & $\begin{array}{l}2012 \\
2013 \\
2014 \\
2015\end{array}$ & 2014 & & \\
\hline $\begin{array}{c}\text { Eastern Red Bat } \\
\text { (Lasiurus borealis) }\end{array}$ & & & & & & & & \begin{tabular}{|l|}
2014 \\
2015 \\
\end{tabular} & 2015 & & & \\
\hline $\begin{array}{l}\text { Western Small-footed Myotis } \\
\text { (Myotis ciliolabrum) }\end{array}$ & & & & 2014 & $\begin{array}{l}2013 \\
2014 \\
2016\end{array}$ & $\begin{array}{l}2013 \\
2014 \\
2015 \\
2016\end{array}$ & $\begin{array}{l}2013 \\
2014 \\
2015\end{array}$ & \begin{tabular}{l|}
2012 \\
2013 \\
2014 \\
2015
\end{tabular} & $\begin{array}{l}2012 \\
2013 \\
2014 \\
2015\end{array}$ & $\begin{array}{l}2013 \\
2014\end{array}$ & & \\
\hline $\begin{array}{l}\text { Long-eared Myotis } \\
\text { (Myotis evotis) }\end{array}$ & & & & & $\begin{array}{l}2014 \\
2016\end{array}$ & $\begin{array}{c}2013 \\
2014 \\
215\end{array}$ & $\begin{array}{c}2013 \\
2014 \\
215\end{array}$ & $\begin{array}{l}2012 \\
2013 \\
2014 \\
2015\end{array}$ & $\begin{array}{l}2012 \\
2013 \\
2014 \\
2015\end{array}$ & 2013 & & \\
\hline $\begin{array}{l}\text { Little Brown Myotis } \\
\text { (Myotis lucifugus) }\end{array}$ & & & & & $\begin{array}{l}2014 \\
2015 \\
2016\end{array}$ & $\begin{array}{l}2013 \\
2014 \\
0215\end{array}$ & $\begin{array}{l}2014 \\
2015\end{array}$ & \begin{tabular}{|l|}
2012 \\
2013 \\
2014 \\
2015 \\
\end{tabular} & $\begin{array}{l}2012 \\
2014 \\
2015\end{array}$ & 2014 & & \\
\hline
\end{tabular}




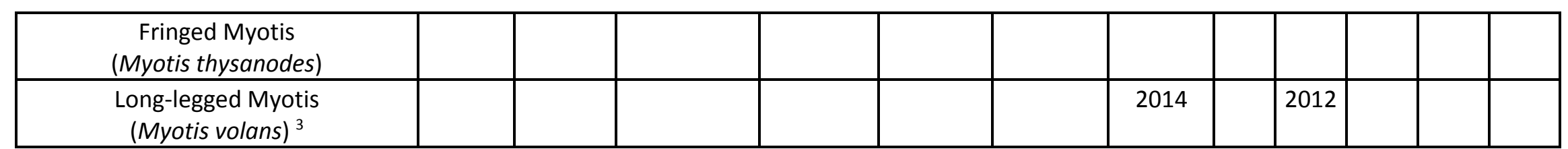

Table 7g. Species definitively detected by month each year of the study at the Signal Peak Mine Reservoir 1 and Busse Water Reservoir sites

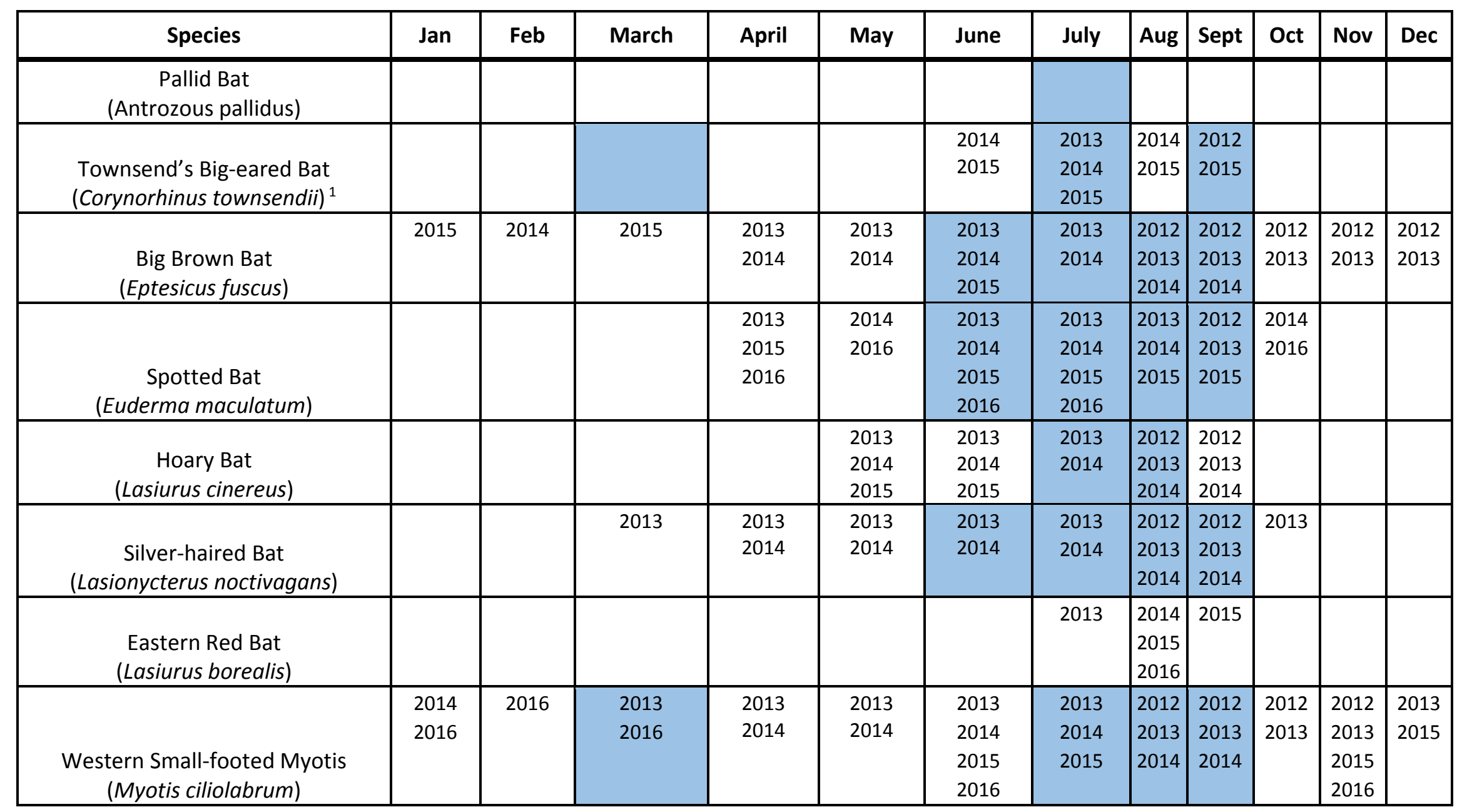




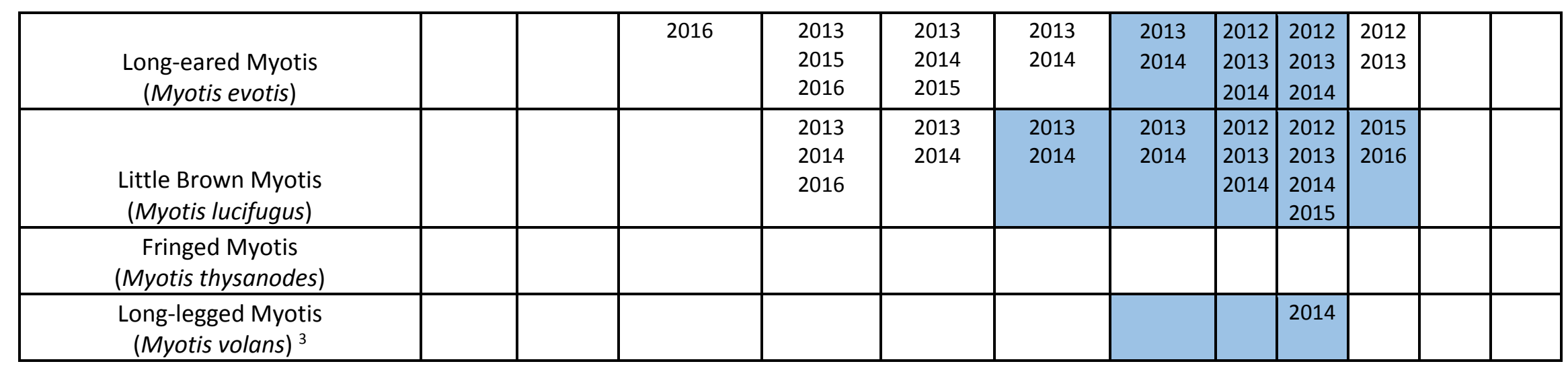


Table 8. Species definitively detected by month across the acoustic detector network (blue cells) and at project detectors (X)

\begin{tabular}{|c|c|c|c|c|c|c|c|c|c|c|c|c|}
\hline Species & Jan & Feb & March & April & May & June & July & Aug & Sept & Oct & Nov & Dec \\
\hline $\begin{array}{c}\text { Pallid Bat } \\
\text { (Antrozous pallidus) }^{1}\end{array}$ & & & & $x$ & $x$ & $x$ & & & & & & \\
\hline $\begin{array}{l}\text { Townsend's Big-eared Bat } \\
\text { (Corynorhinus townsendii) }^{1}\end{array}$ & & & & & & $x$ & $x$ & $x$ & $x$ & & & \\
\hline $\begin{array}{c}\text { Big Brown Bat } \\
\text { (Eptesicus fuscus) }\end{array}$ & $x$ & $x$ & $x$ & $x$ & $x$ & $x$ & $x$ & $x$ & $x$ & $x$ & $x$ & $x$ \\
\hline $\begin{array}{c}\text { Spotted Bat } \\
\text { (Euderma maculatum) }\end{array}$ & & & & $x$ & $x$ & $x$ & $x$ & $x$ & $x$ & $x$ & & \\
\hline $\begin{array}{c}\text { Hoary Bat } \\
\text { (Lasiurus cinereus) } \\
\end{array}$ & & & $x$ & $x$ & $x$ & $x$ & $x$ & $x$ & $x$ & $x$ & $x$ & \\
\hline $\begin{array}{c}\text { Silver-haired Bat } \\
\text { (Lasionycteris noctivagans) }\end{array}$ & $x$ & $x$ & $x$ & $x$ & $x$ & $x$ & $x$ & $x$ & $x$ & $x$ & $x$ & $x$ \\
\hline $\begin{array}{l}\text { Western Small-footed Myotis } \\
\text { (Myotis ciliolabrum) }\end{array}$ & $x$ & $x$ & $x$ & $x$ & $x$ & $x$ & $x$ & $x$ & $x$ & $x$ & $x$ & $x$ \\
\hline $\begin{array}{c}\text { Eastern Red Bat } \\
\text { (Lasiurus borealis) }\end{array}$ & & & & $x$ & & $x$ & $x$ & $x$ & $x$ & $x$ & & \\
\hline $\begin{array}{l}\text { Long-eared Myotis } \\
\text { (Myotis evotis) }\end{array}$ & & & & $x$ & $x$ & $x$ & $x$ & $x$ & $x$ & $x$ & & \\
\hline $\begin{array}{l}\text { Little Brown Myotis } \\
\text { (Myotis lucifugus) }\end{array}$ & & & & $x$ & $x$ & $x$ & $x$ & $x$ & $x$ & $x$ & & \\
\hline $\begin{array}{c}\text { Fringed Myotis } \\
\text { (Myotis thysanodes) }\end{array}$ & & & & & & & $x$ & & & & & \\
\hline 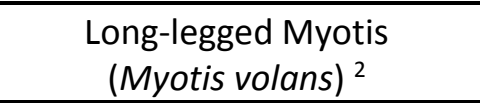 & & & & & $x$ & & $x$ & $x$ & $x$ & $x$ & & \\
\hline
\end{tabular}

${ }^{1}$ Species is relatively quiet and often does not create fully definitive echolocation call recordings on bat detectors.

2 Long-legged Myotis calls can overlap with Western Small-footed Myotis, Long-eared Myotis, Little Brown Myotis, and Fringed Myotis calls and rarely have call characteristics recorded that allow them to be definitively identified as Long-legged Myotis (Maxell 2015). Several call sequences were auto-identified as Long-legged Myotis. However, these call sequences lacked the definitive characteristics necessary to confirm the species' presence. 
Table 9a. Bat Passes summarized by month across all species at the West Decker Mine

\begin{tabular}{|c|c|c|c|c|c|c|c|}
\hline Year & Month & Bat Passes & Sample Nights & $\begin{array}{l}\text { Avg Number } \\
\text { of Bat Passes }\end{array}$ & $\begin{array}{l}\text { StDev of Bat } \\
\text { Passes }\end{array}$ & $\begin{array}{c}\text { Min Count of } \\
\text { Bat Passes }\end{array}$ & $\begin{array}{c}\text { Max Count of } \\
\text { Bat Passes }\end{array}$ \\
\hline 2012 & 8 & 11951 & 11 & 1086.5 & 476.1 & 454 & 1850 \\
\hline 2012 & 9 & 14460 & 30 & 482 & 521.3 & 10 & 2108 \\
\hline 2012 & 10 & 32 & 31 & 1 & 2.3 & 0 & 12 \\
\hline 2012 & 11 & 0 & 30 & 0 & 0 & 0 & 0 \\
\hline 2012 & 12 & 0 & 31 & 0 & 0 & 0 & 0 \\
\hline 2013 & 1 & 0 & 31 & 0 & 0 & 0 & 0 \\
\hline 2013 & 2 & 0 & 28 & 0 & 0 & 0 & 0 \\
\hline 2013 & 3 & 0 & 31 & 0 & 0 & 0 & 0 \\
\hline 2013 & 4 & 1568 & 30 & 52.3 & 140.4 & 0 & 680 \\
\hline 2013 & 5 & 2117 & 31 & 68.3 & 160.8 & 0 & 897 \\
\hline 2013 & 6 & 3033 & 30 & 101.1 & 87.4 & 2 & 273 \\
\hline 2013 & 7 & 1676 & 31 & 54.1 & 134.5 & 0 & 572 \\
\hline 2013 & 8 & 0 & 31 & 0 & 0 & 0 & 0 \\
\hline 2013 & 9 & 1 & 28 & 0 & 0.2 & 0 & 1 \\
\hline 2013 & 10 & 36 & 31 & 1.2 & 4.3 & 0 & 21 \\
\hline 2013 & 11 & 0 & 29 & 0 & 0 & 0 & 0 \\
\hline 2013 & 12 & 0 & 29 & 0 & 0 & 0 & 0 \\
\hline 2014 & 1 & 0 & 31 & 0 & 0 & 0 & 0 \\
\hline 2014 & 2 & 0 & 28 & 0 & 0 & 0 & 0 \\
\hline 2014 & 3 & 0 & 31 & 0 & 0 & 0 & 0 \\
\hline 2014 & 4 & 2037 & 30 & 67.9 & 149.5 & 0 & 773 \\
\hline 2014 & 5 & 7296 & 31 & 235.4 & 195.2 & 0 & 856 \\
\hline 2014 & 6 & 2818 & 30 & 93.9 & 171.2 & 0 & 761 \\
\hline 2014 & 7 & 1283 & 31 & 41.4 & 100.1 & 0 & 386 \\
\hline 2014 & 8 & 11492 & 31 & 370.7 & 1023.6 & 0 & 4010 \\
\hline 2014 & 9 & 31909 & 30 & 1063.6 & 1352.1 & 1 & 5324 \\
\hline 2014 & 10 & 78 & 31 & 2.5 & 5.2 & 0 & 20 \\
\hline 2014 & 11 & 3 & 30 & 0.1 & 0.4 & 0 & 2 \\
\hline
\end{tabular}




\begin{tabular}{|c|c|c|c|c|c|c|c|}
\hline Year & Month & Bat Passes & Sample Nights & $\begin{array}{l}\text { Avg Number } \\
\text { of Bat Passes }\end{array}$ & $\begin{array}{c}\text { StDev of Bat } \\
\text { Passes }\end{array}$ & $\begin{array}{c}\text { Min Count of } \\
\text { Bat Passes }\end{array}$ & $\begin{array}{c}\text { Max Count of } \\
\text { Bat Passes }\end{array}$ \\
\hline 2014 & 12 & 3 & 31 & 0.1 & 0.4 & 0 & 2 \\
\hline 2015 & 1 & 38 & 31 & 1.2 & 6.8 & 0 & 38 \\
\hline 2015 & 2 & 54 & 28 & 1.9 & 8.5 & 0 & 45 \\
\hline 2015 & 3 & 293 & 31 & 9.5 & 25.7 & 0 & 110 \\
\hline 2015 & 4 & 11075 & 30 & 369.2 & 396.7 & 0 & 1246 \\
\hline 2015 & 5 & 30240 & 31 & 975.5 & 1215.8 & 0 & 4413 \\
\hline 2015 & 6 & 19841 & 8 & 2480.1 & 1890.8 & 7 & 4225 \\
\hline 2015 & 6 & 32426 & 22 & 1473.9 & 559 & 517 & 2447 \\
\hline 2015 & 7 & 21401 & 28 & 764.3 & 547.3 & 50 & 1920 \\
\hline 2015 & 8 & 58297 & 31 & 1880.5 & 1529.6 & 14 & 5088 \\
\hline 2015 & 9 & 6647 & 30 & 221.6 & 312.6 & 3 & 1422 \\
\hline 2015 & 10 & 71 & 31 & 2.3 & 5.1 & 0 & 23 \\
\hline 2015 & 11 & 0 & 30 & 0 & 0 & 0 & 0 \\
\hline 2015 & 12 & 19 & 31 & 0.6 & 3.4 & 0 & 19 \\
\hline 2016 & 1 & 2 & 31 & 0.1 & 0.2 & 0 & 1 \\
\hline 2016 & 2 & 0 & 29 & 0 & 0 & 0 & 0 \\
\hline 2016 & 3 & 2 & 31 & 0.1 & 0.2 & 0 & 1 \\
\hline 2016 & 4 & 6166 & 30 & 205.5 & 370 & 0 & 1618 \\
\hline 2016 & 5 & 47534 & 31 & 1533.4 & 962.1 & 0 & 3338 \\
\hline 2016 & 6 & 72849 & 30 & 2428.3 & 872.1 & 1142 & 3965 \\
\hline 2016 & 7 & 46684 & 23 & 2029.7 & 1185.1 & 243 & 4079 \\
\hline
\end{tabular}

Table 9b. Bat Passes summarized by month across all species at the Spring Creek Mine

\begin{tabular}{|c|c|c|c|c|c|c|c|}
\hline Year & Month & Bat Passes & Sample Nights & $\begin{array}{c}\text { Avg Number } \\
\text { of Bat Passes }\end{array}$ & $\begin{array}{c}\text { StDev of Bat } \\
\text { Passes }\end{array}$ & $\begin{array}{c}\text { Min Count of } \\
\text { Bat Passes }\end{array}$ & $\begin{array}{c}\text { Max Count of } \\
\text { Bat Passes }\end{array}$ \\
\hline 2012 & 8 & 13932 & 11 & 1266.5 & 653.2 & 595 & 2744 \\
\hline 2012 & 9 & 2454 & 30 & 81.8 & 215.6 & 0 & 708 \\
\hline 2012 & 10 & 0 & 22 & 0 & 0 & 0 & 0 \\
\hline
\end{tabular}




\begin{tabular}{|c|c|c|c|c|c|c|c|}
\hline Year & Month & Bat Passes & Sample Nights & $\begin{array}{l}\text { Avg Number } \\
\text { of Bat Passes }\end{array}$ & $\begin{array}{l}\text { StDev of Bat } \\
\text { Passes }\end{array}$ & $\begin{array}{c}\text { Min Count of } \\
\text { Bat Passes }\end{array}$ & $\begin{array}{c}\text { Max Count of } \\
\text { Bat Passes }\end{array}$ \\
\hline 2013 & 1 & 0 & 1 & 0 & & 0 & 0 \\
\hline 2013 & 4 & 952 & 28 & 34 & 47 & 0 & 180 \\
\hline 2013 & 5 & 28360 & 31 & 914.8 & 654.3 & 0 & 2105 \\
\hline 2013 & 6 & 27402 & 30 & 913.4 & 486 & 25 & 1976 \\
\hline 2013 & 7 & 0 & 31 & 0 & 0 & 0 & 0 \\
\hline 2013 & 8 & 0 & 31 & 0 & 0 & 0 & 0 \\
\hline 2013 & 9 & 0 & 30 & 0 & 0 & 0 & 0 \\
\hline 2013 & 10 & 65 & 31 & 2.1 & 4.2 & 0 & 18 \\
\hline 2013 & 11 & 20 & 30 & 0.7 & 1.7 & 0 & 8 \\
\hline 2013 & 12 & 6 & 31 & 0.2 & 0.7 & 0 & 4 \\
\hline 2014 & 1 & 0 & 31 & 0 & 0 & 0 & 0 \\
\hline 2014 & 2 & 0 & 28 & 0 & 0 & 0 & 0 \\
\hline 2014 & 3 & 2 & 31 & 0.1 & 0.4 & 0 & 2 \\
\hline 2014 & 4 & 307 & 30 & 10.2 & 29.3 & 0 & 127 \\
\hline 2014 & 5 & 4755 & 31 & 153.4 & 203.3 & 0 & 892 \\
\hline 2014 & 6 & 36944 & 30 & 1231.5 & 583 & 475 & 2489 \\
\hline 2014 & 7 & 46231 & 31 & 1491.3 & 876.8 & 289 & 3018 \\
\hline 2014 & 8 & 24803 & 31 & 800.1 & 798.7 & 0 & 2620 \\
\hline 2014 & 9 & 381 & 30 & 12.7 & 21.8 & 0 & 109 \\
\hline 2014 & 10 & 2 & 31 & 0.1 & 0.4 & 0 & 2 \\
\hline 2014 & 11 & 29 & 30 & 1 & 2.4 & 0 & 11 \\
\hline 2014 & 12 & 21 & 31 & 0.7 & 1.4 & 0 & 5 \\
\hline 2015 & 1 & 1 & 31 & 0 & 0.2 & 0 & 1 \\
\hline 2015 & 2 & 1 & 28 & 0 & 0.2 & 0 & 1 \\
\hline 2015 & 3 & 6 & 31 & 0.2 & 0.6 & 0 & 3 \\
\hline 2015 & 4 & 99 & 30 & 3.3 & 8.1 & 0 & 43 \\
\hline 2015 & 5 & 744 & 31 & 24 & 30.4 & 0 & 131 \\
\hline 2015 & 6 & 469 & 9 & 52.1 & 36.9 & 0 & 108 \\
\hline 2015 & 6 & 5735 & 21 & 273.1 & 97 & 47 & 458 \\
\hline 2015 & 7 & 7561 & 31 & 243.9 & 172.6 & 26 & 889 \\
\hline
\end{tabular}




\begin{tabular}{|c|c|c|c|c|c|c|c|}
\hline Year & Month & Bat Passes & Sample Nights & $\begin{array}{l}\text { Avg Number } \\
\text { of Bat Passes }\end{array}$ & $\begin{array}{l}\text { StDev of Bat } \\
\text { Passes }\end{array}$ & $\begin{array}{c}\text { Min Count of } \\
\text { Bat Passes }\end{array}$ & $\begin{array}{c}\text { Max Count of } \\
\text { Bat Passes }\end{array}$ \\
\hline 2015 & 8 & 8418 & 31 & 271.5 & 194 & 29 & 872 \\
\hline 2015 & 9 & 3858 & 30 & 128.6 & 115.9 & 3 & 427 \\
\hline 2015 & 10 & 155 & 31 & 5 & 10.4 & 0 & 57 \\
\hline 2015 & 11 & 46 & 30 & 1.5 & 2.7 & 0 & 9 \\
\hline 2015 & 12 & 45 & 31 & 1.5 & 3.9 & 0 & 16 \\
\hline 2016 & 1 & 39 & 31 & 1.3 & 2.4 & 0 & 11 \\
\hline 2016 & 2 & 44 & 29 & 1.5 & 4.1 & 0 & 21 \\
\hline 2016 & 3 & 18 & 31 & 0.6 & 1.4 & 0 & 7 \\
\hline 2016 & 4 & 316 & 30 & 10.5 & 16.4 & 0 & 71 \\
\hline 2016 & 5 & 1796 & 31 & 57.9 & 45.4 & 0 & 151 \\
\hline 2016 & 6 & 5173 & 30 & 172.4 & 91.2 & 28 & 460 \\
\hline 2016 & 7 & 6727 & 31 & 217 & 150.9 & 26 & 686 \\
\hline 2016 & 8 & 7291 & 31 & 235.2 & 262.9 & 10 & 1100 \\
\hline 2016 & 9 & 1135 & 30 & 37.8 & 46.6 & 0 & 181 \\
\hline 2016 & 10 & 16 & 31 & 0.5 & 0.9 & 0 & 3 \\
\hline 2016 & 11 & 8 & 30 & 0.3 & 0.6 & 0 & 2 \\
\hline 2016 & 12 & 1 & 12 & 0.1 & 0.3 & 0 & 1 \\
\hline
\end{tabular}

Table 9c. Bat Passes summarized by month across all species at the Otter Creek Coal Tract

\begin{tabular}{|c|c|c|c|c|c|c|c|}
\hline Year & Month & Bat Passes & Sample Nights & $\begin{array}{c}\text { Avg Number } \\
\text { of Bat Passes }\end{array}$ & $\begin{array}{c}\text { StDev of Bat } \\
\text { Passes }\end{array}$ & $\begin{array}{c}\text { Min Count of } \\
\text { Bat Passes }\end{array}$ & $\begin{array}{c}\text { Max Count of } \\
\text { Bat Passes }\end{array}$ \\
\hline 2013 & 10 & 20 & 16 & 1.3 & 3.2 & 0 & 12 \\
\hline 2013 & 11 & 1 & 30 & 0 & 0.2 & 0 & 1 \\
\hline 2013 & 12 & 0 & 31 & 0 & 0 & 0 & 0 \\
\hline 2014 & 1 & 0 & 31 & 0 & 0 & 0 & 0 \\
\hline 2014 & 2 & 1 & 28 & 0 & 0.2 & 0 & 1 \\
\hline 2014 & 3 & 4 & 31 & 0.1 & 0.7 & 0 & 4 \\
\hline 2014 & 4 & 0 & 1 & 0 & & 0 & 0 \\
\hline
\end{tabular}




\begin{tabular}{|c|c|c|c|c|c|c|c|}
\hline Year & Month & Bat Passes & Sample Nights & $\begin{array}{l}\text { Avg Number } \\
\text { of Bat Passes }\end{array}$ & $\begin{array}{l}\text { StDev of Bat } \\
\text { Passes }\end{array}$ & $\begin{array}{c}\text { Min Count of } \\
\text { Bat Passes }\end{array}$ & $\begin{array}{c}\text { Max Count o } \\
\text { Bat Passes }\end{array}$ \\
\hline 2014 & 5 & 32695 & 17 & 1923.2 & 802.4 & 915 & 3545 \\
\hline 2014 & 6 & 36315 & 30 & 1210.5 & 690 & 225 & 3031 \\
\hline 2014 & 7 & 19609 & 31 & 632.5 & 379.9 & 13 & 1348 \\
\hline 2014 & 8 & 8025 & 31 & 258.9 & 220.4 & 0 & 847 \\
\hline 2014 & 9 & 3261 & 6 & 543.5 & 163.5 & 310 & 813 \\
\hline 2014 & 10 & 0 & 4 & 0 & 0 & 0 & 0 \\
\hline 2014 & 11 & 2 & 30 & 0.1 & 0.3 & 0 & 1 \\
\hline 2014 & 12 & 2 & 31 & 0.1 & 0.4 & 0 & 2 \\
\hline 2015 & 1 & 0 & 31 & 0 & 0 & 0 & 0 \\
\hline 2015 & 2 & 2 & 28 & 0.1 & 0.3 & 0 & 1 \\
\hline 2015 & 3 & 125 & 31 & 4 & 8.5 & 0 & 39 \\
\hline 2015 & 4 & 6613 & 30 & 220.4 & 451.7 & 0 & 2025 \\
\hline 2015 & 5 & 28503 & 31 & 919.5 & 711 & 0 & 2264 \\
\hline 2015 & 6 & 11242 & 10 & 1124.2 & 366.4 & 459 & 1656 \\
\hline 2015 & 6 & 18985 & 20 & 949.3 & 543.3 & 295 & 2083 \\
\hline 2015 & 7 & 17014 & 31 & 548.8 & 415.1 & 46 & 1628 \\
\hline 2015 & 8 & 29612 & 31 & 955.2 & 551.6 & 26 & 2079 \\
\hline 2015 & 9 & 13501 & 30 & 450 & 293.7 & 7 & 1062 \\
\hline 2015 & 10 & 253 & 31 & 8.2 & 18 & 0 & 62 \\
\hline 2015 & 11 & 17 & 30 & 0.6 & 3.1 & 0 & 17 \\
\hline 2015 & 12 & 92 & 31 & 3 & 7 & 0 & 31 \\
\hline 2016 & 1 & 50 & 31 & 1.6 & 4.6 & 0 & 19 \\
\hline 2016 & 2 & 16 & 29 & 0.6 & 1.3 & 0 & 4 \\
\hline 2016 & 3 & 87 & 31 & 2.8 & 6.7 & 0 & 28 \\
\hline 2016 & 4 & 5855 & 30 & 195.2 & 302.4 & 0 & 1405 \\
\hline 2016 & 5 & 38930 & 31 & 1255.8 & 859.1 & 0 & 3350 \\
\hline 2016 & 6 & 24716 & 21 & 1177 & 376.1 & 632 & 2156 \\
\hline
\end{tabular}


Table 9d. Bat Passes summarized by month across all species at the Big Sky Mine

\begin{tabular}{|c|c|c|c|c|c|c|c|}
\hline Year & Month & Bat Passes & Sample Nights & $\begin{array}{l}\text { Avg Number } \\
\text { of Bat Passes }\end{array}$ & $\begin{array}{l}\text { StDev of Bat } \\
\text { Passes }\end{array}$ & $\begin{array}{c}\text { Min Count of } \\
\text { Bat Passes }\end{array}$ & $\begin{array}{c}\text { Max Count of } \\
\text { Bat Passes }\end{array}$ \\
\hline 2013 & 10 & 1699 & 16 & 106.2 & 128.3 & 2 & 369 \\
\hline 2013 & 11 & 3007 & 30 & 100.2 & 94 & 0 & 342 \\
\hline 2013 & 12 & 621 & 31 & 20 & 33.4 & 0 & 119 \\
\hline 2014 & 1 & 886 & 31 & 28.6 & 43.4 & 0 & 203 \\
\hline 2014 & 2 & 323 & 28 & 11.5 & 23.2 & 0 & 105 \\
\hline 2014 & 3 & 1313 & 31 & 42.4 & 84 & 0 & 422 \\
\hline 2014 & 4 & 12294 & 30 & 409.8 & 612.3 & 0 & 2451 \\
\hline 2014 & 5 & 922 & 9 & 102.4 & 130.2 & 0 & 408 \\
\hline 2014 & 7 & 756 & 2 & 378 & 274.4 & 184 & 572 \\
\hline 2014 & 8 & 5108 & 31 & 164.8 & 166.4 & 0 & 607 \\
\hline 2014 & 9 & 585 & 30 & 19.5 & 38 & 0 & 168 \\
\hline 2014 & 10 & 0 & 31 & 0 & 0 & 0 & 0 \\
\hline 2014 & 11 & 0 & 30 & 0 & 0 & 0 & 0 \\
\hline 2014 & 12 & 0 & 31 & 0 & 0 & 0 & 0 \\
\hline 2015 & 1 & 0 & 31 & 0 & 0 & 0 & 0 \\
\hline 2015 & 2 & 0 & 28 & 0 & 0 & 0 & 0 \\
\hline 2015 & 3 & 23169 & 31 & 747.4 & 951.5 & 0 & 2984 \\
\hline 2015 & 4 & 12140 & 30 & 404.7 & 460.4 & 14 & 2049 \\
\hline 2015 & 5 & 13263 & 31 & 427.8 & 339.9 & 3 & 1082 \\
\hline 2015 & 6 & 7468 & 10 & 746.8 & 280.3 & 263 & 1176 \\
\hline 2015 & 6 & 26656 & 20 & 1332.8 & 676.4 & 219 & 2587 \\
\hline 2015 & 7 & 42178 & 31 & 1360.6 & 721.8 & 128 & 3186 \\
\hline 2015 & 8 & 24190 & 31 & 780.3 & 471.1 & 0 & 2210 \\
\hline 2015 & 9 & 17334 & 30 & 577.8 & 390.1 & 42 & 1362 \\
\hline 2015 & 10 & 7901 & 31 & 254.9 & 214.7 & 0 & 1086 \\
\hline 2015 & 11 & 5672 & 30 & 189.1 & 192.8 & 0 & 656 \\
\hline 2015 & 12 & 3137 & 31 & 101.2 & 139.4 & 0 & 451 \\
\hline 2016 & 1 & 2309 & 31 & 74.5 & 90.9 & 0 & 276 \\
\hline
\end{tabular}




\begin{tabular}{|c|c|c|c|c|c|c|c|}
\hline Year & Month & Bat Passes & Sample Nights & $\begin{array}{c}\text { Avg Number } \\
\text { of Bat Passes }\end{array}$ & $\begin{array}{c}\text { StDev of Bat } \\
\text { Passes }\end{array}$ & $\begin{array}{c}\text { Min Count of } \\
\text { Bat Passes }\end{array}$ & $\begin{array}{c}\text { Max Count of } \\
\text { Bat Passes }\end{array}$ \\
\hline 2016 & 2 & 9637 & 29 & 332.3 & 319.4 & 0 & 1580 \\
\hline 2016 & 3 & 13073 & 31 & 421.7 & 347.6 & 0 & 1321 \\
\hline 2016 & 4 & 14092 & 30 & 469.7 & 517.3 & 2 & 1797 \\
\hline 2016 & 5 & 25963 & 31 & 837.5 & 548.5 & 1 & 1831 \\
\hline 2016 & 6 & 45155 & 30 & 1505.2 & 494.5 & 544 & 2427 \\
\hline 2016 & 7 & 37735 & 31 & 1217.3 & 656.8 & 81 & 2450 \\
\hline 2016 & 8 & 17140 & 31 & 552.9 & 230.3 & 205 & 1226 \\
\hline 2016 & 9 & 7966 & 30 & 265.5 & 220.6 & 0 & 727 \\
\hline 2016 & 10 & 3861 & 31 & 124.5 & 107.7 & 0 & 344 \\
\hline 2016 & 11 & 5184 & 30 & 172.8 & 113.7 & 0 & 420 \\
\hline 2016 & 12 & 478 & 31 & 15.4 & 34.3 & 0 & 129 \\
\hline 2017 & 1 & 476 & 31 & 15.4 & 30.4 & 0 & 93 \\
\hline 2017 & 2 & 1543 & 28 & 55.1 & 60.4 & 0 & 162 \\
\hline 2017 & 3 & 4999 & 31 & 161.3 & 298 & 0 & 1620 \\
\hline 2017 & 4 & 7534 & 30 & 251.1 & 308.5 & 0 & 1204 \\
\hline 2017 & 5 & 15131 & 31 & 488.1 & 369.3 & 5 & 1430 \\
\hline 2017 & 6 & 24334 & 30 & 811.1 & 367.2 & 67 & 1592 \\
\hline 2017 & 7 & 37131 & 31 & 1197.8 & 373 & 451 & 2100 \\
\hline 2017 & 8 & 8609 & 14 & 614.9 & 207.8 & 226 & 1032 \\
\hline
\end{tabular}


Table 9e. Bat Passes summarized by month across all species at the Absaloka Mine

\begin{tabular}{|c|c|c|c|c|c|c|c|}
\hline Year & Month & Bat Passes & Sample Nights & $\begin{array}{l}\text { Avg Number } \\
\text { of Bat Passes }\end{array}$ & $\begin{array}{l}\text { StDev of Bat } \\
\text { Passes }\end{array}$ & $\begin{array}{c}\text { Min Count of } \\
\text { Bat Passes }\end{array}$ & $\begin{array}{c}\text { Max Count of } \\
\text { Bat Passes }\end{array}$ \\
\hline 2012 & 8 & 4403 & 10 & 440.3 & 316.7 & 111 & 1228 \\
\hline 2012 & 9 & 6608 & 30 & 220.3 & 398.8 & 8 & 2046 \\
\hline 2012 & 10 & 35 & 31 & 1.1 & 2.9 & 0 & 15 \\
\hline 2012 & 11 & 0 & 30 & 0 & 0 & 0 & 0 \\
\hline 2012 & 12 & 0 & 31 & 0 & 0 & 0 & 0 \\
\hline 2013 & 1 & 0 & 31 & 0 & 0 & 0 & 0 \\
\hline 2013 & 2 & 0 & 28 & 0 & 0 & 0 & 0 \\
\hline 2013 & 3 & 0 & 31 & 0 & 0 & 0 & 0 \\
\hline 2013 & 4 & 34 & 30 & 1.1 & 2.5 & 0 & 9 \\
\hline 2013 & 5 & 890 & 31 & 28.7 & 43.4 & 0 & 181 \\
\hline 2013 & 6 & 668 & 30 & 22.3 & 39 & 0 & 189 \\
\hline 2013 & 7 & 193 & 31 & 6.2 & 7.5 & 0 & 26 \\
\hline 2013 & 8 & 0 & 31 & 0 & 0 & 0 & 0 \\
\hline 2013 & 9 & 0 & 30 & 0 & 0 & 0 & 0 \\
\hline 2013 & 10 & 30 & 31 & 1 & 4.2 & 0 & 23 \\
\hline 2013 & 11 & 1 & 30 & 0 & 0.2 & 0 & 1 \\
\hline 2013 & 12 & 5 & 31 & 0.2 & 0.9 & 0 & 5 \\
\hline 2014 & 1 & 0 & 31 & 0 & 0 & 0 & 0 \\
\hline 2014 & 2 & 0 & 28 & 0 & 0 & 0 & 0 \\
\hline 2014 & 3 & 0 & 31 & 0 & 0 & 0 & 0 \\
\hline 2014 & 4 & 153 & 30 & 5.1 & 11.7 & 0 & 55 \\
\hline 2014 & 5 & 1616 & 31 & 52.1 & 89.9 & 0 & 338 \\
\hline 2014 & 6 & 1068 & 30 & 35.6 & 65.5 & 0 & 275 \\
\hline 2014 & 7 & 12 & 31 & 0.4 & 0.7 & 0 & 2 \\
\hline 2014 & 8 & 2001 & 31 & 64.5 & 155.5 & 0 & 572 \\
\hline 2014 & 9 & 1532 & 30 & 51.1 & 94.2 & 0 & 432 \\
\hline 2014 & 10 & 21 & 31 & 0.7 & 1.8 & 0 & 7 \\
\hline 2014 & 11 & 1 & 30 & 0 & 0.2 & 0 & 1 \\
\hline
\end{tabular}




\begin{tabular}{|c|c|c|c|c|c|c|c|}
\hline Year & Month & Bat Passes & Sample Nights & $\begin{array}{c}\text { Avg Number } \\
\text { of Bat Passes }\end{array}$ & $\begin{array}{c}\text { StDev of Bat } \\
\text { Passes }\end{array}$ & $\begin{array}{c}\text { Min Count of } \\
\text { Bat Passes }\end{array}$ & $\begin{array}{c}\text { Max Count of } \\
\text { Bat Passes }\end{array}$ \\
\hline 2014 & 12 & 4 & 31 & 0.1 & 0.5 & 0 & 2 \\
\hline 2015 & 1 & 1 & 31 & 0 & 0.2 & 0 & 1 \\
\hline 2015 & 2 & 2 & 28 & 0.1 & 0.3 & 0 & 1 \\
\hline 2015 & 3 & 48 & 31 & 1.5 & 5.8 & 0 & 30 \\
\hline 2015 & 4 & 466 & 30 & 15.5 & 25.8 & 0 & 102 \\
\hline 2015 & 5 & 2199 & 31 & 70.9 & 103.3 & 0 & 430 \\
\hline 2015 & 6 & 1250 & 10 & 125 & 162.5 & 0 & 469 \\
\hline 2015 & 6 & 9863 & 20 & 493.1 & 305.1 & 134 & 1107 \\
\hline 2015 & 7 & 8131 & 23 & 353.5 & 443.6 & 0 & 1352 \\
\hline 2015 & 8 & 522 & 14 & 37.3 & 32.5 & 5 & 118 \\
\hline 2015 & 9 & 16 & 10 & 1.6 & 2 & 0 & 6 \\
\hline 2015 & 10 & 0 & 10 & 0 & 0 & 0 & 0 \\
\hline 2015 & 11 & 0 & 4 & 0 & 0 & 0 & 0 \\
\hline
\end{tabular}

Table 9f. Bat Passes summarized by month across all species at the Rosebud Mine Area F Pond 7

\begin{tabular}{|c|c|c|c|c|c|c|c|}
\hline Year & Month & Bat Passes & Sample Nights & $\begin{array}{c}\text { Avg Number } \\
\text { of Bat Passes }\end{array}$ & $\begin{array}{c}\text { StDev of Bat } \\
\text { Passes }\end{array}$ & $\begin{array}{c}\text { Min Count of } \\
\text { Bat Passes }\end{array}$ & $\begin{array}{c}\text { Max Count of } \\
\text { Bat Passes }\end{array}$ \\
\hline 2012 & 8 & 1188 & 10 & 118.8 & 76.6 & 26 & 274 \\
\hline 2012 & 9 & 1845 & 30 & 61.5 & 85.6 & 5 & 384 \\
\hline 2012 & 10 & 40 & 31 & 1.3 & 5.5 & 0 & 30 \\
\hline 2012 & 11 & 2 & 30 & 0.1 & 0.3 & 0 & 1 \\
\hline 2012 & 12 & 0 & 31 & 0 & 0 & 0 & 0 \\
\hline 2013 & 1 & 1 & 31 & 0 & 0.2 & 0 & 1 \\
\hline 2013 & 2 & 1 & 28 & 0 & 0.2 & 0 & 1 \\
\hline 2013 & 3 & 0 & 31 & 0 & 0 & 0 & 0 \\
\hline 2013 & 4 & 9 & 30 & 0.3 & 1.1 & 0 & 5 \\
\hline 2013 & 5 & 131 & 31 & 4.2 & 6.9 & 0 & 28 \\
\hline 2013 & 6 & 260 & 30 & 8.7 & 6.4 & 0 & 25 \\
\hline 2013 & 7 & 373 & 31 & 12 & 7.3 & 3 & 2 \\
\hline
\end{tabular}




\begin{tabular}{|c|c|c|c|c|c|c|c|}
\hline 2013 & 8 & 326 & 31 & 10.5 & 9 & 1 & 34 \\
\hline 2013 & 9 & 72 & 30 & 2.4 & 2.5 & 0 & 11 \\
\hline 2013 & 10 & 4 & 15 & 0.3 & 0.6 & 0 & 2 \\
\hline
\end{tabular}

Table 9g. Bat Passes summarized by month across all species at the Rosebud Mine Area C Pond

\begin{tabular}{|c|c|c|c|c|c|c|c|}
\hline Year & Month & Bat Passes & Sample Nights & $\begin{array}{l}\text { Avg Number } \\
\text { of Bat Passes }\end{array}$ & $\begin{array}{l}\text { StDev of Bat } \\
\text { Passes }\end{array}$ & $\begin{array}{c}\text { Min Count of } \\
\text { Bat Passes }\end{array}$ & $\begin{array}{c}\text { Max Count of } \\
\text { Bat Passes }\end{array}$ \\
\hline 2013 & 10 & 11 & 16 & 0.7 & 1.9 & 0 & 7 \\
\hline 2013 & 11 & 3 & 30 & 0.1 & 0.5 & 0 & 3 \\
\hline 2013 & 12 & 3 & 31 & 0.1 & 0.4 & 0 & 2 \\
\hline 2014 & 1 & 0 & 31 & 0 & 0 & 0 & 0 \\
\hline 2014 & 2 & 4 & 28 & 0.1 & 0.8 & 0 & 4 \\
\hline 2014 & 3 & 4 & 31 & 0.1 & 0.6 & 0 & 3 \\
\hline 2014 & 4 & 477 & 30 & 15.9 & 29.4 & 0 & 110 \\
\hline 2014 & 5 & 3796 & 31 & 122.5 & 151.3 & 0 & 485 \\
\hline 2014 & 6 & 10728 & 30 & 357.6 & 246.7 & 6 & 917 \\
\hline 2014 & 7 & 18955 & 31 & 611.5 & 376.4 & 72 & 1633 \\
\hline 2014 & 8 & 11093 & 31 & 357.8 & 318.6 & 0 & 1126 \\
\hline 2014 & 9 & 3590 & 30 & 119.7 & 206.2 & 0 & 965 \\
\hline 2014 & 10 & 125 & 31 & 4 & 6.6 & 0 & 24 \\
\hline 2014 & 11 & 6 & 30 & 0.2 & 0.9 & 0 & 5 \\
\hline 2014 & 12 & 4 & 31 & 0.1 & 0.6 & 0 & 3 \\
\hline 2015 & 1 & 1 & 31 & 0 & 0.2 & 0 & 1 \\
\hline 2015 & 2 & 0 & 28 & 0 & 0 & 0 & 0 \\
\hline 2015 & 3 & 122 & 31 & 3.9 & 15.3 & 0 & 78 \\
\hline 2015 & 4 & 178 & 24 & 7.4 & 34.9 & 0 & 171 \\
\hline 2015 & 5 & 3722 & 31 & 120.1 & 173.1 & 0 & 773 \\
\hline 2015 & 6 & 3525 & 10 & 352.5 & 285.8 & 54 & 1065 \\
\hline 2015 & 6 & 24894 & 20 & 1244.7 & 618.9 & 385 & 2543 \\
\hline 2015 & 7 & 41127 & 30 & 1370.9 & 642.6 & 16 & 2204 \\
\hline 2015 & 8 & 16179 & 31 & 521.9 & 538.3 & 12 & 2318 \\
\hline
\end{tabular}




\begin{tabular}{|c|c|c|c|c|c|c|c|}
\hline Year & Month & Bat Passes & Sample Nights & $\begin{array}{c}\text { Avg Number } \\
\text { of Bat Passes }\end{array}$ & $\begin{array}{c}\text { StDev of Bat } \\
\text { Passes }\end{array}$ & $\begin{array}{c}\text { Min Count of } \\
\text { Bat Passes }\end{array}$ & $\begin{array}{c}\text { Max Count of } \\
\text { Bat Passes }\end{array}$ \\
\hline 2015 & 9 & 3939 & 30 & 131.3 & 161.8 & 0 & 821 \\
\hline 2015 & 10 & 53 & 31 & 1.7 & 3.7 & 0 & 18 \\
\hline 2015 & 11 & 4 & 30 & 0.1 & 0.6 & 0 & 3 \\
\hline 2015 & 12 & 14 & 31 & 0.5 & 1.5 & 0 & 8 \\
\hline 2016 & 1 & 14 & 31 & 0.5 & 1.5 & 0 & 8 \\
\hline 2016 & 2 & 1 & 29 & 0 & 0.2 & 0 & 1 \\
\hline 2016 & 3 & 55 & 31 & 1.8 & 8.3 & 0 & 46 \\
\hline 2016 & 4 & 783 & 30 & 26.1 & 68.7 & 0 & 341 \\
\hline 2016 & 5 & 5659 & 31 & 182.5 & 216.5 & 0 & 742 \\
\hline 2016 & 6 & 8947 & 12 & 745.6 & 424.3 & 57 & 1482 \\
\hline
\end{tabular}

Table 9h. Bat Passes summarized by month across all species at the Signal Peak Mine Reservoir 1

\begin{tabular}{|c|c|c|c|c|c|c|c|}
\hline Year & Month & Bat Passes & Sample Nights & $\begin{array}{c}\text { Avg Number } \\
\text { of Bat Passes }\end{array}$ & $\begin{array}{c}\text { StDev of Bat } \\
\text { Passes }\end{array}$ & $\begin{array}{c}\text { Min Count of } \\
\text { Bat Passes }\end{array}$ & $\begin{array}{c}\text { Max Count of } \\
\text { Bat Passes }\end{array}$ \\
\hline 2012 & 8 & 4851 & 9 & 539 & 101.7 & 383 & 666 \\
\hline 2012 & 9 & 12423 & 30 & 414.1 & 443.5 & 25 & 2309 \\
\hline 2012 & 10 & 1172 & 31 & 37.8 & 65.2 & 0 & 314 \\
\hline 2012 & 11 & 1108 & 30 & 36.9 & 35.4 & 0 & 119 \\
\hline 2012 & 12 & 572 & 31 & 18.5 & 41.3 & 0 & 166 \\
\hline 2013 & 1 & 466 & 31 & 15 & 37.4 & 0 & 157 \\
\hline 2013 & 2 & 276 & 28 & 9.9 & 17.8 & 0 & 66 \\
\hline 2013 & 3 & 1312 & 31 & 42.3 & 75.1 & 0 & 255 \\
\hline 2013 & 4 & 7493 & 30 & 249.8 & 417.8 & 0 & 1275 \\
\hline 2013 & 5 & 8163 & 31 & 263.3 & 386.5 & 0 & 1576 \\
\hline 2013 & 6 & 9084 & 30 & 302.8 & 238.7 & 0 & 898 \\
\hline 2013 & 7 & 7246 & 31 & 233.7 & 124.6 & 30 & 487 \\
\hline 2013 & 8 & 10952 & 31 & 353.3 & 291.1 & 5 & 1635 \\
\hline 2013 & 9 & 2154 & 30 & 71.8 & 81.7 & & 0 \\
\hline 2013 & 10 & 550 & 31 & 17.7 & 29.5 & 0 & 262 \\
\hline
\end{tabular}




\begin{tabular}{|c|c|c|c|c|c|c|c|}
\hline Year & Month & Bat Passes & Sample Nights & $\begin{array}{l}\text { Avg Number } \\
\text { of Bat Passes }\end{array}$ & $\begin{array}{l}\text { StDev of Bat } \\
\text { Passes }\end{array}$ & $\begin{array}{l}\text { Min Count of } \\
\text { Bat Passes }\end{array}$ & $\begin{array}{c}\text { Max Count of } \\
\text { Bat Passes }\end{array}$ \\
\hline 2013 & 11 & 541 & 30 & 18 & 24.8 & 0 & 107 \\
\hline 2013 & 12 & 94 & 31 & 3 & 6.5 & 0 & 21 \\
\hline 2014 & 1 & 43 & 31 & 1.4 & 4.2 & 0 & 21 \\
\hline 2014 & 2 & 4 & 28 & 0.1 & 0.4 & 0 & 2 \\
\hline 2014 & 4 & 231 & 1 & 231 & & 231 & 231 \\
\hline 2014 & 5 & 4746 & 31 & 153.1 & 200.5 & 0 & 740 \\
\hline 2014 & 6 & 6790 & 30 & 226.3 & 195.4 & 1 & 738 \\
\hline 2014 & 7 & 17224 & 31 & 555.6 & 210.5 & 141 & 1057 \\
\hline 2014 & 8 & 10306 & 31 & 332.5 & 270.3 & 0 & 1163 \\
\hline 2014 & 9 & 2021 & 30 & 67.4 & 130.3 & 0 & 690 \\
\hline 2014 & 10 & 285 & 31 & 9.2 & 13.8 & 0 & 60 \\
\hline 2014 & 11 & 406 & 30 & 13.5 & 32.9 & 0 & 146 \\
\hline 2014 & 12 & 410 & 31 & 13.2 & 20.1 & 0 & 82 \\
\hline 2015 & 1 & 97 & 31 & 3.1 & 7.3 & 0 & 28 \\
\hline 2015 & 2 & 59 & 28 & 2.1 & 4.6 & 0 & 19 \\
\hline 2015 & 3 & 3874 & 31 & 125 & 277.7 & 0 & 1120 \\
\hline 2015 & 4 & 2649 & 30 & 88.3 & 159.8 & 0 & 707 \\
\hline 2015 & 5 & 1484 & 31 & 47.9 & 106 & 0 & 490 \\
\hline 2015 & 6 & 392 & 11 & 35.6 & 39.4 & 0 & 122 \\
\hline 2015 & 6 & 12112 & 19 & 637.5 & 478.4 & 146 & 1674 \\
\hline 2015 & 7 & 23690 & 31 & 764.2 & 426.4 & 147 & 1843 \\
\hline 2015 & 8 & 13740 & 31 & 443.2 & 447 & 21 & 2413 \\
\hline 2015 & 9 & 9316 & 30 & 310.5 & 268.9 & 3 & 1071 \\
\hline 2015 & 10 & 1388 & 31 & 44.8 & 41.3 & 0 & 151 \\
\hline 2015 & 11 & 1313 & 30 & 43.8 & 76.1 & 0 & 314 \\
\hline 2015 & 12 & 970 & 31 & 31.3 & 46.3 & 0 & 147 \\
\hline 2016 & 1 & 550 & 31 & 17.7 & 29.3 & 0 & 122 \\
\hline 2016 & 2 & 1073 & 29 & 37 & 72.3 & 0 & 311 \\
\hline 2016 & 3 & 8 & 21 & 0.4 & 1.1 & 0 & 5 \\
\hline 2016 & 4 & 2231 & 21 & 106.2 & 180.8 & 0 & 648 \\
\hline
\end{tabular}




\begin{tabular}{|c|c|c|c|c|c|c|c|}
\hline Year & Month & Bat Passes & Sample Nights & $\begin{array}{c}\text { Avg Number } \\
\text { of Bat Passes }\end{array}$ & $\begin{array}{c}\text { StDev of Bat } \\
\text { Passes }\end{array}$ & $\begin{array}{c}\text { Min Count of } \\
\text { Bat Passes }\end{array}$ & $\begin{array}{c}\text { Max Count of } \\
\text { Bat Passes }\end{array}$ \\
\hline 2016 & 5 & 3099 & 31 & 100 & 103 & 0 & 370 \\
\hline 2016 & 6 & 26577 & 30 & 885.9 & 572.9 & 90 & 2485 \\
\hline 2016 & 7 & 13255 & 31 & 427.6 & 390 & 18 & 1893 \\
\hline 2016 & 8 & 6879 & 31 & 221.9 & 130.1 & 22 & 523 \\
\hline 2016 & 9 & 2698 & 30 & 89.9 & 137.8 & 0 & 534 \\
\hline 2016 & 10 & 661 & 31 & 21.3 & 18 & 0 & 68 \\
\hline 2016 & 11 & 1298 & 30 & 43.3 & 44.2 & 0 & 141 \\
\hline 2016 & 12 & 137 & 31 & 4.4 & 14.3 & 0 & 71 \\
\hline 2017 & 1 & 327 & 31 & 10.5 & 22.7 & 0 & 77 \\
\hline 2017 & 2 & 558 & 28 & 19.9 & 39.7 & 0 & 186 \\
\hline 2017 & 3 & 779 & 31 & 25.1 & 42.6 & 0 & 188 \\
\hline 2017 & 4 & 1537 & 30 & 51.2 & 69 & 0 & 295 \\
\hline 2017 & 5 & 5865 & 31 & 189.2 & 216.7 & 0 & 736 \\
\hline 2017 & 6 & 22021 & 30 & 734 & 488.2 & 78 & 1957 \\
\hline 2017 & 7 & 26120 & 31 & 842.6 & 499.7 & 231 & 2013 \\
\hline 2017 & 8 & 6345 & 13 & 488.1 & 265.6 & 21 & 1008 \\
\hline
\end{tabular}

Table 9i. Bat Passes summarized by month across all species at the Signal Peak Mine Busse Water Reservoir

\begin{tabular}{|c|c|c|c|c|c|c|c|}
\hline Year & Month & Bat Passes & Sample Nights & $\begin{array}{c}\text { Avg Number } \\
\text { of Bat Passes }\end{array}$ & $\begin{array}{c}\text { StDev of Bat } \\
\text { Passes }\end{array}$ & $\begin{array}{c}\text { Min Count of } \\
\text { Bat Passes }\end{array}$ & $\begin{array}{c}\text { Max Count of } \\
\text { Bat Passes }\end{array}$ \\
\hline 2012 & 8 & 24895 & 9 & 2766.1 & 697 & 1735 & 3873 \\
\hline 2012 & 9 & 39185 & 30 & 1306.2 & 904.8 & 90 & 3153 \\
\hline 2012 & 10 & 360 & 31 & 11.6 & 28 & 0 & 118 \\
\hline 2012 & 11 & 81 & 30 & 2.7 & 4.7 & 0 & 23 \\
\hline 2012 & 12 & 98 & 31 & 3.2 & 7.2 & 0 & 35 \\
\hline 2013 & 1 & 11 & 9 & 1.2 & 2.6 & 0 & 8 \\
\hline 2013 & 4 & 2704 & 29 & 93.2 & 168.7 & 0 & 595 \\
\hline 2013 & 5 & 13831 & 31 & 446.2 & 388.3 & 0 & 1630 \\
\hline 2013 & 6 & 59236 & 30 & 1974.5 & 1493.9 & 28 & 4533 \\
\hline
\end{tabular}




\begin{tabular}{|c|c|c|c|c|c|c|c|}
\hline Year & Month & Bat Passes & Sample Nights & $\begin{array}{l}\text { Avg Number } \\
\text { of Bat Passes }\end{array}$ & $\begin{array}{l}\text { StDev of Bat } \\
\text { Passes }\end{array}$ & $\begin{array}{c}\text { Min Count of } \\
\text { Bat Passes }\end{array}$ & $\begin{array}{c}\text { Max Count of } \\
\text { Bat Passes }\end{array}$ \\
\hline 2013 & 7 & 69941 & 26 & 2690 & 474.7 & 1664 & 3614 \\
\hline 2013 & 8 & 21481 & 27 & 795.6 & 344.4 & 284 & 1500 \\
\hline 2013 & 9 & 4693 & 30 & 156.4 & 199 & 0 & 823 \\
\hline 2013 & 10 & 518 & 31 & 16.7 & 36.7 & 0 & 165 \\
\hline 2013 & 11 & 138 & 30 & 4.6 & 8.1 & 0 & 27 \\
\hline 2013 & 12 & 36 & 31 & 1.2 & 2.8 & 0 & 11 \\
\hline 2014 & 1 & 26 & 31 & 0.8 & 1.8 & 0 & 7 \\
\hline 2014 & 2 & 21 & 28 & 0.8 & 1.7 & 0 & 5 \\
\hline 2014 & 3 & 20 & 31 & 0.6 & 1.6 & 0 & 7 \\
\hline 2014 & 4 & 4480 & 30 & 149.3 & 231.3 & 0 & 828 \\
\hline 2014 & 5 & 28045 & 31 & 904.7 & 998.3 & 0 & 3855 \\
\hline 2014 & 6 & 47713 & 30 & 1590.4 & 727.7 & 240 & 2934 \\
\hline 2014 & 7 & 66688 & 31 & 2151.2 & 467.3 & 1206 & 3290 \\
\hline 2014 & 8 & 34026 & 31 & 1097.6 & 671.8 & 0 & 2673 \\
\hline 2014 & 9 & 5678 & 30 & 189.3 & 288.5 & 0 & 1352 \\
\hline 2014 & 10 & 126 & 31 & 4.1 & 7.2 & 0 & 27 \\
\hline 2014 & 11 & 28 & 30 & 0.9 & 1.8 & 0 & 6 \\
\hline 2014 & 12 & 150 & 31 & 4.8 & 8.5 & 0 & 30 \\
\hline 2015 & 1 & 20 & 31 & 0.6 & 1.3 & 0 & 5 \\
\hline 2015 & 2 & 77 & 28 & 2.8 & 11.1 & 0 & 59 \\
\hline 2015 & 3 & 2191 & 31 & 70.7 & 170.4 & 0 & 682 \\
\hline 2015 & 4 & 5288 & 30 & 176.3 & 255.8 & 0 & 930 \\
\hline 2015 & 5 & 16851 & 31 & 543.6 & 380.7 & 0 & 1172 \\
\hline 2015 & 6 & 60207 & 30 & 2006.9 & 508.2 & 855 & 2851 \\
\hline 2015 & 7 & 65667 & 31 & 2118.3 & 665.9 & 429 & 3141 \\
\hline 2015 & 8 & 37383 & 17 & 2199 & 967.3 & 494 & 3901 \\
\hline 2015 & 9 & 21991 & 30 & 733 & 1012.2 & 6 & 3555 \\
\hline 2015 & 10 & 104 & 18 & 5.8 & 8 & 0 & 25 \\
\hline 2015 & 6 & 24315 & 10 & 2431.5 & 386.7 & 1875 & 2938 \\
\hline 2015 & 8 & 26506 & 11 & 2409.6 & 1082.4 & 543 & 4060 \\
\hline
\end{tabular}




\begin{tabular}{|c|c|c|c|c|c|c|c|}
\hline Year & Month & Bat Passes & Sample Nights & $\begin{array}{l}\text { Avg Number } \\
\text { of Bat Passes }\end{array}$ & $\begin{array}{c}\text { StDev of Bat } \\
\text { Passes }\end{array}$ & $\begin{array}{c}\text { Min Count of } \\
\text { Bat Passes }\end{array}$ & $\begin{array}{c}\text { Max Count of } \\
\text { Bat Passes }\end{array}$ \\
\hline 2015 & 9 & 28220 & 30 & 940.7 & 994.4 & 25 & 3702 \\
\hline 2015 & 10 & 386 & 31 & 12.5 & 20 & 0 & 93 \\
\hline 2015 & 11 & 247 & 30 & 8.2 & 16.5 & 0 & 64 \\
\hline 2015 & 12 & 409 & 31 & 13.2 & 25.1 & 0 & 106 \\
\hline 2016 & 1 & 369 & 31 & 11.9 & 17.2 & 0 & 66 \\
\hline 2016 & 2 & 259 & 29 & 8.9 & 13.3 & 0 & 52 \\
\hline 2016 & 3 & 629 & 31 & 20.3 & 40.4 & 0 & 154 \\
\hline 2016 & 4 & 14756 & 30 & 491.9 & 715.7 & 0 & 3383 \\
\hline 2016 & 5 & 23256 & 30 & 775.2 & 502 & 0 & 1819 \\
\hline 2016 & 9 & 1242 & 3 & 414 & 137.9 & 286 & 560 \\
\hline 2016 & 10 & 538 & 31 & 17.4 & 34.7 & 0 & 158 \\
\hline 2016 & 11 & 413 & 30 & 13.8 & 21.4 & 0 & 94 \\
\hline 2016 & 12 & 119 & 31 & 3.8 & 11.4 & 0 & 56 \\
\hline 2017 & 1 & 122 & 31 & 3.9 & 8.3 & 0 & 33 \\
\hline 2017 & 2 & 311 & 28 & 11.1 & 20.3 & 0 & 73 \\
\hline 2017 & 3 & 455 & 31 & 14.7 & 26.3 & 0 & 103 \\
\hline 2017 & 4 & 2818 & 30 & 93.9 & 134.9 & 0 & 496 \\
\hline 2017 & 5 & 31531 & 31 & 1017.1 & 526.5 & 39 & 1976 \\
\hline 2017 & 6 & 67669 & 30 & 2255.6 & 818.3 & 607 & 3622 \\
\hline 2017 & 7 & 99795 & 31 & 3219.2 & 432 & 2224 & 4149 \\
\hline 2017 & 8 & 38330 & 13 & 2948.5 & 867 & 1714 & 4482 \\
\hline
\end{tabular}


Table 10a. Nightly background and bat pass temperatures summarized by month at the West Decker Mine ${ }^{1}$

\begin{tabular}{|c|c|c|c|c|c|c|c|}
\hline ঐँ & 돌 & $\begin{array}{c}\text { Background } \\
\text { Temp C } \\
\text { Avg (SD) N }\end{array}$ & $\begin{array}{c}\text { Bat Pass } \\
\text { Temp C } \\
\text { Avg (SD) N }\end{array}$ & $\begin{array}{c}\text { Background } \\
\text { Min } \\
\text { Temp C }\end{array}$ & $\begin{array}{c}\text { Bat Pass } \\
\text { Min Temp C }\end{array}$ & $\begin{array}{c}\text { Background } \\
\text { Max } \\
\text { Temp C }\end{array}$ & $\begin{array}{c}\text { Bat Pass } \\
\text { Max Temp C }\end{array}$ \\
\hline 2012 & 8 & $\begin{array}{c}15.7(4.4) \\
1311\end{array}$ & $\begin{array}{c}17.3(3.4) \\
8968\end{array}$ & 5.5 & 5.7 & 25.7 & 24.7 \\
\hline 2012 & 9 & $\begin{array}{c}10.5(4.9) \\
5355\end{array}$ & $\begin{array}{c}13.6(3.3) \\
10927\end{array}$ & -0.8 & 0.3 & 24.4 & 22.9 \\
\hline 2012 & 10 & $\begin{array}{c}4.4(4.3) \\
10931\end{array}$ & $9.7(4.1) 33$ & -6.7 & 1.3 & 20.1 & 17.3 \\
\hline 2012 & 11 & $\begin{array}{c}-0.4(4.9) \\
16328\end{array}$ & - & -16.2 & - & 15.8 & - \\
\hline 2012 & 12 & $\begin{array}{c}-5.5(6.8) \\
9830\end{array}$ & - & -20.5 & - & 6.7 & - \\
\hline 2013 & 1 & $\begin{array}{c}-8.2(5.4) \\
8826\end{array}$ & - & -20.5 & - & 5.9 & - \\
\hline 2013 & 2 & $\begin{array}{c}-2.8(3.5) \\
4560\end{array}$ & - & -11.2 & - & 5.4 & - \\
\hline 2013 & 3 & $-1(5) 4499$ & - & -14.8 & - & 12.5 & - \\
\hline 2013 & 4 & 2 (5.1) 3785 & $\begin{array}{c}10.8(3.5) \\
1065\end{array}$ & -9.6 & -1.5 & 15 & 15 \\
\hline 2013 & 5 & $\begin{array}{c}10.5(5.2) \\
3400\end{array}$ & $\begin{array}{c}12.1(3.5) \\
1492\end{array}$ & -3.9 & -1.3 & 26.5 & 22.1 \\
\hline 2013 & 6 & $\begin{array}{c}14.6(4.4) \\
3034\end{array}$ & $\begin{array}{c}16.3(2.6) \\
2538\end{array}$ & 2.2 & 7 & 25.1 & 24.4 \\
\hline 2013 & 7 & $\begin{array}{c}18.6(3.2) \\
3294\end{array}$ & $\begin{array}{c}18.2(1.6) \\
1422\end{array}$ & 11.7 & 12.7 & 27.7 & 24.9 \\
\hline 2013 & 8 & $\begin{array}{c}18.4(3.4) \\
3731\end{array}$ & - & 10.3 & - & 27.4 & - \\
\hline 2013 & 9 & $\begin{array}{c}14.3(5.8) \\
3781\end{array}$ & - & 0.6 & - & 30.3 & - \\
\hline 2013 & 10 & $\begin{array}{c}5.2(3.8) \\
4894\end{array}$ & $6.1(0.1) 4$ & -4.6 & 6 & 16.6 & 6.2 \\
\hline 2013 & 11 & $-1.2(5) 5002$ & - & -16 & - & 10.3 & - \\
\hline 2013 & 12 & $\begin{array}{c}-9.1(7.5) \\
5219\end{array}$ & - & -20.5 & - & 6 & - \\
\hline 2014 & 1 & $-3.5(7) 5513$ & - & -20.5 & - & 11 & - \\
\hline 2014 & 2 & $\begin{array}{c}-8.7(8.1) \\
4577\end{array}$ & - & -20.5 & - & 4.9 & - \\
\hline 2014 & 3 & $\begin{array}{c}-1.1(5.9) \\
4503\end{array}$ & - & -20.5 & - & 9.7 & - \\
\hline
\end{tabular}




\begin{tabular}{|c|c|c|c|c|c|c|c|}
\hline ঐँ & $\frac{\text { 厂 }}{\tilde{z}}$ & $\begin{array}{l}\text { Background } \\
\text { Temp C } \\
\text { Avg (SD) N }\end{array}$ & $\begin{array}{c}\text { Bat Pass } \\
\text { Temp C } \\
\text { Avg (SD) N }\end{array}$ & $\begin{array}{c}\text { Background } \\
\text { Min } \\
\text { Temp C }\end{array}$ & $\begin{array}{c}\text { Bat Pass } \\
\text { Min Temp C }\end{array}$ & $\begin{array}{c}\text { Background } \\
\text { Max } \\
\text { Temp C }\end{array}$ & $\begin{array}{c}\text { Bat Pass } \\
\text { Max Temp C }\end{array}$ \\
\hline 2014 & 4 & $\begin{array}{c}4.1(4.6) \\
3789\end{array}$ & $\begin{array}{c}11.9(4.2) \\
1475\end{array}$ & -5.9 & -0.3 & 18.6 & 18.6 \\
\hline 2014 & 5 & $10(5.1) 3429$ & $\begin{array}{c}13.2(3.6) \\
5567\end{array}$ & -2.6 & 1.1 & 25.2 & 24.9 \\
\hline 2014 & 6 & 13 (3.3) 3085 & $\begin{array}{c}12.8(2.6) \\
2497\end{array}$ & 3.7 & 5.2 & 22.6 & 18.6 \\
\hline 2014 & 7 & $\begin{array}{c}16.9(3.8) \\
3291\end{array}$ & $\begin{array}{c}17.6(2.3) \\
1055\end{array}$ & 7.2 & 12.7 & 26.5 & 25.2 \\
\hline 2014 & 8 & $\begin{array}{c}16.6(3.8) \\
3766\end{array}$ & $\begin{array}{c}14.6(1.8) \\
8140\end{array}$ & 6.5 & 11.3 & 29.7 & 20.3 \\
\hline 2014 & 9 & 11 (4.9) 4162 & $\begin{array}{c}10.8(4) \\
25643\end{array}$ & -2 & 0.1 & 27.7 & 23.1 \\
\hline 2014 & 10 & $6.2(5) 4878$ & $12.6(3.8) 74$ & -4.6 & 0.6 & 22.7 & 19.6 \\
\hline 2014 & 11 & $\begin{array}{c}-3.5(8.3) \\
5241\end{array}$ & $9.8(3.4) 2$ & -20.5 & 7.4 & 12.7 & 12.2 \\
\hline 2014 & 12 & $\begin{array}{c}-4.9(6.4) \\
5694\end{array}$ & - & -20.5 & - & 8 & - \\
\hline 2015 & 1 & $\begin{array}{c}-6.1(6.9) \\
5547\end{array}$ & $5(0.1) 20$ & -20.5 & 4.9 & 9.8 & 5.1 \\
\hline 2015 & 2 & $\begin{array}{c}-1.8(5.7) \\
4598\end{array}$ & 4.9 (2) 34 & -17.3 & 4.2 & 13.5 & 12.7 \\
\hline 2015 & 3 & $2(7.2) 4533$ & 10 (3) 208 & -18 & 3.7 & 17.6 & 15.1 \\
\hline 2015 & 4 & $\begin{array}{c}4.9(4.9) \\
3817\end{array}$ & $\begin{array}{c}9.6(2.8) \\
7665\end{array}$ & -8.6 & 0.3 & 22.1 & 20.8 \\
\hline 2015 & 5 & $\begin{array}{c}9.7(4.1) \\
3426\end{array}$ & $\begin{array}{c}11.2(3.4) \\
21295\end{array}$ & -2.1 & -0.8 & 20.9 & 20.1 \\
\hline 2015 & 6 & $\begin{array}{c}19.1(3.2) \\
2182\end{array}$ & $\begin{array}{c}18.8(2.8) \\
33219\end{array}$ & 11.8 & 12.2 & 27.7 & 27.5 \\
\hline 2015 & 6 & $\begin{array}{c}15.4(2.4) \\
884\end{array}$ & $\begin{array}{c}15.2(1.7) \\
11014\end{array}$ & 9.8 & 10.8 & 24.7 & 20.9 \\
\hline 2015 & 7 & $\begin{array}{c}19.4(3.3) \\
2887\end{array}$ & $\begin{array}{c}18(2.8) \\
22230\end{array}$ & 10.3 & 10.8 & 28.7 & 27.9 \\
\hline 2015 & 8 & 18.7 (5) 3731 & $\begin{array}{c}14.9(3.4) \\
60490\end{array}$ & 3.4 & 3.6 & 30.3 & 28.5 \\
\hline 2015 & 9 & $\begin{array}{c}15.4(4.5) \\
4174\end{array}$ & $\begin{array}{c}17.9(3.2) \\
7376\end{array}$ & 6 & 8.2 & 28.7 & 27 \\
\hline 2015 & 10 & $\begin{array}{c}10.1(4.7) \\
4895\end{array}$ & $17.7(3.1) 75$ & -2.1 & 8.5 & 25.1 & 22.6 \\
\hline 2015 & 11 & $0.4(6) 5232$ & - & -16.5 & - & 12.8 & 0 \\
\hline
\end{tabular}




\begin{tabular}{|c|c|c|c|c|c|c|c|}
\hline$\stackrel{\frac{1}{d}}{\frac{1}{2}}$ & $\frac{\text { c }}{\text { 亡 }}$ & $\begin{array}{c}\text { Background } \\
\text { Temp C } \\
\text { Avg (SD) N }\end{array}$ & $\begin{array}{c}\text { Bat Pass } \\
\text { Temp C } \\
\text { Avg (SD) N }\end{array}$ & $\begin{array}{c}\text { Background } \\
\text { Min } \\
\text { Temp C }\end{array}$ & $\begin{array}{c}\text { Bat Pass } \\
\text { Min Temp C }\end{array}$ & $\begin{array}{c}\text { Background } \\
\text { Max } \\
\text { Temp C }\end{array}$ & $\begin{array}{c}\text { Bat Pass } \\
\text { Max Temp C }\end{array}$ \\
\hline 2015 & 12 & $\begin{array}{c}-4.2(6.5) \\
5732\end{array}$ & $3.1(0) 22$ & -20.3 & 3.1 & 16.5 & 3.1 \\
\hline 2016 & 1 & $\begin{array}{c}-3.6(5.9) \\
5578 \\
\end{array}$ & 0.9 () 1 & -17.3 & 0.9 & 10.2 & 0.9 \\
\hline 2016 & 2 & $\begin{array}{c}2.2(4.9) \\
4843\end{array}$ & - & -11.5 & - & 19.3 & - \\
\hline 2016 & 3 & 2.9 (1.6) 236 & - & 0.6 & - & 8 & - \\
\hline
\end{tabular}

${ }^{1}$ Temperatures should only be regarded as being indicative of the general temperature at the time of detection. Temperatures were recorded at the detector approximately 1-meter above ground level while the microphone was mounted at approximately 3-meters above ground level and bats were in flight at an unknown altitude, but probably typically within 30-meters of ground level. Temperatures of the bat's roost environment at the time flights were initiated are also obviously unknown.

Table 10b. Nightly background and bat pass temperatures summarized by month at the Spring Creek Mine

\begin{tabular}{|c|c|c|c|c|c|c|c|}
\hline$\frac{1}{\stackrel{\pi}{\pi}}$ & 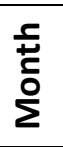 & $\begin{array}{c}\text { Background } \\
\text { Temp C } \\
\text { Avg (SD) } \mathbf{N}\end{array}$ & $\begin{array}{c}\text { Bat Pass } \\
\text { Temp C } \\
\text { Avg (SD) N }\end{array}$ & $\begin{array}{c}\text { Background } \\
\text { Min } \\
\text { Temp C }\end{array}$ & $\begin{array}{c}\text { Bat Pass } \\
\text { Min Temp C }\end{array}$ & $\begin{array}{c}\text { Background } \\
\text { Max } \\
\text { Temp C }\end{array}$ & $\begin{array}{c}\text { Bat Pass } \\
\text { Max Temp C }\end{array}$ \\
\hline 2012 & 8 & $\begin{array}{r}20.3(4.5) \\
131 \\
4 \\
\end{array}$ & $\begin{array}{r}21(4.3) \\
119 \\
10 \\
\end{array}$ & 8.4 & 8.7 & 31.7 & 30.7 \\
\hline 2012 & 9 & 15 (4.4) 4164 & $\begin{array}{r}19.2(3.4) \\
208 \\
2 \\
\end{array}$ & 5.2 & 12 & 29 & 26.4 \\
\hline 2012 & 10 & 9 (4.7) 3495 & - & -3.6 & - & 24.4 & - \\
\hline 2013 & 1 & $\begin{array}{r}-12.8(2.3) \\
114\end{array}$ & - & -15.7 & - & -8.9 & - \\
\hline 2013 & 4 & $\begin{array}{r}4.7(5.8) \\
344 \\
1\end{array}$ & $\begin{array}{r}11.7(3.2) \\
602\end{array}$ & -9.2 & 3.2 & 18.1 & 17.8 \\
\hline 2013 & 5 & $\begin{array}{r}12.9(5.1) \\
339 \\
9 \\
\end{array}$ & $\begin{array}{r}14.5(4.4) \\
226 \\
68 \\
\end{array}$ & -0.1 & 2.1 & 26.2 & 26.2 \\
\hline 2013 & 6 & $\begin{array}{r}16.8(4.6) \\
300 \\
5 \\
\end{array}$ & $\begin{array}{r}18.4(3.5) \\
226 \\
65 \\
\end{array}$ & 4.7 & 4.7 & 28.2 & 28 \\
\hline 2013 & 7 & 21 (3.6) 3276 & - & 13.5 & - & 32 & - \\
\hline 2013 & 8 & $\begin{array}{r}21.7(3.7) \\
372 \\
3\end{array}$ & - & 14.3 & - & 31.8 & - \\
\hline
\end{tabular}




\begin{tabular}{|c|c|c|c|c|c|c|c|}
\hline$\stackrel{\frac{1}{\pi}}{\frac{\pi}{2}}$ & 돈 & $\begin{array}{c}\text { Background } \\
\text { Temp C } \\
\text { Avg (SD) N }\end{array}$ & $\begin{array}{c}\text { Bat Pass } \\
\text { Temp C } \\
\text { Avg (SD) N }\end{array}$ & $\begin{array}{c}\text { Background } \\
\text { Min } \\
\text { Temp C }\end{array}$ & $\begin{array}{c}\text { Bat Pass } \\
\text { Min Temp C }\end{array}$ & $\begin{array}{c}\text { Background } \\
\text { Max } \\
\text { Temp C }\end{array}$ & $\begin{array}{c}\text { Bat Pass } \\
\text { Max Temp }\end{array}$ \\
\hline 2013 & 9 & $16.5(6) 4142$ & - & 3.2 & - & 33.6 & - \\
\hline 2013 & 10 & $\begin{array}{r}8.1(3.8) \\
496 \\
7\end{array}$ & $11.8(2.4) 49$ & -1 & 6 & 18.4 & 15 \\
\hline 2013 & 11 & $\begin{array}{r}2.4(4.9) \\
524 \\
0\end{array}$ & $6.2(2.4) 17$ & -12.7 & 1.3 & 13.8 & 9 \\
\hline 2013 & 12 & $\begin{array}{r}-3.1(9.5) \\
566 \\
7\end{array}$ & $6.1(2.9) 6$ & -20.5 & 4.1 & 12.7 & 10 \\
\hline 2014 & 1 & $\begin{array}{r}0.7(7.2) \\
551 \\
9 \\
\end{array}$ & - & -20.5 & 0 & 13.6 & 0 \\
\hline 2014 & 2 & $-5(8.7) 4597$ & - & -20.5 & 0 & 10.2 & 0 \\
\hline 2014 & 3 & $\begin{array}{r}2.2(6.5) \\
452 \\
8\end{array}$ & $6($ () 1 & -20.5 & 6 & 13.3 & 6 \\
\hline 2014 & 4 & $\begin{array}{r}7.1(4.5) \\
381 \\
0 \\
\end{array}$ & $\begin{array}{r}16.1(3.2) \\
238\end{array}$ & -3.9 & 4.9 & 19.9 & 19.6 \\
\hline 2014 & 5 & $\begin{array}{r}12.2(4.9) \\
344 \\
5\end{array}$ & $\begin{array}{r}16.9(3.8) \\
338 \\
0\end{array}$ & 2.9 & 4.6 & 27.5 & 27.5 \\
\hline 2014 & 6 & $\begin{array}{r}15.3(3.3) \\
308 \\
2 \\
\end{array}$ & $\begin{array}{r}15.4(3) \\
247 \\
74 \\
\end{array}$ & 6.9 & 6.9 & 25.5 & 25.5 \\
\hline 2014 & 7 & $\begin{array}{r}19.9(4.1) \\
330 \\
8\end{array}$ & $\begin{array}{r}18.9(3.7) \\
268 \\
85\end{array}$ & 10.5 & 10.5 & 29.8 & 29.8 \\
\hline 2014 & 8 & $\begin{array}{r}19.1(3.7) \\
363 \\
6 \\
\end{array}$ & $\begin{array}{r}19.2(2.5) \\
153 \\
43 \\
\end{array}$ & 8.9 & 14.3 & 29.3 & 26.9 \\
\hline 2014 & 9 & $14.2(5) 4175$ & $\begin{array}{r}18.5(4.2) \\
364 \\
\end{array}$ & 0 & 6.7 & 28.5 & 25.9 \\
\hline 2014 & 10 & $\begin{array}{c}9.9(4.6) \\
491 \\
4\end{array}$ & - & -1.6 & - & 22.9 & - \\
\hline 2014 & 11 & $\begin{array}{r}0.3(9.4) \\
518 \\
1\end{array}$ & 8.5 (5.1) 23 & -20.5 & 3.2 & 17.9 & 15.1 \\
\hline 2014 & 12 & $\begin{array}{r}-0.2(6.7) \\
569 \\
3\end{array}$ & $6.3(1.7) 15$ & -20.5 & 2.9 & 13.5 & 8.9 \\
\hline
\end{tabular}




\begin{tabular}{|c|c|c|c|c|c|c|c|}
\hline$\stackrel{\frac{1}{d}}{2}$ & 岳 & $\begin{array}{c}\text { Background } \\
\text { Temp C } \\
\text { Avg (SD) } \mathbf{N}\end{array}$ & $\begin{array}{c}\text { Bat Pass } \\
\text { Temp C } \\
\text { Avg (SD) N }\end{array}$ & $\begin{array}{c}\text { Background } \\
\text { Min } \\
\text { Temp C }\end{array}$ & $\begin{array}{c}\text { Bat Pass } \\
\text { Min Temp C }\end{array}$ & $\begin{array}{c}\text { Background } \\
\text { Max } \\
\text { Temp C }\end{array}$ & $\begin{array}{c}\text { Bat Pass } \\
\text { Max Temp C }\end{array}$ \\
\hline 2015 & 1 & 0.1 (7) 5544 & 4.2 () 1 & -18.3 & 4.2 & 16.1 & 4.2 \\
\hline 2015 & 2 & $\begin{array}{r}1.6(7.2) \\
460 \\
1 \\
\end{array}$ & - & -17.5 & - & 17.4 & - \\
\hline 2015 & 3 & $\begin{array}{r}6.2(7.3) \\
453 \\
3 \\
\end{array}$ & $13.1(4.9) 5$ & -15.3 & 7.5 & 20.9 & 17.3 \\
\hline 2015 & 4 & $\begin{array}{r}7.1(4.3) \\
381 \\
7\end{array}$ & $17.2(6.2) 92$ & -3.9 & 5.1 & 24.6 & 24.6 \\
\hline 2015 & 5 & $\begin{array}{r}11.6(4.3) \\
343 \\
7\end{array}$ & $\begin{array}{r}16.7(3.6) \\
677\end{array}$ & 2.4 & 3.1 & 22.6 & 22.4 \\
\hline 2015 & 6 & $\begin{array}{r}18.2(2.5) \\
985\end{array}$ & $\begin{array}{r}19.4(2.3) \\
461\end{array}$ & 12 & 14.5 & 24.9 & 24.7 \\
\hline 2015 & 6 & $\begin{array}{r}20.9(3.2) \\
209 \\
6\end{array}$ & $\begin{array}{r}21.9(2.9) \\
581 \\
2 \\
\end{array}$ & 14.1 & 14.5 & 29.5 & 29.2 \\
\hline 2015 & 7 & $\begin{array}{r}21.3(3.4) \\
332 \\
7 \\
\end{array}$ & $\begin{array}{r}23.2(3.3) \\
778 \\
5 \\
\end{array}$ & 13.5 & 14.8 & 31 & 31 \\
\hline 2015 & 8 & $\begin{array}{r}21.4(5.4) \\
376 \\
3\end{array}$ & $\begin{array}{r}25.6(4.6) \\
869 \\
0\end{array}$ & 5.5 & 8 & 33 & 33 \\
\hline 2015 & 9 & 18 (4.6) 4192 & $\begin{array}{r}21.4(3.8) \\
399 \\
0\end{array}$ & 8.9 & 10.8 & 30 & 30 \\
\hline 2015 & 10 & $\begin{array}{r}13.1(4.8) \\
490 \\
9\end{array}$ & 19.2 (5) 168 & 0.4 & 5.7 & 26 & 24.4 \\
\hline 2015 & 11 & $\begin{array}{r}3.7(6.2) \\
524 \\
1 \\
\end{array}$ & $6.4(2.9) 49$ & -13.4 & 0.6 & 15.8 & 11.8 \\
\hline 2015 & 12 & $0.8(6) 5704$ & $5.4(3.1) 49$ & -13.4 & 0 & 19.8 & 12.8 \\
\hline 2016 & 1 & $\begin{array}{r}1.8(4.8) \\
552 \\
7\end{array}$ & $5.2(3.6) 40$ & -8.7 & 0.9 & 14.1 & 10.2 \\
\hline 2016 & 2 & $\begin{array}{r}5.8(4.4) \\
480 \\
2\end{array}$ & $6.9(2.3) 46$ & -5.9 & 3.1 & 21.7 & 11.3 \\
\hline 2016 & 3 & 7.2 (5) 4537 & $13.2(4.3) 18$ & -7.2 & 7.7 & 19.8 & 17.9 \\
\hline
\end{tabular}




\begin{tabular}{|c|c|c|c|c|c|c|c|}
\hline さ & $\stackrel{\text { f }}{\stackrel{5}{0}}$ & $\begin{array}{c}\text { Background } \\
\text { Temp C } \\
\text { Avg (SD) N }\end{array}$ & $\begin{array}{c}\text { Bat Pass } \\
\text { Temp C } \\
\text { Avg (SD) N }\end{array}$ & $\begin{array}{c}\text { Background } \\
\text { Min } \\
\text { Temp C }\end{array}$ & $\begin{array}{c}\text { Bat Pass } \\
\text { Min Temp C }\end{array}$ & $\begin{array}{c}\text { Background } \\
\text { Max } \\
\text { Temp C }\end{array}$ & $\begin{array}{c}\text { Bat Pass } \\
\text { Max Temp C }\end{array}$ \\
\hline 2016 & 4 & $\begin{array}{r}11.9(4.5) \\
560\end{array}$ & $16.9(4.7) 19$ & 2.4 & 10 & 21.4 & 21.4 \\
\hline
\end{tabular}

Table 10c. Nightly background and bat pass temperatures summarized by month at the Otter Creek Coal Tract

\begin{tabular}{|c|c|c|c|c|c|c|c|}
\hline 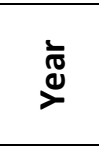 & 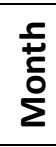 & $\begin{array}{c}\text { Background } \\
\text { Temp C } \\
\text { Avg (SD) N }\end{array}$ & $\begin{array}{c}\text { Bat Pass } \\
\text { Temp C } \\
\text { Avg (SD) } \mathbf{N}\end{array}$ & $\begin{array}{c}\text { Background } \\
\text { Min } \\
\text { Temp C }\end{array}$ & $\begin{array}{c}\text { Bat Pass } \\
\text { Min Temp C }\end{array}$ & $\begin{array}{c}\text { Background } \\
\text { Max } \\
\text { Temp C }\end{array}$ & $\begin{array}{c}\text { Bat Pass } \\
\text { Max Temp C }\end{array}$ \\
\hline 2013 & 10 & $\begin{array}{r}4.5(4.1) \\
253 \\
3 \\
\end{array}$ & $5.3(5.1) 14$ & -3.6 & 1.9 & 14 & 13.5 \\
\hline 2013 & 11 & $-3(5.2) 5260$ & - & -17.7 & - & 9.5 & - \\
\hline 2013 & 12 & $\begin{array}{r}-7.7(8.5) \\
570 \\
6\end{array}$ & - & -20.5 & - & 8.9 & - \\
\hline 2014 & 1 & $\begin{array}{r}-3.9(7.3) \\
555 \\
6\end{array}$ & - & -20.5 & - & 11.5 & - \\
\hline 2014 & 2 & $\begin{array}{r}-9.1(9.1) \\
460 \\
2\end{array}$ & - & -20.5 & - & 8.9 & - \\
\hline 2014 & 3 & $-2(6.4) 4509$ & $0.8(0) 3$ & -20.5 & 0.8 & 12.8 & 0.8 \\
\hline 2014 & 4 & $-4.4(5.5) 201$ & - & -12.9 & - & 1.7 & - \\
\hline 2014 & 5 & $\begin{array}{r}12.2(3.6) \\
177 \\
4\end{array}$ & $\begin{array}{r}11.9(2.9) \\
179 \\
36\end{array}$ & 3.6 & 4.4 & 25.9 & 25.9 \\
\hline 2014 & 6 & $\begin{array}{r}12.2(3.7) \\
309 \\
1\end{array}$ & $\begin{array}{r}13(2.6) \\
202 \\
05\end{array}$ & 2.1 & 6 & 21.9 & 19.8 \\
\hline 2014 & 7 & $\begin{array}{r}15.8(4.6) \\
329 \\
1 \\
\end{array}$ & $\begin{array}{r}14.5(2.7) \\
139 \\
31 \\
\end{array}$ & 6.4 & 6.5 & 30.7 & 25.4 \\
\hline 2014 & 8 & 16 (4.1) 3720 & 12.6 (2) 6988 & 8.5 & 8.2 & 29.2 & 26 \\
\hline 2014 & 9 & $8.1(4) 778$ & $9.6(2) 2678$ & 0.6 & 3.9 & 21.2 & 21.2 \\
\hline 2014 & 10 & $2.4(6.9) 702$ & - & -6.4 & - & 21.4 & - \\
\hline 2014 & 11 & $\begin{array}{r}-4.7(8.8) \\
523 \\
8 \\
\end{array}$ & $-0.7(3) 2$ & -20.5 & -2.8 & 14.6 & 1.4 \\
\hline
\end{tabular}




\begin{tabular}{|c|c|c|c|c|c|c|c|}
\hline$\stackrel{\frac{1}{\pi}}{\frac{1}{2}}$ & 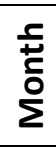 & $\begin{array}{c}\text { Background } \\
\text { Temp C } \\
\text { Avg (SD) } \mathbf{N}\end{array}$ & $\begin{array}{c}\text { Bat Pass } \\
\text { Temp C } \\
\text { Avg (SD) N }\end{array}$ & $\begin{array}{c}\text { Background } \\
\text { Min } \\
\text { Temp C }\end{array}$ & $\begin{array}{c}\text { Bat Pass } \\
\text { Min Temp C }\end{array}$ & $\begin{array}{c}\text { Background } \\
\text { Max } \\
\text { Temp C }\end{array}$ & $\begin{array}{c}\text { Bat Pass } \\
\text { Max Temp C }\end{array}$ \\
\hline 2014 & 12 & $\begin{array}{r}-4.5(6.3) \\
568 \\
1\end{array}$ & $1.6(0.9) 3$ & -20.5 & 0.9 & 7.5 & 2.7 \\
\hline 2015 & 1 & $\begin{array}{r}-6.2(7.6) \\
552 \\
6\end{array}$ & - & -20.5 & - & 12.5 & - \\
\hline 2015 & 2 & $\begin{array}{r}-3.6(7.2) \\
458 \\
2\end{array}$ & $1(0.6) 2$ & -20.5 & 0.6 & 12.8 & 1.4 \\
\hline 2015 & 3 & $\begin{array}{r}1.2(7.1) \\
450 \\
3\end{array}$ & $\begin{array}{r}10.3(3.6) \\
115\end{array}$ & -18.1 & 3.7 & 20.3 & 18.3 \\
\hline 2015 & 4 & $\begin{array}{r}3.2(5.5) \\
378 \\
1\end{array}$ & $\begin{array}{r}7.3(3.6) \\
528 \\
8\end{array}$ & -9.4 & -1.8 & 18.9 & 17.9 \\
\hline 2015 & 5 & $\begin{array}{r}8.8(4.5) \\
340 \\
6\end{array}$ & $\begin{array}{r}11.1(3.4) \\
196 \\
01\end{array}$ & -4.3 & -1.6 & 20.4 & 19.3 \\
\hline 2015 & 6 & $\begin{array}{r}15.5(2.6) \\
108 \\
4\end{array}$ & $\begin{array}{r}15.6(2.2) \\
798 \\
8\end{array}$ & 10.7 & 10.7 & 24.7 & 22.7 \\
\hline 2015 & 6 & $\begin{array}{r}17.7(2.9) \\
200 \\
3\end{array}$ & $\begin{array}{r}17.4(2.3) \\
194 \\
18\end{array}$ & 10.7 & 11.7 & 25.2 & 24.4 \\
\hline 2015 & 7 & 18 (3.9) 3334 & $\begin{array}{r}15.9(2.4) \\
172 \\
20\end{array}$ & 7.7 & 8.9 & 30.5 & 27.9 \\
\hline 2015 & 8 & $17.1(5) 3309$ & $\begin{array}{r}15.3(3) \\
284 \\
84\end{array}$ & 1.6 & 3.2 & 29 & 27.9 \\
\hline 2015 & 9 & $\begin{array}{r}13.6(4.7) \\
263 \\
4\end{array}$ & $\begin{array}{r}14.8(3.1) \\
125 \\
54\end{array}$ & 4.2 & 6 & 27.9 & 27.4 \\
\hline
\end{tabular}

Table 10d. Nightly background and bat pass temperatures summarized by month at the Big Sky Mine

\begin{tabular}{|c|c|c|c|c|c|c|c|}
\hline 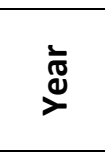 & 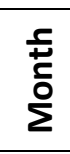 & $\begin{array}{c}\text { Background } \\
\text { Temp C } \\
\text { Avg (SD) N }\end{array}$ & $\begin{array}{c}\text { Bat Pass } \\
\text { Temp C } \\
\text { Avg (SD) N }\end{array}$ & $\begin{array}{c}\text { Background } \\
\text { Min } \\
\text { Temp C } \\
\end{array}$ & $\begin{array}{c}\text { Bat Pass } \\
\text { Min Temp C }\end{array}$ & $\begin{array}{c}\text { Background } \\
\text { Max } \\
\text { Temp C }\end{array}$ & $\begin{array}{c}\text { Bat Pass } \\
\text { Max Temp C }\end{array}$ \\
\hline 2013 & 10 & 7.2 (4) 2538 & $\begin{array}{r}9.3(3.5) \\
117 \\
1\end{array}$ & -2.1 & 0 & 15.5 & 15 \\
\hline 2013 & 11 & $\begin{array}{r}0.8(5.1) \\
527 \\
1\end{array}$ & $\begin{array}{r}3.3(3.4) \\
197 \\
0\end{array}$ & -14.7 & -4.1 & 12.3 & 12 \\
\hline
\end{tabular}




\begin{tabular}{|c|c|c|c|c|c|c|c|}
\hline$\stackrel{\frac{1}{\pi}}{\frac{\pi}{2}}$ & 岳 & $\begin{array}{c}\text { Background } \\
\text { Temp C } \\
\text { Avg (SD) } \mathbf{N}\end{array}$ & $\begin{array}{c}\text { Bat Pass } \\
\text { Temp C } \\
\text { Avg (SD) N }\end{array}$ & $\begin{array}{c}\text { Background } \\
\text { Min } \\
\text { Temp C }\end{array}$ & $\begin{array}{c}\text { Bat Pass } \\
\text { Min Temp C }\end{array}$ & $\begin{array}{c}\text { Background } \\
\text { Max } \\
\text { Temp C }\end{array}$ & $\begin{array}{c}\text { Bat Pass } \\
\text { Max Temp C }\end{array}$ \\
\hline 2013 & 12 & $\begin{array}{r}-3.4(10) \\
570 \\
7\end{array}$ & $5.7(2.8) 411$ & -20.5 & -0.5 & 12.7 & 12.3 \\
\hline 2014 & 1 & $0.1(8) 5534$ & $5.3(2.8) 596$ & -20.5 & -3.3 & 13.8 & 12.8 \\
\hline 2014 & 2 & $\begin{array}{r}-6.1(9.5) \\
458 \\
9\end{array}$ & $4.6(2) 199$ & -20.5 & 0.8 & 11 & 10.5 \\
\hline 2014 & 3 & $\begin{array}{r}1.2(7.2) \\
449 \\
8\end{array}$ & $8.9(4.5) 748$ & -20.5 & -1.1 & 15.3 & 15.1 \\
\hline 2014 & 4 & $\begin{array}{r}5.9(5.4) \\
377 \\
1\end{array}$ & $\begin{array}{r}11.2(4.6) \\
591 \\
8\end{array}$ & -11.7 & 0.6 & 22.1 & 22.1 \\
\hline 2014 & 5 & $\begin{array}{r}8.8(3.3) \\
103 \\
9\end{array}$ & $\begin{array}{r}11.1(2.2) \\
732\end{array}$ & 0.4 & 3.1 & 14.8 & 14.5 \\
\hline 2014 & 7 & $\begin{array}{r}18.3(4.2) \\
165 \\
\end{array}$ & $\begin{array}{r}19.9(2.8) \\
598 \\
\end{array}$ & 13 & 13.2 & 25.7 & 23.4 \\
\hline 2014 & 8 & $\begin{array}{r}18.9(4.1) \\
370 \\
8 \\
\end{array}$ & $20(3.9) 3985$ & 10 & 10.7 & 31.2 & 30.7 \\
\hline 2014 & 9 & $\begin{array}{r}12.3(5.1) \\
415 \\
5\end{array}$ & $\begin{array}{r}15.3(2.9) \\
351\end{array}$ & 0.6 & 7.9 & 26.7 & 21.4 \\
\hline 2014 & 10 & $\begin{array}{r}8.5(4.5) \\
490 \\
2 \\
\end{array}$ & - & -3.3 & - & 22.1 & - \\
\hline 2014 & 11 & $\begin{array}{r}-1.1(9.5) \\
525 \\
0 \\
\end{array}$ & - & -20.5 & - & 18.9 & - \\
\hline 2014 & 12 & $-1(6.2) 5687$ & - & -20.5 & - & 11 & - \\
\hline 2015 & 1 & $\begin{array}{r}-1.8(8.4) \\
553 \\
6 \\
\end{array}$ & - & -20.5 & - & 16.1 & - \\
\hline 2015 & 2 & $\begin{array}{r}-0.3(7.5) \\
458 \\
8\end{array}$ & - & -20.5 & - & 16.6 & - \\
\hline 2015 & 3 & $\begin{array}{r}4.8(7.2) \\
450 \\
6\end{array}$ & $\begin{array}{r}11.4(4.6) \\
115 \\
07\end{array}$ & -16.3 & -0.1 & 22.1 & 22.1 \\
\hline 2015 & 4 & $\begin{array}{r}5.9(4.9) \\
377 \\
3\end{array}$ & $\begin{array}{r}10.9(4.2) \\
638 \\
4\end{array}$ & -5.6 & -1.3 & 20.8 & 20.4 \\
\hline
\end{tabular}




\begin{tabular}{|c|c|c|c|c|c|c|c|}
\hline ঐ & 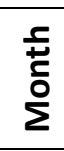 & $\begin{array}{l}\text { Background } \\
\text { Temp C } \\
\text { Avg (SD) } N\end{array}$ & $\begin{array}{c}\text { Bat Pass } \\
\text { Temp C } \\
\text { Avg (SD) N }\end{array}$ & $\begin{array}{c}\text { Background } \\
\text { Min } \\
\text { Temp C }\end{array}$ & $\begin{array}{c}\text { Bat Pass } \\
\text { Min Temp C }\end{array}$ & $\begin{array}{c}\text { Background } \\
\text { Max } \\
\text { Temp C }\end{array}$ & $\begin{array}{c}\text { Bat Pass } \\
\text { Max Temp }\end{array}$ \\
\hline 2015 & 5 & $\begin{array}{r}11.2(4.3) \\
338 \\
4\end{array}$ & $\begin{array}{r}14.3(3.7) \\
926 \\
7\end{array}$ & -2.1 & 0.6 & 22.9 & 21.9 \\
\hline 2015 & 6 & 18 (2.8) 1068 & $\begin{array}{r}18.4(2.3) \\
545 \\
7 \\
\end{array}$ & 11.5 & 12.3 & 26.9 & 26.4 \\
\hline 2015 & 6 & $\begin{array}{r}20.3(3.6) \\
197 \\
1\end{array}$ & $\begin{array}{r}21.1(3.4) \\
266 \\
64\end{array}$ & 11 & 11.3 & 30.8 & 30.5 \\
\hline 2015 & 7 & $\begin{array}{r}20.6(3.9) \\
325 \\
2 \\
\end{array}$ & $\begin{array}{r}20.8(3) \\
427 \\
25 \\
\end{array}$ & 12 & 12.5 & 33.8 & 33.8 \\
\hline 2015 & 8 & $20(5.2) 3703$ & $\begin{array}{r}20.4(3) \\
250 \\
93\end{array}$ & 4.6 & 5.5 & 33.6 & 30.7 \\
\hline 2015 & 9 & $17(4.8) 4161$ & $\begin{array}{r}20.7(3.9) \\
178 \\
29\end{array}$ & 7 & 8.4 & 30.5 & 29.3 \\
\hline 2015 & 10 & $\begin{array}{r}11.7(5.2) \\
488 \\
5\end{array}$ & $15.3(5) 8034$ & -3.6 & 0.1 & 25.4 & 23.4 \\
\hline 2015 & 11 & $\begin{array}{r}2.4(7.3) \\
524 \\
7\end{array}$ & 6.8 (4) 5764 & -16.2 & -1.5 & 20.9 & 17.4 \\
\hline 2015 & 12 & -0.2 (7) 5694 & $\begin{array}{r}6.2(4.4) \\
321 \\
1 \\
\end{array}$ & -15.3 & -7.1 & 19.8 & 17.3 \\
\hline 2016 & 1 & $\begin{array}{r}0.4(6.2) \\
554 \\
3 \\
\end{array}$ & $\begin{array}{r}6.3(3.4) \\
235 \\
6 \\
\end{array}$ & -12.7 & -4.9 & 14.8 & 14.3 \\
\hline 2016 & 2 & $\begin{array}{r}4.4(4.5) \\
476 \\
9\end{array}$ & $\begin{array}{r}6.3(3.1) \\
981 \\
0\end{array}$ & -6.6 & -2.1 & 16.1 & 15.8 \\
\hline 2016 & 3 & $\begin{array}{r}5.7(4.8) \\
446 \\
9\end{array}$ & $\begin{array}{r}8.7(3.5) \\
131 \\
83 \\
\end{array}$ & -8.4 & -1.6 & 18.1 & 17.3 \\
\hline 2016 & 4 & $\begin{array}{r}9.3(4.5) \\
146 \\
8 \\
\end{array}$ & $\begin{array}{r}11.4(2.7) \\
614 \\
0 \\
\end{array}$ & -0.8 & 0.3 & 19.6 & 18.9 \\
\hline
\end{tabular}


Table 10e. Nightly background and bat pass temperatures summarized by month at the Absaloka Mine

\begin{tabular}{|c|c|c|c|c|c|c|c|}
\hline ঠ্ঠ & & $\begin{array}{c}\text { Background } \\
\text { Temp C } \\
\text { Avg (SD) N }\end{array}$ & $\begin{array}{c}\text { Bat Pass } \\
\text { Temp C } \\
\text { Avg (SD) N }\end{array}$ & $\begin{array}{c}\text { Background } \\
\text { Min } \\
\text { Temp C }\end{array}$ & $\begin{array}{c}\text { Bat Pass } \\
\text { Min Temp C }\end{array}$ & $\begin{array}{c}\text { Background } \\
\text { Max } \\
\text { Temp C }\end{array}$ & $\begin{array}{c}\text { Bat Pass } \\
\text { Max Temp C }\end{array}$ \\
\hline 2012 & 8 & $\begin{array}{r}14.9(5.6) \\
119 \\
1\end{array}$ & $\begin{array}{r}17.2(4.4) \\
403 \\
5\end{array}$ & 1.4 & 2.4 & 26.2 & 26.2 \\
\hline 2012 & 9 & $9(5.7) 4160$ & $\begin{array}{r}15.6(5.3) \\
552 \\
8 \\
\end{array}$ & -3.3 & -1.8 & 24.7 & 24.7 \\
\hline 2012 & 10 & 4.1 (5) 5104 & $12.2(5.5) 30$ & -8.1 & -1.3 & 19.4 & 18.8 \\
\hline 2012 & 11 & $\begin{array}{r}-1.7(4.7) \\
126 \\
73 \\
\end{array}$ & - & -19 & - & 18.3 & - \\
\hline 2012 & 12 & $\begin{array}{r}-4.9(7.1) \\
104 \\
35\end{array}$ & - & -20.5 & - & 13.3 & - \\
\hline 2013 & 1 & $\begin{array}{r}-6.8(6.3) \\
708 \\
0\end{array}$ & - & -20.5 & - & 8.5 & - \\
\hline 2013 & 2 & $\begin{array}{r}-4.2(4.8) \\
532 \\
1\end{array}$ & - & -19.1 & - & 7.5 & - \\
\hline 2013 & 3 & $\begin{array}{r}-3.1(5.9) \\
532 \\
8\end{array}$ & - & -20.5 & - & 15.1 & - \\
\hline 2013 & 4 & $\begin{array}{r}0.3(5.6) \\
453 \\
5\end{array}$ & 9.1 (3.3) 36 & -13.2 & 2.7 & 14.6 & 13.8 \\
\hline 2013 & 5 & $\begin{array}{r}10.1(5.3) \\
663 \\
4\end{array}$ & $\begin{array}{r}14.3(3.6) \\
956\end{array}$ & -8.1 & 3.6 & 19.6 & 19.4 \\
\hline 2013 & 6 & 12.9 (5) 4030 & $\begin{array}{r}16.8(3.6) \\
533\end{array}$ & -1 & 6.7 & 25.2 & 25.2 \\
\hline 2013 & 7 & $\begin{array}{r}16.4(3.5) \\
682\end{array}$ & - & 7.2 & - & 22.4 & - \\
\hline 2013 & 8 & $\begin{array}{r}15.8(4.3) \\
431 \\
1\end{array}$ & - & 7 & - & 27 & - \\
\hline 2013 & 9 & $\begin{array}{r}11.8(6.1) \\
481 \\
0\end{array}$ & - & -0.1 & - & 31.7 & - \\
\hline
\end{tabular}




\begin{tabular}{|c|c|c|c|c|c|c|c|}
\hline$\stackrel{\bar{\varpi}}{\stackrel{\nu}{\nu}}$ & 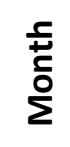 & $\begin{array}{c}\text { Background } \\
\text { Temp C } \\
\text { Avg (SD) N }\end{array}$ & $\begin{array}{c}\text { Bat Pass } \\
\text { Temp C } \\
\text { Avg (SD) N }\end{array}$ & $\begin{array}{c}\text { Background } \\
\text { Min } \\
\text { Temp C }\end{array}$ & $\begin{array}{c}\text { Bat Pass } \\
\text { Min Temp C }\end{array}$ & $\begin{array}{c}\text { Background } \\
\text { Max } \\
\text { Temp C }\end{array}$ & $\begin{array}{c}\text { Bat Pass } \\
\text { Max Temp C }\end{array}$ \\
\hline 2013 & 10 & $\begin{array}{r}3.5(4.6) \\
543 \\
3\end{array}$ & 11.7 (1.5) 24 & -7.1 & 8.2 & 16.3 & 13.3 \\
\hline 2013 & 11 & $\begin{array}{r}-2.5(5.8) \\
528 \\
5\end{array}$ & 1.7 () 1 & -18.1 & 1.7 & 9.5 & 1.7 \\
\hline 2013 & 12 & $\begin{array}{r}-7.2(8.4) \\
571 \\
7\end{array}$ & - & -20.5 & - & 8.9 & - \\
\hline 2014 & 1 & $\begin{array}{r}-4.5(7.5) \\
557 \\
2\end{array}$ & - & -20.5 & - & 10.2 & - \\
\hline 2014 & 2 & $\begin{array}{r}-8.6(8.8) \\
468 \\
5 \\
\end{array}$ & - & -20.5 & - & 7.9 & - \\
\hline 2014 & 3 & -3.4 (7) 5304 & - & -20.5 & - & 11 & - \\
\hline 2014 & 4 & $\begin{array}{r}3.1(5.7) \\
378 \\
2\end{array}$ & 12.6 (5.5) 98 & -12.7 & 0.4 & 18.6 & 18.6 \\
\hline 2014 & 5 & $\begin{array}{r}8.8(5.5) \\
341 \\
2\end{array}$ & 15.7 (5) 1476 & -4.3 & 3.1 & 27.2 & 27.2 \\
\hline 2014 & 6 & $\begin{array}{r}11.5(4.4) \\
304 \\
7\end{array}$ & $\begin{array}{r}13.5(2.4) \\
112 \\
1\end{array}$ & -0.3 & 4.4 & 19.9 & 19.1 \\
\hline 2014 & 7 & $\begin{array}{r}14.5(4.4) \\
328 \\
4 \\
\end{array}$ & 17.7 (6.1) 20 & 4.9 & 8 & 26.9 & 26 \\
\hline 2014 & 8 & $\begin{array}{r}15.4(3.6) \\
374 \\
7\end{array}$ & $\begin{array}{r}13.7(2.8) \\
133 \\
7\end{array}$ & 8.5 & 7.5 & 31.5 & 23.9 \\
\hline 2014 & 9 & $\begin{array}{r}8.8(5.1) \\
417 \\
6\end{array}$ & $\begin{array}{r}13.5(4.4) \\
141 \\
5\end{array}$ & -4.1 & -0.8 & 22.7 & 22.2 \\
\hline 2014 & 10 & $5.2(5) 4912$ & $14.6(2.9) 20$ & -6.4 & 10.5 & 19.8 & 18.8 \\
\hline 2014 & 11 & $\begin{array}{r}-4.5(9.1) \\
524 \\
6\end{array}$ & 10.8() 1 & -20.5 & 10.8 & 14.8 & 10.8 \\
\hline 2014 & 12 & $-4(6.4) 5695$ & $2(1.6) 4$ & -20.5 & 0.3 & 7.4 & 3.4 \\
\hline
\end{tabular}




\begin{tabular}{|c|c|c|c|c|c|c|c|}
\hline$\stackrel{\bar{\varpi}}{\nu}$ & 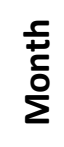 & $\begin{array}{c}\text { Background } \\
\text { Temp C } \\
\text { Avg (SD) N }\end{array}$ & $\begin{array}{c}\text { Bat Pass } \\
\text { Temp C } \\
\text { Avg (SD) N }\end{array}$ & $\begin{array}{c}\text { Background } \\
\text { Min } \\
\text { Temp C }\end{array}$ & $\begin{array}{c}\text { Bat Pass } \\
\text { Min Temp C }\end{array}$ & $\begin{array}{c}\text { Background } \\
\text { Max } \\
\text { Temp C }\end{array}$ & $\begin{array}{c}\text { Bat Pass } \\
\text { Max Temp C }\end{array}$ \\
\hline 2015 & 1 & $\begin{array}{r}-6.4(6.7) \\
554 \\
8 \\
\end{array}$ & 1.1() 1 & -20.5 & 1.1 & 7.9 & 1.1 \\
\hline 2015 & 2 & $\begin{array}{r}-2.8(6.7) \\
458 \\
9 \\
\end{array}$ & $3.3(1.6) 2$ & -20.3 & 2.1 & 14.8 & 4.4 \\
\hline 2015 & 3 & $\begin{array}{r}0.9(6.9) \\
450 \\
6\end{array}$ & 10.3 (5) 32 & -16.5 & 0.9 & 18.3 & 16.1 \\
\hline 2015 & 4 & $\begin{array}{r}2.6(5.5) \\
378 \\
3 \\
\end{array}$ & $9.6(4.7) 352$ & -9.6 & -1.6 & 17.4 & 17 \\
\hline 2015 & 5 & $\begin{array}{r}8.8(4.6) \\
338 \\
9 \\
\end{array}$ & $\begin{array}{r}13.2(2.8) \\
196 \\
2\end{array}$ & -5.6 & 2.1 & 19.3 & 19.3 \\
\hline 2015 & 6 & $\begin{array}{r}17.6(3.8) \\
198 \\
7 \\
\end{array}$ & $\begin{array}{r}19.2(3.8) \\
998 \\
0 \\
\end{array}$ & 7.9 & 8.4 & 29 & 29 \\
\hline 2015 & 6 & $\begin{array}{r}15.3(2.8) \\
106 \\
8\end{array}$ & $\begin{array}{r}16.8(2.7) \\
113 \\
5\end{array}$ & 10.2 & 10.3 & 23.1 & 22.6 \\
\hline 2015 & 7 & $\begin{array}{r}18.2(3.8) \\
214 \\
9 \\
\end{array}$ & $\begin{array}{r}19.7(3.6) \\
836 \\
5 \\
\end{array}$ & 10.5 & 10.5 & 29.8 & 29.7 \\
\hline 2015 & 8 & 19.6 (4) 1584 & $\begin{array}{r}21.4(4.6) \\
674\end{array}$ & 11.2 & 12.5 & 29.2 & 29.2 \\
\hline 2015 & 9 & $\begin{array}{r}12.9(4.9) \\
129 \\
5 \\
\end{array}$ & $17.1(5.6) 23$ & 4.4 & 7.2 & 26 & 25.7 \\
\hline 2015 & 10 & $\begin{array}{r}8.2(5.4) \\
156 \\
2 \\
\end{array}$ & - & -3.4 & - & 17.3 & - \\
\hline 2015 & 11 & $\begin{array}{r}10.6(2.7) \\
627\end{array}$ & - & 2.2 & - & 17.9 & - \\
\hline
\end{tabular}


Table 10f. Nightly background and bat pass temperatures summarized by month at the Rosebud Mine Area F Pond 7

\begin{tabular}{|c|c|c|c|c|c|c|c|}
\hline 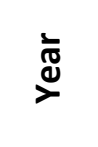 & & $\begin{array}{l}\text { Background } \\
\text { Temp C } \\
\text { Avg (SD) N }\end{array}$ & $\begin{array}{c}\text { Bat Pass } \\
\text { Temp C } \\
\text { Avg (SD) N }\end{array}$ & $\begin{array}{c}\text { Background } \\
\text { Min } \\
\text { Temp C }\end{array}$ & $\begin{array}{c}\text { Bat Pass } \\
\text { Min Temp C }\end{array}$ & $\begin{array}{c}\text { Background } \\
\text { Max } \\
\text { Temp C }\end{array}$ & $\begin{array}{c}\text { Bat Pass } \\
\text { Max } \\
\text { Temp C }\end{array}$ \\
\hline 2012 & 8 & $19(6.3) 1196$ & $22(4.4) 1133$ & 5.9 & 8.2 & 32.3 & 32 \\
\hline 2012 & 9 & $\begin{array}{r}14.9(4.8) \\
416 \\
6 \\
\end{array}$ & $\begin{array}{r}18.9(4.3) \\
140 \\
1 \\
\end{array}$ & 4.1 & 4.1 & 27.2 & 26.7 \\
\hline 2012 & 10 & $8.8(6) 4894$ & $17.5(4.2) 30$ & -3.6 & 4.9 & 22.7 & 21.9 \\
\hline 2012 & 11 & $\begin{array}{r}5.8(6.1) \\
524 \\
4\end{array}$ & 13.4 (7) 2 & -12 & 8.5 & 20.3 & 18.4 \\
\hline 2012 & 12 & $\begin{array}{r}-0.4(7.2) \\
570 \\
0\end{array}$ & - & -18.1 & - & 18.6 & - \\
\hline 2013 & 1 & $\begin{array}{r}-0.2(6.4) \\
553 \\
4\end{array}$ & - & -18.3 & - & 13.6 & - \\
\hline 2013 & 2 & $\begin{array}{r}1.4(4.5) \\
457 \\
2 \\
\end{array}$ & - & -13 & - & 9.5 & - \\
\hline 2013 & 3 & 1.9 (7) 4478 & - & -15.8 & - & 17.9 & - \\
\hline 2013 & 4 & $\begin{array}{r}5.1(6.4) \\
374 \\
7 \\
\end{array}$ & - & -8.7 & - & 18.9 & - \\
\hline 2013 & 5 & $\begin{array}{r}12.3(4.9) \\
337 \\
2\end{array}$ & 18.5 (2.9) 39 & -3.9 & 9.5 & 27 & 20.9 \\
\hline 2013 & 6 & $\begin{array}{r}16.2(4.3) \\
303 \\
6\end{array}$ & $\begin{array}{r}18.1(3.6) \\
218\end{array}$ & 6.9 & 9.5 & 27.5 & 27.5 \\
\hline 2013 & 7 & $\begin{array}{r}20.6(3.8) \\
326 \\
9\end{array}$ & $\begin{array}{r}21.2(3.3) \\
295\end{array}$ & 12.8 & 13.2 & 29.8 & 29.3 \\
\hline 2013 & 8 & $\begin{array}{r}20.8(4.4) \\
369 \\
6 \\
\end{array}$ & $\begin{array}{r}20.7(3.6) \\
317\end{array}$ & 11.3 & 11.5 & 31.8 & 31.5 \\
\hline 2013 & 9 & $\begin{array}{r}16.6(5.5) \\
414 \\
6\end{array}$ & $19.8(4.5) 60$ & 6 & 9.7 & 31.2 & 28.9 \\
\hline 2013 & 10 & $\begin{array}{r}8.1(3.9) \\
236 \\
6\end{array}$ & 16.1 (1) 3 & 0.3 & 15 & 18.1 & 17 \\
\hline
\end{tabular}


Table 10g. Nightly background and bat pass temperatures summarized by month at the Rosebud Mine Area C

\begin{tabular}{|c|c|c|c|c|c|c|c|}
\hline 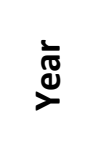 & 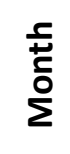 & $\begin{array}{c}\text { Background } \\
\text { Temp C } \\
\text { Avg (SD) N }\end{array}$ & $\begin{array}{c}\text { Bat Pass } \\
\text { Temp C } \\
\text { Avg (SD) N }\end{array}$ & $\begin{array}{c}\text { Background } \\
\text { Min } \\
\text { Temp C }\end{array}$ & $\begin{array}{c}\text { Bat Pass } \\
\text { Min Temp C }\end{array}$ & $\begin{array}{c}\text { Background } \\
\text { Max } \\
\text { Temp C }\end{array}$ & $\begin{array}{r}\text { Bat Pass } \\
\text { Max Temp } \\
\text { C }\end{array}$ \\
\hline 2013 & 10 & $\begin{array}{r}6.3(4.3) \\
252 \\
7 \\
\end{array}$ & $10.7(2.3) 8$ & -4.4 & 7 & 14.1 & 14 \\
\hline 2013 & 11 & $\begin{array}{r}0.4(5.9) \\
529 \\
9\end{array}$ & $4.3(0.8) 2$ & -16.8 & 3.7 & 17 & 4.9 \\
\hline 2013 & 12 & $\begin{array}{r}-3.3(10) \\
572 \\
7\end{array}$ & - & -20.5 & - & 12.3 & - \\
\hline 2014 & 1 & $\begin{array}{r}-0.3(8.1) \\
557 \\
3 \\
\end{array}$ & - & -20.5 & - & 12.5 & - \\
\hline 2014 & 2 & $-7(9.3) 4596$ & $3(0.1) 3$ & -20.5 & 2.9 & 9.5 & 3.1 \\
\hline 2014 & 3 & $\begin{array}{r}0.5(7.5) \\
463 \\
3\end{array}$ & - & -20.5 & - & 14.1 & - \\
\hline 2014 & 4 & $\begin{array}{r}4.8(5.1) \\
379 \\
9 \\
\end{array}$ & 11 (4.4) 326 & -11.7 & 1.4 & 19.3 & 18.9 \\
\hline 2014 & 5 & $10(5.3) 3418$ & $\begin{array}{r}14.8(3.5) \\
249 \\
1\end{array}$ & -1.5 & 1.4 & 25.7 & 25.7 \\
\hline 2014 & 6 & $\begin{array}{r}13.2(3.8) \\
306 \\
6\end{array}$ & $\begin{array}{r}14.7(2.9) \\
722 \\
2\end{array}$ & 1.7 & 4.7 & 20.3 & 20.3 \\
\hline 2014 & 7 & $\begin{array}{r}17.1(4.3) \\
328 \\
4\end{array}$ & $\begin{array}{r}17.6(3.4) \\
147 \\
28\end{array}$ & 8.5 & 9.4 & 28 & 27.5 \\
\hline 2014 & 8 & $\begin{array}{r}17.4(3.6) \\
372 \\
8\end{array}$ & $16.1(3) 9654$ & 8.2 & 9.8 & 27.2 & 27.2 \\
\hline 2014 & 9 & $11(5) 4162$ & $\begin{array}{r}12.9(2.7) \\
314 \\
6 \\
\end{array}$ & 0.4 & 1.4 & 24.2 & 21.2 \\
\hline 2014 & 10 & $\begin{array}{r}8.2(4.3) \\
488 \\
2\end{array}$ & $11.3(4.8) 96$ & -4.6 & 2.6 & 20.8 & 19.8 \\
\hline 2014 & 11 & $\begin{array}{r}-1.5(9.9) \\
525 \\
1\end{array}$ & $9.4(0) 4$ & -20.5 & 9.4 & 17.4 & 9.4 \\
\hline
\end{tabular}




\begin{tabular}{|c|c|c|c|c|c|c|c|}
\hline ঠ & 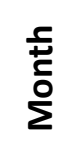 & $\begin{array}{c}\text { Background } \\
\text { Temp C } \\
\text { Avg (SD) N }\end{array}$ & $\begin{array}{c}\text { Bat Pass } \\
\text { Temp C } \\
\text { Avg (SD) } \mathbf{N}\end{array}$ & $\begin{array}{c}\text { Background } \\
\text { Min } \\
\text { Temp C }\end{array}$ & $\begin{array}{c}\text { Bat Pass } \\
\text { Min Temp C }\end{array}$ & $\begin{array}{c}\text { Background } \\
\text { Max } \\
\text { Temp C }\end{array}$ & $\begin{array}{r}\text { Bat Pass } \\
\text { Max Temp } \\
\text { C }\end{array}$ \\
\hline 2014 & 12 & $\begin{array}{r}-0.8(6.4) \\
573 \\
0 \\
\end{array}$ & $8.9(0) 4$ & -19.3 & 8.9 & 12 & 8.9 \\
\hline 2015 & 1 & $\begin{array}{r}-1.4(8.4) \\
557 \\
5\end{array}$ & - & -20.5 & - & 16.3 & - \\
\hline 2015 & 2 & $-0.6(8) 4615$ & - & -20.5 & - & 16 & - \\
\hline 2015 & 3 & 4.8 (7) 4519 & 15.9 (5.9) 70 & -14.5 & 4.9 & 21.6 & 21.6 \\
\hline 2015 & 4 & $\begin{array}{r}4.9(5.1) \\
306 \\
6 \\
\end{array}$ & $\begin{array}{r}12.2(2.2) \\
117\end{array}$ & -6.6 & -4.9 & 18.3 & 17.1 \\
\hline 2015 & 5 & 10 (4.1) 3390 & $\begin{array}{r}14.5(3.6) \\
240 \\
9\end{array}$ & -3.3 & 2.1 & 20.9 & 20.9 \\
\hline 2015 & 6 & $\begin{array}{r}18.2(3.5) \\
305 \\
8\end{array}$ & $\begin{array}{r}18.7(3.7) \\
290 \\
4\end{array}$ & 9.2 & 12 & 28.2 & 25.2 \\
\hline 2015 & 7 & $\begin{array}{r}19.4(3.4) \\
315 \\
0 \\
\end{array}$ & - & 10.8 & - & 29.2 & - \\
\hline 2015 & 8 & 19 (5.5) 3699 & - & 2.7 & - & 29.5 & - \\
\hline 2015 & 9 & 15.7 (5) 4147 & - & 4.9 & - & 28 & - \\
\hline 2015 & 10 & $\begin{array}{r}11.1(5.6) \\
489 \\
9\end{array}$ & - & -4.4 & - & 26.7 & - \\
\hline 2015 & 11 & $\begin{array}{r}1.9(7.9) \\
525 \\
7\end{array}$ & - & -17.5 & - & 20.8 & - \\
\hline 2015 & 12 & $\begin{array}{r}-2.1(5.7) \\
415 \\
3\end{array}$ & 2.1 (1) 12 & -17.3 & 0.4 & 18.3 & 2.7 \\
\hline 2015 & 12 & $\begin{array}{r}6.5(5.1) \\
155 \\
5\end{array}$ & - & -1.5 & - & 18.1 & - \\
\hline 2016 & 1 & $\begin{array}{r}-0.1(5.8) \\
494 \\
8 \\
\end{array}$ & 4.7 (1.3) 13 & -15.2 & 3.9 & 11.5 & 7 \\
\hline 2016 & 4 & $\begin{array}{r}8.4(3.9) \\
214 \\
8\end{array}$ & $\begin{array}{r}15.2(2.6) \\
605\end{array}$ & -0.6 & 2.9 & 18.6 & 18.6 \\
\hline
\end{tabular}




\begin{tabular}{|c|c|c|c|c|c|c|c|}
\hline ঐ্ঠ & 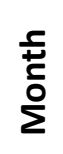 & $\begin{array}{l}\text { Background } \\
\text { Temp C } \\
\text { Avg (SD) N }\end{array}$ & $\begin{array}{c}\text { Bat Pass } \\
\text { Temp C } \\
\text { Avg (SD) N }\end{array}$ & $\begin{array}{c}\text { Background } \\
\text { Min } \\
\text { Temp C }\end{array}$ & $\begin{array}{c}\text { Bat Pass } \\
\text { Min Temp C }\end{array}$ & $\begin{array}{c}\text { Background } \\
\text { Max } \\
\text { Temp C }\end{array}$ & $\begin{array}{r}\text { Bat Pass } \\
\text { Max Temp } \\
\text { C }\end{array}$ \\
\hline 2016 & 5 & $\begin{array}{r}10.7(4.8) \\
282 \\
1\end{array}$ & $\begin{array}{r}15.4(3.2) \\
373 \\
2\end{array}$ & -0.6 & 1.6 & 22.4 & 22.4 \\
\hline
\end{tabular}

Table 10h. Nightly background and bat pass temperatures summarized by month at the Signal Peak Mine Reservoir 1

\begin{tabular}{|c|c|c|c|c|c|c|c|}
\hline$\stackrel{\frac{\pi}{0}}{\searrow}$ & $\frac{c}{\stackrel{5}{0}}$ & $\begin{array}{c}\text { Background } \\
\text { Temp C } \\
\text { Avg (SD) N }\end{array}$ & $\begin{array}{c}\text { Bat Pass } \\
\text { Temp C } \\
\text { Avg (SD) N }\end{array}$ & $\begin{array}{c}\text { Background } \\
\text { Min } \\
\text { Temp C }\end{array}$ & $\begin{array}{c}\text { Bat Pass } \\
\text { Min Temp C }\end{array}$ & $\begin{array}{c}\text { Background } \\
\text { Max } \\
\text { Temp C }\end{array}$ & $\begin{array}{r}\text { Bat Pass } \\
\text { Max Temp } \\
\text { C }\end{array}$ \\
\hline 2012 & 8 & $\begin{array}{r}21.6(4.9) \\
106 \\
7\end{array}$ & $\begin{array}{r}23.3(3.9) \\
355 \\
3 \\
\end{array}$ & 7.7 & 7.9 & 29.3 & 29.3 \\
\hline 2012 & 9 & $\begin{array}{r}16.1(4.1) \\
416 \\
1\end{array}$ & $\begin{array}{r}18.6(3.4) \\
788 \\
4\end{array}$ & 4.4 & 5.1 & 26.5 & 26.5 \\
\hline 2012 & 10 & $\begin{array}{r}7.9(5.6) \\
492 \\
2\end{array}$ & $\begin{array}{r}13.3(4.2) \\
790\end{array}$ & -3.9 & 0.9 & 21.6 & 20.3 \\
\hline 2012 & 11 & $\begin{array}{r}5.7(5.9) \\
527 \\
3 \\
\end{array}$ & $8.6(3.6) 901$ & -11.9 & -0.6 & 18.8 & 17 \\
\hline 2012 & 12 & $-0.5(6) 5714$ & 4.8 (3.6) 421 & -16.5 & -2.1 & 15.6 & 12 \\
\hline 2013 & 1 & $\begin{array}{r}0.1(6.1) \\
555 \\
2 \\
\end{array}$ & 6.5 (2.3) 290 & -16.2 & 0 & 10.7 & 10.2 \\
\hline 2013 & 2 & $\begin{array}{r}1.2(3.6) \\
457 \\
5\end{array}$ & $3.6(1.8) 183$ & -10.4 & -1.6 & 8.9 & 7.7 \\
\hline 2013 & 3 & 2.5 (6) 4506 & 7.6 (3.7) 727 & -10.7 & -0.6 & 16.3 & 16.3 \\
\hline 2013 & 4 & $\begin{array}{r}4.3(6.3) \\
374 \\
1\end{array}$ & $\begin{array}{r}12.6(3.7) \\
326 \\
6\end{array}$ & -7.9 & 1.1 & 20.4 & 20.3 \\
\hline 2013 & 5 & $\begin{array}{r}12.3(4.4) \\
335 \\
6\end{array}$ & $\begin{array}{r}15.1(3.2) \\
562 \\
8\end{array}$ & -2.4 & -0.3 & 27.2 & 27 \\
\hline 2013 & 6 & 15.8 (4) 2986 & $\begin{array}{r}17.6(2.7) \\
599 \\
8\end{array}$ & 4.6 & 5.9 & 26 & 24.9 \\
\hline
\end{tabular}




\begin{tabular}{|c|c|c|c|c|c|c|c|}
\hline$\stackrel{\bar{\varpi}}{\nu}$ & 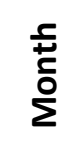 & $\begin{array}{c}\text { Background } \\
\text { Temp C } \\
\text { Avg (SD) N }\end{array}$ & $\begin{array}{c}\text { Bat Pass } \\
\text { Temp C } \\
\text { Avg (SD) N }\end{array}$ & $\begin{array}{c}\text { Background } \\
\text { Min } \\
\text { Temp C }\end{array}$ & $\begin{array}{c}\text { Bat Pass } \\
\text { Min Temp C }\end{array}$ & $\begin{array}{c}\text { Background } \\
\text { Max } \\
\text { Temp C }\end{array}$ & $\begin{array}{r}\text { Bat Pass } \\
\text { Max Temp } \\
\text { C }\end{array}$ \\
\hline 2013 & 7 & $\begin{array}{r}20.4(2.9) \\
324 \\
4 \\
\end{array}$ & 20.9 (3) 5076 & 13.6 & 13.6 & 27.7 & 27.7 \\
\hline 2013 & 8 & 21 (3.1) 3689 & $\begin{array}{r}21.7(3.1) \\
760 \\
9\end{array}$ & 13 & 13.3 & 27.9 & 27.7 \\
\hline 2013 & 9 & 16.4 (5) 4153 & $\begin{array}{r}21.6(3.5) \\
152 \\
7\end{array}$ & 5.7 & 7.9 & 27.5 & 27.5 \\
\hline 2013 & 10 & $\begin{array}{r}7.3(4.2) \\
491 \\
1 \\
\end{array}$ & $\begin{array}{r}11.7(2.9) \\
406\end{array}$ & -5.1 & 3.7 & 17.8 & 17.6 \\
\hline 2013 & 11 & $\begin{array}{r}3.3(5.3) \\
528 \\
5 \\
\end{array}$ & $6.9(3.8) 447$ & -14.5 & 0.6 & 14.1 & 14 \\
\hline 2013 & 12 & $\begin{array}{r}-2.2(9.6) \\
571 \\
5\end{array}$ & $7.4(2.6) 67$ & -20.5 & 3.1 & 12.3 & 11.3 \\
\hline 2014 & 1 & $1(6.6) 5554$ & $5.3(2.1) 31$ & -20.5 & 1.9 & 12.5 & 8.5 \\
\hline 2014 & 2 & $\begin{array}{r}-4.8(8.4) \\
458 \\
7 \\
\end{array}$ & $6.2(2.8) 5$ & -20.5 & 3.9 & 9.5 & 9.2 \\
\hline 2014 & 4 & $8.3(1.8) 56$ & $9.6(1.5) 164$ & 6.2 & 6.4 & 11.8 & 11.8 \\
\hline 2014 & 5 & $11.5(5) 3363$ & $\begin{array}{r}15.4(3.5) \\
400 \\
4\end{array}$ & 0.1 & 4.1 & 25.5 & 25.5 \\
\hline 2014 & 6 & $\begin{array}{r}14.5(3.1) \\
300 \\
3 \\
\end{array}$ & $\begin{array}{r}16.4(2.3) \\
579 \\
2 \\
\end{array}$ & 4.9 & 7.9 & 22.2 & 22.2 \\
\hline 2014 & 7 & $\begin{array}{r}20.6(3.4) \\
323 \\
8 \\
\end{array}$ & $\begin{array}{r}21.1(3.4) \\
147 \\
38 \\
\end{array}$ & 11.2 & 11.2 & 30.7 & 28.2 \\
\hline 2014 & 8 & $\begin{array}{r}19.1(4.2) \\
369 \\
6 \\
\end{array}$ & $\begin{array}{r}21.6(3.3) \\
851 \\
7 \\
\end{array}$ & 8.2 & 9.7 & 30.7 & 30.7 \\
\hline 2014 & 9 & $\begin{array}{r}14.3(5.2) \\
414 \\
2\end{array}$ & $\begin{array}{r}16.7(3.5) \\
151 \\
9\end{array}$ & 2.1 & 4.2 & 25.4 & 24.2 \\
\hline 2014 & 10 & $\begin{array}{r}1.4(4.3) \\
489 \\
0 \\
\end{array}$ & $\begin{array}{r}14.5(2.7) \\
286\end{array}$ & -2.4 & 5.1 & 21.7 & 21.6 \\
\hline
\end{tabular}




\begin{tabular}{|c|c|c|c|c|c|c|c|}
\hline d & 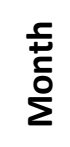 & $\begin{array}{c}\text { Background } \\
\text { Temp C } \\
\text { Avg (SD) N }\end{array}$ & $\begin{array}{c}\text { Bat Pass } \\
\text { Temp C } \\
\text { Avg (SD) N }\end{array}$ & $\begin{array}{c}\text { Background } \\
\text { Min } \\
\text { Temp C }\end{array}$ & $\begin{array}{c}\text { Bat Pass } \\
\text { Min Temp C }\end{array}$ & $\begin{array}{c}\text { Background } \\
\text { Max } \\
\text { Temp C }\end{array}$ & $\begin{array}{r}\text { Bat Pass } \\
\text { Max Temp } \\
\text { C }\end{array}$ \\
\hline 2014 & 11 & $\begin{array}{r}0.6(9.9) \\
525 \\
3 \\
\end{array}$ & $8.4(3.4) 297$ & -19.3 & 0.8 & 17.8 & 17.6 \\
\hline 2014 & 12 & $1.6(7) 5716$ & $7(3.6) 163$ & -20.5 & -1.5 & 13.6 & 13.5 \\
\hline 2015 & 1 & $1(7.3) 5553$ & $6.7(3.7) 30$ & -19 & -1.5 & 16 & 12.7 \\
\hline 2015 & 2 & $\begin{array}{r}1.8(7.7) \\
460 \\
6\end{array}$ & 5.7 (3) 60 & -16.5 & 2.7 & 17 & 11.7 \\
\hline 2015 & 3 & $6.8(7) 4533$ & $\begin{array}{r}13.2(2.7) \\
168 \\
2\end{array}$ & -14.8 & 5.2 & 20.6 & 20.6 \\
\hline 2015 & 4 & $\begin{array}{r}7.3(4.5) \\
380 \\
2\end{array}$ & $\begin{array}{r}11.8(2.4) \\
162 \\
1\end{array}$ & -3.8 & 1.6 & 21.2 & 21.1 \\
\hline 2015 & 5 & $\begin{array}{r}10.7(3.4) \\
337 \\
4\end{array}$ & $\begin{array}{r}11.9(2.6) \\
144 \\
9\end{array}$ & 0.8 & 2.6 & 20.9 & 20.6 \\
\hline 2015 & 6 & $\begin{array}{r}20.3(3.6) \\
185 \\
6\end{array}$ & $\begin{array}{r}22.4(3.4) \\
123 \\
90\end{array}$ & 12.7 & 14.1 & 29.5 & 29.5 \\
\hline 2015 & 6 & $18(3.4) 1163$ & $\begin{array}{r}20.4(2.4) \\
481\end{array}$ & 11.8 & 13.5 & 26.4 & 26.4 \\
\hline 2015 & 7 & 21 (3.3) 3291 & $\begin{array}{r}22.5(2.9) \\
242 \\
10\end{array}$ & 10.5 & 10.5 & 30.3 & 30.3 \\
\hline 2015 & 8 & $\begin{array}{r}21.8(5.3) \\
371 \\
4\end{array}$ & $\begin{array}{r}23.8(3.9) \\
142 \\
55\end{array}$ & 7.9 & 7.9 & 33.5 & 33.5 \\
\hline 2015 & 9 & $\begin{array}{r}18.7(4.6) \\
418 \\
1 \\
\end{array}$ & 21.5 (4) 9275 & 8.4 & 8.9 & 30 & 30 \\
\hline 2015 & 10 & $\begin{array}{r}13.9(4.8) \\
490 \\
4\end{array}$ & $\begin{array}{r}16.4(6.4) \\
143 \\
6\end{array}$ & 3.6 & 3.9 & 28.5 & 28.2 \\
\hline 2015 & 11 & $\begin{array}{r}4.7(6.4) \\
528 \\
1\end{array}$ & $\begin{array}{r}10.1(3.2) \\
135 \\
5\end{array}$ & -11.5 & 2.9 & 17.9 & 16.8 \\
\hline 2015 & 12 & $\begin{array}{r}2.5(5.8) \\
576 \\
4\end{array}$ & $\begin{array}{r}8.3(3.5) \\
100 \\
9\end{array}$ & -10.7 & -0.8 & 17 & 15.6 \\
\hline
\end{tabular}




\begin{tabular}{|c|c|c|c|c|c|c|c|}
\hline 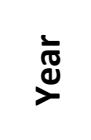 & 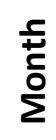 & $\begin{array}{c}\text { Background } \\
\text { Temp C } \\
\text { Avg (SD) N }\end{array}$ & $\begin{array}{c}\text { Bat Pass } \\
\text { Temp C } \\
\text { Avg (SD) N }\end{array}$ & $\begin{array}{c}\text { Background } \\
\text { Min } \\
\text { Temp C }\end{array}$ & $\begin{array}{c}\text { Bat Pass } \\
\text { Min Temp C }\end{array}$ & $\begin{array}{c}\text { Background } \\
\text { Max } \\
\text { Temp C }\end{array}$ & $\begin{array}{r}\text { Bat Pass } \\
\text { Max Temp } \\
\text { C }\end{array}$ \\
\hline 2016 & 1 & $\begin{array}{r}3.6(4.4) \\
557 \\
5 \\
\end{array}$ & $6.9(2.7) 558$ & -10.7 & -1.3 & 13.6 & 12 \\
\hline 2016 & 2 & $\begin{array}{r}6.6(4.4) \\
359 \\
8\end{array}$ & $\begin{array}{r}9.3(3.6) \\
108 \\
2\end{array}$ & -4.1 & 2.2 & 15.6 & 14.8 \\
\hline
\end{tabular}

Table 10i. Nightly background and bat pass temperatures summarized by month at the Signal Peak Mine Busse Water Reservoir

\begin{tabular}{|c|c|c|c|c|c|c|c|}
\hline ֻ & & $\begin{array}{l}\text { Background } \\
\text { Temp C } \\
\text { Avg (SD) N }\end{array}$ & $\begin{array}{c}\text { Bat Pass } \\
\text { Temp C } \\
\text { Avg (SD) N }\end{array}$ & $\begin{array}{c}\text { Background } \\
\text { Min } \\
\text { Temp C }\end{array}$ & $\begin{array}{c}\text { Bat Pass } \\
\text { Min Temp C }\end{array}$ & $\begin{array}{c}\text { Background } \\
\text { Max } \\
\text { Temp C }\end{array}$ & $\begin{array}{c}\text { Bat Pass } \\
\text { Max } \\
\text { Temp C }\end{array}$ \\
\hline 2012 & 8 & $\begin{array}{r}19.7(5.5) \\
105 \\
6 \\
\end{array}$ & $\begin{array}{r}20.9(4.4) \\
137 \\
68\end{array}$ & 5.4 & 6.2 & 30.2 & 30.2 \\
\hline 2012 & 9 & $\begin{array}{r}14.3(4.6) \\
412 \\
9\end{array}$ & $\begin{array}{r}16.6(3.8) \\
235 \\
76\end{array}$ & 3.6 & 3.6 & 26.9 & 26.9 \\
\hline 2012 & 10 & $\begin{array}{r}6.6(5.2) \\
486 \\
8\end{array}$ & $\begin{array}{r}14.3(3.8) \\
257\end{array}$ & -4.4 & 1.1 & 21.2 & 20.1 \\
\hline 2012 & 11 & $4.1(6) 5248$ & $6.8(3) 57$ & -15.8 & 1.3 & 17.9 & 13.6 \\
\hline 2012 & 12 & $\begin{array}{r}-2.5(6.6) \\
573 \\
5 \\
\end{array}$ & $4.6(4.5) 74$ & -20.3 & -2.9 & 15.3 & 11.7 \\
\hline 2013 & 1 & $\begin{array}{r}0.1(5.5) \\
174 \\
4\end{array}$ & $6.7(1.9) 7$ & -13.9 & 4.6 & 9.2 & 8.7 \\
\hline 2013 & 4 & $\begin{array}{r}2.2(6.4) \\
356 \\
0\end{array}$ & $\begin{array}{r}10.4(2.9) \\
136 \\
6\end{array}$ & -10.7 & 0.8 & 17.9 & 17 \\
\hline 2013 & 5 & $\begin{array}{r}10.3(4.3) \\
336 \\
3\end{array}$ & 13.3 (3) 7956 & -3.9 & 0.6 & 24.7 & 24.4 \\
\hline 2013 & 6 & $14(4.3) 2939$ & $\begin{array}{r}15.9(2.8) \\
308 \\
80\end{array}$ & 2.1 & 2.7 & 24.4 & 23.9 \\
\hline 2013 & 7 & $\begin{array}{r}18.9(3.2) \\
267 \\
3\end{array}$ & $\begin{array}{r}18.9(3) \\
414 \\
51\end{array}$ & 10.5 & 10.7 & 26.7 & 26.7 \\
\hline
\end{tabular}




\begin{tabular}{|c|c|c|c|c|c|c|c|}
\hline 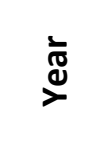 & 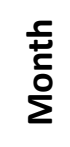 & $\begin{array}{c}\text { Background } \\
\text { Temp C } \\
\text { Avg (SD) N }\end{array}$ & $\begin{array}{c}\text { Bat Pass } \\
\text { Temp C } \\
\text { Avg (SD) N }\end{array}$ & $\begin{array}{c}\text { Background } \\
\text { Min } \\
\text { Temp C }\end{array}$ & $\begin{array}{c}\text { Bat Pass } \\
\text { Min Temp C }\end{array}$ & $\begin{array}{c}\text { Background } \\
\text { Max } \\
\text { Temp C }\end{array}$ & $\begin{array}{c}\text { Bat Pass } \\
\text { Max } \\
\text { Temp C }\end{array}$ \\
\hline 2013 & 8 & $\begin{array}{r}19.2(3.6) \\
314 \\
4 \\
\end{array}$ & $\begin{array}{r}18.5(3.5) \\
125 \\
66 \\
\end{array}$ & 11 & 11.7 & 28 & 28 \\
\hline 2013 & 9 & $\begin{array}{r}14.3(5.1) \\
414 \\
6\end{array}$ & $\begin{array}{r}17.6(3.1) \\
372 \\
8\end{array}$ & 2.7 & 5.5 & 26 & 25.9 \\
\hline 2013 & 10 & $\begin{array}{r}4.9(4.1) \\
491 \\
4\end{array}$ & $\begin{array}{r}11.6(3.3) \\
343\end{array}$ & -6.6 & -1.5 & 17.3 & 17 \\
\hline 2013 & 11 & $\begin{array}{r}0.7(5.5) \\
529 \\
0\end{array}$ & 4.6 (3.7) 91 & -20 & -1.6 & 11.7 & 10.8 \\
\hline 2013 & 12 & $\begin{array}{r}-4.5(9.2) \\
573 \\
2 \\
\end{array}$ & $4.5(3.3) 22$ & -20.5 & -2 & 9 & 9 \\
\hline 2014 & 1 & -1.7 (7) 5553 & $2.4(3.7) 20$ & -20.5 & -1.1 & 10 & 9.8 \\
\hline 2014 & 2 & $\begin{array}{r}-7.4(8.2) \\
459 \\
7 \\
\end{array}$ & $4.6(2.5) 9$ & -20.5 & 0.8 & 7 & 7 \\
\hline 2014 & 3 & $-0.8(7) 4510$ & $4.2(3.9) 12$ & -20.5 & -1.6 & 10.8 & 10.7 \\
\hline 2014 & 4 & $\begin{array}{r}4.5(4.8) \\
379 \\
8\end{array}$ & 11 (3.1) 2204 & -8.6 & -0.5 & 17.3 & 17.3 \\
\hline 2014 & 5 & $\begin{array}{r}9.7(5.4) \\
341 \\
1\end{array}$ & $\begin{array}{r}14.4(3.7) \\
158 \\
52 \\
\end{array}$ & -2.1 & 1.1 & 23.1 & 23.1 \\
\hline 2014 & 6 & $\begin{array}{r}12.3(3.4) \\
301 \\
2 \\
\end{array}$ & $\begin{array}{r}13.6(2.9) \\
306 \\
86 \\
\end{array}$ & 1.9 & 2.1 & 20.4 & 20.4 \\
\hline 2014 & 7 & $\begin{array}{r}17.7(3.7) \\
325 \\
0 \\
\end{array}$ & $\begin{array}{r}17.8(3.3) \\
437 \\
47\end{array}$ & 8.9 & 8.9 & 26.9 & 26.5 \\
\hline 2014 & 8 & $\begin{array}{r}16.6(3.9) \\
370 \\
1\end{array}$ & $\begin{array}{r}18(3.2) \\
229 \\
65\end{array}$ & 6 & 6.4 & 28.5 & 28.5 \\
\hline 2014 & 9 & 11.3 (5) 4141 & $\begin{array}{r}13.7(3.7) \\
428 \\
4\end{array}$ & 0.3 & 3.9 & 23.7 & 23.7 \\
\hline 2014 & 10 & $\begin{array}{r}8.4(4.5) \\
489 \\
4\end{array}$ & $13.1(4.6) 99$ & -5.3 & 0.6 & 20.8 & 20.6 \\
\hline
\end{tabular}




\begin{tabular}{|c|c|c|c|c|c|c|c|}
\hline$\stackrel{\frac{1}{d}}{\frac{\pi}{2}}$ & $\frac{5}{\Sigma}$ & $\begin{array}{c}\text { Background } \\
\text { Temp C } \\
\text { Avg (SD) N }\end{array}$ & $\begin{array}{c}\text { Bat Pass } \\
\text { Temp C } \\
\text { Avg (SD) N }\end{array}$ & $\begin{array}{c}\text { Background } \\
\text { Min } \\
\text { Temp C }\end{array}$ & $\begin{array}{c}\text { Bat Pass } \\
\text { Min Temp C }\end{array}$ & $\begin{array}{c}\text { Background } \\
\text { Max } \\
\text { Temp C }\end{array}$ & $\begin{array}{c}\text { Bat Pass } \\
\text { Max } \\
\text { Temp C }\end{array}$ \\
\hline 2014 & 11 & $\begin{array}{r}-2.1(9.9) \\
527 \\
2 \\
\end{array}$ & $5.1(4.1) 25$ & -20.5 & -4.4 & 15.3 & 13.2 \\
\hline 2014 & 12 & $\begin{array}{r}-1.3(7.1) \\
571 \\
8\end{array}$ & $4.4(4.4) 104$ & -20.5 & -5.8 & 12.2 & 12 \\
\hline 2015 & 1 & $\begin{array}{r}-1.9(7.3) \\
555 \\
7\end{array}$ & 4.4 (4.1) 17 & -20.5 & -1.6 & 12.8 & 12 \\
\hline 2015 & 2 & $\begin{array}{r}-0.7(7.4) \\
461 \\
9\end{array}$ & $10.1(5.2) 31$ & -19.1 & 0.3 & 15.6 & 14.1 \\
\hline 2015 & 3 & 4 (7) 4535 & $\begin{array}{r}11.6(3.7) \\
105 \\
4\end{array}$ & -17.3 & -1.6 & 19.1 & 18.4 \\
\hline 2015 & 4 & $5.3(5) 3788$ & $\begin{array}{r}11.1(3.5) \\
240 \\
0\end{array}$ & -6.3 & 0 & 17.4 & 17.1 \\
\hline 2015 & 5 & $\begin{array}{r}8.8(3.5) \\
337 \\
7\end{array}$ & $\begin{array}{r}10.6(3) \\
102 \\
30 \\
\end{array}$ & -1 & 0.4 & 20.6 & 20.6 \\
\hline 2015 & 6 & $\begin{array}{r}16.5(3.5) \\
304 \\
0\end{array}$ & $\begin{array}{r}17(3.4) \\
485 \\
46\end{array}$ & 9.4 & 9.4 & 27.4 & 27.4 \\
\hline 2015 & 6 & $\begin{array}{r}16.5(2.8) \\
100 \\
0\end{array}$ & $\begin{array}{c}16.7(2.7) \\
246 \\
55\end{array}$ & 11.2 & 11.2 & 24.9 & 24.9 \\
\hline 2015 & 7 & $17(3.4) 3288$ & $\begin{array}{r}17.6(2.8) \\
688 \\
47\end{array}$ & 8.2 & 8.4 & 27.9 & 27.9 \\
\hline 2015 & 8 & $\begin{array}{r}18.8(5.8) \\
196 \\
1\end{array}$ & $\begin{array}{r}19.9(4.3) \\
297 \\
47\end{array}$ & 3.7 & 3.9 & 29.8 & 29.8 \\
\hline 2015 & 8 & $\begin{array}{r}20.3(6.6) \\
132 \\
1\end{array}$ & $\begin{array}{r}22.2(4.7) \\
270 \\
61\end{array}$ & 5.2 & 5.4 & 30.7 & 30.7 \\
\hline 2015 & 9 & 15.7 (5) 4141 & $\begin{array}{r}18.9(4.6) \\
288 \\
98\end{array}$ & 5.2 & 5.4 & 28 & 28 \\
\hline 2015 & 9 & 14.2 (5) 4148 & $\begin{array}{r}16.2(4.4) \\
161 \\
50\end{array}$ & 3.7 & 3.9 & 26.7 & 26.7 \\
\hline
\end{tabular}




\begin{tabular}{|c|c|c|c|c|c|c|c|}
\hline$\stackrel{\frac{\pi}{d}}{\check{2}}$ & 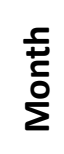 & $\begin{array}{c}\text { Background } \\
\text { Temp C } \\
\text { Avg (SD) N }\end{array}$ & $\begin{array}{c}\text { Bat Pass } \\
\text { Temp C } \\
\text { Avg (SD) N }\end{array}$ & $\begin{array}{c}\text { Background } \\
\text { Min } \\
\text { Temp C }\end{array}$ & $\begin{array}{c}\text { Bat Pass } \\
\text { Min Temp C }\end{array}$ & $\begin{array}{c}\text { Background } \\
\text { Max } \\
\text { Temp C }\end{array}$ & $\begin{array}{c}\text { Bat Pass } \\
\text { Max } \\
\text { Temp C }\end{array}$ \\
\hline 2015 & 10 & $\begin{array}{r}11.1(5.1) \\
284 \\
2\end{array}$ & $\begin{array}{r}16.2(4.4) \\
100\end{array}$ & 0 & 4.2 & 25.5 & 25.2 \\
\hline 2015 & 10 & $11.1(5) 4889$ & $\begin{array}{r}17.4(4.5) \\
394\end{array}$ & 0 & 4.7 & 26.5 & 26.5 \\
\hline 2015 & 11 & $\begin{array}{r}1.6(6.9) \\
526 \\
2\end{array}$ & $6.7(3.4) 255$ & -16.8 & 0 & 16 & 14.8 \\
\hline 2015 & 12 & $\begin{array}{r}-0.6(6.5) \\
573 \\
1\end{array}$ & $5.8(4.3) 413$ & -16.3 & -2.1 & 14.8 & 14.8 \\
\hline 2016 & 1 & $\begin{array}{r}0.5(4.8) \\
557 \\
1\end{array}$ & 4.8 (2.7) 372 & -13.4 & -4.6 & 11 & 10.3 \\
\hline 2016 & 2 & $\begin{array}{r}3.6(4.3) \\
359 \\
3\end{array}$ & 6.9 (3) 218 & -7.9 & 0.3 & 12.7 & 11 \\
\hline
\end{tabular}


Table 11a. Monthly minimum bat pass temperatures $\left({ }^{\circ} \mathrm{C}\right)$ recorded for individual species hand confirmed as definitively present at the West Decker Mine

\begin{tabular}{|c|c|c|c|c|c|c|c|c|c|c|c|c|c|}
\hline Species & Year & Jan & Feb & Mar & Apr & May & June & July & Aug & Sep & Oct & Nov & Dec \\
\hline Epfu & 2012 & & & & & & & & 17.4 & 13.8 & & & \\
\hline Epfu & 2013 & & & & 13.6 & 14.1 & 18.6 & 21.1 & & & & & \\
\hline Epfu & 2014 & & & & & 16.1 & 14.6 & & 15.3 & 16.1 & & & \\
\hline Labo & 2012 & & & & & & & & 14.5 & & & & \\
\hline Labo & 2014 & & & & & & & & & 6.5 & & & \\
\hline Laci & 2012 & & & & & & & & 9 & 9.8 & & & \\
\hline Laci & 2013 & & & & & & 18.9 & & & & & & \\
\hline Laci & 2014 & & & & & 12.7 & & 20.1 & 13 & 10.8 & & & \\
\hline Lano & 2012 & & & & & & & & 13.5 & 7.7 & 2.9 & & \\
\hline Lano & 2013 & & & & 12.2 & 8.5 & & & & & & & \\
\hline Lano & 2014 & & & & & 11.3 & 11.7 & & 16.3 & 7.4 & 15.5 & & \\
\hline Myci & 2012 & & & & & & & & 7.4 & 13.3 & & & \\
\hline Myci & 2013 & & & & 13.8 & 14.1 & 18.1 & 18.8 & & & & & \\
\hline Myci & 2014 & & & & 8.7 & 14 & 12.2 & 18.1 & 13.3 & 8.7 & & & \\
\hline Myev & 2012 & & & & & & & & 9.7 & 9.2 & & & \\
\hline Myev & 2013 & & & & 7.2 & 15 & 11 & & & & & & \\
\hline Myev & 2014 & & & & & 13.6 & 17.4 & & 17 & 5.4 & & & \\
\hline Mylu & 2012 & & & & & & & & 11.3 & 0.4 & 9 & & \\
\hline Mylu & 2013 & & & & & 12.7 & 17.1 & 17.1 & & & & & \\
\hline Mylu & 2014 & & & & & 11.2 & 17.4 & 17.1 & 12.8 & 16.1 & & & \\
\hline Mylu & 2015 & & & & 14.6 & 18.6 & 16.3 & & & & & & \\
\hline Epfu & 2016 & & & & 15 & & & & & & & & \\
\hline Labo & 2015 & & & & & & & & 19.1 & 16.6 & 22.6 & & \\
\hline Laci & 2015 & & & & & & 16.1 & 13.8 & 17.1 & 13.3 & & & \\
\hline Laci & 2016 & & & & & 14.5 & 15 & 20.4 & & & & & \\
\hline Lano & 2015 & & & & & & 15.6 & 13.8 & 16.1 & 13.2 & & & \\
\hline Lano & 2016 & & & & 13.5 & 10 & 15 & 20.9 & & & & & \\
\hline Myci & 2015 & & & & & & 14.6 & 11 & & & 17.6 & & \\
\hline Myev & 2015 & & & & & & 17.6 & 16.1 & 17 & 11 & & & \\
\hline Myev & 2016 & & & & & & & 21.7 & & & & & \\
\hline
\end{tabular}

Table 11b. Monthly minimum bat pass temperatures $\left({ }^{\circ} \mathrm{C}\right)$ recorded for individual species hand confirmed as definitively present at the Spring Creek Mine

\begin{tabular}{|c|c|c|c|c|c|c|c|c|c|c|c|c|c|}
\hline Species & Year & Jan & Feb & Mar & Apr & May & June & July & Aug & Sep & Oct & Nov & Dec \\
\hline Epfu & 2012 & & & & & & & & 22.6 & & & & \\
\hline Epfu & 2013 & & & & & 12.5 & 21.4 & & & & & & \\
\hline Epfu & 2014 & & & & & 24.6 & 17 & 23.9 & 20.8 & & & & \\
\hline Labo & 2012 & & & & & & & & 21.6 & & & & \\
\hline
\end{tabular}




\begin{tabular}{|c|c|c|c|c|c|c|c|c|c|c|c|c|c|}
\hline Species & Year & Jan & Feb & Mar & Apr & May & June & July & Aug & Sep & Oct & Nov & Dec \\
\hline Labo & 2014 & & & & & & & & 19.8 & & & & \\
\hline Laci & 2012 & & & & & & & & 20.6 & 17.9 & & & \\
\hline Laci & 2013 & & & & & 11.7 & 17.6 & & & & & & \\
\hline Laci & 2014 & & & & & 13.5 & 21.2 & 25.9 & 20.9 & 8.9 & & & \\
\hline Lano & 2012 & & & & & & & & 23.7 & 16.5 & & & \\
\hline Lano & 2013 & & & & 12 & 12.2 & 11.3 & & & & & 8.2 & \\
\hline Lano & 2014 & & & & 12 & 12.7 & 16.5 & 12 & 21.4 & 15.8 & & 13.5 & \\
\hline Myci & 2012 & & & & & & & & 20.6 & 17.1 & & & \\
\hline Myci & 2013 & & & & 7.5 & 16.5 & 17 & & & & & & \\
\hline Myci & 2014 & & & & 18.4 & 19.4 & 16.8 & 16.8 & 20.8 & 24.2 & & & \\
\hline Myev & 2012 & & & & & & & & 24.9 & 16.6 & & & \\
\hline Myev & 2013 & & & & & 6.5 & 14.5 & & & & & & \\
\hline Myev & 2014 & & & & 11.7 & 12.2 & 8.9 & 18.8 & 22.4 & 8.7 & & & \\
\hline Myev & 2015 & & & & & 16.6 & & & & & & & \\
\hline Mylu & 2012 & & & & & & & & 17.4 & 19.8 & & & \\
\hline Mylu & 2013 & & & & 15.1 & 6 & 12.3 & & & & & & \\
\hline Mylu & 2014 & & & & & 15.1 & 16 & 19.9 & 22.7 & 13.3 & & & \\
\hline Myvo & 2014 & & & & & & & & 22.4 & & & & \\
\hline Epfu & 2015 & & & & & & & 28.2 & 28.4 & & & & \\
\hline Labo & 2016 & & & & 12.5 & & & & & & & & \\
\hline Laci & 2015 & & & & & & 23.9 & 17.8 & 15.6 & 13.8 & & & \\
\hline Laci & 2016 & & & & & & 22.9 & 21.7 & 15.3 & 17.4 & & & \\
\hline Lano & 2015 & & & & & & 19.8 & 21.7 & 23.2 & & & & \\
\hline Lano & 2016 & & & & & & & 26.9 & & 17.8 & & & \\
\hline Myci & 2015 & & & & & & & 22.1 & & & & & \\
\hline Myev & 2015 & & & & & & 19.8 & 19.1 & 17.9 & 19.9 & & & \\
\hline Mylu & 2015 & & & & & & 23.6 & 19.3 & & & & & \\
\hline Myth & 2016 & & & & & & & 19.4 & & & & & \\
\hline
\end{tabular}

Table 11c. Monthly minimum bat pass temperatures $\left({ }^{\circ} \mathrm{C}\right)$ recorded for individual species hand confirmed as definitively present at the Otter Creek Coal Tract

\begin{tabular}{|c|l|l|l|l|l|l|l|l|l|l|l|l|l|}
\hline Species & Year & Jan & Feb & Mar & Apr & May & June & July & Aug & Sep & Oct & Nov & Dec \\
\hline Labo & 2014 & & & & & & & & 14 & & & & \\
\hline Laci & 2014 & & & & & 10.2 & 14.3 & 21.6 & 8.9 & 8.9 & & & \\
\hline Laci & 2015 & & & & & 17.1 & & & & & & & \\
\hline Lano & 2014 & & & & & 8.9 & & & 10.8 & 5.4 & & & \\
\hline Lano & 2015 & & & & & 16.1 & & & & & & & \\
\hline Myci & 2013 & & & & & & & & & & 13 & & \\
\hline Myci & 2014 & & & & & 9.2 & & 13.8 & 11.2 & & & & \\
\hline Myci & 2015 & & & & 8.7 & & & & & & & & \\
\hline
\end{tabular}




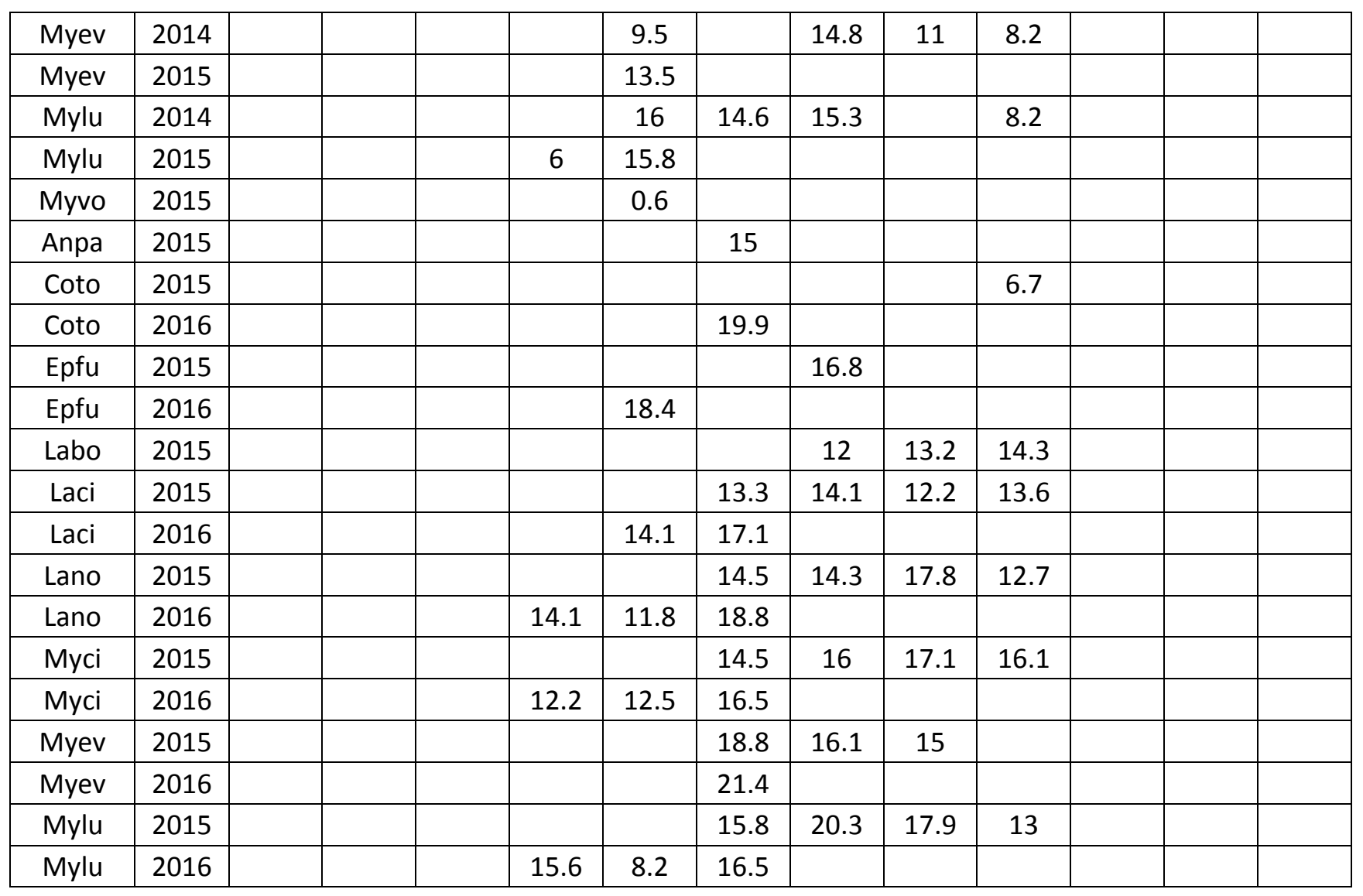

Table 11d. Monthly minimum bat pass temperatures $\left({ }^{\circ} \mathrm{C}\right)$ recorded for individual species hand confirmed as definitively present at the Big Sky Mine

\begin{tabular}{|c|c|c|c|c|c|c|c|c|c|c|c|c|c|}
\hline Species & Year & Jan & Feb & Mar & Apr & May & June & July & Aug & Sep & Oct & Nov & Dec \\
\hline Anpa & 2013 & & & & & & & & & & & 0.8 & \\
\hline Anpa & 2014 & & & & 5.2 & & & & & & & & \\
\hline Epfu & 2013 & & & & & & & & & & 15 & 8 & \\
\hline Epfu & 2014 & 5.1 & 3.7 & 6.5 & 13.3 & 12.7 & & 21.9 & 17.8 & 19.3 & & & \\
\hline Labo & 2014 & & & & & & & & 13.6 & & & & \\
\hline Laci & 2015 & & & 14.5 & 6.7 & 7.7 & 17.8 & & & & & & \\
\hline Lano & 2013 & & & & & & & & & & 14.1 & -4.1 & 0.4 \\
\hline Lano & 2014 & -0.5 & 4.9 & 10.7 & 8.2 & 12.3 & & & 18.8 & & & & \\
\hline Lano & 2015 & & & 6.4 & 12.2 & 9.2 & 17 & & & & & & \\
\hline Myci & 2013 & & & & & & & & & & 4.1 & -0.6 & \\
\hline Myci & 2014 & -1 & 4.6 & 2.9 & 7 & 12.7 & & & 12.3 & & & & \\
\hline Myev & 2014 & & & & & & & 22.9 & 12.2 & & & & \\
\hline Myev & 2015 & & & & & 9.2 & 18.1 & & & & & & \\
\hline Mylu & 2014 & & & & 18.3 & 3.9 & & & 19.6 & 18.3 & & & \\
\hline Anpa & 2016 & & & & & 11.7 & & & & & & & \\
\hline
\end{tabular}




\begin{tabular}{|c|l|l|l|l|l|l|l|l|l|l|l|l|l|}
\hline Epfu & 2015 & & & & & & 18.4 & & & & & & \\
\hline Euma & 2015 & & & & & & 23.6 & & & & & & \\
\hline Labo & 2015 & & & & & & 29.3 & & & & & & \\
\hline Laci & 2015 & & & & & & 20.9 & 14.5 & 15.6 & 12 & 8.5 & 2.2 & \\
\hline Laci & 2016 & & & & & 11.5 & 14.6 & 14 & 15.3 & 17.1 & & & \\
\hline Lano & 2015 & & & & & & 20.1 & 18.6 & 17.4 & 21.2 & 15.1 & 1.7 & 2.1 \\
\hline Lano & 2016 & & & 13.5 & 14.6 & 17.1 & & 21.4 & 18.9 & 12.2 & 16 & & \\
\hline Myci & 2015 & & & & & & 18.3 & 17.9 & 18.6 & 19.9 & 14.8 & 6.9 & 2.9 \\
\hline Myci & 2016 & 3.7 & 10.8 & 11.7 & 10.3 & 11.7 & 10.7 & 22.4 & & 22.4 & & & \\
\hline Myev & 2015 & & & & & & 12.3 & 14.1 & 16 & 18.3 & & & \\
\hline Myev & 2016 & & & & & 11.7 & & & & 21.1 & & & \\
\hline Mylu & 2015 & & & & & & 18.4 & 22.2 & 15.8 & 19.8 & & & \\
\hline Mylu & 2016 & & & & 18.6 & 18.3 & 21.9 & 26.2 & 17.4 & 18.1 & & & \\
\hline Myvo & 2015 & & & & & & & & & & 18.4 & & \\
\hline
\end{tabular}

Table 11e. Monthly minimum bat pass temperatures $\left({ }^{\circ} \mathrm{C}\right)$ recorded for individual species hand confirmed as definitively present at the Absaloka Mine

\begin{tabular}{|c|l|l|l|l|l|l|l|l|l|l|l|l|l|}
\hline Species & Year & Jan & Feb & Mar & Apr & May & June & July & Aug & Sep & Oct & Nov & Dec \\
\hline Epfu & 2012 & & & & & & & & & 17.9 & & & \\
\hline Epfu & 2013 & & & & & 12.3 & 18.8 & & & & & & \\
\hline Epfu & 2014 & & & & & 11.7 & & & & & & & \\
\hline Labo & 2012 & & & & & & & & 15.8 & & & & \\
\hline Labo & 2014 & & & & & & & & & 1.6 & & & \\
\hline Laci & 2012 & & & & & & & & 14.5 & -0.1 & & & \\
\hline Laci & 2013 & & & & & 17.9 & 16.6 & & & & & & \\
\hline Laci & 2014 & & & & & 20.3 & 4.6 & 19.6 & 10.3 & 0.8 & & & \\
\hline Laci & 2015 & & & & 2.7 & 12.3 & 17.6 & & & & & & \\
\hline Lano & 2012 & & & & & & & & 20.6 & -1.8 & 12.2 & & \\
\hline Lano & 2013 & & & & & 4.6 & 19.8 & & & & & & \\
\hline Lano & 2014 & & & & 15.6 & 6.5 & & & 17.3 & -0.8 & & & \\
\hline Lano & 2015 & & & & & & 17.4 & & & & & & \\
\hline Myci & 2012 & & & & & & & & 16.8 & & & & \\
\hline Myci & 2013 & & & & & & 16.3 & & & & & & \\
\hline Myev & 2012 & & & & & & & & 13.3 & 3.4 & & & \\
\hline Myev & 2013 & & & & & 10 & 17 & & & & & & \\
\hline Myev & 2014 & & & & & 10.2 & 13 & & 16.1 & 0.3 & & & \\
\hline Mylu & 2012 & & & & & & & & 14 & -0.5 & & & \\
\hline Laci & 2015 & & & & & & 15.1 & 14 & 14.1 & & & & \\
\hline Lano & 2015 & & & & & & 13.8 & 15.5 & & & & & \\
\hline Myci & 2015 & & & & & & 13.8 & 18.9 & & & & & \\
\hline Myev & 2015 & & & & & & 13.5 & 15.3 & & & & & \\
\hline
\end{tabular}


Table 11f. Monthly minimum bat pass temperatures $\left({ }^{\circ} \mathrm{C}\right)$ recorded for individual species hand confirmed as definitively present at the Rosebud Mine Area F Pond 7

\begin{tabular}{|c|l|l|l|l|l|l|l|l|l|l|l|l|l|}
\hline Species & Year & Jan & Feb & Mar & Apr & May & June & July & Aug & Sep & Oct & Nov & Dec \\
\hline Epfu & 2012 & & & & & & & & 26.7 & 10.8 & & 8.5 & \\
\hline Epfu & 2013 & & & & & 20.9 & 19.8 & 24.7 & 18.4 & & & & \\
\hline Laci & 2013 & & & & & & 16.5 & 18.6 & 13 & & & & \\
\hline Lano & 2012 & & & & & & & & 11.5 & 14.1 & & & \\
\hline Lano & 2013 & & & & & 16.3 & 16.1 & 16.5 & 12.3 & 27.9 & & & \\
\hline Myci & 2012 & & & & & & & & 23.7 & 15.5 & & & \\
\hline Myci & 2013 & & & & & 20.9 & 16.8 & 22.6 & 17.4 & 21.9 & & & \\
\hline Myev & 2012 & & & & & & & & 13.8 & 6.2 & & & \\
\hline Myev & 2013 & & & & & & 19.1 & 17.1 & 12.7 & 26.5 & 15 & & \\
\hline Mylu & 2012 & & & & & & & & 19.3 & 19.3 & & & \\
\hline Mylu & 2013 & & & & & & 14 & & 17.3 & & & & \\
\hline Myvo & 2012 & & & & & & & & & 9.4 & & & \\
\hline
\end{tabular}

Table 11g. Monthly minimum bat pass temperatures $\left({ }^{\circ} \mathrm{C}\right)$ recorded for individual species hand confirmed as definitively present at the Rosebud Mine Area C Pond

\begin{tabular}{|c|l|l|l|l|c|c|c|c|c|c|c|c|c|}
\hline Species & Year & Jan & Feb & Mar & Apr & May & June & July & Aug & Sep & Oct & Nov & Dec \\
\hline Epfu & 2014 & & & & 7 & 9.7 & 11 & 15.8 & 15 & 15.1 & 16 & & \\
\hline Labo & 2014 & & & & & & & & 12.5 & & & & \\
\hline Laci & 2014 & & & & & 9.8 & 7.7 & 10.7 & 14 & 9.7 & & & \\
\hline Lano & 2014 & & & & 5.7 & 10.2 & 10.5 & 12.2 & 12.2 & 11 & 14.5 & & \\
\hline Lano & 2015 & & & & & 11.7 & 13.3 & & & & & & \\
\hline Myci & 2013 & & & & & & & & & & 14 & & \\
\hline Myci & 2014 & & & & 16.3 & 10.3 & 13.2 & 11.7 & 11 & 13.2 & 10 & & \\
\hline Myev & 2014 & & & & & 6 & 19.3 & 11 & 14.6 & 5.7 & & & \\
\hline Mylu & 2014 & & & & & 8 & 11.5 & 12.3 & 9.8 & 5.4 & 8.2 & & \\
\hline Mylu & 2015 & & & & & 18.6 & & & & & & & \\
\hline Myvo & 2014 & & & & & & & 22.2 & & & & & \\
\hline Bug & 2015 & & & & & & & & 15.6 & & & & \\
\hline Epfu & 2016 & & & & & 15.1 & & & & & & & \\
\hline Labo & 2015 & & & & & & & & 16.1 & 12.8 & & & \\
\hline Laci & 2015 & & & & & & 21.9 & 15 & 10.5 & 17 & & & \\
\hline Laci & 2016 & & & & & 14.1 & & & & & & & \\
\hline Lano & 2015 & & & & & 17.8 & 18.8 & 17 & 19.1 & & & \\
\hline Lano & 2016 & & & & 11.7 & 16.1 & & & & & & & \\
\hline Myci & 2015 & & & & & & 21.7 & 19.3 & 26.7 & 15.6 & & & \\
\hline Myci & 2016 & & & & & 18.4 & 16 & & & & & & \\
\hline Myev & 2015 & & & & & & 16.3 & 23.2 & 15.6 & 12.7 & & & \\
\hline
\end{tabular}




\begin{tabular}{|l|l|l|l|l|l|l|l|l|l|l|l|l|l|}
\hline Myev & 2016 & & & & & 11.2 & & & & & & & \\
\hline Mylu & 2015 & & & & & & 18.1 & 20.3 & 18.8 & 12.2 & & & \\
\hline Mylu & 2016 & & & & & 11.5 & & & & & & & \\
\hline
\end{tabular}

Table 11h. Monthly minimum bat pass temperatures $\left({ }^{\circ} \mathrm{C}\right)$ recorded for individual species hand confirmed as definitively present at Signal Peak Reservoir 1

\begin{tabular}{|c|c|c|c|c|c|c|c|c|c|c|c|c|c|}
\hline Species & Year & Jan & Feb & Mar & Apr & May & June & July & Aug & Sep & Oct & Nov & Dec \\
\hline Coto & 2013 & & & & & & & 22.9 & & & & & \\
\hline Coto & 2014 & & & & & & 16.5 & 19.3 & 25.7 & & & & \\
\hline Epfu & 2012 & & & & & & & & 28.2 & 21.4 & 17.4 & 4.2 & -1.6 \\
\hline Epfu & 2013 & & & & 13.5 & 14 & 19.9 & 17.6 & 21.2 & 19.8 & 13 & & \\
\hline Epfu & 2014 & & & & & 10.8 & 17.8 & 21.6 & 26 & 16.1 & & & \\
\hline Epfu & 2015 & 5.7 & & & & & & & & & & & \\
\hline Euma & 2012 & & & & & & & & & 20.4 & & & \\
\hline Euma & 2013 & & & & 12 & & 15.8 & 23.6 & 23.9 & & & & \\
\hline Euma & 2014 & & & & & 21.2 & 13.6 & 13.2 & 15.3 & & 15.5 & & \\
\hline Euma & 2015 & & & & 11.5 & & & & & & & & \\
\hline Labo & 2013 & & & & & & & 17.4 & & & & & \\
\hline Labo & 2014 & & & & & & & & 15.8 & & & & \\
\hline Laci & 2012 & & & & & & & & 24.9 & 18.9 & & & \\
\hline Laci & 2013 & & & & & 14.6 & 12.5 & 16 & 16.5 & 18.3 & & & \\
\hline Laci & 2014 & & & & & 10 & 12.2 & 16.6 & 10.8 & 11 & & & \\
\hline Lano & 2012 & & & & & & & & 19.6 & 21.7 & & & \\
\hline Lano & 2013 & & & 0.3 & 13.6 & 8.9 & 14.3 & 18.6 & 18.8 & 18.8 & 8.2 & & \\
\hline Lano & 2014 & & & & 10.7 & 16.8 & 13.6 & 15.6 & 10.7 & 14 & & & \\
\hline Myci & 2012 & & & & & & & & 17.6 & 17.6 & 10.5 & 10.2 & \\
\hline Myci & 2013 & & & -0.6 & & 14 & 13.8 & 20.3 & & 22.9 & 10.3 & & \\
\hline Myci & 2014 & & & & & 12.7 & 13 & & 11.3 & 15.8 & & & \\
\hline Myev & 2012 & & & & & & & & 21.9 & 16.3 & & & \\
\hline Myev & 2013 & & & & 12.2 & -0.3 & 13 & 22.4 & 14 & 14.8 & 11.3 & & \\
\hline Myev & 2014 & & & & & 11 & 11 & 18.3 & 18.8 & 14.1 & & & \\
\hline Myev & 2015 & & & & & 13.6 & & & & & & & \\
\hline Mylu & 2012 & & & & & & & & 20.9 & 17.6 & & & \\
\hline Mylu & 2013 & & & & 12.2 & 13.2 & 14.1 & 17.6 & 17.6 & 21.2 & & & \\
\hline Mylu & 2014 & & & & & 14 & 15.8 & 14.1 & 20.4 & 15.6 & & & \\
\hline Myvo & 2014 & & & & & & & & & 17 & & & \\
\hline Bird & 2016 & & & & 5.9 & & & & & & & & \\
\hline Coto & 2015 & & & & & & 16.3 & 20.6 & 23.7 & 22.9 & & & \\
\hline Euma & 2015 & & & & & & 15.3 & 14.8 & 19.3 & 14.8 & & & \\
\hline Euma & 2016 & & & & 15 & 12.5 & 16 & 23.6 & & & 16 & & \\
\hline Labo & 2016 & & & & & & & & 18.4 & & & & \\
\hline
\end{tabular}




\begin{tabular}{|c|c|c|c|c|c|c|c|c|c|c|c|c|c|}
\hline Species & Year & Jan & Feb & Mar & Apr & May & June & July & Aug & Sep & Oct & Nov & Dec \\
\hline Myci & 2015 & & & & & & & 17.4 & & & & 7.9 & \\
\hline Myci & 2016 & & & & & & 17.9 & & & & & 18.8 & \\
\hline
\end{tabular}

Table 11i. Monthly minimum bat pass temperatures $\left({ }^{\circ} \mathrm{C}\right)$ recorded for individual species hand confirmed as definitively present at the Signal Peak Busse Water Reservoir

\begin{tabular}{|c|c|c|c|c|c|c|c|c|c|c|c|c|c|}
\hline Species & Year & Jan & Feb & Mar & Apr & May & June & July & Aug & Sep & Oct & Nov & Dec \\
\hline Coto & 2012 & & & & & & & & & 11.7 & & & \\
\hline Epfu & 2012 & & & & & & & & 23.6 & & 19.4 & & \\
\hline Epfu & 2013 & & & & 7.9 & 10 & 15.1 & 16.8 & 22.6 & 18.6 & 12.7 & -1.3 & -2 \\
\hline Epfu & 2014 & & 0.8 & & 5.5 & 5.9 & & 23.2 & & 9.7 & & & \\
\hline Epfu & 2015 & & & -1.3 & & & & & & & & & \\
\hline Euma & 2013 & & & & & & 14.1 & 17.1 & 12.7 & 21.7 & & & \\
\hline Euma & 2014 & & & & & 12.5 & 13.6 & 16 & 11.3 & & & & \\
\hline Euma & 2015 & & & & & & 10.8 & 8.9 & 17 & & & & \\
\hline Labo & 2014 & & & & & & & & 23.1 & & & & \\
\hline Labo & 2015 & & & & & & & & & 12.3 & & & \\
\hline Laci & 2012 & & & & & & & & 6.7 & 4.7 & & & \\
\hline Laci & 2013 & & & & & 8.5 & 13.6 & 18.3 & 16.3 & 17.1 & & & \\
\hline Laci & 2014 & & & & & 10.8 & 8.7 & 11.5 & 14.8 & 9 & & & \\
\hline Laci & 2015 & & & & & 9 & & & & & & & \\
\hline Lano & 2012 & & & & & & & & 25.4 & 17.1 & & & \\
\hline Lano & 2013 & & & & 13.2 & 4.4 & 12.7 & 19.3 & 12.8 & 10.7 & 12.5 & & \\
\hline Lano & 2014 & & & & 2.6 & 7.7 & 10.5 & 11.3 & 8 & 13.6 & & & \\
\hline Myci & 2012 & & & & & & & & 25.7 & & 19.6 & & \\
\hline Myci & 2013 & & & & 8.4 & 12.2 & & & 13.2 & 25.2 & 12.7 & 10.8 & 3.4 \\
\hline Myci & 2014 & -1 & & & 4.1 & 9.4 & 18.4 & 11 & 17.9 & 12.5 & & & \\
\hline Myev & 2012 & & & & & & & & 14.1 & 3.9 & 8.2 & & \\
\hline Myev & 2013 & & & & & 11 & 12.2 & 12.8 & 12.7 & 14.3 & 6.5 & & \\
\hline Myev & 2014 & & & & & 8.5 & 8 & 11.5 & 15.6 & 8.4 & & & \\
\hline Myev & 2015 & & & & 0 & & & & & & & & \\
\hline Mylu & 2012 & & & & & & & & 21.1 & 17.9 & & & \\
\hline Mylu & 2013 & & & & & 12.5 & 13 & 16.5 & 13.3 & 14 & & & \\
\hline Mylu & 2014 & & & & 5.9 & 9.4 & 9.8 & 12 & 18.9 & 10 & & & \\
\hline Mylu & 2015 & & & & & & & & & 20.8 & 17.3 & & \\
\hline Epfu & 2015 & & & & & & 15.1 & & & & & & \\
\hline Euma & 2015 & & & & & & 12.7 & & 20.3 & 19.8 & & & \\
\hline Euma & 2016 & & & & 17.6 & 18.4 & & & & & & & \\
\hline Labo & 2015 & & & & & & & & 19.1 & 14.1 & & & \\
\hline Laci & 2015 & & & & & & 13.2 & & & & & & \\
\hline Myci & 2015 & & & & & & 24.2 & & & & & 4.6 & 7 \\
\hline Мусі & 2016 & 2.9 & 9 & 5.9 & & & & & & & & & \\
\hline
\end{tabular}




\begin{tabular}{|c|c|c|c|c|c|c|c|c|l|l|l|l|l|}
\hline Myci & 2017 & 9.4 & 2.4 & & & & & & & & & & \\
\hline Myev & 2016 & & & 12.2 & 14.3 & & & & & & & & \\
\hline Mylu & 2016 & & & & 20.6 & & & & & & 19.6 & & \\
\hline
\end{tabular}


Table 12. Monthly Minimum bat pass temperatures recorded for definitive call sequences of species across the detector network compared to mine sites ${ }^{1}$

\begin{tabular}{|c|c|c|c|c|c|c|c|c|}
\hline \multirow[b]{2}{*}{ Species } & \multicolumn{8}{|c|}{ Minimum Temperature Recorded $\left({ }^{\circ} \mathrm{C}\right)$} \\
\hline & $\begin{array}{l}\text { Across } \\
\text { Network }\end{array}$ & $\begin{array}{l}\text { West } \\
\text { Decker } \\
\text { Mine }\end{array}$ & $\begin{array}{l}\text { Spring } \\
\text { Creek } \\
\text { Mine }\end{array}$ & $\begin{array}{c}\text { Otter } \\
\text { Creek } \\
\text { Coal } \\
\text { Tract }\end{array}$ & $\begin{array}{l}\text { Big } \\
\text { Sky } \\
\text { Mine }\end{array}$ & $\begin{array}{l}\text { Absaloka } \\
\text { Mine }\end{array}$ & $\begin{array}{c}\text { Rosebud } \\
\text { Mine }\end{array}$ & $\begin{array}{l}\text { Signal } \\
\text { Peak } \\
\text { Mine }\end{array}$ \\
\hline $\begin{array}{c}\text { Pallid Bat } \\
\text { (Antrozous } \\
\text { pallidus) }\end{array}$ & 5.2 & & & 15 & 5.2 & & & \\
\hline $\begin{array}{c}\text { Townsend's } \\
\text { Big-eared Bat } \\
\text { (Corynorhinus } \\
\text { townsendii) }\end{array}$ & 5.7 & & & 6.7 & & & & 11.7 \\
\hline $\begin{array}{l}\text { Big Brown } \\
\text { Bat } \\
\text { (Eptesicus } \\
\text { fuscus) }\end{array}$ & -4.8 & 11.7 & 17 & 16.8 & 3.7 & 11.7 & 7 & -2.0 \\
\hline $\begin{array}{l}\text { Spotted Bat } \\
\text { (Euderma } \\
\text { maculatum) }\end{array}$ & 1.1 & & & & & & & 8.9 \\
\hline $\begin{array}{c}\text { Eastern Red } \\
\text { Bat } \\
\text { (Lasiurus } \\
\text { borealis) }\end{array}$ & 1.6 & 6.5 & 19.8 & 14 & 13.6 & 1.6 & 12.5 & 15.8 \\
\hline $\begin{array}{l}\text { Hoary Bat } \\
\text { (Lasiurus } \\
\text { cinereus) }\end{array}$ & -0.6 & 9 & 8.9 & 8.9 & 6.7 & 0.8 & 7.4 & 4.7 \\
\hline $\begin{array}{l}\text { Silver-haired } \\
\text { Bat } \\
\text { (Lasionycteris } \\
\text { noctivagans) }\end{array}$ & -4.9 & 2.9 & 8.2 & & -4.1 & -1.8 & 5.7 & 0.3 \\
\hline $\begin{array}{c}\text { Western } \\
\text { Small-footed } \\
\text { Myotis } \\
\text { (Myotis } \\
\text { ciliolabrum) }\end{array}$ & -4.8 & 7.4 & 7.5 & 13 & -1.0 & 16.3 & 10 & -1.0 \\
\hline $\begin{array}{l}\text { Long-eared } \\
\text { Myotis } \\
\text { (Myotis } \\
\text { evotis) }\end{array}$ & -2.1 & 5.4 & 6.5 & 8.2 & 9.2 & 0.3 & 6 & -0.3 \\
\hline $\begin{array}{l}\text { Little Brown } \\
\text { Myotis }\end{array}$ & -0.5 & 0.4 & 6.0 & 15.3 & 3.9 & -0.5 & 5.4 & 5.9 \\
\hline
\end{tabular}




\begin{tabular}{|c|c|c|c|c|c|c|c|c|}
\hline \multirow[b]{2}{*}{ Species } & \multicolumn{8}{|c|}{ Minimum Temperature Recorded $\left({ }^{\circ} \mathrm{C}\right)$} \\
\hline & $\begin{array}{c}\text { Across } \\
\text { Network }\end{array}$ & $\begin{array}{l}\text { West } \\
\text { Decker } \\
\text { Mine }\end{array}$ & $\begin{array}{l}\text { Spring } \\
\text { Creek } \\
\text { Mine }\end{array}$ & $\begin{array}{c}\text { Otter } \\
\text { Creek } \\
\text { Coal } \\
\text { Tract }\end{array}$ & $\begin{array}{c}\text { Big } \\
\text { Sky } \\
\text { Mine }\end{array}$ & $\begin{array}{c}\text { Absaloka } \\
\text { Mine }\end{array}$ & $\begin{array}{c}\text { Rosebud } \\
\text { Mine }\end{array}$ & $\begin{array}{c}\text { Signal } \\
\text { Peak } \\
\text { Mine }\end{array}$ \\
\hline $\begin{array}{c}\text { (Myotis } \\
\text { lucifugus) }\end{array}$ & & & & & & & & \\
\hline $\begin{array}{c}\text { Fringed } \\
\text { Myotis } \\
\text { (Myotis } \\
\text { thysanodes) }\end{array}$ & 3.1 & & & & & & & \\
\hline $\begin{array}{c}\text { Long-legged } \\
\text { Myotis } \\
\text { (Myotis } \\
\text { volans) }\end{array}$ & 5.5 & & & & & & 9.4 & 16.6 \\
\hline
\end{tabular}

${ }^{1}$ Temperatures should only be regarded as being indicative of the general temperature at the time of detection. Temperatures were recorded at the detector approximately 1-meter above ground level while the microphone was mounted at approximately 3-meters above ground level and bats were in flight at an unknown altitude, but probably typically within 30-meters of ground level. Temperatures of the bat's roost environment at the time flights were initiated are also obviously unknown. 
Figure 1. Network of long term ultrasonic acoustic detectors as of Summer 2017

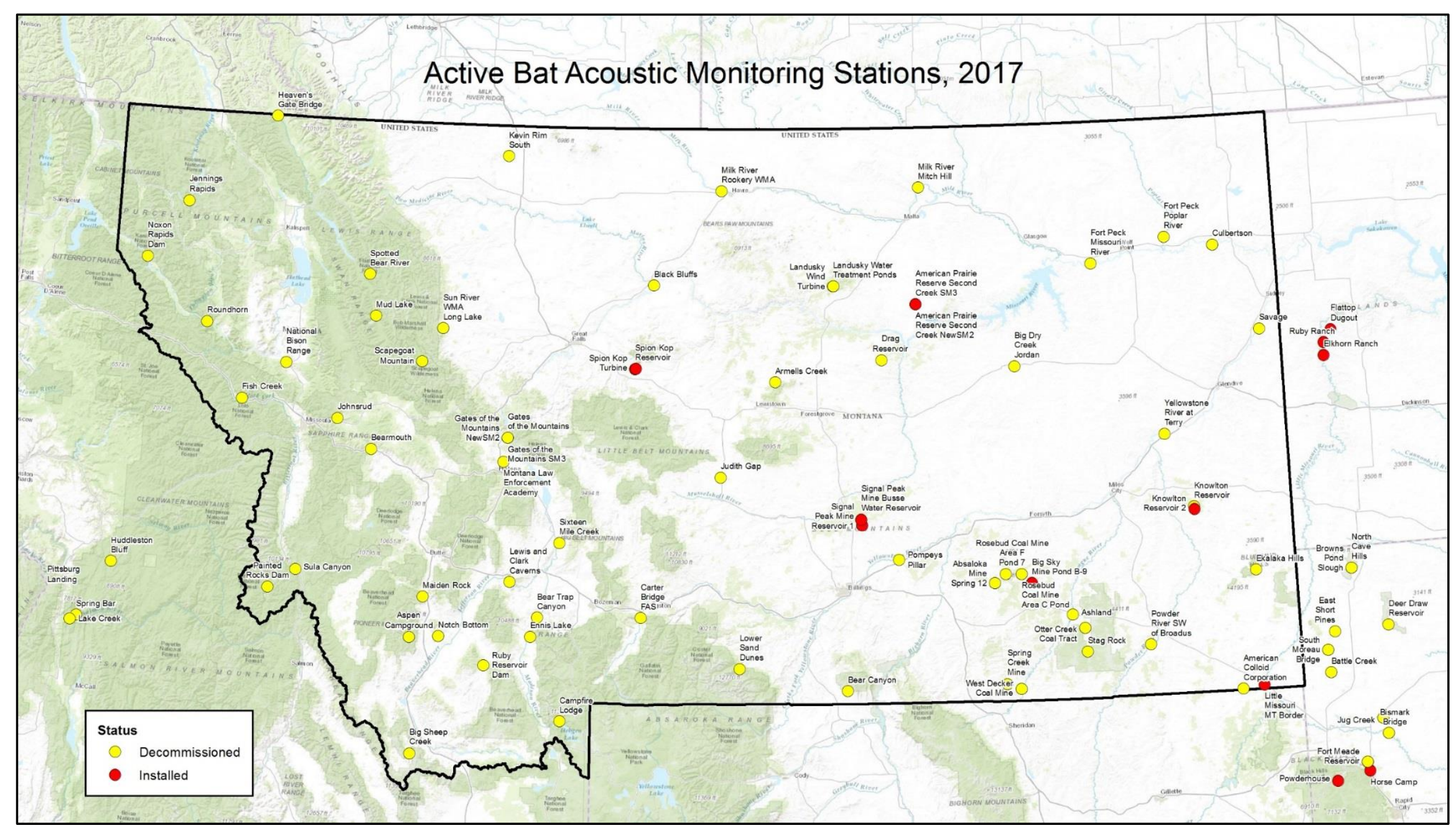


Figure 2a. Overview showing the locations of the West Decker Mine, Spring Creek Mine, and Otter Creek Coal Tract detector recorders (blue pins).

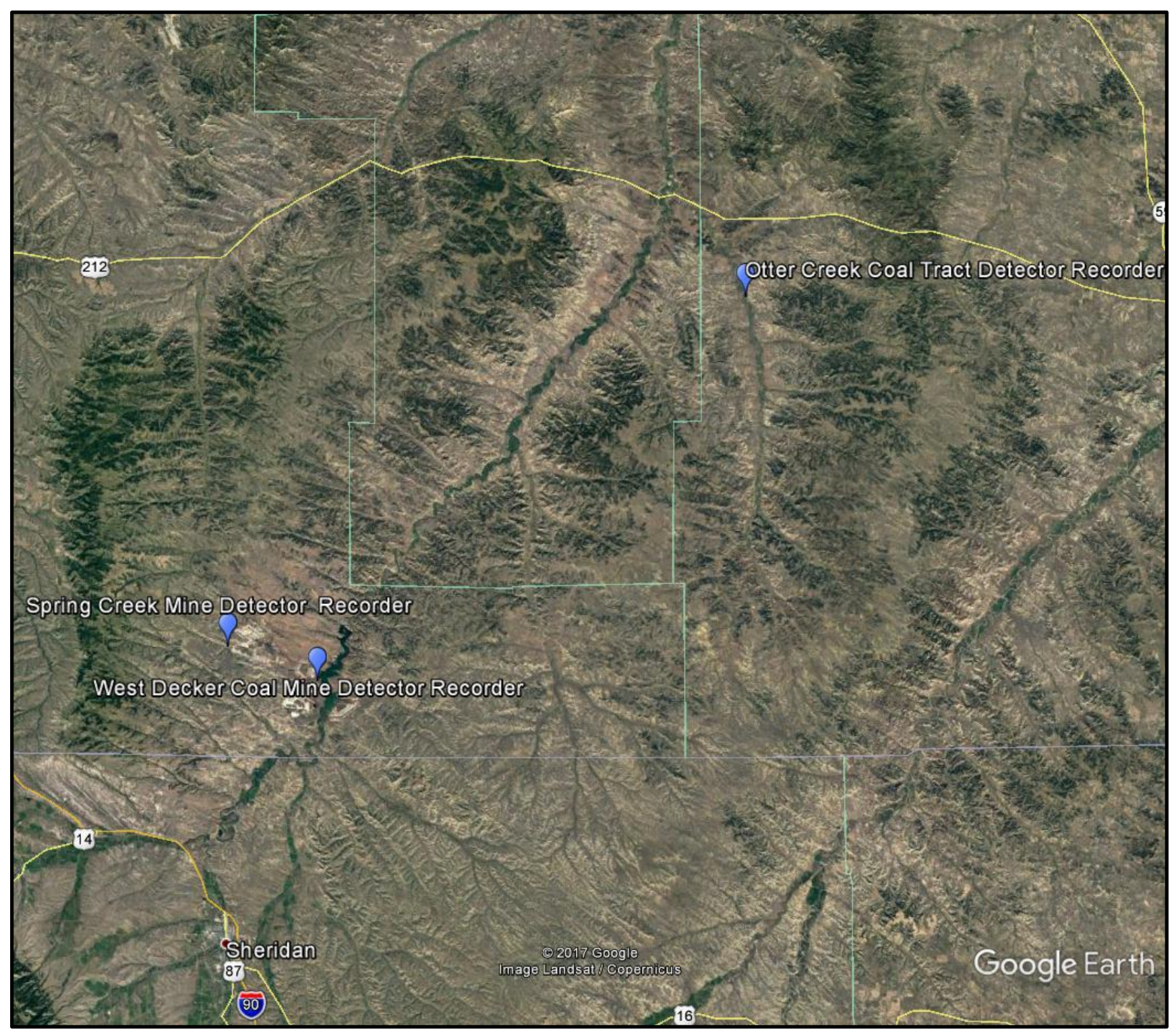


Figure 2b. Overview showing the locations of the detector recorders placed at the Big Sky, Absaloka, and Rosebud mines (blue pins).

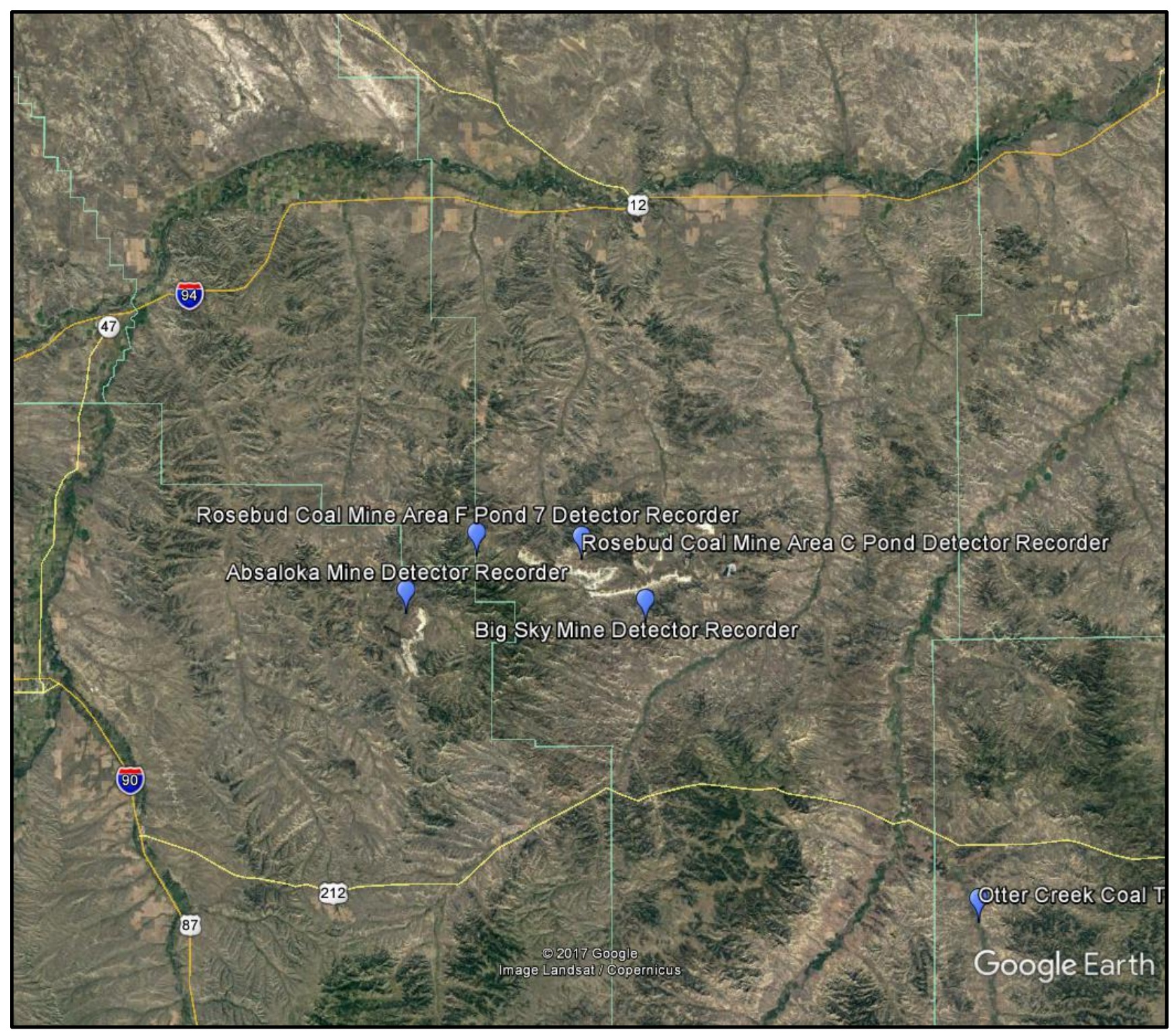


Figure 2c. Overview showing the locations of the detector recorders placed at the Signal Peak Mine (blue pins).

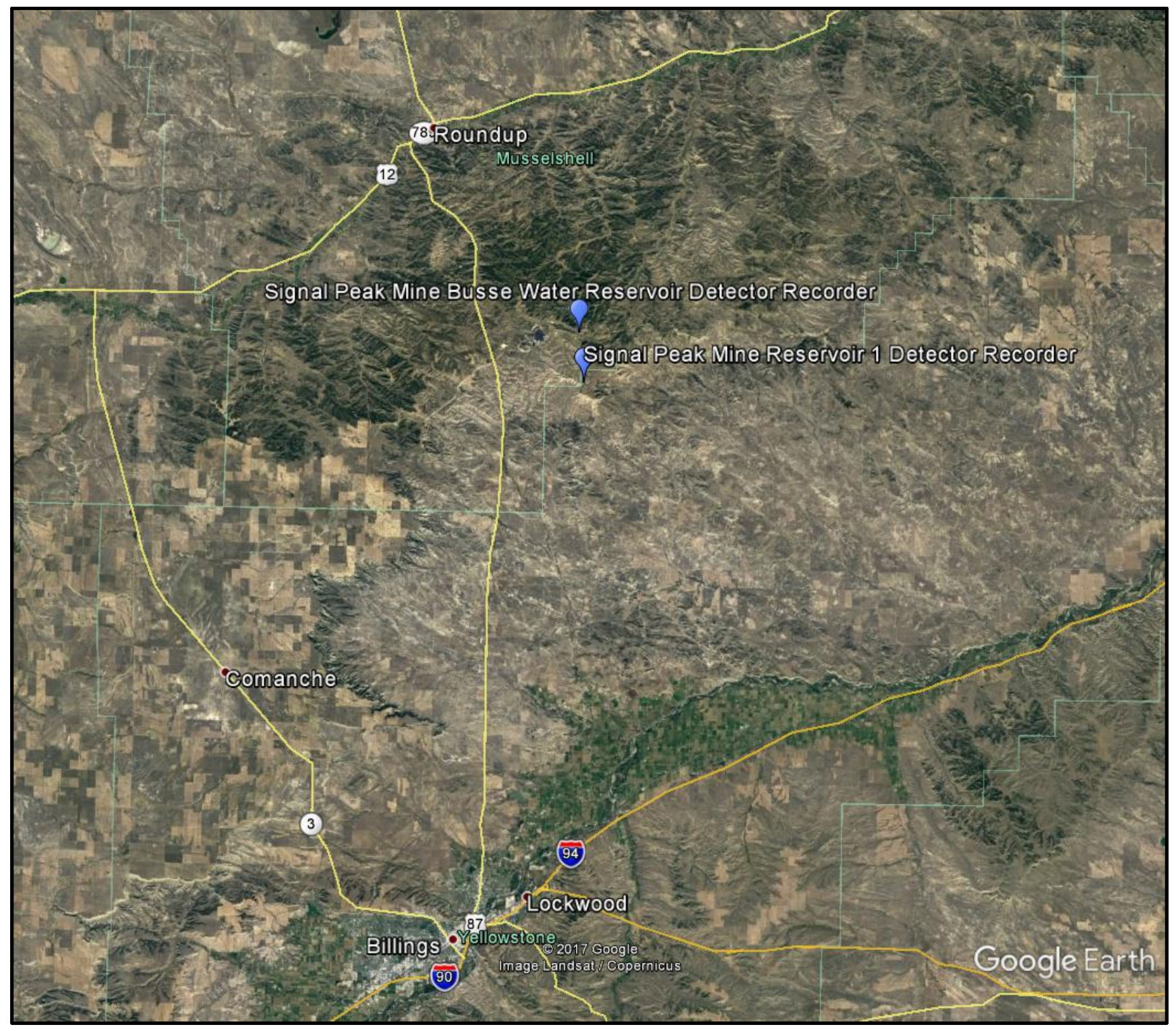


Figure 3. Local images of the placements of detector recorders at: (a) West Decker Coal Mine, (b) Spring Creek Mine, (c) Otter Creek Coal Tract, (d) Big Sky Mine, (e) Absaloka Mine, (f) Rosebud Mine, (g) Signal Peak Mine.

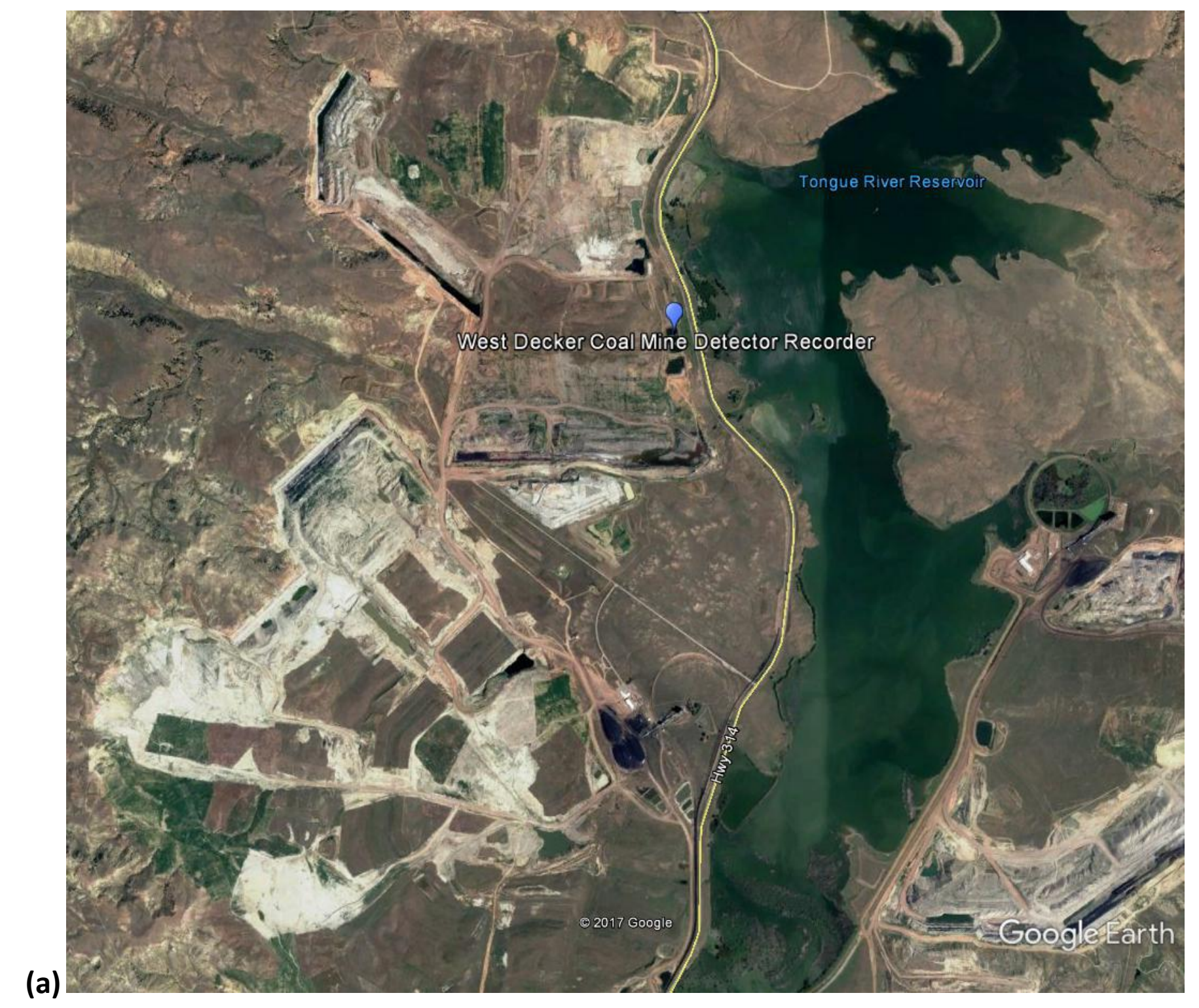




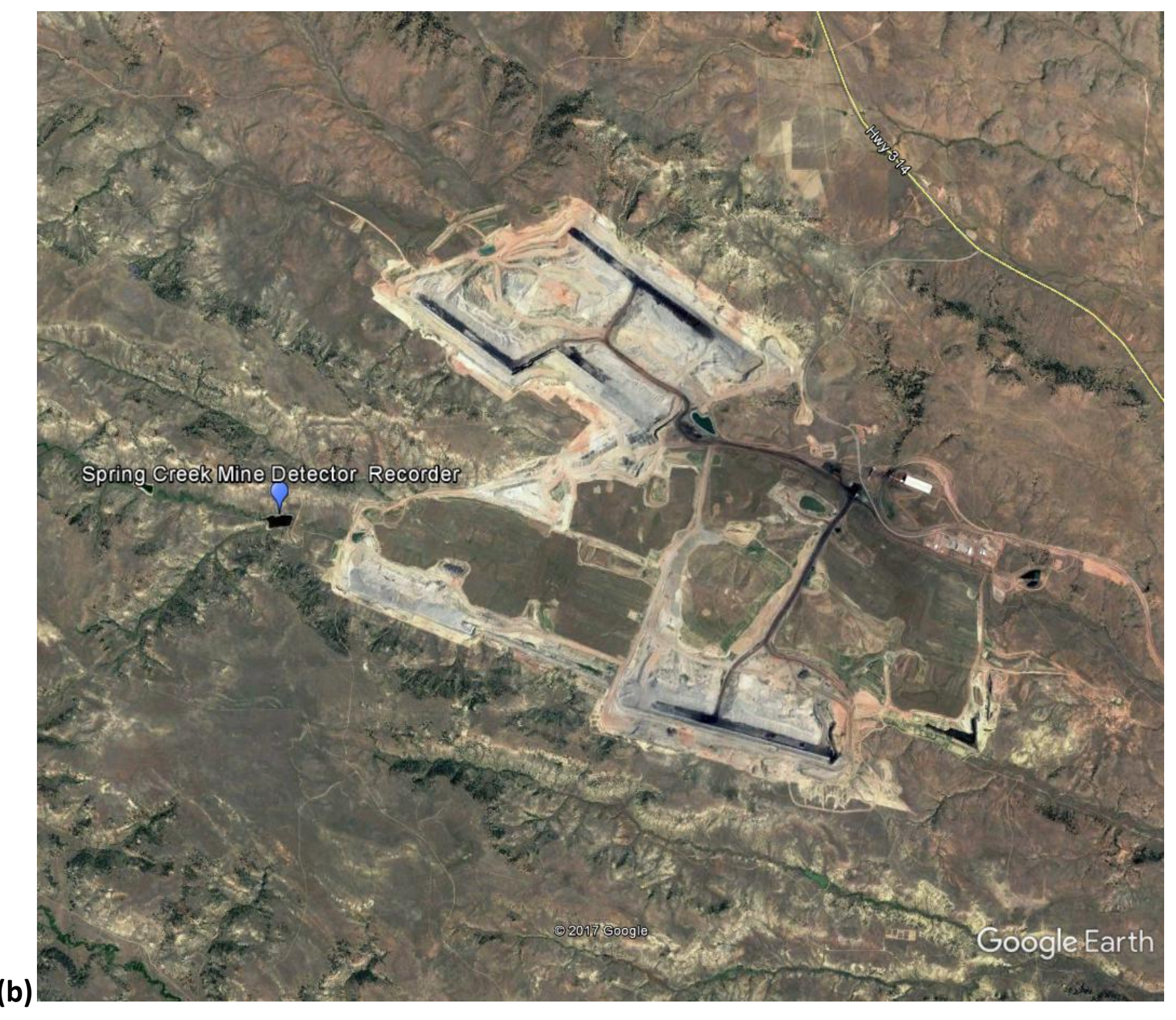




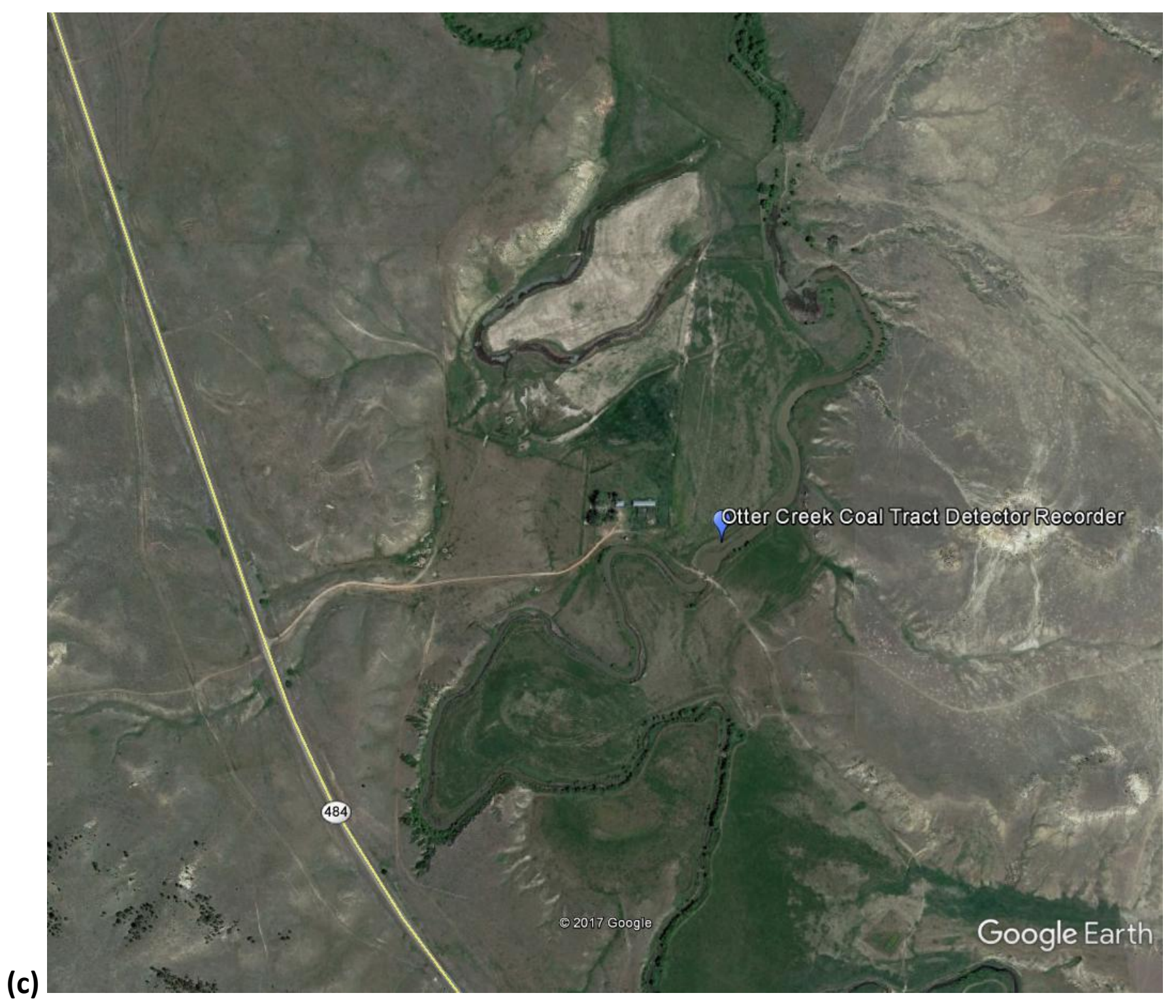




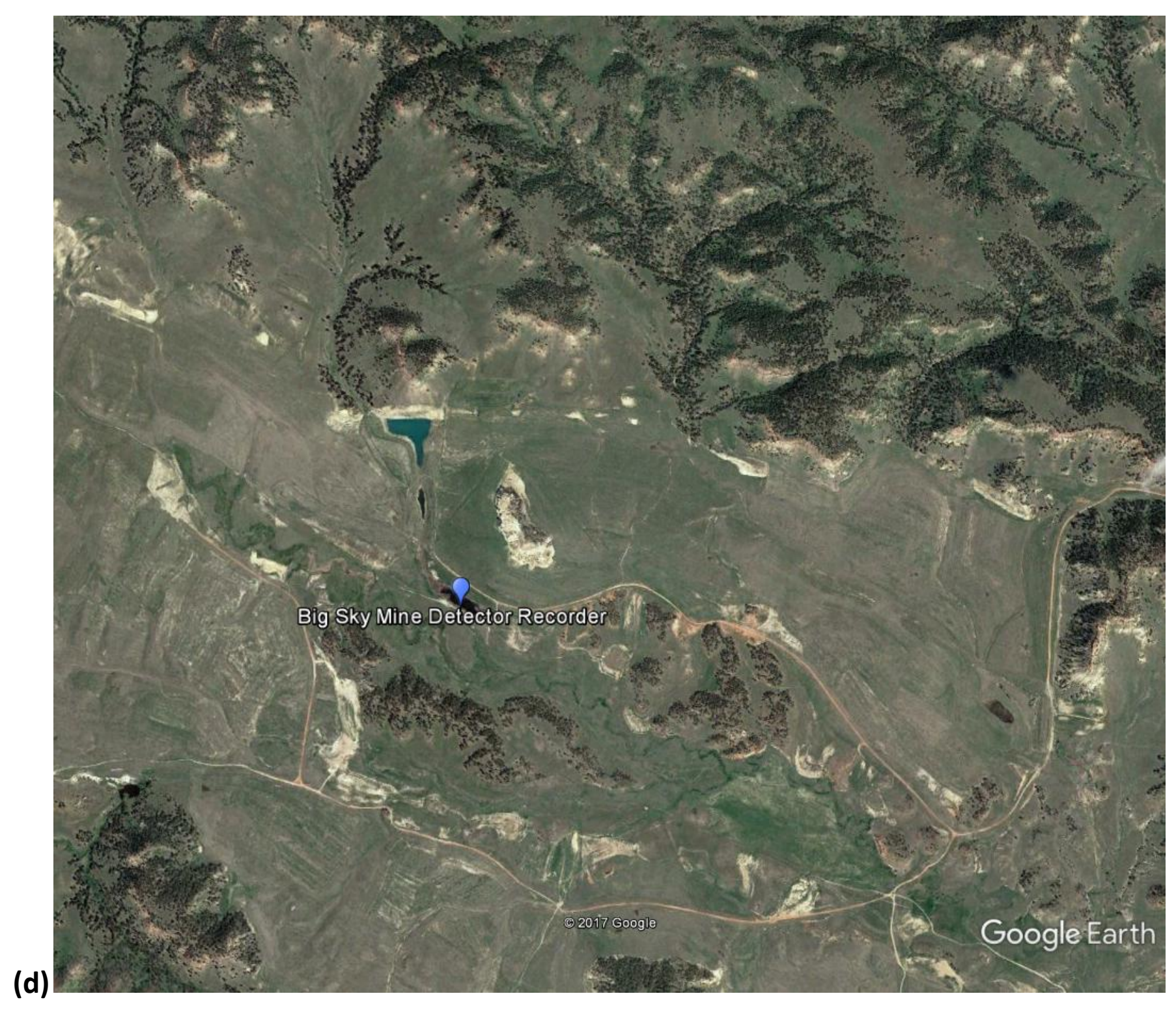




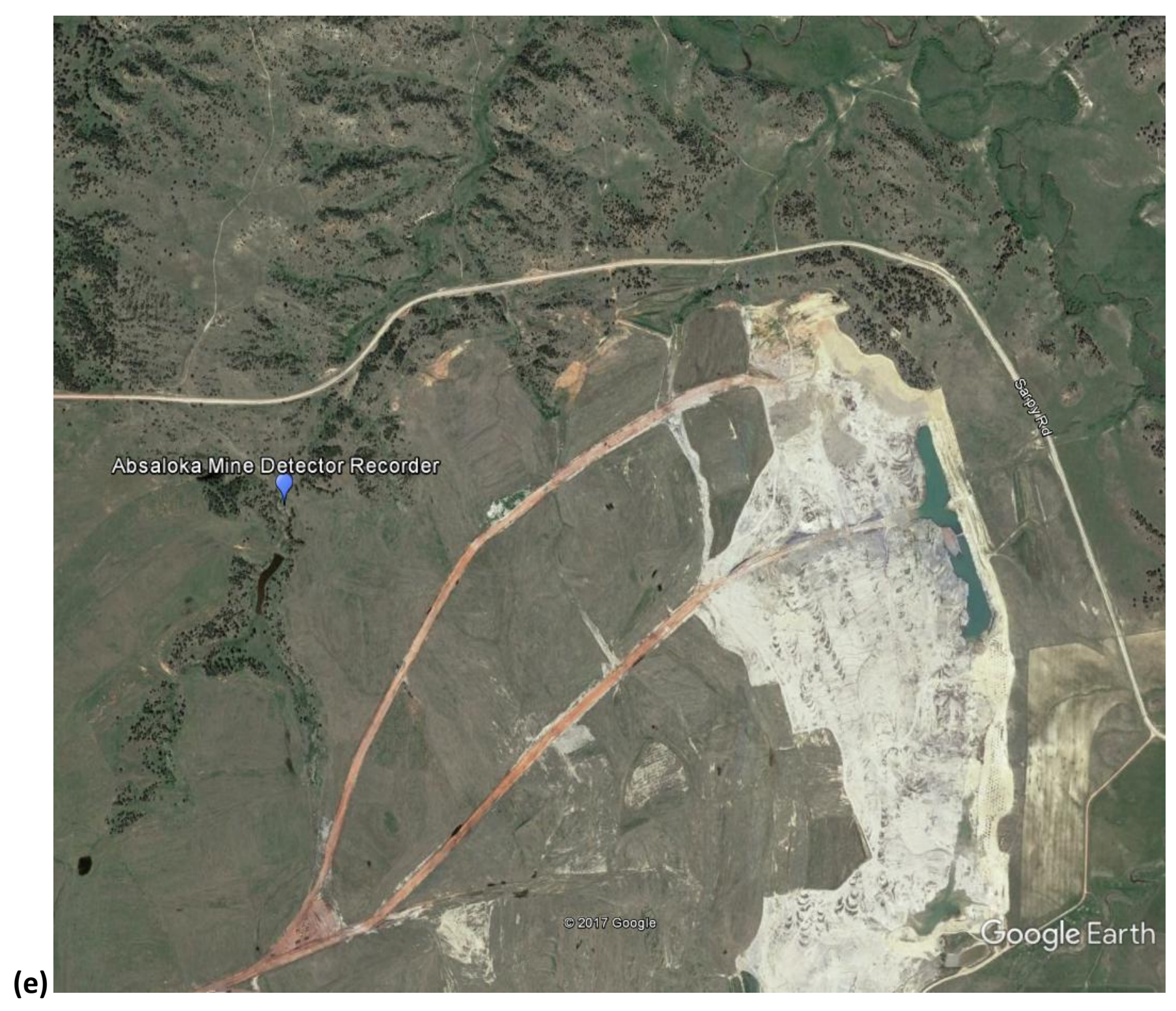




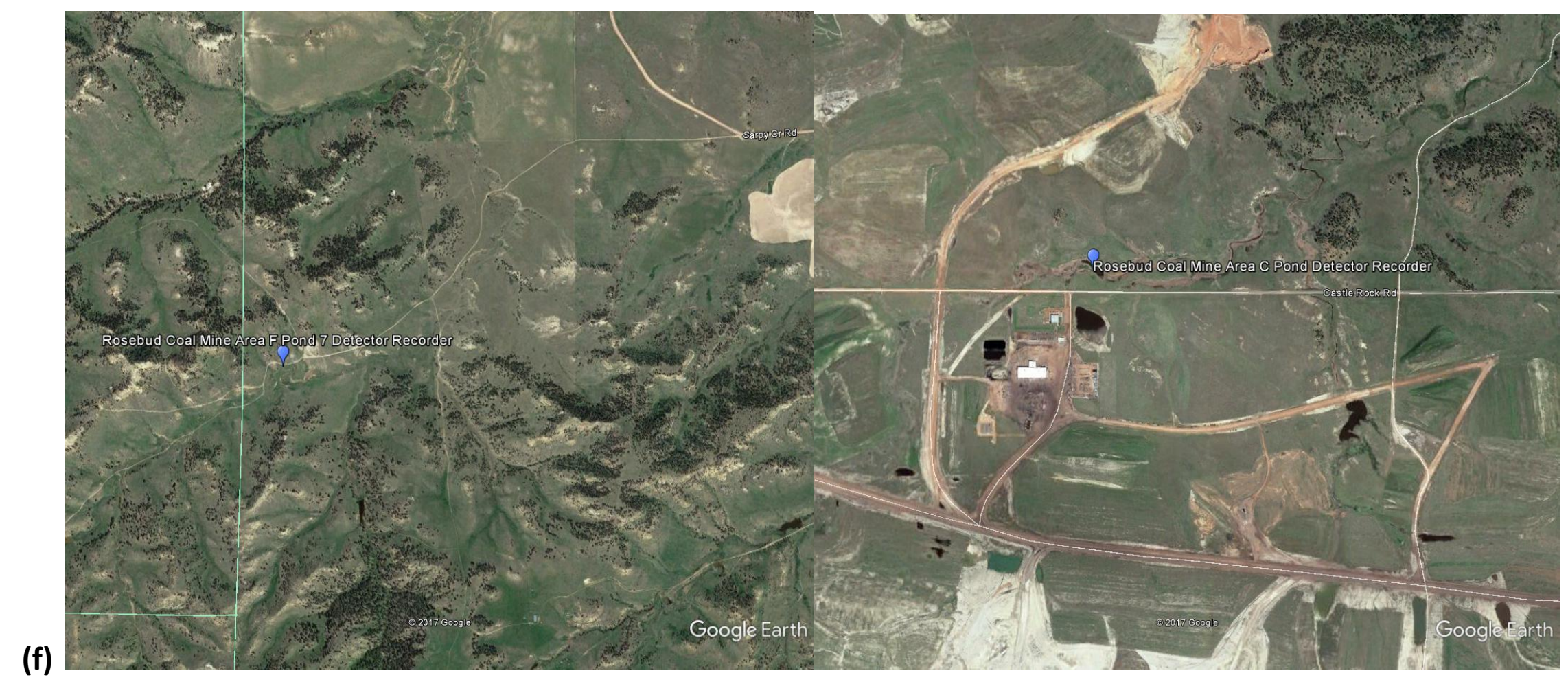




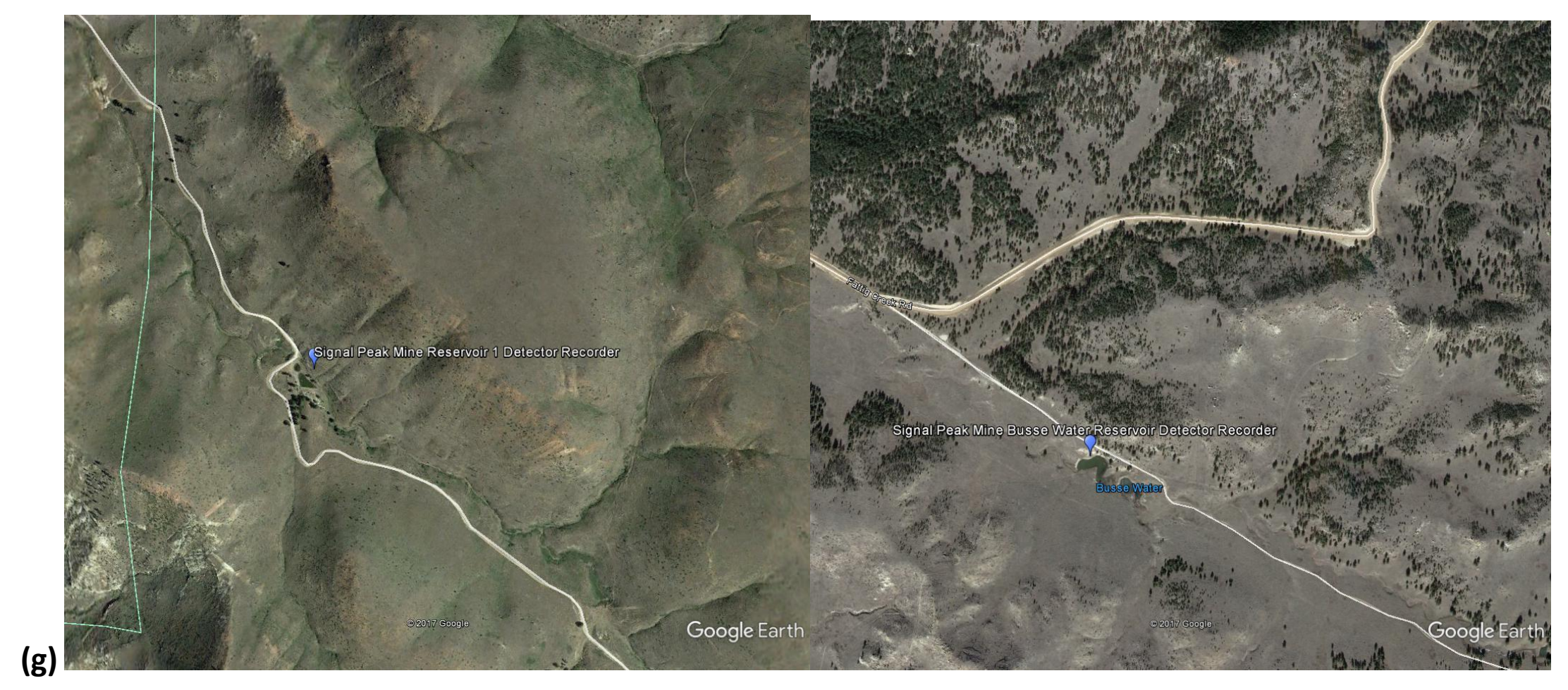


Figure 4. Site photos of detector placements (red star) at: (a) West Decker Coal Mine, (b) Spring Creek Mine, (c) Otter Creek Coal Tract, (d) Big Sky Mine, (e) Absaloka Mine, (f) Rosebud Mine, (g) Signal Peak Mine.

(a) West Decker

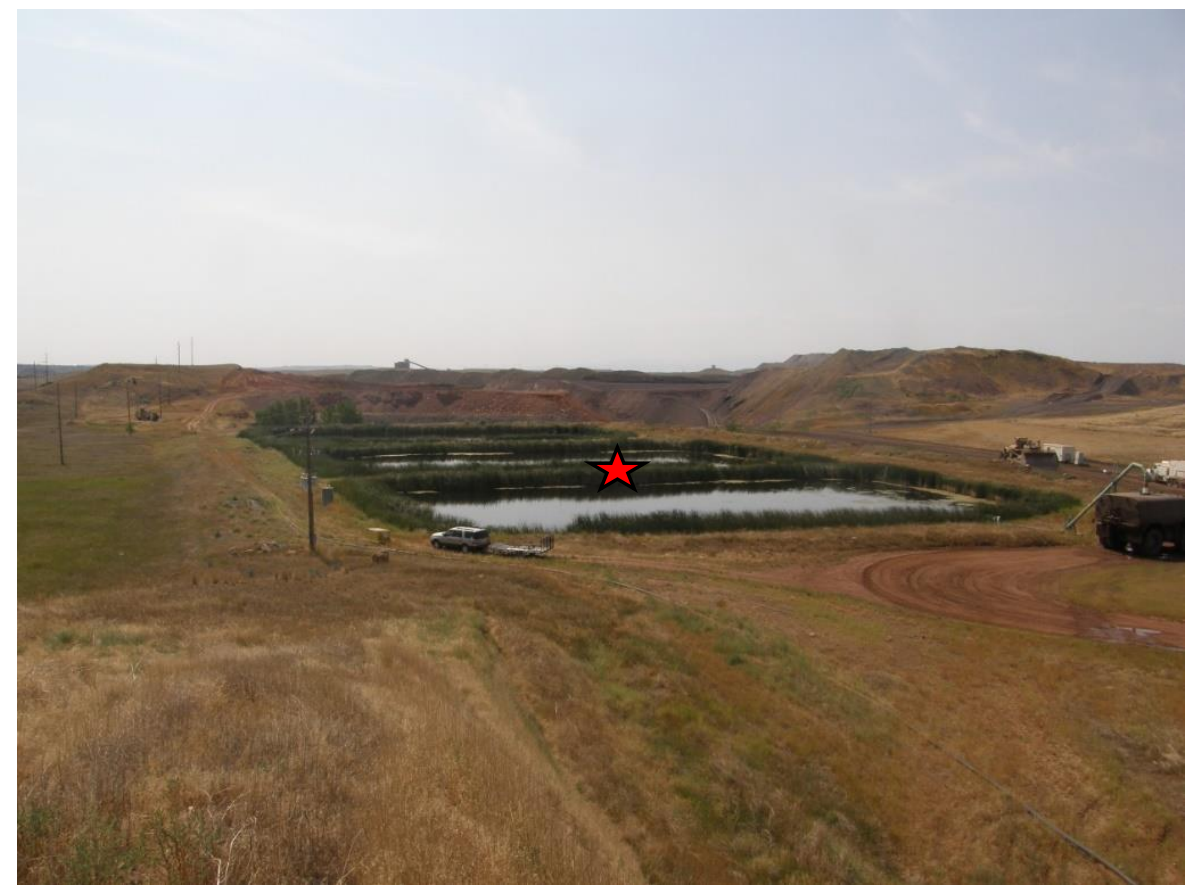

(b) Spring Creek Mine

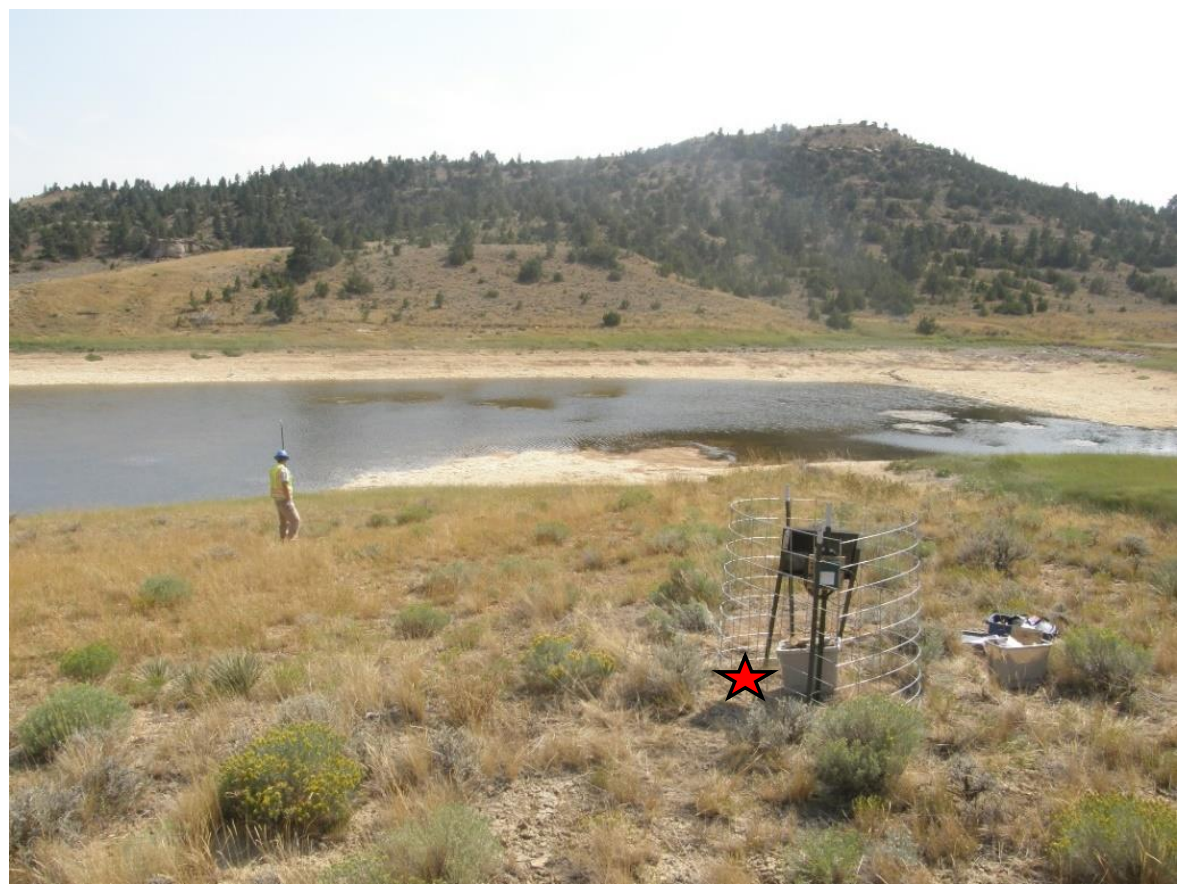


(c) Otter Creek Coal Tract

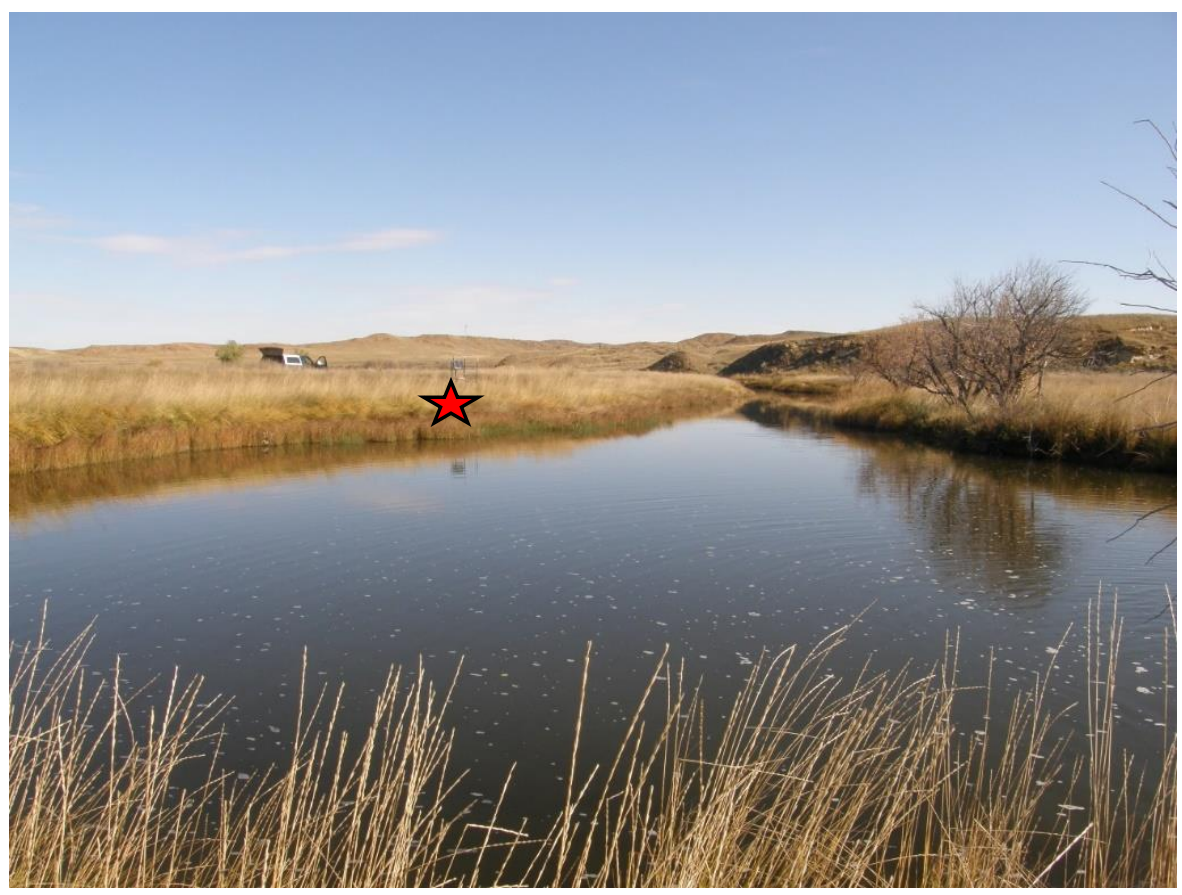

(d) Big Sky Mine

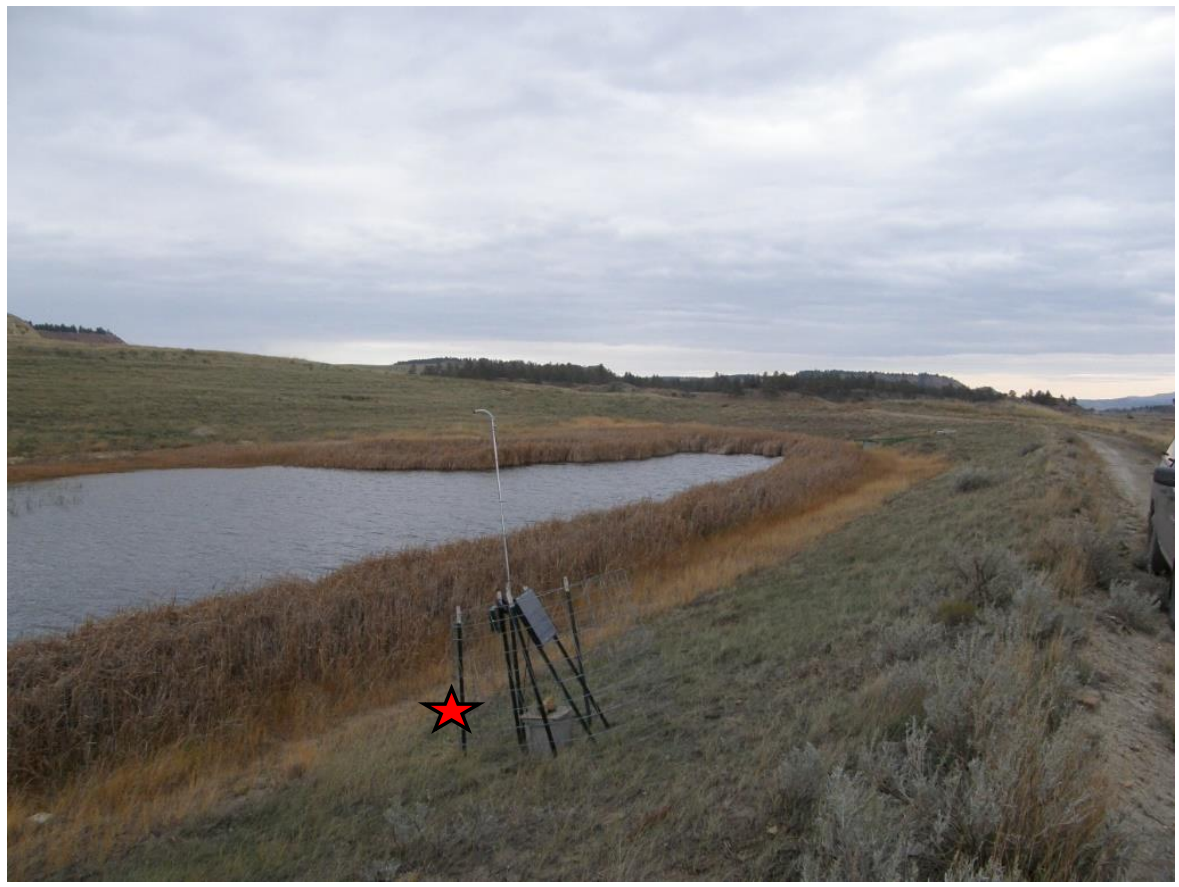


(e) Absaloka Mine

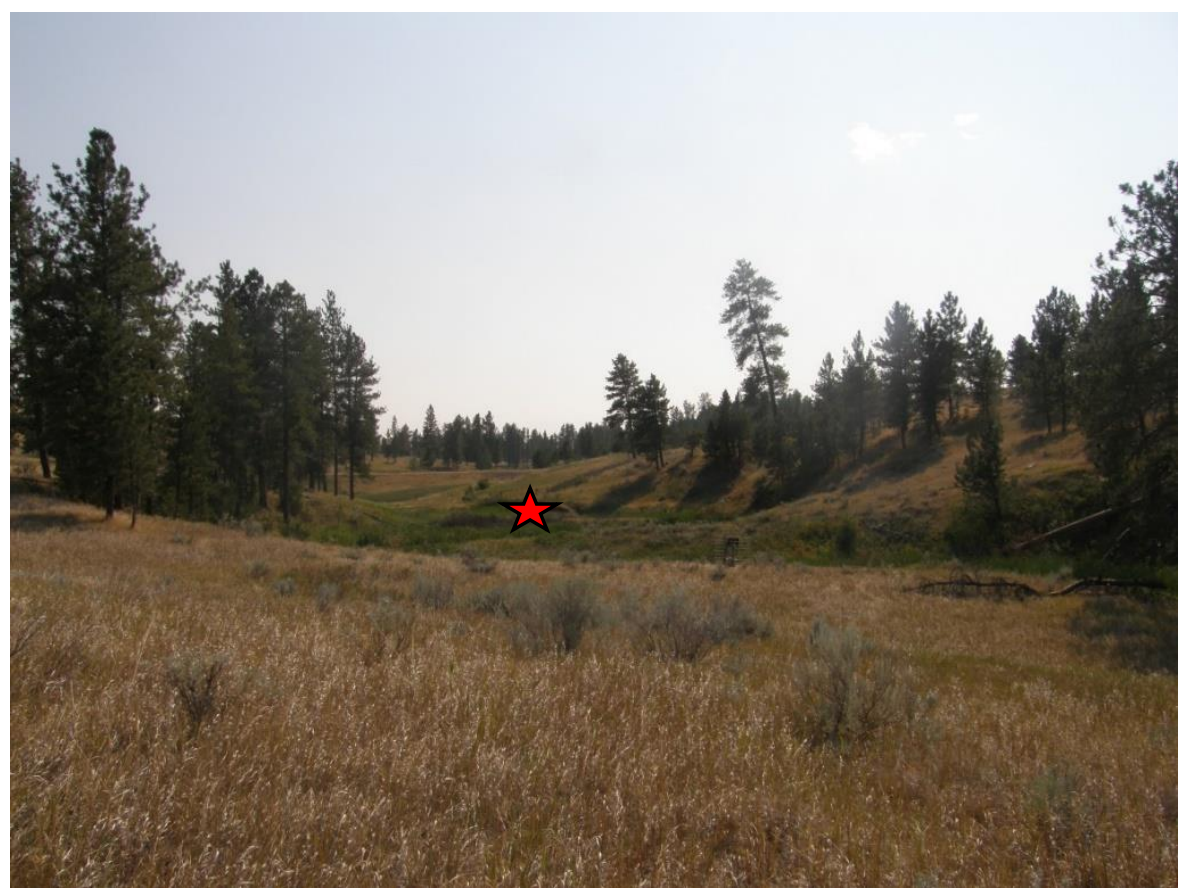

(f) Rosebud Mine Area F

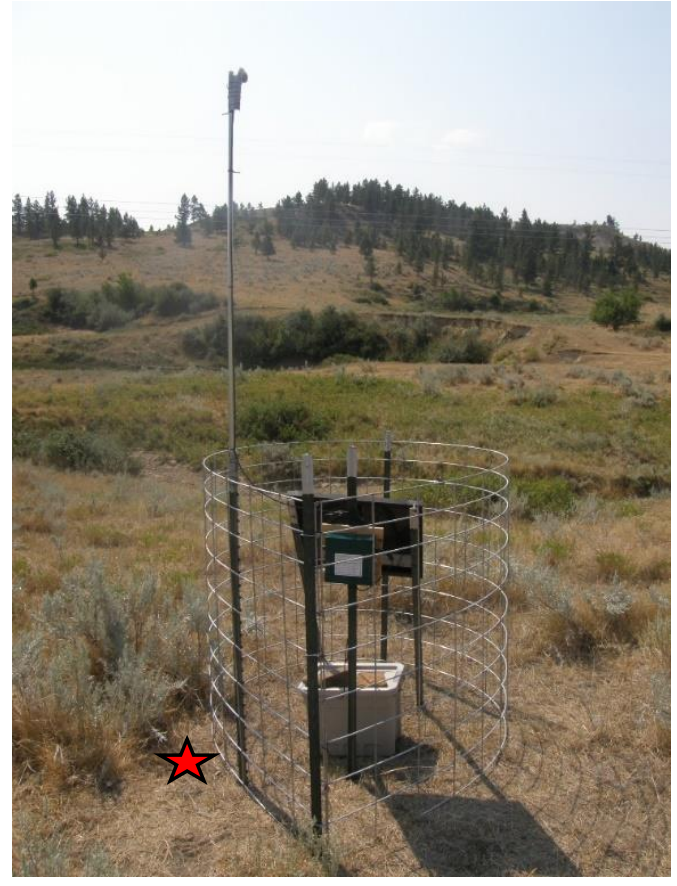


(f cont.) Rosebud Mine Area C

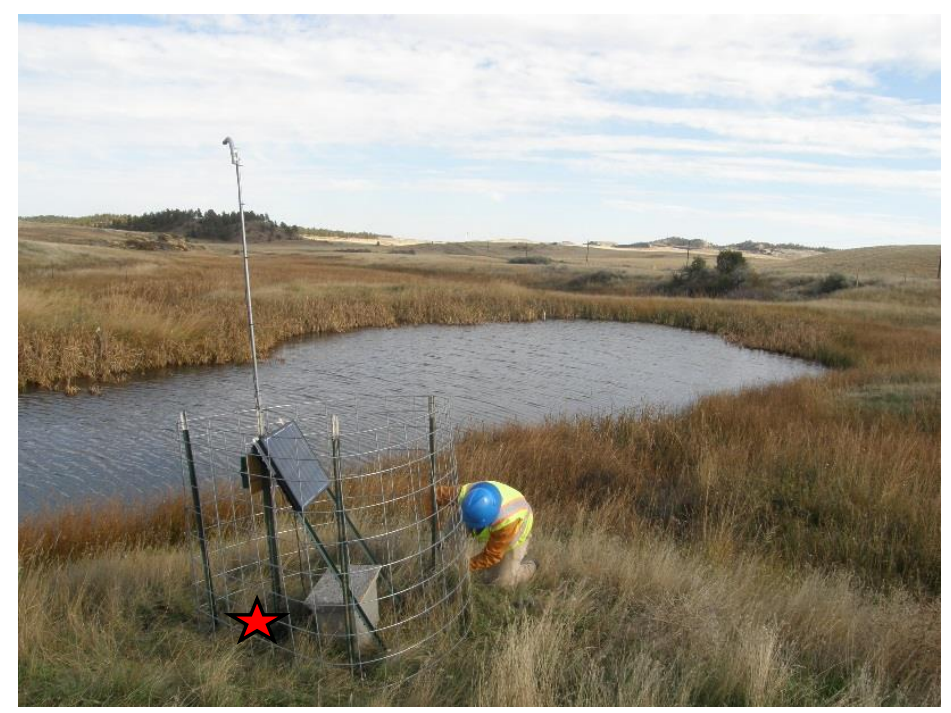

(g) Signal Peak Reservoir 1

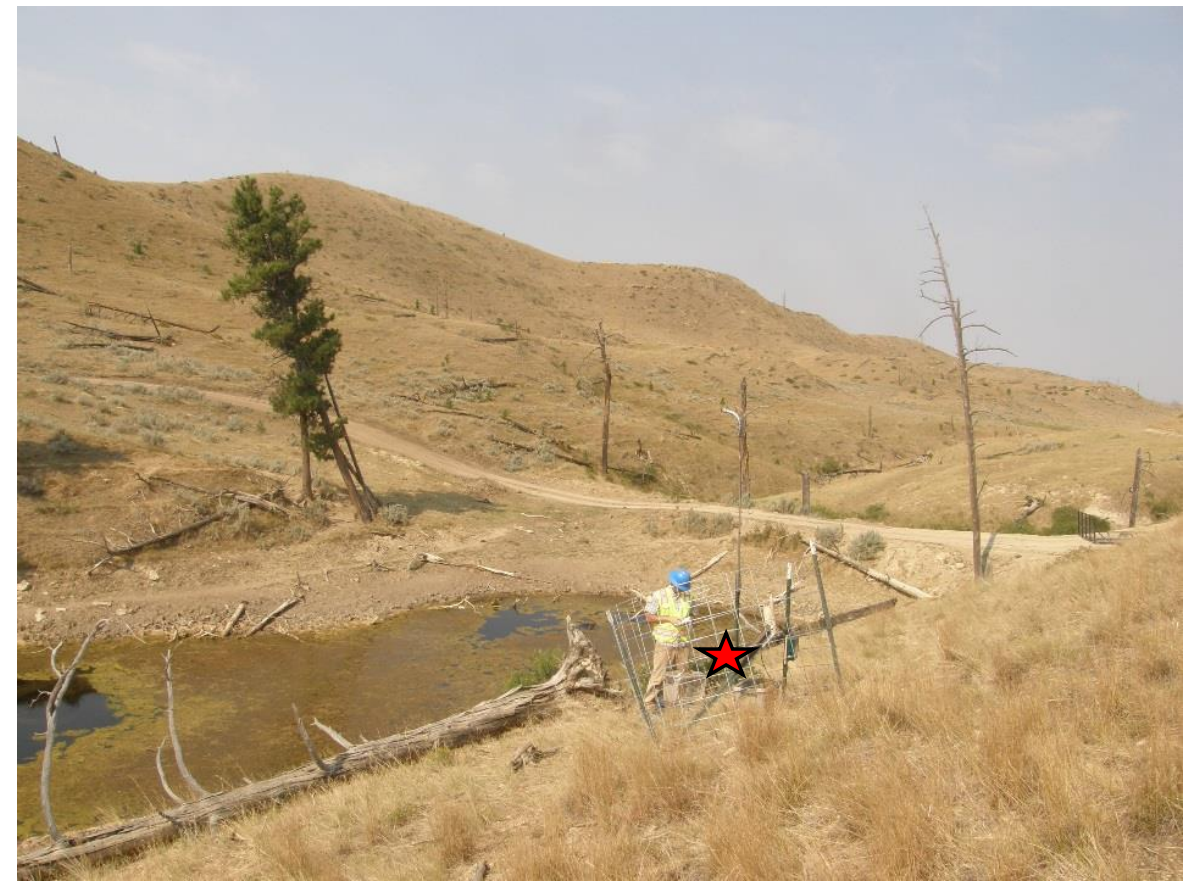


(g cont.) Signal Peak Mine Busse Water Reservoir

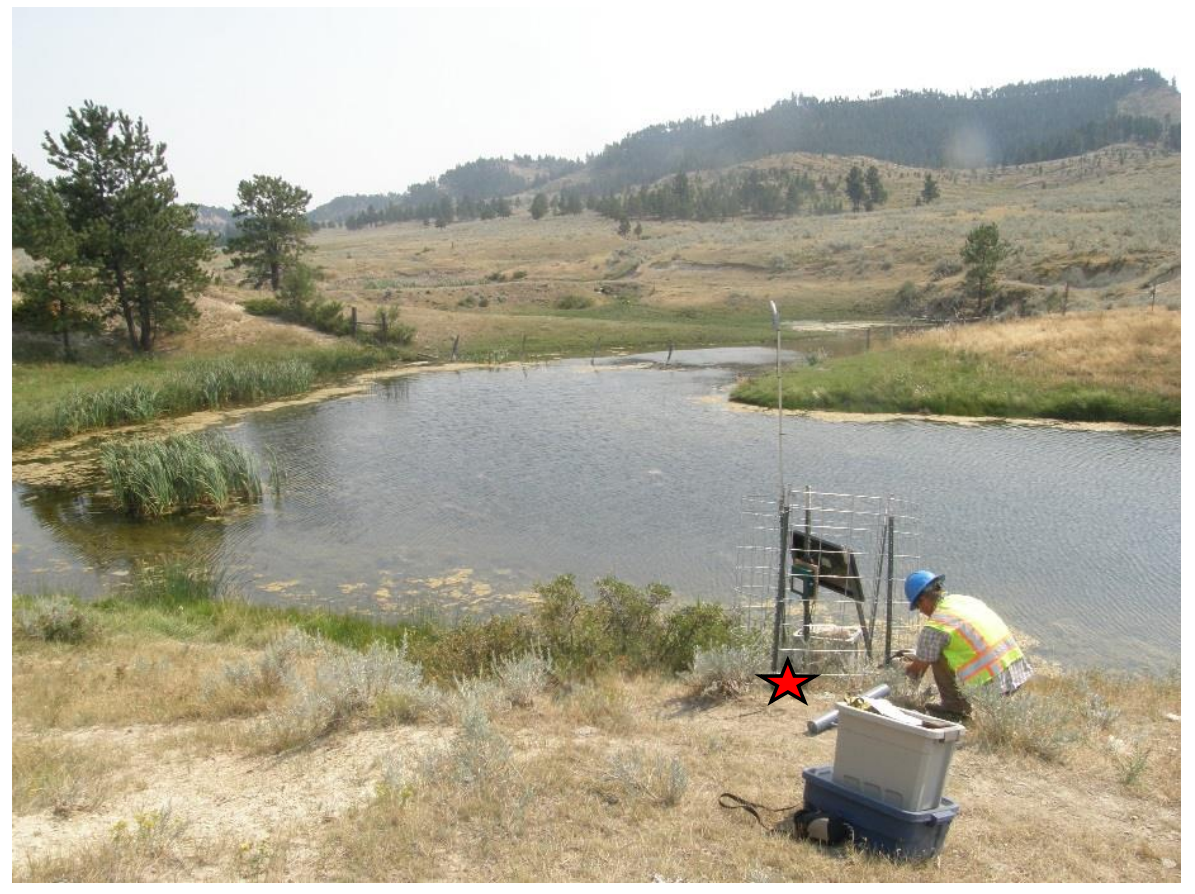


Figure 5. Percent of call sequences auto-identified to species each month for: (a) West Decker Coal Mine, (b) Spring Creek Mine, (c) Otter Creek Coal Tract, (d) Big Sky Mine, (e) Absaloka Mine, (f) Rosebud Mine, (g) Signal Peak Mine.

(a)

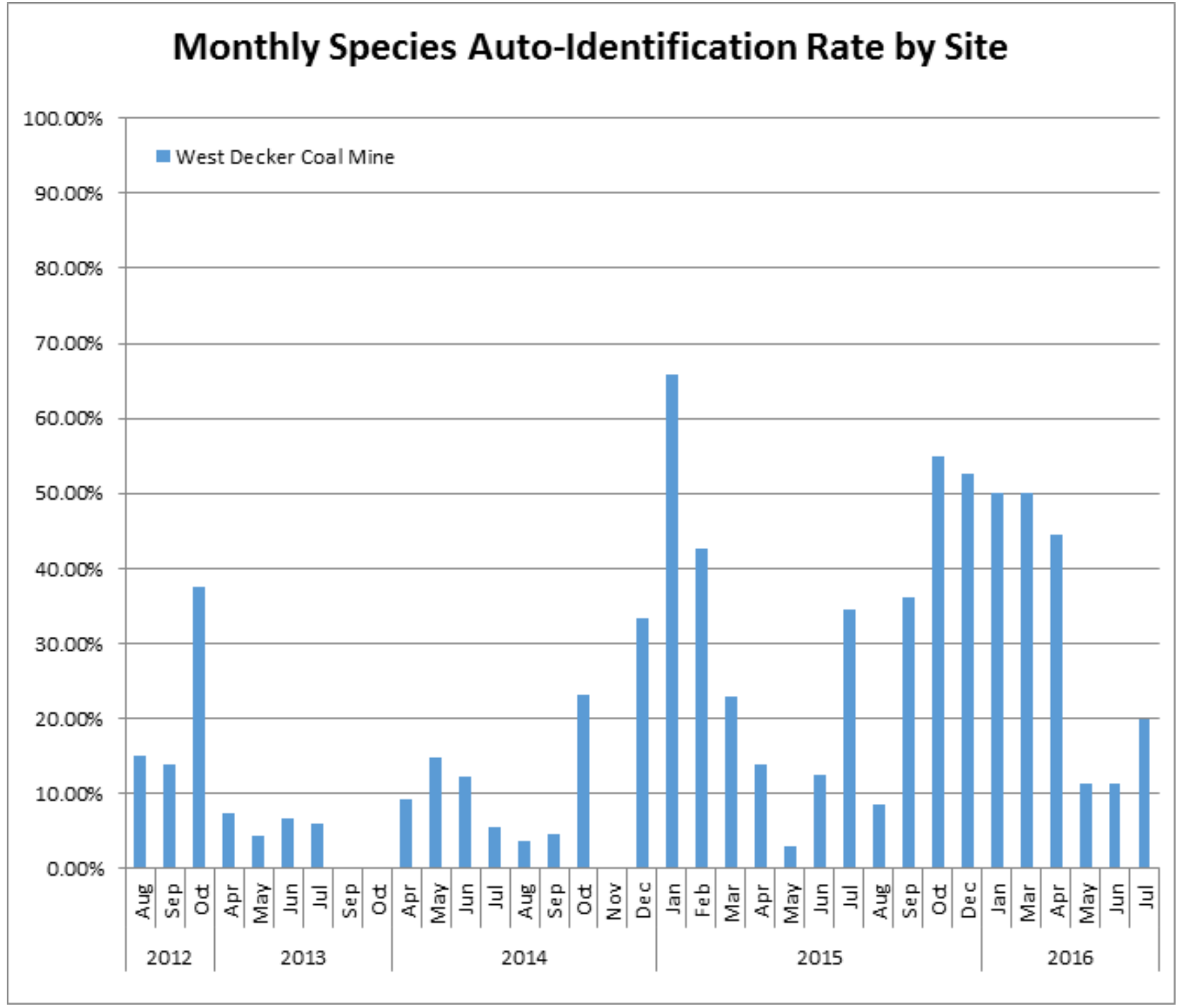


(b)

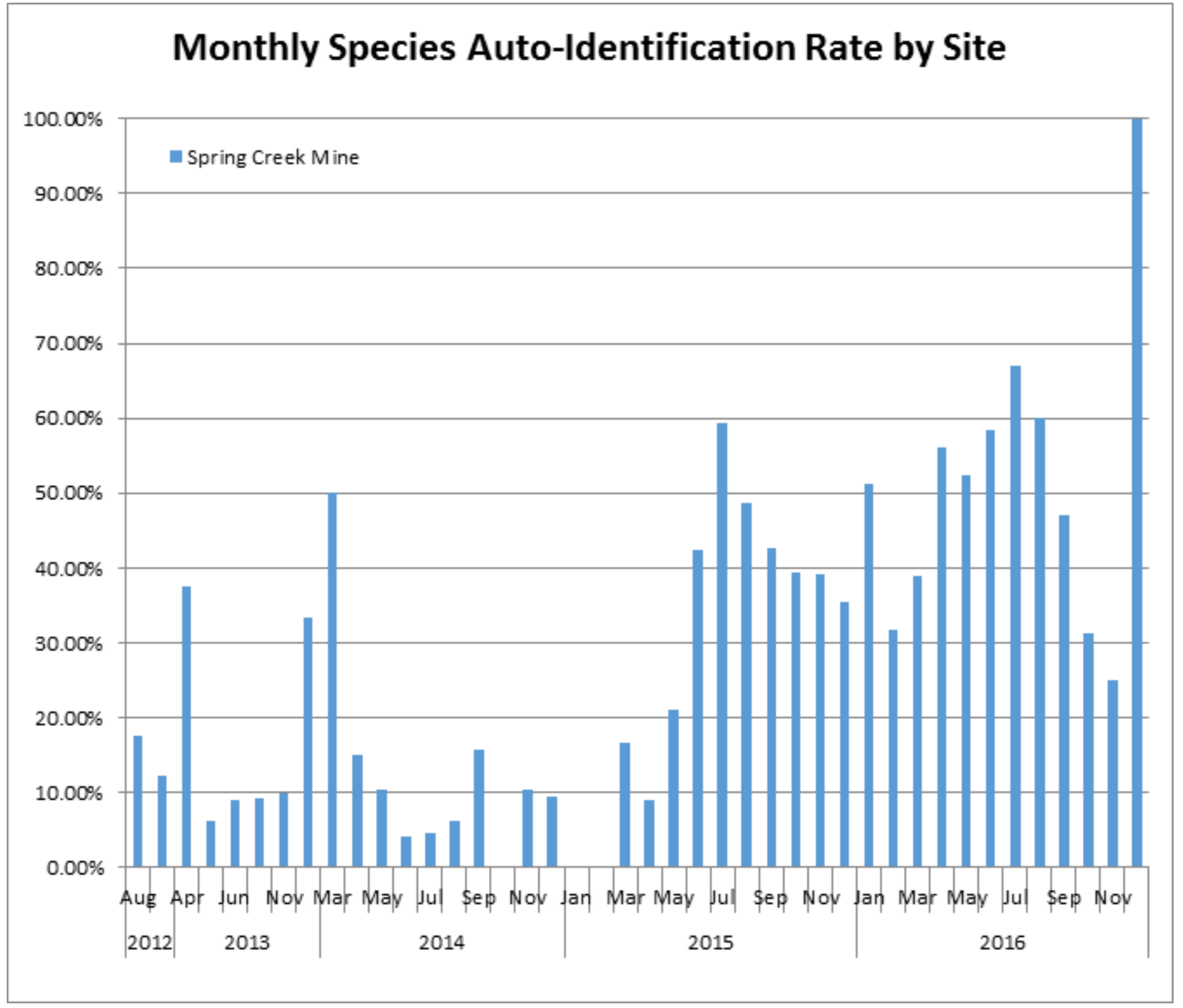


(c)

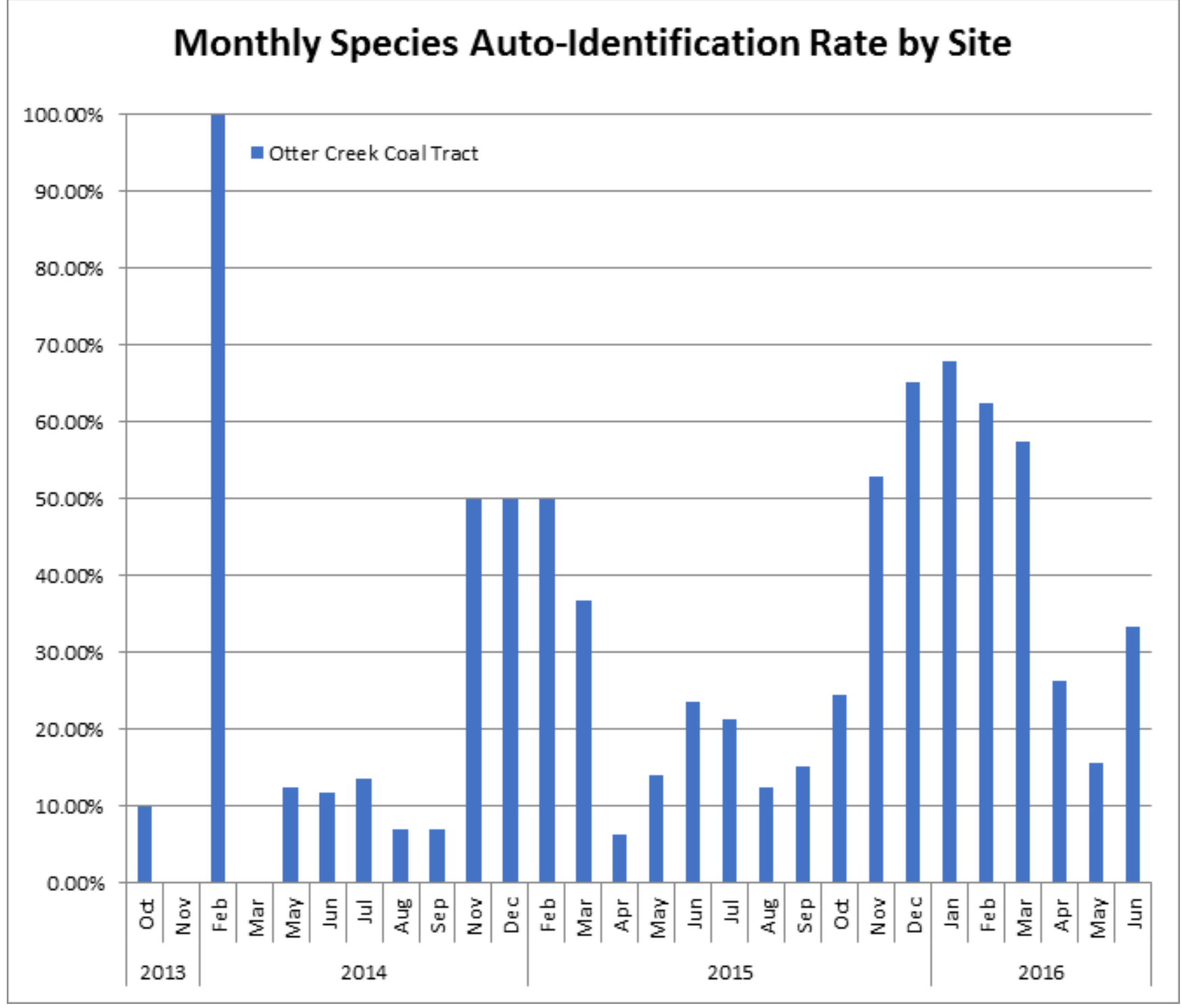


(d)

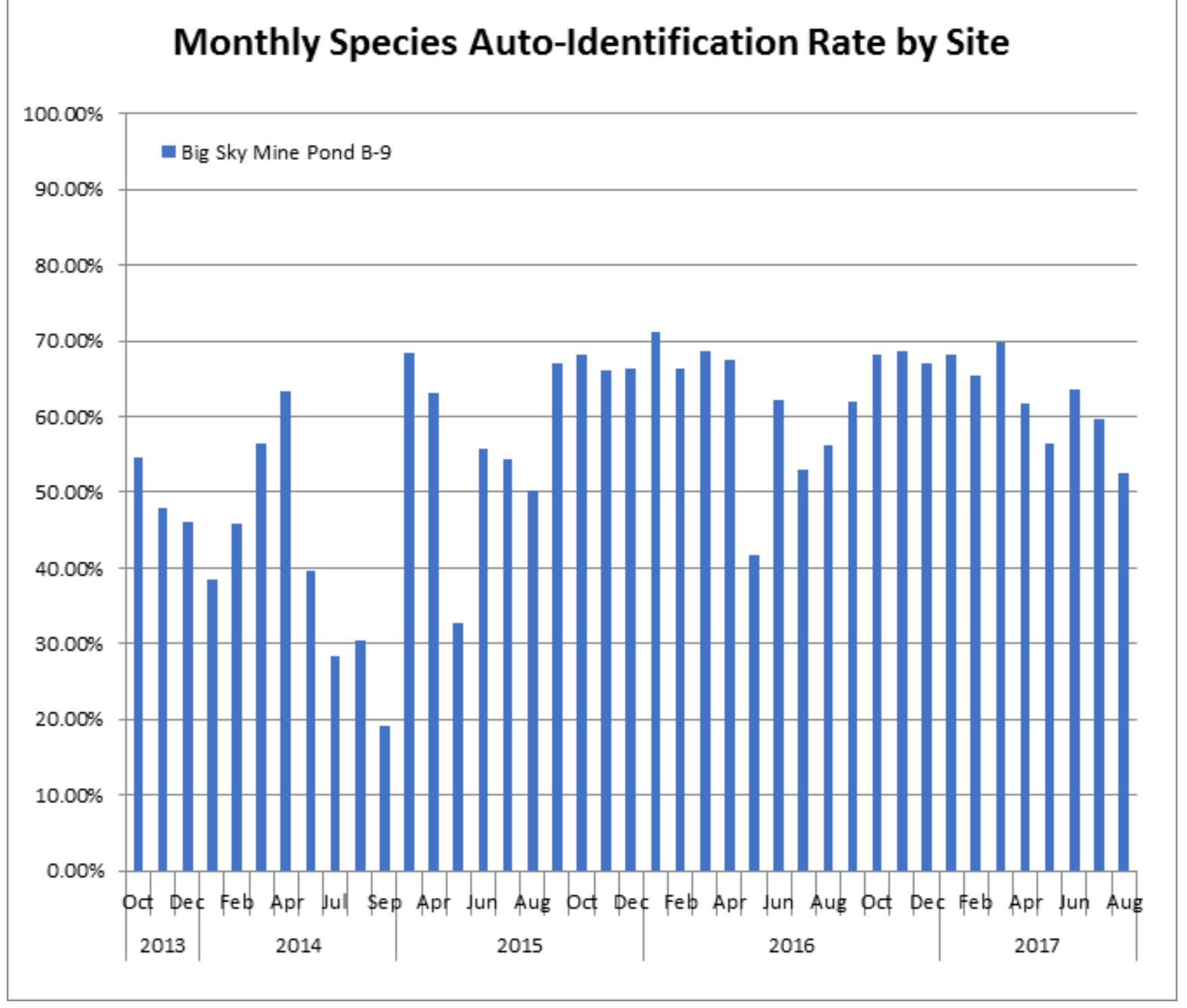


(e)

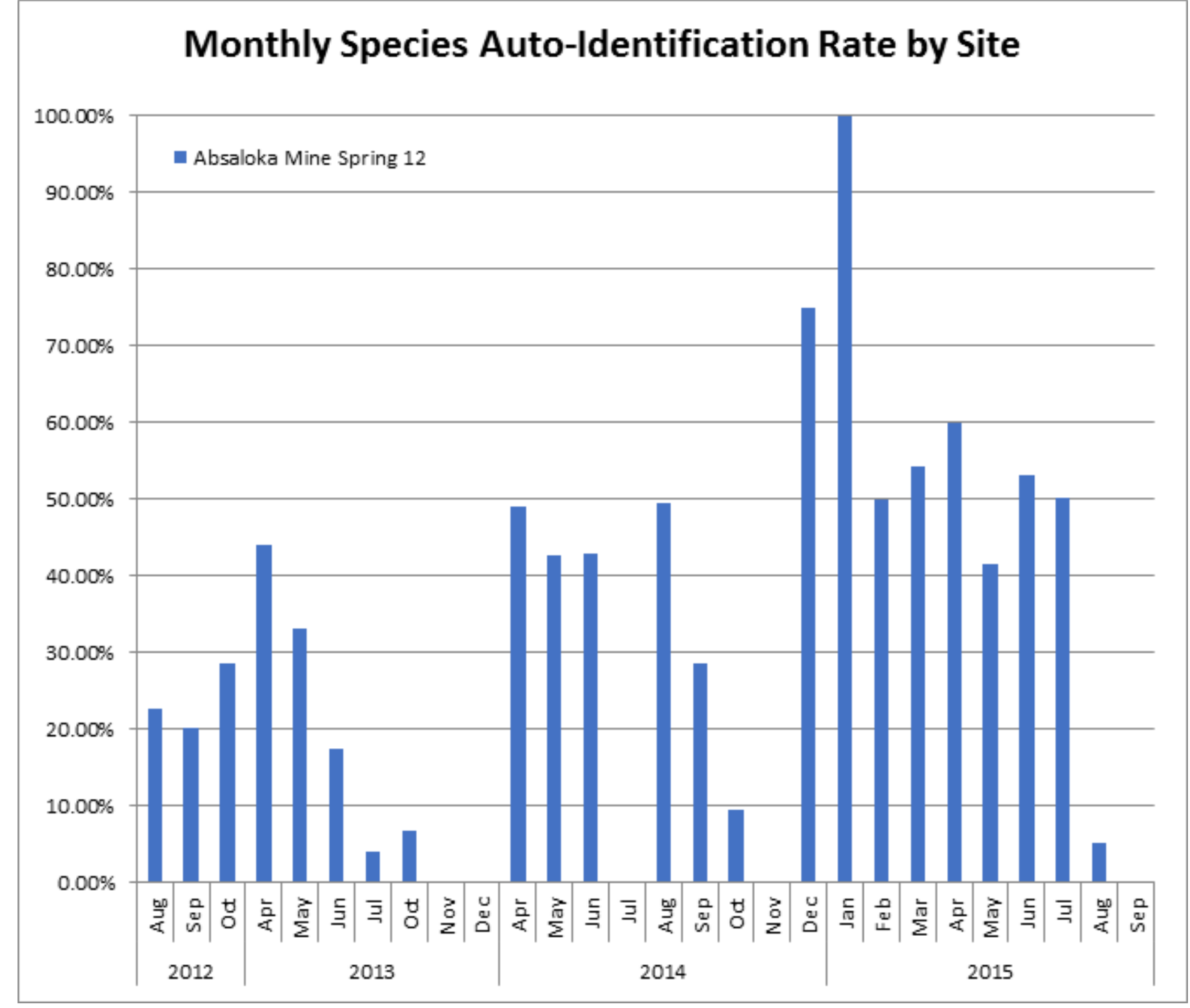




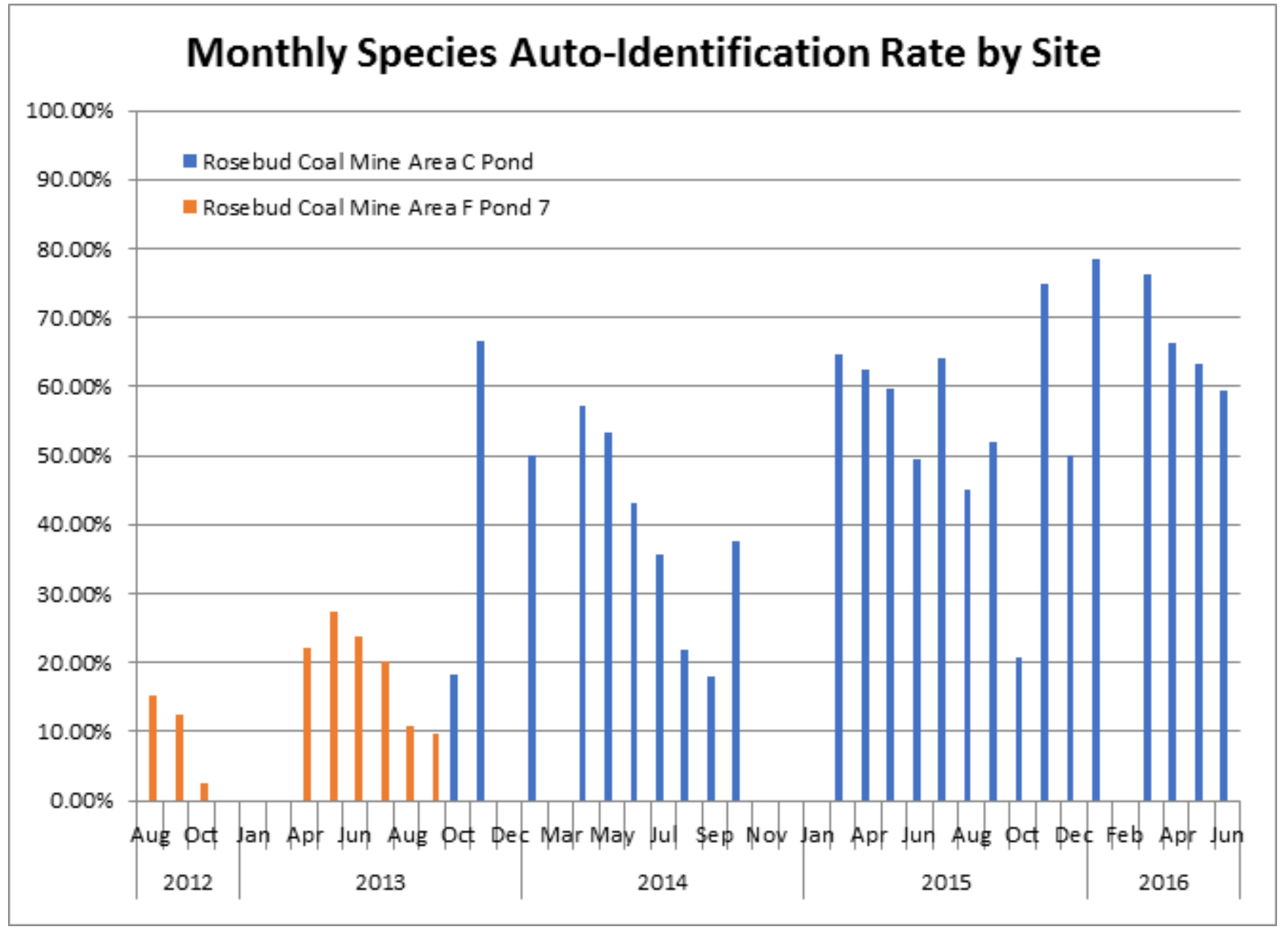


(g)

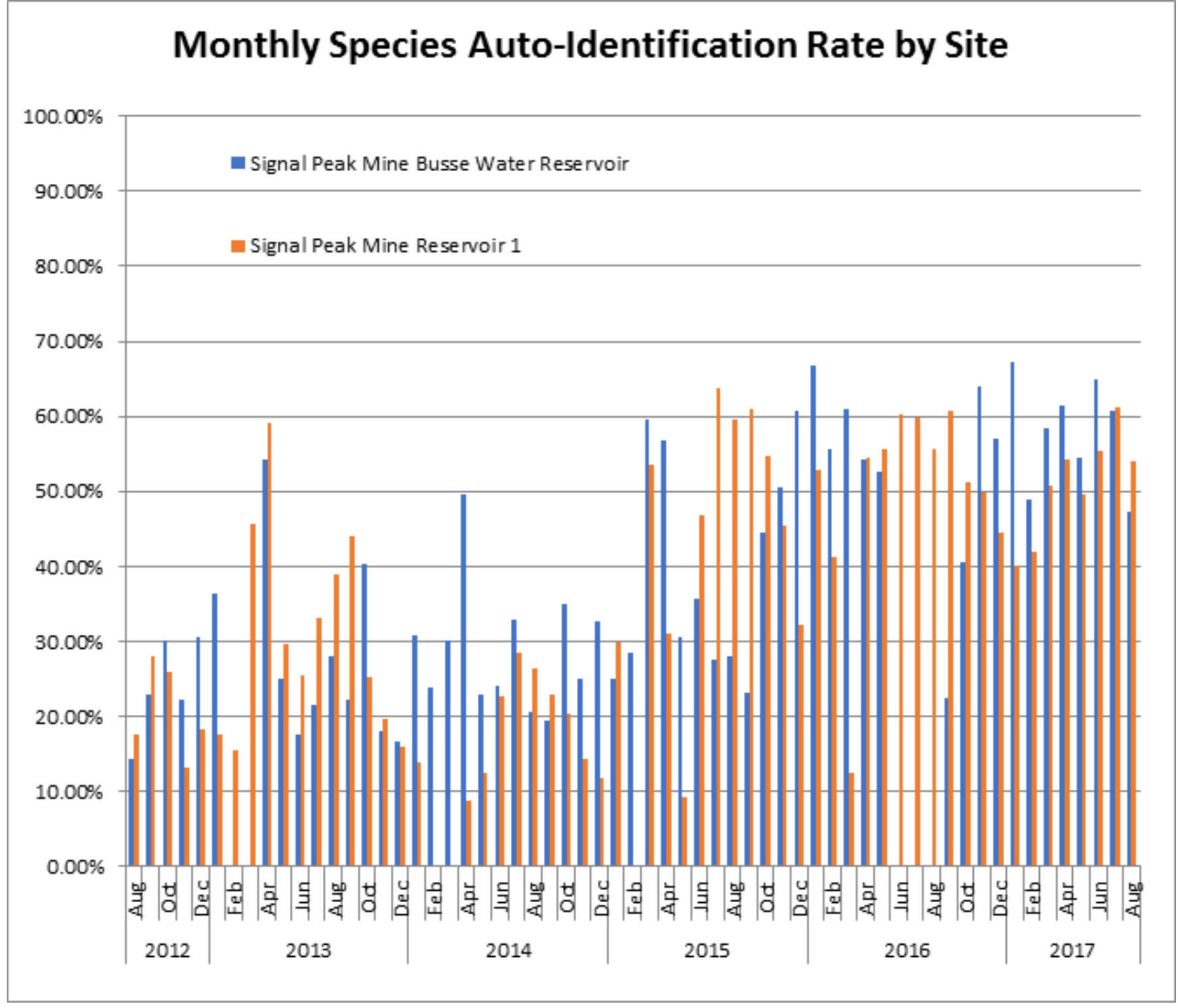


Figure 6. Average (blue) and maximum counts (red) of bat passes per night by month for: (a) West Decker Coal Mine, (b) Spring Creek Mine, (c) Otter Creek Coal Tract, (d) Big Sky Mine, (e) Absaloka Mine, (f) Rosebud Mine, (g) Signal Peak Mine. Numbers on X-axis are years and months.

(a)

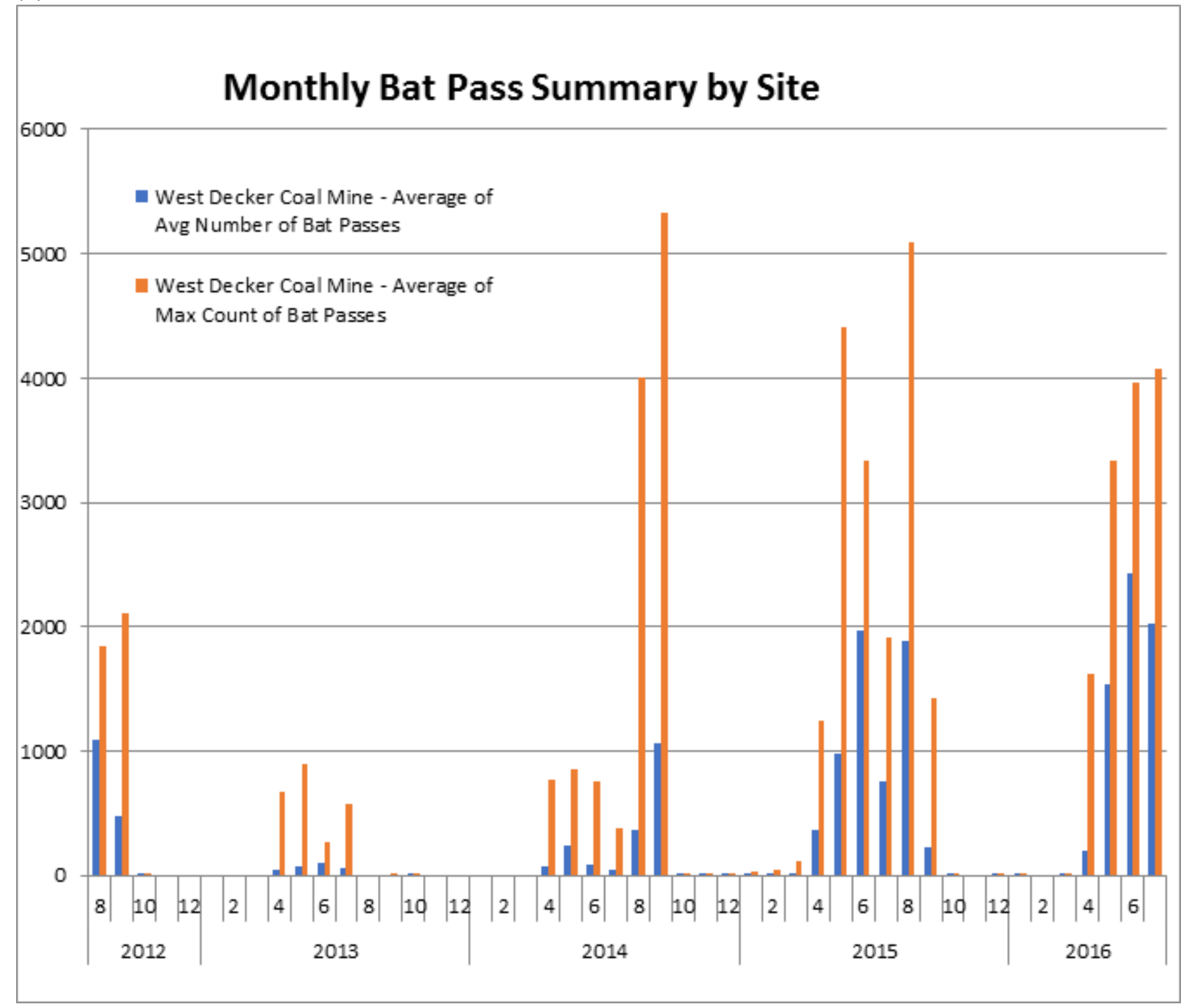


(b)

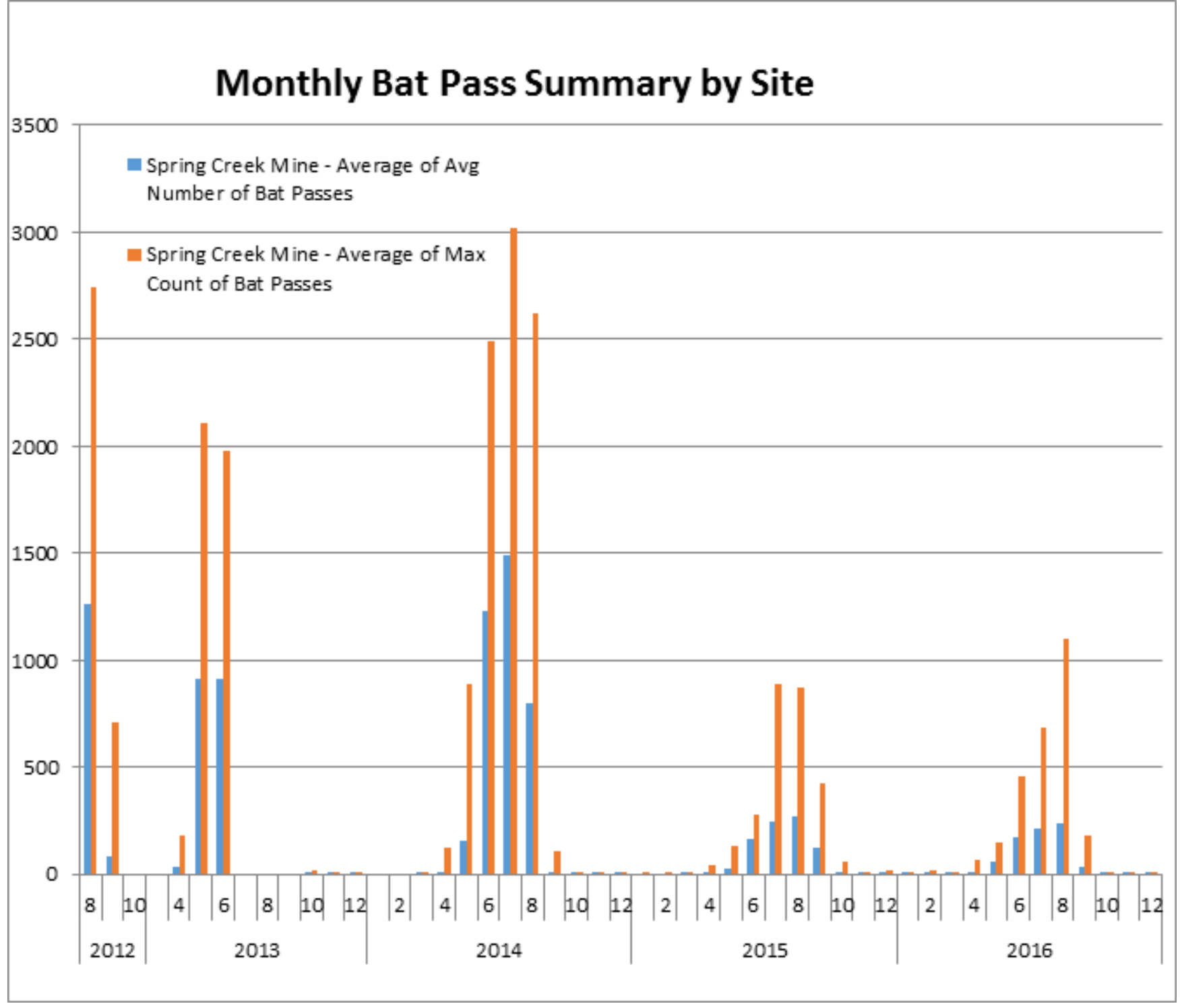


(c)

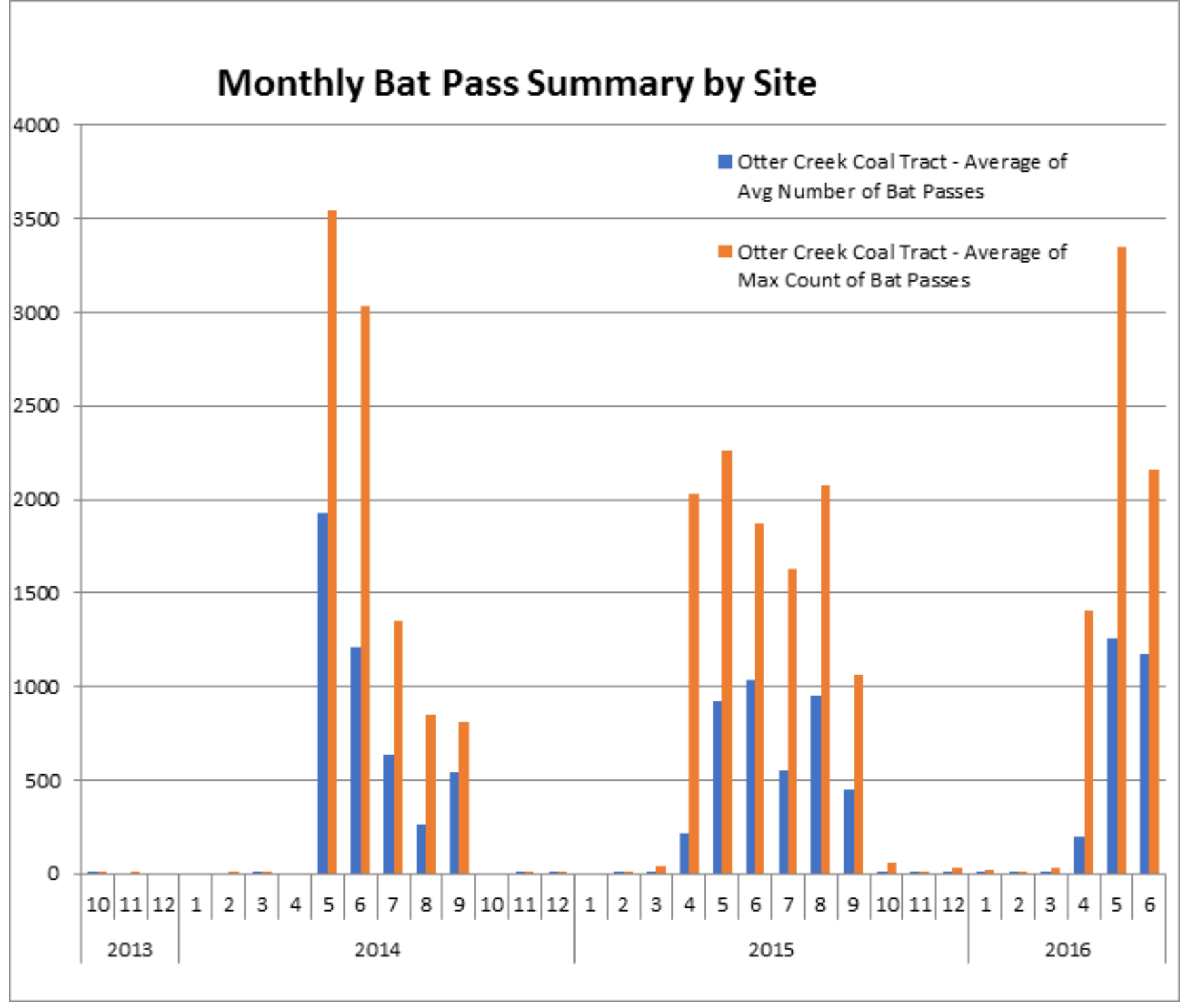


(d)

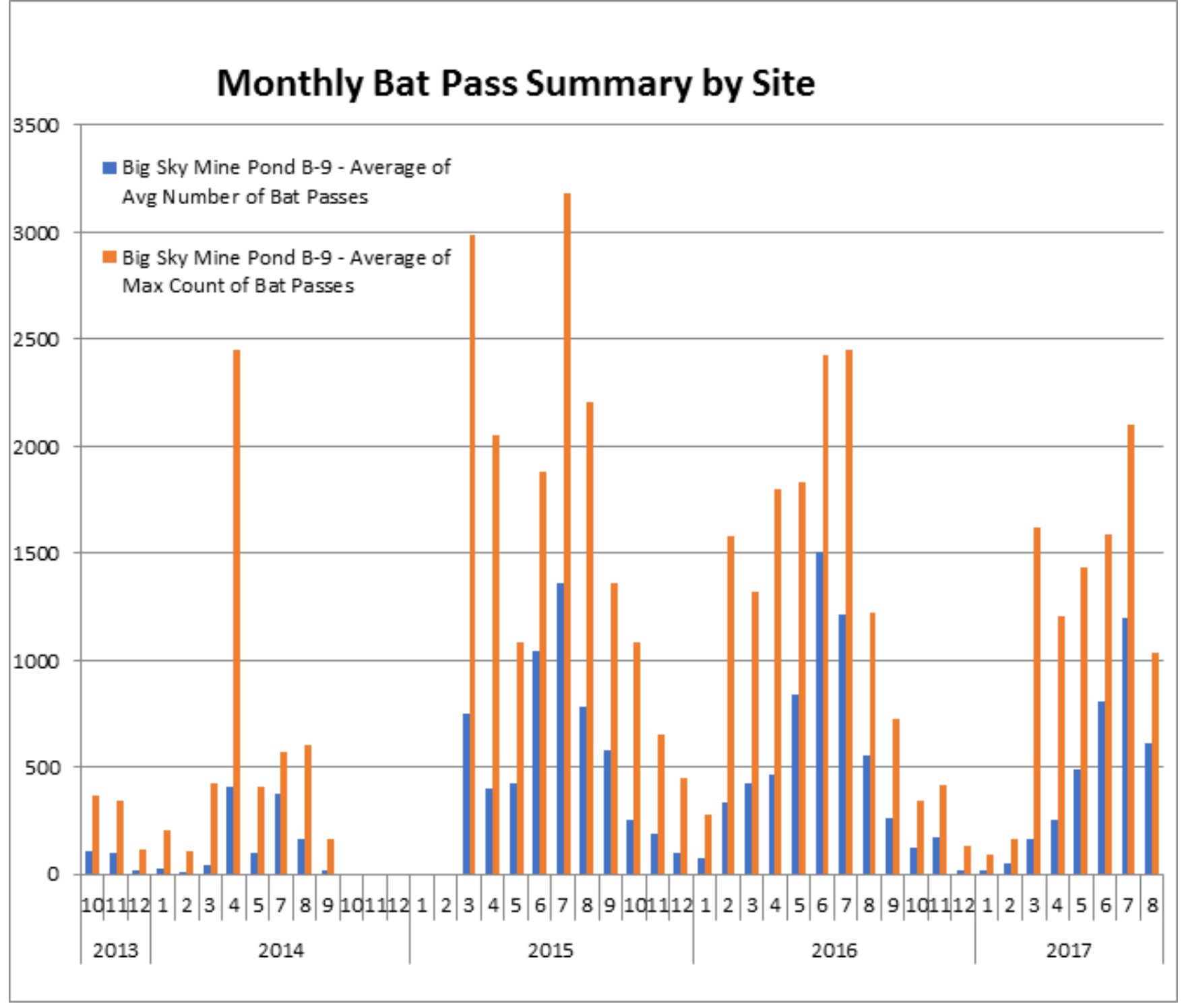


(e)

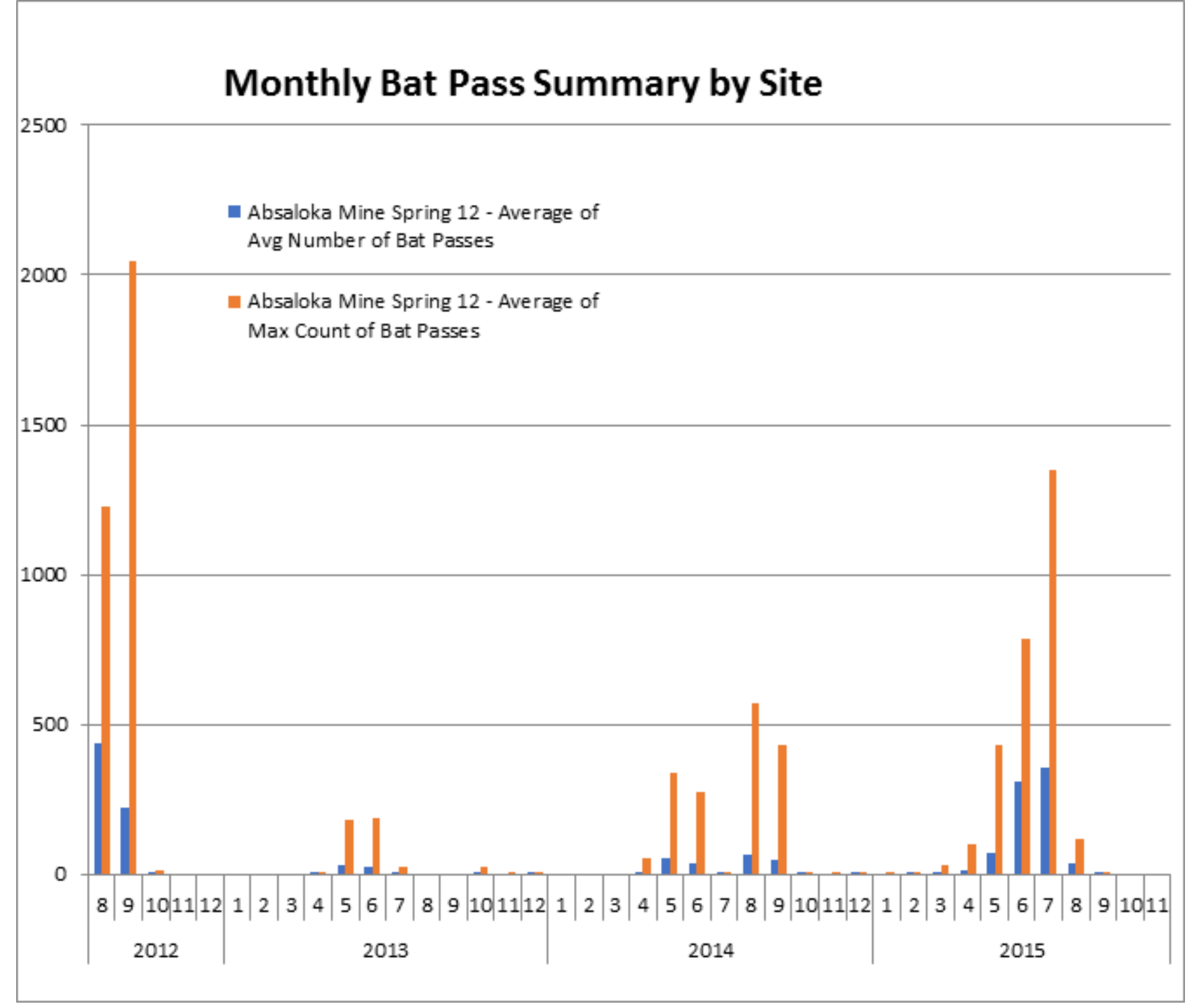




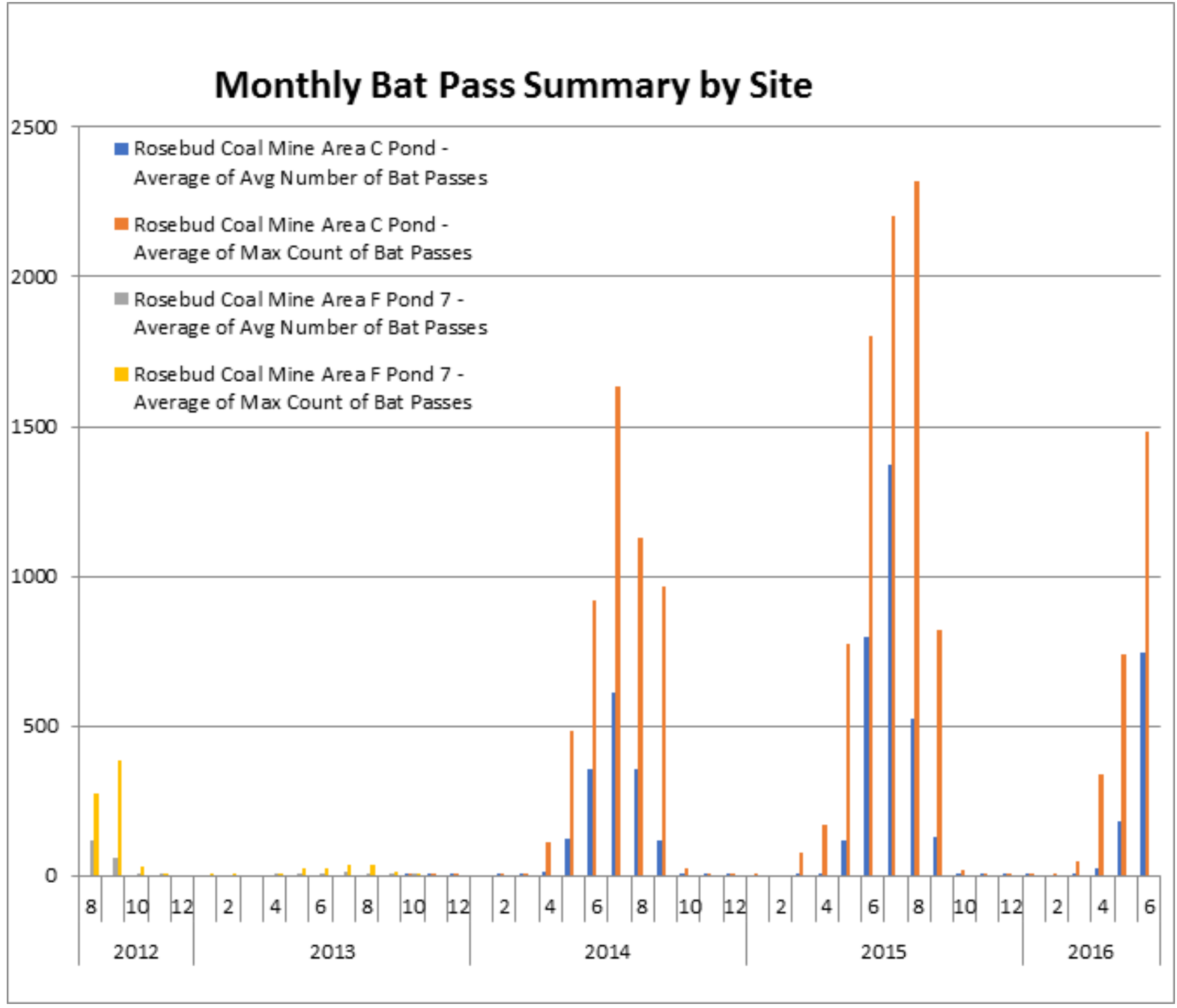


(g)

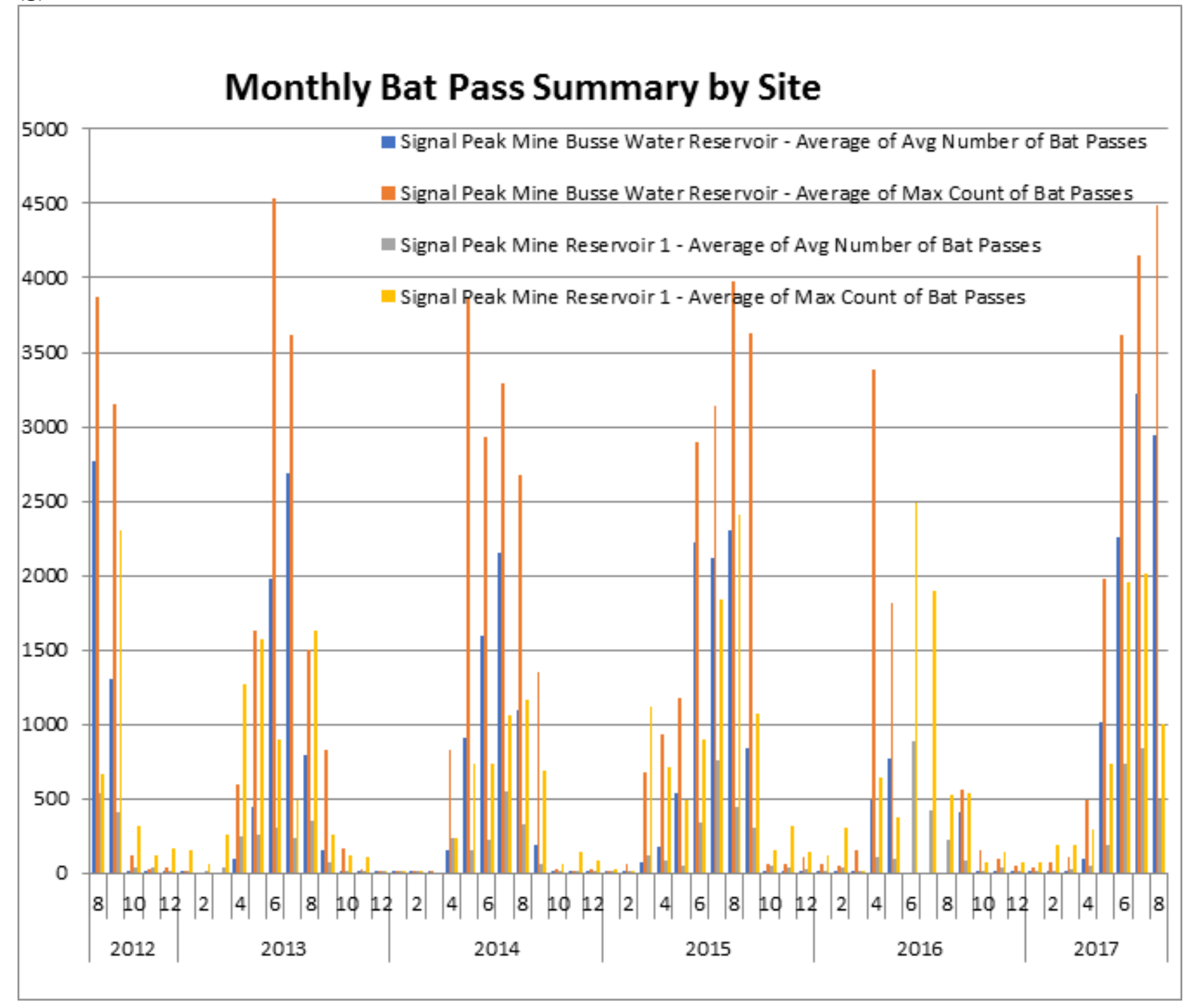


Figure 7. Average number of bat passes per night by week for the active and inactive season at: (a) West Decker Coal Mine, (b) Spring Creek Mine, (c) Otter Creek Coal Tract, (d) Big Sky Mine, (e) Absaloka Mine, (f) Rosebud Mine, (g) Signal Peak Mine. Numbers on $X$ axis are years, months, and weeks.

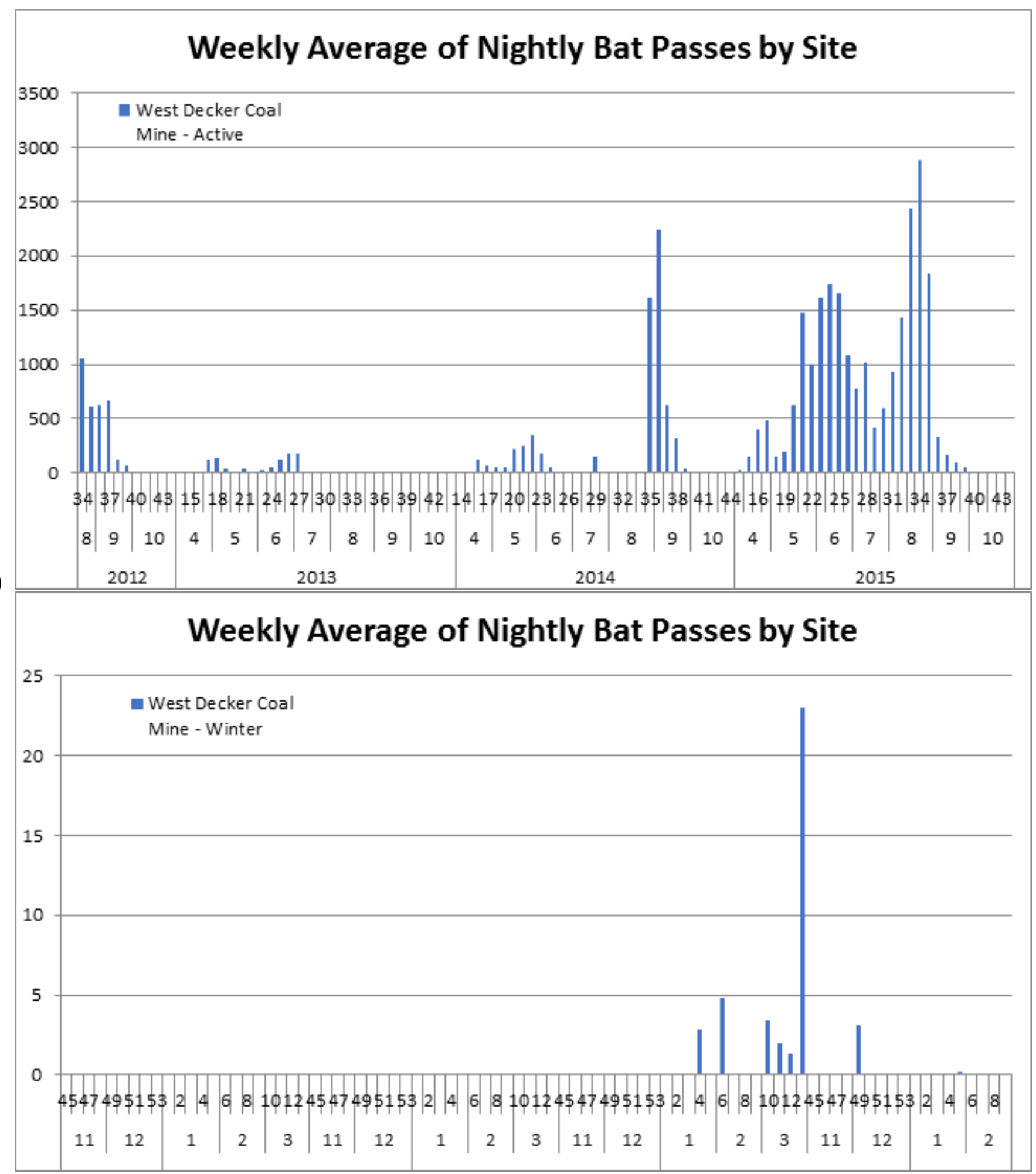




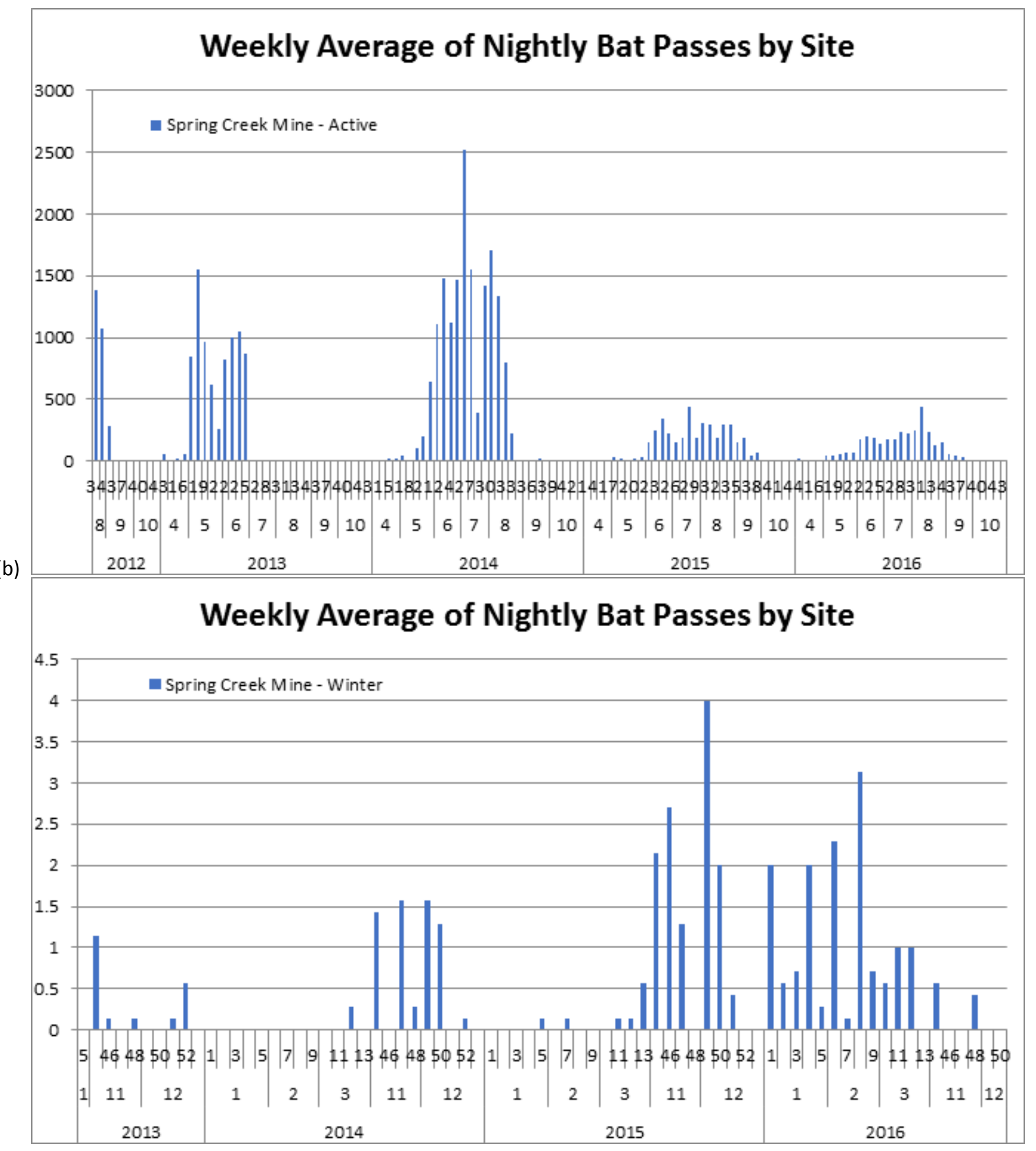




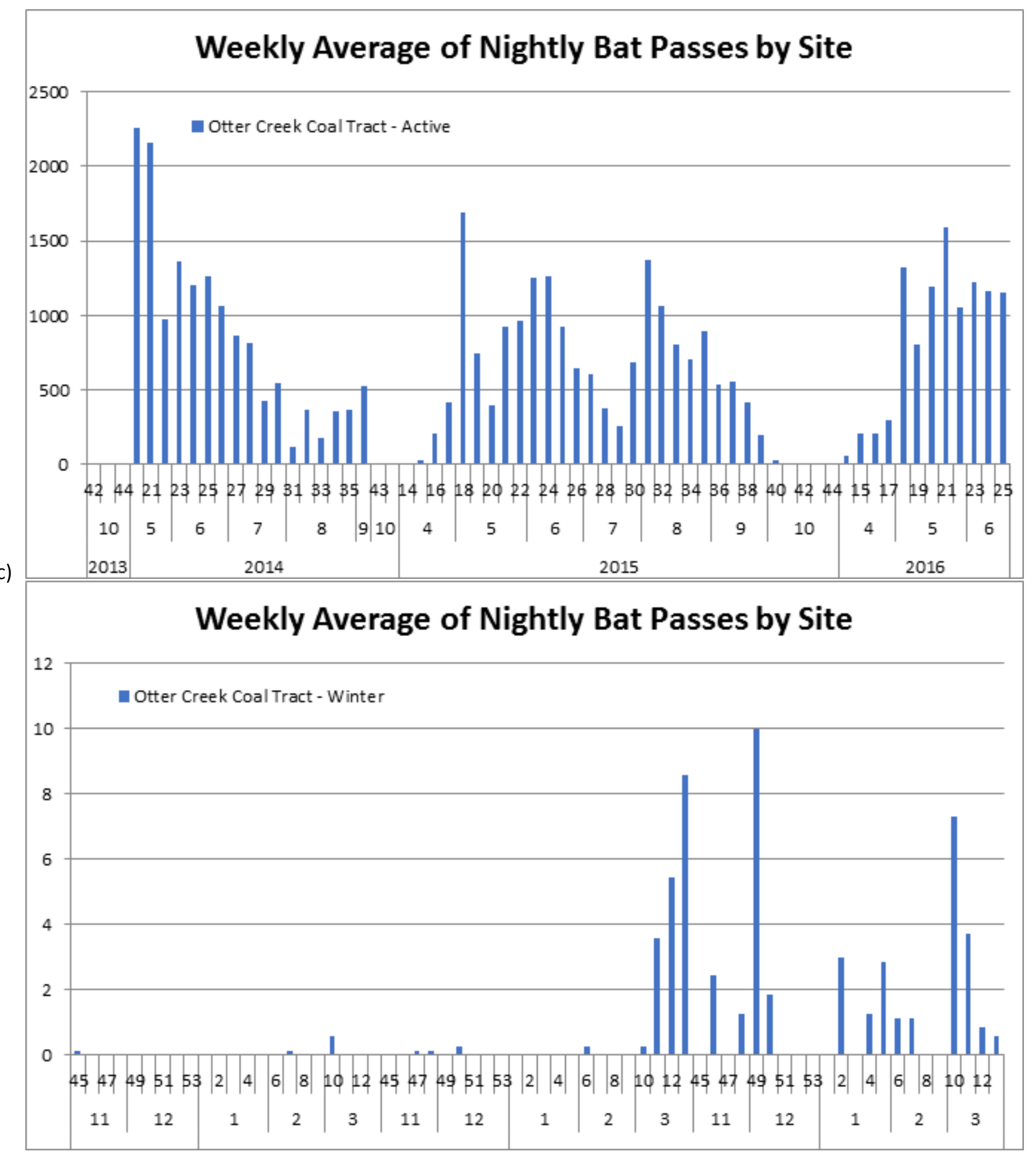




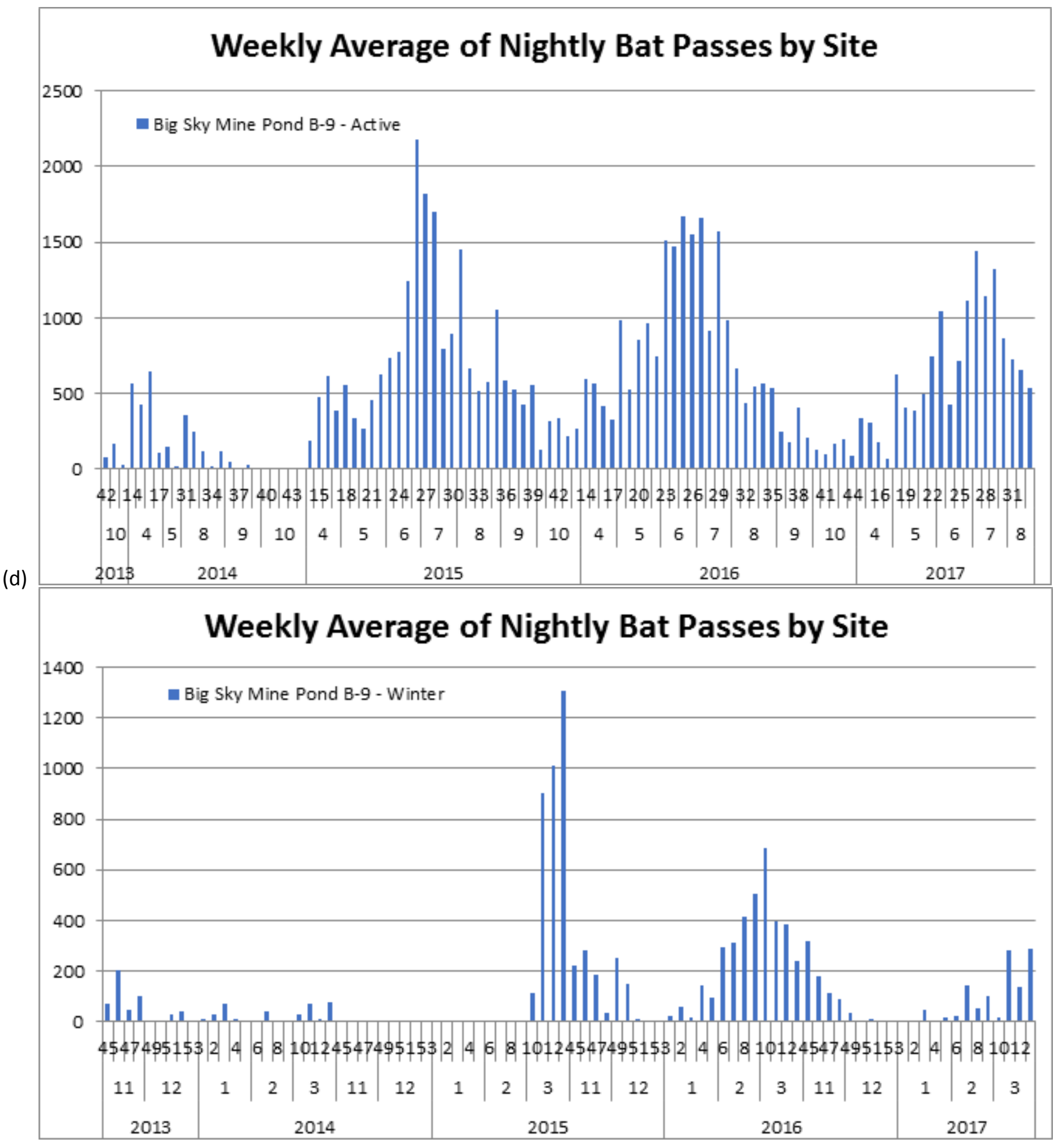


(e)

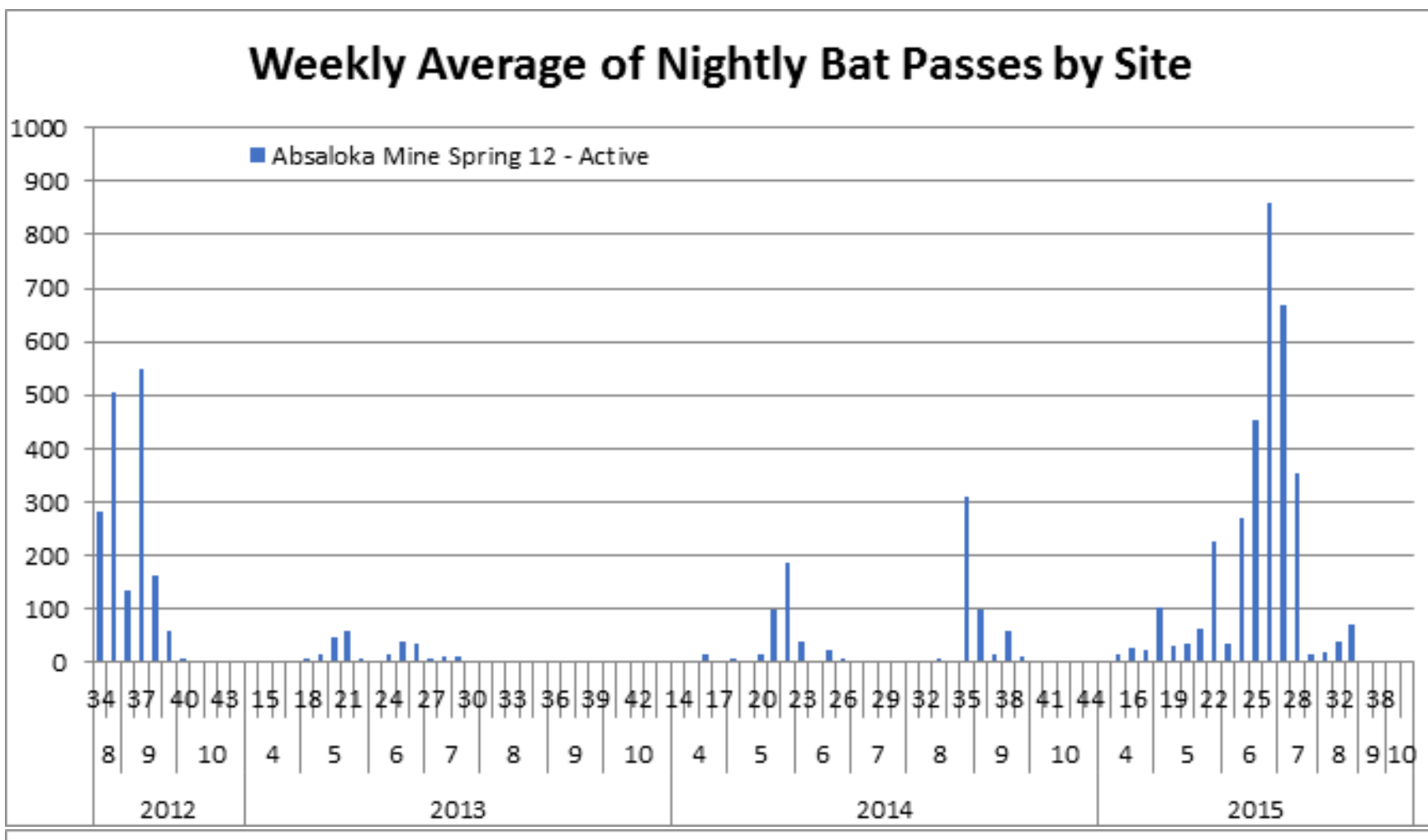

Weekly Average of Nightly Bat Passes by Site

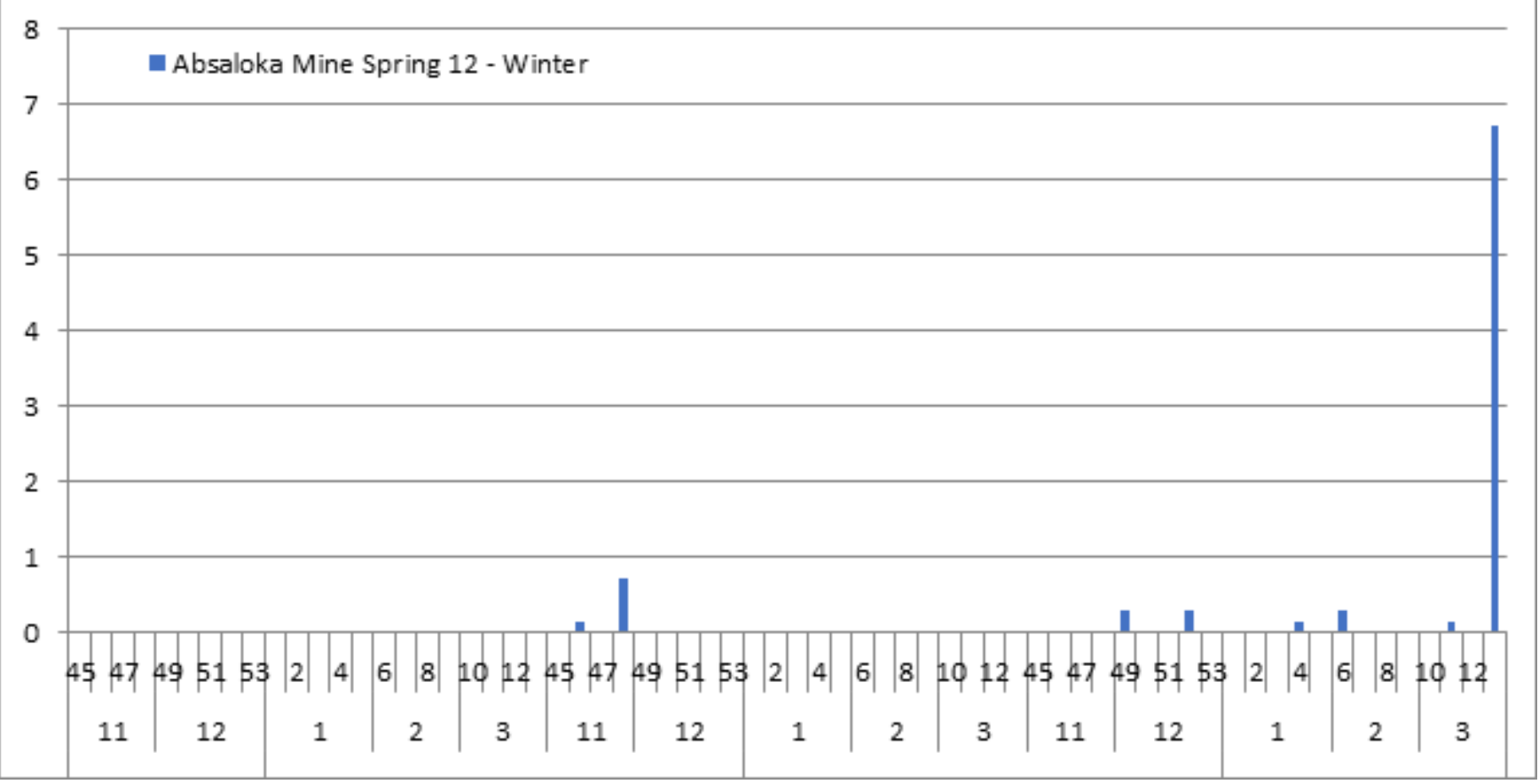




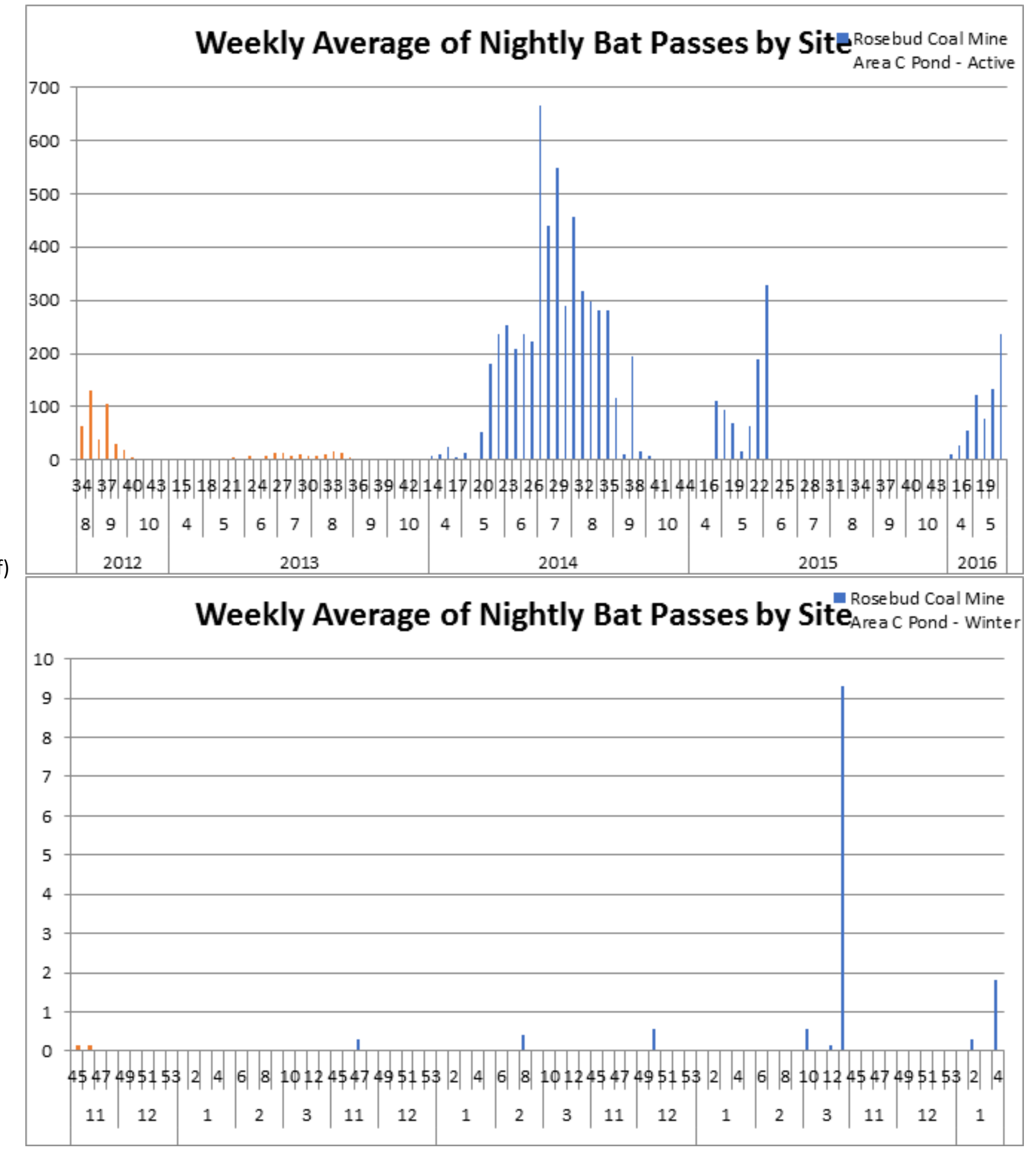




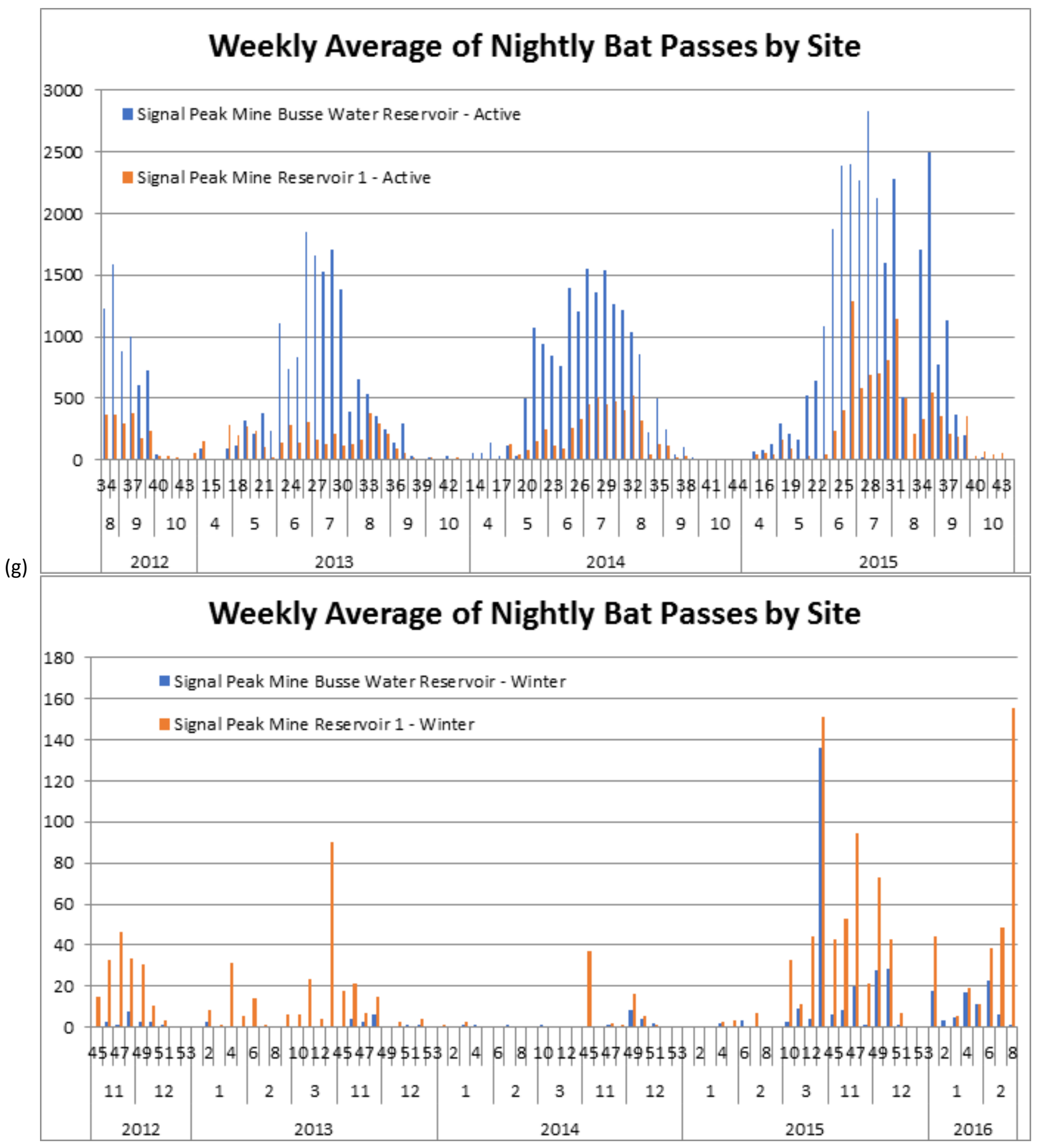


Figure 8. Average number of bat passes per night by week across the detector network for active season (a) and inactive season (b). Numbers on $\mathrm{X}$ axis are years, months, and weeks.

(a)

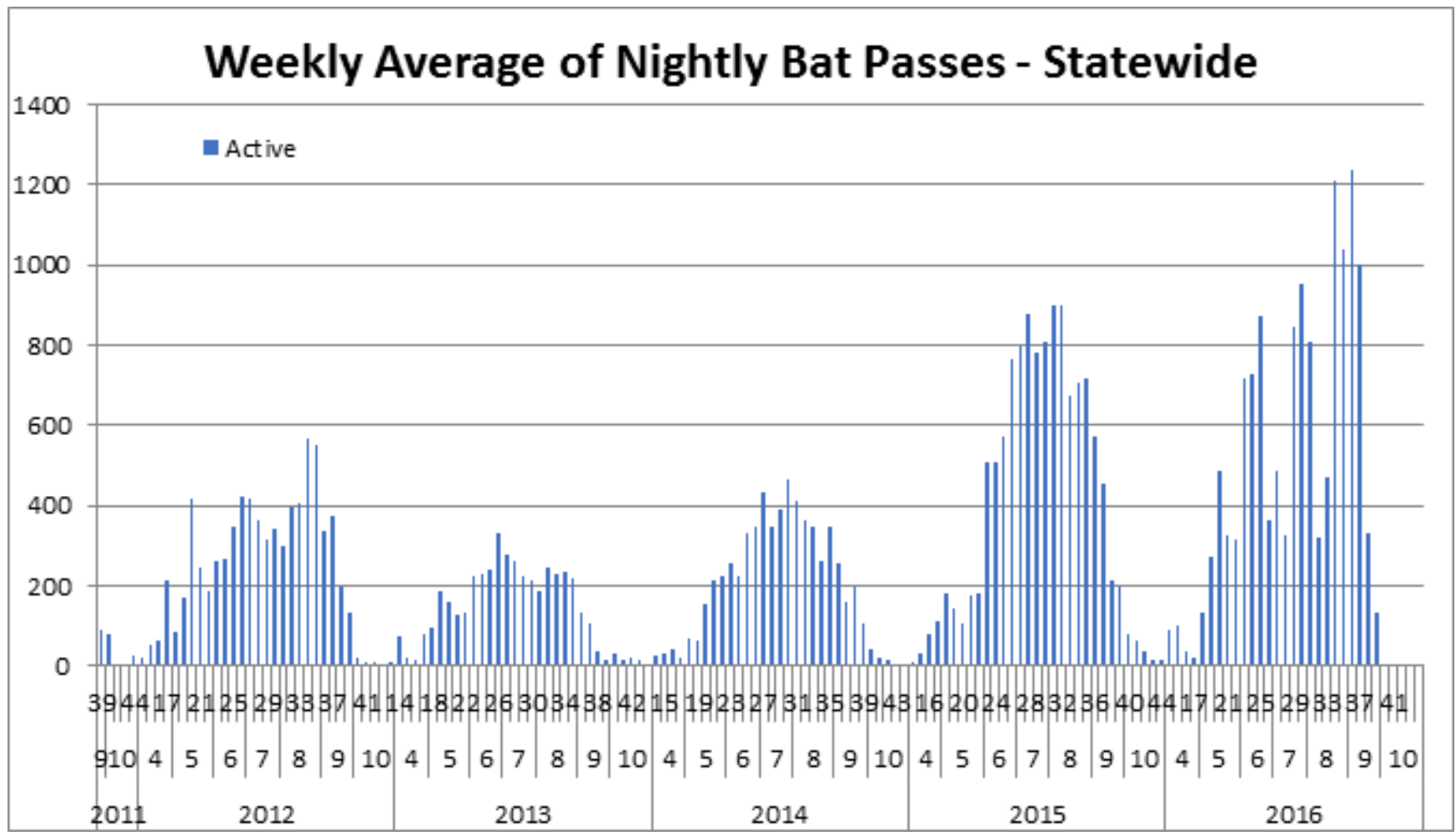

(b)

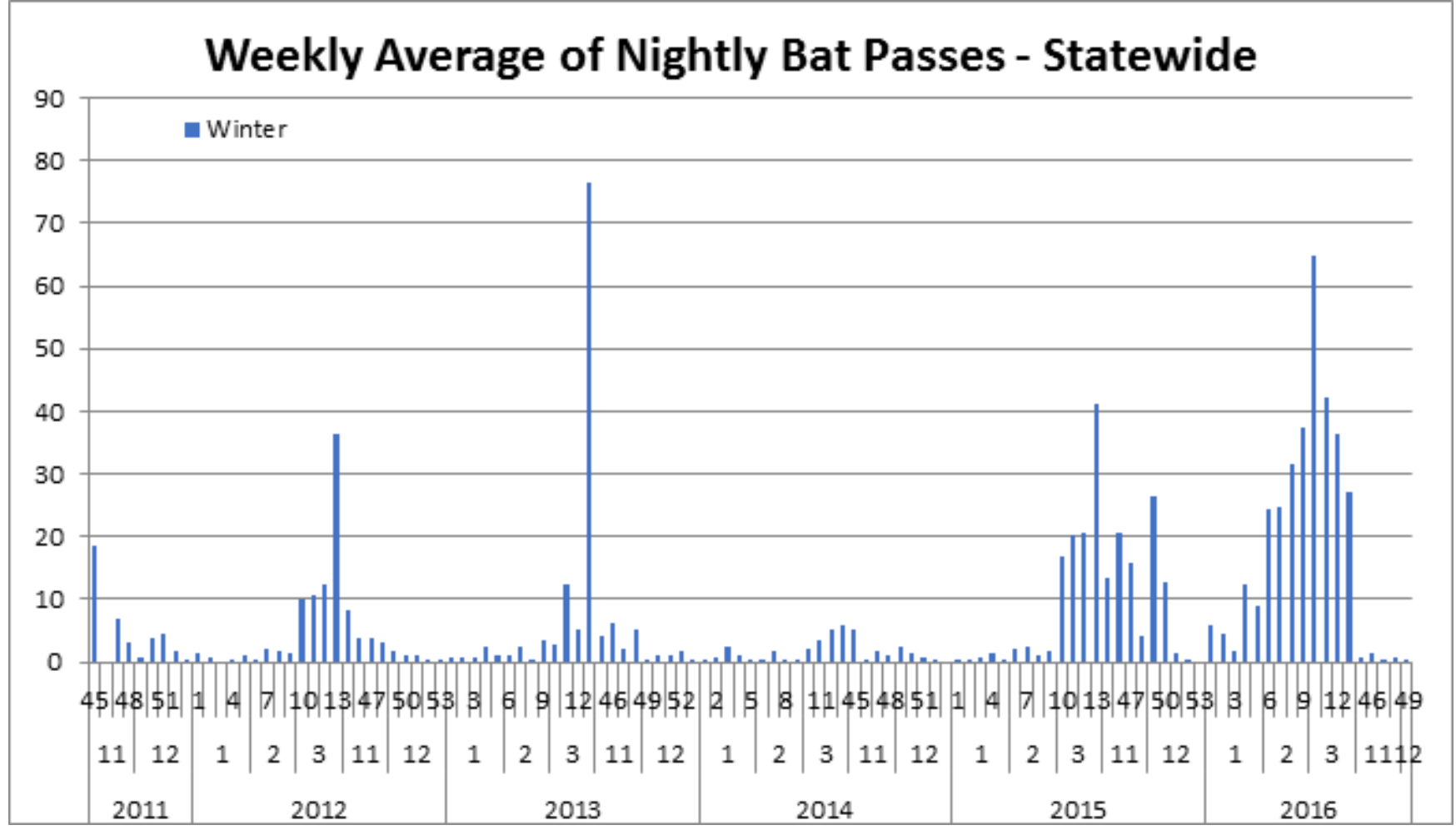


Figure 9. Total number of bat passes per night by week across the detector network across all years for active season (a) and inactive season (b). Numbers on $\mathrm{X}$ axis are weeks.

a)

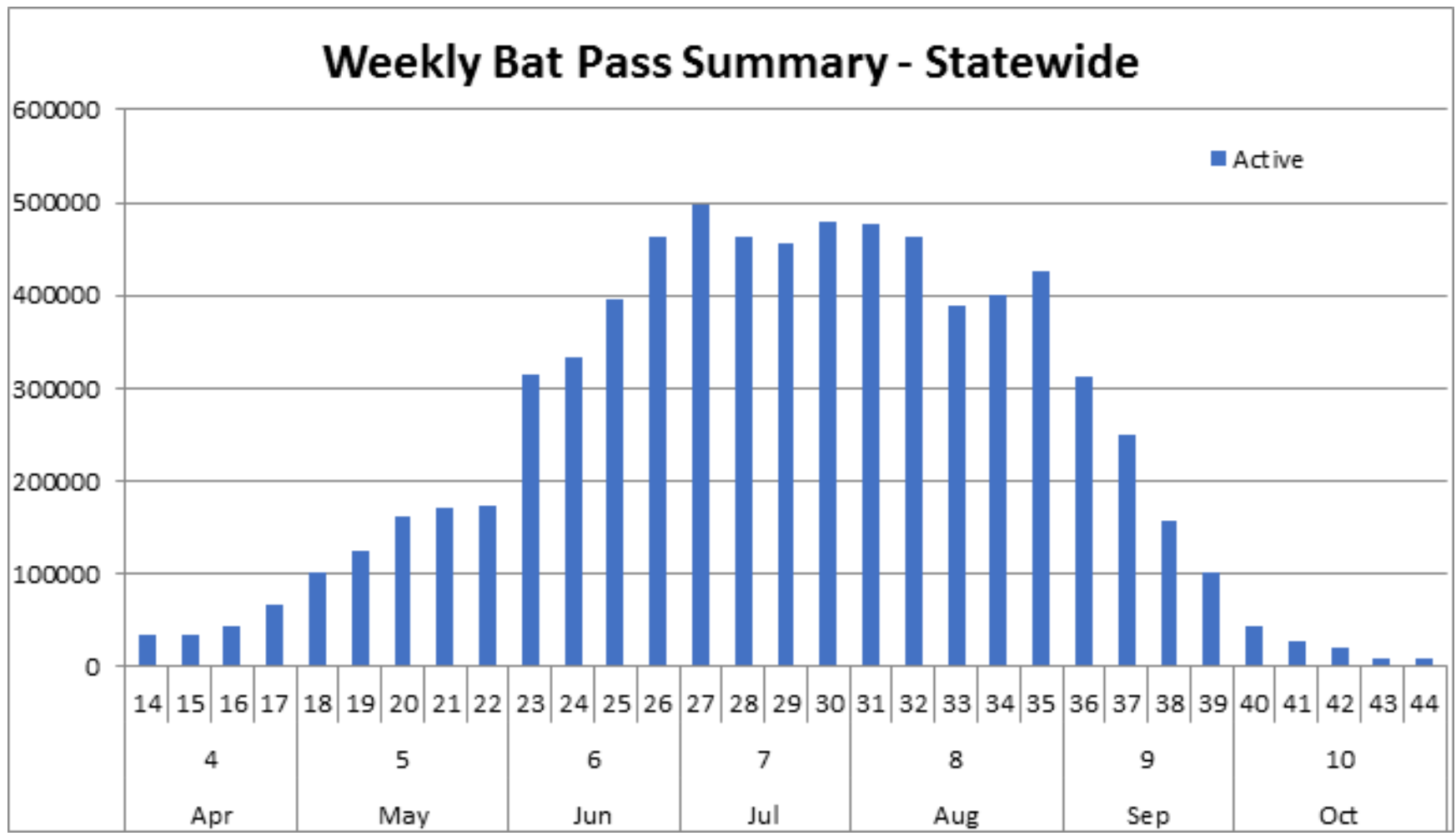

(b)

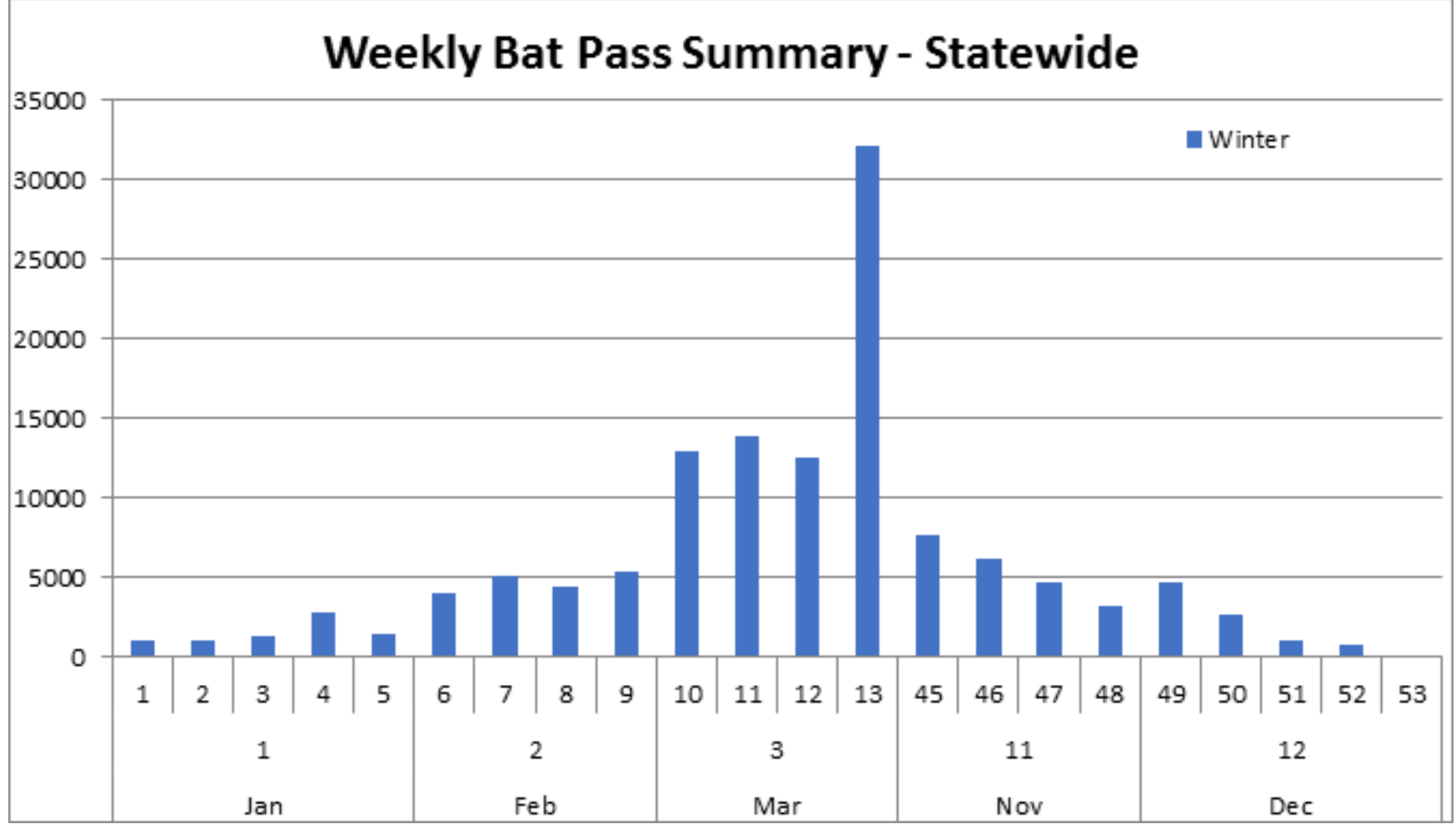


Figure 10. Average number of bat passes each hour after sunset across all years during active (a) and inactive season (b). Numbers on $\mathrm{X}$ axis are weeks.

(a)

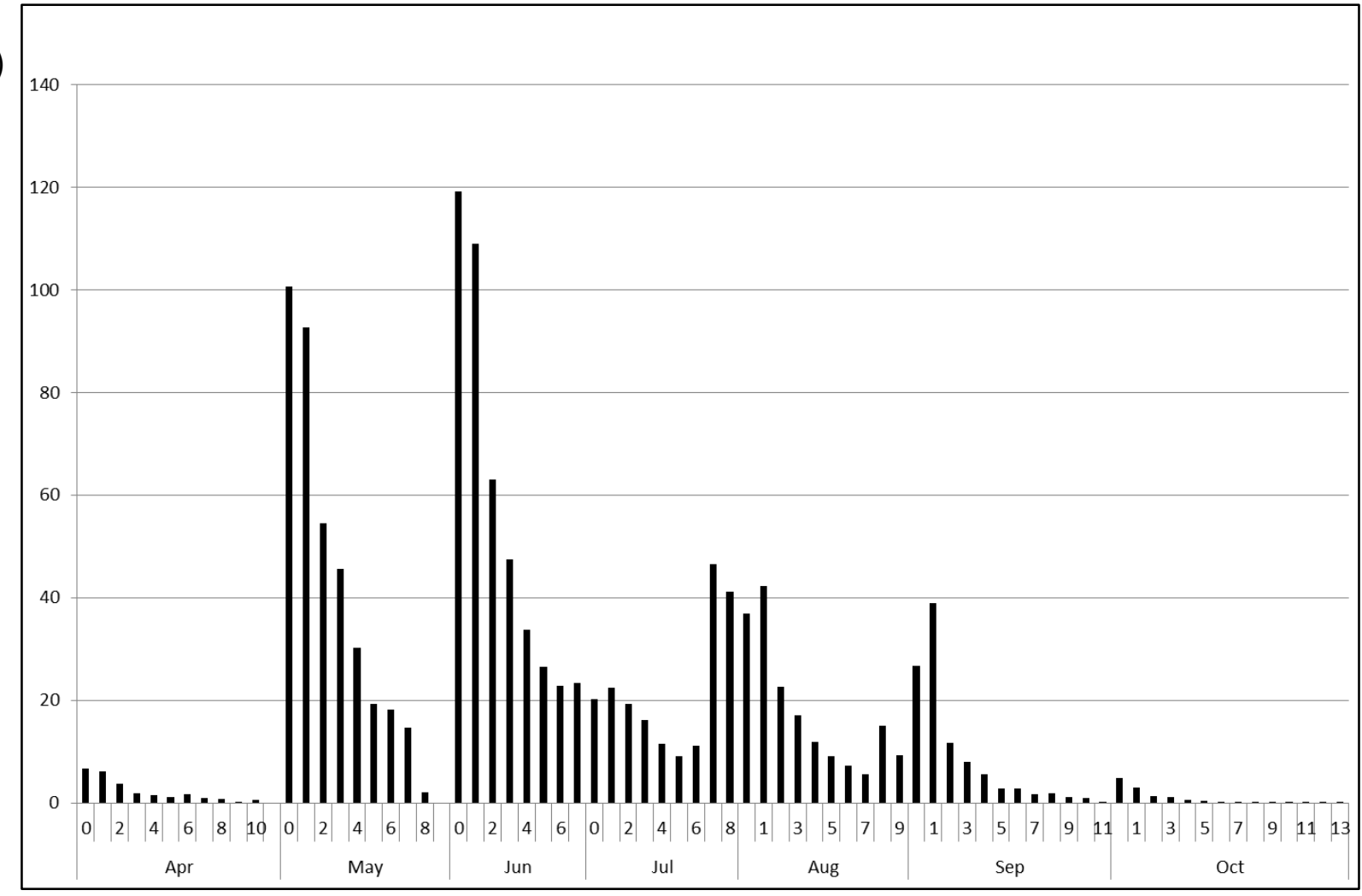

(b)

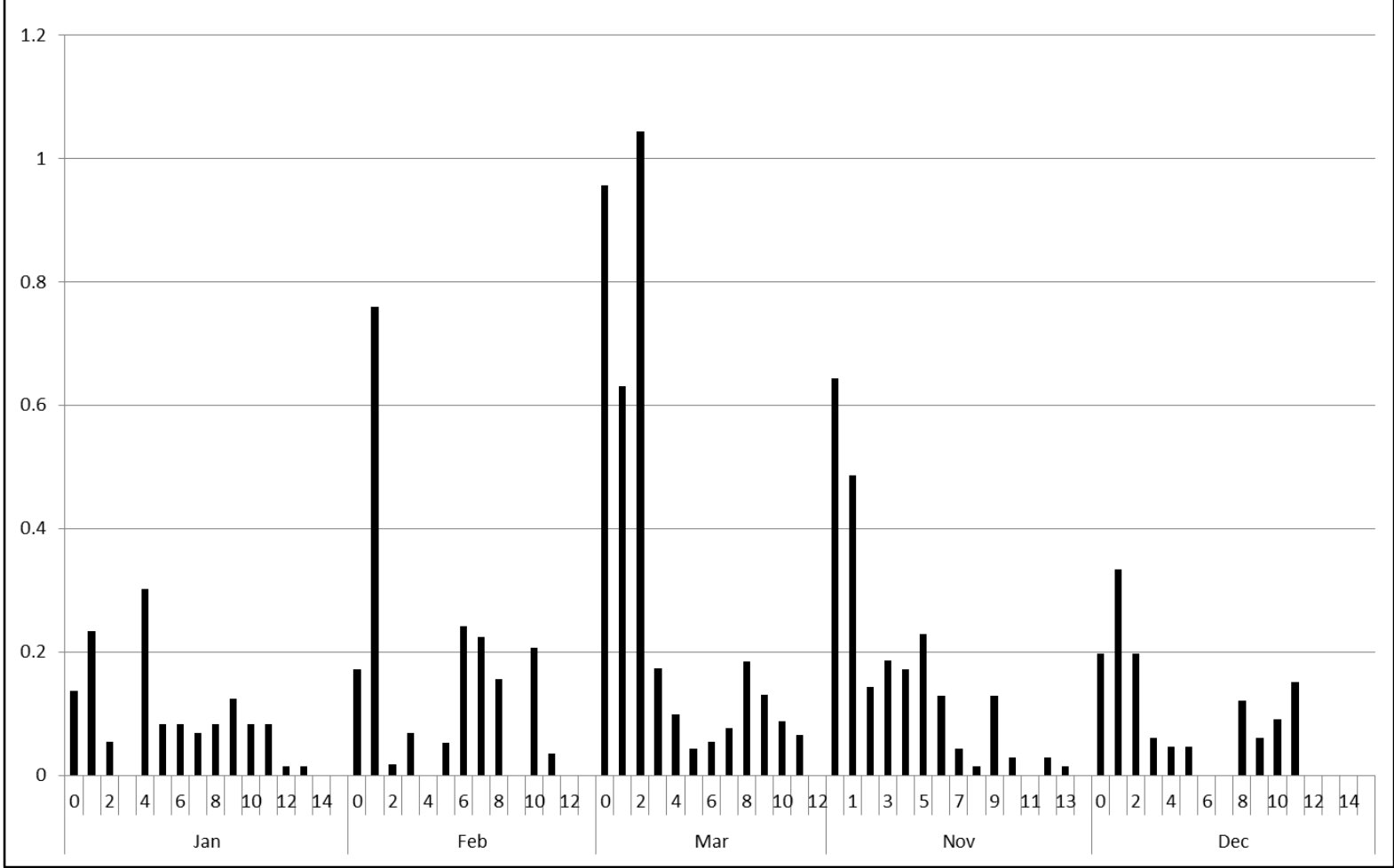


Figure 11. Average number of bat passes per night by week across the detector network and across all years for active season (a) and inactive season (b) in rugged and non-rugged landscapes with and without trees. Numbers on $\mathrm{X}$ axis are months and weeks.

(a)

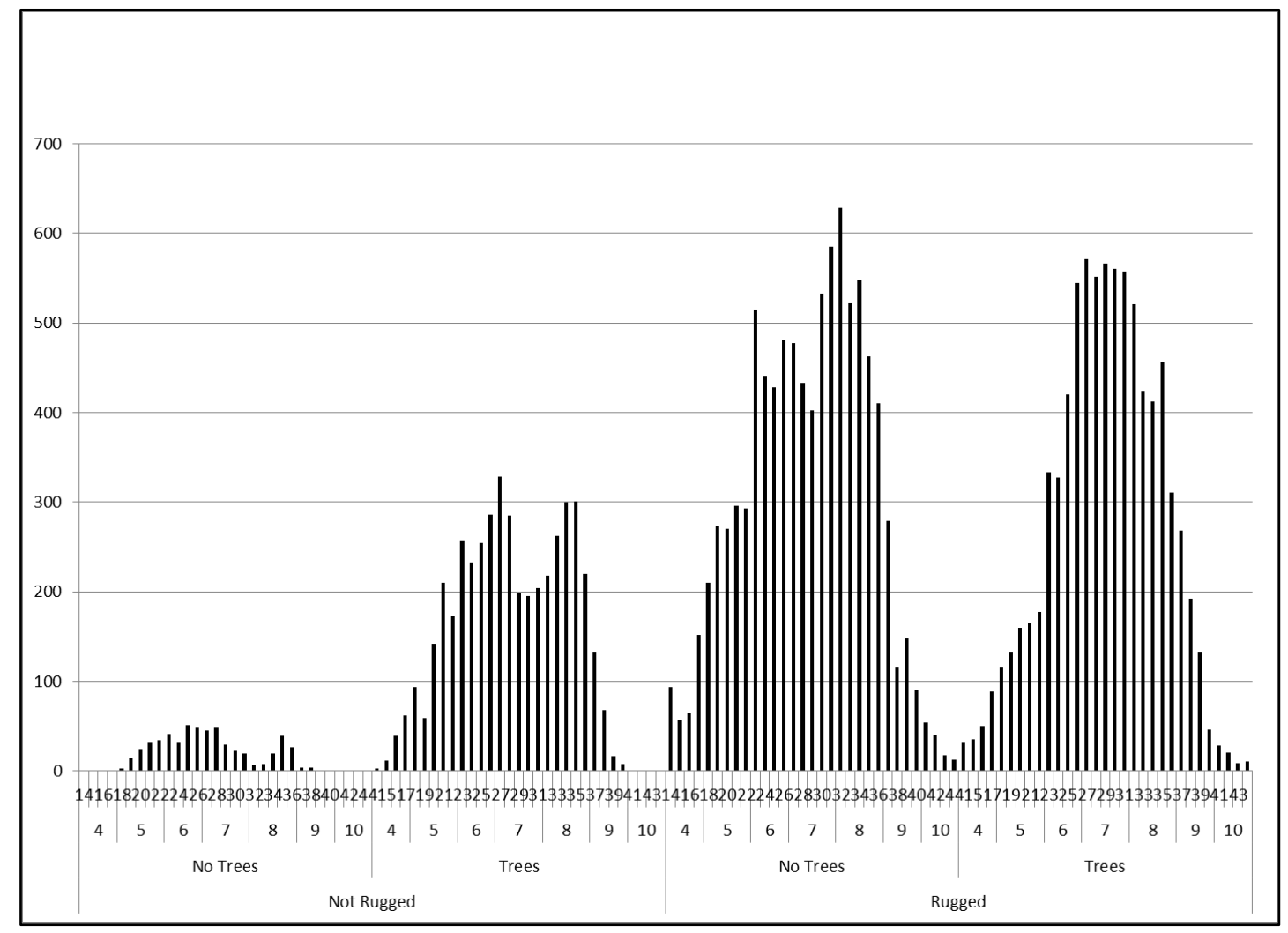

(b)

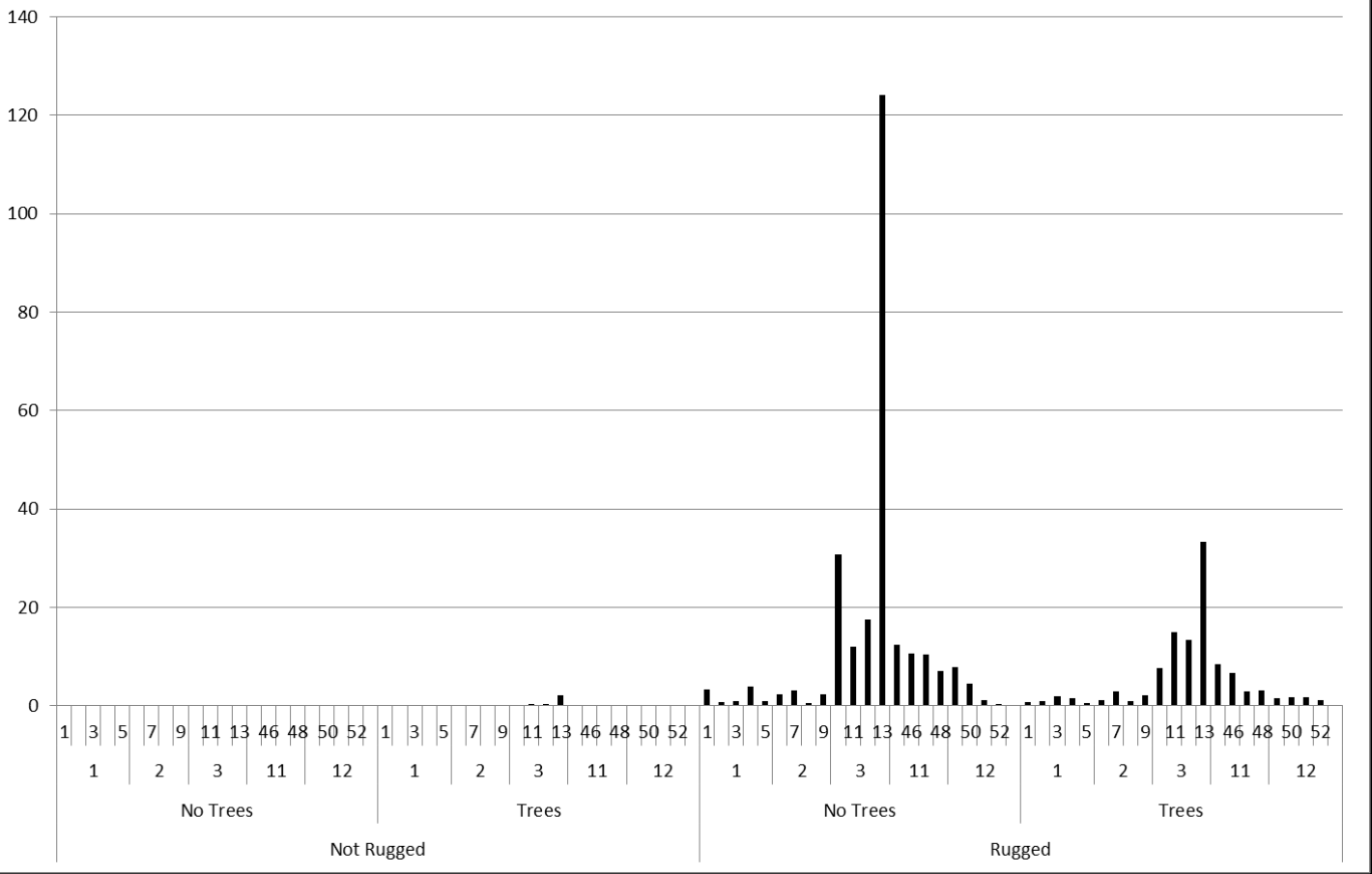


Figure 12. Average number of bat passes per night by week across the detector network and across all years for active season (a) and inactive season (b) at different water body types. Numbers on X axis are months and weeks.

(a)

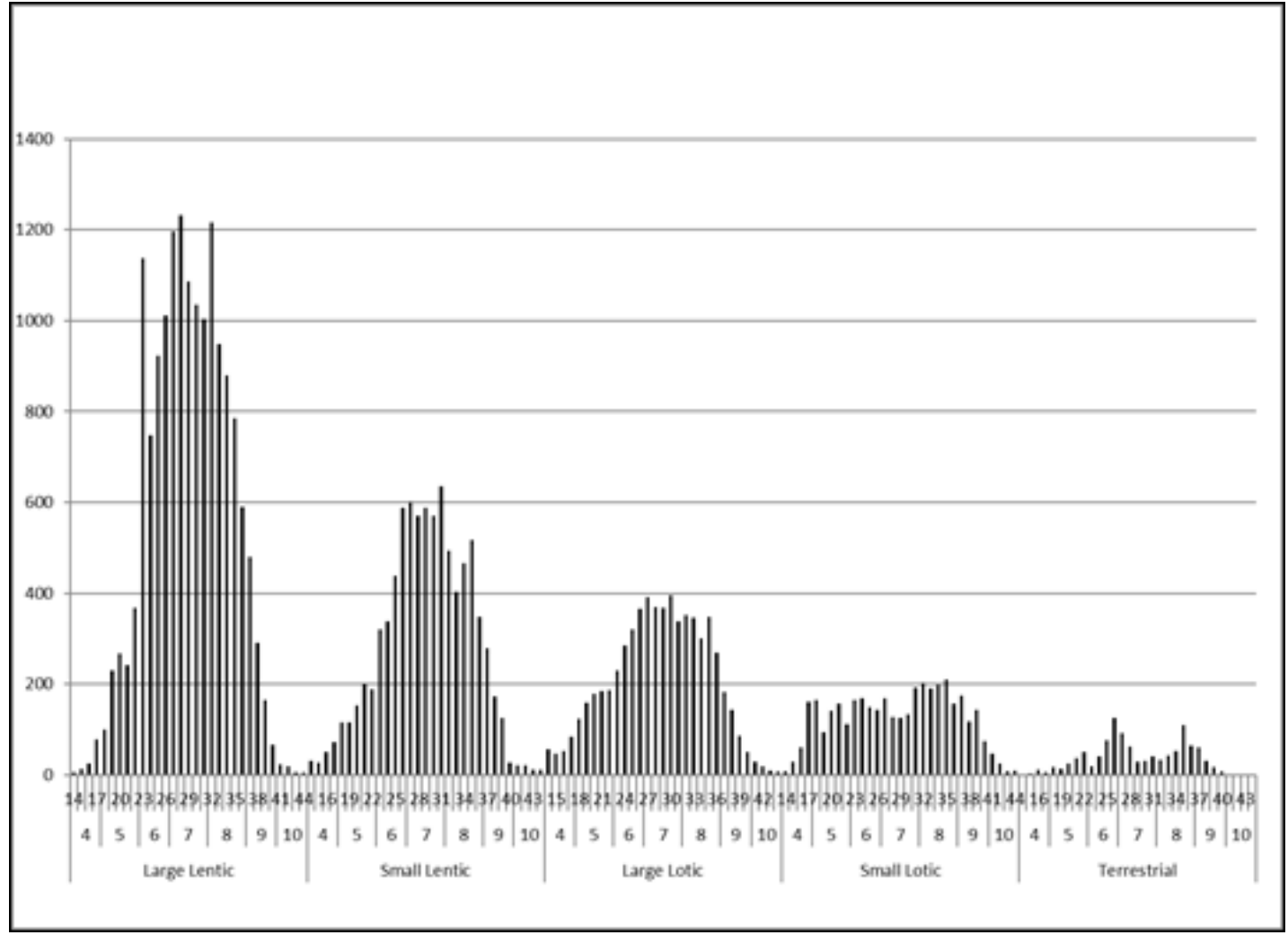

(b)

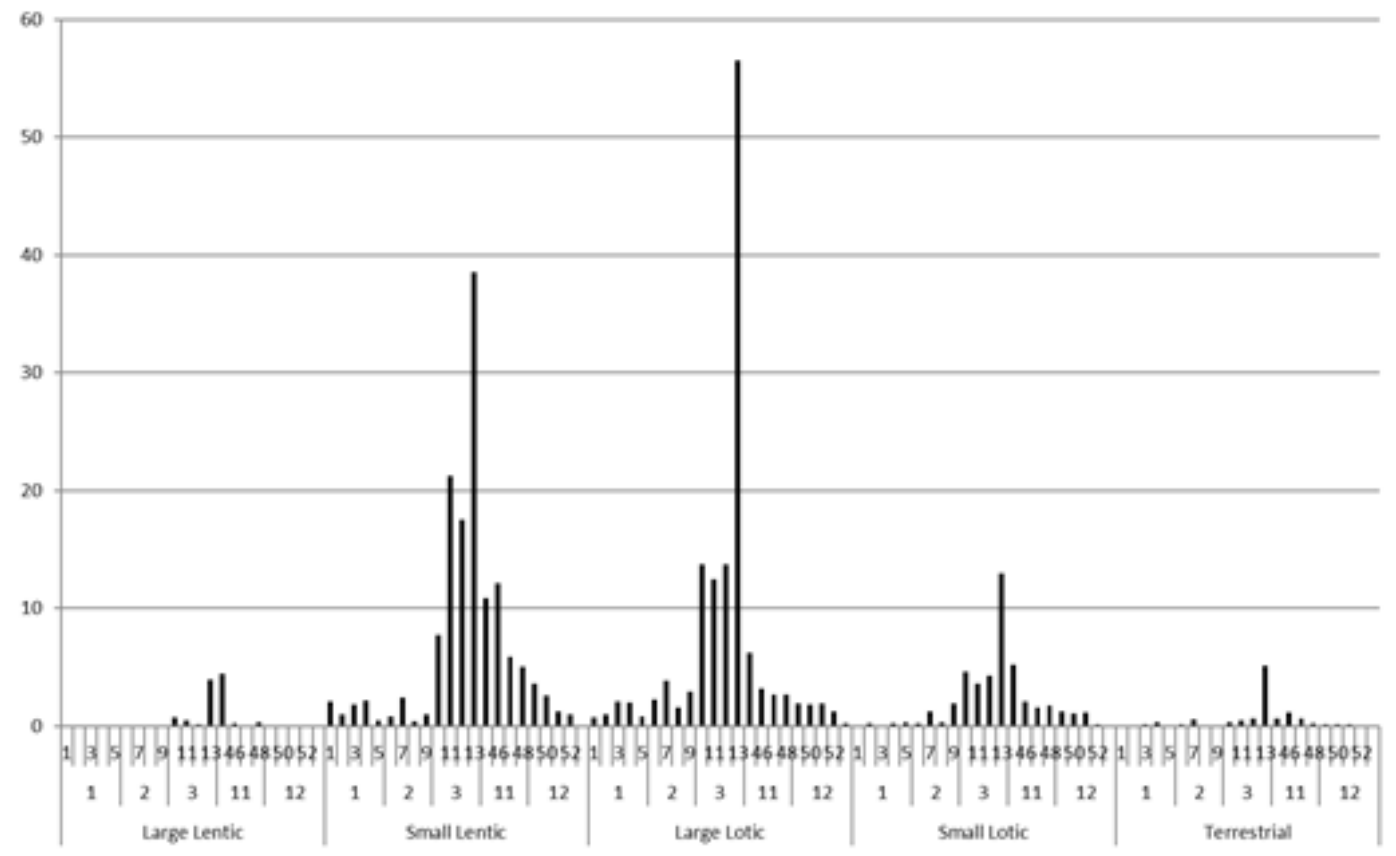


Figure 13. Average nightly background (blue) and bat pass (red) temperatures by month at: (a) West Decker Coal Mine, (b) Spring Creek Mine, (c) Otter Creek Coal Tract, (d) Big Sky Mine, (e) Absaloka Mine, (f) Rosebud Mine, (g) Signal Peak Mine. Numbers on $\mathrm{X}$ axis are years and months.

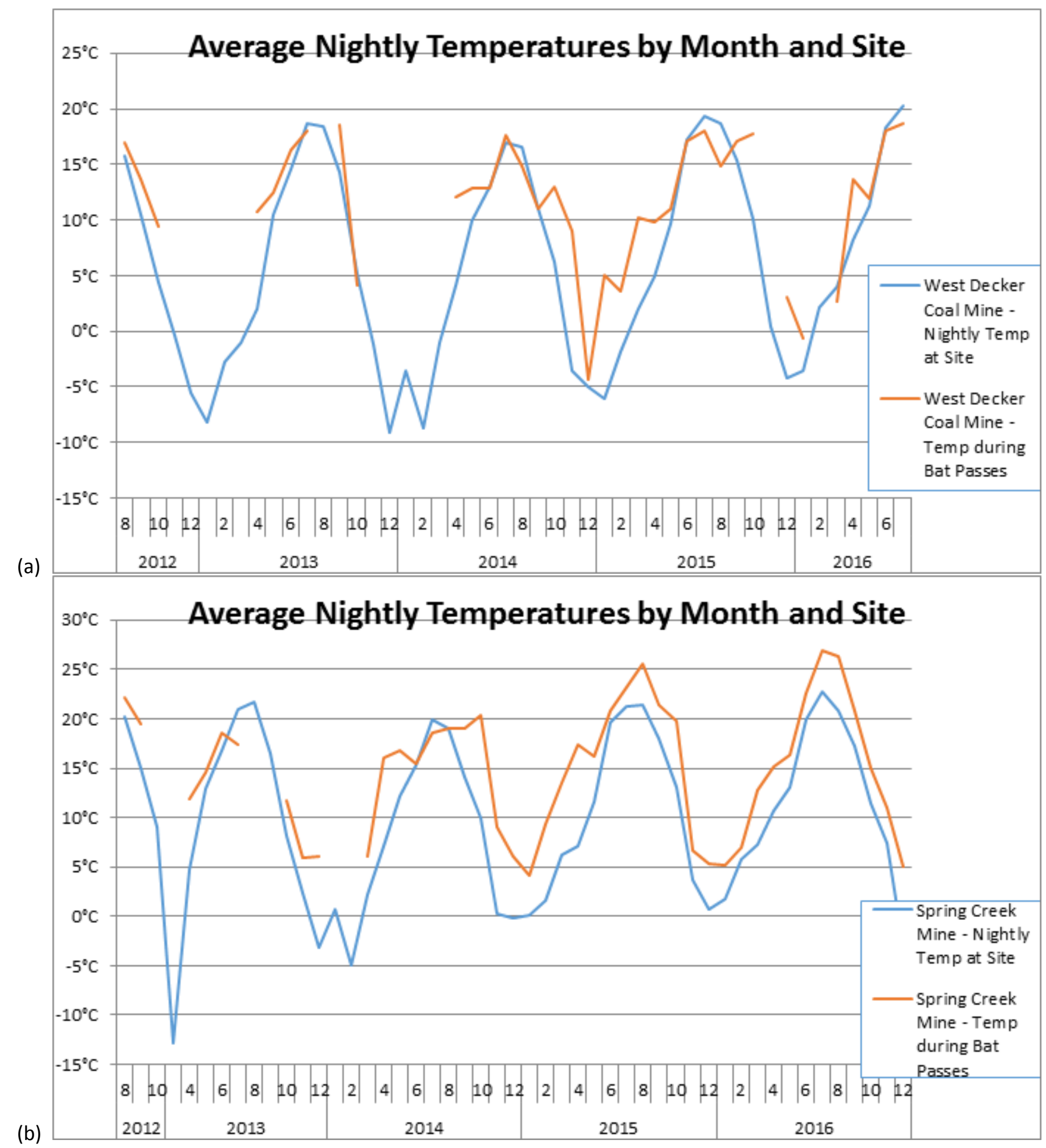




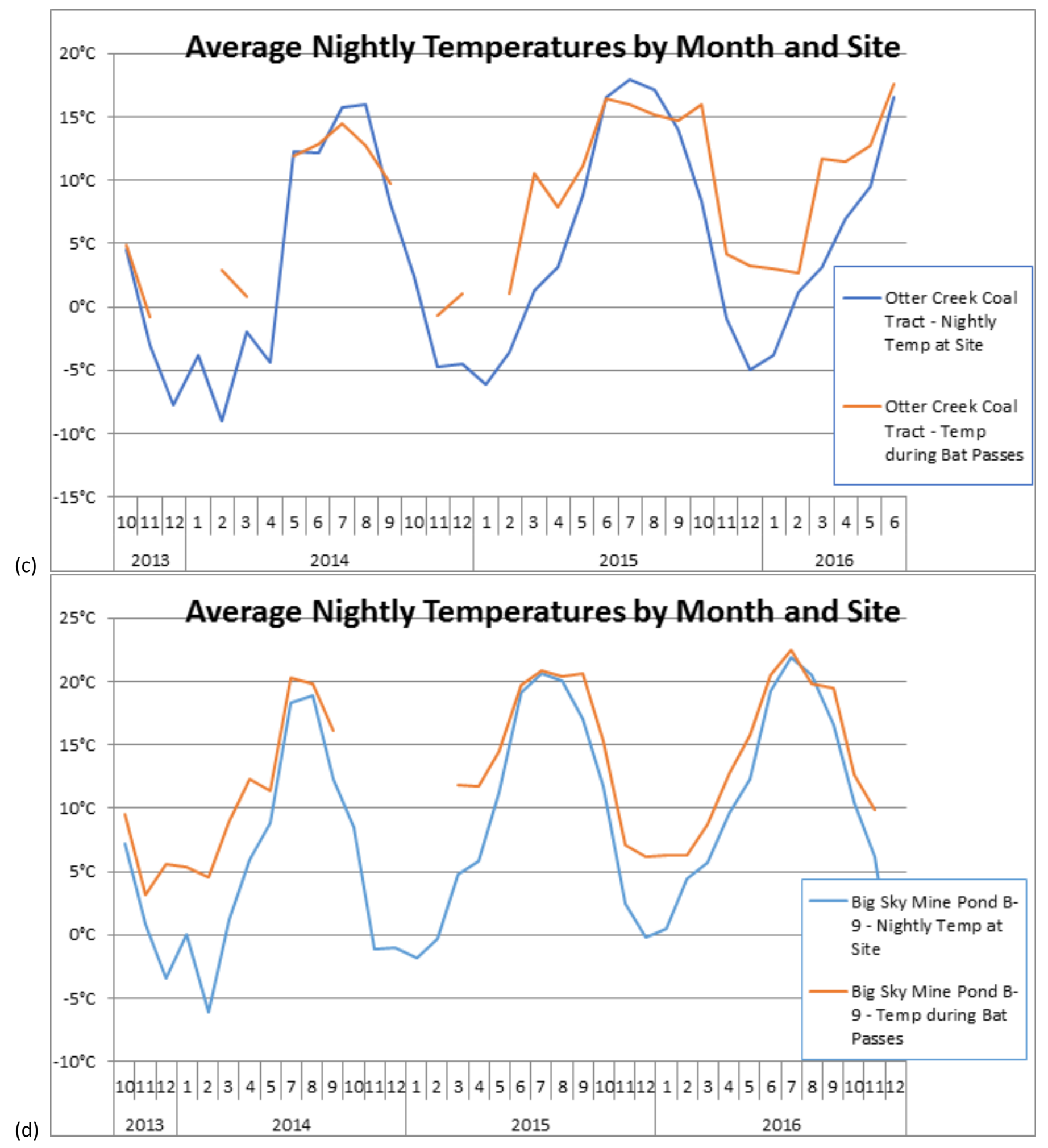




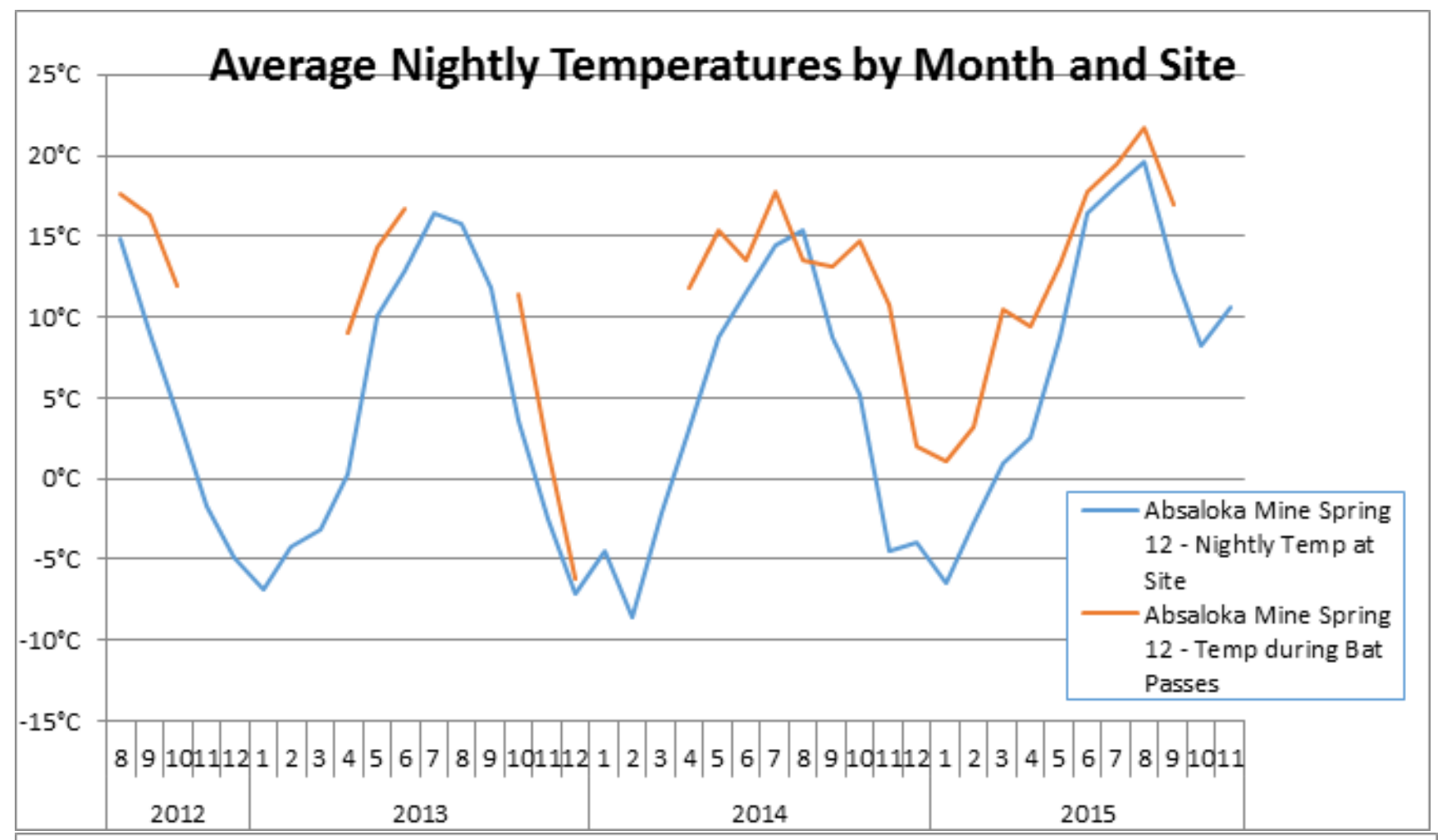

(e)

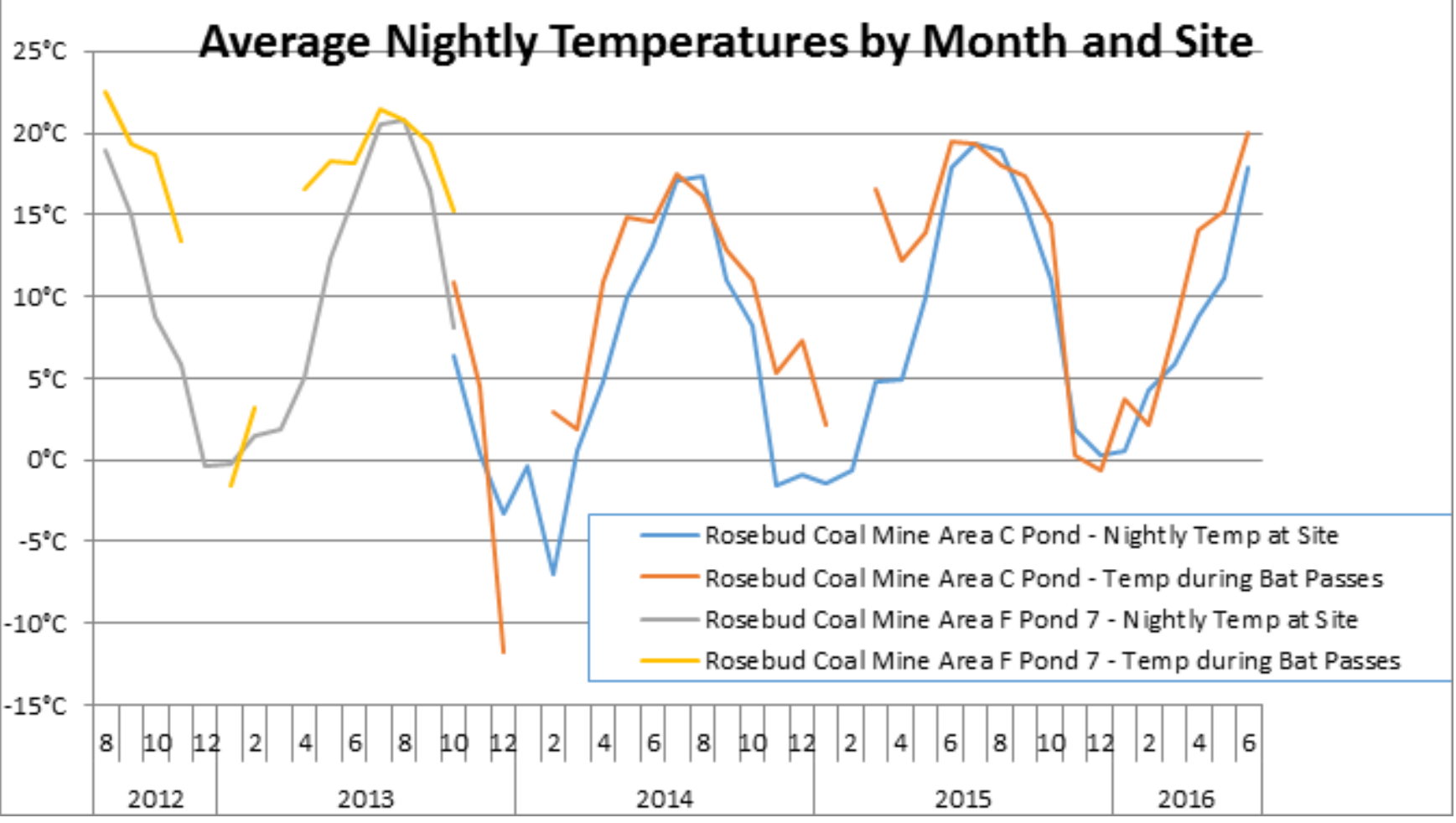




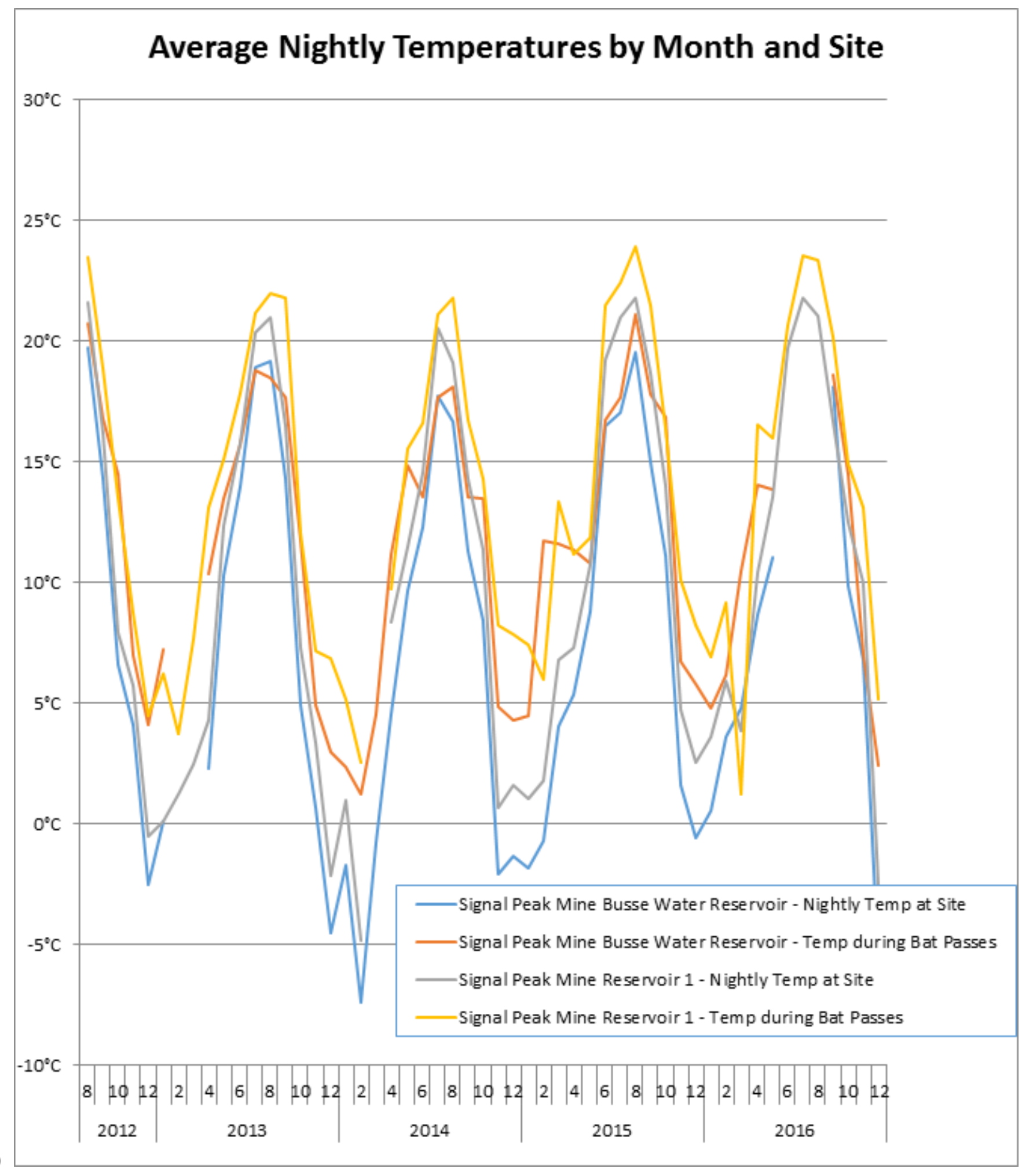


Figure 14. Percent of nightly hours with average background temperatures (blue) and average temperatures associated with bat passes (red) for the closest weather station at: (a) West Decker Coal Mine, (b) Spring Creek Mine, (c) Otter Creek Coal Tract, (d) Big Sky Mine, (e) Absaloka Mine, (f) Rosebud Mine, (g) Signal Peak Mine. Numbers are lower ends of ${ }^{\circ} \mathrm{C}$ temperature bins.

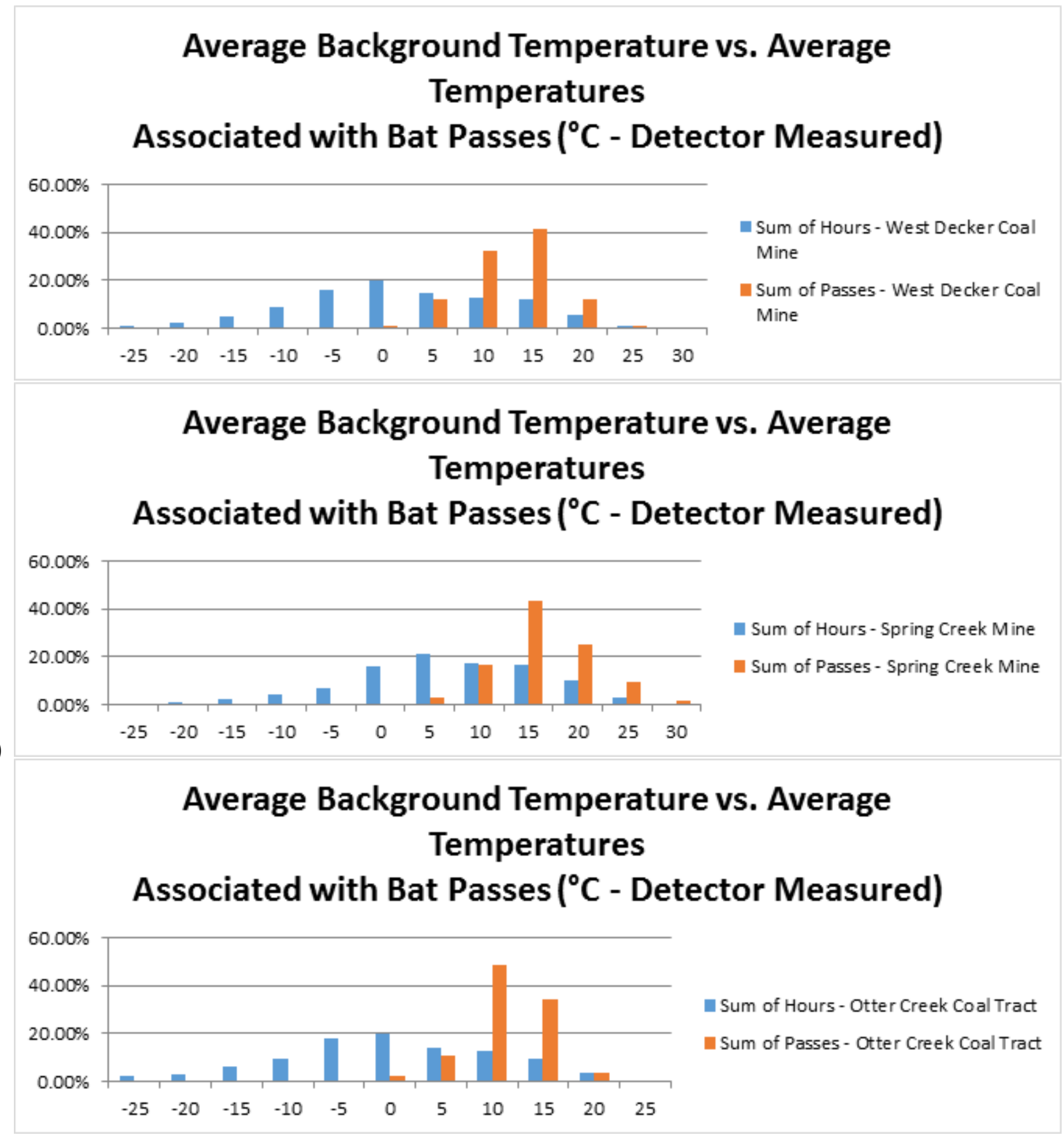


(d)

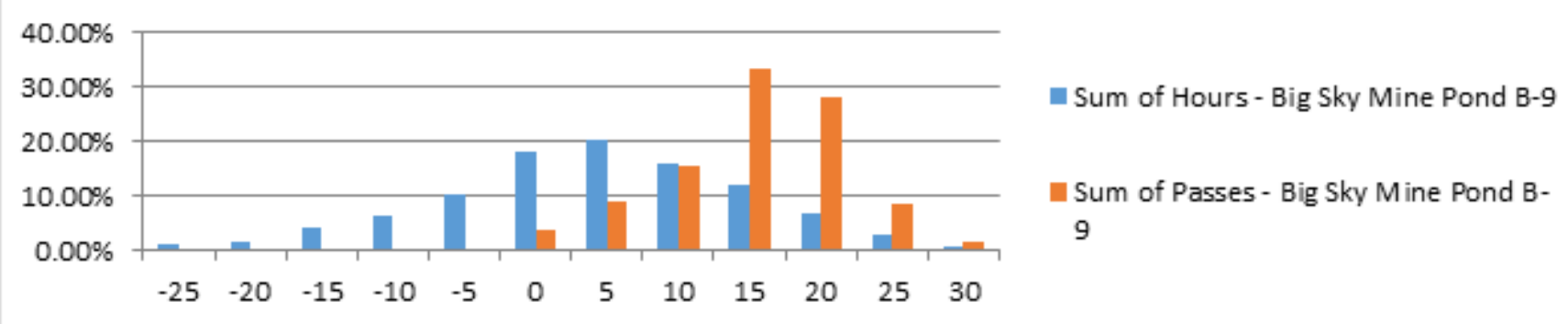

(e)

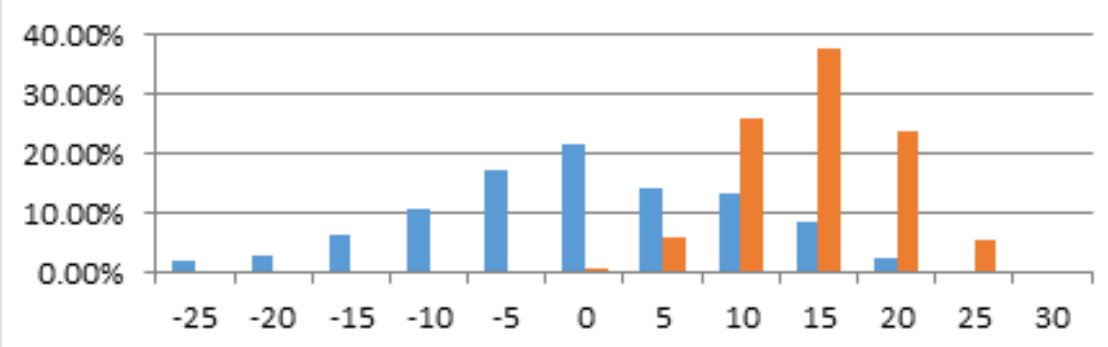

\section{Average Background Temperature vs. Average} Temperatures

Associated with Bat Passes $\left({ }^{\circ} \mathrm{C}\right.$ - Detector Measured)

\section{Average Background Temperature vs. Average} Temperatures

\section{Associated with Bat Passes $\left({ }^{\circ} \mathrm{C}\right.$ - Detector Measured)}

$$
\begin{aligned}
& \text { Sum of Hours - Absaloka Mine Spring } \\
& 12 \\
& \text { Sum of Passes - Absaloka M ine Spring } \\
& 12
\end{aligned}
$$

Average Background Temperature vs. Average Temperatures

Associated with Bat Passes $\left({ }^{\circ} \mathrm{C}\right.$ - Detector Measured)

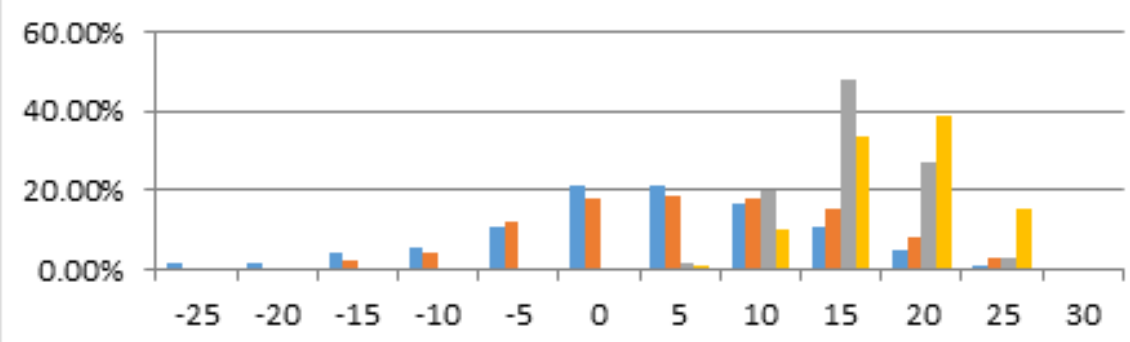

- Sum of Hours - Rosebud Coal Mine Area $\mathrm{C}$ Pond

- Sum of Hours - Rosebud Coal Mine Area $\mathrm{F}$ Pond 7

- Sum of Passes - Rosebud Coal Mine Area $\mathrm{C}$ Pond

Sum of Passes - Rosebud Coal Mine Area F Pond 7 (f) 


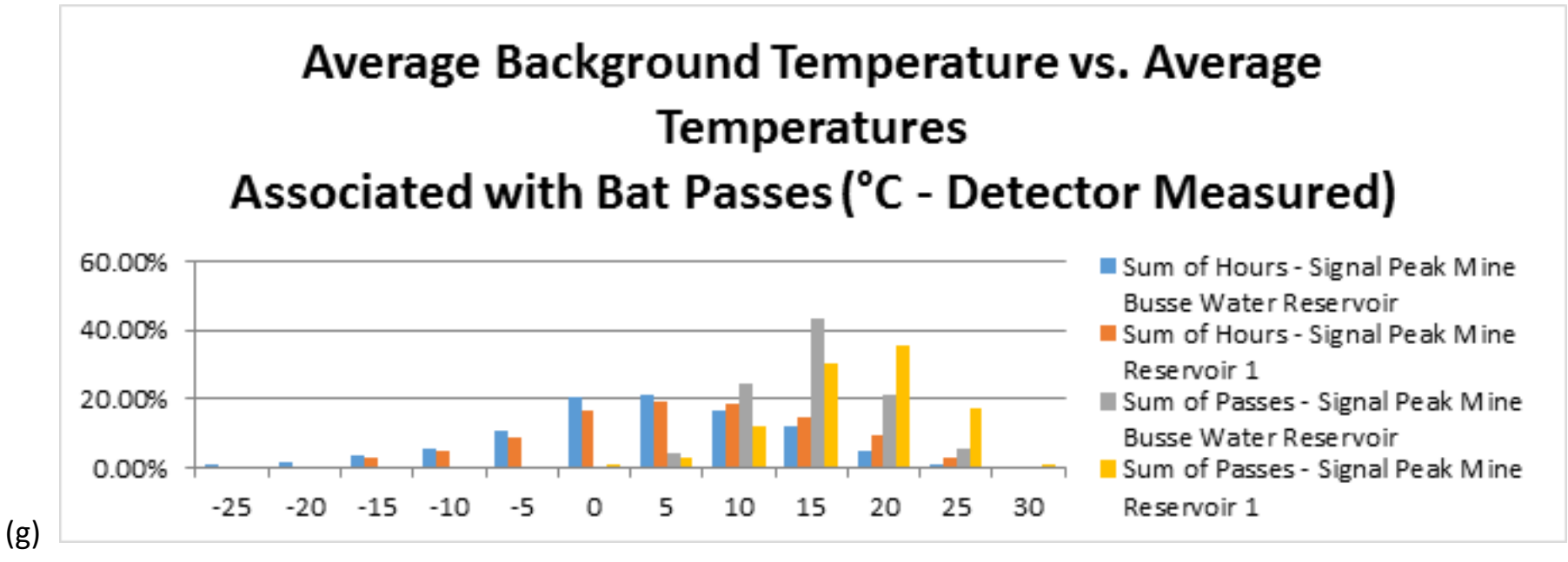


Figure 15. Percent of nightly hours with average background temperatures (blue) and average temperatures associated with bat passes (red) across the regional network of detectors. Numbers are lower ends of ${ }^{\circ} \mathrm{C}$ temperature bins. Of the 572,897 hours that detectors have been deployed, temperature data was available from nearby weather stations for 559,321 hours $(98 \%)$. Note that some detectors were up to 43 kilometers from the weather station where temperatures were recorded $(X=15.9$ $\mathrm{km}, \mathrm{SD}=10.5 \mathrm{~km})$.

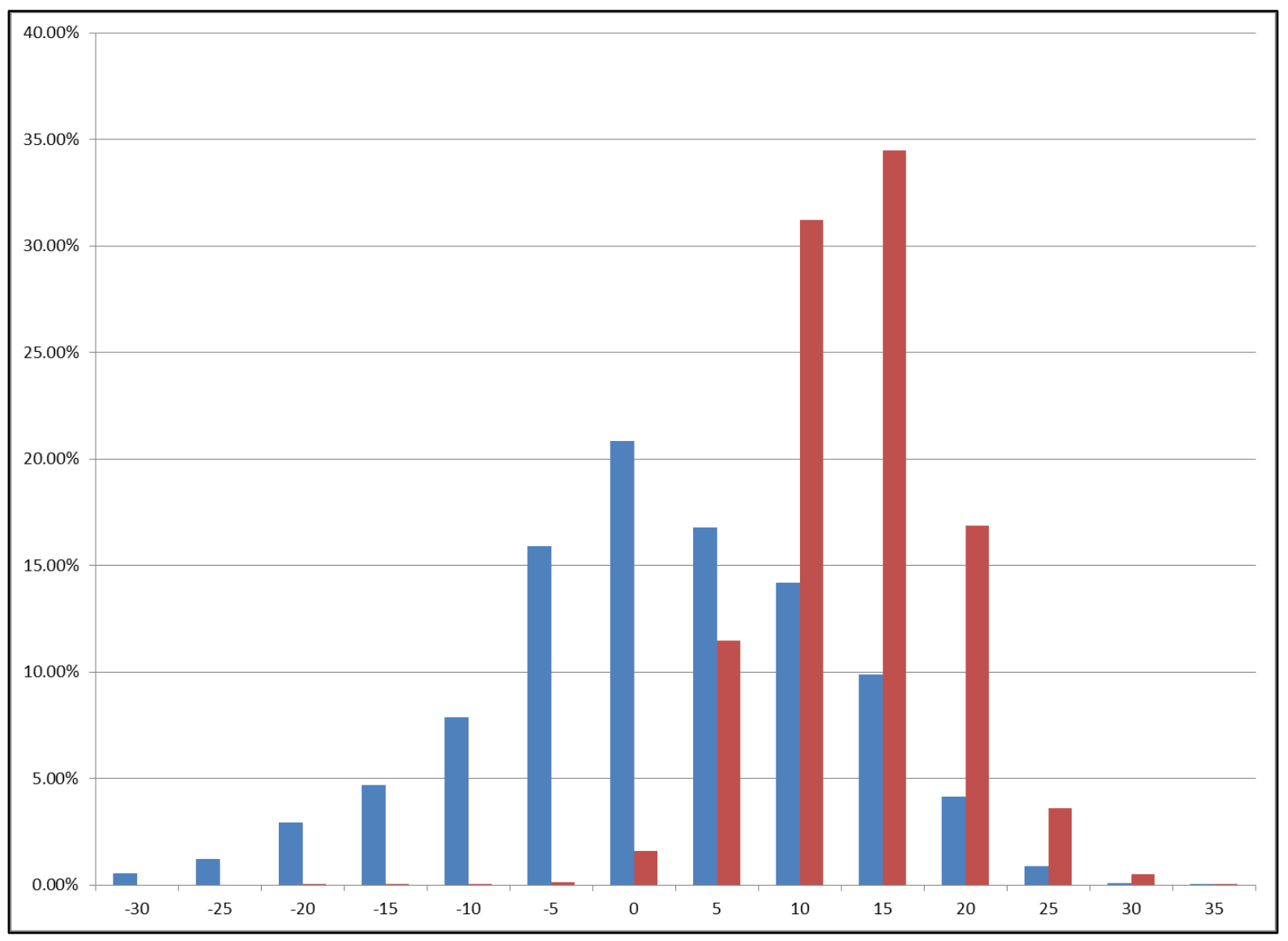


Figure 16. Percent of hours with average background wind speeds (blue) and average wind speeds associated with bat passes (red) at the closest associated weather station at: (a) West Decker Coal Mine, (b) Spring Creek Mine, (c) Otter Creek Coal Tract, (d) Big Sky Mine, (e) Absaloka Mine, (f) Rosebud Mine, (g) Signal Peak Mine. Wind speed categories are meters per second. Numbers are lower ends of wind speed bins.

\section{Average Background Wind Speeds vs. Average Wind Speeds Associated with Bat Passes (m/s - Wind Site}

(a)

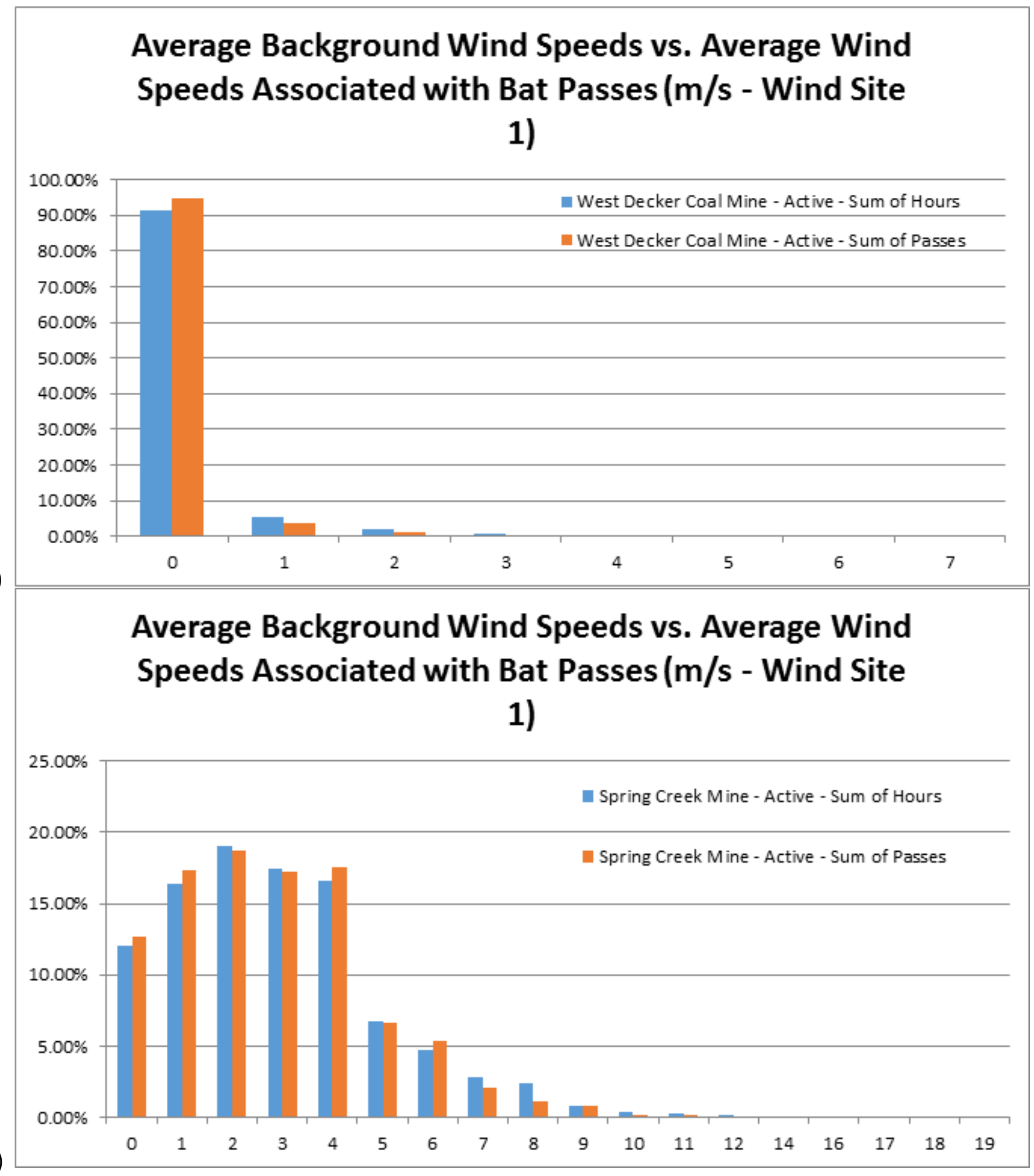




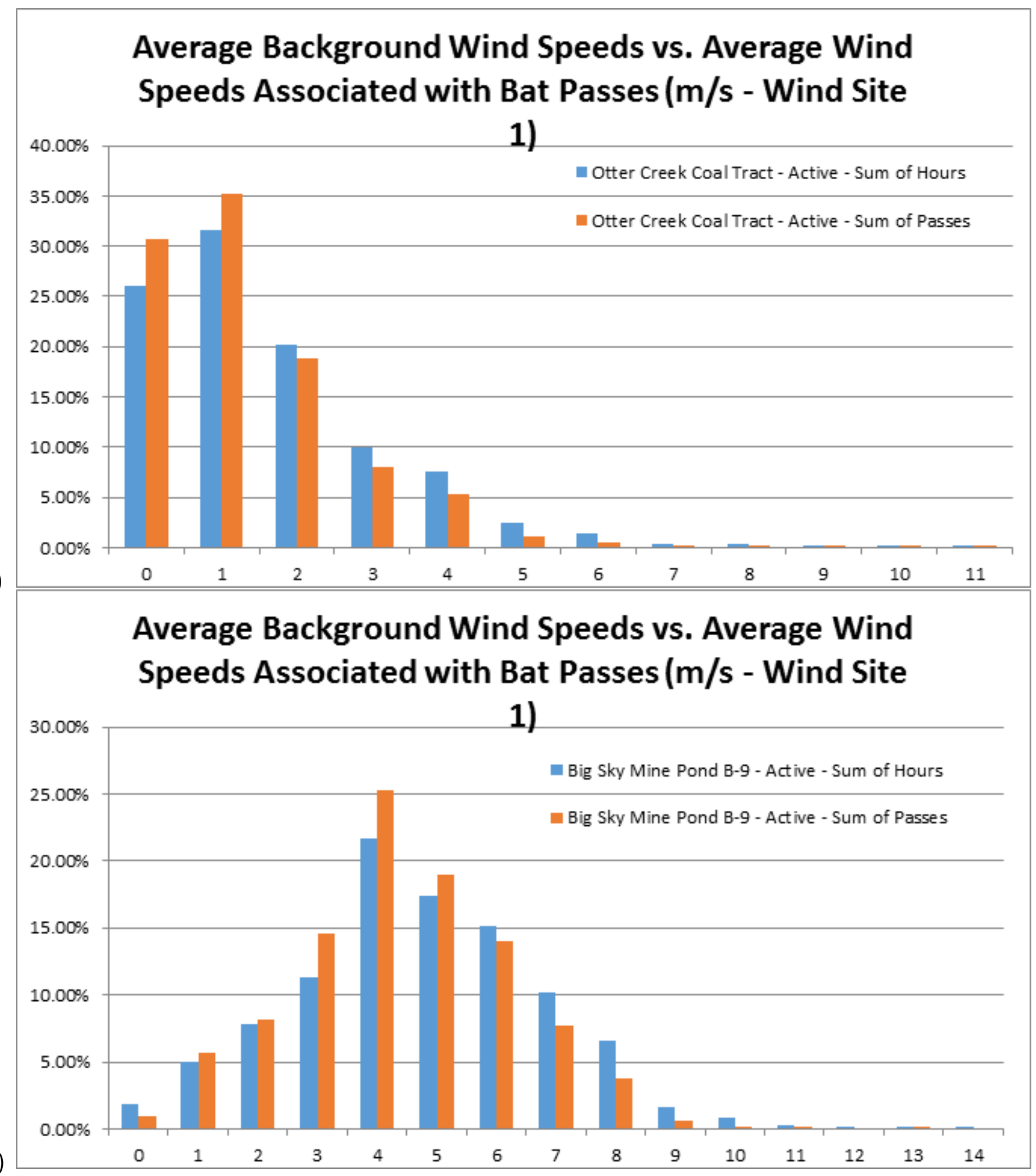




\section{Average Background Wind Speeds vs. Average Wind Speeds Associated with Bat Passes (m/s - Wind Site}

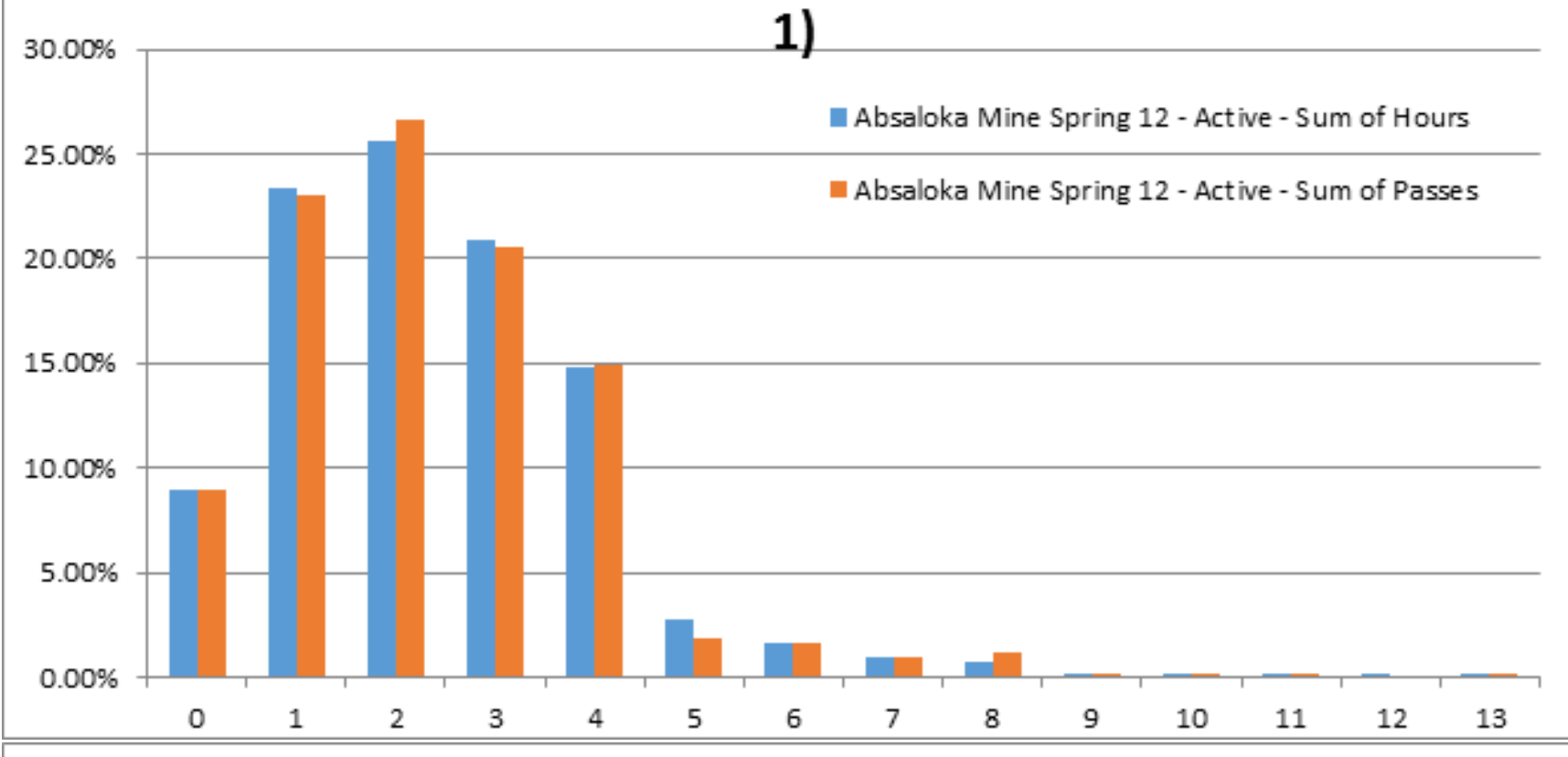

(e)

\section{Average Background Wind Speeds vs. Average Wind Speeds Associated with Bat Passes (m/s - Wind Site} 1)

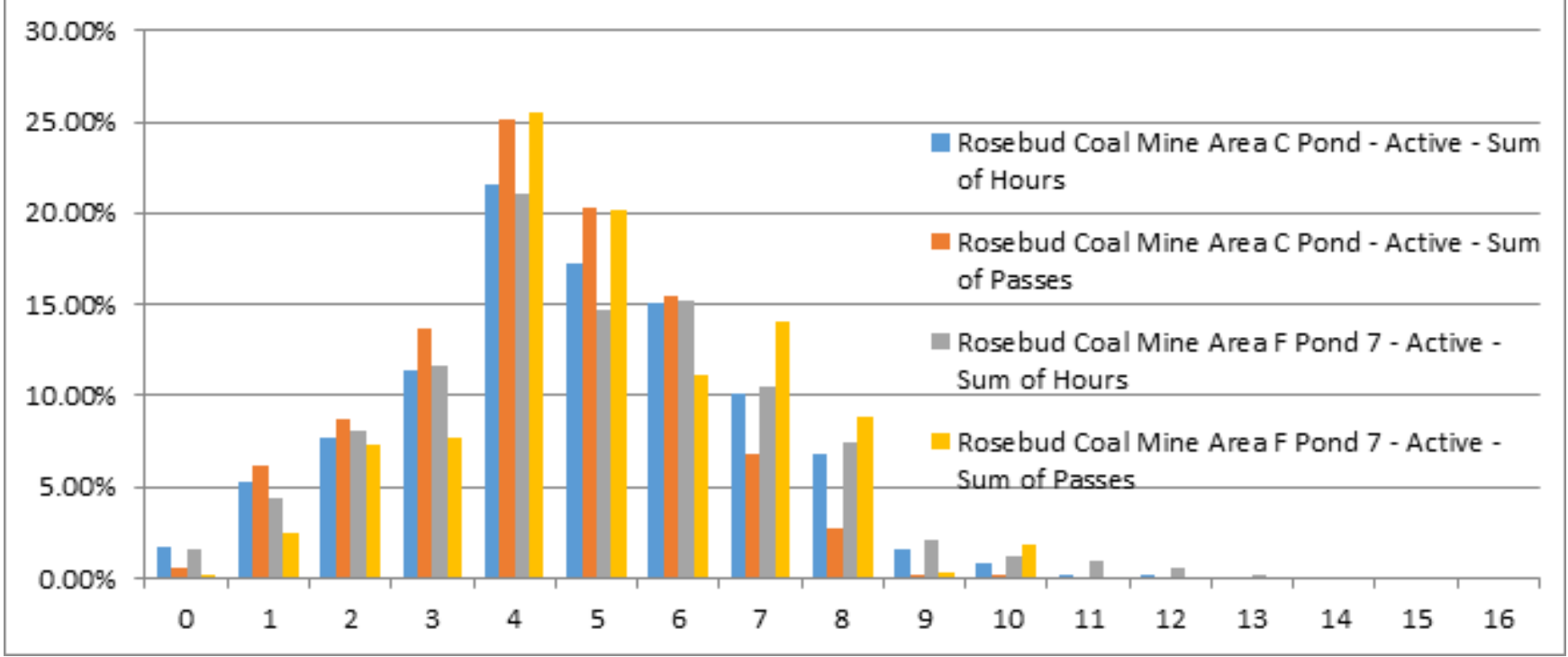




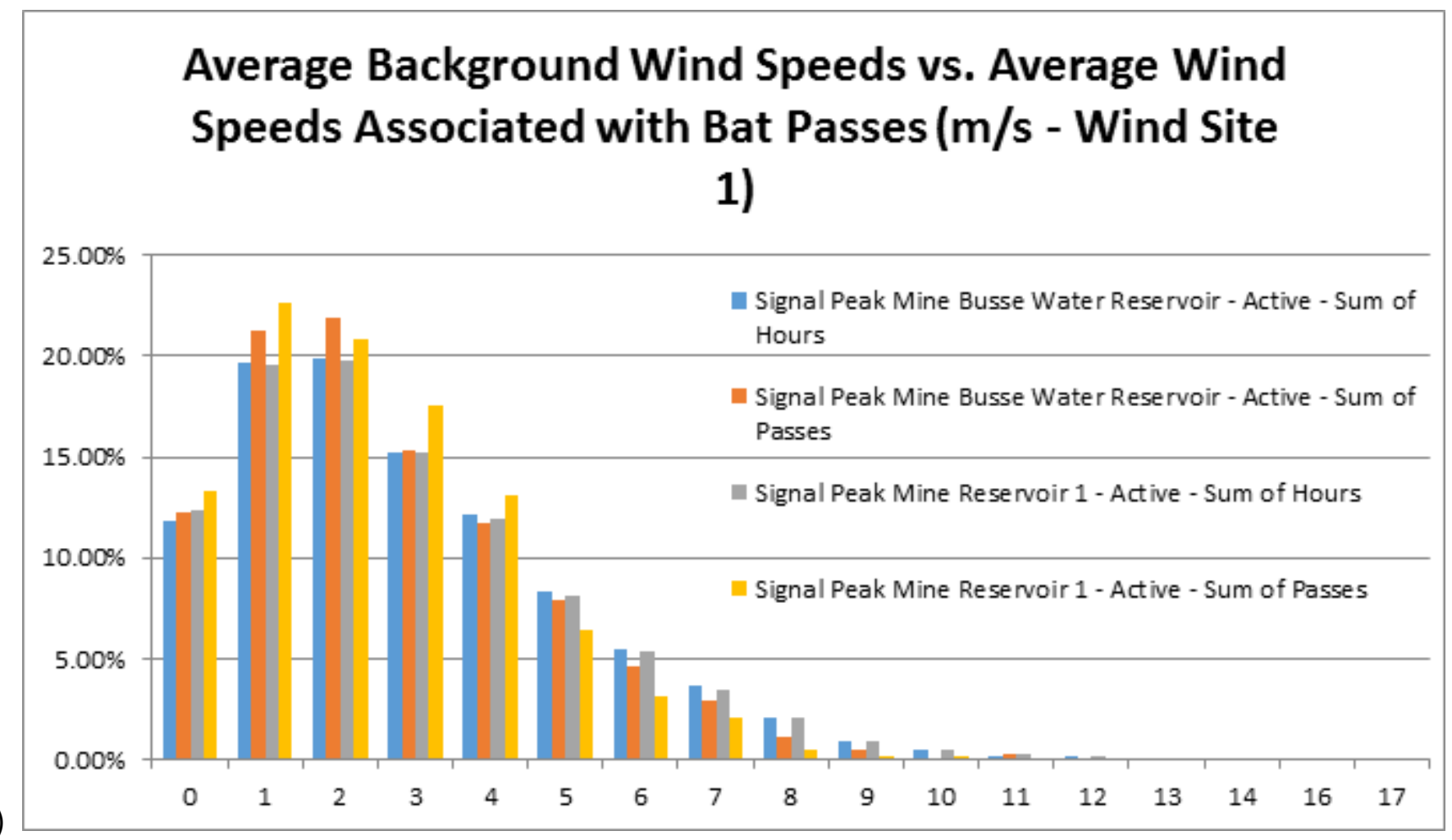


Figure 17. Percent of hours with average background wind speeds (blue) and average wind speeds associated with bat passes (red) across the regional network of detectors. Wind speed categories are meters per second. Numbers are lower ends of wind speed bins. Of the 572,897 hours that detectors have been deployed, wind speed data was available from nearby weather stations for 556,720 hours $(97 \%)$. Note that some detectors were up to 43 kilometers from the weather station where wind speeds were recorded $(X=17.9 \mathrm{~km}, \mathrm{SD}=10.5 \mathrm{~km})$.

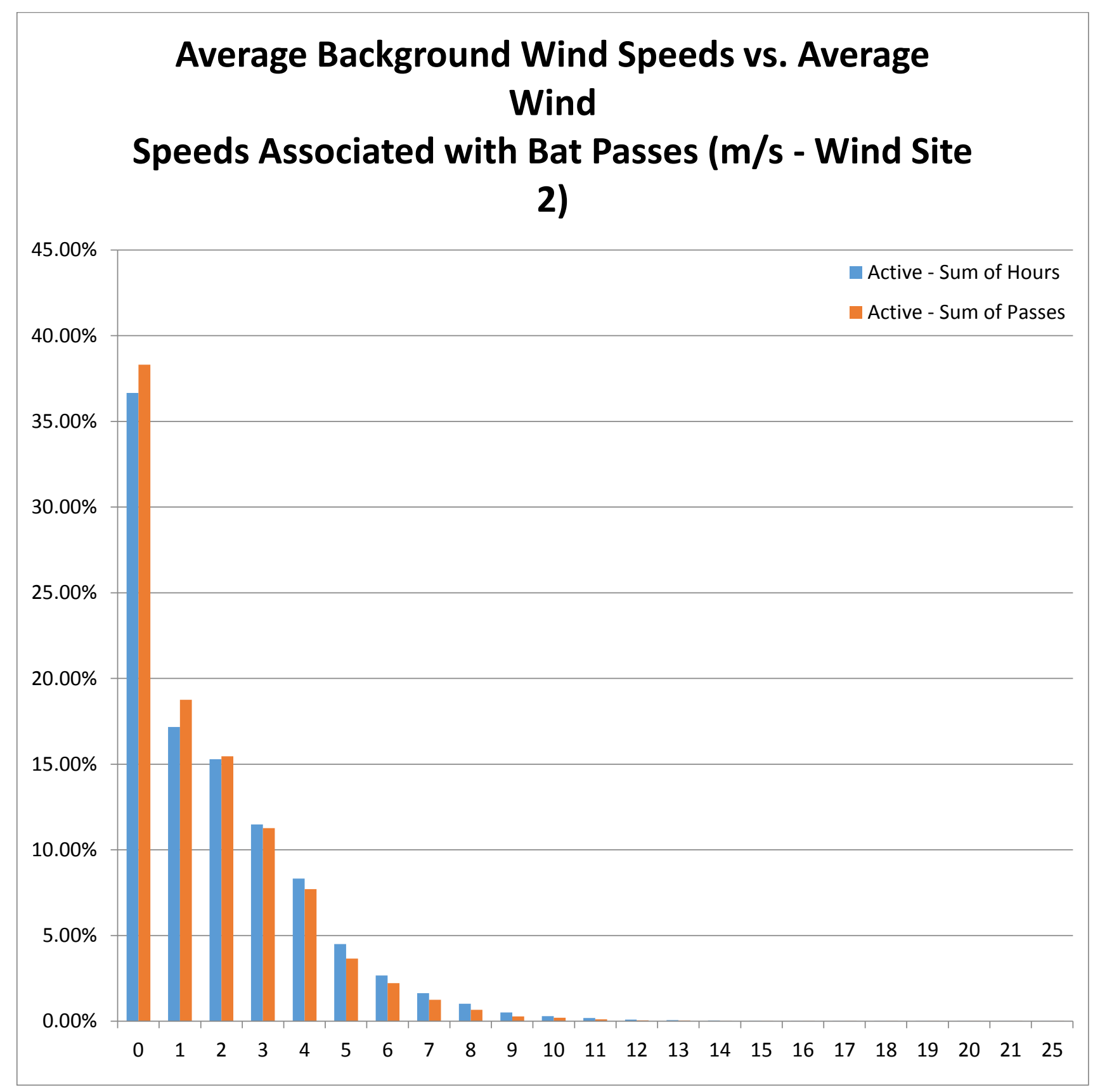


Figure 18. Percent of hours with background barometric pressure changes (blue) and barometric pressure changes associated with bat passes (red) at the closest associated weather station at: (a) West Decker Coal Mine, (b) Spring Creek Mine, (c) Otter Creek Coal Tract, (d) Big Sky Mine, (e) Absaloka Mine, (f) Rosebud Mine. Numbers shown are the lower ends of categories of millibars of change per hour. Pressure data were unavailable for both Signal Peak Mine Detectors, so this relationship is not shown for detectors places at this mine.

(a)

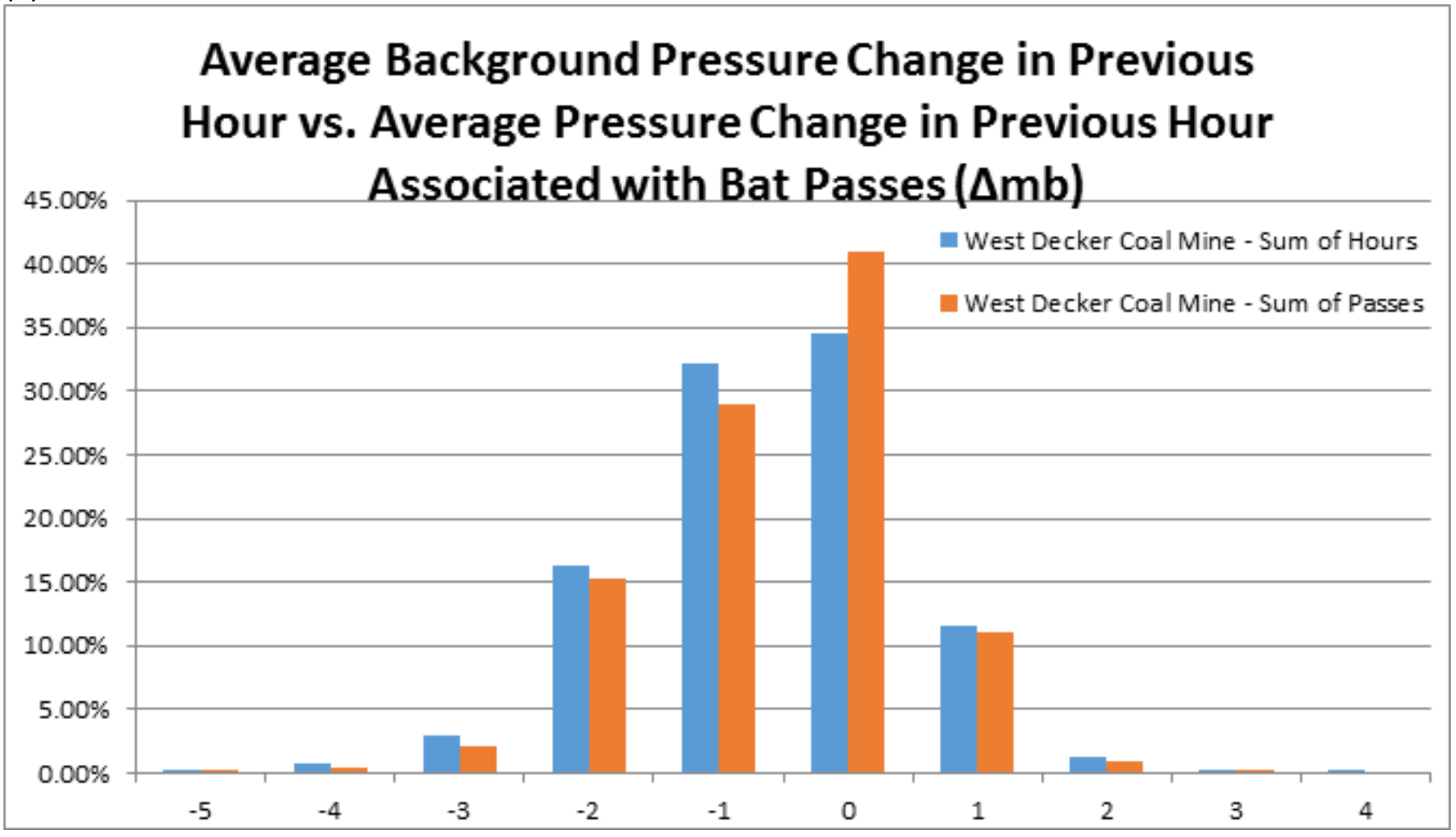

(b)

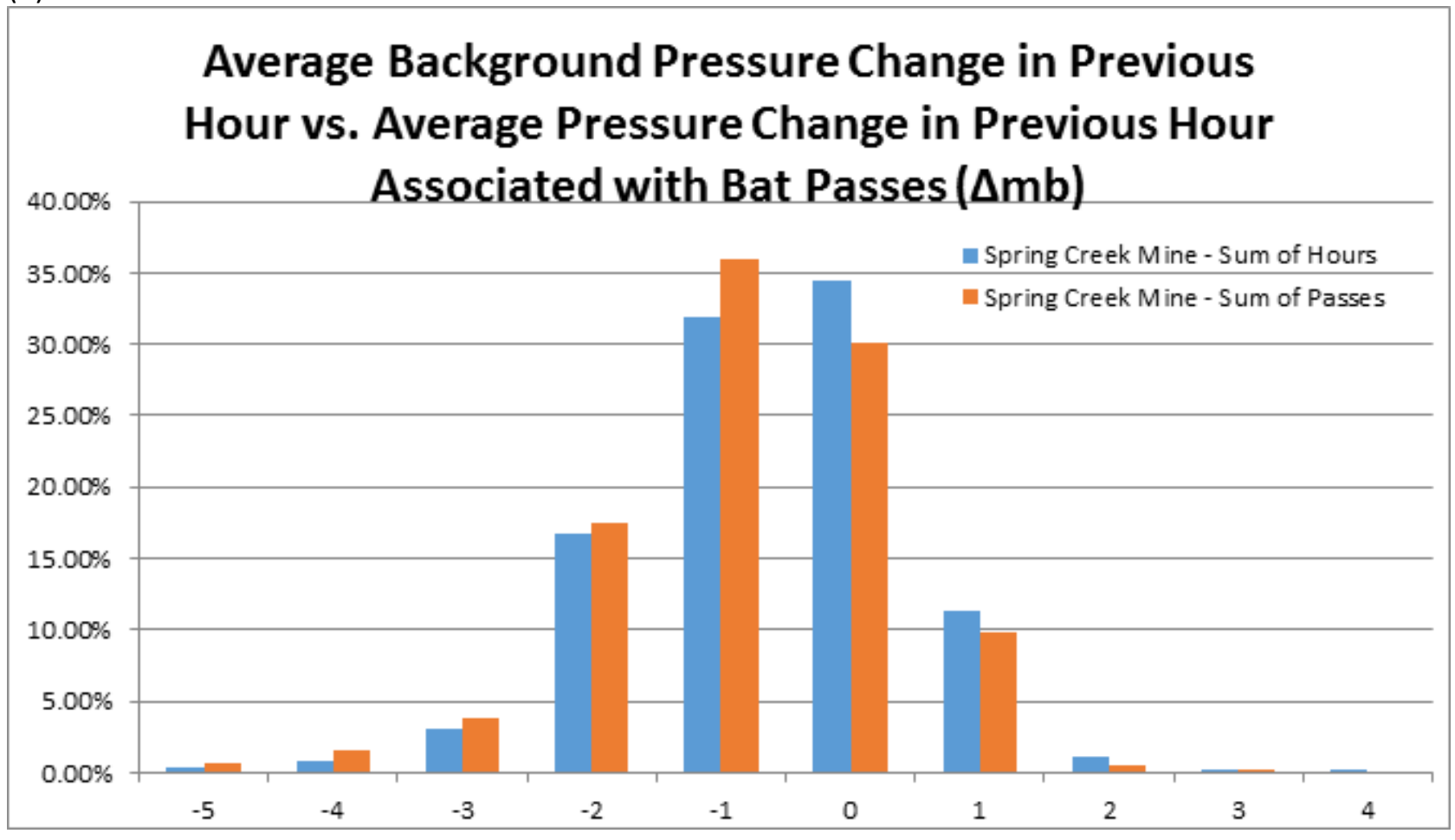


(c)

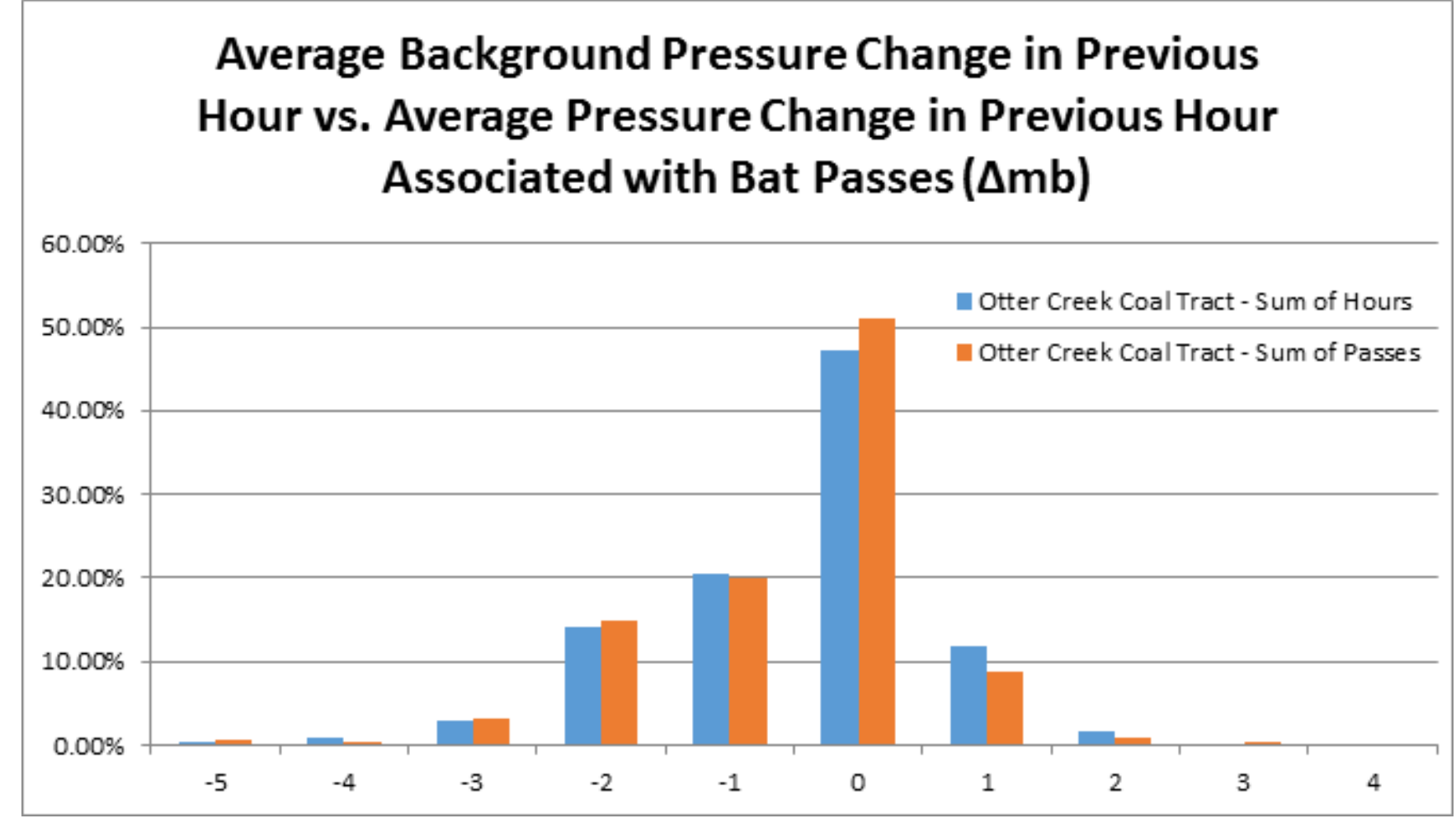

(d)

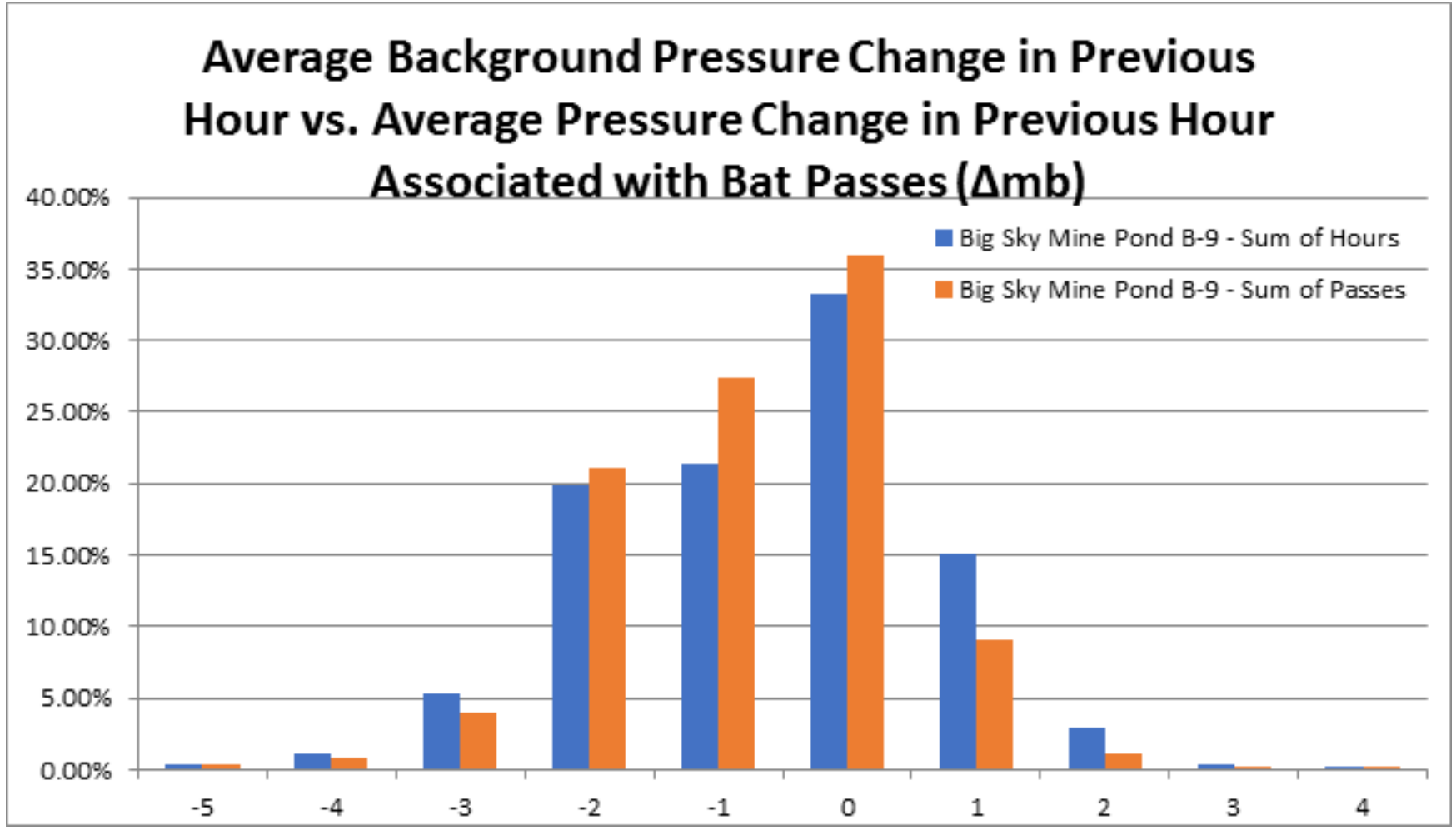


(e)

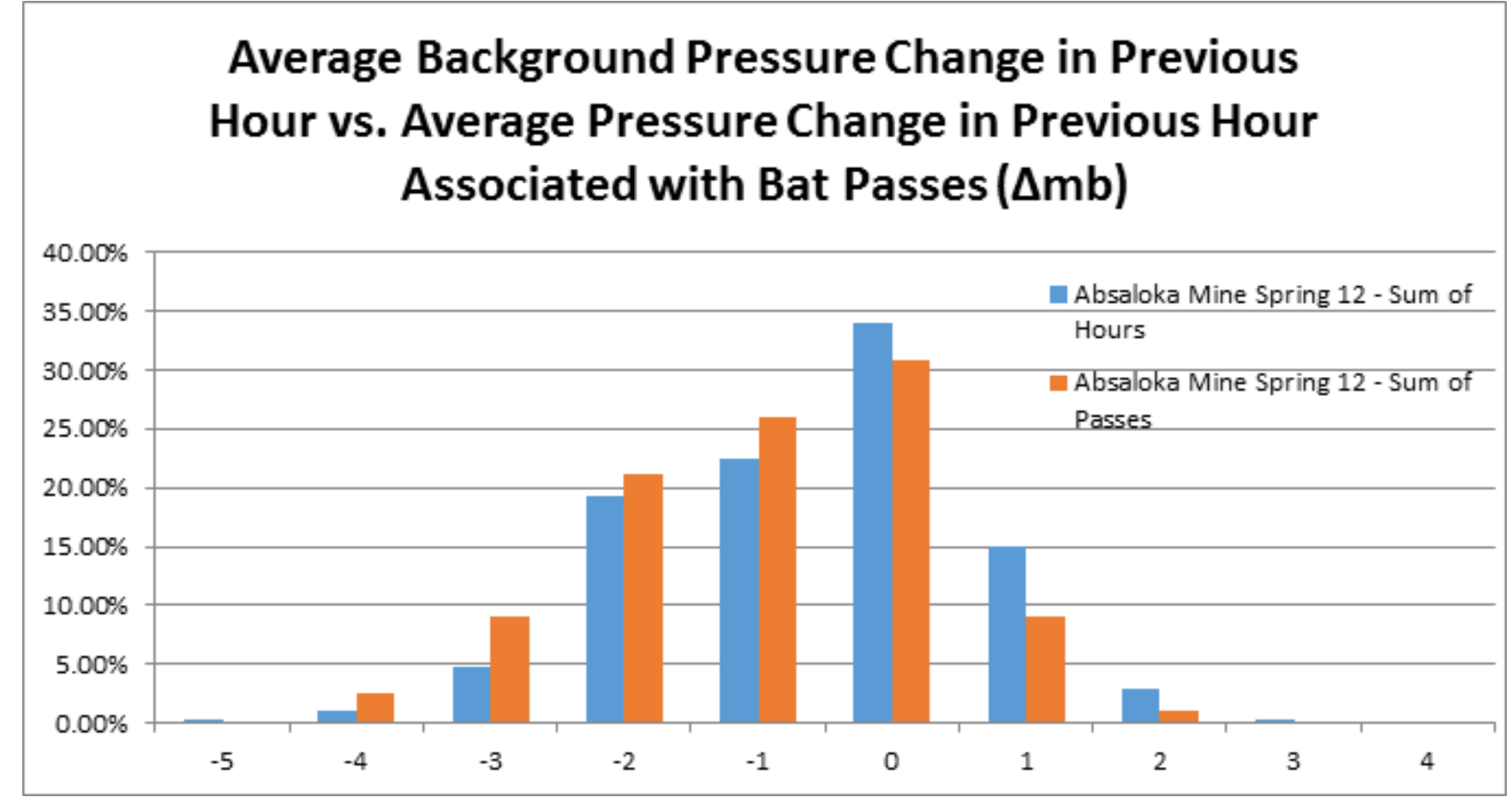

(f)

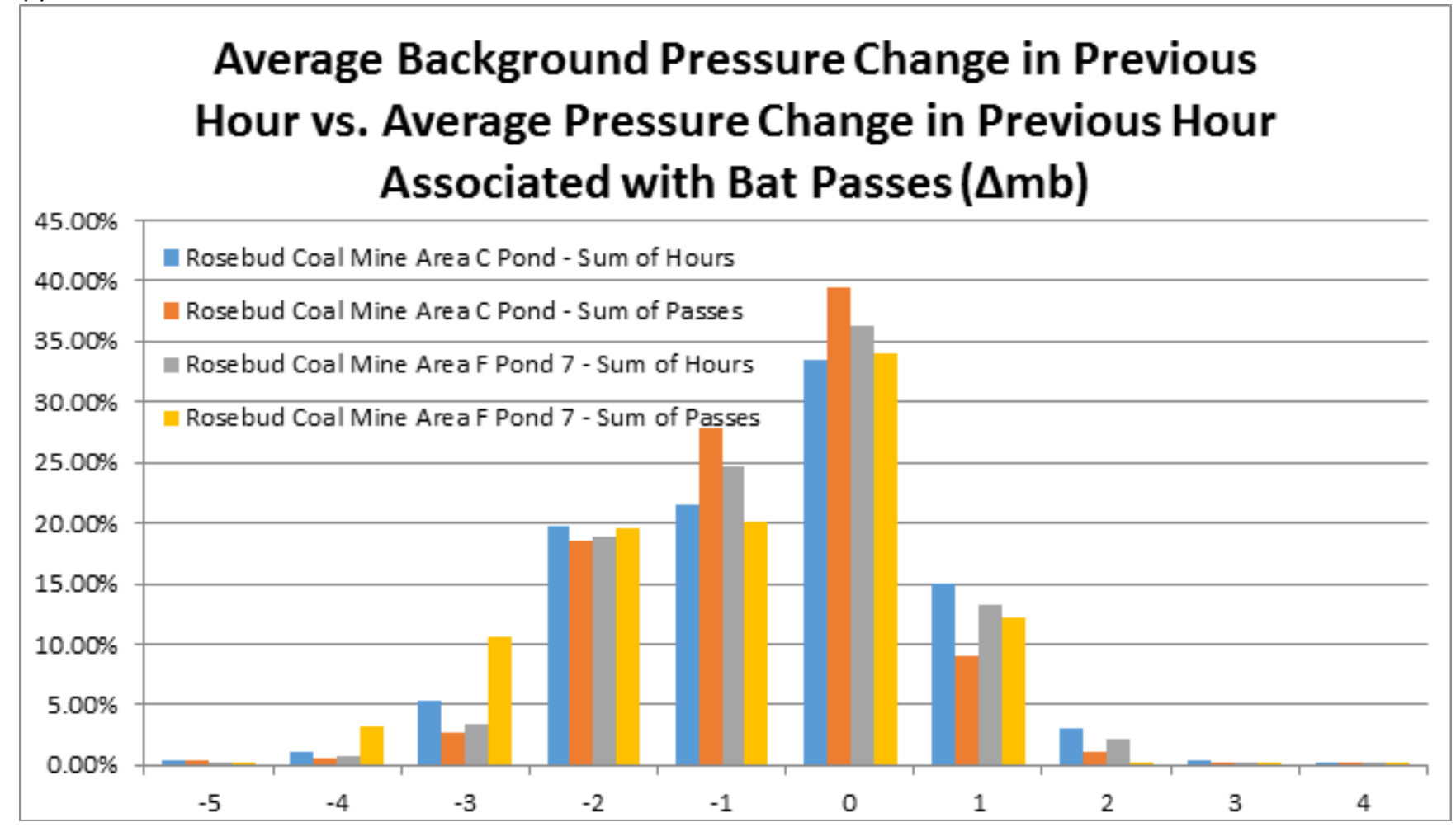


Figure 19. Percent of hours with background barometric pressure changes (blue) and barometric pressure changes associated with bat passes (red) across the regional network of detectors. Numbers shown are the lower ends of categories of millibars of change per hour. Of the 572,897 hours that detectors have been deployed, barometric pressure data was available from nearby weather stations for 517,468 hours $(90 \%)$. Note that some detectors were up to 94 kilometers from the weather station where barometric pressures were recorded $(X=37.1 \mathrm{~km}, S D=21.5$ $\mathrm{km})$.

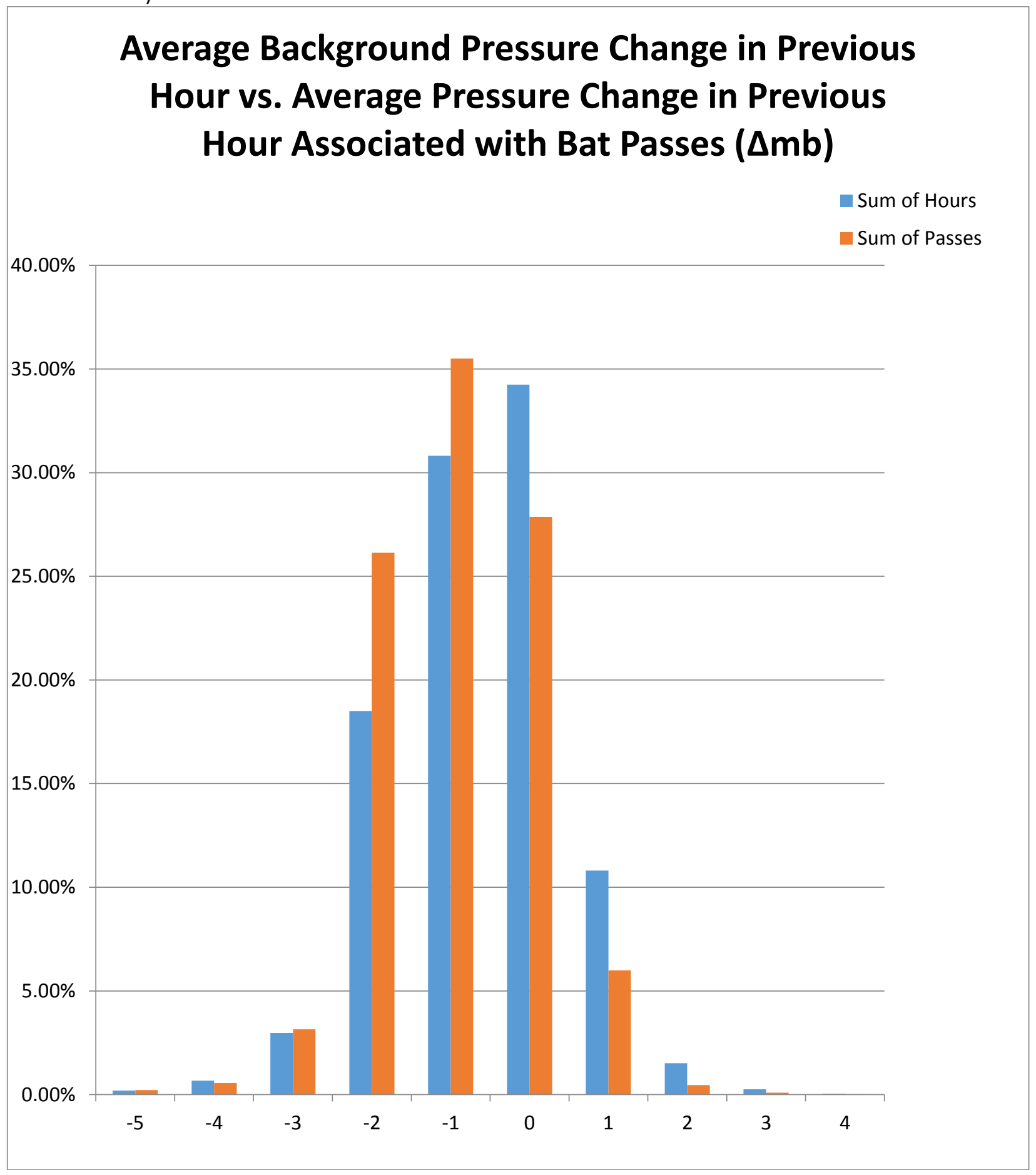


Figure 20. Percent of background hours (blue) and hours with bat passes (red) with (1) and without (0) precipitation at the closest weather station to: (a) West Decker Coal Mine, (b) Spring Creek Mine, (c) Otter Creek Coal Tract, (d) Big Sky Mine, (e) Absaloka Mine, (f) Rosebud Mine. Precipitation data was not available for the Signal Peak Mine, so the figure for this site is not provided.

\section{Background Hours vs. Bat Passes With (1) or Without (0) Precipitation in the Previous Hour}

(a)

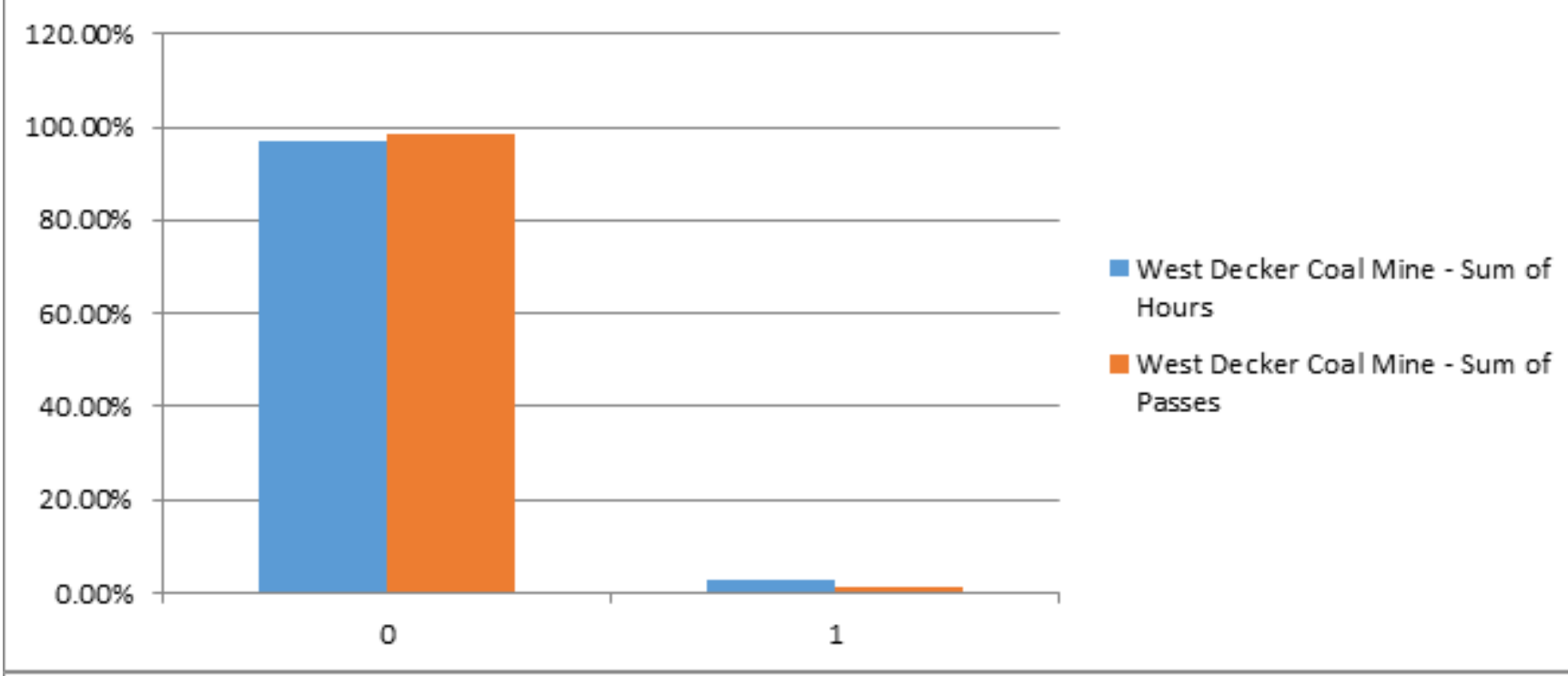

(b)

\section{Background Hours vs. Bat Passes With (1) or Without (0) Precipitation in the Previous Hour}

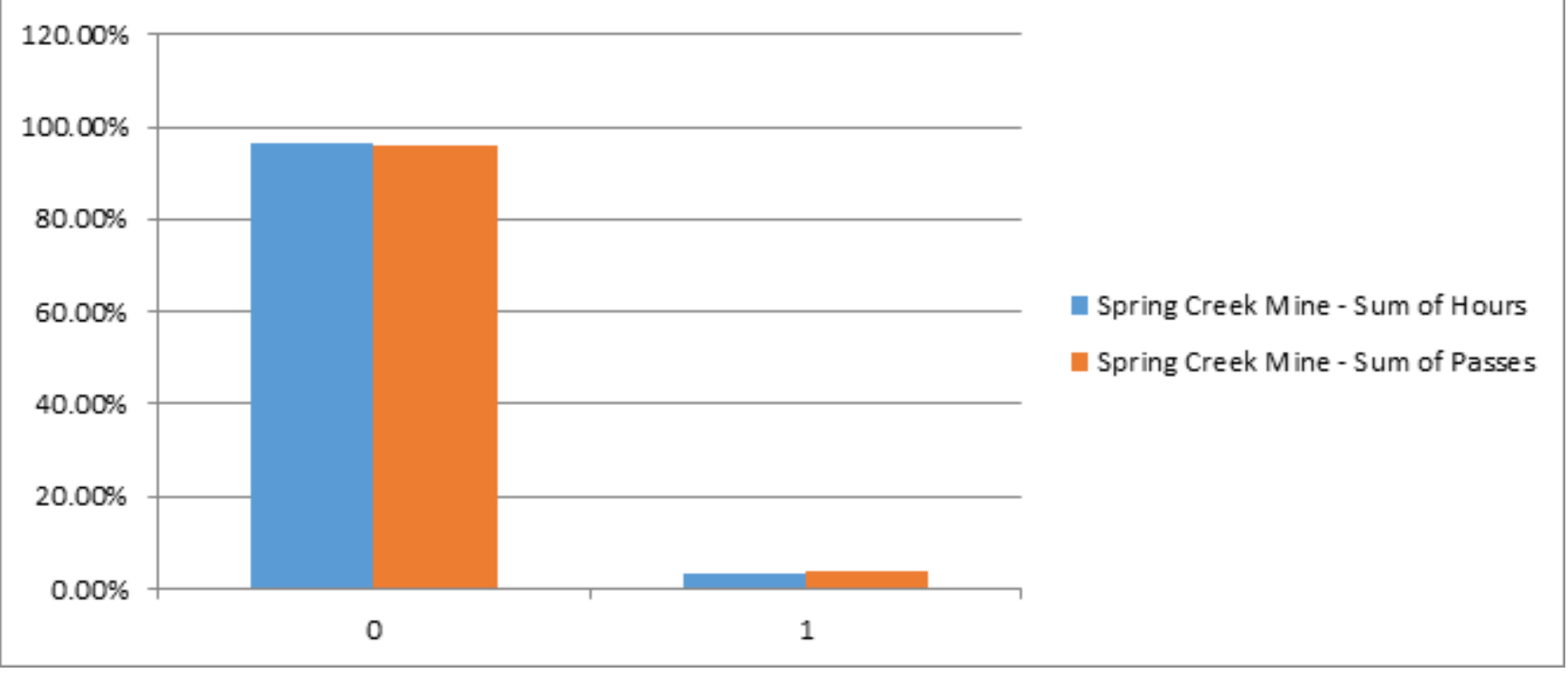




\section{Background Hours vs. Bat Passes With (1) or Without}

(0)

\section{Precipitation in the Previous Hour}

(c)

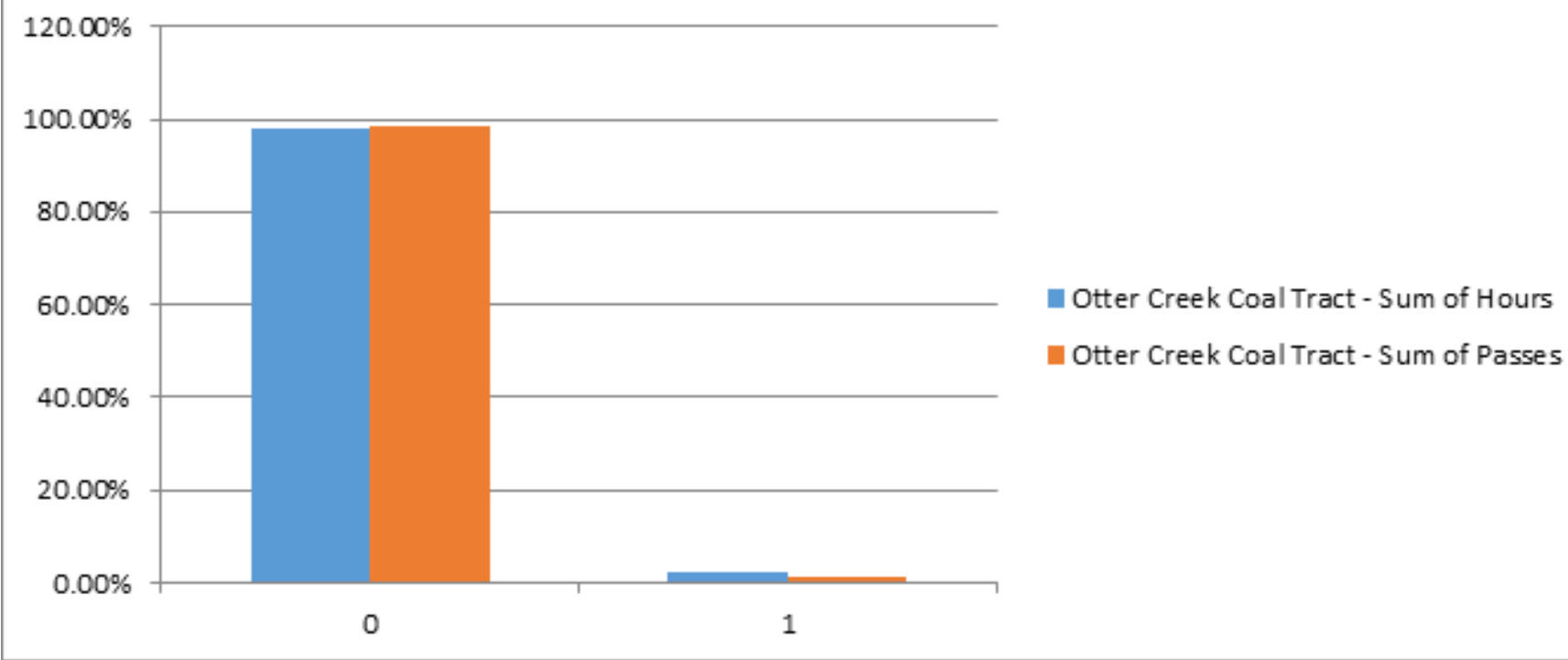

\section{Background Hours vs. Bat Passes With (1) or Without (0)} Precipitation in the Previous Hour

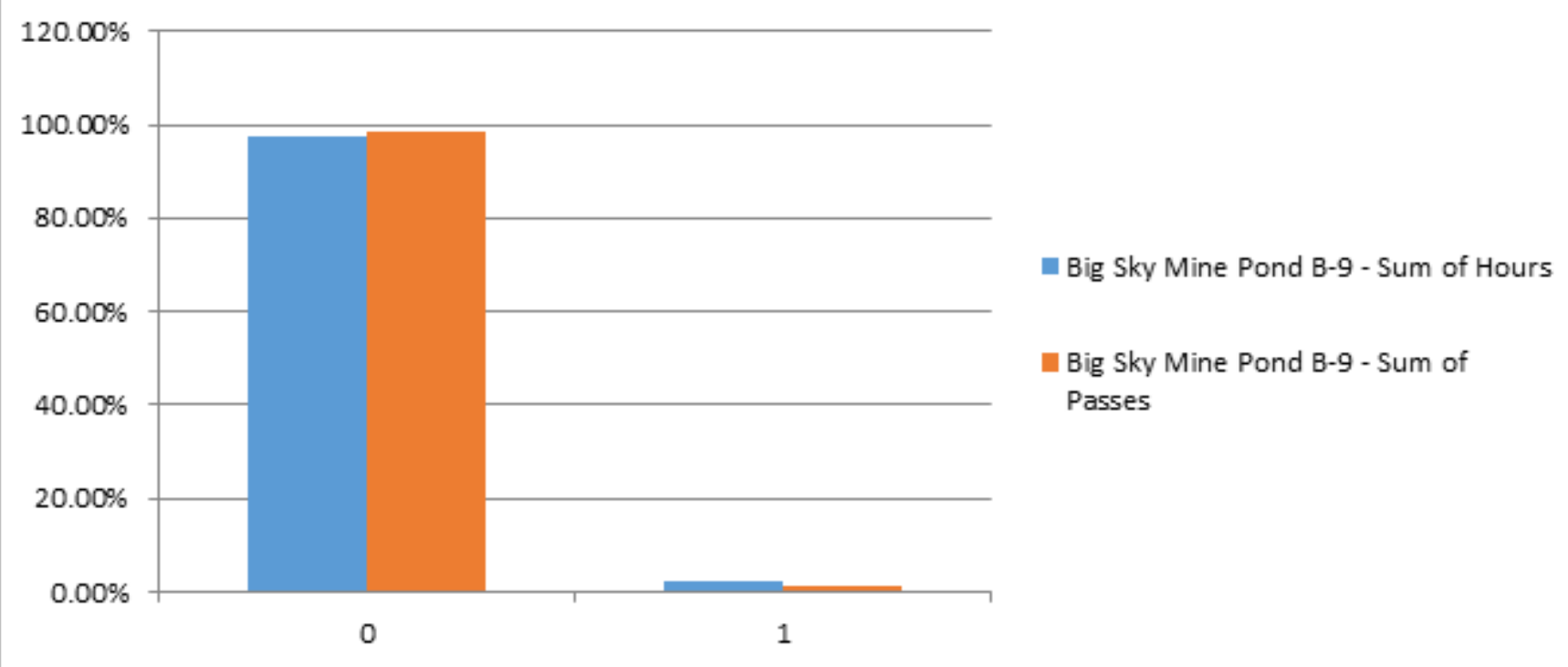




\section{Background Hours vs. Bat Passes With (1) or Without (0) Precipitation in the Previous Hour}

(e)

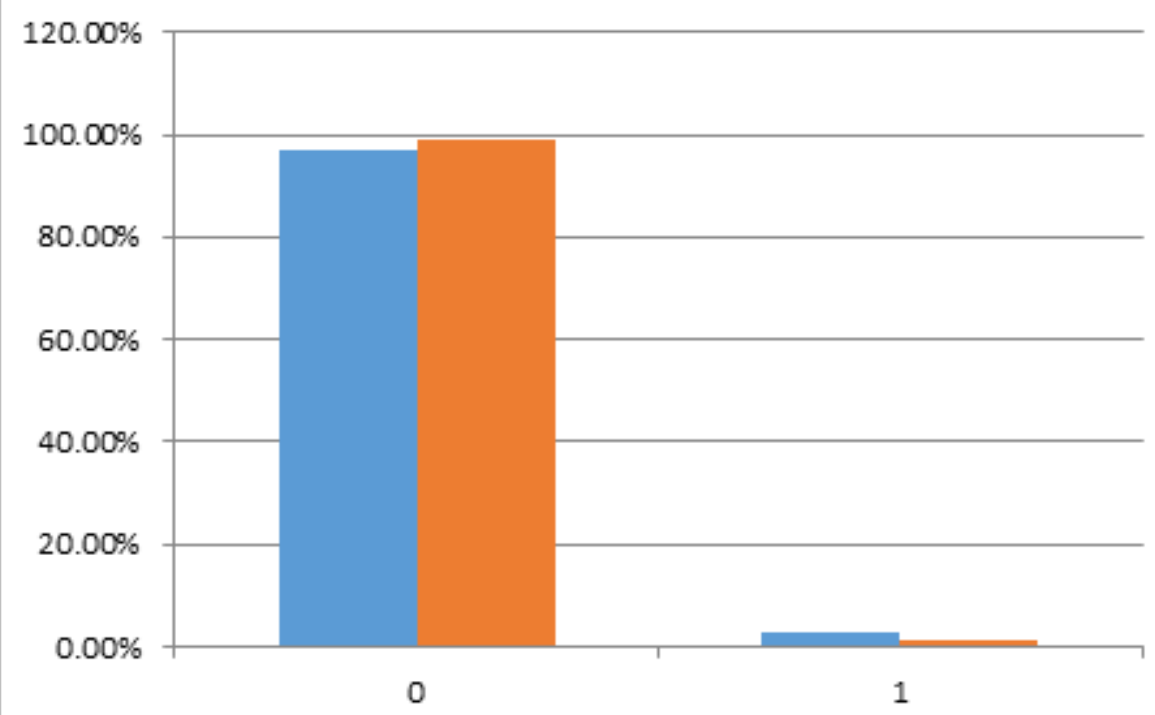

Absaloka Mine Spring 12 - Sum of Hours

Absaloka Mine Spring 12 - Sum of Passes

\section{Background Hours vs. Bat Passes With (1) or Without (0)}

Precipitation in the Previous Hour

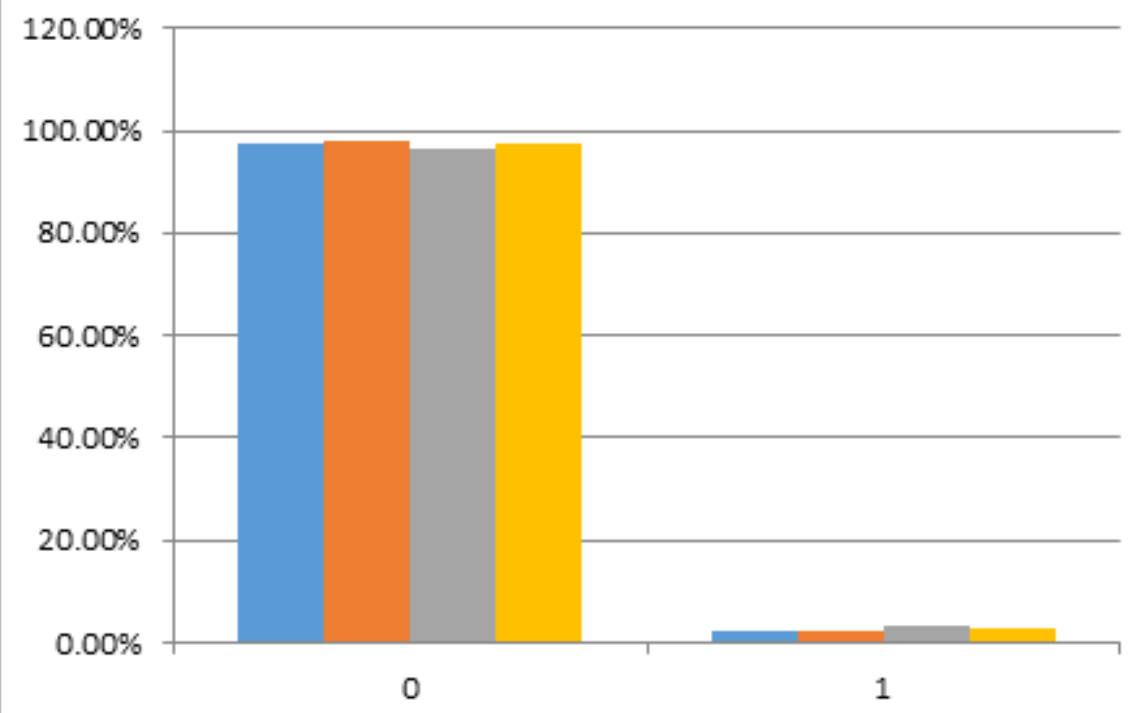

- Rosebud Coal Mine Area C Pond Sum of Hours

nosebud Coal Mine Area C Pond Sum of Passes

Rosebud Coal Mine Area F Pond 7 Sum of Hours

Rosebud Coal Mine Area F Pond 7 Sum of Passes 
Figure 21. Percent of background hours (blue) and hours with bat passes (red) with (1) and without (0) precipitation across the regional network of detectors. Of the 572,897 hours that detectors have been deployed, precipitation data was available from nearby weather stations for 556,881 hours $(97 \%)$. Note that some detectors were up to 75 kilometers from the weather station where precipitation events were recorded $(X=20.70 \mathrm{~km}, \mathrm{SD}=15.2 \mathrm{~km})$ and bats are capable of flight within minutes of the passing of a rain shower.

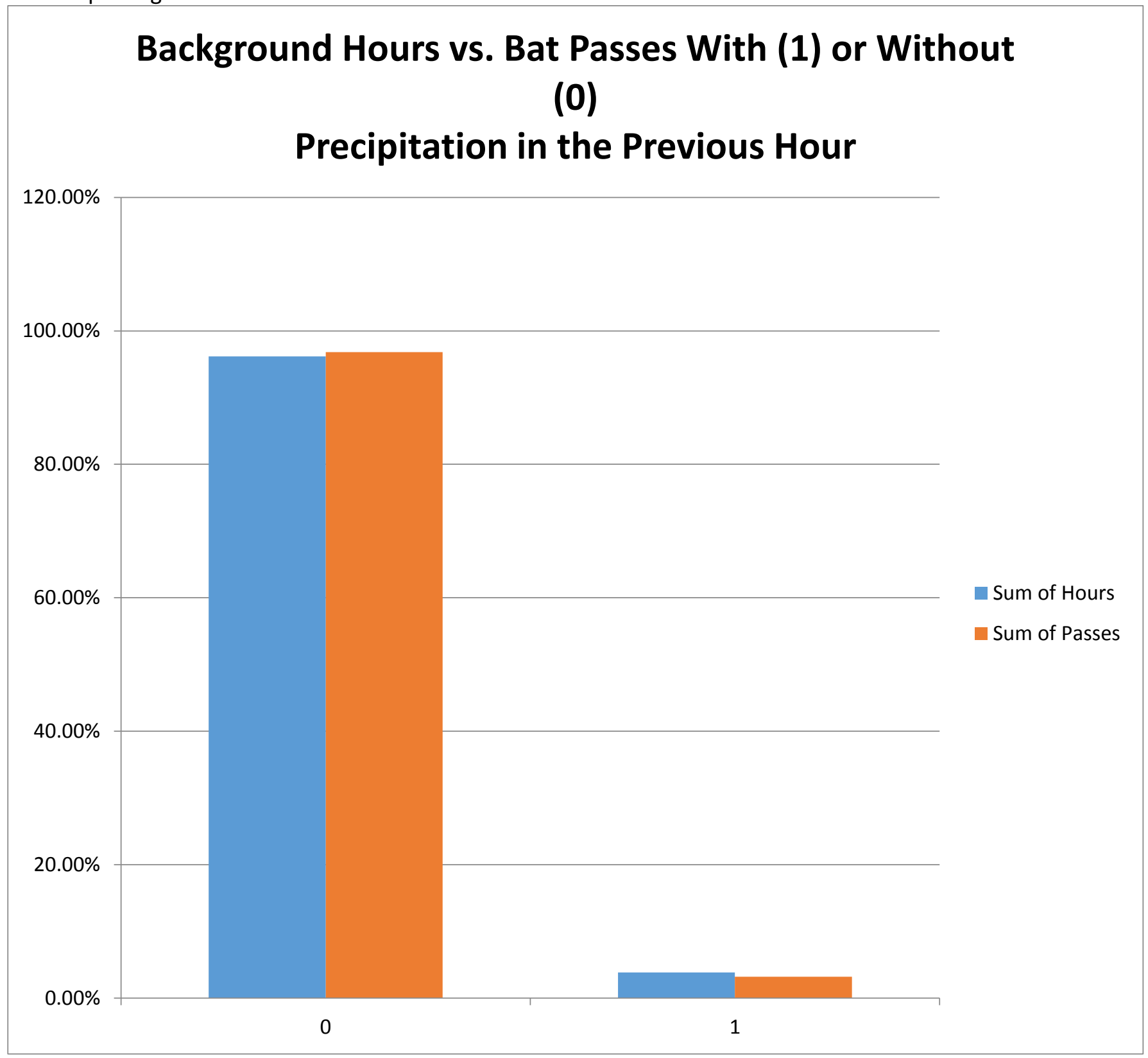


Figure 22. Percent of background hours (blue) and hours with bat passes (red) at various moon illumination categories $(0=$ no illumination and $1=$ full moon) and with the moon above and below the horizon at: (a) West Decker Coal Mine, (b) Spring Creek Mine, (c) Otter Creek Coal Tract, (d) Big Sky Mine, (e) Absaloka Mine, (f) Rosebud Mine, (g) Signal Peak Mine.

(a)

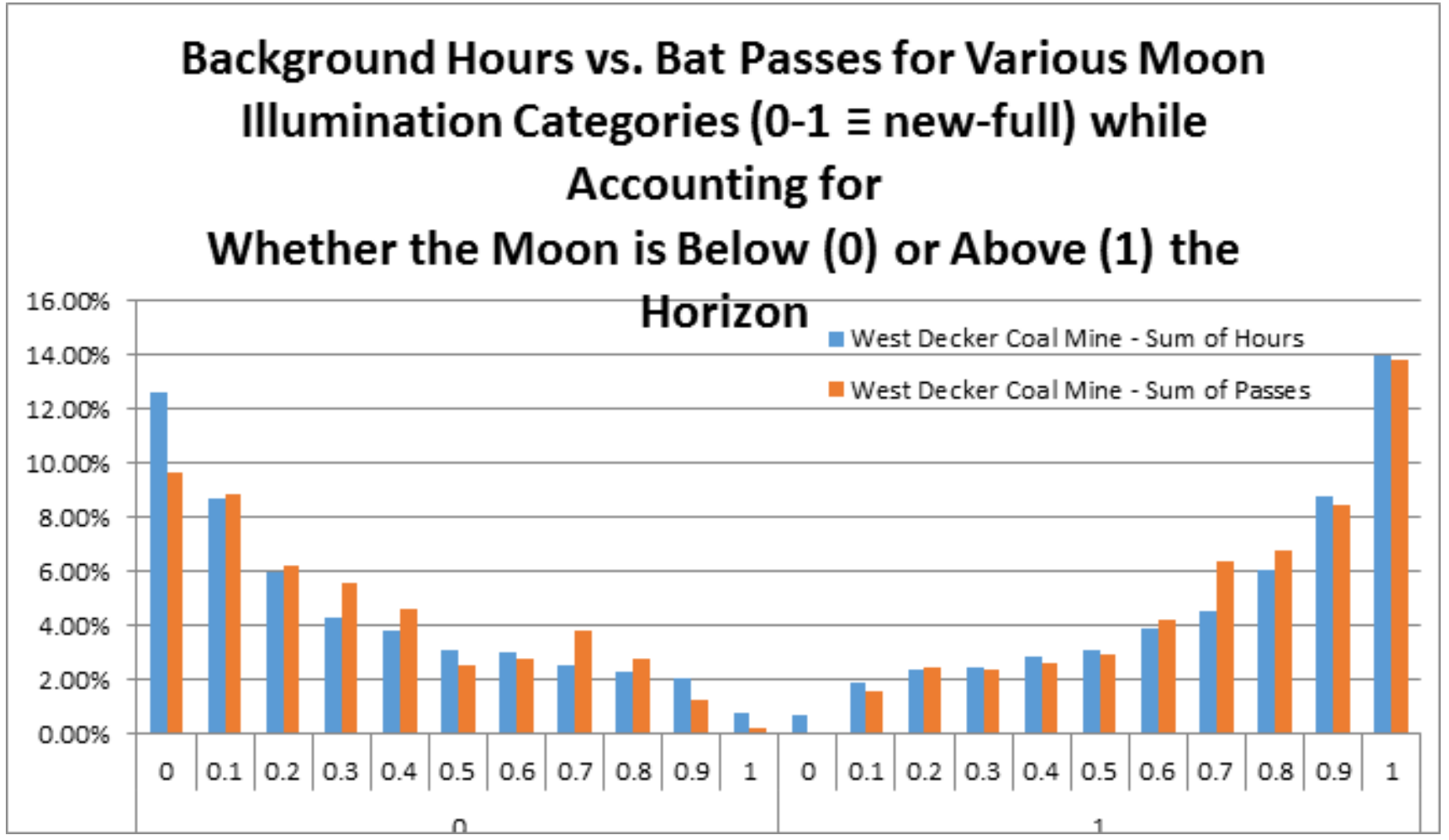

(b)

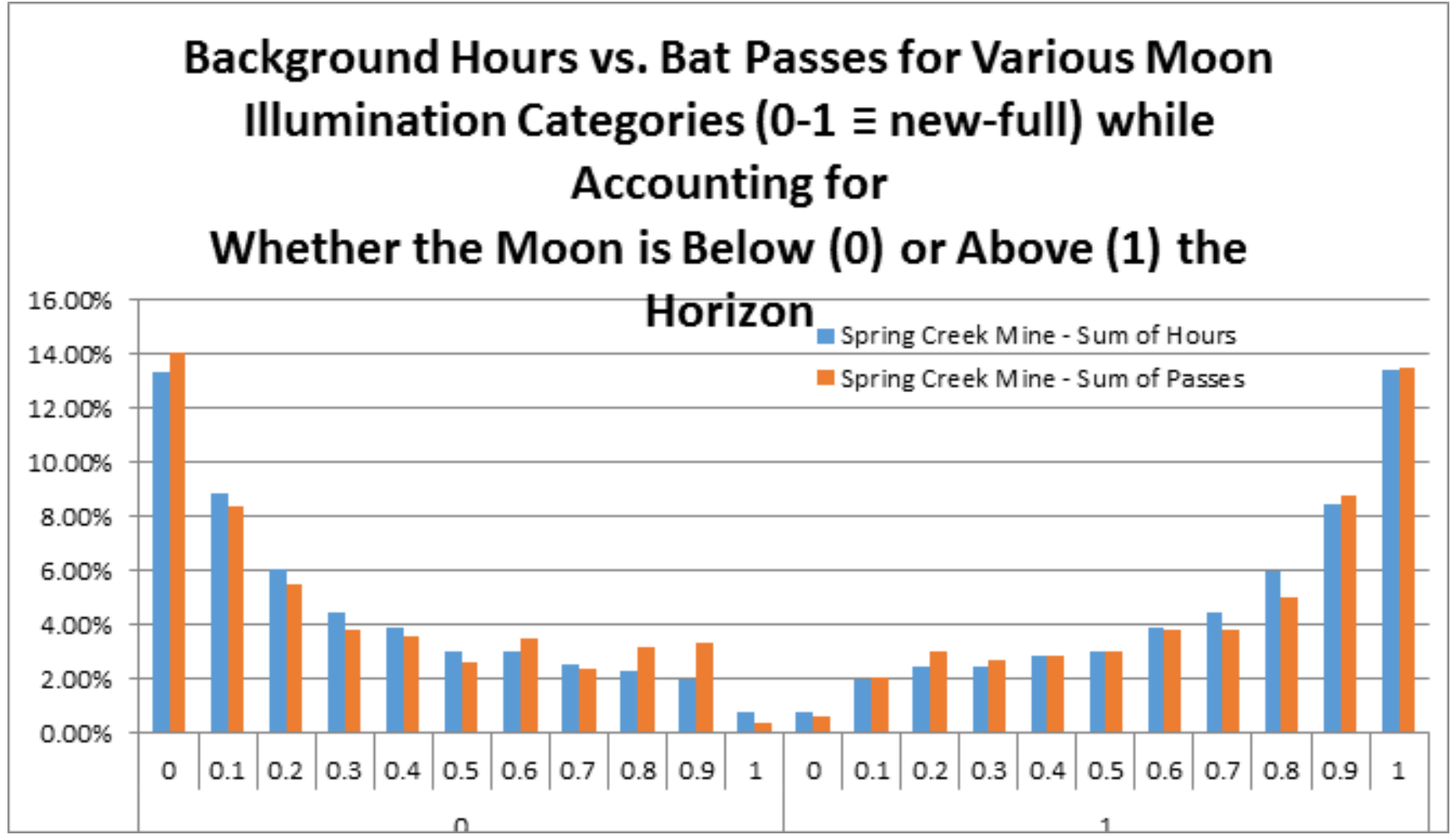


(c)

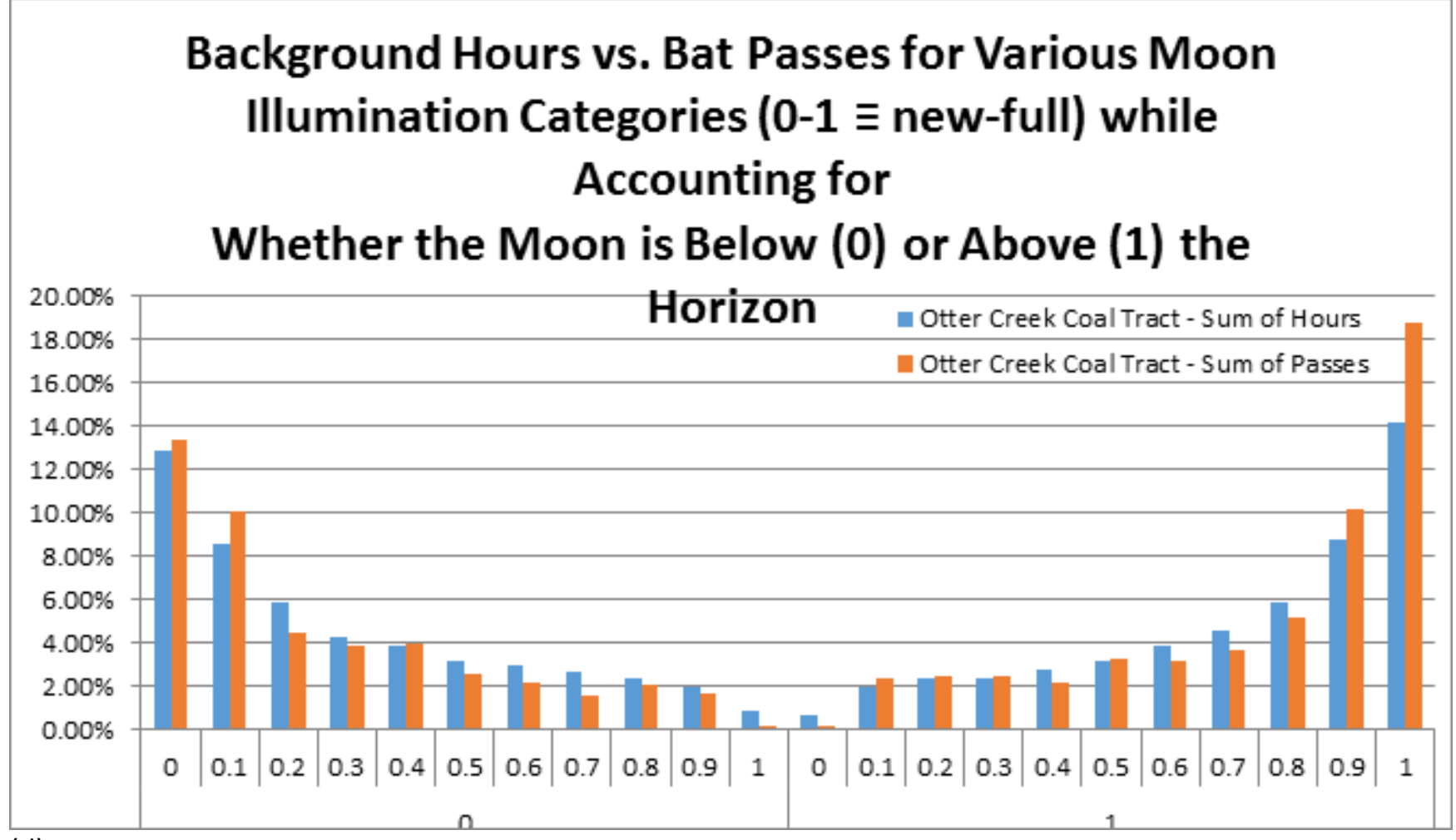

(d)

\section{Background Hours vs. Bat Passes for Various Moon Illumination Categories (0-1 $\equiv$ new-full) while} Accounting for

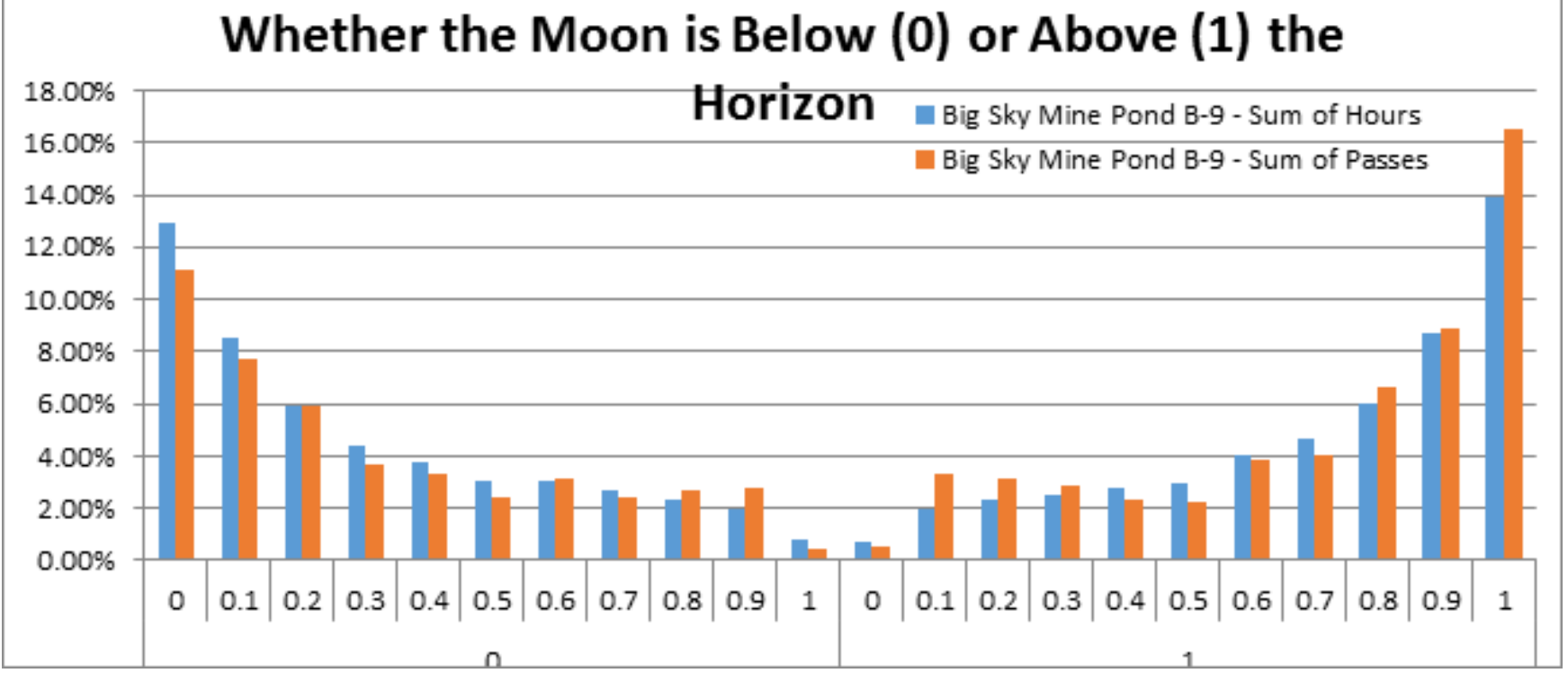


(e)

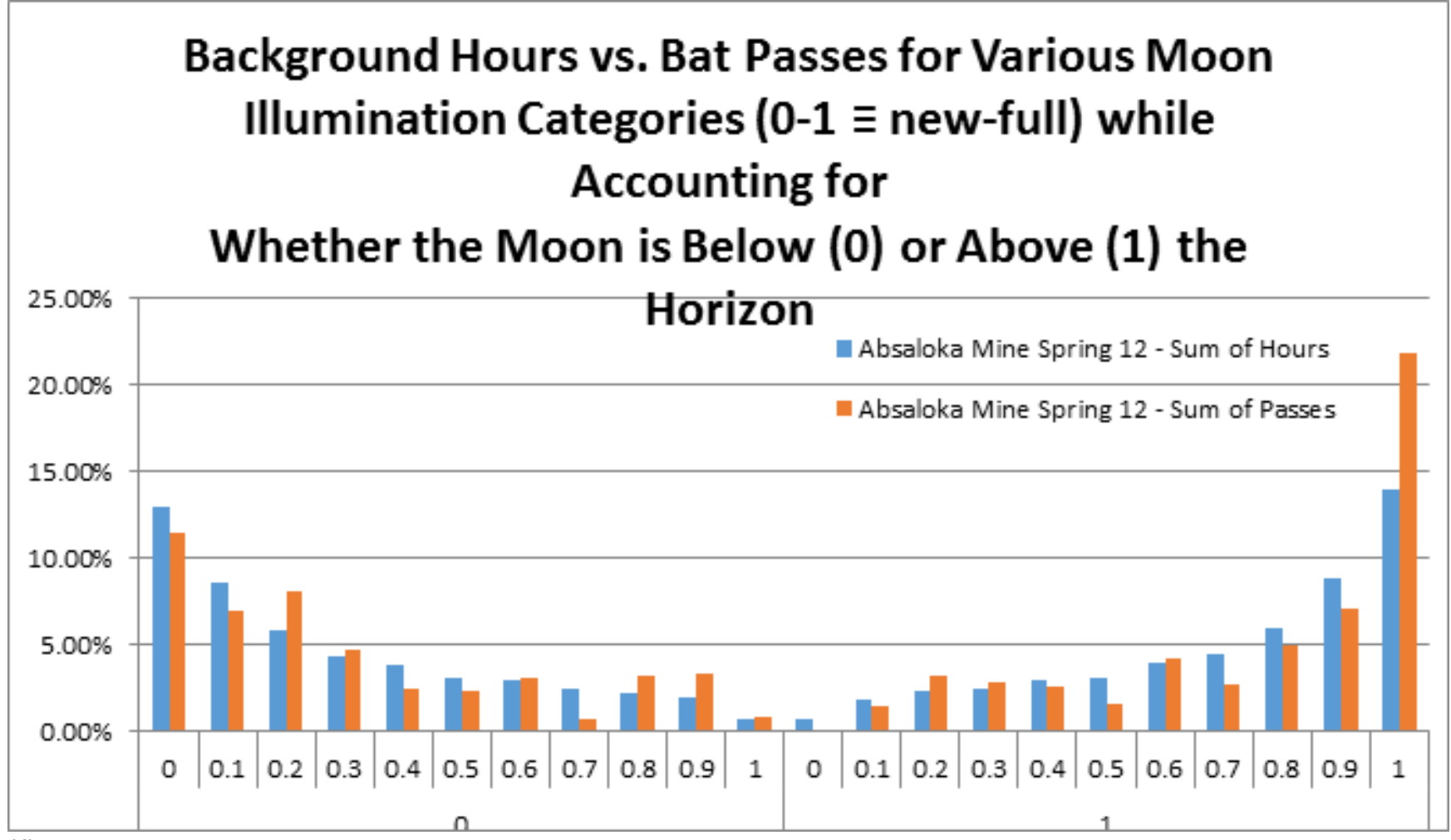

(f)

\section{Background Hours vs. Bat Passes for Various Moon Illumination Categories (0-1 $\equiv$ new-full) while Accounting for Whether the Moon is Below (0) or Above (1) the Horizon}

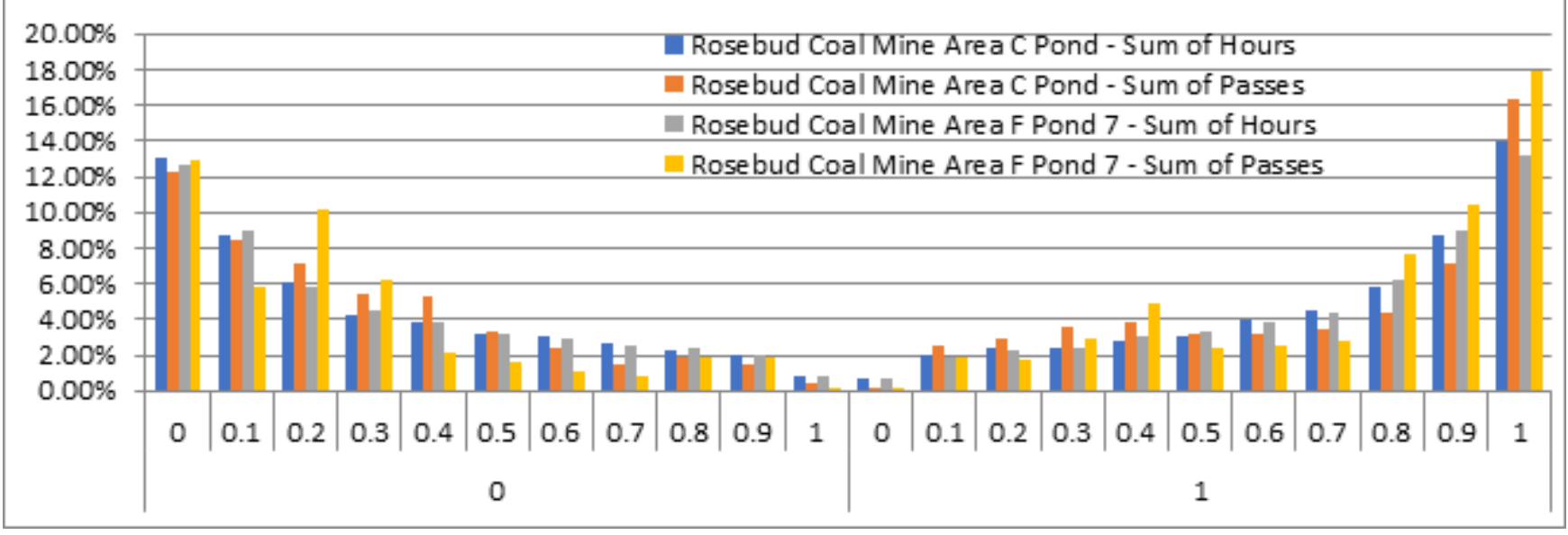


(g)

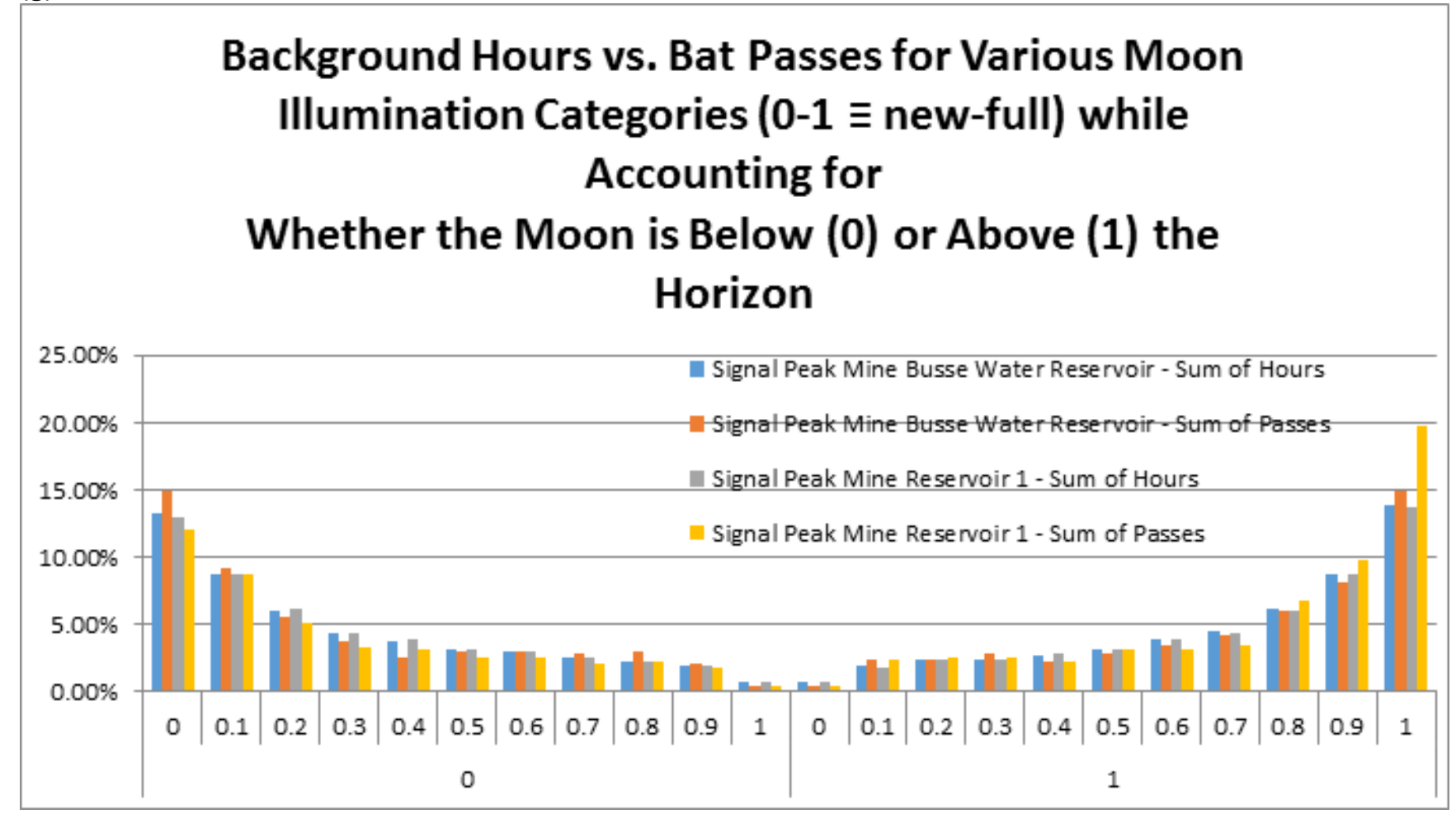


Figure 23. Percent of background hours (blue) and hours with bat passes (red) associated with various moon illumination categories ( $0=$ no illumination and $1=$ full moon) and with the moon below or above the horizon across the regional network of detectors. Moon illumination values could be calculated for $100 \%$ of the 572,897 hours that detectors have been deployed.

\section{Background Hours vs. Bat Passes for Various Moon Illumination Categories (0-1 $\equiv$ new-full) while Accounting for Whether the Moon is Below (0) or Above (1) the Horizon}

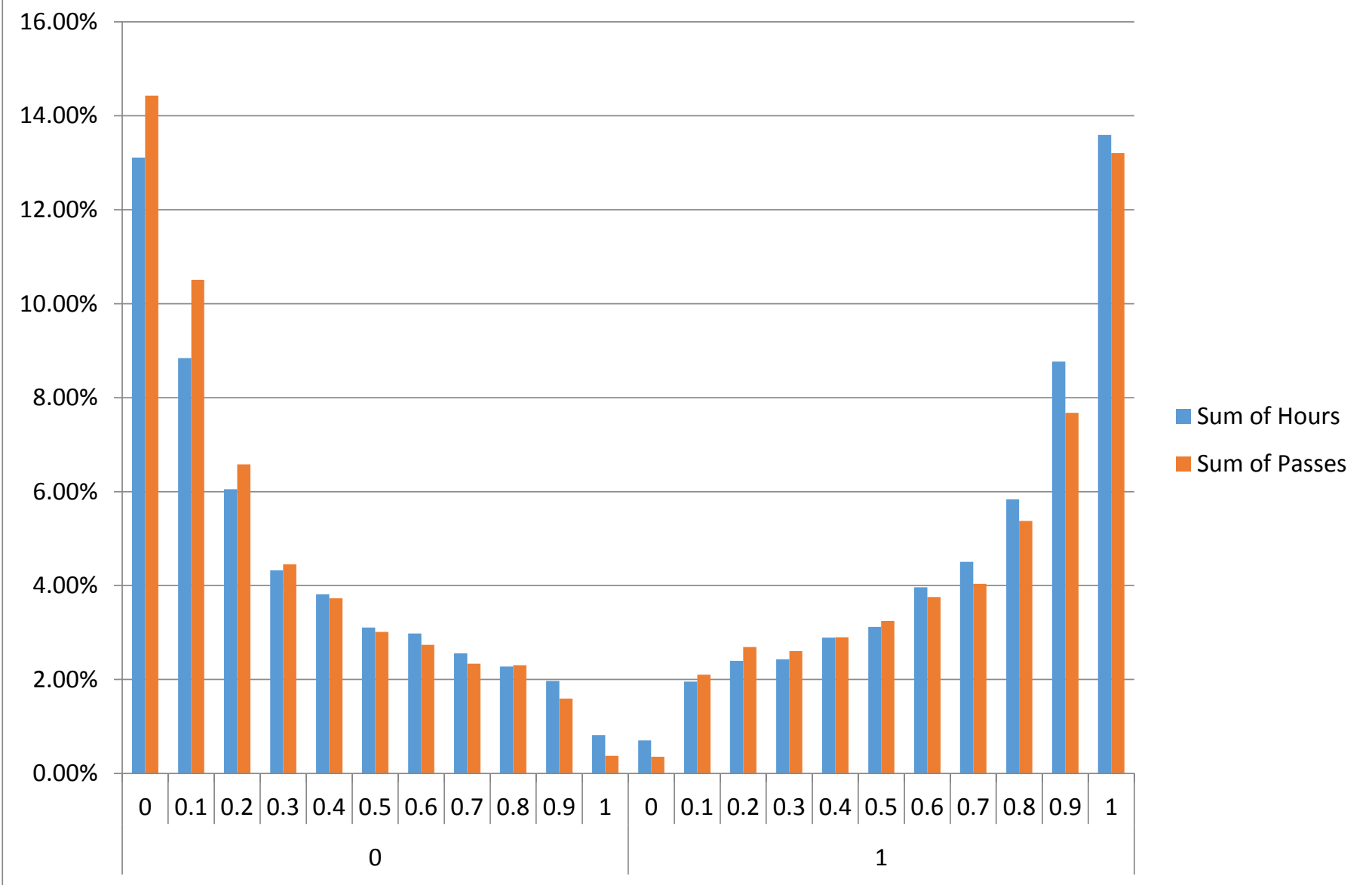


Figure 24. Average number of nightly bat passes each week auto-identified by species at: (a) West Decker Coal Mine, (b) Spring Creek Mine, (c) Otter Creek Coal Tract, (d) Big Sky Mine, (e) Absaloka Mine, (f) Rosebud Mine, (g) Signal Peak Mine. Numbers on X axis are years and weeks.

(a)

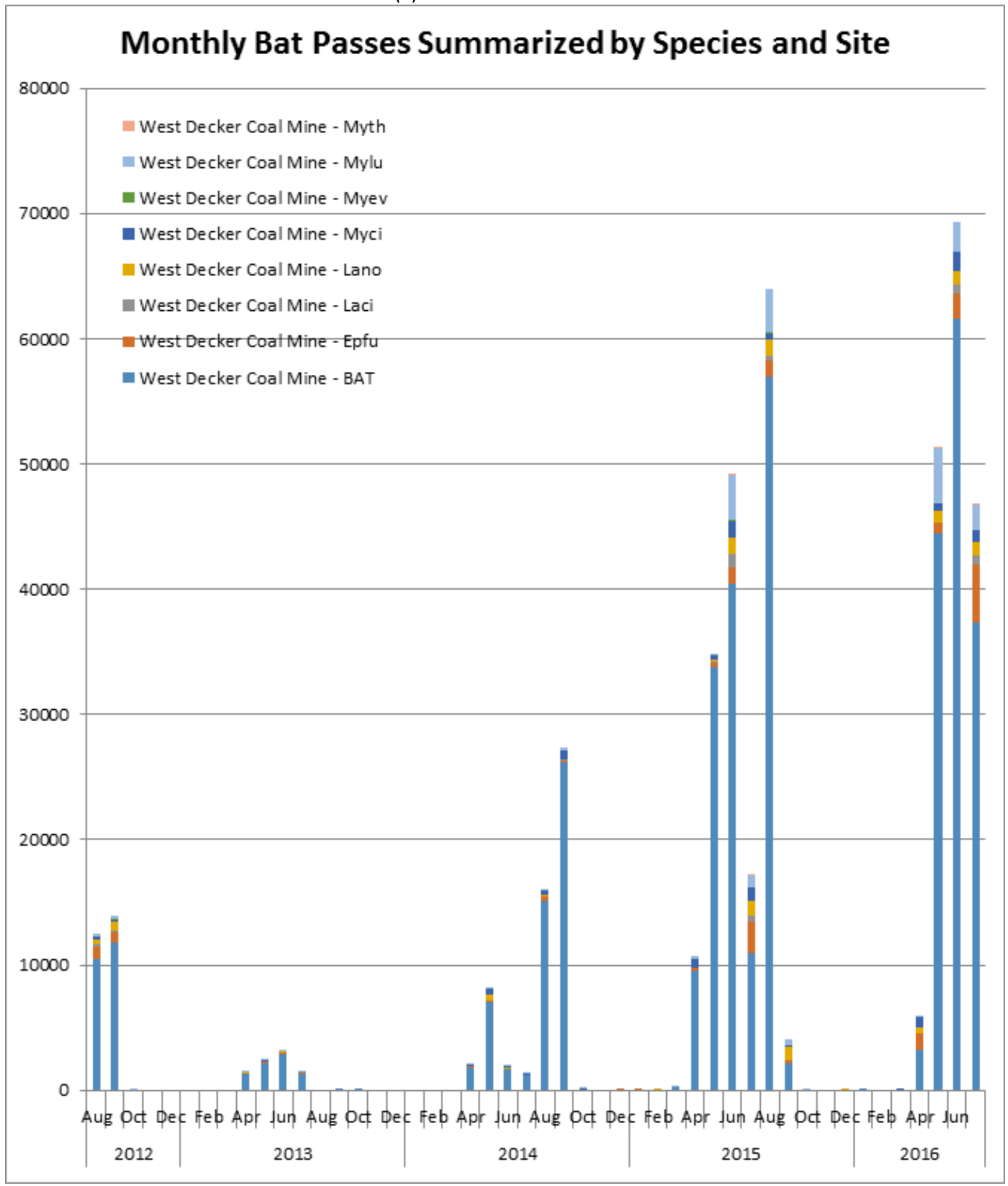


(b)

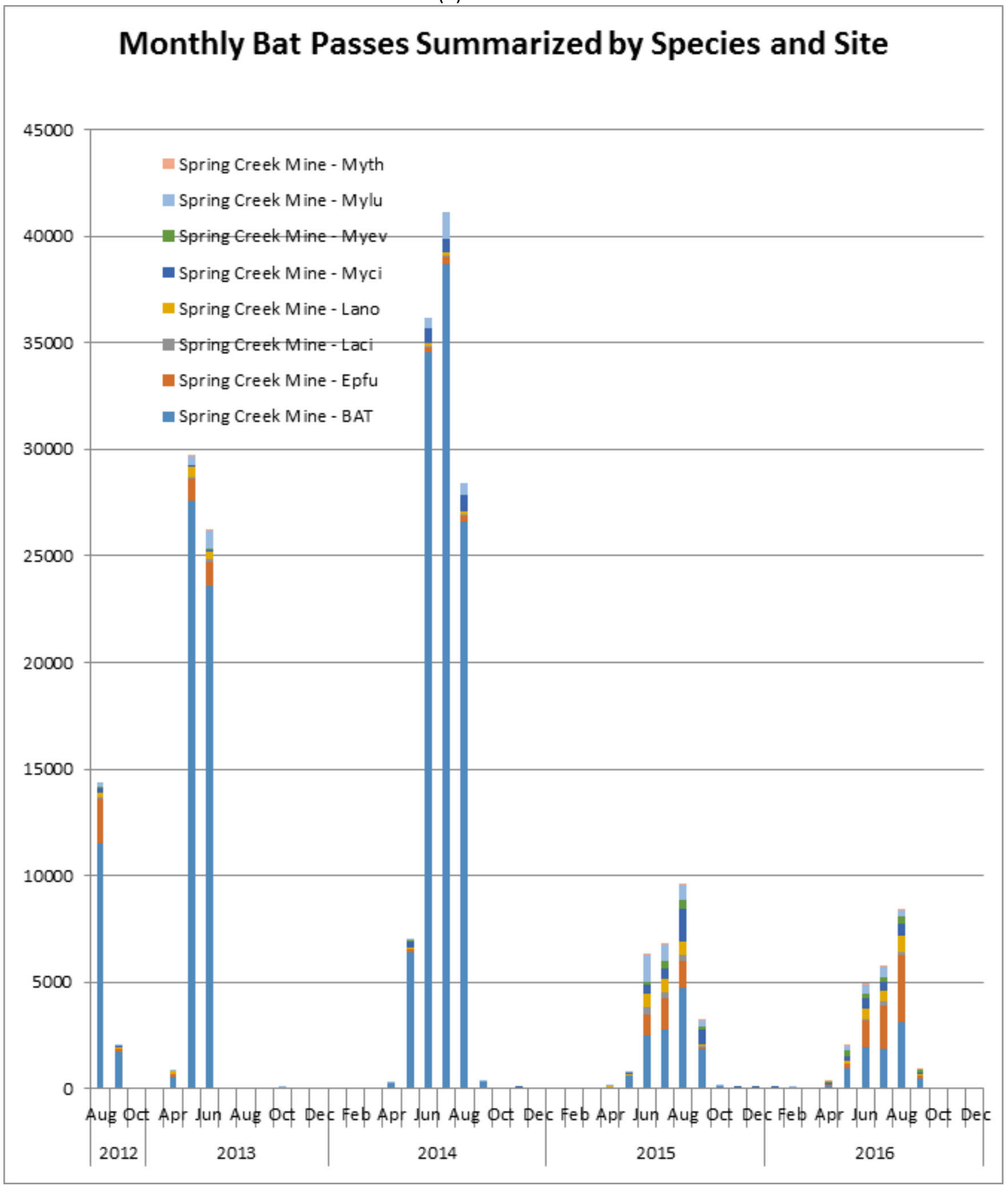


(c)

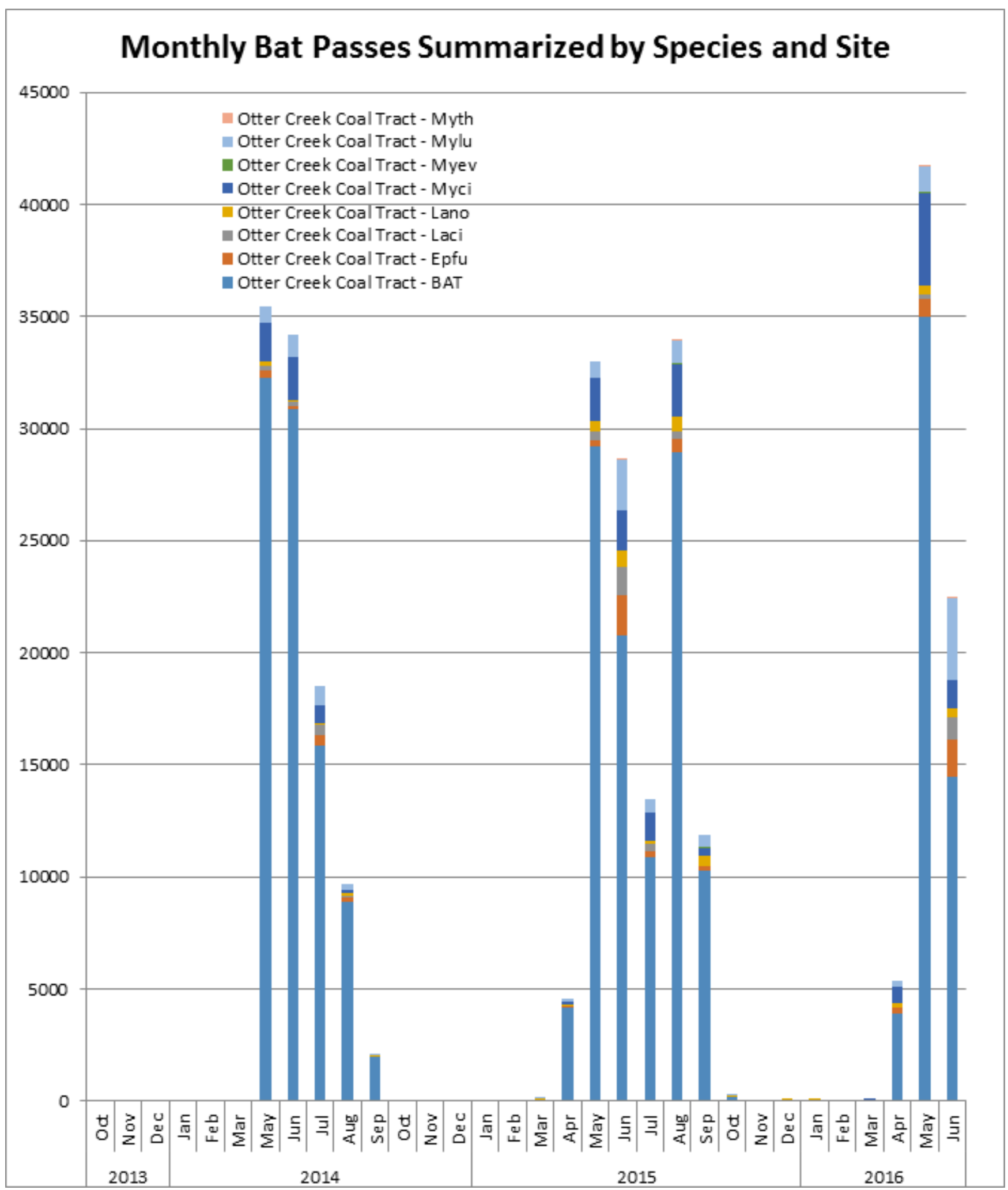


(d)

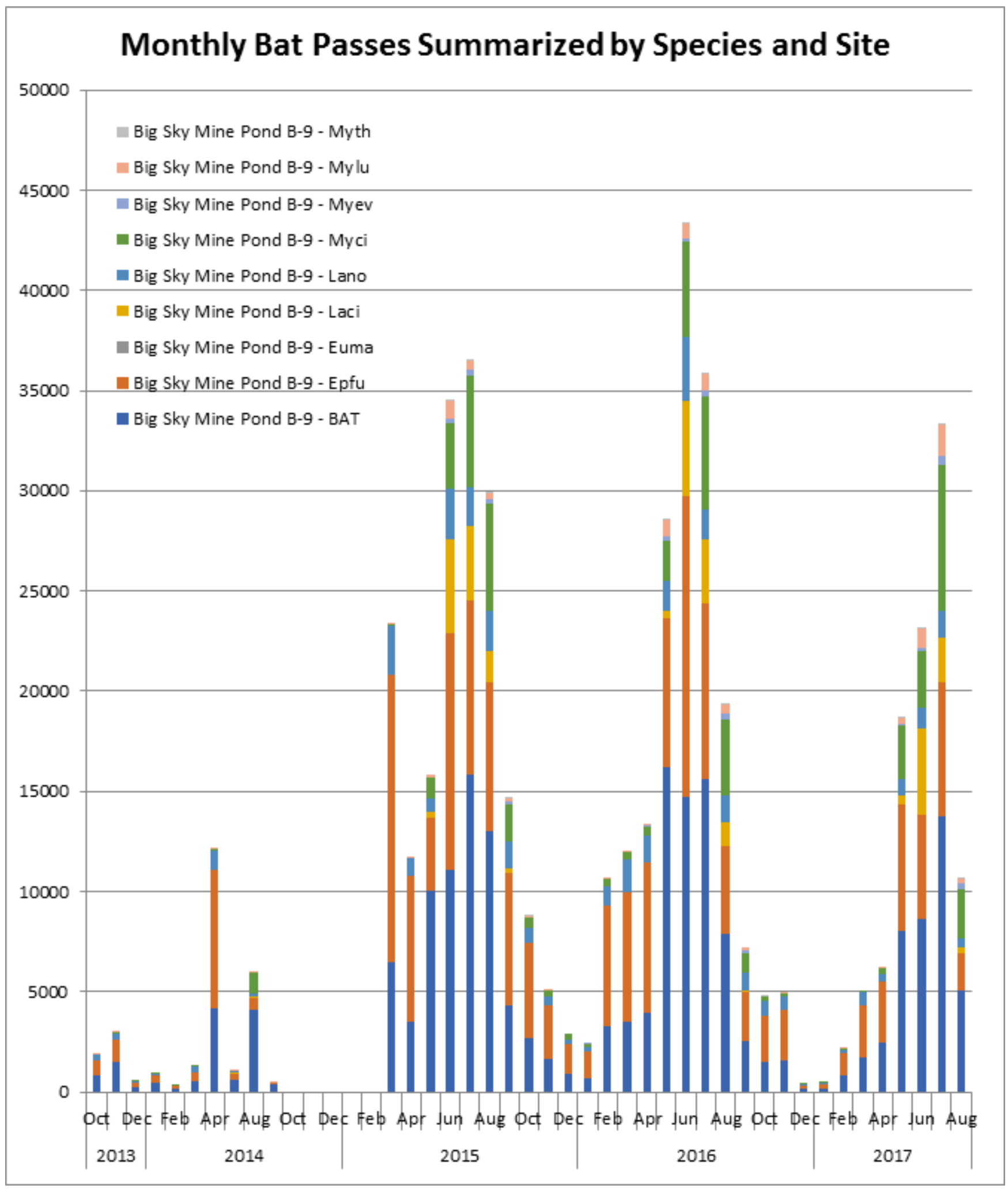


(e)

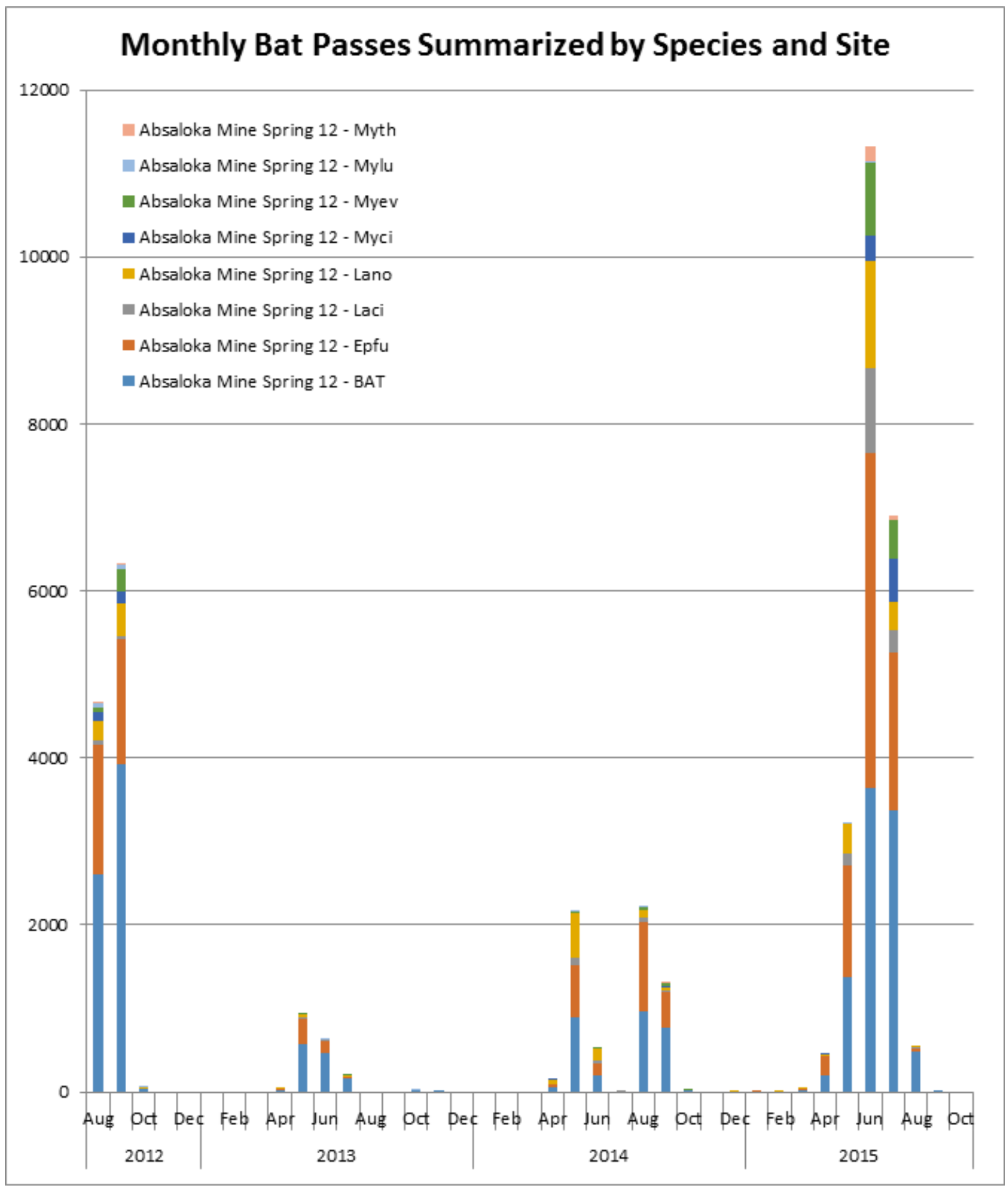




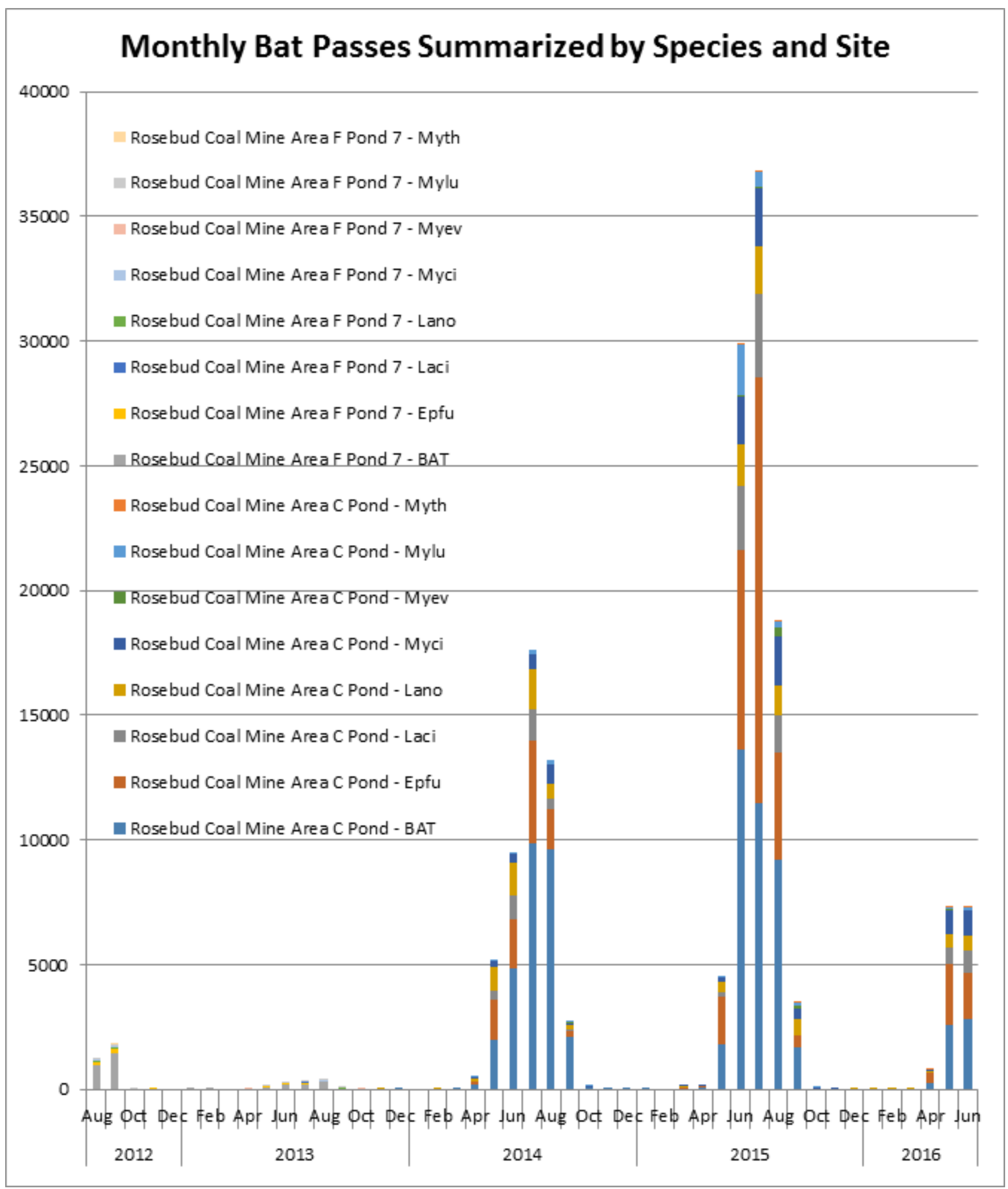




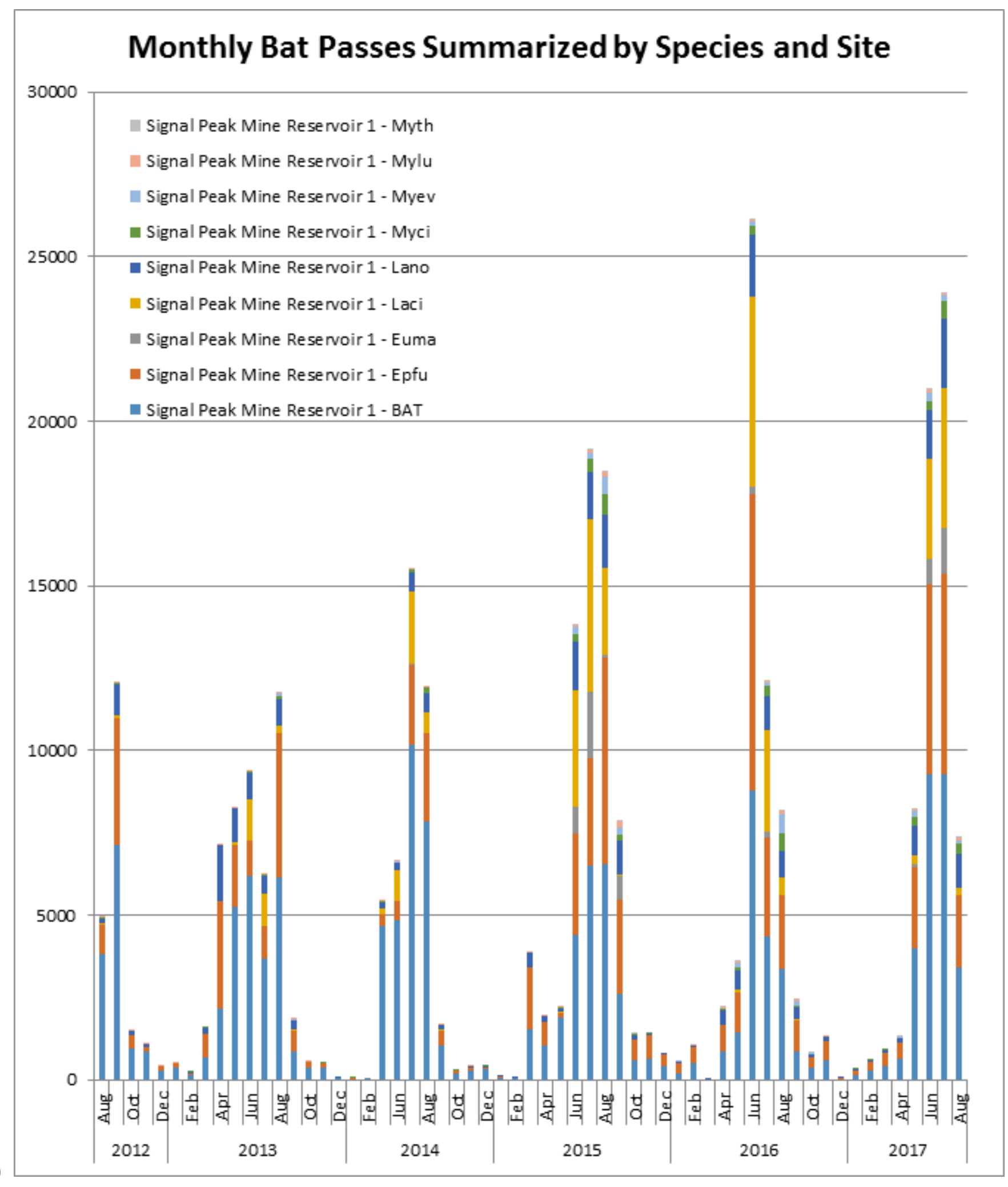




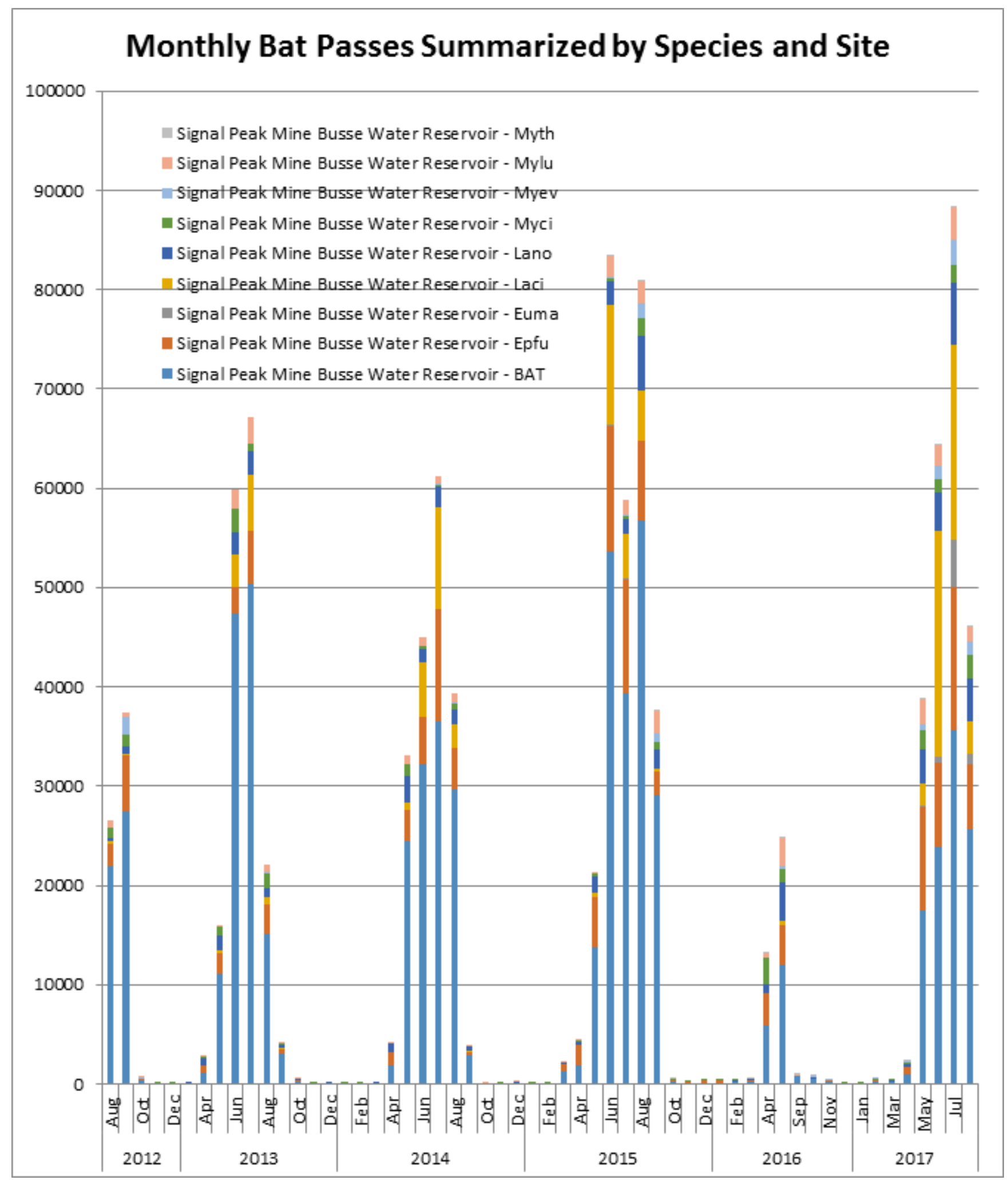




\title{
Appendix A
}

\section{References on Wind Turbine and other Human Structure Impacts on Bats}

\author{
Compiled by Bryce A. Maxell, Senior Zoologist, Montana Natural Heritage Program
}

September 2015

An * in front of a citation, indicates the article has particular value for wind turbine impacts to bats and turbine management in Montana. Additional information on wind turbine impacts to bats and other wildlife can be found at the Wind-Wildlife Impacts Literature Database (WILD) at http://wild.nrel.gov

Ahlén, I. 2003. Wind turbines and bats-a pilot study. Uppsala, Sweden. http://publikationer.slu.se/Filer/08WindBat FinalReport.pdf

Anderson, R.L., D. Strickland, J. Tom, N. Neumann, W. Erickson, J. Cleckler, G. Mayorga, G. Nuhn, A. Leuders, J. Schneider, L. Backus, P. Becker and N. Flagg. 2000. Avian monitoring and risk assessment at Tehachapi Pass and San Gorgonio Pass wind resource areas, California: Phase 1 preliminary results. Proceedings of the National Avian-Wind Power Planning Meeting 3:31-46. National Wind Coordinating Committee, Washington, D.C.

Arnett, E. B. (Tech. ed.). 2005. Relationships between bats and wind turbines in Pennsylvania and West Virginia: An assessment of bat fatality search protocols, patterns of fatality, and behavioral interactions with wind turbines. A final report submitted to the Bats and Wind Energy Cooperative. Bat Conservation International.

*Arnett, E.B. 2006. A preliminary evaluation on the use of dogs to recover bat fatalities at wind energy facilities. Wildlife Society Bulletin 34(5):1440-1445.

*Arnett, E.B., W.K. Brown, W.P. Erickson, J.K. Fiedler, B.L. Hamilton, T.H. Henry, A. Jain, G.D. Johnson, J. Kerns, R.R. Koford, C.P.
Nicholson, T.J. O'Connell, M.D. Piorkowski, and R.D. Tankersley, Jr. 2008. Patterns of bat fatalities at wind energy facilities in North America. Journal of Wildlife Management 72(1):61-78.

Arnett E.B., J.P. Hayes, M.M.P. Huso. 2006. An evaluation of the use of acoustic monitoring to predict bat fatality at a proposed wind facility in southcentral Pennsylvania. An annual report submitted to the bats and wind energy cooperative. Austin, Texas, USA. http://www.batsandwind.org/pdf/precon pa.pdf

*Arnett E.B., C. Hein, M. Schirmacher, M.M.P. Huso, and J. Szewczak. 2013. Evaluating the effectiveness of an ultrasonic acoustic deterrent for reducing bat fatalities at wind turbines. PLoS ONE 8(6):e65794. doi:10.1371/journal.pone.0065794

Arnett, E.B., M.M.P. Huso, D.S. Reynolds, and M. Schirmacher. 2007. Patterns of preconstruction bat activity at a proposed wind facility in northwest Massachusetts. Annual report prepared for the Bats and Wind Energy Cooperative. Bat Conservation International. Austin, Texas, USA. 35 p.

*Arnett, E.B., M.M.P. Huso, M.R. Schirmacher, and J.P. Hayes. 2011. Altering turbine speed reduces bat mortality at wind-energy facilities. Frontiers in Ecology and the Environment 9(4):209-214. 
Avery, M. and T. Clement. 1972. Bird mortality at four towers in eastern North Dakota: Fall 1972. Prairie Naturalist 4:87-95.

*Baerwald, E.F. and R.M.R. Barclay. 2009. Geographic variation in activity and fatality of migratory bats at wind energy facilities. Journal of Mammalogy 90(6):1341-1349.

*Baerwald, E.F. and R.M.R. Barclay. 2011. Patterns of activity and fatality of migratory bats at a wind energy facility in Alberta, Canada. Journal of Wildlife Management 75(5):1103-1114.

Baerwald, E.F., G.H. D'Amours, B.J. Klug, and R.M.R. Barclay. 2008. Barotrauma is a significant cause of bat fatalities at wind turbines. Current Biology 18(16):R695R696.

*Baerwald, E.F., J. Edworthy, M. Holder, and R.M.R. Barclay. 2009. A large-scale mitigation experiment to reduce bat fatalities at wind energy facilities. Journal of Wildlife Management 73(7):1077-1081.

*Barclay, R.M.R., E.F. Baerwald, and J.C. Gruver. 2007. Variation in bat and bird fatalities at wind energy facilities: assessing the effects of rotor size and tower height. Canadian Journal of Zoology 85:381-387.

Bennett, V.J. and A.M. Hale. 2014. Red aviation lights on wind turbines do not increase bat-turbine collisions. Animal Conservation 17:354-358.

Bernardino, J., R. Bispo, H. Costa, and M. Mascarenhas. 2013. Estimating bird and bat fatality at wind farms: a practical overview of estimators, their assumptions and limitations. New Zealand Journal of Zoology 40(1):63-74.

Chang, T. E. Nielson, W. Auberle, F.I. Solop. 2013. A quantitative method to analyze the quality of EIA information in wind energy development and avian/bat assessments. Environmental Impact Assessment Review 38:142-150.

Crawford, R.L. and W.W. Baker. 1981. Bats killed at a north Florida television tower: a 25-year record. Journal of Mammalogy 62:651-652.

*Cryan, P.M. 2008. Mating behavior as a possible cause of bat fatalities at wind turbines. Journal of Wildlife Management 72(3): 845-849.

Cryan, P.M. and R.M.R. Barclay. 2009. Causes of bat fatalities at wind turbines: hypotheses and predictions. Journal of Mammalogy 90(6):1330-1340.

Cryan, P.M. and A.C. Brown. 2007. Migration of bats past a remote island offers clues toward the problem of bat fatalities at wind turbines. Biological Conservation 139:1-11.

*Cryan, P.M., P.M. Gorresen, C.D. Hein, M.R. Schirmacher, R.H. Diehl, M.M. Huso, D.T.S. Hayman, P.D. Fricker, F.J. Bonaccorso, D.H. Johnson, K. Heist, and D.C. Dalton. 2014. Behavior of bats at wind turbines. Proceedings of the National Academy of Sciences 111(42):15126-15131.

Cryan, P.M., J.W. Jameson, E.F. Baerwald, C.K.R. Willis, R.M.R. Barclay, E.A. Snider, and E.G. Chrichton. 2012. Evidence of latesummer mating readiness and early sexual maturation in migratory tree-roosting bats found dead at wind turbines. PLoS One 7(10):e47586. Doi:10.1371/journal.pone.0047586

Cryan, P.M., C.A. Stricker, and M.B. Wunder. 2014. Continental-scale, seasonal movements of a heterothermic migratory tree bat. Ecological Applications 24(4):602616. 
Cullinan, V.I., S. Matzner, and C.A. Duberstein. 2015. Classification of birds and bats using flight tracks. Ecological Informatics 27:5563.

DeBlase, A.F. and J.B. Cope. 1967. An Indiana bat impaled on barbed wire. American Midland Naturalist 77:238.

Dedon, M., S. Byrne, J. Aycrigg, and P. Hartman. 1989. Bird mortality in relation to the Mare Island 115-kV transmission line: progress report 1988/1989. Department of the Navy, Western Division, Naval Facilities Engineering Command, Office of Environmental Management, San Bruno, California. Report 443-89.3. 150pp.

Denys, G.A. 1972. Hoary bat impaled on barbed wire. Jack-Pine Warbler 50:63.

Diehl, R.H. 2013. The airspace is habitat. Trends in Ecology and Evolution 28(7):377379. doi.org/10.1016/j.tree.2013.02.015

Doty, A.C. and A.P. Martin. 2013. Assessment of bat and avian mortality at a pilot wind turbine at Coega, Port Elizabeth, Eastern Cape, South Africa. New Zealand Journal of Zoology 40(1):75-80.

*Drake, D., C.S. Jennelle, J.N. Liu, S.M. Grodsky, S. Schumacher, and M. Sponsler. 2015. Regional analysis of wind turbinecaused bat mortality. Acta Chiropterologica 17(1)179-188.

Erickson, W.P., B. Gritski, and K. Kronner, 2003. Nine Canyon Wind Power Project Avian and Bat Monitoring Annual Report. Technical report submitted to Energy Northwest and the Nine Canyon Technical Advisory Committee.

Erickson, W.P., J. Jeffrey, K. Kronner, and K. Bay. 2003. Stateline Wind Project Wildlife Monitoring Annual Report, Results for the Period July 2001 - December 2002.
Technical report submitted to FPL Energy, the Oregon Office of Energy, and the Stateline Technical Advisory Committee.

Erickson, W.P., G.D. Johnson, M.D. Strickland, and K. Kronner. 2000. Avian and bat mortality associated with the Vansycle Wind Project, Umatilla County, Oregon: 1999 study year. Technical Report prepared by WEST, Inc. for Umatilla County Department of Resource Services and Development, Pendleton, Oregon. 21p.

Erickson, W., G. Johnson, D. Young, D. Stickland, R. Good, M. Bourassa, K. Bay, K. Sernka. 2002. Synthesis and comparison of baseline avian and bat use, raptor nesting and mortality information from proposed and existing wind developments. Report to Bonneville Power Administration. West Inc., Cheyenne, Wyoming. 124 p.

Ferreira, D., C. Frexio, J.A. Cabral, R. Santos, and M. Santos. 2015. Do habitat characteristics determine mortality risk for bats at wind farms? Modelling susceptible species activity patterns and anticipating possible mortality events. Ecological Informatics 28:7-18.

Fiedler, J.K. 2004. Assessment of bat mortality and activity at Buffalo Mountain Windfarm, eastern Tennessee. M.S. Thesis, University of Tennessee, Knoxville.

Fiedler J.K., T.H. Henry, R.D. Tankersley, and C.P. Nicholson. 2007. Results of bat and bird mortality monitoring at the expanded Buffalo Mountain Windfarm, 2005. Tennessee Valley Authority. http://www.tva.gov/environment/bmw re port/results.pdf

Ganier, A.F. 1962. Bird casualties at a Nashville TV tower. Migrant 33:58-60.

Gollop, M.A. 1965. Bird migration collision casualties at Saskatoon. Blue Jay 23:15-17. 
Grodsky, S.M., M.J. Behr, A. Gendler, D. Drake, B.D. Dieterle, R.J. Rudd, and N.L. Walrath. 2011. Investigating the causes of death for wind turbine-associated bat fatalities. Journal of Mammalogy 92(5):917925.

*Grodsky, S.M., C.S. Jennelle, D. Drake, T. Virzi. 2012. Bat mortality at a wind-energy facility in southeastern Wisconsin. Wildlife Society Bulletin 36(4):773-783.

Hayes, J.P. and D.L. Waldien. 2000. Potential influences of the proposed Condon Wind Project on bats. Unpublished report prepared for $\mathrm{CH} 2 \mathrm{MHILL}$, Portland, Oregon. $14 p p$.

Hayes, J.P. and D.L. Waldien. 2000. Potential influences of the Stateline wind project on bats. Unpublished report prepared for $\mathrm{CH} 2 \mathrm{MHILL}$, Portland, Oregon.

*Hayes, M. 2013. Bats killed in large numbers at United States wind energy facilities. BioScience 63(12):975-979.

Higgins, K.F., R.G. Osborn, C.D. Dieter, and R.E. Usgaard. 1996. Monitoring of seasonal bird activity and mortality at the Buffalo Ridge Wind Resource Area, Minnesota, 1994-1995. Completion Report for the Research Period May 1, 1994 - December 31, 1995. Unpubl. report prepared for Kenetech Wind power, Inc. by the South Dakota Cooperative Fish and Wildlife Research Unit, Brookings, SD. 84pp.

*Horn, J.W., E.B. Arnett, and T.H. Kunz. 2008. Behavioral responses of bats to operating wind turbines. Journal of Wildlife Management 72(1):123-132.

Howe, R.W., W. Evans, and A.T. Wolf. 2002. Effects of wind turbines on birds and bats in northeastern Wisconsin. Wisconsin Public Service Corporation, Madison, Wisconsin
Howell, J.A. 1997. Bird mortality at rotor swept area equivalents, Altamont Pass and Montezuma Hills, California. Transactions of the Western Section of the Wildlife Society 33:24-29.

Howell, J.A. and J.E. Didonato. 1991. Assessment of avian use and mortality related to wind turbine operations, Altamont Pass, Alameda and Contra Costa Counties, California, September 1998 through August 1989. Final report submitted to U.S. Wind power, Inc.

Hull, C.L. and L. Cawthen. 2013. Bat fatalities at two wind farms in Tasmania, Australia: bat characteristics, and spatial and temporal patterns. New Zealand Journal of Zoology 40(1):5-15.

Huso, M.M.P. and D. Dalthrop. 2014. Accounting for unsearched areas in estimating wind turbine-caused fatality. Journal of Wildlife Management 78(2):347358.

Huso, M.M.P. and D. Dalthrop. 2014. A comment on "Bats killed in large numbers at United States wind energy facilities". BioScience 64(6):546-547.

James, R.D. 2002. Pickering Wind Turbine, Bird monitoring program in 2002. Report to Ontario Power Generation, December 2002.

Jameson, J.W. and C.K.R. Willis. 2012. Bat mortality at a wind power facility in central Canada. Northwestern Naturalist 93:194202.

*Jameson, J.W. and C.K.R. Willis. 2014. Activity of tree bats at anthropogenic tall structures: implications for mortality of bats at wind turbines. Animal Behaviour 97:145152. 
Johnson, G.D. and E. Arnett. 2004. A bibliography of bat interactions with wind turbines. Unpublished. $9 \mathrm{p}$.

Johnson, G.D., W.P. Erickson, M.D. Strickland, M.F. Shepherd and D.A. Shepherd. 2000. Avian Monitoring Studies at the Buffalo Ridge Wind Resource Area, Minnesota: Results of a 4-year study. Technical report prepared for Northern States Power Co., Minneapolis, MN. 212pp.

Johnson, G.D., W.P. Erickson, M.D. Strickland, M.F. Shepherd, D.A. Shepherd, and S.A. Sarappo. 2003. Mortality of bats at a largescale wind power development at Buffalo Ridge, Minnesota. The American Midland Naturalist 150(2):332-342.

Johnson, G.D., W.P. Erickson, and J. White. 2003. Avian and bat mortality at the Klondike, Oregon Phase I Wind Plant. Technical report prepared for Northwestern Wind Power by WEST, Inc.

Johnson, G.D., M.K. Perlik, W.P. Erickson, and M.D. Strickland. 2004. Bat activity, composition, and collision mortality at large wind plant in Minnesota. Wildlife Society Bulletin 32(4):1278-1288.

Johnson, G.D., M.K. Perlik, W.P. Erickson, M.D. Strickland, D.A. Shepherd, and P. Sutherland, Jr. 2003. Bat interactions with wind turbines at the Buffalo Ridge, Minnesota Wind Resource Area: An assessment of bat activity, species composition, and collision mortality. Electric Power Research Institute, Palo Alto, California, and Xcel Energy, Minneapolis, Minnesota. EPRI report \# 1009178.

Johnson, G.D. and M.D. Strickland. 2003. Biological assessment for the federally endangered Indiana bat (Myotis sodalis) and Virginia big-eared bat (Corynorhinus townsendii virginianus), NedPower Mount Storm Wind Project, Grant County, West
Virginia. Unpublished report prepared by WEST, Inc. for NedPower Mount Storm, Chantilly, Virginia.

Johnson, G.D., D.P. Young, Jr., W.P. Erickson, M.D. Strickland, R.E. Good and P. Becker. 2000. Avian and bat mortality associated with the initial phase of the Foote Creek Rim Wind power Project, Carbon County, Wyoming: November 3, 1998 - October 31, 1999. Technical Report prepared for SeaWest Energy Corporation and Bureau of Land Management. 32pp.

Johnson, J.S., K.S. Watrous, G.J. Giumarro, T.S. Peterson, S.A. Boyden, and M.J. Lacki. 2011. Seasonal and geographic trends in acoustic detection of tree-roosting bats. Acta Chiropterologica 13(1):157-168.

Johnson, P.B. 1933. Accidents to bats. Journal of Mammalogy 14:156-157.

Keeley, B., S. Ugoretz, and D. Strickland. 2001. Bat ecology and wind turbine considerations. Proceedings of the National Avian-Wind Power Planning Meeting, 4:135-146. National Wind Coordinating Committee, Washington, D.C.

Kelm, D.H., J. Lenski, V. Kelm, U. Toelch, and F. Dziock. 2014. Seasonal bat activity in relation to distance to hedgerows in an agricultural landscape in central Europe and implications for wind energy development. 16(1):65-73.

Kerlinger, P., R. Curry, and R. Ryder. 2000. Ponnequin wind energy project avian studies, Weld County, Colorado: Summary of activities during 2000. Prepared for Public Service Company of Colorado, Denver, Colorado.

Kiefer, A., H. Merz, W. Rackow, H. Roer, and D. Schlegel. 1995. Bats as traffic casualties in Germany. Myotis 32-33:215-220. 
*Kiesecker, J.M., J.S. Evans, J. Fargione, K. Doherty, K.R. Foresman, T.H. Kunz, D. Naugle, N.P. Nibbelink, and N.D. Niemuth. 2011. Win-win for wind and wildlife: a vision to facilitate sustainable development. PLoS One 6:4:e17566.

Doi:10.1371/journal.pone.0017566.

Klug, B.J. and E.F. Baerwald. 2010. Incidence and management of live and injured bats at wind energy facilities. Journal of Wildlife Rehabilitation 30(2):11-16.

Koford, R., A. Jain, G. Zenner and A. Hancock. 2004. Avian mortality associated with the Top of lowa Wind Farm: Progress Report, Calendar Year 2003. lowa Cooperative Fish and Wildlife Research Unit, lowa State University, Ames, lowa. 9pp.

Korner-Nievergelt, F., P. Korner-Nievergelt, O. Behr, I. Niermann, R. Brinkmann, and B. Hellriegel. 2011. A new method to determine bird and bat fatality at wind energy turbines from carcass searches. Wildlife Biology 17:350-363.

Korstian, J.M., A.M. Hale, V.J. Bennett, and D.A. Williams. 2013. Advances in sex determination in bats and its utility in windwildlife studies. Molecular Ecology 13:776780.

Krenz, J.D., and B.R. McMillan. 2000. Final Report: Wind-turbine related bat mortality in southwestern Minnesota. Minnesota Department of Natural Resources, St. Paul.

*Kunz, T.H., E.B. Arnett, B.M. Cooper, W.P. Erickson, R.P. Larkin, T. Mabee, M.L. Morrison, M.D. Strickland, and J.M. Szewczak. 2007. Assessing impacts of wind-energy development on nocturnally active birds and bats: a guidance document. Journal of Wildlife Management 71(8):2449-2486.
*Kunz, T.H., E.B. Arnett, W.P. Erickson, A.R. Hoar, G.D. Johnson, R.P. Larkin, M.D. Strickland, R.W. Thresher, and M.D. Tuttle. 2007. Ecological impacts of wind energy development on bats: questions, research needs, and hypotheses. Frontiers in Ecology and the Environment 5(6):315-324.

Mabee, T.J., B.A. Cooper, and J.H. Plissner. 2004. A radar study of nocturnal bird migration at the proposed Mount Storm wind power development, West Virginia, Fall 2003. Unpublished report prepared by ABR, Inc. for WEST, Inc. and Nedpower.

* Mathews, F., M. Swindells, R. Goodhead, T.A. August, P. Hardman, D.M. Linton, D.J. Hosken. 2013. Effectiveness of search dogs compared with human observers in locating bat carcasses at wind-turbine sites: a blinded randomized trial. Wildlife Society Bulletin 37(1):34-40.

Millon, L., J.F. Julen, R. Julliard, and C. Kerbiriou. 2015. Bat activity in intensively farmed landscapes with wind turbines and offset measures. Ecological Engineering 75:250-257.

*Minderman, J., C.J. Pendlebury, J.W. PearceHiggins, and K.J. Park. 2012. Experimental evidence for the effect of small wind turbine proximity and operation on bird and bat activity. PLoS One 7(7):e41177. Doi:10.1371/journal.pone.0041177.

Nicholson, C.P. 2003. Buffalo Mountain Windfarm bird and bat mortality monitoring report: October 2001 - September 2002. Tennessee Valley Authority, Knoxville.

Nicholson, C.P. 2001. Buffalo Mountain Windfarm bird and bat mortality monitoring report: October 2000 - September 2001. Tennessee Valley Authority, Knoxville.

Orloff, S. and A. Flannery. 1992. Wind turbine effects on avian activity, habitat use, and mortality in Altamont Pass and Solano 
County Wind Resource Areas, 1989-1991. Final report to Alameda, Costra Costa and Solano Counties and the California Energy Commission by Biosystems Analysis, Inc., Tiburon, CA.

Osborn, R.G., K.F. Higgins, C.D. Dieter, and R.E. Usgaard. 1996. Bat collisions with wind turbines in southwestern Minnesota. Bat Research News 37:105-108.

Pandion Systems, Inc. 2003. White paper on bats and wind turbines with reference to the Backbone Mountain site. Unpublished report prepared for Florida Power \& Light, Juno Beach, Florida.

Péron, G., J.E. Hines, J.D. Nichols, W.L. Kendall, K.A. Peters, and D.S. Misrahi. 2013. Estimation of bird and bat mortality at wind-power farms with superpopulation models. Journal of Applied Ecology 50:902911.

Peste, F., A. Paula, L.P. da Silva, J. Bernardino, P. Pereira, M. Mascarenhas, H. Costa, J. Vieira, C. Bastos, C. Fonseca, M.J.R. Pereira. 2015. How to mitigate impacts of wind farms on bats? A review of potential conservation measures in the European context. Environmental Impact Assessment Review 51:10-22.

Piorkowski, M.D. and T.J. O'Connell. 2010. Spatial pattern of summer bat mortality from collisions with wind turbines in mixedgrass prairie. American Midland Naturalist 164(2):260-269.

Poulton, V. and W. Erickson. 2010. Postconstruction bat and bird fatality study Judith Gap Wind Farm Wheatland County, Montana. Final Report. Results from JuneOctober 2009 study and comparison with 2006-2007 study. Western Ecosystems Technology, Inc. 2003 Central Avenue, Cheyenne, WY. 35 p.
Puzen, S.C. 2002. Bat interactions with wind turbines in northeastern Wisconsin. Wisconsin Public Service Commission, Madison, Wisconsin.

Redell D., E.B. Arnett, J.P. Hayes, M.M.P. Huso. 2006. Patterns of preconstruction bat activity determined using acoustic monitoring at a proposed wind facility in south-central Wisconsin. A final report submitted to the Bats and Wind Energy Cooperative. Bat Conservation International. Austin, Texas, USA. http://www.batsandwind.org/pdf/precon wi.pdf

Reynolds, D.S. 2006. Monitoring the potential impact of a wind development site on bats in the northeast. Journal of Wildlife Management 70(5):1219-1227.

*Rollins, K.E., D.K. Meyerholz, G.D. Johnson, A.P. Capparella, and S.S. Loew. 2012. A forensic investigation into the etiology of bat mortality at a wind farm: barotrauma or traumatic injury? Veterinary Pathology 49(2):362-371.

Rocioni, F., H. Rebelo, D. Russo, M.L. Carranza, M.D. Febbraro, and A. Loy. 2014. A modelling approach to infer the effects of wind farms on landscape connectivity for bats. Landscape Ecology 29:891-903.

Rydell J., L. Bach, M. Dubourg-Savage, M. Green, L. Rodrigues, and A. Hedenström. 2010. Bat mortality at wind turbines in northwestern Europe. Acta Chiropterologica 12(2):261-274. doi:10.3161/150811010X537846

Rydell, J., L. Bach, M. Dubourg-Savage, M. Green, L. Rodrigues, and A. Hedenstrom. 2010. Mortality of bats at wind turbines links to nocturnal insect migration. European Journal of Wildlife Research 56:823-827. 
Saunders, W.E. 1930. Bats in migration. Journal of Mammalogy 11:225.

Schmidt, E., A.J. Piaggio, C.E. Bock, and D.M. Armstrong. 2003. National Wind Technology Center site environmental assessment: bird and bat use and fatalities Final report NREL/SR-500-32981, National Renewable Energy Laboratory, Golden, Colorado. 21pp.

*Schuster, E., L. Bulling, and J. Koppel. 2015. Consolidating the state of knowledge: a synoptical review of wind energy's wildlife effects. Environmental Management 56:300-331.

Sjollema, A.L., J.E. Gats, R.H. Hilderbrand, and J. Sherwell. 2014. Offshore activity of bats along the mid-Atlantic Coast. Northeastern Naturalist 21(2):154-163.

Smallwood, K.S. 2013. Comparing bird and bat fatality-rate estimates among North American wind-energy projects. Wildlife Society Bulletin 37(1):19-33.

Smallwood, K.S., D.A. Bell, S.A. Snyder, and J.E. Didonato. 2010. Novel scavenger removal trials increase wind turbine-caused avian fatality estimates. Journal of Wildlife Management 74(5):1089-1097.

Smallwood, K.S. and B. Karas. 2009. Avian and bat fatality rates at old-generation and repowered wind turbines in California. Journal of Wildlife Management 73(7):1062-1071.

Tennessee Valley Authority. 2002. Draft Environmental Assessment - 20-MW Windfarm and Associated Energy Storage Facility. Tennessee Valley Authority, Knoxville, Tennessee.

Terres, J.K. 1956. Migration records of the red bat, Lasiurus borealis. Journal of Mammalogy 37:442.
Thelander, C.G. and L. Rugge. 2000. Bird risk behaviors and fatalities at the Altamont Wind Resource Area. Pp. 5-14 in Proceedings of the National Avian-Wind Power Planning Meeting III. National Wind Coordinating Committee/RESOLVE. Washington, D.C.

Tuttle, M.D. 2004. Wind energy and the threat to bats. BATS 22(2):4-5.

U.S. Department of Energy. 2002. Draft SiteWide Environmental Assessment of National Renewable Energy Laboratory's National Wind Technology Center. U.S. Department of Energy, Golden, Colorado.

Van Gelder, R.G. 1956. Echo-location failure in migratory bats. Transactions of the Kansas Academy of Science 59:220-222.

Villegas-Patraca, R., S. Macias-Sanchez, I. MacGregor-Fors, and C. Munoz-Robles. 2012. Scavenger removal: bird and bat carcass persistence in a tropical wind farm. Acta Oecologica 43:121-125.

Voigt, C.C., L.S. Lehnert, G. Petersons, F. Adorf, and L. Bach. 2015. Wildlife and renewable energy: German politics cross migratory bats. European Journal of Wildlife Research 61:213-219.

*Voigt, C.C., A.G. Popa-Lisseanu, I. Niermann, and S. Kramer-Schadt. 2012. The catchment area of wind farms for European bats: a plea for international regulations. Biological Conservation 153:80-86.

*Weller, T.J. and J.A. Baldwin. 2012. Using echolocation monitoring to model bat occupancy and inform mitigations at wind energy facilities. Journal of Wildlife Management 76(3):619-631. 
Williams, W. 2004. When blade meets bat: Unexpected bat kills threaten future wind farms. Scientific American. February 2004.

Williams, W. 2003. Alarming evidence of bat kills in eastern U.S. Windpower Monthly 19: 21-23.

Winhold, L., A. Kurta, and R. Foster. 2008. Long-term change in an assemblage of North American bats: are eastern red bats declining? Acta Chiropterologica 10(2):359366.

Wisely, A.N. 1978. Bat dies on barbed wire fence. Blue Jay 36:53.

Wolbert, S.J., A.S. Zellner, H.P. Whidden. 2014. Bat activity, insect biomass, and temperature along an elevational gradient. Northeastern Naturalist 21(1):72-85.

Young, D.P., Jr., W.P. Erickson, R.E. Good, M.D. Strickland, and G.D. Johnson. 2003. Avian and bat mortality associated with the initial phase of the Foote Creek Rim wind power project, Carbon County, Wyoming: November 1998 - June 2002. Tech. Rept. prepared for SeaWest Energy Corporation and Bureau of Land Management.

Young, D.P., Jr., W.P. Erickson, M.D. Strickland, and R.E. Good. 2002. Comparison of avian effects from UV light reflective paint applied to wind turbines: Foote Creek Rim Wind Plant, Carbon County, Wyoming. National Renewable Energy Laboratory, Golden, Colorado. 


\section{Appendix B}

\section{Bat Pass temperatures summarized by species and month for all project detectors}

\begin{tabular}{|c|c|c|c|c|c|c|}
\hline Location & $\begin{array}{l}\frac{\mathscr{d}}{u} \\
\stackrel{d}{n}\end{array}$ & $\stackrel{\frac{1}{2}}{\frac{1}{2}}$ & $\begin{array}{l}\frac{c}{亡} \\
\stackrel{0}{\Sigma}\end{array}$ & $\begin{array}{c}\text { Bat Pass Temp C } \\
\text { Avg (SD) N }\end{array}$ & Bat Pass Min Temp C & Bat Pass Max Temp C \\
\hline Absaloka Mine Spring 12 & Epfu & 2012 & 8 & $17.2(4.4) 1446$ & 2.4 & 26.2 \\
\hline Absaloka Mine Spring 12 & Epfu & 2012 & 9 & $16.5(4.7) 1616$ & 1.6 & 24.7 \\
\hline Absaloka Mine Spring 12 & Epfu & 2012 & 10 & $14(4.3) 8$ & 9.2 & 18.3 \\
\hline Absaloka Mine Spring 12 & Epfu & 2013 & 4 & $9.6(3.1) 14$ & 5.4 & 13.6 \\
\hline Absaloka Mine Spring 12 & Epfu & 2013 & 5 & $15.1(3.4) 302$ & 5.5 & 19.1 \\
\hline Absaloka Mine Spring 12 & Epfu & 2013 & 6 & $17.6(3.6) 105$ & 8.4 & 25.2 \\
\hline Absaloka Mine Spring 12 & Epfu & 2013 & 10 & $8.7(0.7) 2$ & 8.2 & 9.2 \\
\hline Absaloka Mine Spring 12 & Epfu & 2014 & 4 & $12.2(5.6) 40$ & -0.1 & 18.6 \\
\hline Absaloka Mine Spring 12 & Epfu & 2014 & 5 & $16.1(4.6) 472$ & 2.2 & 27.2 \\
\hline Absaloka Mine Spring 12 & Epfu & 2014 & 6 & $14.1(2) 277$ & 7.7 & 19.1 \\
\hline Absaloka Mine Spring 12 & Epfu & 2014 & 8 & $12.4(2.6) 981$ & 7.5 & 22.4 \\
\hline Absaloka Mine Spring 12 & Epfu & 2014 & 9 & $13.8(4.2) 496$ & 1.3 & 22.2 \\
\hline Absaloka Mine Spring 12 & Epfu & 2014 & 12 & $0.7(0.6) 2$ & 0.3 & 1.1 \\
\hline Absaloka Mine Spring 12 & Epfu & 2015 & 1 & 1.1() 1 & 1.1 & 1.1 \\
\hline Absaloka Mine Spring 12 & Epfu & 2015 & 3 & $10.2(4.5) 19$ & 0.9 & 16 \\
\hline Absaloka Mine Spring 12 & Epfu & 2015 & 4 & $9.1(4.5) 232$ & 0.6 & 17 \\
\hline Absaloka Mine Spring 12 & Epfu & 2015 & 5 & $13.4(3.1) 882$ & 2.1 & 19.1 \\
\hline Absaloka Mine Spring 12 & Epfu & 2015 & 6 & $16.8(2.9) 549$ & 10.3 & 22.6 \\
\hline Absaloka Mine Spring 12 & Epfu & 2015 & 6 & $18.9(3.8) 3670$ & 8.4 & 29 \\
\hline Absaloka Mine Spring 12 & Epfu & 2015 & 7 & 17.8 (3.7) 2147 & 10.7 & 29.7 \\
\hline Absaloka Mine Spring 12 & Epfu & 2015 & 8 & $23.5(4.3) 35$ & 12.7 & 28.9 \\
\hline Absaloka Mine Spring 12 & Laci & 2012 & 8 & $17.2(5.4) 37$ & 5.7 & 24.4 \\
\hline Absaloka Mine Spring 12 & Laci & 2012 & 9 & $13.1(5.5) 53$ & -0.1 & 20.4 \\
\hline Absaloka Mine Spring 12 & Laci & 2013 & 4 & $6(0) 2$ & 6 & 6 \\
\hline Absaloka Mine Spring 12 & Laci & 2013 & 5 & $16(3.7) 8$ & 7.9 & 18.9 \\
\hline Absaloka Mine Spring 12 & Laci & 2013 & 6 & $15.9(2.7) 9$ & 12.2 & 20.4 \\
\hline Absaloka Mine Spring 12 & Laci & 2014 & 4 & $13.1(6.3) 4$ & 6.4 & 18.6 \\
\hline Absaloka Mine Spring 12 & Laci & 2014 & 5 & $15.9(4.3) 77$ & 9 & 26.4 \\
\hline Absaloka Mine Spring 12 & Laci & 2014 & 6 & $13.4(2.3) 64$ & 4.6 & 18.6 \\
\hline Absaloka Mine Spring 12 & Laci & 2014 & 7 & 19.6() 1 & 19.6 & 19.6 \\
\hline Absaloka Mine Spring 12 & Laci & 2014 & 8 & $14.3(2.6) 57$ & 7.5 & 17.6 \\
\hline Absaloka Mine Spring 12 & Laci & 2014 & 9 & $10.4(5.2) 32$ & 0.8 & 21.9 \\
\hline Absaloka Mine Spring 12 & Laci & 2015 & 3 & $9.1(5.2) 11$ & 2.4 & 15.1 \\
\hline Absaloka Mine Spring 12 & Laci & 2015 & 4 & $8.7(4.6) 64$ & -1.6 & 16.3 \\
\hline Absaloka Mine Spring 12 & Laci & 2015 & 5 & 13.1 (3) 145 & 2.7 & 18.9 \\
\hline
\end{tabular}




\begin{tabular}{|c|c|c|c|c|c|c|}
\hline Absaloka Mine Spring 12 & Laci & 2015 & 6 & $17.8(1.2) 28$ & 15 & 19.6 \\
\hline Absaloka Mine Spring 12 & Laci & 2015 & 6 & 18.7 (3.3) 949 & 9.4 & 29 \\
\hline Absaloka Mine Spring 12 & Laci & 2015 & 7 & $18.4(3.8) 305$ & 10.7 & 29.7 \\
\hline Absaloka Mine Spring 12 & Laci & 2015 & 8 & $17.8(3.2) 3$ & 14.1 & 19.9 \\
\hline Absaloka Mine Spring 12 & Lano & 2012 & 8 & $18.1(4.5) 232$ & 3.6 & 25.7 \\
\hline Absaloka Mine Spring 12 & Lano & 2012 & 9 & $10.6(5.2) 402$ & -1.8 & 24.1 \\
\hline Absaloka Mine Spring 12 & Lano & 2012 & 10 & $8.8(2.5) 4$ & 6.9 & 12.2 \\
\hline Absaloka Mine Spring 12 & Lano & 2013 & 4 & 6() 1 & 6 & 6 \\
\hline Absaloka Mine Spring 12 & Lano & 2013 & 5 & $14.4(3.5) 34$ & 4.6 & 18.9 \\
\hline Absaloka Mine Spring 12 & Lano & 2013 & 6 & $17.9(5.5) 3$ & 11.7 & 22.2 \\
\hline Absaloka Mine Spring 12 & Lano & 2014 & 4 & $11.1(5.4) 55$ & 1.9 & 18.6 \\
\hline Absaloka Mine Spring 12 & Lano & 2014 & 5 & $14.1(4.9) 394$ & 4.1 & 27.2 \\
\hline Absaloka Mine Spring 12 & Lano & 2014 & 6 & $13.4(2.3) 281$ & 4.4 & 19.1 \\
\hline Absaloka Mine Spring 12 & Lano & 2014 & 8 & $14.3(2.3) 64$ & 8.2 & 18.1 \\
\hline Absaloka Mine Spring 12 & Lano & 2014 & 9 & $9.2(4.6) 60$ & -0.8 & 17.9 \\
\hline Absaloka Mine Spring 12 & Lano & 2014 & 10 & $10.5(0) 2$ & 10.5 & 10.5 \\
\hline Absaloka Mine Spring 12 & Lano & 2014 & 12 & $3.4(0) 2$ & 3.4 & 3.4 \\
\hline Absaloka Mine Spring 12 & Lano & 2015 & 2 & 2.1() 1 & 2.1 & 2.1 \\
\hline Absaloka Mine Spring 12 & Lano & 2015 & 3 & 15.6() 1 & 15.6 & 15.6 \\
\hline Absaloka Mine Spring 12 & Lano & 2015 & 4 & $10.4(4.3) 29$ & 2.6 & 16.1 \\
\hline Absaloka Mine Spring 12 & Lano & 2015 & 5 & $13.6(3.1) 183$ & 2.1 & 19.3 \\
\hline Absaloka Mine Spring 12 & Lano & 2015 & 6 & $17(2.4) 187$ & 11 & 21.9 \\
\hline Absaloka Mine Spring 12 & Lano & 2015 & 6 & $18(3.3) 1201$ & 8.4 & 29 \\
\hline Absaloka Mine Spring 12 & Lano & 2015 & 7 & $17.7(3.5) 406$ & 10.7 & 29.7 \\
\hline Absaloka Mine Spring 12 & Lano & 2015 & 8 & $27.1(1.6) 2$ & 26 & 28.2 \\
\hline Absaloka Mine Spring 12 & Myci & 2012 & 8 & 19.5 (4.1) 94 & 7.9 & 25.5 \\
\hline Absaloka Mine Spring 12 & Myci & 2012 & 9 & $19.7(4.3) 141$ & 6 & 24.1 \\
\hline Absaloka Mine Spring 12 & Myci & 2012 & 10 & $15.9(3.8) 3$ & 11.5 & 18.4 \\
\hline Absaloka Mine Spring 12 & Myci & 2013 & 6 & $16.9(2.8) 4$ & 14.3 & 20.8 \\
\hline Absaloka Mine Spring 12 & Myci & 2013 & 10 & $12.3(0) 3$ & 12.3 & 12.3 \\
\hline Absaloka Mine Spring 12 & Myci & 2014 & 4 & $16.9(1.1) 3$ & 15.6 & 17.6 \\
\hline Absaloka Mine Spring 12 & Myci & 2014 & 5 & 15.6 () 1 & 15.6 & 15.6 \\
\hline Absaloka Mine Spring 12 & Myci & 2014 & 6 & $13.7(3.5) 2$ & 11.2 & 16.1 \\
\hline Absaloka Mine Spring 12 & Myci & 2014 & 8 & $16.8(1.3) 7$ & 14.1 & 17.9 \\
\hline Absaloka Mine Spring 12 & Myci & 2014 & 9 & $16.2(3) 14$ & 12.5 & 21.4 \\
\hline Absaloka Mine Spring 12 & Myci & 2015 & 4 & 10.3 () 1 & 10.3 & 10.3 \\
\hline Absaloka Mine Spring 12 & Myci & 2015 & 5 & 17.6() 1 & 17.6 & 17.6 \\
\hline Absaloka Mine Spring 12 & Myci & 2015 & 6 & 18.8() 1 & 18.8 & 18.8 \\
\hline Absaloka Mine Spring 12 & Myci & 2015 & 6 & $22(4.2) 282$ & 10.3 & 29 \\
\hline Absaloka Mine Spring 12 & Myci & 2015 & 7 & $21.3(2.9) 539$ & 13.3 & 29 \\
\hline Absaloka Mine Spring 12 & Myev & 2012 & 8 & $17.4(3.8) 56$ & 11.7 & 24.1 \\
\hline Absaloka Mine Spring 12 & Myev & 2012 & 9 & 10.5 (4.8) 266 & 0.4 & 23.9 \\
\hline Absaloka Mine Spring 12 & Myev & 2012 & 10 & -1.3() 1 & -1.3 & -1.3 \\
\hline Absaloka Mine Spring 12 & Myev & 2013 & 5 & $11.3(1.8) 2$ & 10 & 12.5 \\
\hline
\end{tabular}




\begin{tabular}{|c|c|c|c|c|c|c|}
\hline Absaloka Mine Spring 12 & Myev & 2013 & 6 & $18.4(1.7) 4$ & 16.8 & 19.9 \\
\hline Absaloka Mine Spring 12 & Myev & 2014 & 5 & $10.4(0.4) 2$ & 10.2 & 10.7 \\
\hline Absaloka Mine Spring 12 & Myev & 2014 & 6 & 13() 1 & 13 & 13 \\
\hline Absaloka Mine Spring 12 & Myev & 2014 & 8 & $16.4(1.3) 33$ & 9.4 & 17.8 \\
\hline Absaloka Mine Spring 12 & Myev & 2014 & 9 & $11.9(5.9) 34$ & -0.1 & 18.1 \\
\hline Absaloka Mine Spring 12 & Myev & 2014 & 10 & $11.7(0.9) 2$ & 11 & 12.3 \\
\hline Absaloka Mine Spring 12 & Myev & 2015 & 5 & $14.7(4.8) 2$ & 11.3 & 18.1 \\
\hline Absaloka Mine Spring 12 & Myev & 2015 & 6 & $20.6(3.8) 590$ & 12.5 & 29 \\
\hline Absaloka Mine Spring 12 & Myev & 2015 & 7 & $20.3(2.4) 739$ & 12.2 & 28.5 \\
\hline Absaloka Mine Spring 12 & Mylu & 2012 & 8 & $15.8(3.7) 56$ & 12.3 & 22.7 \\
\hline Absaloka Mine Spring 12 & Mylu & 2012 & 9 & $19.8(5.9) 66$ & -0.5 & 23.4 \\
\hline Absaloka Mine Spring 12 & Mylu & 2012 & 10 & 18.4() 1 & 18.4 & 18.4 \\
\hline Absaloka Mine Spring 12 & Mylu & 2013 & 6 & 15() 1 & 15 & 15 \\
\hline Absaloka Mine Spring 12 & Mylu & 2013 & 10 & 12.2 () 1 & 12.2 & 12.2 \\
\hline Absaloka Mine Spring 12 & Mylu & 2014 & 5 & $11.7(2.3) 8$ & 10.2 & 17.1 \\
\hline Absaloka Mine Spring 12 & Mylu & 2014 & 8 & $15.9(2) 3$ & 13.6 & 17.4 \\
\hline Absaloka Mine Spring 12 & Mylu & 2014 & 9 & $16.3(3.5) 6$ & 10 & 19.9 \\
\hline Absaloka Mine Spring 12 & Mylu & 2015 & 5 & $12.7(3.7) 4$ & 9.8 & 17.6 \\
\hline Absaloka Mine Spring 12 & Mylu & 2015 & 6 & $20.7(4.3) 20$ & 13.5 & 26.7 \\
\hline Absaloka Mine Spring 12 & Mylu & 2015 & 7 & $23.6(0.6) 6$ & 22.9 & 24.2 \\
\hline Absaloka Mine Spring 12 & Myth & 2012 & 8 & $19.2(4.6) 5$ & 13 & 25.1 \\
\hline Absaloka Mine Spring 12 & Myth & 2012 & 9 & $18.3(3.9) 5$ & 13 & 22.1 \\
\hline Absaloka Mine Spring 12 & Myth & 2014 & 9 & $14.9(3.2) 6$ & 11.8 & 20.9 \\
\hline Absaloka Mine Spring 12 & Myth & 2015 & 6 & $20.6(4.1) 168$ & 8.5 & 29 \\
\hline Absaloka Mine Spring 12 & Myth & 2015 & 7 & 18.7 (3) 56 & 14.3 & 25.5 \\
\hline Big Sky Mine Pond B-9 & Epfu & 2013 & 10 & $9.7(3.3) 720$ & 0 & 15 \\
\hline Big Sky Mine Pond B-9 & Epfu & 2013 & 11 & $3.5(3.3) 1113$ & -2.9 & 11 \\
\hline Big Sky Mine Pond B-9 & Epfu & 2013 & 12 & $5.6(2.7) 237$ & 0 & 12.3 \\
\hline Big Sky Mine Pond B-9 & Epfu & 2014 & 1 & $5.6(2.7) 307$ & -2.6 & 11.5 \\
\hline Big Sky Mine Pond B-9 & Epfu & 2014 & 2 & $4.9(2) 124$ & 0.8 & 10.3 \\
\hline Big Sky Mine Pond B-9 & Epfu & 2014 & 3 & $8.5(4.6) 448$ & -1.1 & 15.1 \\
\hline Big Sky Mine Pond B-9 & Epfu & 2014 & 4 & $12.9(4.4) 6951$ & 0.6 & 22.1 \\
\hline Big Sky Mine Pond B-9 & Epfu & 2014 & 5 & $12.5(0.8) 283$ & 9.7 & 14.5 \\
\hline Big Sky Mine Pond B-9 & Epfu & 2014 & 7 & $18.4(3.9) 56$ & 13.3 & 23.4 \\
\hline Big Sky Mine Pond B-9 & Epfu & 2014 & 8 & $19.2(4) 548$ & 13.3 & 29.8 \\
\hline Big Sky Mine Pond B-9 & Epfu & 2014 & 9 & $14.1(3) 62$ & 9.7 & 21.4 \\
\hline Big Sky Mine Pond B-9 & Epfu & 2015 & 3 & $12.3(4.5) 14296$ & -0.1 & 22.1 \\
\hline Big Sky Mine Pond B-9 & Epfu & 2015 & 4 & $12.5(4.1) 7490$ & -1.3 & 19.1 \\
\hline Big Sky Mine Pond B-9 & Epfu & 2015 & 5 & $14.5(3.7) 3006$ & 4.9 & 21.9 \\
\hline Big Sky Mine Pond B-9 & Epfu & 2015 & 6 & $18.6(2.5) 1536$ & 12.5 & 25.9 \\
\hline Big Sky Mine Pond B-9 & Epfu & 2015 & 6 & $21(3.5) 10169$ & 11.3 & 30.5 \\
\hline Big Sky Mine Pond B-9 & Epfu & 2015 & 7 & 19.9 (3.1) 10342 & 12.5 & 30.7 \\
\hline Big Sky Mine Pond B-9 & Epfu & 2015 & 8 & $19.5(2.9) 5006$ & 8.9 & 30.2 \\
\hline Big Sky Mine Pond B-9 & Epfu & 2015 & 9 & $21.1(3.7) 7945$ & 8.5 & 29.3 \\
\hline
\end{tabular}




\begin{tabular}{|c|c|c|c|c|c|c|}
\hline Big Sky Mine Pond B-9 & Epfu & 2015 & 10 & $15.7(4.9) 4269$ & 0.3 & 23.4 \\
\hline Big Sky Mine Pond B-9 & Epfu & 2015 & 11 & 7 (3.9) 2985 & -1.3 & 17.4 \\
\hline Big Sky Mine Pond B-9 & Epfu & 2015 & 12 & $6.5(4.4) 1589$ & -3.9 & 17.3 \\
\hline Big Sky Mine Pond B-9 & Epfu & 2016 & 1 & $6.4(3.4) 1335$ & -4.9 & 14.3 \\
\hline Big Sky Mine Pond B-9 & Epfu & 2016 & 2 & 6.5 (3) 5479 & -1.8 & 15.8 \\
\hline Big Sky Mine Pond B-9 & Epfu & 2016 & 3 & 9 (3.4) 7043 & -0.3 & 17.1 \\
\hline Big Sky Mine Pond B-9 & Epfu & 2016 & 4 & $13(3.1) 7616$ & 0.3 & 19.4 \\
\hline Big Sky Mine Pond B-9 & Epfu & 2016 & 5 & $15.9(2.7) 6844$ & 4.4 & 24.6 \\
\hline Big Sky Mine Pond B-9 & Epfu & 2016 & 6 & $20.1(4) 15390$ & 10.8 & 32 \\
\hline Big Sky Mine Pond B-9 & Epfu & 2016 & 7 & $21.4(3.8) 9213$ & 12 & 34.6 \\
\hline Big Sky Mine Pond B-9 & Epfu & 2016 & 8 & $19.7(3.7) 3914$ & 10.7 & 32.3 \\
\hline Big Sky Mine Pond B-9 & Epfu & 2016 & 9 & $19.3(3.5) 2719$ & 9.2 & 30.3 \\
\hline Big Sky Mine Pond B-9 & Epfu & 2016 & 10 & $12.2(4.5) 1939$ & 2.1 & 22.7 \\
\hline Big Sky Mine Pond B-9 & Epfu & 2016 & 11 & $10.3(3) 1938$ & 2.6 & 19.8 \\
\hline Big Sky Mine Pond B-9 & Euma & 2015 & 6 & 23.6() 1 & 23.6 & 23.6 \\
\hline Big Sky Mine Pond B-9 & Laci & 2013 & 10 & $10.2(3.9) 81$ & 0 & 15 \\
\hline Big Sky Mine Pond B-9 & Laci & 2013 & 11 & $2.3(3.4) 236$ & -2.9 & 10 \\
\hline Big Sky Mine Pond B-9 & Laci & 2013 & 12 & $5.3(2.3) 15$ & 1.6 & 9.2 \\
\hline Big Sky Mine Pond B-9 & Laci & 2014 & 1 & $4.9(2.9) 35$ & -0.6 & 10.5 \\
\hline Big Sky Mine Pond B-9 & Laci & 2014 & 2 & $4.6(1.6) 15$ & 2.2 & 8 \\
\hline Big Sky Mine Pond B-9 & Laci & 2014 & 3 & $7.4(4.7) 55$ & -0.6 & 14.3 \\
\hline Big Sky Mine Pond B-9 & Laci & 2014 & 4 & $10.1(4.4) 532$ & 1.1 & 22.1 \\
\hline Big Sky Mine Pond B-9 & Laci & 2014 & 5 & $11.3(2.4) 41$ & 4.7 & 14.3 \\
\hline Big Sky Mine Pond B-9 & Laci & 2014 & 7 & $16.3(5.1) 3$ & 13.2 & 22.2 \\
\hline Big Sky Mine Pond B-9 & Laci & 2014 & 8 & $16.4(2.8) 80$ & 12.3 & 26 \\
\hline Big Sky Mine Pond B-9 & Laci & 2014 & 9 & $11.7(1.4) 3$ & 10 & 12.5 \\
\hline Big Sky Mine Pond B-9 & Laci & 2015 & 3 & $10.7(4.7) 701$ & -0.1 & 21.2 \\
\hline Big Sky Mine Pond B-9 & Laci & 2015 & 4 & $9.3(4.9) 212$ & -1.3 & 18.1 \\
\hline Big Sky Mine Pond B-9 & Laci & 2015 & 5 & $16(4.2) 199$ & 6 & 21.1 \\
\hline Big Sky Mine Pond B-9 & Laci & 2015 & 6 & $18.4(3) 453$ & 12.5 & 26.4 \\
\hline Big Sky Mine Pond B-9 & Laci & 2015 & 6 & $21.2(3.5) 4081$ & 11.8 & 30.5 \\
\hline Big Sky Mine Pond B-9 & Laci & 2015 & 7 & $19.6(3.8) 4218$ & 12.7 & 31.3 \\
\hline Big Sky Mine Pond B-9 & Laci & 2015 & 8 & $17.8(2.1) 1266$ & 7.2 & 29.5 \\
\hline Big Sky Mine Pond B-9 & Laci & 2015 & 9 & $19.9(4.5) 254$ & 8.7 & 29.3 \\
\hline Big Sky Mine Pond B-9 & Laci & 2015 & 10 & 12.9 (5.6) 200 & 2.2 & 23.1 \\
\hline Big Sky Mine Pond B-9 & Laci & 2015 & 11 & $5.1(3.6) 176$ & -0.8 & 14.5 \\
\hline Big Sky Mine Pond B-9 & Laci & 2015 & 12 & 3.6 (3.9) 105 & -1.1 & 14.3 \\
\hline Big Sky Mine Pond B-9 & Laci & 2016 & 1 & $6.5(3.8) 73$ & -4.8 & 11.8 \\
\hline Big Sky Mine Pond B-9 & Laci & 2016 & 2 & $4.9(2.7) 347$ & -1.3 & 11.8 \\
\hline Big Sky Mine Pond B-9 & Laci & 2016 & 3 & $6.3(3.6) 373$ & -1.6 & 16.1 \\
\hline Big Sky Mine Pond B-9 & Laci & 2016 & 4 & 12.4 (3.6) 445 & 0.6 & 19.3 \\
\hline Big Sky Mine Pond B-9 & Laci & 2016 & 5 & $15.8(4) 316$ & 7.5 & 24.6 \\
\hline Big Sky Mine Pond B-9 & Laci & 2016 & 6 & $19.3(4.3) 4843$ & 10.7 & 32.2 \\
\hline Big Sky Mine Pond B-9 & Laci & 2016 & 7 & $20.6(3.8) 3294$ & 11.7 & 34.6 \\
\hline
\end{tabular}




\begin{tabular}{|c|c|c|c|c|c|c|}
\hline Big Sky Mine Pond B-9 & Laci & 2016 & 8 & $17.2(2.1) 1121$ & 10.3 & 32.3 \\
\hline Big Sky Mine Pond B-9 & Laci & 2016 & 9 & $18(2.3) 110$ & 11 & 23.9 \\
\hline Big Sky Mine Pond B-9 & Laci & 2016 & 10 & $12.3(4.1) 102$ & 5.4 & 22.4 \\
\hline Big Sky Mine Pond B-9 & Laci & 2016 & 11 & 8.8 (2.9) 110 & 2.6 & 15 \\
\hline Big Sky Mine Pond B-9 & Lano & 2013 & 10 & $8.6(3.6) 235$ & 0 & 15 \\
\hline Big Sky Mine Pond B-9 & Lano & 2013 & 11 & $1.8(3.1) 309$ & -4.1 & 10.7 \\
\hline Big Sky Mine Pond B-9 & Lano & 2013 & 12 & $3.7(2.3) 88$ & -0.5 & 10.2 \\
\hline Big Sky Mine Pond B-9 & Lano & 2014 & 1 & $3.9(3.5) 83$ & -2.6 & 11 \\
\hline Big Sky Mine Pond B-9 & Lano & 2014 & 2 & $3.7(1.1) 36$ & 2.2 & 5.4 \\
\hline Big Sky Mine Pond B-9 & Lano & 2014 & 3 & $10.1(3.9) 332$ & -0.6 & 14.8 \\
\hline Big Sky Mine Pond B-9 & Lano & 2014 & 4 & 9.9 (3.8) 997 & 1.9 & 22.1 \\
\hline Big Sky Mine Pond B-9 & Lano & 2014 & 5 & $12.1(1.2) 65$ & 4.7 & .5 \\
\hline Big Sky Mine Pond B-9 & Lano & 2014 & 7 & $16.1(3.2) 26$ & 13.3 & 22.4 \\
\hline Big Sky Mine Pond B-9 & Lano & 2014 & 8 & $16.7(3.6) 154$ & 13.3 & 28.7 \\
\hline Big Sky Mine Pond B-9 & Lano & 2014 & 9 & $13.8(2.3) 14$ & 10.2 & 17.3 \\
\hline Big Sky Mine Pond B-9 & Lano & 2015 & 3 & 10.4 (3.7) 2427 & -0.1 & 22. \\
\hline Big Sky Mine Pond B-9 & Lano & 2015 & 4 & $10.4(3.2) 881$ & -0.6 & 18.6 \\
\hline Big Sky Mine Pond B-9 & Lano & 2015 & 5 & $14.1(4.1) 627$ & 5.2 & 21.9 \\
\hline Big Sky Mine Pond B-9 & Lano & 2015 & 6 & $18.4(2.6) 466$ & 12.3 & 24.9 \\
\hline Big Sky Mine Pond B-9 & Lano & 2015 & 6 & 20.6 (3.3) 2045 & 11.8 & 30.2 \\
\hline Big Sky Mine Pond B-9 & Lano & 2015 & 7 & 19.4 (3.1) 2289 & 12.5 & 30.7 \\
\hline Big Sky Mine Pond B-9 & Lano & 2015 & 8 & 18.3 (3) 1478 & 5.5 & 27.4 \\
\hline Big Sky Mine Pond B-9 & Lano & 2015 & 9 & $17.8(3.8) 1621$ & 8.4 & 28.7 \\
\hline Big Sky Mine Pond B-9 & Lano & 2015 & 10 & $15.2(4.5) 733$ & 2.2 & 23.2 \\
\hline Big Sky Mine Pond B-9 & Lano & 2015 & 11 & 4.9 (3.8) 504 & -1.5 & 16.8 \\
\hline Big Sky Mine Pond B-9 & Lano & 2015 & 12 & $3.6(4.1) 286$ & -4.1 & 16.8 \\
\hline Big Sky Mine Pond B-9 & Lano & 2016 & 1 & $5.5(3.8) 182$ & -4.8 & 13.3 \\
\hline Big Sky Mine Pond B-9 & Lano & 2016 & 2 & $5.3(3.1) 792$ & -2.1 & 14. \\
\hline Big Sky Mine Pond B-9 & Lano & 2016 & 3 & 8.7 (3.8) 1837 & -1.6 & 16.5 \\
\hline Big Sky Mine Pond B-9 & Lano & 2016 & 4 & $12.1(3.4) 1388$ & 0.3 & 19.1 \\
\hline Big Sky Mine Pond B-9 & Lano & 2016 & 5 & $15.4(3.2) 1371$ & 4.4 & 24.4 \\
\hline Big Sky Mine Pond B-9 & Lano & 2016 & 6 & $19.7(3.8) 3311$ & 10.8 & 31.7 \\
\hline Big Sky Mine Pond B-9 & Lano & 2016 & 7 & $20.5(3.5) 1545$ & 12 & 33.6 \\
\hline Big Sky Mine Pond B-9 & Lano & 2016 & 8 & $17.5(2.8) 1201$ & 10 & 30.8 \\
\hline Big Sky Mine Pond B-9 & Lano & 2016 & 9 & $16.6(3.3) 949$ & 8.5 & 29.8 \\
\hline Big Sky Mine Pond B-9 & Lano & 2016 & 10 & $11.7(4.6) 540$ & 3.2 & 22.7 \\
\hline Big Sky Mine Pond B-9 & Lano & 2016 & 11 & $9(3.6) 557$ & 2.6 & 17.6 \\
\hline Big Sky Mine Pond B-9 & Myci & 2013 & 10 & $5.7(1.7) 3$ & 4.1 & 7.4 \\
\hline Big Sky Mine Pond B-9 & Myci & 2013 & 11 & $2.4(4.1) 28$ & -3.9 & 12 \\
\hline Big Sky Mine Pond B-9 & Myci & 2013 & 12 & $6(1.8) 5$ & 3.6 & 8.4 \\
\hline Big Sky Mine Pond B-9 & Myci & 2014 & 1 & $4.3(3.5) 11$ & -1 & 8.9 \\
\hline Big Sky Mine Pond B-9 & Myci & 2014 & 2 & $4.3(1.1) 11$ & 2.1 & 5.1 \\
\hline Big Sky Mine Pond B-9 & Myci & 2014 & 3 & $4.7(2.8) 8$ & 0.3 & 7.2 \\
\hline Big Sky Mine Pond B-9 & Myci & 2014 & 4 & $14.3(5.5) 58$ & 3.6 & 21 \\
\hline
\end{tabular}




\begin{tabular}{|c|c|c|c|c|c|c|}
\hline Big Sky Mine Pond B-9 & Myci & 2014 & 5 & $12.2(0.6) 12$ & 10.8 & 12.7 \\
\hline Big Sky Mine Pond B-9 & Myci & 2014 & 7 & $21(2.1) 144$ & 13.3 & 23.4 \\
\hline Big Sky Mine Pond B-9 & Myci & 2014 & 8 & 20.9 (3.3) 822 & 11 & 30.7 \\
\hline Big Sky Mine Pond B-9 & Myci & 2014 & 9 & $16.5(2.4) 35$ & 10.5 & 20.8 \\
\hline Big Sky Mine Pond B-9 & Myci & 2015 & 3 & $13.3(4.7) 47$ & 2.2 & 17.9 \\
\hline Big Sky Mine Pond B-9 & Myci & 2015 & 4 & $9.6(4.2) 47$ & 2.9 & 17 \\
\hline Big Sky Mine Pond B-9 & Myci & 2015 & 5 & $15.9(2) 765$ & 6.5 & 21.6 \\
\hline Big Sky Mine Pond B-9 & Myci & 2015 & 6 & $18.3(1.6) 873$ & 14 & 24.9 \\
\hline Big Sky Mine Pond B-9 & Myci & 2015 & 6 & $21.7(3.3) 2123$ & 12.2 & 30.3 \\
\hline Big Sky Mine Pond B-9 & Myci & 2015 & 7 & $21.7(2.4) 6402$ & 12.8 & 30.2 \\
\hline Big Sky Mine Pond B-9 & Myci & 2015 & 8 & $21.4(2.8) 4604$ & 10.3 & 30.7 \\
\hline Big Sky Mine Pond B-9 & Myci & 2015 & 9 & $22(3.7) 2252$ & 9.7 & 29.2 \\
\hline Big Sky Mine Pond B-9 & Myci & 2015 & 10 & $14.5(5.4) 484$ & 0.1 & 23.1 \\
\hline Big Sky Mine Pond B-9 & Myci & 2015 & 11 & $7.2(3.8) 329$ & -0.6 & 17.4 \\
\hline Big Sky Mine Pond B-9 & Myci & 2015 & 12 & $6.2(4) 260$ & -0.8 & 17.3 \\
\hline Big Sky Mine Pond B-9 & Myci & 2016 & 1 & $6.3(2.6) 153$ & 0.8 & 13.5 \\
\hline Big Sky Mine Pond B-9 & Myci & 2016 & 2 & $6(3.2) 401$ & -0.8 & 14 \\
\hline Big Sky Mine Pond B-9 & Myci & 2016 & 3 & $7.7(2.9) 358$ & 1.7 & 15.3 \\
\hline Big Sky Mine Pond B-9 & Myci & 2016 & 4 & 13.5 (3.1) 504 & 2.2 & 18.8 \\
\hline Big Sky Mine Pond B-9 & Myci & 2016 & 5 & $17.5(2.4) 1759$ & 8.4 & 24.7 \\
\hline Big Sky Mine Pond B-9 & Myci & 2016 & 6 & $21.9(3.5) 4938$ & 10.7 & 32.2 \\
\hline Big Sky Mine Pond B-9 & Myci & 2016 & 7 & $23.5(3.5) 6040$ & 13.3 & 34.3 \\
\hline Big Sky Mine Pond B-9 & Myci & 2016 & 8 & $20.8(4.3) 3375$ & 11.5 & 32.2 \\
\hline Big Sky Mine Pond B-9 & Myci & 2016 & 9 & $21(3.4) 1127$ & 9.2 & 29.8 \\
\hline Big Sky Mine Pond B-9 & Myci & 2016 & 10 & $14.5(4.4) 193$ & 2.7 & 22.7 \\
\hline Big Sky Mine Pond B-9 & Myci & 2016 & 11 & $10.4(3.4) 120$ & 2.9 & 18.6 \\
\hline Big Sky Mine Pond B-9 & Myev & 2014 & 7 & $22.9(0) 2$ & 22.9 & 22.9 \\
\hline Big Sky Mine Pond B-9 & Myev & 2014 & 8 & $20.2(6.5) 8$ & 12.2 & 30 \\
\hline Big Sky Mine Pond B-9 & Myev & 2014 & 9 & 15.3() 1 & 15.3 & 15.3 \\
\hline Big Sky Mine Pond B-9 & Myev & 2015 & 4 & 4.2() 1 & 4.2 & 4.2 \\
\hline Big Sky Mine Pond B-9 & Myev & 2015 & 5 & 14.3 (3) 26 & 8.9 & 20.8 \\
\hline Big Sky Mine Pond B-9 & Myev & 2015 & 6 & $18.2(1.6) 7$ & 14.6 & 19.3 \\
\hline Big Sky Mine Pond B-9 & Myev & 2015 & 6 & 21.7 (3.3) 240 & 12.3 & 29.8 \\
\hline Big Sky Mine Pond B-9 & Myev & 2015 & 7 & 21.7 (3.3) 323 & 12.8 & 28.7 \\
\hline Big Sky Mine Pond B-9 & Myev & 2015 & 8 & 20.5 (3.7) 172 & 10.2 & 29.2 \\
\hline Big Sky Mine Pond B-9 & Myev & 2015 & 9 & 19.4 (4.6) 156 & 10.2 & 28.4 \\
\hline Big Sky Mine Pond B-9 & Myev & 2016 & 1 & 7.5() 1 & 7.5 & 7.5 \\
\hline Big Sky Mine Pond B-9 & Myev & 2016 & 3 & 12.3() 1 & 12.3 & 12.3 \\
\hline Big Sky Mine Pond B-9 & Myev & 2016 & 4 & $14(2.1) 6$ & 11.3 & 17.9 \\
\hline Big Sky Mine Pond B-9 & Myev & 2016 & 5 & 13.2 (3.5) 230 & 6.4 & 23.9 \\
\hline Big Sky Mine Pond B-9 & Myev & 2016 & 6 & 20.9 (4.9) 184 & 12.3 & 31.5 \\
\hline Big Sky Mine Pond B-9 & Myev & 2016 & 7 & 22.5 (4.1) 292 & 15.3 & 34 \\
\hline Big Sky Mine Pond B-9 & Myev & 2016 & 8 & 20.2 (4.6) 257 & 10.3 & 31 \\
\hline Big Sky Mine Pond B-9 & Myev & 2016 & 9 & $21.2(4.8) 88$ & 10 & 29.8 \\
\hline
\end{tabular}




\begin{tabular}{|c|c|c|c|c|c|c|}
\hline Big Sky Mine Pond B-9 & Myev & 2016 & 10 & $18.9(0) 2$ & 18.9 & 18.9 \\
\hline Big Sky Mine Pond B-9 & Myev & 2016 & 11 & 14.5 () 1 & 14.5 & 14.5 \\
\hline Big Sky Mine Pond B-9 & Mylu & 2013 & 10 & $12(2.3) 3$ & 10.3 & 14.6 \\
\hline Big Sky Mine Pond B-9 & Mylu & 2013 & 11 & $2.7(3.1) 4$ & 0 & 5.5 \\
\hline Big Sky Mine Pond B-9 & Mylu & 2014 & 4 & $16.5(5.3) 26$ & 1.7 & 21.6 \\
\hline Big Sky Mine Pond B-9 & Mylu & 2014 & 5 & $10.4(4.4) 10$ & 3.9 & 14.1 \\
\hline Big Sky Mine Pond B-9 & Mylu & 2014 & 7 & 20.4() 1 & 20.4 & 20.4 \\
\hline Big Sky Mine Pond B-9 & Mylu & 2014 & 8 & $21.8(3.3) 42$ & 14.1 & 28.7 \\
\hline Big Sky Mine Pond B-9 & Mylu & 2014 & 9 & $15.2(3.2) 8$ & 9.5 & 18.3 \\
\hline Big Sky Mine Pond B-9 & Mylu & 2015 & 3 & $11.4(4.9) 16$ & 5.4 & 17 \\
\hline Big Sky Mine Pond B-9 & Mylu & 2015 & 4 & $10.9(4.3) 13$ & 2.9 & 18.4 \\
\hline Big Sky Mine Pond B-9 & Mylu & 2015 & 5 & $15.2(2.5) 98$ & 6.2 & 20.8 \\
\hline Big Sky Mine Pond B-9 & Mylu & 2015 & 6 & $18.4(2) 119$ & 14.5 & 24.4 \\
\hline Big Sky Mine Pond B-9 & Mylu & 2015 & 6 & $21.8(2.2) 749$ & 15.3 & 30 \\
\hline Big Sky Mine Pond B-9 & Mylu & 2015 & 7 & 22.8 (3) 524 & 12.7 & 30.5 \\
\hline Big Sky Mine Pond B-9 & Mylu & 2015 & 8 & $20.8(3.5) 235$ & 9.8 & 30.7 \\
\hline Big Sky Mine Pond B-9 & Mylu & 2015 & 9 & 20.5 (3.8) 177 & 11.5 & 27.9 \\
\hline Big Sky Mine Pond B-9 & Mylu & 2015 & 10 & $18.8(2.2) 14$ & 13.8 & 23.1 \\
\hline Big Sky Mine Pond B-9 & Mylu & 2015 & 11 & $6.6(4.3) 5$ & 2.6 & 13.6 \\
\hline Big Sky Mine Pond B-9 & Mylu & 2016 & 2 & $9.1(2.5) 3$ & 6.4 & 11.2 \\
\hline Big Sky Mine Pond B-9 & Mylu & 2016 & 3 & $10.1(2) 2$ & 8.7 & 11.5 \\
\hline Big Sky Mine Pond B-9 & Mylu & 2016 & 4 & $11.3(2.7) 116$ & 7.5 & 18.6 \\
\hline Big Sky Mine Pond B-9 & Mylu & 2016 & 5 & $14.4(4.1) 653$ & 3.6 & 24.7 \\
\hline Big Sky Mine Pond B-9 & Mylu & 2016 & 6 & $22.4(3.4) 805$ & 11.3 & 32.2 \\
\hline Big Sky Mine Pond B-9 & Mylu & 2016 & 7 & $25.1(3.5) 872$ & 14.6 & 33.6 \\
\hline Big Sky Mine Pond B-9 & Mylu & 2016 & 8 & $23.1(4.7) 361$ & 13 & 32.2 \\
\hline Big Sky Mine Pond B-9 & Mylu & 2016 & 9 & 20.4 (4) 204 & 10.3 & 30 \\
\hline Big Sky Mine Pond B-9 & Mylu & 2016 & 10 & $17.6(3.8) 9$ & 13 & 21.9 \\
\hline Big Sky Mine Pond B-9 & Mylu & 2016 & 11 & $16.6(0.1) 3$ & 16.5 & 16.6 \\
\hline Big Sky Mine Pond B-9 & Myth & 2015 & 6 & $22(3.8) 15$ & 15 & 27.7 \\
\hline Big Sky Mine Pond B-9 & Myth & 2015 & 6 & $17.4(2.5) 2$ & 15.6 & 19.1 \\
\hline Big Sky Mine Pond B-9 & Myth & 2015 & 7 & $23.1(3.2) 22$ & 12.7 & 27.4 \\
\hline Big Sky Mine Pond B-9 & Myth & 2015 & 8 & $17.8(0.7) 9$ & 16 & 18.3 \\
\hline Big Sky Mine Pond B-9 & Myth & 2015 & 9 & $20.5(4.9) 3$ & 16.6 & 26 \\
\hline Big Sky Mine Pond B-9 & Myth & 2015 & 10 & $17(1.2) 2$ & 16.1 & 17.8 \\
\hline Big Sky Mine Pond B-9 & Myth & 2016 & 5 & $15.4(2.2) 17$ & 12.8 & 21.1 \\
\hline Big Sky Mine Pond B-9 & Myth & 2016 & 6 & $20.8(4.9) 11$ & 14.1 & 27 \\
\hline Big Sky Mine Pond B-9 & Myth & 2016 & 7 & $24.6(3.5) 14$ & 19.3 & 30.7 \\
\hline Big Sky Mine Pond B-9 & Myth & 2016 & 8 & $18.2(4.7) 9$ & 13.6 & 28.9 \\
\hline Big Sky Mine Pond B-9 & Myth & 2016 & 10 & $10.2(0.5) 2$ & 9.8 & 10.5 \\
\hline Otter Creek Coal Tract & Epfu & 2013 & 10 & $8.3(7.4) 2$ & 3.1 & 13.5 \\
\hline Otter Creek Coal Tract & Epfu & 2014 & 3 & $0.8(0) 3$ & 0.8 & 0.8 \\
\hline Otter Creek Coal Tract & Epfu & 2014 & 5 & $14.4(2.8) 303$ & 8 & 22.1 \\
\hline Otter Creek Coal Tract & Epfu & 2014 & 6 & $13.8(2.4) 207$ & 8.4 & 18.9 \\
\hline
\end{tabular}




\begin{tabular}{|c|c|c|c|c|c|c|}
\hline Otter Creek Coal Tract & Epfu & 2014 & 7 & $16.2(3.8) 460$ & 9.4 & 23.1 \\
\hline Otter Creek Coal Tract & Epfu & 2014 & 8 & $12.8(1.7) 190$ & 9.2 & 22.6 \\
\hline Otter Creek Coal Tract & Epfu & 2014 & 9 & $9.3(2.1) 38$ & 5.7 & 14.5 \\
\hline Otter Creek Coal Tract & Epfu & 2015 & 2 & 0.6() 1 & 0.6 & 0.6 \\
\hline Otter Creek Coal Tract & Epfu & 2015 & 3 & $10.4(5.9) 5$ & 4.1 & 17.8 \\
\hline Otter Creek Coal Tract & Epfu & 2015 & 4 & $9.8(2.8) 40$ & 5.5 & 15.6 \\
\hline Otter Creek Coal Tract & Epfu & 2015 & 5 & 13.5 (2.9) 267 & 6.4 & 18.9 \\
\hline Otter Creek Coal Tract & Epfu & 2015 & 6 & $18.2(1.8) 214$ & 13.3 & 21.1 \\
\hline Otter Creek Coal Tract & Epfu & 2015 & 6 & $19.3(2.5) 1545$ & 12.7 & 24.2 \\
\hline Otter Creek Coal Tract & Epfu & 2015 & 7 & $17(3.4) 418$ & 11.3 & 27.7 \\
\hline Otter Creek Coal Tract & Epfu & 2015 & 8 & $16.4(3.9) 505$ & 9.4 & 27.9 \\
\hline Otter Creek Coal Tract & Epfu & 2015 & 9 & 17.9 (3.6) 240 & 6.5 & 26 \\
\hline Otter Creek Coal Tract & Epfu & 2015 & 10 & $14.2(3.8) 34$ & 8.7 & 19.1 \\
\hline Otter Creek Coal Tract & Epfu & 2015 & 11 & $4.2(0) 2$ & 4.2 & 4.2 \\
\hline Otter Creek Coal Tract & Epfu & 2015 & 12 & $2.6(2) 50$ & -0.1 & 6.4 \\
\hline Otter Creek Coal Tract & Epfu & 2016 & 1 & $3(1.7) 25$ & 0.8 & 6.5 \\
\hline Otter Creek Coal Tract & Epfu & 2016 & 2 & $2.6(1.2) 9$ & 0.8 & 4.9 \\
\hline Otter Creek Coal Tract & Epfu & 2016 & 3 & $11.4(2.2) 34$ & 7 & 14.3 \\
\hline Otter Creek Coal Tract & Epfu & 2016 & 4 & $13.6(2.6) 289$ & 5.2 & 17.4 \\
\hline Otter Creek Coal Tract & Epfu & 2016 & 5 & $15(2.5) 778$ & 3.2 & 19.1 \\
\hline Otter Creek Coal Tract & Epfu & 2016 & 6 & $19.2(3.9) 1668$ & 9.2 & 29.5 \\
\hline Otter Creek Coal Tract & Euma & 2015 & 8 & $16.8(1.3) 7$ & 14.5 & 18.6 \\
\hline Otter Creek Coal Tract & Euma & 2015 & 9 & $16.2(1.2) 4$ & 14.8 & 17.4 \\
\hline Otter Creek Coal Tract & Euma & 2015 & 10 & $16.6(1) 21$ & 14.6 & 18.8 \\
\hline Otter Creek Coal Tract & Laci & 2014 & 5 & 13.7 (3) 155 & 7.5 & 22.6 \\
\hline Otter Creek Coal Tract & Laci & 2014 & 6 & 14.5 (2.9) 205 & 6.7 & 19.8 \\
\hline Otter Creek Coal Tract & Laci & 2014 & 7 & $16.5(4.2) 434$ & 7.5 & 23.4 \\
\hline Otter Creek Coal Tract & Laci & 2014 & 8 & $12.3(1.4) 80$ & 8 & 15.8 \\
\hline Otter Creek Coal Tract & Laci & 2014 & 9 & $8.1(1.7) 29$ & 5.7 & 12.7 \\
\hline Otter Creek Coal Tract & Laci & 2015 & 3 & $6.4(3.1) 5$ & 3.9 & 11.5 \\
\hline Otter Creek Coal Tract & Laci & 2015 & 4 & $10.3(3.4) 29$ & 5.5 & 14.6 \\
\hline Otter Creek Coal Tract & Laci & 2015 & 5 & $12.3(2.3) 341$ & 5.5 & 19.3 \\
\hline Otter Creek Coal Tract & Laci & 2015 & 6 & $17.4(2.7) 185$ & 10.8 & 21.1 \\
\hline Otter Creek Coal Tract & Laci & 2015 & 6 & 17.9 (2.7) 1059 & 11.7 & 24.2 \\
\hline Otter Creek Coal Tract & Laci & 2015 & 7 & 15.4 (3.1) 406 & 9.7 & 27.9 \\
\hline Otter Creek Coal Tract & Laci & 2015 & 8 & 15 (2.7) 247 & 7.4 & 26.4 \\
\hline Otter Creek Coal Tract & Laci & 2015 & 9 & 14.7 (3.9) 39 & 6.2 & 22.2 \\
\hline Otter Creek Coal Tract & Laci & 2015 & 10 & $14.6(1.5) 3$ & 13.2 & 16.1 \\
\hline Otter Creek Coal Tract & Laci & 2015 & 11 & $4.2(0) 8$ & 4.2 & 4.2 \\
\hline Otter Creek Coal Tract & Laci & 2015 & 12 & $4.6(0.2) 11$ & 4.4 & 4.9 \\
\hline Otter Creek Coal Tract & Laci & 2016 & 1 & $2.9(0) 13$ & 2.9 & 2.9 \\
\hline Otter Creek Coal Tract & Laci & 2016 & 2 & $3.5(0.8) 2$ & 2.9 & 4.1 \\
\hline Otter Creek Coal Tract & Laci & 2016 & 3 & 11.9 (1.9) 14 & 7 & 13.5 \\
\hline Otter Creek Coal Tract & Laci & 2016 & 4 & $12(4.2) 60$ & 3.1 & 16.1 \\
\hline
\end{tabular}




\begin{tabular}{|c|c|c|c|c|c|c|}
\hline Otter Creek Coal Tract & Laci & 2016 & 5 & 14.5 (2.4) 117 & 5.1 & 18.8 \\
\hline Otter Creek Coal Tract & Laci & 2016 & 6 & $18.3(4.6) 1074$ & 9.2 & 29.2 \\
\hline Otter Creek Coal Tract & Lano & 2013 & 10 & 3.4() 1 & 3.4 & 3.4 \\
\hline Otter Creek Coal Tract & Lano & 2014 & 2 & 2.9() 1 & 2.9 & 2.9 \\
\hline Otter Creek Coal Tract & Lano & 2014 & 5 & 13.1 (3) 242 & 6.7 & 21.4 \\
\hline Otter Creek Coal Tract & Lano & 2014 & 6 & $13.8(2.2) 84$ & 7.5 & 18.6 \\
\hline Otter Creek Coal Tract & Lano & 2014 & 7 & $15.1(3.9) 113$ & 7.7 & 22.4 \\
\hline Otter Creek Coal Tract & Lano & 2014 & 8 & $12.3(1) 51$ & 8.9 & 15.3 \\
\hline Otter Creek Coal Tract & Lano & 2014 & 9 & $8.5(2.1) 87$ & 4.9 & 12.7 \\
\hline Otter Creek Coal Tract & Lano & 2014 & 11 & -2.8() 1 & -2.8 & -2.8 \\
\hline Otter Creek Coal Tract & Lano & 2014 & 12 & 1.3() 1 & 1.3 & 1.3 \\
\hline Otter Creek Coal Tract & Lano & 2015 & 3 & $10.3(3.9) 36$ & 3.7 & 18.3 \\
\hline Otter Creek Coal Tract & Lano & 2015 & 4 & $10(3.1) 115$ & 1.7 & 17.9 \\
\hline Otter Creek Coal Tract & Lano & 2015 & 5 & $11.8(2.8) 501$ & 5.7 & 18.9 \\
\hline Otter Creek Coal Tract & Lano & 2015 & 6 & $18.1(2) 131$ & 12.8 & 21.1 \\
\hline Otter Creek Coal Tract & Lano & 2015 & 6 & $18.3(2.4) 586$ & 12.3 & 24.1 \\
\hline Otter Creek Coal Tract & Lano & 2015 & 7 & $14.6(2.4) 123$ & 10.3 & 21.9 \\
\hline Otter Creek Coal Tract & Lano & 2015 & 8 & $15.7(3.2) 479$ & 2.2 & 26.4 \\
\hline Otter Creek Coal Tract & Lano & 2015 & 9 & $15.2(3.8) 627$ & 6.7 & 27.2 \\
\hline Otter Creek Coal Tract & Lano & 2015 & 10 & $15.3(5) 8$ & 8.7 & 19.4 \\
\hline Otter Creek Coal Tract & Lano & 2015 & 12 & $4.2(0.8) 8$ & 2.7 & 5.5 \\
\hline Otter Creek Coal Tract & Lano & 2016 & 1 & 4.2() 1 & 4.2 & 4.2 \\
\hline Otter Creek Coal Tract & Lano & 2016 & 3 & $11.6(0.4) 7$ & 10.8 & 11.8 \\
\hline Otter Creek Coal Tract & Lano & 2016 & 4 & 11.9 (3) 187 & 4.4 & 17.6 \\
\hline Otter Creek Coal Tract & Lano & 2016 & 5 & 14 (3) 393 & 4.2 & 19.1 \\
\hline Otter Creek Coal Tract & Lano & 2016 & 6 & $19(3.8) 399$ & 10.7 & 29.5 \\
\hline Otter Creek Coal Tract & Myci & 2013 & 10 & 13() 1 & 13 & 13 \\
\hline Otter Creek Coal Tract & Myci & 2014 & 5 & 11.9 (2.6) 1589 & 6 & 22.1 \\
\hline Otter Creek Coal Tract & Myci & 2014 & 6 & $12.3(2.7) 2001$ & 7 & 18.9 \\
\hline Otter Creek Coal Tract & Myci & 2014 & 7 & $14.7(2.1) 815$ & 9.2 & 21.7 \\
\hline Otter Creek Coal Tract & Myci & 2014 & 8 & 14.4 (1.9) 104 & 10.7 & 20.6 \\
\hline Otter Creek Coal Tract & Myci & 2014 & 9 & $12.2(1.9) 35$ & 4.9 & 15 \\
\hline Otter Creek Coal Tract & Myci & 2015 & 3 & 10.5 () 1 & 10.5 & 10.5 \\
\hline Otter Creek Coal Tract & Myci & 2015 & 4 & $10.6(2.4) 111$ & 3.4 & 16.1 \\
\hline Otter Creek Coal Tract & Myci & 2015 & 5 & $12.3(1.8) 1774$ & 3.2 & 17.6 \\
\hline Otter Creek Coal Tract & Myci & 2015 & 6 & $14.6(1.7) 714$ & 10.7 & 20.4 \\
\hline Otter Creek Coal Tract & Myci & 2015 & 6 & $17.2(1.8) 1161$ & 12.8 & 24.2 \\
\hline Otter Creek Coal Tract & Myci & 2015 & 7 & 16.8 (1.9) 1785 & 11.7 & 27 \\
\hline Otter Creek Coal Tract & Myci & 2015 & 8 & $17.4(2.4) 1684$ & 9.2 & 26.9 \\
\hline Otter Creek Coal Tract & Myci & 2015 & 9 & $18.1(2.5) 541$ & 11 & 25.9 \\
\hline Otter Creek Coal Tract & Myci & 2015 & 10 & $16(3.3) 3$ & 12.2 & 18.1 \\
\hline Otter Creek Coal Tract & Myci & 2016 & 3 & $13.6(1.3) 5$ & 12.3 & 15.3 \\
\hline Otter Creek Coal Tract & Myci & 2016 & 4 & $14.1(2.1) 786$ & 7.7 & 18.3 \\
\hline Otter Creek Coal Tract & Myci & 2016 & 5 & $14.3(2.3) 3887$ & 7.2 & 19.1 \\
\hline
\end{tabular}




\begin{tabular}{|c|c|c|c|c|c|c|}
\hline Otter Creek Coal Tract & Myci & 2016 & 6 & $17.9(2.8) 1487$ & 9 & 29.7 \\
\hline Otter Creek Coal Tract & Myev & 2014 & 5 & 9.5() 1 & 9.5 & 9.5 \\
\hline Otter Creek Coal Tract & Myev & 2014 & 6 & 12.8() 1 & 12.8 & 12.8 \\
\hline Otter Creek Coal Tract & Myev & 2014 & 7 & 14.8() 1 & 14.8 & 14.8 \\
\hline Otter Creek Coal Tract & Myev & 2014 & 8 & $11.2(0.2) 3$ & 11 & 11.3 \\
\hline Otter Creek Coal Tract & Myev & 2014 & 9 & 8.2() 1 & 8.2 & 8.2 \\
\hline Otter Creek Coal Tract & Myev & 2015 & 3 & 17.3() 1 & 17.3 & 17.3 \\
\hline Otter Creek Coal Tract & Myev & 2015 & 5 & $12.1(2) 2$ & 10.7 & 13.5 \\
\hline Otter Creek Coal Tract & Myev & 2015 & 6 & $17.7(2.7) 38$ & 14.3 & 23.6 \\
\hline Otter Creek Coal Tract & Myev & 2015 & 7 & $16.9(2.6) 23$ & 11.5 & 20.8 \\
\hline Otter Creek Coal Tract & Myev & 2015 & 8 & $15.2(3.3) 28$ & 10.5 & 22.2 \\
\hline Otter Creek Coal Tract & Myev & 2015 & 9 & $14.2(2.1) 21$ & 11.2 & 19.1 \\
\hline Otter Creek Coal Tract & Myev & 2016 & 4 & $10.8(5.7) 3$ & 7.4 & 17.4 \\
\hline Otter Creek Coal Tract & Myev & 2016 & 5 & 8.8 (3) 31 & 3.9 & 15.3 \\
\hline Otter Creek Coal Tract & Myev & 2016 & 6 & $18(3.8) 24$ & 10.3 & 24.9 \\
\hline Otter Creek Coal Tract & Mylu & 2013 & 10 & 12.8() 1 & 12.8 & 12.8 \\
\hline Otter Creek Coal Tract & Mylu & 2014 & 5 & $11.9(3.2) 676$ & 6.4 & 22.7 \\
\hline Otter Creek Coal Tract & Mylu & 2014 & 6 & $13.4(2.2) 1008$ & 7.7 & 18.6 \\
\hline Otter Creek Coal Tract & Mylu & 2014 & 7 & $14.5(2) 886$ & 7.9 & 22.7 \\
\hline Otter Creek Coal Tract & Mylu & 2014 & 8 & 13.4 (1.8) 222 & 9.4 & 19.3 \\
\hline Otter Creek Coal Tract & Mylu & 2014 & 9 & $10.3(2.2) 69$ & 6.5 & 15.3 \\
\hline Otter Creek Coal Tract & Mylu & 2015 & 3 & $11.6(0.7) 4$ & 10.7 & 12.3 \\
\hline Otter Creek Coal Tract & Mylu & 2015 & 4 & $8.5(2.8) 159$ & 0.9 & 15.6 \\
\hline Otter Creek Coal Tract & Mylu & 2015 & 5 & $11.3(2) 658$ & 0 & 18.6 \\
\hline Otter Creek Coal Tract & Mylu & 2015 & 6 & $15.5(2) 183$ & 11 & 21.1 \\
\hline Otter Creek Coal Tract & Mylu & 2015 & 6 & $17.7(2.3) 1784$ & 12 & 24.4 \\
\hline Otter Creek Coal Tract & Mylu & 2015 & 7 & $16.8(2.3) 1004$ & 9 & 27.9 \\
\hline Otter Creek Coal Tract & Mylu & 2015 & 8 & $15.3(3.3) 814$ & 4.4 & 25.2 \\
\hline Otter Creek Coal Tract & Mylu & 2015 & 9 & $14.2(3.5) 652$ & 6.4 & 24.6 \\
\hline Otter Creek Coal Tract & Mylu & 2015 & 10 & 16.6() 1 & 16.6 & 16.6 \\
\hline Otter Creek Coal Tract & Mylu & 2016 & 4 & 10.2 (4) 278 & 2.7 & 17.1 \\
\hline Otter Creek Coal Tract & Mylu & 2016 & 5 & $9.8(4.2) 1045$ & 1.1 & 19.1 \\
\hline Otter Creek Coal Tract & Mylu & 2016 & 6 & $18(2.5) 3713$ & 9.5 & 29.3 \\
\hline Otter Creek Coal Tract & Myth & 2015 & 6 & $19.7(2.3) 9$ & 16 & 22.9 \\
\hline Otter Creek Coal Tract & Myth & 2015 & 8 & $15.3(3.9) 4$ & 9.4 & 17.8 \\
\hline Otter Creek Coal Tract & Myth & 2016 & 5 & 12() 1 & 12 & 12 \\
\hline Otter Creek Coal Tract & Myth & 2016 & 6 & $19.4(5.3) 9$ & 9.7 & 24.4 \\
\hline $\begin{array}{c}\text { Rosebud Coal Mine Area C } \\
\text { Pond }\end{array}$ & Epfu & 2014 & 4 & $11.2(4.9) 95$ & 5.4 & 18.8 \\
\hline $\begin{array}{c}\text { Rosebud Coal Mine Area C } \\
\text { Pond }\end{array}$ & Epfu & 2014 & 5 & 14.9 (3.9) 1177 & 3.6 & 25.7 \\
\hline $\begin{array}{l}\text { Rosebud Coal Mine Area C } \\
\text { Pond }\end{array}$ & Epfu & 2014 & 6 & $14.1(2.5) 2301$ & 6.7 & 20.3 \\
\hline
\end{tabular}




\begin{tabular}{|c|c|c|c|c|c|c|}
\hline $\begin{array}{l}\text { Rosebud Coal Mine Area C } \\
\text { Pond }\end{array}$ & Epfu & 2014 & 7 & 18.3 (3.5) 4341 & 9.5 & 27.5 \\
\hline $\begin{array}{l}\text { Rosebud Coal Mine Area C } \\
\text { Pond }\end{array}$ & Epfu & 2014 & 8 & 17.6 (2.9) 1381 & 10.5 & 26.7 \\
\hline $\begin{array}{c}\text { Rosebud Coal Mine Area C } \\
\text { Pond }\end{array}$ & Epfu & 2014 & 9 & 13.2 (3.3) 320 & 6 & 19.6 \\
\hline $\begin{array}{c}\text { Rosebud Coal Mine Area C } \\
\text { Pond }\end{array}$ & Epfu & 2014 & 10 & $16.2(3.5) 19$ & 3.4 & 19.8 \\
\hline $\begin{array}{c}\text { Rosebud Coal Mine Area C } \\
\text { Pond }\end{array}$ & Epfu & 2015 & 3 & $16.4(5.7) 68$ & 8 & 21.6 \\
\hline $\begin{array}{c}\text { Rosebud Coal Mine Area C } \\
\text { Pond }\end{array}$ & Epfu & 2015 & 4 & $12.2(2.1) 88$ & -4.9 & 14.1 \\
\hline $\begin{array}{c}\text { Rosebud Coal Mine Area C } \\
\text { Pond }\end{array}$ & Epfu & 2015 & 5 & 14.6 (3.8) 1766 & 3.7 & 20.9 \\
\hline $\begin{array}{c}\text { Rosebud Coal Mine Area C } \\
\text { Pond }\end{array}$ & Epfu & 2015 & 6 & 19.5 (3.6) 1263 & 12 & 25.1 \\
\hline $\begin{array}{c}\text { Rosebud Coal Mine Area C } \\
\text { Pond }\end{array}$ & Epfu & 2015 & 6 & 21.6 (3.2) 6137 & 12 & 28.2 \\
\hline $\begin{array}{c}\text { Rosebud Coal Mine Area C } \\
\text { Pond }\end{array}$ & Epfu & 2015 & 7 & 19.6 (3.3) 18553 & 12 & 28.5 \\
\hline $\begin{array}{c}\text { Rosebud Coal Mine Area C } \\
\text { Pond }\end{array}$ & Epfu & 2015 & 8 & 18.6 (3.2) 3496 & 6.7 & 28.7 \\
\hline $\begin{array}{c}\text { Rosebud Coal Mine Area C } \\
\text { Pond }\end{array}$ & Epfu & 2015 & 9 & $18.4(3.3) 578$ & 9.8 & 25.7 \\
\hline $\begin{array}{c}\text { Rosebud Coal Mine Area C } \\
\text { Pond }\end{array}$ & Epfu & 2015 & 11 & $5.9(0) 3$ & 5.9 & 5.9 \\
\hline $\begin{array}{c}\text { Rosebud Coal Mine Area C } \\
\text { Pond }\end{array}$ & Epfu & 2015 & 12 & 0.4 () 1 & 0.4 & 0.4 \\
\hline $\begin{array}{c}\text { Rosebud Coal Mine Area C } \\
\text { Pond }\end{array}$ & Epfu & 2016 & 1 & $5(1.4) 10$ & 3.9 & 7 \\
\hline $\begin{array}{c}\text { Rosebud Coal Mine Area C } \\
\text { Pond }\end{array}$ & Epfu & 2016 & 3 & $8.2(0.2) 38$ & 7.9 & 8.4 \\
\hline $\begin{array}{c}\text { Rosebud Coal Mine Area C } \\
\text { Pond }\end{array}$ & Epfu & 2016 & 4 & 14 (3) 405 & 5.7 & 17.8 \\
\hline $\begin{array}{c}\text { Rosebud Coal Mine Area C } \\
\text { Pond }\end{array}$ & Epfu & 2016 & 5 & 15.3 (3.1) 1976 & 5.9 & 22.4 \\
\hline $\begin{array}{c}\text { Rosebud Coal Mine Area C } \\
\text { Pond }\end{array}$ & Epfu & 2016 & 6 & $21.1(4.2) 2329$ & 12.7 & 29.2 \\
\hline $\begin{array}{c}\text { Rosebud Coal Mine Area C } \\
\text { Pond }\end{array}$ & Laci & 2014 & 4 & 9.3 (3.7) 64 & 3.7 & 17.6 \\
\hline $\begin{array}{c}\text { Rosebud Coal Mine Area C } \\
\text { Pond }\end{array}$ & Laci & 2014 & 5 & 15.1 (3.7) 300 & 5.2 & 25.7 \\
\hline $\begin{array}{c}\text { Rosebud Coal Mine Area C } \\
\text { Pond }\end{array}$ & Laci & 2014 & 6 & 14.5 (2.7) 1012 & 6.7 & 20.1 \\
\hline $\begin{array}{c}\text { Rosebud Coal Mine Area C } \\
\text { Pond }\end{array}$ & Laci & 2014 & 7 & 16.3 (4.3) 1245 & 9.5 & 25.4 \\
\hline $\begin{array}{c}\text { Rosebud Coal Mine Area C } \\
\text { Pond }\end{array}$ & Laci & 2014 & 8 & 14.7 (2.7) 375 & 9.8 & 27.2 \\
\hline
\end{tabular}




\begin{tabular}{|c|c|c|c|c|c|c|}
\hline $\begin{array}{c}\text { Rosebud Coal Mine Area C } \\
\text { Pond }\end{array}$ & Laci & 2014 & 9 & 10.9 (3.4) 64 & 4.2 & 18.4 \\
\hline $\begin{array}{c}\text { Rosebud Coal Mine Area C } \\
\text { Pond }\end{array}$ & Laci & 2014 & 10 & 11.6 (11.6) 2 & 3.4 & 19.8 \\
\hline $\begin{array}{c}\text { Rosebud Coal Mine Area C } \\
\text { Pond }\end{array}$ & Laci & 2015 & 1 & 2.1 () 1 & 2.1 & 2.1 \\
\hline $\begin{array}{c}\text { Rosebud Coal Mine Area C } \\
\text { Pond }\end{array}$ & Laci & 2015 & 3 & 4.9 () 1 & 4.9 & 4.9 \\
\hline $\begin{array}{c}\text { Rosebud Coal Mine Area C } \\
\text { Pond }\end{array}$ & Laci & 2015 & 4 & 10.9 (5.4) 11 & -4.9 & 13.5 \\
\hline $\begin{array}{l}\text { Rosebud Coal Mine Area C } \\
\text { Pond }\end{array}$ & Laci & 2015 & 5 & $15.2(2.6) 113$ & 7 & 20.6 \\
\hline $\begin{array}{c}\text { Rosebud Coal Mine Area C } \\
\text { Pond }\end{array}$ & Laci & 2015 & 6 & 16.9 (3.5) 182 & 12 & 24.6 \\
\hline $\begin{array}{c}\text { Rosebud Coal Mine Area C } \\
\text { Pond }\end{array}$ & Laci & 2015 & 6 & 19.8 (3.6) 2202 & 9.8 & 28.2 \\
\hline $\begin{array}{c}\text { Rosebud Coal Mine Area C } \\
\text { Pond }\end{array}$ & Laci & 2015 & 7 & 17.9 (3.2) 3884 & 10.8 & 28.7 \\
\hline $\begin{array}{c}\text { Rosebud Coal Mine Area C } \\
\text { Pond }\end{array}$ & Laci & 2015 & 8 & $16.4(2.8) 1114$ & 3.9 & 26.4 \\
\hline $\begin{array}{l}\text { Rosebud Coal Mine Area C } \\
\text { Pond }\end{array}$ & Laci & 2015 & 9 & $17.3(3.4) 50$ & 7.4 & 24.7 \\
\hline $\begin{array}{c}\text { Rosebud Coal Mine Area C } \\
\text { Pond }\end{array}$ & Laci & 2016 & 4 & $14.5(2.8) 38$ & 5.7 & 18.6 \\
\hline $\begin{array}{c}\text { Rosebud Coal Mine Area C } \\
\text { Pond }\end{array}$ & Laci & 2016 & 5 & 14.3 (2.4) 484 & 5.9 & 22.2 \\
\hline $\begin{array}{c}\text { Rosebud Coal Mine Area C } \\
\text { Pond }\end{array}$ & Laci & 2016 & 6 & 19.4 (3.7) 1074 & 10 & 29.2 \\
\hline $\begin{array}{c}\text { Rosebud Coal Mine Area C } \\
\text { Pond }\end{array}$ & Lano & 2013 & 11 & $4.9(0) 2$ & 4.9 & 4.9 \\
\hline $\begin{array}{c}\text { Rosebud Coal Mine Area C } \\
\text { Pond }\end{array}$ & Lano & 2014 & 2 & $2.9(0) 2$ & 2.9 & 2.9 \\
\hline $\begin{array}{c}\text { Rosebud Coal Mine Area C } \\
\text { Pond }\end{array}$ & Lano & 2014 & 4 & $12(3.7) 145$ & 5.2 & 18.8 \\
\hline $\begin{array}{c}\text { Rosebud Coal Mine Area C } \\
\text { Pond }\end{array}$ & Lano & 2014 & 5 & 15 (3.3) 690 & 3.7 & 25.1 \\
\hline $\begin{array}{l}\text { Rosebud Coal Mine Area C } \\
\text { Pond }\end{array}$ & Lano & 2014 & 6 & $14.2(2.2) 1585$ & 6.7 & 20.3 \\
\hline $\begin{array}{c}\text { Rosebud Coal Mine Area C } \\
\text { Pond }\end{array}$ & Lano & 2014 & 7 & $16.2(3.5) 1711$ & 9.5 & 26.7 \\
\hline $\begin{array}{c}\text { Rosebud Coal Mine Area C } \\
\text { Pond }\end{array}$ & Lano & 2014 & 8 & $15.2(2.4) 476$ & 10.7 & 27 \\
\hline $\begin{array}{c}\text { Rosebud Coal Mine Area C } \\
\text { Pond }\end{array}$ & Lano & 2014 & 9 & $12.1(2.8) 181$ & 1.6 & 18.1 \\
\hline $\begin{array}{l}\text { Rosebud Coal Mine Area C } \\
\text { Pond }\end{array}$ & Lano & 2014 & 10 & 6.2 (5) 16 & 3.4 & 16 \\
\hline $\begin{array}{l}\text { Rosebud Coal Mine Area C } \\
\text { Pond }\end{array}$ & Lano & 2015 & 3 & $17.2(5.2) 11$ & 9.2 & 20.9 \\
\hline
\end{tabular}




\begin{tabular}{|c|c|c|c|c|c|c|}
\hline $\begin{array}{c}\text { Rosebud Coal Mine Area C } \\
\text { Pond }\end{array}$ & Lano & 2015 & 4 & $13.2(0.9) 2$ & 12.5 & 13.8 \\
\hline $\begin{array}{c}\text { Rosebud Coal Mine Area C } \\
\text { Pond }\end{array}$ & Lano & 2015 & 5 & 15.3 (3.5) 384 & 4.1 & 20.8 \\
\hline $\begin{array}{c}\text { Rosebud Coal Mine Area C } \\
\text { Pond }\end{array}$ & Lano & 2015 & 6 & 19.4 (3.9) 534 & 12 & 25.1 \\
\hline $\begin{array}{c}\text { Rosebud Coal Mine Area C } \\
\text { Pond }\end{array}$ & Lano & 2015 & 6 & 20.5 (3.3) 1106 & 12 & 28.2 \\
\hline $\begin{array}{c}\text { Rosebud Coal Mine Area C } \\
\text { Pond }\end{array}$ & Lano & 2015 & 7 & 18 (3) 2201 & 11.7 & 28.2 \\
\hline $\begin{array}{c}\text { Rosebud Coal Mine Area C } \\
\text { Pond }\end{array}$ & Lano & 2015 & 8 & 17.1 (3) 1005 & 3.4 & 27.4 \\
\hline $\begin{array}{c}\text { Rosebud Coal Mine Area C } \\
\text { Pond }\end{array}$ & Lano & 2015 & 9 & $16.4(4.3) 689$ & 7.7 & 26.2 \\
\hline $\begin{array}{l}\text { Rosebud Coal Mine Area C } \\
\text { Pond }\end{array}$ & Lano & 2015 & 10 & $16.5(0.2) 3$ & 16.3 & 16.6 \\
\hline $\begin{array}{c}\text { Rosebud Coal Mine Area C } \\
\text { Pond }\end{array}$ & Lano & 2015 & 12 & 0.4 () 1 & 0.4 & 0.4 \\
\hline $\begin{array}{c}\text { Rosebud Coal Mine Area C } \\
\text { Pond }\end{array}$ & Lano & 2016 & 1 & $3.9(0) 2$ & 3.9 & 3.9 \\
\hline $\begin{array}{c}\text { Rosebud Coal Mine Area C } \\
\text { Pond }\end{array}$ & Lano & 2016 & 3 & $8.1(0.3) 5$ & 7.9 & 8.4 \\
\hline $\begin{array}{c}\text { Rosebud Coal Mine Area C } \\
\text { Pond }\end{array}$ & Lano & 2016 & 4 & 14.5 (2.8) 62 & 1.1 & 17.8 \\
\hline $\begin{array}{c}\text { Rosebud Coal Mine Area C } \\
\text { Pond }\end{array}$ & Lano & 2016 & 5 & 16.2 (3.4) 468 & 6.2 & 22.4 \\
\hline $\begin{array}{c}\text { Rosebud Coal Mine Area C } \\
\text { Pond }\end{array}$ & Lano & 2016 & 6 & 20.6 (4.6) 717 & 11.8 & 29.2 \\
\hline $\begin{array}{c}\text { Rosebud Coal Mine Area C } \\
\text { Pond }\end{array}$ & Мyсі & 2013 & 10 & $12.3(2.9) 3$ & 9 & 14 \\
\hline $\begin{array}{c}\text { Rosebud Coal Mine Area C } \\
\text { Pond }\end{array}$ & Myci & 2014 & 4 & 11.6 (4.6) 24 & 6.2 & 17.8 \\
\hline $\begin{array}{c}\text { Rosebud Coal Mine Area C } \\
\text { Pond }\end{array}$ & Мyci & 2014 & 5 & 14.7 (2.7) 189 & 5.7 & 24.6 \\
\hline $\begin{array}{c}\text { Rosebud Coal Mine Area C } \\
\text { Pond }\end{array}$ & Мyсі & 2014 & 6 & $13.2(2.3) 372$ & 7.9 & 19.8 \\
\hline $\begin{array}{c}\text { Rosebud Coal Mine Area C } \\
\text { Pond }\end{array}$ & Мyсі & 2014 & 7 & 16.8 (3) 729 & 9.8 & 27.5 \\
\hline $\begin{array}{c}\text { Rosebud Coal Mine Area C } \\
\text { Pond }\end{array}$ & Мyсі & 2014 & 8 & 17.2 (3) 662 & 10.5 & 26.7 \\
\hline $\begin{array}{c}\text { Rosebud Coal Mine Area C } \\
\text { Pond }\end{array}$ & Мyсі & 2014 & 9 & 12.4 (2.7) 126 & 2.2 & 19.3 \\
\hline $\begin{array}{c}\text { Rosebud Coal Mine Area C } \\
\text { Pond }\end{array}$ & Мyсі & 2014 & 10 & 9.1 (3.3) 19 & 2.6 & 14.8 \\
\hline $\begin{array}{c}\text { Rosebud Coal Mine Area C } \\
\text { Pond }\end{array}$ & Мyci & 2015 & 3 & $16.6(6.2) 3$ & 9.5 & 20.9 \\
\hline $\begin{array}{c}\text { Rosebud Coal Mine Area C } \\
\text { Pond }\end{array}$ & Myci & 2015 & 4 & $12.4(0.8) 17$ & 9.8 & 13.2 \\
\hline
\end{tabular}




\begin{tabular}{|c|c|c|c|c|c|c|}
\hline $\begin{array}{l}\text { Rosebud Coal Mine Area C } \\
\text { Pond }\end{array}$ & Myci & 2015 & 5 & 13.5 (1.9) 144 & 7.4 & 17.8 \\
\hline $\begin{array}{l}\text { Rosebud Coal Mine Area C } \\
\text { Pond }\end{array}$ & Myci & 2015 & 6 & 19.1 (3.7) 64 & 13.2 & 25.1 \\
\hline $\begin{array}{c}\text { Rosebud Coal Mine Area C } \\
\text { Pond }\end{array}$ & Myci & 2015 & 6 & 19 (2.5) 1666 & 9.8 & 27.9 \\
\hline $\begin{array}{c}\text { Rosebud Coal Mine Area C } \\
\text { Pond }\end{array}$ & Myсi & 2015 & 7 & $19.3(2.6) 2595$ & 11.3 & 28 \\
\hline $\begin{array}{c}\text { Rosebud Coal Mine Area C } \\
\text { Pond }\end{array}$ & Myci & 2015 & 8 & 18.4 (3) 1711 & 8.5 & 28.4 \\
\hline $\begin{array}{c}\text { Rosebud Coal Mine Area C } \\
\text { Pond }\end{array}$ & Myci & 2015 & 9 & $18(3.2) 481$ & 9.8 & 24.7 \\
\hline $\begin{array}{c}\text { Rosebud Coal Mine Area C } \\
\text { Pond }\end{array}$ & Myci & 2015 & 10 & $17.6(3.8) 9$ & 13.6 & 24.2 \\
\hline $\begin{array}{c}\text { Rosebud Coal Mine Area C } \\
\text { Pond }\end{array}$ & Myсi & 2015 & 12 & $2.7(0) 5$ & 2.7 & 2.7 \\
\hline $\begin{array}{c}\text { Rosebud Coal Mine Area C } \\
\text { Pond }\end{array}$ & Myсi & 2016 & 4 & $12.8(4) 33$ & 4.9 & 19.9 \\
\hline $\begin{array}{c}\text { Rosebud Coal Mine Area C } \\
\text { Pond }\end{array}$ & Myсi & 2016 & 5 & $15.6(2.2) 709$ & 2.7 & 22.1 \\
\hline $\begin{array}{c}\text { Rosebud Coal Mine Area C } \\
\text { Pond }\end{array}$ & Myci & 2016 & 6 & $18.8(2.6) 1235$ & 11 & 28.5 \\
\hline $\begin{array}{c}\text { Rosebud Coal Mine Area C } \\
\text { Pond }\end{array}$ & Myev & 2014 & 5 & $6.4(4.7) 3$ & 1.9 & 11.3 \\
\hline $\begin{array}{c}\text { Rosebud Coal Mine Area C } \\
\text { Pond }\end{array}$ & Myev & 2014 & 6 & 19.3 () 1 & 19.3 & 19.3 \\
\hline $\begin{array}{c}\text { Rosebud Coal Mine Area C } \\
\text { Pond }\end{array}$ & Myev & 2014 & 7 & $14(2.5) 5$ & 11 & 17.9 \\
\hline $\begin{array}{c}\text { Rosebud Coal Mine Area C } \\
\text { Pond }\end{array}$ & Myev & 2014 & 8 & $17.1(3.7) 7$ & 12 & 22.7 \\
\hline $\begin{array}{c}\text { Rosebud Coal Mine Area C } \\
\text { Pond }\end{array}$ & Myev & 2014 & 9 & $9.8(3) 4$ & 5.7 & 12.8 \\
\hline $\begin{array}{c}\text { Rosebud Coal Mine Area C } \\
\text { Pond }\end{array}$ & Myev & 2015 & 6 & 20.4 (2.3) 91 & 16.3 & 27 \\
\hline $\begin{array}{c}\text { Rosebud Coal Mine Area C } \\
\text { Pond }\end{array}$ & Myev & 2015 & 7 & 19.3 (2.5) 105 & 11.5 & 23.9 \\
\hline $\begin{array}{l}\text { Rosebud Coal Mine Area C } \\
\text { Pond }\end{array}$ & Myev & 2015 & 8 & 16.9 (2.9) 320 & 10.2 & 24.7 \\
\hline $\begin{array}{c}\text { Rosebud Coal Mine Area C } \\
\text { Pond }\end{array}$ & Myev & 2015 & 9 & 15.5 (4.2) 98 & 8.4 & 27.2 \\
\hline $\begin{array}{c}\text { Rosebud Coal Mine Area C } \\
\text { Pond }\end{array}$ & Myev & 2016 & 4 & 6 (1.1) 2 & 5.2 & 6.7 \\
\hline $\begin{array}{c}\text { Rosebud Coal Mine Area C } \\
\text { Pond }\end{array}$ & Myev & 2016 & 5 & $15.6(3.4) 14$ & 8.4 & 19.6 \\
\hline $\begin{array}{c}\text { Rosebud Coal Mine Area C } \\
\text { Pond }\end{array}$ & Myev & 2016 & 6 & $19.9(4.8) 15$ & 11.7 & 26.7 \\
\hline $\begin{array}{c}\text { Rosebud Coal Mine Area C } \\
\text { Pond }\end{array}$ & Mylu & 2014 & 4 & 15.5 (1.1) 3 & 14.3 & 16.5 \\
\hline
\end{tabular}




\begin{tabular}{|c|c|c|c|c|c|c|}
\hline $\begin{array}{c}\text { Rosebud Coal Mine Area C } \\
\text { Pond }\end{array}$ & Mylu & 2014 & 5 & 11.9 (4) 16 & 3.9 & 18.3 \\
\hline $\begin{array}{c}\text { Rosebud Coal Mine Area C } \\
\text { Pond }\end{array}$ & Mylu & 2014 & 6 & $14.1(3.3) 80$ & 8.7 & 20.3 \\
\hline $\begin{array}{c}\text { Rosebud Coal Mine Area C } \\
\text { Pond }\end{array}$ & Mylu & 2014 & 7 & $17.1(3.2) 166$ & 11.2 & 23.2 \\
\hline $\begin{array}{c}\text { Rosebud Coal Mine Area C } \\
\text { Pond }\end{array}$ & Mylu & 2014 & 8 & $17.4(3.2) 117$ & 9.8 & 25.7 \\
\hline $\begin{array}{c}\text { Rosebud Coal Mine Area C } \\
\text { Pond }\end{array}$ & Mylu & 2014 & 9 & 11.8 (3.2) 34 & 2.9 & 16.8 \\
\hline $\begin{array}{c}\text { Rosebud Coal Mine Area C } \\
\text { Pond }\end{array}$ & Mylu & 2014 & 10 & $8.7(1.4) 5$ & 6.7 & 10.2 \\
\hline $\begin{array}{c}\text { Rosebud Coal Mine Area C } \\
\text { Pond }\end{array}$ & Mylu & 2015 & 5 & $12.9(3.4) 15$ & 9 & 18.6 \\
\hline $\begin{array}{c}\text { Rosebud Coal Mine Area C } \\
\text { Pond }\end{array}$ & Mylu & 2015 & 6 & 19.6 (3.1) 30 & 14.6 & 24.1 \\
\hline $\begin{array}{c}\text { Rosebud Coal Mine Area C } \\
\text { Pond }\end{array}$ & Mylu & 2015 & 6 & $19.4(2) 1766$ & 12 & 28.2 \\
\hline $\begin{array}{c}\text { Rosebud Coal Mine Area C } \\
\text { Pond }\end{array}$ & Mylu & 2015 & 7 & $20.3(2.3) 846$ & 11.7 & 26.5 \\
\hline $\begin{array}{c}\text { Rosebud Coal Mine Area C } \\
\text { Pond }\end{array}$ & Mylu & 2015 & 8 & 17.8 (3.1) 217 & 8.5 & 26.5 \\
\hline $\begin{array}{c}\text { Rosebud Coal Mine Area C } \\
\text { Pond }\end{array}$ & Mylu & 2015 & 9 & $17(3.1) 158$ & 9.4 & 23.2 \\
\hline $\begin{array}{c}\text { Rosebud Coal Mine Area C } \\
\text { Pond }\end{array}$ & Mylu & 2015 & 10 & $20.8(4.8) 2$ & 17.4 & 24.2 \\
\hline $\begin{array}{c}\text { Rosebud Coal Mine Area C } \\
\text { Pond }\end{array}$ & Mylu & 2016 & 4 & $17.5(0.7) 4$ & 17.1 & 18.6 \\
\hline $\begin{array}{c}\text { Rosebud Coal Mine Area C } \\
\text { Pond }\end{array}$ & Mylu & 2016 & 5 & 13.7 (3.1) 41 & 4.1 & 18.9 \\
\hline $\begin{array}{c}\text { Rosebud Coal Mine Area C } \\
\text { Pond }\end{array}$ & Mylu & 2016 & 6 & 20.8 (3.7) 174 & 13 & 28.7 \\
\hline $\begin{array}{c}\text { Rosebud Coal Mine Area C } \\
\text { Pond }\end{array}$ & Myth & 2015 & 6 & $21.4(2) 6$ & 19.3 & 23.7 \\
\hline $\begin{array}{c}\text { Rosebud Coal Mine Area C } \\
\text { Pond }\end{array}$ & Myth & 2015 & 7 & 20.3 (1.6) 3 & 19.4 & 22.1 \\
\hline $\begin{array}{c}\text { Rosebud Coal Mine Area C } \\
\text { Pond }\end{array}$ & Myth & 2015 & 8 & $16.4(3.3) 3$ & 13.3 & 19.8 \\
\hline $\begin{array}{c}\text { Rosebud Coal Mine Area C } \\
\text { Pond }\end{array}$ & Myth & 2015 & 9 & $12.4(3.8) 6$ & 9.8 & 18.4 \\
\hline $\begin{array}{c}\text { Rosebud Coal Mine Area C } \\
\text { Pond }\end{array}$ & Myth & 2016 & 4 & $11.6(4.3) 3$ & 6.7 & 14.5 \\
\hline $\begin{array}{c}\text { Rosebud Coal Mine Area C } \\
\text { Pond }\end{array}$ & Myth & 2016 & 6 & 18.6 () 1 & 18.6 & 18.6 \\
\hline $\begin{array}{l}\text { Rosebud Coal Mine Area F } \\
\text { Pond } 7\end{array}$ & Epfu & 2012 & 8 & 22.9 (4.9) 116 & 11.5 & 31.8 \\
\hline $\begin{array}{l}\text { Rosebud Coal Mine Area F } \\
\text { Pond } 7\end{array}$ & Epfu & 2012 & 9 & 17.8 (4.9) 175 & 4.1 & 26.7 \\
\hline
\end{tabular}




\begin{tabular}{|c|c|c|c|c|c|c|}
\hline $\begin{array}{l}\text { Rosebud Coal Mine Area F } \\
\text { Pond } 7\end{array}$ & Epfu & 2012 & 11 & 8.5 () 1 & 8.5 & 8.5 \\
\hline $\begin{array}{l}\text { Rosebud Coal Mine Area F } \\
\text { Pond } 7\end{array}$ & Epfu & 2013 & 4 & 18.4 () 1 & 18.4 & 18.4 \\
\hline $\begin{array}{l}\text { Rosebud Coal Mine Area F } \\
\text { Pond } 7\end{array}$ & Epfu & 2013 & 5 & 20.3 (5.5) 24 & 11.7 & 25.7 \\
\hline $\begin{array}{l}\text { Rosebud Coal Mine Area F } \\
\text { Pond } 7\end{array}$ & Epfu & 2013 & 6 & $19.1(3.4) 63$ & 11.7 & 27.5 \\
\hline $\begin{array}{c}\text { Rosebud Coal Mine Area F } \\
\text { Pond } 7\end{array}$ & Epfu & 2013 & 7 & $22(2.9) 63$ & 15.6 & 27.2 \\
\hline $\begin{array}{l}\text { Rosebud Coal Mine Area F } \\
\text { Pond } 7\end{array}$ & Epfu & 2013 & 8 & 20.6 (3.3) 24 & 15.8 & 28.2 \\
\hline $\begin{array}{l}\text { Rosebud Coal Mine Area F } \\
\text { Pond } 7\end{array}$ & Epfu & 2013 & 9 & 15.9 (4.1) 8 & 9.7 & 22.4 \\
\hline $\begin{array}{l}\text { Rosebud Coal Mine Area F } \\
\text { Pond } 7\end{array}$ & Epfu & 2013 & 10 & 12.7 () 1 & 12.7 & 12.7 \\
\hline $\begin{array}{c}\text { Rosebud Coal Mine Area F } \\
\text { Pond } 7\end{array}$ & Laci & 2012 & 9 & 13.7 (3.7) 9 & 8.5 & 19.9 \\
\hline $\begin{array}{c}\text { Rosebud Coal Mine Area F } \\
\text { Pond } 7\end{array}$ & Laci & 2013 & 5 & 19.5 (6.6) 3 & 12.5 & 25.5 \\
\hline $\begin{array}{c}\text { Rosebud Coal Mine Area F } \\
\text { Pond } 7\end{array}$ & Laci & 2013 & 6 & $18.9(2.4) 6$ & 16.1 & 21.7 \\
\hline $\begin{array}{l}\text { Rosebud Coal Mine Area F } \\
\text { Pond } 7\end{array}$ & Laci & 2013 & 7 & 19.8 (1.5) 7 & 18.4 & 22.7 \\
\hline $\begin{array}{l}\text { Rosebud Coal Mine Area F } \\
\text { Pond } 7\end{array}$ & Laci & 2013 & 8 & 16.4 (1.7) 7 & 13 & 17.8 \\
\hline $\begin{array}{c}\text { Rosebud Coal Mine Area F } \\
\text { Pond } 7\end{array}$ & Lano & 2012 & 8 & 22.9 (4.2) 54 & 11.5 & 27.2 \\
\hline $\begin{array}{c}\text { Rosebud Coal Mine Area F } \\
\text { Pond } 7\end{array}$ & Lano & 2012 & 9 & 17.5 (4.3) 64 & 8.9 & 24.6 \\
\hline $\begin{array}{l}\text { Rosebud Coal Mine Area F } \\
\text { Pond } 7\end{array}$ & Lano & 2013 & 4 & 15.6 () 1 & 15.6 & 15.6 \\
\hline $\begin{array}{c}\text { Rosebud Coal Mine Area F } \\
\text { Pond } 7\end{array}$ & Lano & 2013 & 5 & 21.9 (3.5) 19 & 15.5 & 25.7 \\
\hline $\begin{array}{c}\text { Rosebud Coal Mine Area F } \\
\text { Pond } 7\end{array}$ & Lano & 2013 & 6 & $17.1(3.3) 16$ & 10.2 & 20.3 \\
\hline $\begin{array}{c}\text { Rosebud Coal Mine Area F } \\
\text { Pond } 7\end{array}$ & Lano & 2013 & 7 & 21.4 (3.5) 28 & 16.3 & 27.2 \\
\hline $\begin{array}{c}\text { Rosebud Coal Mine Area F } \\
\text { Pond } 7\end{array}$ & Lano & 2013 & 8 & 19.2 (3.9) 11 & 12.3 & 25.9 \\
\hline $\begin{array}{l}\text { Rosebud Coal Mine Area F } \\
\text { Pond } 7\end{array}$ & Lano & 2013 & 9 & $19.4(6.8) 4$ & 12.2 & 27.9 \\
\hline $\begin{array}{l}\text { Rosebud Coal Mine Area F } \\
\text { Pond } 7\end{array}$ & Myсi & 2012 & 8 & $21.2(4.6) 49$ & 10.8 & 32 \\
\hline $\begin{array}{c}\text { Rosebud Coal Mine Area F } \\
\text { Pond } 7\end{array}$ & Мyсі & 2012 & 9 & $18.8(4.1) 59$ & 10.3 & 26.4 \\
\hline $\begin{array}{c}\text { Rosebud Coal Mine Area F } \\
\text { Pond } 7\end{array}$ & Myсi & 2012 & 10 & 18.3 (3.7) 4 & 13.5 & 21.9 \\
\hline
\end{tabular}




\begin{tabular}{|c|c|c|c|c|c|c|}
\hline $\begin{array}{l}\text { Rosebud Coal Mine Area F } \\
\text { Pond } 7\end{array}$ & Myci & 2013 & 5 & $20(1.2) 5$ & 18.1 & 20.9 \\
\hline $\begin{array}{l}\text { Rosebud Coal Mine Area F } \\
\text { Pond } 7\end{array}$ & Myci & 2013 & 6 & 16.7 (3.6) 10 & 11 & 20.9 \\
\hline $\begin{array}{l}\text { Rosebud Coal Mine Area F } \\
\text { Pond } 7\end{array}$ & Myci & 2013 & 7 & $22.5(3.6) 9$ & 16.1 & 27.4 \\
\hline $\begin{array}{l}\text { Rosebud Coal Mine Area F } \\
\text { Pond } 7\end{array}$ & Myci & 2013 & 8 & 20.7 (3.7) 15 & 17 & 29.8 \\
\hline $\begin{array}{l}\text { Rosebud Coal Mine Area F } \\
\text { Pond } 7\end{array}$ & Myci & 2013 & 9 & $19.9(4.2) 3$ & 15 & 22.7 \\
\hline $\begin{array}{l}\text { Rosebud Coal Mine Area F } \\
\text { Pond } 7\end{array}$ & Myev & 2012 & 8 & $19.7(6.2) 22$ & 8.2 & 32 \\
\hline $\begin{array}{l}\text { Rosebud Coal Mine Area F } \\
\text { Pond } 7\end{array}$ & Myev & 2012 & 9 & $14.5(5) 19$ & 6.2 & 22.6 \\
\hline $\begin{array}{l}\text { Rosebud Coal Mine Area F } \\
\text { Pond } 7\end{array}$ & Myev & 2013 & 4 & 14.1 () 1 & 14.1 & 14.1 \\
\hline $\begin{array}{l}\text { Rosebud Coal Mine Area F } \\
\text { Pond } 7\end{array}$ & Myev & 2013 & 5 & 9.2 () 1 & 9.2 & 9.2 \\
\hline $\begin{array}{l}\text { Rosebud Coal Mine Area F } \\
\text { Pond } 7\end{array}$ & Myev & 2013 & 6 & $14.4(4.1) 3$ & 12 & 19.1 \\
\hline $\begin{array}{c}\text { Rosebud Coal Mine Area F } \\
\text { Pond } 7\end{array}$ & Myev & 2013 & 7 & $19.5(1.4) 6$ & 17.1 & 20.9 \\
\hline $\begin{array}{c}\text { Rosebud Coal Mine Area F } \\
\text { Pond } 7\end{array}$ & Myev & 2013 & 8 & $17.4(4.8) 6$ & 12.7 & 25.9 \\
\hline $\begin{array}{l}\text { Rosebud Coal Mine Area F } \\
\text { Pond } 7\end{array}$ & Myev & 2013 & 9 & 26.5 () 1 & 26.5 & 26.5 \\
\hline $\begin{array}{l}\text { Rosebud Coal Mine Area F } \\
\text { Pond } 7\end{array}$ & Myev & 2013 & 10 & 15 () 1 & 15 & 15 \\
\hline $\begin{array}{l}\text { Rosebud Coal Mine Area F } \\
\text { Pond } 7\end{array}$ & Mylu & 2012 & 8 & $22(3.5) 12$ & 14.6 & 25.2 \\
\hline $\begin{array}{l}\text { Rosebud Coal Mine Area F } \\
\text { Pond } 7\end{array}$ & Mylu & 2012 & 9 & $17.8(4.7) 28$ & 9.2 & 24.7 \\
\hline $\begin{array}{l}\text { Rosebud Coal Mine Area F } \\
\text { Pond } 7\end{array}$ & Mylu & 2013 & 5 & $13.6($ () 1 & 13.6 & 13.6 \\
\hline $\begin{array}{l}\text { Rosebud Coal Mine Area F } \\
\text { Pond } 7\end{array}$ & Mylu & 2013 & 6 & $14.5(3.1) 3$ & 11.7 & 17.8 \\
\hline $\begin{array}{l}\text { Rosebud Coal Mine Area F } \\
\text { Pond } 7\end{array}$ & Mylu & 2013 & 7 & 25.4 () 1 & 25.4 & 25.4 \\
\hline $\begin{array}{l}\text { Rosebud Coal Mine Area F } \\
\text { Pond } 7\end{array}$ & Mylu & 2013 & 8 & $19.4(6.2) 6$ & 11.5 & 30.3 \\
\hline $\begin{array}{l}\text { Rosebud Coal Mine Area F } \\
\text { Pond } 7\end{array}$ & Mylu & 2013 & 9 & $21(4.5) 2$ & 17.8 & 24.1 \\
\hline $\begin{array}{l}\text { Rosebud Coal Mine Area F } \\
\text { Pond } 7\end{array}$ & Myth & 2012 & 9 & 20.8 () 1 & 20.8 & 20.8 \\
\hline $\begin{array}{c}\text { Signal Peak Mine Busse } \\
\text { Water Reservoir }\end{array}$ & Epfu & 2012 & 8 & $21.1(4.2) 1887$ & 7 & 30.2 \\
\hline $\begin{array}{l}\text { Signal Peak Mine Busse } \\
\text { Water Reservoir }\end{array}$ & Epfu & 2012 & 9 & $17.2(3.4) 6229$ & 3.9 & 26.9 \\
\hline
\end{tabular}


Signal Peak Mine Busse Water Reservoir

Signal Peak Mine Busse Water Reservoir

Signal Peak Mine Busse Water Reservoir

Signal Peak Mine Busse Water Reservoir

Signal Peak Mine Busse Water Reservoir

Signal Peak Mine Busse Water Reservoir

Signal Peak Mine Busse Water Reservoir

Signal Peak Mine Busse Water Reservoir

Signal Peak Mine Busse Water Reservoir

Signal Peak Mine Busse Water Reservoir

Signal Peak Mine Busse Water Reservoir

Signal Peak Mine Busse Water Reservoir

Signal Peak Mine Busse Water Reservoir

Signal Peak Mine Busse Water Reservoir

Signal Peak Mine Busse Water Reservoir

Signal Peak Mine Busse Water Reservoir

Signal Peak Mine Busse Water Reservoir

Signal Peak Mine Busse Water Reservoir

Signal Peak Mine Busse Water Reservoir

Signal Peak Mine Busse Water Reservoir

Signal Peak Mine Busse Water Reservoir

Signal Peak Mine Busse Water Reservoir

Signal Peak Mine Busse Water Reservoir

Signal Peak Mine Busse Water Reservoir

\begin{tabular}{|c|c|c|c|c|c|}
\hline Epfu & 2012 & 10 & 14.5 (3.4) 79 & 5.2 & 19.9 \\
\hline Epfu & 2012 & 11 & $7.8(4.5) 11$ & 1.3 & 13.6 \\
\hline Epfu & 2012 & 12 & $2.1(3.2) 33$ & -2.3 & 5.7 \\
\hline Epfu & 2013 & 4 & $10.8(2.8) 709$ & 3.4 & 17.6 \\
\hline Epfu & 2013 & 5 & $14.5(4.5) 1737$ & 2.4 & 24.4 \\
\hline Epfu & 2013 & 6 & $15.8(2.7) 2856$ & 5.7 & 23.7 \\
\hline Epfu & 2013 & 7 & $18.4(3.2) 5388$ & 10.7 & 26 \\
\hline Epfu & 2013 & 8 & $19.3(3.8) 2844$ & 11.8 & 28 \\
\hline Epfu & 2013 & 9 & 19 (3) 590 & 9 & 25.9 \\
\hline Epfu & 2013 & 10 & $12.3(2.2) 99$ & 4.7 & 17 \\
\hline Epfu & 2013 & 11 & $3.9(4.4) 26$ & -1.3 & 10.7 \\
\hline Epfu & 2013 & 12 & $-2(0) 5$ & -2 & -2 \\
\hline Epfu & 2014 & 1 & $5.4(2.8) 2$ & 3.4 & 7.4 \\
\hline Epfu & 2014 & 2 & $1.9(1.9) 3$ & 0.8 & 4.1 \\
\hline Epfu & 2014 & 3 & $7(0.1) 5$ & 7 & 7.2 \\
\hline Epfu & 2014 & 4 & 11.5 (2.7) 1264 & 0 & 17.3 \\
\hline Epfu & 2014 & 5 & $15.1(4) 2660$ & 3.7 & 23.1 \\
\hline Epfu & 2014 & 6 & $12.5(3.8) 5005$ & 2.2 & 20.4 \\
\hline Epfu & 2014 & 7 & 17 (3.7) 12051 & 8.9 & 26.5 \\
\hline Epfu & 2014 & 8 & 19 (3.6) 3660 & 7 & 28.5 \\
\hline Epfu & 2014 & 9 & 13.7 (4.1) 418 & 4.6 & 22.4 \\
\hline Epfu & 2014 & 10 & $15.8(5.6) 28$ & 0.6 & 20.6 \\
\hline Epfu & 2014 & 11 & $1.7(3.7) 5$ & -4.4 & 5.1 \\
\hline Epfu & 2014 & 12 & $3.9(3.3) 43$ & -1.3 & 10.2 \\
\hline
\end{tabular}


Signal Peak Mine Busse Water Reservoir

Signal Peak Mine Busse Water Reservoir

Signal Peak Mine Busse Water Reservoir

Signal Peak Mine Busse Water Reservoir

Signal Peak Mine Busse Water Reservoir

Signal Peak Mine Busse Water Reservoir

Signal Peak Mine Busse Water Reservoir

Signal Peak Mine Busse Water Reservoir

Signal Peak Mine Busse Water Reservoir

Signal Peak Mine Busse Water Reservoir

Signal Peak Mine Busse Water Reservoir

Signal Peak Mine Busse Water Reservoir

Signal Peak Mine Busse Water Reservoir

Signal Peak Mine Busse Water Reservoir

Signal Peak Mine Busse Water Reservoir

Signal Peak Mine Busse Water Reservoir

Signal Peak Mine Busse Water Reservoir

Signal Peak Mine Busse Water Reservoir

Signal Peak Mine Busse Water Reservoir

Signal Peak Mine Busse Water Reservoir

Signal Peak Mine Busse Water Reservoir

Signal Peak Mine Busse Water Reservoir

Signal Peak Mine Busse Water Reservoir

Signal Peak Mine Busse Water Reservoir

\begin{tabular}{|c|c|c|c|c|c|}
\hline Epfu & 2015 & 1 & $2.9(3) 5$ & -0.8 & 5.1 \\
\hline Epfu & 2015 & 2 & $13.5(2.8) 24$ & 0.3 & 14.1 \\
\hline Epfu & 2015 & 3 & $11(3.4) 779$ & -1.6 & 18.4 \\
\hline Epfu & 2015 & 4 & $11.2(3.3) 2612$ & 2.4 & 17.1 \\
\hline Epfu & 2015 & 5 & $10.6(3.1) 4025$ & 0.4 & 20.6 \\
\hline Epfu & 2015 & 6 & $17.2(3.2) 10157$ & 9.5 & 27.4 \\
\hline Epfu & 2015 & 6 & $17(3.2) 2661$ & 11.2 & 24.9 \\
\hline Epfu & 2015 & 7 & 17.2 (2.9) 12908 & 8.7 & 27.9 \\
\hline Epfu & 2015 & 8 & 20.7 (3.9) 3690 & 4.6 & 29.5 \\
\hline Epfu & 2015 & 8 & 23.3 (4.2) 2087 & 7.5 & 30.7 \\
\hline Epfu & 2015 & 9 & $20.4(3.5) 1987$ & 9.7 & 28 \\
\hline Epfu & 2015 & 9 & 18.2 (3.9) 1698 & 5.5 & 26.7 \\
\hline Epfu & 2015 & 10 & $16.9(3.4) 32$ & 6.7 & 24.1 \\
\hline Epfu & 2015 & 10 & 18.5 (4) 141 & 9.5 & 26.4 \\
\hline Epfu & 2015 & 11 & 5.9 (2.7) 103 & 1.9 & 9 \\
\hline Epfu & 2015 & 12 & 7.5 (4.7) 209 & -2 & 14.8 \\
\hline Epfu & 2016 & 1 & 4.9 (2.9) 175 & -4.6 & 10.3 \\
\hline Epfu & 2016 & 2 & 7.8 (3) 104 & 0.4 & 12.3 \\
\hline Epfu & 2016 & 3 & 10.5 (6.2) 148 & -0.6 & 17.8 \\
\hline Epfu & 2016 & 4 & 13.9 (3.2) 3321 & 2.7 & 21.9 \\
\hline Epfu & 2016 & 5 & 14 (4) 3996 & 6.5 & 22.1 \\
\hline Epfu & 2016 & 9 & $18.8(1.6) 31$ & 16.8 & 21.9 \\
\hline Epfu & 2016 & 10 & $13.5(4.3) 51$ & 6.9 & 19.4 \\
\hline Epfu & 2016 & 11 & $5(4.1) 172$ & -0.3 & 15.8 \\
\hline
\end{tabular}


Signal Peak Mine Busse Water Reservoir

Signal Peak Mine Busse Water Reservoir

Signal Peak Mine Busse Water Reservoir

Signal Peak Mine Busse Water Reservoir

Signal Peak Mine Busse Water Reservoir

Signal Peak Mine Busse Water Reservoir

Signal Peak Mine Busse Water Reservoir

Signal Peak Mine Busse Water Reservoir

Signal Peak Mine Busse Water Reservoir

Signal Peak Mine Busse Water Reservoir

Signal Peak Mine Busse Water Reservoir

Signal Peak Mine Busse Water Reservoir

Signal Peak Mine Busse Water Reservoir

Signal Peak Mine Busse Water Reservoir

Signal Peak Mine Busse Water Reservoir

Signal Peak Mine Busse Water Reservoir

Signal Peak Mine Busse Water Reservoir

Signal Peak Mine Busse Water Reservoir

Signal Peak Mine Busse Water Reservoir

Signal Peak Mine Busse Water Reservoir

Signal Peak Mine Busse Water Reservoir

Signal Peak Mine Busse Water Reservoir

Signal Peak Mine Busse Water Reservoir

Signal Peak Mine Busse Water Reservoir

\begin{tabular}{|c|c|c|c|c|c|}
\hline Epfu & 2016 & 12 & $3.2(2.1) 56$ & 0.8 & 5.7 \\
\hline Epfu & 2017 & 1 & $4.8(3.1) 53$ & -2 & 8.4 \\
\hline Epfu & 2017 & 2 & 4.9 (3) 134 & 0.3 & 10.8 \\
\hline Epfu & 2017 & 3 & $8.8(2.8) 60$ & 1.4 & 12.2 \\
\hline Euma & 2013 & 6 & $15.6(0.9) 29$ & 14.1 & 17.3 \\
\hline Euma & 2013 & 7 & $18.3(2) 7$ & 17 & 22.4 \\
\hline Euma & 2013 & 8 & $18(3.5) 4$ & 12.7 & 19.8 \\
\hline Euma & 2013 & 9 & 21.7 () 1 & 21.7 & 21.7 \\
\hline Euma & 2014 & 5 & 12.5 () 1 & 12.5 & 12.5 \\
\hline Euma & 2014 & 6 & $13.8(0.4) 2$ & 13.6 & 14.1 \\
\hline Euma & 2014 & 7 & $19.2(2.5) 18$ & 16 & 24.9 \\
\hline Euma & 2014 & 8 & 20.6 (3.8) 19 & 11.3 & 28.2 \\
\hline Euma & 2015 & 6 & $18.3(4.4) 15$ & 10.8 & 24.7 \\
\hline Euma & 2015 & 6 & $15.4(3.9) 9$ & 12.7 & 20.6 \\
\hline Euma & 2015 & 7 & $17.7(2.4) 117$ & 8.9 & 23.6 \\
\hline Euma & 2015 & 8 & $19.4(2.6) 21$ & 17 & 26.9 \\
\hline Euma & 2015 & 8 & 23.3 (3.1) 14 & 20.3 & 28.2 \\
\hline Euma & 2015 & 9 & $19.8(0) 2$ & 19.8 & 19.8 \\
\hline Euma & 2016 & 4 & $17.8(0.2) 2$ & 17.6 & 17.9 \\
\hline Euma & 2016 & 5 & $18.4(0) 4$ & 18.4 & 18.4 \\
\hline Laci & 2012 & 8 & $19.4(6.2) 151$ & 6.2 & 28 \\
\hline Laci & 2012 & 9 & $17.4(4.6) 197$ & 4.7 & 26.5 \\
\hline Laci & 2012 & 10 & $14.4(2.5) 4$ & 12.2 & 17 \\
\hline Laci & 2012 & 11 & $4.5(4.5) 2$ & 1.4 & 7.7 \\
\hline
\end{tabular}


Signal Peak Mine Busse Water Reservoir

Signal Peak Mine Busse Water Reservoir

Signal Peak Mine Busse Water Reservoir

Signal Peak Mine Busse Water Reservoir

Signal Peak Mine Busse Water Reservoir

Signal Peak Mine Busse Water Reservoir

Signal Peak Mine Busse Water Reservoir

Signal Peak Mine Busse Water Reservoir

Signal Peak Mine Busse Water Reservoir

Signal Peak Mine Busse Water Reservoir

Signal Peak Mine Busse Water Reservoir

Signal Peak Mine Busse Water Reservoir

Signal Peak Mine Busse Water Reservoir

Signal Peak Mine Busse Water Reservoir

Signal Peak Mine Busse Water Reservoir

Signal Peak Mine Busse Water Reservoir

Signal Peak Mine Busse Water Reservoir

Signal Peak Mine Busse Water Reservoir

Signal Peak Mine Busse Water Reservoir

Signal Peak Mine Busse Water Reservoir

Signal Peak Mine Busse Water Reservoir

Signal Peak Mine Busse Water Reservoir

Signal Peak Mine Busse Water Reservoir

Signal Peak Mine Busse Water Reservoir

\begin{tabular}{|c|c|c|c|c|c|}
\hline Laci & 2012 & 12 & 0.9() 1 & 0.9 & 0.9 \\
\hline Laci & 2013 & 4 & $12.6(2.3) 155$ & 3.6 & 17.8 \\
\hline Laci & 2013 & 5 & 11.6 (3.1) 177 & 4.4 & 24.2 \\
\hline Laci & 2013 & 6 & 17.1 (3) 3107 & 5.7 & 23.9 \\
\hline Laci & 2013 & 7 & $18.8(3.4) 5893$ & 10.8 & 26 \\
\hline Laci & 2013 & 8 & $18.4(3.5) 651$ & 11.8 & 28 \\
\hline Laci & 2013 & 9 & $19.3(3.3) 92$ & 8.9 & 25.4 \\
\hline Laci & 2013 & 10 & $9.8(3.7) 4$ & 4.7 & 13.5 \\
\hline Laci & 2013 & 11 & $4.1(4.4) 4$ & 1.9 & 10.7 \\
\hline Laci & 2013 & 12 & -2 () 1 & -2 & -2 \\
\hline Laci & 2014 & 1 & $6.1(2.3) 3$ & 3.4 & 7.4 \\
\hline Laci & 2014 & 4 & $10(3.1) 278$ & 0 & 17.3 \\
\hline Laci & 2014 & 5 & $12.9(4.5) 611$ & 4.4 & 23.1 \\
\hline Laci & 2014 & 6 & 13.6 (2.6) 5194 & 2.2 & 20.4 \\
\hline Laci & 2014 & 7 & $18.1(3.5) 10937$ & 8.9 & 26.5 \\
\hline Laci & 2014 & 8 & 18.2 (3) 1923 & 7 & 28.4 \\
\hline Laci & 2014 & 9 & $13.1(3.4) 176$ & 6.7 & 20.4 \\
\hline Laci & 2014 & 10 & $11.7(3.3) 2$ & 9.4 & 14 \\
\hline Laci & 2014 & 11 & $3(1.5) 3$ & 1.3 & 4.2 \\
\hline Laci & 2014 & 12 & $6.4(5.8) 9$ & -1.3 & 10.3 \\
\hline Laci & 2015 & 2 & $12.3(4.9) 8$ & 0.3 & 14.1 \\
\hline Laci & 2015 & 3 & 11.5 (3.3) 482 & 5.9 & 18.4 \\
\hline Laci & 2015 & 4 & $12(2.8) 307$ & 3.7 & 17.1 \\
\hline Laci & 2015 & 5 & $10.3(2.6) 339$ & 0.4 & 20.4 \\
\hline
\end{tabular}


Signal Peak Mine Busse Water Reservoir

Signal Peak Mine Busse Water Reservoir

Signal Peak Mine Busse Water Reservoir

Signal Peak Mine Busse Water Reservoir

Signal Peak Mine Busse Water Reservoir

Signal Peak Mine Busse Water Reservoir

Signal Peak Mine Busse Water Reservoir

Signal Peak Mine Busse Water Reservoir

Signal Peak Mine Busse Water Reservoir

Signal Peak Mine Busse Water Reservoir

Signal Peak Mine Busse Water Reservoir

Signal Peak Mine Busse Water Reservoir

Signal Peak Mine Busse Water Reservoir

Signal Peak Mine Busse Water Reservoir

Signal Peak Mine Busse Water Reservoir

Signal Peak Mine Busse Water Reservoir

Signal Peak Mine Busse Water Reservoir

Signal Peak Mine Busse Water Reservoir

Signal Peak Mine Busse Water Reservoir

Signal Peak Mine Busse Water Reservoir

Signal Peak Mine Busse Water Reservoir

Signal Peak Mine Busse Water Reservoir

Signal Peak Mine Busse Water Reservoir

Signal Peak Mine Busse Water Reservoir

\begin{tabular}{|c|c|c|c|c|c|}
\hline Laci & 2015 & 6 & $17.1(3.5) 6047$ & 9.4 & 27 \\
\hline Laci & 2015 & 6 & 16.4 (3) 5902 & 11.2 & 24.9 \\
\hline Laci & 2015 & 7 & $18.1(3.2) 5386$ & 8.5 & 27.9 \\
\hline Laci & 2015 & 8 & $20.4(4.4) 1161$ & 4.9 & 29.8 \\
\hline Laci & 2015 & 8 & 23.7 (4.7) 2254 & 6.2 & 30.7 \\
\hline Laci & 2015 & 9 & 22.5 (3.1) 1018 & 5.4 & 28 \\
\hline Laci & 2015 & 9 & 19.6 (3.8) 223 & 7.9 & 24.1 \\
\hline Laci & 2015 & 10 & $19.3(5.5) 5$ & 9.5 & 22.2 \\
\hline Laci & 2015 & 12 & $5.2(2.3) 4$ & 2.9 & 7.2 \\
\hline Laci & 2016 & 1 & $4.4(2.9) 29$ & -1.3 & 7.4 \\
\hline Laci & 2016 & 2 & $4(3.2) 9$ & 1.1 & 9 \\
\hline Laci & 2016 & 3 & 7.8 (6.2) 21 & -0.6 & 17.8 \\
\hline Laci & 2016 & 4 & 13.9 (3.1) 463 & 5.1 & 21.7 \\
\hline Laci & 2016 & 5 & $12.4(3.2) 511$ & 5.7 & 22.1 \\
\hline Laci & 2016 & 9 & 15 () 1 & 15 & 15 \\
\hline Laci & 2016 & 10 & 10.7 () 1 & 10.7 & 10.7 \\
\hline Laci & 2016 & 11 & $8.8(1.3) 22$ & 4.2 & 10.2 \\
\hline Laci & 2016 & 12 & $1.8(0.2) 5$ & 1.6 & 1.9 \\
\hline Laci & 2017 & 1 & $-2(0) 4$ & -2 & -2 \\
\hline Laci & 2017 & 2 & $5.2(1.5) 6$ & 2.6 & 6.5 \\
\hline Lano & 2012 & 8 & 21.3 (4.3) 273 & 6.4 & 30.2 \\
\hline Lano & 2012 & 9 & 15.9 (4.1) 751 & 5.1 & 26.5 \\
\hline Lano & 2012 & 10 & $10.5(2.4) 31$ & 8 & 16.1 \\
\hline Lano & 2012 & 11 & $5.1(3.3) 12$ & 2.4 & 10.5 \\
\hline
\end{tabular}


Signal Peak Mine Busse Water Reservoir

Signal Peak Mine Busse Water Reservoir

Signal Peak Mine Busse Water Reservoir

Signal Peak Mine Busse Water Reservoir

Signal Peak Mine Busse Water Reservoir

Signal Peak Mine Busse Water Reservoir

Signal Peak Mine Busse Water Reservoir

Signal Peak Mine Busse Water Reservoir

Signal Peak Mine Busse Water Reservoir

Signal Peak Mine Busse Water Reservoir

Signal Peak Mine Busse Water Reservoir

Signal Peak Mine Busse Water Reservoir

Signal Peak Mine Busse Water Reservoir

Signal Peak Mine Busse Water Reservoir

Signal Peak Mine Busse Water Reservoir

Signal Peak Mine Busse Water Reservoir

Signal Peak Mine Busse Water Reservoir

Signal Peak Mine Busse Water Reservoir

Signal Peak Mine Busse Water Reservoir

Signal Peak Mine Busse Water Reservoir

Signal Peak Mine Busse Water Reservoir

Signal Peak Mine Busse Water Reservoir

Signal Peak Mine Busse Water Reservoir

Signal Peak Mine Busse Water Reservoir

\begin{tabular}{|c|c|c|c|c|c|}
\hline Lano & 2012 & 12 & 4.4 (3) 7 & -2.3 & 5.7 \\
\hline Lano & 2013 & 1 & $8.7(0) 4$ & 8.7 & 8.7 \\
\hline Lano & 2013 & 4 & $9.1(2.7) 851$ & 0.8 & 17 \\
\hline Lano & 2013 & 5 & 12.9 (3.4) 1364 & 0.6 & 24.4 \\
\hline Lano & 2013 & 6 & 15.5 (2.9) 2376 & 6.2 & 23.7 \\
\hline Lano & 2013 & 7 & 19.8 (3.1) 2524 & 10.8 & 25.7 \\
\hline Lano & 2013 & 8 & 18.4 (3.4) 831 & 11.7 & 28 \\
\hline Lano & 2013 & 9 & 17.4 (3.9) 437 & 6.9 & 25.4 \\
\hline Lano & 2013 & 10 & 13.9 (3) 131 & 5.7 & 17 \\
\hline Lano & 2013 & 11 & $4.9(5.2) 12$ & -0.8 & 10.7 \\
\hline Lano & 2013 & 12 & 6.2 (3.3) 2 & 3.9 & 8.5 \\
\hline Lano & 2014 & 1 & $1.9(3.7) 8$ & -0.8 & 7.4 \\
\hline Lano & 2014 & 2 & 7 () 1 & 7 & 7 \\
\hline Lano & 2014 & 3 & $5(2.5) 4$ & 2.9 & 7.2 \\
\hline Lano & 2014 & 4 & $10.6(3.2) 948$ & -0.5 & 17.1 \\
\hline Lano & 2014 & 5 & $14.2(3.5) 2398$ & 5.2 & 23.1 \\
\hline Lano & 2014 & 6 & 13.6 (3.3) 1551 & 2.2 & 20.4 \\
\hline Lano & 2014 & 7 & $18(3.8) 2235$ & 8.9 & 26.5 \\
\hline Lano & 2014 & 8 & 17.6 (3.9) 1377 & 7 & 28.5 \\
\hline Lano & 2014 & 9 & 11.5 (3.5) 506 & 3.9 & 22.1 \\
\hline Lano & 2014 & 10 & 6.3 (5.7) 13 & 0.6 & 15.5 \\
\hline Lano & 2014 & 12 & 5.6 (5.5) 16 & -1.3 & 10.3 \\
\hline Lano & 2015 & 2 & 10.6 (6.9) 4 & 0.3 & 14.1 \\
\hline Lano & 2015 & 3 & 10.3 (3.2) 127 & -1.6 & 18.4 \\
\hline
\end{tabular}


Signal Peak Mine Busse Water Reservoir

Signal Peak Mine Busse Water Reservoir

Signal Peak Mine Busse Water Reservoir

Signal Peak Mine Busse Water Reservoir

Signal Peak Mine Busse Water Reservoir

Signal Peak Mine Busse Water Reservoir

Signal Peak Mine Busse Water Reservoir

Signal Peak Mine Busse Water Reservoir

Signal Peak Mine Busse Water Reservoir

Signal Peak Mine Busse Water Reservoir

Signal Peak Mine Busse Water Reservoir

Signal Peak Mine Busse Water Reservoir

Signal Peak Mine Busse Water Reservoir

Signal Peak Mine Busse Water Reservoir

Signal Peak Mine Busse Water Reservoir

Signal Peak Mine Busse Water Reservoir

Signal Peak Mine Busse Water Reservoir

Signal Peak Mine Busse Water Reservoir

Signal Peak Mine Busse Water Reservoir

Signal Peak Mine Busse Water Reservoir

Signal Peak Mine Busse Water Reservoir

Signal Peak Mine Busse Water Reservoir

Signal Peak Mine Busse Water Reservoir

Signal Peak Mine Busse Water Reservoir

\begin{tabular}{|c|c|c|c|c|c|}
\hline Lano & 2015 & 4 & 10.9 (4) 331 & 2.9 & 17.1 \\
\hline Lano & 2015 & 5 & $11(2.6) 1224$ & 0.4 & 20.1 \\
\hline Lano & 2015 & 6 & 17.6 (3.9) 1704 & 9.4 & 27.2 \\
\hline Lano & 2015 & 6 & 16.7 (3.2) 936 & 11.2 & 24.9 \\
\hline Lano & 2015 & 7 & 18 (3) 1688 & 9.5 & 27.9 \\
\hline Lano & 2015 & 8 & 21.1 (4.3) 1652 & 5.1 & 29.5 \\
\hline Lano & 2015 & 8 & $23.1(4.6) 2883$ & 6 & 30.7 \\
\hline Lano & 2015 & 9 & $17.3(4.9) 1870$ & 5.4 & 27.9 \\
\hline Lano & 2015 & 9 & $15.6(5) 728$ & 4.1 & 26.5 \\
\hline Lano & 2015 & 10 & 15.4 (4) 6 & 10.7 & 18.9 \\
\hline Lano & 2015 & 10 & 19 (3.1) 21 & 15 & 25.4 \\
\hline Lano & 2015 & 11 & $7.5(4.3) 5$ & 4.2 & 14.8 \\
\hline Lano & 2015 & 12 & $5.2(1.5) 9$ & 3.6 & 8.4 \\
\hline Lano & 2016 & 1 & 5 (1.8) 33 & 1.3 & 10.2 \\
\hline Lano & 2016 & 2 & $5.6(3.8) 16$ & 0.1 & 10.7 \\
\hline Lano & 2016 & 3 & $11(6.8) 179$ & -0.6 & 17.8 \\
\hline Lano & 2016 & 4 & 14.8 (3.2) 908 & 6.5 & 21.2 \\
\hline Lano & 2016 & 5 & 13.8 (3) 3800 & 4.4 & 21.9 \\
\hline Lano & 2016 & 9 & 18.9 (1.3) 18 & 17 & 19.9 \\
\hline Lano & 2016 & 10 & 12.7 (3.7) 20 & 6.9 & 19.4 \\
\hline Lano & 2016 & 11 & $5.4(4.5) 24$ & 0.4 & 13.6 \\
\hline Lano & 2016 & 12 & $1.7(2) 5$ & 0.8 & 5.2 \\
\hline Lano & 2017 & 1 & 4.5 (3.4) 14 & -2 & 8.4 \\
\hline Lano & 2017 & 2 & 7.1 (3.9) 26 & 2.2 & 10.8 \\
\hline
\end{tabular}


Signal Peak Mine Busse Water Reservoir

Signal Peak Mine Busse Water Reservoir

Signal Peak Mine Busse Water Reservoir

Signal Peak Mine Busse Water Reservoir

Signal Peak Mine Busse Water Reservoir

Signal Peak Mine Busse Water Reservoir

Signal Peak Mine Busse Water Reservoir

Signal Peak Mine Busse Water Reservoir

Signal Peak Mine Busse Water Reservoir

Signal Peak Mine Busse Water Reservoir

Signal Peak Mine Busse Water Reservoir

Signal Peak Mine Busse Water Reservoir

Signal Peak Mine Busse Water Reservoir

Signal Peak Mine Busse Water Reservoir

Signal Peak Mine Busse Water Reservoir

Signal Peak Mine Busse Water Reservoir

Signal Peak Mine Busse Water Reservoir

Signal Peak Mine Busse Water Reservoir

Signal Peak Mine Busse Water Reservoir

Signal Peak Mine Busse Water Reservoir

Signal Peak Mine Busse Water Reservoir

Signal Peak Mine Busse Water Reservoir

Signal Peak Mine Busse Water Reservoir

Signal Peak Mine Busse Water Reservoir

\begin{tabular}{|c|c|c|c|c|c|}
\hline Lano & 2017 & 3 & $10.2(2.3) 7$ & 7.5 & 12.2 \\
\hline Мyсi & 2012 & 8 & 21.6 (4.3) 954 & 6.4 & 30.2 \\
\hline Myci & 2012 & 9 & 17.8 (3.4) 1201 & 6.4 & 26.7 \\
\hline Myci & 2012 & 10 & $17.2(3.2) 12$ & 11.3 & 19.6 \\
\hline Myсі & 2012 & 11 & $8(2.2) 9$ & 5.9 & 11.5 \\
\hline Мyci & 2012 & 12 & 5.6 (5) 7 & 1.1 & 11 \\
\hline Мyсi & 2013 & 4 & 11.9 (2.8) 29 & 4.1 & 16.6 \\
\hline Мyсi & 2013 & 5 & 13.9 (2) 848 & 6.9 & 24.1 \\
\hline Мyсi & 2013 & 6 & 15.7 (2.1) 2367 & 9 & 23.7 \\
\hline Myci & 2013 & 7 & 19 (2.6) 644 & 11 & 26 \\
\hline Мyсi & 2013 & 8 & $18.1(3.3) 1574$ & 11.7 & 27.5 \\
\hline Мyсi & 2013 & 9 & $18.8(3.4) 96$ & 8.4 & 26 \\
\hline Мyсi & 2013 & 10 & 10.8 (1.6) 10 & 7 & 12.7 \\
\hline Мyсi & 2013 & 11 & $4.5(4.4) 12$ & -1.6 & 10.8 \\
\hline Мyсі & 2013 & 12 & 3.4 () 1 & 3.4 & 3.4 \\
\hline Myсі & 2014 & 1 & $-0.7(0.4) 4$ & -1 & -0.3 \\
\hline Мyсі & 2014 & 2 & $4.5(1.2) 3$ & 3.1 & 5.2 \\
\hline Мyсi & 2014 & 4 & 13 (3) 57 & 4.1 & 17 \\
\hline Мyсi & 2014 & 5 & $16.4(2.6) 909$ & 5.9 & 22.6 \\
\hline Myсі & 2014 & 6 & $14(2.2) 499$ & 5.7 & 19.4 \\
\hline Мyсi & 2014 & 7 & 17.9 (3.2) 256 & 9 & 26.4 \\
\hline Мyсi & 2014 & 8 & $18(3.3) 550$ & 8.9 & 28.5 \\
\hline Мyсі & 2014 & 9 & $15.3(3.8) 124$ & 4.9 & 21.2 \\
\hline Мyсі & 2014 & 10 & $13.6(2.6) 7$ & 10.7 & 17.3 \\
\hline
\end{tabular}


Signal Peak Mine Busse Water Reservoir

Signal Peak Mine Busse Water Reservoir

Signal Peak Mine Busse Water Reservoir

Signal Peak Mine Busse Water Reservoir

Signal Peak Mine Busse Water Reservoir

Signal Peak Mine Busse Water Reservoir

Signal Peak Mine Busse Water Reservoir

Signal Peak Mine Busse Water Reservoir

Signal Peak Mine Busse Water Reservoir

Signal Peak Mine Busse Water Reservoir

Signal Peak Mine Busse Water Reservoir

Signal Peak Mine Busse Water Reservoir

Signal Peak Mine Busse Water Reservoir

Signal Peak Mine Busse Water Reservoir

Signal Peak Mine Busse Water Reservoir

Signal Peak Mine Busse Water Reservoir

Signal Peak Mine Busse Water Reservoir

Signal Peak Mine Busse Water Reservoir

Signal Peak Mine Busse Water Reservoir

Signal Peak Mine Busse Water Reservoir

Signal Peak Mine Busse Water Reservoir

Signal Peak Mine Busse Water Reservoir

Signal Peak Mine Busse Water Reservoir

Signal Peak Mine Busse Water Reservoir
Myci $2014 \quad 11$

$-0.1(0) 2$

$-0.1$

$-0.1$

$\begin{array}{lll}\text { Myci } 2014 \quad 12 & -0.8(0) 2\end{array}$

$-0.8$

$-0.8$

Myci $2015 \quad 1$

9.7 () 1

9.7

9.7

Myci $2015 \quad 2$

2.7 () 1

2.7

2.7

Myci $2015 \quad 3$

12.7 (3.3) 21

8.2

18.3

Myci $2015 \quad 4 \quad 11.4(4.4) 70$

1.9

17.1

Myci $2015 \quad 5 \quad 12.9(2) 278$

2.7

16.1

Myci $2015 \quad 6 \quad 16.8(3.3) 204$

9.7

27.2

Myci $2015 \quad 6 \quad 17(2.8) 154$

11.3

24.7

$\begin{array}{lllll}\text { Myci } & 2015 \quad 7 & 17.6(2.7) 467 & 8.9\end{array}$

25.5

$\begin{array}{lllll}\text { Myci } & 2015 & 8 & 20.8(4.3) & 519\end{array}$

29.8

$\begin{array}{lllll}\text { Myci } & 2015 & 8 & 22.3(4.4) 865 & 9.4\end{array}$

30.7

Myci $2015 \quad 9 \quad 20.6(4.6) 980$

9.7

28

$\begin{array}{lllll}\text { Myci } 2015 \quad 9 & 18(4.3) 186\end{array}$

25.5

Myci $2015 \quad 10 \quad 13.4(1.7) 3$

11.8

15.1

$\begin{array}{lllll}\text { Myci } & 2015 & 10 & 17.1(5.2) & 57\end{array}$

26.5

$\begin{array}{lllll}\text { Myci } & 2015 & 11 & 8.3(4.1) 38 & 0.1\end{array}$

13.6

$\begin{array}{lllll}\text { Myci } & 2015 & 12 & 3(2.3) 64 & -2.1\end{array}$

8.7

$\begin{array}{lllll}\text { Myci } & 2016 & 1 & 3.5(2.3) 26 & -1\end{array}$

7.2

Myci $2016 \quad 2 \quad 2.7(2.6) 33$

$-0.5$

9

Myci $2016 \quad 3 \quad 12.3(6) 43$

0.6

18.1

Myci $2016 \quad 4 \quad 17.1(3.1) 2653$

21.9

Myci $2016 \quad 5 \quad 17.6(2.6) 1334$

8.2

22.1

Myci $2016 \quad 9 \quad 19(2.4) 47$

9.8 
Signal Peak Mine Busse Water Reservoir

Signal Peak Mine Busse Water Reservoir

Signal Peak Mine Busse Water Reservoir

Signal Peak Mine Busse Water Reservoir

Signal Peak Mine Busse Water Reservoir

Signal Peak Mine Busse Water Reservoir

Signal Peak Mine Busse Water Reservoir

Signal Peak Mine Busse Water Reservoir

Signal Peak Mine Busse Water Reservoir

Signal Peak Mine Busse Water Reservoir

Signal Peak Mine Busse Water Reservoir

Signal Peak Mine Busse Water Reservoir

Signal Peak Mine Busse Water Reservoir

Signal Peak Mine Busse Water Reservoir

Signal Peak Mine Busse Water Reservoir

Signal Peak Mine Busse Water Reservoir

Signal Peak Mine Busse Water Reservoir

Signal Peak Mine Busse Water Reservoir

Signal Peak Mine Busse Water Reservoir

Signal Peak Mine Busse Water Reservoir

Signal Peak Mine Busse Water Reservoir

Signal Peak Mine Busse Water Reservoir

Signal Peak Mine Busse Water Reservoir

Signal Peak Mine Busse Water Reservoir $\begin{array}{llll}\text { Myci } 2016 \quad 10 & 14.7(2.7) 75\end{array}$

Myci $2016 \quad 11 \quad 10.5$ (4.9) 66

17.8

Myci $2016 \quad 12 \quad 1(0.8) 11$

Myci $2017 \quad 1$

$6.4(3.3) 17$

9.4

Myci $2017 \quad 2$

$4.1(4.4) 19$

0.1

11

Myci $2017 \quad 3$

$7.1(1.8) 8$

3.2

8.4

Myev $2012 \quad 8 \quad 20.1(4.1) 62$

14.1

27

$\begin{array}{lllll}\text { Myev } 2012 & 9 & 14.4(2.3) & 1931 & 3.6\end{array}$

25.1

$\begin{array}{llll}\text { Myev } 2012 \quad 10 & 15.9(1.9) & 23 & 8.2\end{array}$

19.8

$\begin{array}{lllll}\text { Myev } & 2013 \quad 5 & 11.7(1.4) 6 & 9.4\end{array}$

13.5

$\begin{array}{lllll}\text { Myev } & 2013 & 6 & 14.1(2.2) 34 & 8.9\end{array}$

$\begin{array}{lllll}\text { Myev } & 2013 & 7 & 18.2(3.7) 5 & 12.8\end{array}$

22.7

12.7

23.6

Myev $2013 \quad 8 \quad 19.5(2.8) 20$

7.7

24.7

Myev $2013 \quad 9 \quad 16.8(4.7) 18$

$\begin{array}{lllll}\text { Myev } & 2013 \quad 10 & 8(2.4) 3 & 6.5\end{array}$

10.8

3.9

21.4

Myev $2014 \quad 5 \quad 13(5.7) 9$

$\begin{array}{lllll}\text { Myev } & 2014 & 6 & 11.5(3.4) 9 & 8\end{array}$

18.4

$\begin{array}{lllll}\text { Myev } & 2014 & 7 & 19(3.1) 43 & 11\end{array}$

26.4

$\begin{array}{lllll}\text { Myev } & 2014 & 8 & 18.8(4.1) 49 & 7\end{array}$

27

$\begin{array}{lllll}\text { Myev } & 2014 & 9 & 13.7(4.4) 14 & 8.4\end{array}$

19.3

$\begin{array}{lll}\text { Myev } 2014 \quad 10 & 16() 1\end{array}$

16

16

$\begin{array}{lllll}\text { Myev } 2015 \quad 4 & 8.5(6.1) 6 & 0\end{array}$

16

$\begin{array}{lllll}\text { Myev } & 2015 \quad 5 & 12.3(2.8) 15\end{array}$

$\begin{array}{llll}\text { Myev } 2015 \quad 6 & 18.6(4) 34\end{array}$

9.7
15.5

26.4 
Signal Peak Mine Busse Water Reservoir

Signal Peak Mine Busse Water Reservoir

Signal Peak Mine Busse Water Reservoir

Signal Peak Mine Busse Water Reservoir

Signal Peak Mine Busse Water Reservoir

Signal Peak Mine Busse Water Reservoir

Signal Peak Mine Busse Water Reservoir

Signal Peak Mine Busse Water Reservoir

Signal Peak Mine Busse Water Reservoir

Signal Peak Mine Busse Water Reservoir

Signal Peak Mine Busse Water Reservoir

Signal Peak Mine Busse Water Reservoir

Signal Peak Mine Busse Water Reservoir

Signal Peak Mine Busse Water Reservoir

Signal Peak Mine Busse Water Reservoir

Signal Peak Mine Busse Water Reservoir

Signal Peak Mine Busse Water Reservoir

Signal Peak Mine Busse Water Reservoir

Signal Peak Mine Busse Water Reservoir

Signal Peak Mine Busse Water Reservoir

Signal Peak Mine Busse Water Reservoir

Signal Peak Mine Busse Water Reservoir

Signal Peak Mine Busse Water Reservoir

Signal Peak Mine Busse Water Reservoir
Myev $2015 \quad 6 \quad 17.8(3.8) 69$

11.3

24.7

$\begin{array}{lllll}\text { Myev } & 2015 \quad 7 & 17.9(3) & 59 & 11.5\end{array}$

24.6

$\begin{array}{lllll}\text { Myev } & 2015 \quad 8 & 21.3(4.2) 150 & 10\end{array}$

5.4

Myev $2015 \quad 8 \quad 22.8(4.7) 1006$

5.9

5.2

7.7

$\begin{array}{llll}\text { Myev } \quad 2015 \quad 10 & 15.2(6.3) 21\end{array}$

$9.2(1.3) 3$

7.7

10

Myev $2015 \quad 11$

$9.5(4.6) 9$

1.4

12.2

Myev $2016 \quad 4 \quad 12.4(4.9) 112$

1.3

21.7

$\begin{array}{lllll}\text { Myev } & 2016 \quad 5 & 14(4.1) 272 & 4.7\end{array}$

22.1

$\begin{array}{lllll}\text { Myev } & 2016 \quad 9 & 19.5(1.6) 47 & 16.8\end{array}$

22.9

Myev $2016 \quad 10 \quad 15.8(3.4) 23$

5.7

18.9

Myev $2016 \quad 11$

3.4() 1

3.4

3.4

Myev $2017 \quad 3$

9.7 () 1

9.7

9.7

$\begin{array}{lllll}\text { Mylu } & 2012 \quad 8 & 21.4(4.5) 618 & 14\end{array}$

29.3

Mylu $2012 \quad 9 \quad 18.7(3.6) 335$

9

26.7

Mylu $2012 \quad 10 \quad 14.4(4.9) 5$

19.6

Mylu $2013 \quad 4 \quad 12.5(0.3) 5$

12.3

13

Mylu $2013 \quad 5 \quad 13.6(2.2) 212 \quad 5.7$

23.6

Mylu $2013 \quad 6 \quad 15.9(2) 1755$

23.6

$\begin{array}{lllll}\text { Mylu } 2013 \quad 7 & 19.4(2.9) 2886\end{array}$

26

Mylu $2013 \quad 8 \quad 17.6(3.3) 773$

11.7

25.5

Mylu $2013 \quad 9 \quad 17.7(3.2) 68$

10.3 
Signal Peak Mine Busse Water Reservoir

Signal Peak Mine Busse Water Reservoir

Signal Peak Mine Busse Water Reservoir

Signal Peak Mine Busse Water Reservoir

Signal Peak Mine Busse Water Reservoir

Signal Peak Mine Busse Water Reservoir

Signal Peak Mine Busse Water Reservoir

Signal Peak Mine Busse Water Reservoir

Signal Peak Mine Busse Water Reservoir

Signal Peak Mine Busse Water Reservoir

Signal Peak Mine Busse Water Reservoir

Signal Peak Mine Busse Water Reservoir

Signal Peak Mine Busse Water Reservoir

Signal Peak Mine Busse Water Reservoir

Signal Peak Mine Busse Water Reservoir

Signal Peak Mine Busse Water Reservoir

Signal Peak Mine Busse Water Reservoir

Signal Peak Mine Busse Water Reservoir

Signal Peak Mine Busse Water Reservoir

Signal Peak Mine Busse Water Reservoir

Signal Peak Mine Busse Water Reservoir

Signal Peak Mine Busse Water Reservoir

Signal Peak Mine Busse Water Reservoir

Signal Peak Mine Busse Water Reservoir

\begin{tabular}{|c|c|c|c|c|c|}
\hline Mylu & 2013 & 10 & $8.7(1.3) 5$ & 7 & 10.3 \\
\hline Mylu & 2014 & 4 & 9.8 (3.6) 9 & 2.9 & 14.5 \\
\hline Mylu & 2014 & 5 & $16.3(2.7) 826$ & 1.9 & 23.1 \\
\hline Mylu & 2014 & 6 & $14.4(2) 1005$ & 2.4 & 20.4 \\
\hline Mylu & 2014 & 7 & $17.8(2.7) 834$ & 8.9 & 26.2 \\
\hline Mylu & 2014 & 8 & $18.2(2.9) 883$ & 7.2 & 28.5 \\
\hline Mylu & 2014 & 9 & 13.5 (3.2) 81 & 5.5 & 20.4 \\
\hline Mylu & 2014 & 10 & $13.7(4.6) 3$ & 8.4 & 17 \\
\hline Mylu & 2014 & 12 & $3.8(5.2) 2$ & 0.1 & 7.5 \\
\hline Mylu & 2015 & 3 & $15.3(3.4) 11$ & 11.2 & 18.4 \\
\hline Mylu & 2015 & 4 & $12(4.3) 10$ & 4.2 & 15.8 \\
\hline Mylu & 2015 & 5 & $12.3(2.3) 186$ & 2.9 & 15.8 \\
\hline Mylu & 2015 & 6 & 17.7 (4) 583 & 9.5 & 27.4 \\
\hline Mylu & 2015 & 6 & $17(2.3) 1488$ & 11.2 & 24.2 \\
\hline Mylu & 2015 & 7 & $18.2(2.3) 1661$ & 8.9 & 27.5 \\
\hline Mylu & 2015 & 8 & 19.5 (3.9) 462 & 5.4 & 29.5 \\
\hline Mylu & 2015 & 8 & 21.5 (4.7) 1396 & 6 & 30.7 \\
\hline Mylu & 2015 & 9 & $18(5) 2174$ & 5.4 & 28 \\
\hline Mylu & 2015 & 9 & $14.2(4.5) 385$ & 7 & 26 \\
\hline Mylu & 2015 & 10 & $13.6(3.2) 3$ & 11.8 & 17.3 \\
\hline Mylu & 2015 & 10 & $18.6(0.2) 6$ & 18.4 & 18.8 \\
\hline Mylu & 2016 & 4 & $8.6(4.6) 942$ & 3.4 & 21.6 \\
\hline Mylu & 2016 & 5 & 12.7 (3.7) 2325 & 4.2 & 21.6 \\
\hline Mylu & 2016 & 9 & $18.2(3.8) 125$ & 10 & 23.1 \\
\hline
\end{tabular}




\begin{tabular}{|c|c|c|c|c|c|c|}
\hline $\begin{array}{l}\text { Signal Peak Mine Busse } \\
\text { Water Reservoir }\end{array}$ & Mylu & 2016 & 10 & 14.2 (3.7) 46 & 6.2 & 19.6 \\
\hline $\begin{array}{c}\text { Signal Peak Mine Busse } \\
\text { Water Reservoir }\end{array}$ & Myth & 2012 & 8 & $19.1(2.4) 2$ & 17.4 & 20.8 \\
\hline $\begin{array}{l}\text { Signal Peak Mine Busse } \\
\text { Water Reservoir }\end{array}$ & Myth & 2012 & 9 & 21.6() 1 & 21.6 & 21.6 \\
\hline $\begin{array}{l}\text { Signal Peak Mine Busse } \\
\text { Water Reservoir }\end{array}$ & Myth & 2015 & 6 & $21.5(3.7) 2$ & 18.8 & 24.1 \\
\hline $\begin{array}{l}\text { Signal Peak Mine Busse } \\
\text { Water Reservoir }\end{array}$ & Myth & 2015 & 8 & 21 (5) 34 & 12.3 & 29.7 \\
\hline $\begin{array}{c}\text { Signal Peak Mine Busse } \\
\text { Water Reservoir }\end{array}$ & Myth & 2015 & 9 & 18.3 (4.3) 45 & 9 & 24.7 \\
\hline $\begin{array}{l}\text { Signal Peak Mine Busse } \\
\text { Water Reservoir }\end{array}$ & Myth & 2015 & 10 & 10.7 () 1 & 10.7 & 10.7 \\
\hline $\begin{array}{l}\text { Signal Peak Mine Busse } \\
\text { Water Reservoir }\end{array}$ & Myth & 2016 & 3 & $11.5(1.9) 6$ & 9.8 & 14 \\
\hline $\begin{array}{l}\text { Signal Peak Mine Busse } \\
\text { Water Reservoir }\end{array}$ & Myth & 2016 & 4 & $9.2(4.5) 12$ & 3.7 & 18.6 \\
\hline $\begin{array}{l}\text { Signal Peak Mine Busse } \\
\text { Water Reservoir }\end{array}$ & Myth & 2016 & 5 & 13.3 (4.7) 16 & 4.6 & 21.9 \\
\hline $\begin{array}{l}\text { Signal Peak Mine Busse } \\
\text { Water Reservoir }\end{array}$ & Myth & 2016 & 9 & 19.4 (1) 25 & 16.8 & 22.6 \\
\hline $\begin{array}{c}\text { Signal Peak Mine Reservoir } \\
1\end{array}$ & Epfu & 2012 & 8 & 24.7 (3.6) 901 & 15.8 & 29.3 \\
\hline $\begin{array}{c}\text { Signal Peak Mine Reservoir } \\
1\end{array}$ & Epfu & 2012 & 9 & $19.1(3.4) 3910$ & 5.1 & 26.5 \\
\hline $\begin{array}{c}\text { Signal Peak Mine Reservoir } \\
1\end{array}$ & Epfu & 2012 & 10 & 13.8 (3.1) 335 & 3.1 & 19.9 \\
\hline $\begin{array}{c}\text { Signal Peak Mine Reservoir } \\
1\end{array}$ & Epfu & 2012 & 11 & $8.2(3.8) 154$ & 2.2 & 16.1 \\
\hline $\begin{array}{c}\text { Signal Peak Mine Reservoir } \\
1\end{array}$ & Epfu & 2012 & 12 & $4(4) 127$ & -2.1 & 11.8 \\
\hline $\begin{array}{c}\text { Signal Peak Mine Reservoir } \\
1\end{array}$ & Epfu & 2013 & 1 & $5.1(2.5) 128$ & 0.1 & 10.2 \\
\hline $\begin{array}{c}\text { Signal Peak Mine Reservoir } \\
1\end{array}$ & Epfu & 2013 & 2 & 3.1 (1.7) 53 & -1.6 & 7.7 \\
\hline $\begin{array}{c}\text { Signal Peak Mine Reservoir } \\
1\end{array}$ & Epfu & 2013 & 3 & $8(2.8) 548$ & 0.4 & 16.3 \\
\hline $\begin{array}{c}\text { Signal Peak Mine Reservoir } \\
1\end{array}$ & Epfu & 2013 & 4 & 12.5 (3.1) 3478 & 1.4 & 20.3 \\
\hline $\begin{array}{c}\text { Signal Peak Mine Reservoir } \\
1\end{array}$ & Epfu & 2013 & 5 & 14.9 (2.6) 1892 & 5.2 & 26.7 \\
\hline $\begin{array}{c}\text { Signal Peak Mine Reservoir } \\
1\end{array}$ & Epfu & 2013 & 6 & $19.3(2.6) 1026$ & 7 & 24.7 \\
\hline $\begin{array}{c}\text { Signal Peak Mine Reservoir } \\
1\end{array}$ & Epfu & 2013 & 7 & $22.1(2.6) 1156$ & 13.6 & 27.7 \\
\hline $\begin{array}{c}\text { Signal Peak Mine Reservoir } \\
1\end{array}$ & Epfu & 2013 & 8 & 22.7 (3) 4154 & 13.6 & 27.4 \\
\hline
\end{tabular}




\begin{tabular}{|c|c|c|c|c|c|c|}
\hline $\begin{array}{c}\text { Signal Peak Mine Reservoir } \\
1\end{array}$ & Epfu & 2013 & 9 & $23(2.8) 801$ & 8.4 & 27.5 \\
\hline $\begin{array}{c}\text { Signal Peak Mine Reservoir } \\
1\end{array}$ & Epfu & 2013 & 10 & 13.3 (2.4) 154 & 6 & 16.1 \\
\hline $\begin{array}{c}\text { Signal Peak Mine Reservoir } \\
1\end{array}$ & Epfu & 2013 & 11 & $8.4(4.2) 128$ & 0.6 & 14 \\
\hline $\begin{array}{c}\text { Signal Peak Mine Reservoir } \\
1\end{array}$ & Epfu & 2013 & 12 & $6.8(1.8) 17$ & 4.2 & 11.3 \\
\hline $\begin{array}{c}\text { Signal Peak Mine Reservoir } \\
1\end{array}$ & Epfu & 2014 & 1 & $6.4(0) 9$ & 6.4 & 6.5 \\
\hline $\begin{array}{c}\text { Signal Peak Mine Reservoir } \\
1\end{array}$ & Epfu & 2014 & 4 & 9.6 (1.6) 20 & 6.4 & 11.5 \\
\hline $\begin{array}{c}\text { Signal Peak Mine Reservoir } \\
1\end{array}$ & Epfu & 2014 & 5 & $16(3.8) 308$ & 6.7 & 25.1 \\
\hline $\begin{array}{c}\text { Signal Peak Mine Reservoir } \\
1\end{array}$ & Epfu & 2014 & 6 & $17(2.3) 554$ & 12 & 22.2 \\
\hline $\begin{array}{c}\text { Signal Peak Mine Reservoir } \\
1\end{array}$ & Epfu & 2014 & 7 & 21.6 (3.4) 2788 & 11.2 & 28 \\
\hline $\begin{array}{c}\text { Signal Peak Mine Reservoir } \\
1\end{array}$ & Epfu & 2014 & 8 & $22(2.9) 2359$ & 9.7 & 30.7 \\
\hline $\begin{array}{c}\text { Signal Peak Mine Reservoir } \\
1\end{array}$ & Epfu & 2014 & 9 & $17.2(3.3) 465$ & 6.2 & 24.2 \\
\hline $\begin{array}{c}\text { Signal Peak Mine Reservoir } \\
1\end{array}$ & Epfu & 2014 & 10 & $14.4(2.4) 85$ & 8 & 17.9 \\
\hline $\begin{array}{c}\text { Signal Peak Mine Reservoir } \\
1\end{array}$ & Epfu & 2014 & 11 & $6.8(2.5) 95$ & 0.8 & 13.6 \\
\hline $\begin{array}{c}\text { Signal Peak Mine Reservoir } \\
1\end{array}$ & Epfu & 2014 & 12 & $8.2(3.1) 56$ & -1.3 & 13 \\
\hline $\begin{array}{c}\text { Signal Peak Mine Reservoir } \\
1\end{array}$ & Epfu & 2015 & 1 & 7.1 (3) 31 & 2.7 & 12.7 \\
\hline $\begin{array}{c}\text { Signal Peak Mine Reservoir } \\
1\end{array}$ & Epfu & 2015 & 2 & 8.7 () 1 & 8.7 & 8.7 \\
\hline $\begin{array}{c}\text { Signal Peak Mine Reservoir } \\
1\end{array}$ & Epfu & 2015 & 3 & 13.4 (2.5) 1877 & 5.9 & 20.4 \\
\hline $\begin{array}{c}\text { Signal Peak Mine Reservoir } \\
1\end{array}$ & Epfu & 2015 & 4 & 10 (3.1) 754 & 1.6 & 21.1 \\
\hline $\begin{array}{c}\text { Signal Peak Mine Reservoir } \\
1\end{array}$ & Epfu & 2015 & 5 & 12.7 (1.8) 118 & 3.9 & 17.6 \\
\hline $\begin{array}{c}\text { Signal Peak Mine Reservoir } \\
1\end{array}$ & Epfu & 2015 & 6 & 20.5 (2.1) 81 & 15.6 & 25.4 \\
\hline $\begin{array}{c}\text { Signal Peak Mine Reservoir } \\
1\end{array}$ & Epfu & 2015 & 6 & 22.6 (3.3) 2825 & 14.1 & 29.5 \\
\hline $\begin{array}{c}\text { Signal Peak Mine Reservoir } \\
1\end{array}$ & Epfu & 2015 & 7 & $23(2.8) 4590$ & 14.5 & 30.3 \\
\hline $\begin{array}{c}\text { Signal Peak Mine Reservoir } \\
1\end{array}$ & Epfu & 2015 & 8 & 24.4 (3.5) 4382 & 7.9 & 33.5 \\
\hline $\begin{array}{c}\text { Signal Peak Mine Reservoir } \\
1\end{array}$ & Epfu & 2015 & 9 & $22.8(3.4) 3572$ & 12.8 & 30 \\
\hline
\end{tabular}




\begin{tabular}{|c|c|c|c|c|c|c|}
\hline $\begin{array}{c}\text { Signal Peak Mine Reservoir } \\
1\end{array}$ & Epfu & 2015 & 10 & 15.8 (6.4) 635 & 3.9 & 28.2 \\
\hline $\begin{array}{c}\text { Signal Peak Mine Reservoir } \\
1\end{array}$ & Epfu & 2015 & 11 & 9.7 (3.1) 672 & 3.4 & 16.6 \\
\hline $\begin{array}{c}\text { Signal Peak Mine Reservoir } \\
1\end{array}$ & Epfu & 2015 & 12 & 8.3 (3) 392 & -0.8 & 15.6 \\
\hline $\begin{array}{c}\text { Signal Peak Mine Reservoir } \\
1\end{array}$ & Epfu & 2016 & 1 & 6.6 (2.6) 318 & 3.1 & 12 \\
\hline $\begin{array}{c}\text { Signal Peak Mine Reservoir } \\
1\end{array}$ & Epfu & 2016 & 2 & 9.3 (3.3) 494 & 1.1 & 14.8 \\
\hline $\begin{array}{c}\text { Signal Peak Mine Reservoir } \\
1\end{array}$ & Epfu & 2016 & 3 & $0.8(0) 2$ & 0.8 & 0.8 \\
\hline $\begin{array}{c}\text { Signal Peak Mine Reservoir } \\
1\end{array}$ & Epfu & 2016 & 4 & 16.5 (4) 818 & 2.7 & 24.1 \\
\hline $\begin{array}{c}\text { Signal Peak Mine Reservoir } \\
1\end{array}$ & Epfu & 2016 & 5 & 15.6 (3.3) 1095 & 10.8 & 25.2 \\
\hline $\begin{array}{c}\text { Signal Peak Mine Reservoir } \\
1\end{array}$ & Epfu & 2016 & 6 & 20.4 (3.9) 9146 & 12 & 30.8 \\
\hline $\begin{array}{c}\text { Signal Peak Mine Reservoir } \\
1\end{array}$ & Epfu & 2016 & 7 & $24.2(2.8) 3326$ & 15 & 30.5 \\
\hline $\begin{array}{c}\text { Signal Peak Mine Reservoir } \\
1\end{array}$ & Epfu & 2016 & 8 & $24.1(3.4) 1577$ & 10 & 30.7 \\
\hline $\begin{array}{c}\text { Signal Peak Mine Reservoir } \\
1\end{array}$ & Epfu & 2016 & 9 & $20.7(2.8) 1053$ & 13 & 28.5 \\
\hline $\begin{array}{c}\text { Signal Peak Mine Reservoir } \\
1\end{array}$ & Epfu & 2016 & 10 & 14.6 (4.6) 285 & 6.2 & 23.2 \\
\hline $\begin{array}{c}\text { Signal Peak Mine Reservoir } \\
1\end{array}$ & Epfu & 2016 & 11 & 12.9 (3.3) 585 & 3.7 & 20.9 \\
\hline $\begin{array}{c}\text { Signal Peak Mine Reservoir } \\
1\end{array}$ & Epfu & 2016 & 12 & $5(1.7) 69$ & 0 & 6.9 \\
\hline $\begin{array}{c}\text { Signal Peak Mine Reservoir } \\
1\end{array}$ & Epfu & 2017 & 1 & 8.9 (3.2) 160 & 1.9 & 13.3 \\
\hline $\begin{array}{c}\text { Signal Peak Mine Reservoir } \\
1\end{array}$ & Epfu & 2017 & 2 & 9.7 (3) 265 & 3.9 & 15 \\
\hline $\begin{array}{c}\text { Signal Peak Mine Reservoir } \\
1\end{array}$ & Epfu & 2017 & 3 & $10.3(0.7) 35$ & 9.7 & 11.7 \\
\hline $\begin{array}{c}\text { Signal Peak Mine Reservoir } \\
1\end{array}$ & Euma & 2012 & 9 & $20.4(0) 2$ & 20.4 & 20.4 \\
\hline $\begin{array}{c}\text { Signal Peak Mine Reservoir } \\
1\end{array}$ & Euma & 2013 & 4 & 12 () 1 & 12 & 12 \\
\hline $\begin{array}{c}\text { Signal Peak Mine Reservoir } \\
1\end{array}$ & Euma & 2013 & 6 & $17.3(2.1) 2$ & 15.8 & 18.8 \\
\hline $\begin{array}{c}\text { Signal Peak Mine Reservoir } \\
1\end{array}$ & Euma & 2013 & 7 & 23.6() 1 & 23.6 & 23.6 \\
\hline $\begin{array}{c}\text { Signal Peak Mine Reservoir } \\
1\end{array}$ & Euma & 2013 & 8 & 23.9 () 1 & 23.9 & 23.9 \\
\hline $\begin{array}{c}\text { Signal Peak Mine Reservoir } \\
1\end{array}$ & Euma & 2014 & 5 & $19(5.4) 6$ & 9 & 24.9 \\
\hline
\end{tabular}




\begin{tabular}{|c|c|c|c|c|c|c|}
\hline $\begin{array}{c}\text { Signal Peak Mine Reservoir } \\
1\end{array}$ & Euma & 2014 & 6 & 16.7 (2) 8 & 13.6 & 19.1 \\
\hline $\begin{array}{c}\text { Signal Peak Mine Reservoir } \\
1\end{array}$ & Euma & 2014 & 7 & 20.3 (3.3) 38 & 13.2 & 27.2 \\
\hline $\begin{array}{c}\text { Signal Peak Mine Reservoir } \\
1\end{array}$ & Euma & 2014 & 8 & 19.3 (1.9) 11 & 15.3 & 21.2 \\
\hline $\begin{array}{c}\text { Signal Peak Mine Reservoir } \\
1\end{array}$ & Euma & 2014 & 10 & $16.5(0.6) 6$ & 15.5 & 17.1 \\
\hline $\begin{array}{c}\text { Signal Peak Mine Reservoir } \\
1\end{array}$ & Euma & 2015 & 4 & 11.5 (0) 2 & 11.5 & 11.5 \\
\hline $\begin{array}{c}\text { Signal Peak Mine Reservoir } \\
1\end{array}$ & Euma & 2015 & 6 & $22.8(2.7) 655$ & 14.3 & 28 \\
\hline $\begin{array}{c}\text { Signal Peak Mine Reservoir } \\
1\end{array}$ & Euma & 2015 & 7 & 21.6 (2.3) 2181 & 14.8 & 28.5 \\
\hline $\begin{array}{c}\text { Signal Peak Mine Reservoir } \\
1\end{array}$ & Euma & 2015 & 8 & 23.8 (4) 81 & 7.9 & 32.5 \\
\hline $\begin{array}{c}\text { Signal Peak Mine Reservoir } \\
1\end{array}$ & Euma & 2015 & 9 & 19.4 (3.1) 706 & 14.3 & 26.4 \\
\hline $\begin{array}{c}\text { Signal Peak Mine Reservoir } \\
1\end{array}$ & Euma & 2016 & 4 & 20.7 (1.9) 29 & 15 & 21.4 \\
\hline $\begin{array}{c}\text { Signal Peak Mine Reservoir } \\
1\end{array}$ & Euma & 2016 & 5 & $13.1(1.6) 9$ & 12.5 & 17.4 \\
\hline $\begin{array}{c}\text { Signal Peak Mine Reservoir } \\
1\end{array}$ & Euma & 2016 & 6 & 20.3 (3.7) 204 & 12.3 & 29.8 \\
\hline $\begin{array}{c}\text { Signal Peak Mine Reservoir } \\
1\end{array}$ & Euma & 2016 & 7 & 23.9 (1.8) 188 & 19.1 & 28.7 \\
\hline $\begin{array}{c}\text { Signal Peak Mine Reservoir } \\
1\end{array}$ & Euma & 2016 & 8 & 19.9 (0) 2 & 19.9 & 19.9 \\
\hline $\begin{array}{c}\text { Signal Peak Mine Reservoir } \\
1\end{array}$ & Euma & 2016 & 10 & $16.1(0.1) 2$ & 16 & 16.1 \\
\hline $\begin{array}{c}\text { Signal Peak Mine Reservoir } \\
1\end{array}$ & Laci & 2012 & 8 & 25.6 (3.1) 39 & 19.1 & 29.3 \\
\hline $\begin{array}{c}\text { Signal Peak Mine Reservoir } \\
1\end{array}$ & Laci & 2012 & 9 & $18.1(2.9) 79$ & 10.7 & 25.7 \\
\hline $\begin{array}{c}\text { Signal Peak Mine Reservoir } \\
1\end{array}$ & Laci & 2012 & 10 & $16.2(4.7) 19$ & 2.6 & 19.6 \\
\hline $\begin{array}{c}\text { Signal Peak Mine Reservoir } \\
1\end{array}$ & Laci & 2012 & 11 & $5.2(4.1) 12$ & -0.3 & 12.3 \\
\hline $\begin{array}{c}\text { Signal Peak Mine Reservoir } \\
1\end{array}$ & Laci & 2012 & 12 & $4.2(1.1) 2$ & 3.4 & 4.9 \\
\hline $\begin{array}{c}\text { Signal Peak Mine Reservoir } \\
1\end{array}$ & Laci & 2013 & 1 & $8.5(0.5) 5$ & 8 & 8.9 \\
\hline $\begin{array}{c}\text { Signal Peak Mine Reservoir } \\
1\end{array}$ & Laci & 2013 & 2 & $3.3(1.3) 6$ & 0.9 & 4.2 \\
\hline $\begin{array}{c}\text { Signal Peak Mine Reservoir } \\
1\end{array}$ & Laci & 2013 & 3 & 9.3 (4.6) 17 & 2.2 & 14.3 \\
\hline $\begin{array}{c}\text { Signal Peak Mine Reservoir } \\
1\end{array}$ & Laci & 2013 & 4 & 14.7 (2.1) 64 & 4.9 & 17.9 \\
\hline
\end{tabular}




\begin{tabular}{|c|c|c|c|c|c|c|}
\hline $\begin{array}{c}\text { Signal Peak Mine Reservoir } \\
1\end{array}$ & Laci & 2013 & 5 & 16.9 (2.6) 69 & 11.8 & 26.4 \\
\hline $\begin{array}{c}\text { Signal Peak Mine Reservoir } \\
1\end{array}$ & Laci & 2013 & 6 & $18.9(3.2) 1151$ & 10 & 24.9 \\
\hline $\begin{array}{c}\text { Signal Peak Mine Reservoir } \\
1\end{array}$ & Laci & 2013 & 7 & 22.7 (3) 1127 & 15.3 & 27.7 \\
\hline $\begin{array}{c}\text { Signal Peak Mine Reservoir } \\
1\end{array}$ & Laci & 2013 & 8 & 22 (3) 208 & 15.8 & 26.5 \\
\hline $\begin{array}{c}\text { Signal Peak Mine Reservoir } \\
1\end{array}$ & Laci & 2013 & 9 & 22.8 (3.7) 34 & 14.5 & 27.5 \\
\hline $\begin{array}{c}\text { Signal Peak Mine Reservoir } \\
1\end{array}$ & Laci & 2013 & 10 & $9.4(2.6) 4$ & 7.2 & 12.2 \\
\hline $\begin{array}{c}\text { Signal Peak Mine Reservoir } \\
1\end{array}$ & Laci & 2013 & 11 & $8.6(2.4) 9$ & 2.4 & 9.5 \\
\hline $\begin{array}{c}\text { Signal Peak Mine Reservoir } \\
1\end{array}$ & Laci & 2014 & 4 & $11.2(0.8) 7$ & 9.5 & 11.5 \\
\hline $\begin{array}{c}\text { Signal Peak Mine Reservoir } \\
1\end{array}$ & Laci & 2014 & 5 & $16(2.8) 183$ & 7.4 & 24.6 \\
\hline $\begin{array}{c}\text { Signal Peak Mine Reservoir } \\
1\end{array}$ & Laci & 2014 & 6 & 17.8 (2.1) 916 & 11.3 & 22.2 \\
\hline $\begin{array}{c}\text { Signal Peak Mine Reservoir } \\
1\end{array}$ & Laci & 2014 & 7 & 22 (3.3) 2360 & 11.2 & 28.2 \\
\hline $\begin{array}{c}\text { Signal Peak Mine Reservoir } \\
1\end{array}$ & Laci & 2014 & 8 & 20.9 (3.5) 473 & 10.8 & 30.2 \\
\hline $\begin{array}{c}\text { Signal Peak Mine Reservoir } \\
1\end{array}$ & Laci & 2014 & 9 & 16.3 (3.5) 60 & 8 & 23.7 \\
\hline $\begin{array}{c}\text { Signal Peak Mine Reservoir } \\
1\end{array}$ & Laci & 2014 & 12 & $5.1(3.2) 6$ & -1.3 & 6.7 \\
\hline $\begin{array}{c}\text { Signal Peak Mine Reservoir } \\
1\end{array}$ & Laci & 2015 & 1 & $12(0) 3$ & 12 & 12 \\
\hline $\begin{array}{c}\text { Signal Peak Mine Reservoir } \\
1\end{array}$ & Laci & 2015 & 2 & 5.7 () 1 & 5.7 & 5.7 \\
\hline $\begin{array}{c}\text { Signal Peak Mine Reservoir } \\
1\end{array}$ & Laci & 2015 & 3 & 14.4 (2.1) 148 & 8.2 & 20.4 \\
\hline $\begin{array}{c}\text { Signal Peak Mine Reservoir } \\
1\end{array}$ & Laci & 2015 & 4 & 11.9 (2.4 51 & 6.9 & 20.6 \\
\hline $\begin{array}{c}\text { Signal Peak Mine Reservoir } \\
1\end{array}$ & Laci & 2015 & 5 & $13(0.9) 11$ & 11.2 & 14.8 \\
\hline $\begin{array}{c}\text { Signal Peak Mine Reservoir } \\
1\end{array}$ & Laci & 2015 & 6 & $20.8(2) 47$ & 14.5 & 24.4 \\
\hline $\begin{array}{c}\text { Signal Peak Mine Reservoir } \\
1\end{array}$ & Laci & 2015 & 6 & 22.9 (3.5) 3145 & 14.1 & 29.3 \\
\hline $\begin{array}{c}\text { Signal Peak Mine Reservoir } \\
1\end{array}$ & Laci & 2015 & 7 & 22.5 (3.1) 6363 & 14.1 & 30.3 \\
\hline $\begin{array}{c}\text { Signal Peak Mine Reservoir } \\
1\end{array}$ & Laci & 2015 & 8 & 23.4 (3.4) 1858 & 8.2 & 33.5 \\
\hline $\begin{array}{c}\text { Signal Peak Mine Reservoir } \\
1\end{array}$ & Laci & 2015 & 9 & $24.4(4.1) 65$ & 14.8 & 29.8 \\
\hline
\end{tabular}




\begin{tabular}{|c|c|c|c|c|c|c|}
\hline $\begin{array}{c}\text { Signal Peak Mine Reservoir } \\
1\end{array}$ & Laci & 2015 & 10 & $10(6.7) 15$ & 3.9 & 19.9 \\
\hline $\begin{array}{c}\text { Signal Peak Mine Reservoir } \\
1\end{array}$ & Laci & 2015 & 11 & 11.1 (1) 49 & 7.7 & 13 \\
\hline $\begin{array}{c}\text { Signal Peak Mine Reservoir } \\
1\end{array}$ & Laci & 2015 & 12 & $9.8(4.6) 24$ & -0.6 & 15.5 \\
\hline $\begin{array}{c}\text { Signal Peak Mine Reservoir } \\
1\end{array}$ & Laci & 2016 & 1 & $6.6(0.8) 11$ & 4.1 & 6.9 \\
\hline $\begin{array}{c}\text { Signal Peak Mine Reservoir } \\
1\end{array}$ & Laci & 2016 & 2 & $9.1(2.6) 14$ & 6.9 & 13.3 \\
\hline $\begin{array}{c}\text { Signal Peak Mine Reservoir } \\
1\end{array}$ & Laci & 2016 & 4 & 15.8 (3.7) 26 & 9.8 & 23.4 \\
\hline $\begin{array}{c}\text { Signal Peak Mine Reservoir } \\
1\end{array}$ & Laci & 2016 & 5 & 16.5 (3.9) 61 & 10.5 & 25.2 \\
\hline $\begin{array}{c}\text { Signal Peak Mine Reservoir } \\
1\end{array}$ & Laci & 2016 & 6 & $21.3(4.2) 5809$ & 11.7 & 30.8 \\
\hline $\begin{array}{c}\text { Signal Peak Mine Reservoir } \\
1\end{array}$ & Laci & 2016 & 7 & 23 (3) 3209 & 14.5 & 30.5 \\
\hline $\begin{array}{c}\text { Signal Peak Mine Reservoir } \\
1\end{array}$ & Laci & 2016 & 8 & $23.1(4.4) 341$ & 14.3 & 30.7 \\
\hline $\begin{array}{c}\text { Signal Peak Mine Reservoir } \\
1\end{array}$ & Laci & 2016 & 9 & 21.7 (4.2) 51 & 10.5 & 28.5 \\
\hline $\begin{array}{c}\text { Signal Peak Mine Reservoir } \\
1\end{array}$ & Laci & 2016 & 11 & $12(4.3) 23$ & 7 & 17 \\
\hline $\begin{array}{c}\text { Signal Peak Mine Reservoir } \\
1\end{array}$ & Laci & 2016 & 12 & $2.3(4) 3$ & 0 & 6.9 \\
\hline $\begin{array}{c}\text { Signal Peak Mine Reservoir } \\
1\end{array}$ & Laci & 2017 & 1 & $12.6(0.8) 2$ & 12 & 13.2 \\
\hline $\begin{array}{c}\text { Signal Peak Mine Reservoir } \\
1\end{array}$ & Laci & 2017 & 2 & $11.2(5.6) 3$ & 4.7 & 14.5 \\
\hline $\begin{array}{c}\text { Signal Peak Mine Reservoir } \\
1\end{array}$ & Laci & 2017 & 3 & 9.7 () 1 & 9.7 & 9.7 \\
\hline $\begin{array}{c}\text { Signal Peak Mine Reservoir } \\
1\end{array}$ & Lano & 2012 & 8 & $22.7(4.5) 127$ & 8.5 & 29.2 \\
\hline $\begin{array}{c}\text { Signal Peak Mine Reservoir } \\
1\end{array}$ & Lano & 2012 & 9 & 17.8 (2.9) 968 & 8 & 26 \\
\hline $\begin{array}{c}\text { Signal Peak Mine Reservoir } \\
1\end{array}$ & Lano & 2012 & 10 & 13.5 (4.4) 96 & 2.6 & 19.8 \\
\hline $\begin{array}{c}\text { Signal Peak Mine Reservoir } \\
1\end{array}$ & Lano & 2012 & 11 & $8(4) 73$ & -0.6 & 14.3 \\
\hline $\begin{array}{c}\text { Signal Peak Mine Reservoir } \\
1\end{array}$ & Lano & 2012 & 12 & $3.7(4.2) 43$ & -2.1 & 11.3 \\
\hline $\begin{array}{c}\text { Signal Peak Mine Reservoir } \\
1\end{array}$ & Lano & 2013 & 1 & 5 (3) 15 & 0.3 & 10.2 \\
\hline $\begin{array}{c}\text { Signal Peak Mine Reservoir } \\
1\end{array}$ & Lano & 2013 & 2 & $3(2.4) 27$ & -1.8 & 7.7 \\
\hline $\begin{array}{c}\text { Signal Peak Mine Reservoir } \\
1\end{array}$ & Lano & 2013 & 3 & $8.1(3.4) 174$ & 0.3 & 15.3 \\
\hline
\end{tabular}




\begin{tabular}{|c|c|c|c|c|c|c|}
\hline $\begin{array}{c}\text { Signal Peak Mine Reservoir } \\
1\end{array}$ & Lano & 2013 & 4 & 14.9 (3.5) 1703 & 5.1 & 20.3 \\
\hline $\begin{array}{c}\text { Signal Peak Mine Reservoir } \\
1\end{array}$ & Lano & 2013 & 5 & $15.7(2.8) 983$ & 7.5 & 26.7 \\
\hline $\begin{array}{c}\text { Signal Peak Mine Reservoir } \\
1\end{array}$ & Lano & 2013 & 6 & $18.3(2.4) 759$ & 10.7 & 24.7 \\
\hline $\begin{array}{c}\text { Signal Peak Mine Reservoir } \\
1\end{array}$ & Lano & 2013 & 7 & 20.3 (2.8) 599 & 14.1 & 27.7 \\
\hline $\begin{array}{c}\text { Signal Peak Mine Reservoir } \\
1\end{array}$ & Lano & 2013 & 8 & 21.2 (3.2) 773 & 13.6 & 27.5 \\
\hline $\begin{array}{c}\text { Signal Peak Mine Reservoir } \\
1\end{array}$ & Lano & 2013 & 9 & 20.5 (4.4) 278 & 7.9 & 27.5 \\
\hline $\begin{array}{c}\text { Signal Peak Mine Reservoir } \\
1\end{array}$ & Lano & 2013 & 10 & 13.8 (3.8) 16 & 6.2 & 17.4 \\
\hline $\begin{array}{c}\text { Signal Peak Mine Reservoir } \\
1\end{array}$ & Lano & 2013 & 11 & 7 (3.5) 22 & 0.6 & 11 \\
\hline $\begin{array}{c}\text { Signal Peak Mine Reservoir } \\
1\end{array}$ & Lano & 2013 & 12 & $5.6(2) 8$ & 3.1 & 8 \\
\hline $\begin{array}{c}\text { Signal Peak Mine Reservoir } \\
1\end{array}$ & Lano & 2014 & 1 & $6.5(0) 2$ & 6.5 & 6.5 \\
\hline $\begin{array}{c}\text { Signal Peak Mine Reservoir } \\
1\end{array}$ & Lano & 2014 & 4 & 10.7 (0) 2 & 10.7 & 10.7 \\
\hline $\begin{array}{c}\text { Signal Peak Mine Reservoir } \\
1\end{array}$ & Lano & 2014 & 5 & 15 (4.1) 154 & 7.5 & 25.5 \\
\hline $\begin{array}{c}\text { Signal Peak Mine Reservoir } \\
1\end{array}$ & Lano & 2014 & 6 & $16.4(2.3) 204$ & 11.2 & 22.2 \\
\hline $\begin{array}{c}\text { Signal Peak Mine Reservoir } \\
1\end{array}$ & Lano & 2014 & 7 & $21(3.9) 647$ & 11.2 & 27.9 \\
\hline $\begin{array}{c}\text { Signal Peak Mine Reservoir } \\
1\end{array}$ & Lano & 2014 & 8 & 20.6 (3.4) 502 & 9.8 & 27.9 \\
\hline $\begin{array}{c}\text { Signal Peak Mine Reservoir } \\
1\end{array}$ & Lano & 2014 & 9 & 16.1 (3.8) 176 & 7.2 & 24.2 \\
\hline $\begin{array}{c}\text { Signal Peak Mine Reservoir } \\
1\end{array}$ & Lano & 2014 & 10 & 14.7 (1.9) 9 & 12.2 & 17.6 \\
\hline $\begin{array}{c}\text { Signal Peak Mine Reservoir } \\
1\end{array}$ & Lano & 2014 & 11 & $5.9(3.1) 7$ & 0.8 & 9.4 \\
\hline $\begin{array}{c}\text { Signal Peak Mine Reservoir } \\
1\end{array}$ & Lano & 2014 & 12 & $4.1(4.3) 20$ & -1.3 & 11 \\
\hline $\begin{array}{c}\text { Signal Peak Mine Reservoir } \\
1\end{array}$ & Lano & 2015 & 1 & $7.2(6.3) 6$ & -0.5 & 12.7 \\
\hline $\begin{array}{c}\text { Signal Peak Mine Reservoir } \\
1\end{array}$ & Lano & 2015 & 2 & 5.7 () 1 & 5.7 & 5.7 \\
\hline $\begin{array}{c}\text { Signal Peak Mine Reservoir } \\
1\end{array}$ & Lano & 2015 & 3 & $12.7(2.2) 461$ & 5.9 & 20.4 \\
\hline $\begin{array}{c}\text { Signal Peak Mine Reservoir } \\
1\end{array}$ & Lano & 2015 & 4 & 10.7 (2.4) 206 & 1.6 & 20.6 \\
\hline $\begin{array}{c}\text { Signal Peak Mine Reservoir } \\
1\end{array}$ & Lano & 2015 & 5 & 11.3 (3) 90 & 2.6 & 15.8 \\
\hline
\end{tabular}




\begin{tabular}{|c|c|c|c|c|c|c|}
\hline $\begin{array}{c}\text { Signal Peak Mine Reservoir } \\
1\end{array}$ & Lano & 2015 & 6 & $20.3(2) 35$ & 15.5 & 23.7 \\
\hline $\begin{array}{c}\text { Signal Peak Mine Reservoir } \\
1\end{array}$ & Lano & 2015 & 6 & 21.8 (3.3) 1161 & 14.1 & 29.3 \\
\hline $\begin{array}{c}\text { Signal Peak Mine Reservoir } \\
1\end{array}$ & Lano & 2015 & 7 & $22.1(2.8) 1856$ & 14.8 & 30.3 \\
\hline $\begin{array}{c}\text { Signal Peak Mine Reservoir } \\
1\end{array}$ & Lano & 2015 & 8 & 23.4 (4.6) 1319 & 8 & 33.5 \\
\hline $\begin{array}{c}\text { Signal Peak Mine Reservoir } \\
1\end{array}$ & Lano & 2015 & 9 & 19.7 (4.6) 1169 & 8.9 & 29.8 \\
\hline $\begin{array}{c}\text { Signal Peak Mine Reservoir } \\
1\end{array}$ & Lano & 2015 & 10 & 16.9 (5.4) 126 & 3.9 & 27 \\
\hline $\begin{array}{c}\text { Signal Peak Mine Reservoir } \\
1\end{array}$ & Lano & 2015 & 11 & 10.2 (3.5) 59 & 3.9 & 16.8 \\
\hline $\begin{array}{c}\text { Signal Peak Mine Reservoir } \\
1\end{array}$ & Lano & 2015 & 12 & $6.7(2.5) 67$ & 0 & 15.5 \\
\hline $\begin{array}{c}\text { Signal Peak Mine Reservoir } \\
1\end{array}$ & Lano & 2016 & 1 & 7.4 (2) 29 & 5.1 & 11.2 \\
\hline $\begin{array}{c}\text { Signal Peak Mine Reservoir } \\
1\end{array}$ & Lano & 2016 & 2 & $9.3(3.6) 50$ & 3.4 & 14 \\
\hline $\begin{array}{c}\text { Signal Peak Mine Reservoir } \\
1\end{array}$ & Lano & 2016 & 3 & 0.8 () 1 & 0.8 & 0.8 \\
\hline $\begin{array}{c}\text { Signal Peak Mine Reservoir } \\
1\end{array}$ & Lano & 2016 & 4 & 16.6 (4.3) 449 & 2.7 & 23.6 \\
\hline $\begin{array}{c}\text { Signal Peak Mine Reservoir } \\
1\end{array}$ & Lano & 2016 & 5 & 15.7 (3.2) 515 & 6.9 & 25.1 \\
\hline $\begin{array}{c}\text { Signal Peak Mine Reservoir } \\
1\end{array}$ & Lano & 2016 & 6 & 20 (3.6) 1927 & 12 & 30.5 \\
\hline $\begin{array}{c}\text { Signal Peak Mine Reservoir } \\
1\end{array}$ & Lano & 2016 & 7 & 23.5 (3) 1133 & 15 & 30.3 \\
\hline $\begin{array}{c}\text { Signal Peak Mine Reservoir } \\
1\end{array}$ & Lano & 2016 & 8 & 22.5 (4) 589 & 10 & 30.7 \\
\hline $\begin{array}{c}\text { Signal Peak Mine Reservoir } \\
1\end{array}$ & Lano & 2016 & 9 & 19 (3.7) 374 & 10 & 28 \\
\hline $\begin{array}{c}\text { Signal Peak Mine Reservoir } \\
1\end{array}$ & Lano & 2016 & 10 & 14.6 (4.1) 56 & 9 & 22.6 \\
\hline $\begin{array}{c}\text { Signal Peak Mine Reservoir } \\
1\end{array}$ & Lano & 2016 & 11 & 12.8 (4.4) 96 & 4.4 & 20.9 \\
\hline $\begin{array}{c}\text { Signal Peak Mine Reservoir } \\
1\end{array}$ & Lano & 2016 & 12 & $6.3(1.3) 13$ & 2.2 & 6.9 \\
\hline $\begin{array}{c}\text { Signal Peak Mine Reservoir } \\
1\end{array}$ & Lano & 2017 & 1 & $8.1(4.9) 29$ & 0.9 & 13.3 \\
\hline $\begin{array}{c}\text { Signal Peak Mine Reservoir } \\
1\end{array}$ & Lano & 2017 & 2 & 10.5 (2.5) 49 & 4.1 & 14.5 \\
\hline $\begin{array}{c}\text { Signal Peak Mine Reservoir } \\
1\end{array}$ & Lano & 2017 & 3 & $9.7(0.1) 6$ & 9.7 & 9.8 \\
\hline $\begin{array}{c}\text { Signal Peak Mine Reservoir } \\
1\end{array}$ & Мyсі & 2012 & 8 & 22.7 (3.7) 36 & 15.5 & 28.7 \\
\hline
\end{tabular}




\begin{tabular}{|c|c|c|c|c|c|c|}
\hline $\begin{array}{c}\text { Signal Peak Mine Reservoir } \\
1\end{array}$ & Myci & 2012 & 9 & $18.6(2.4) 43$ & 13.2 & 24.9 \\
\hline $\begin{array}{c}\text { Signal Peak Mine Reservoir } \\
1\end{array}$ & Мyсi & 2012 & 10 & 13.3 (3.8) 19 & 7.2 & 19.3 \\
\hline $\begin{array}{c}\text { Signal Peak Mine Reservoir } \\
1\end{array}$ & Мyсі & 2012 & 11 & $7.8(1.6) 7$ & 5.9 & 10.2 \\
\hline $\begin{array}{c}\text { Signal Peak Mine Reservoir } \\
1\end{array}$ & Мyсі & 2012 & 12 & $4.5(2.9) 7$ & 3.2 & 11 \\
\hline $\begin{array}{c}\text { Signal Peak Mine Reservoir } \\
1\end{array}$ & Мyсi & 2013 & 1 & $6.4(2.3) 12$ & 0 & 8.2 \\
\hline $\begin{array}{c}\text { Signal Peak Mine Reservoir } \\
1\end{array}$ & Myci & 2013 & 2 & $2.1(1.1) 2$ & 1.4 & 2.9 \\
\hline $\begin{array}{c}\text { Signal Peak Mine Reservoir } \\
1\end{array}$ & Myci & 2013 & 3 & $2(2.4) 6$ & -0.6 & 4.9 \\
\hline $\begin{array}{c}\text { Signal Peak Mine Reservoir } \\
1\end{array}$ & Мyсі & 2013 & 4 & $8.8(3.8) 6$ & 1.7 & 12.2 \\
\hline $\begin{array}{c}\text { Signal Peak Mine Reservoir } \\
1\end{array}$ & Myсi & 2013 & 5 & $15.4(2.9) 18$ & 7.4 & 18.9 \\
\hline $\begin{array}{c}\text { Signal Peak Mine Reservoir } \\
1\end{array}$ & Myci & 2013 & 6 & $17.2(2.7) 42$ & 12.8 & 23.2 \\
\hline $\begin{array}{c}\text { Signal Peak Mine Reservoir } \\
1\end{array}$ & Myci & 2013 & 7 & $21(2.7) 57$ & 13.8 & 26.9 \\
\hline $\begin{array}{c}\text { Signal Peak Mine Reservoir } \\
1\end{array}$ & Мyсі & 2013 & 8 & $22.2(2.8) 93$ & 15.8 & 27.5 \\
\hline $\begin{array}{c}\text { Signal Peak Mine Reservoir } \\
1\end{array}$ & Мyсі & 2013 & 9 & 21.9 (1.1) 9 & 19.6 & 23.4 \\
\hline $\begin{array}{c}\text { Signal Peak Mine Reservoir } \\
1\end{array}$ & Мyсі & 2013 & 10 & 12.8 (1.4) 10 & 10.2 & 14.1 \\
\hline $\begin{array}{c}\text { Signal Peak Mine Reservoir } \\
1\end{array}$ & Myci & 2013 & 11 & $6.2(0.6) 3$ & 5.5 & 6.5 \\
\hline $\begin{array}{c}\text { Signal Peak Mine Reservoir } \\
1\end{array}$ & Мyсі & 2014 & 1 & $2.7(0) 4$ & 2.7 & 2.7 \\
\hline $\begin{array}{c}\text { Signal Peak Mine Reservoir } \\
1\end{array}$ & Мyсi & 2014 & 5 & 16.7 (3) 33 & 9 & 21.2 \\
\hline $\begin{array}{c}\text { Signal Peak Mine Reservoir } \\
1\end{array}$ & Мyсi & 2014 & 6 & $15(2) 36$ & 11.7 & 18.9 \\
\hline $\begin{array}{c}\text { Signal Peak Mine Reservoir } \\
1\end{array}$ & Myсi & 2014 & 7 & 20.8 (3.2) 106 & 11.3 & 27.4 \\
\hline $\begin{array}{c}\text { Signal Peak Mine Reservoir } \\
1\end{array}$ & Myci & 2014 & 8 & 21.5 (3.9) 146 & 9.8 & 30.5 \\
\hline $\begin{array}{c}\text { Signal Peak Mine Reservoir } \\
1\end{array}$ & Мyсі & 2014 & 9 & 16.9 (3.3) 16 & 11 & 23.1 \\
\hline $\begin{array}{c}\text { Signal Peak Mine Reservoir } \\
1\end{array}$ & Мyсi & 2014 & 10 & $19.3(2.3) 2$ & 17.6 & 20.9 \\
\hline $\begin{array}{c}\text { Signal Peak Mine Reservoir } \\
1\end{array}$ & Мyсі & 2014 & 11 & 8.2 (1.1) 2 & 7.4 & 9 \\
\hline $\begin{array}{c}\text { Signal Peak Mine Reservoir } \\
1\end{array}$ & Мyсі & 2014 & 12 & 4.1 () 1 & 4.1 & 4.1 \\
\hline
\end{tabular}




\begin{tabular}{|c|c|c|c|c|c|c|}
\hline $\begin{array}{c}\text { Signal Peak Mine Reservoir } \\
1\end{array}$ & Myci & 2015 & 3 & 13.8() 1 & 13.8 & 13.8 \\
\hline $\begin{array}{c}\text { Signal Peak Mine Reservoir } \\
1\end{array}$ & Myci & 2015 & 4 & $9.6(1.4) 3$ & 8.2 & 11 \\
\hline $\begin{array}{c}\text { Signal Peak Mine Reservoir } \\
1\end{array}$ & Myci & 2015 & 5 & $12.3(1.2) 2$ & 11.5 & 13.2 \\
\hline $\begin{array}{c}\text { Signal Peak Mine Reservoir } \\
1\end{array}$ & Мyсі & 2015 & 6 & $17.8(2.3) 2$ & 16.1 & 19.4 \\
\hline $\begin{array}{c}\text { Signal Peak Mine Reservoir } \\
1\end{array}$ & Мyсi & 2015 & 6 & $21(3.2) 234$ & 14.1 & 28.5 \\
\hline $\begin{array}{c}\text { Signal Peak Mine Reservoir } \\
1\end{array}$ & Myci & 2015 & 7 & 23 (3) 484 & 15.5 & 29.3 \\
\hline $\begin{array}{c}\text { Signal Peak Mine Reservoir } \\
1\end{array}$ & Myci & 2015 & 8 & 23.8 (3.7) 531 & 13.2 & 32.2 \\
\hline $\begin{array}{c}\text { Signal Peak Mine Reservoir } \\
1\end{array}$ & Мyсі & 2015 & 9 & 19.6 (4) 239 & 12.8 & 28.7 \\
\hline $\begin{array}{c}\text { Signal Peak Mine Reservoir } \\
1\end{array}$ & Myсi & 2015 & 10 & 13.9 (7.1) 38 & 4.7 & 26.4 \\
\hline $\begin{array}{c}\text { Signal Peak Mine Reservoir } \\
1\end{array}$ & Мyсi & 2015 & 11 & 11.6 (3.5) 27 & 2.9 & 14.1 \\
\hline $\begin{array}{c}\text { Signal Peak Mine Reservoir } \\
1\end{array}$ & Myci & 2016 & 1 & $3.6(4.4) 3$ & -1.3 & 7 \\
\hline $\begin{array}{c}\text { Signal Peak Mine Reservoir } \\
1\end{array}$ & Мyсі & 2016 & 2 & $6.3(2.2) 21$ & 3.9 & 9.4 \\
\hline $\begin{array}{c}\text { Signal Peak Mine Reservoir } \\
1\end{array}$ & Мyсі & 2016 & 4 & 18.2 (3.6) 23 & 11 & 24.1 \\
\hline $\begin{array}{c}\text { Signal Peak Mine Reservoir } \\
1\end{array}$ & Myci & 2016 & 5 & $19.4(4.2) 47$ & 12.8 & 25.4 \\
\hline $\begin{array}{c}\text { Signal Peak Mine Reservoir } \\
1\end{array}$ & Myci & 2016 & 6 & 20 (4.1) 291 & 12.2 & 29.3 \\
\hline $\begin{array}{c}\text { Signal Peak Mine Reservoir } \\
1\end{array}$ & Мyсі & 2016 & 7 & 23.8 (2.7) 363 & 16.3 & 30.5 \\
\hline $\begin{array}{c}\text { Signal Peak Mine Reservoir } \\
1\end{array}$ & Мyсi & 2016 & 8 & 22.7 (3.5) 405 & 13.8 & 30.7 \\
\hline $\begin{array}{c}\text { Signal Peak Mine Reservoir } \\
1\end{array}$ & Мyсi & 2016 & 9 & 20.1 (3.6) 77 & 13.3 & 28 \\
\hline $\begin{array}{c}\text { Signal Peak Mine Reservoir } \\
1\end{array}$ & Myсi & 2016 & 10 & $15.8(4.4) 12$ & 7 & 23.2 \\
\hline $\begin{array}{c}\text { Signal Peak Mine Reservoir } \\
1\end{array}$ & Myci & 2016 & 11 & $14(3.8) 26$ & 4.1 & 19.8 \\
\hline $\begin{array}{c}\text { Signal Peak Mine Reservoir } \\
1\end{array}$ & Мyсі & 2016 & 12 & 2.4 () 1 & 2.4 & 2.4 \\
\hline $\begin{array}{c}\text { Signal Peak Mine Reservoir } \\
1\end{array}$ & Мyсi & 2017 & 1 & 2.1 () 1 & 2.1 & 2.1 \\
\hline $\begin{array}{c}\text { Signal Peak Mine Reservoir } \\
1\end{array}$ & Мyсі & 2017 & 2 & 7.4 () 1 & 7.4 & 7.4 \\
\hline $\begin{array}{c}\text { Signal Peak Mine Reservoir } \\
1\end{array}$ & Мyсі & 2017 & 3 & 5.7 () 1 & 5.7 & 5.7 \\
\hline
\end{tabular}




\begin{tabular}{|c|c|c|c|c|c|c|}
\hline $\begin{array}{c}\text { Signal Peak Mine Reservoir } \\
1\end{array}$ & Myev & 2012 & 8 & $21.7(4.1) 9$ & 14.5 & 28.2 \\
\hline $\begin{array}{c}\text { Signal Peak Mine Reservoir } \\
1\end{array}$ & Myev & 2012 & 9 & $16.9(3.8) 35$ & 7.5 & 24.9 \\
\hline $\begin{array}{c}\text { Signal Peak Mine Reservoir } \\
1\end{array}$ & Myev & 2012 & 10 & 18.4 () 1 & 18.4 & 18.4 \\
\hline $\begin{array}{c}\text { Signal Peak Mine Reservoir } \\
1\end{array}$ & Myev & 2013 & 4 & $12.3(0.1) 2$ & 12.2 & 12.3 \\
\hline $\begin{array}{c}\text { Signal Peak Mine Reservoir } \\
1\end{array}$ & Myev & 2013 & 5 & 15.8 (5.6) 11 & -0.3 & 19.3 \\
\hline $\begin{array}{c}\text { Signal Peak Mine Reservoir } \\
1\end{array}$ & Myev & 2013 & 6 & 17.7 (2.1) 18 & 13 & 21.4 \\
\hline $\begin{array}{c}\text { Signal Peak Mine Reservoir } \\
1\end{array}$ & Myev & 2013 & 7 & 19.9 (2.2) 14 & 17 & 23.2 \\
\hline $\begin{array}{c}\text { Signal Peak Mine Reservoir } \\
1\end{array}$ & Myev & 2013 & 8 & 21.1 (3.1) 51 & 14 & 26.7 \\
\hline $\begin{array}{c}\text { Signal Peak Mine Reservoir } \\
1\end{array}$ & Myev & 2013 & 9 & $22.1(4.2) 29$ & 14.5 & 27.5 \\
\hline $\begin{array}{c}\text { Signal Peak Mine Reservoir } \\
1\end{array}$ & Myev & 2013 & 10 & $9.5(2.5) 2$ & 7.7 & 11.3 \\
\hline $\begin{array}{c}\text { Signal Peak Mine Reservoir } \\
1\end{array}$ & Myev & 2014 & 5 & $16.2(2.5) 21$ & 11 & 19.4 \\
\hline $\begin{array}{c}\text { Signal Peak Mine Reservoir } \\
1\end{array}$ & Myev & 2014 & 6 & $15.6(3.2) 30$ & 10.8 & 21.2 \\
\hline $\begin{array}{c}\text { Signal Peak Mine Reservoir } \\
1\end{array}$ & Myev & 2014 & 7 & $20.2(2.7) 21$ & 15.1 & 24.6 \\
\hline $\begin{array}{c}\text { Signal Peak Mine Reservoir } \\
1\end{array}$ & Myev & 2014 & 8 & 21.9 (3.4) 20 & 16 & 26.9 \\
\hline $\begin{array}{c}\text { Signal Peak Mine Reservoir } \\
1\end{array}$ & Myev & 2014 & 9 & 15 (3.7) 12 & 8.2 & 20.1 \\
\hline $\begin{array}{c}\text { Signal Peak Mine Reservoir } \\
1\end{array}$ & Myev & 2015 & 5 & 13.6 () 1 & 13.6 & 13.6 \\
\hline $\begin{array}{c}\text { Signal Peak Mine Reservoir } \\
1\end{array}$ & Myev & 2015 & 6 & $15.6(0.1) 2$ & 15.5 & 15.6 \\
\hline $\begin{array}{c}\text { Signal Peak Mine Reservoir } \\
1\end{array}$ & Myev & 2015 & 6 & 21.2 (3) 191 & 14.1 & 27.7 \\
\hline $\begin{array}{c}\text { Signal Peak Mine Reservoir } \\
1\end{array}$ & Myev & 2015 & 7 & 21.9 (2.7) 179 & 15.1 & 30.3 \\
\hline $\begin{array}{c}\text { Signal Peak Mine Reservoir } \\
1\end{array}$ & Myev & 2015 & 8 & 23.9 (4.3) 489 & 8.7 & 33.5 \\
\hline $\begin{array}{c}\text { Signal Peak Mine Reservoir } \\
1\end{array}$ & Myev & 2015 & 9 & 20 (4.3) 267 & 11.7 & 28.9 \\
\hline $\begin{array}{c}\text { Signal Peak Mine Reservoir } \\
1\end{array}$ & Myev & 2015 & 10 & $17.4(4.8) 8$ & 9.2 & 23.2 \\
\hline $\begin{array}{c}\text { Signal Peak Mine Reservoir } \\
1\end{array}$ & Myev & 2016 & 1 & 6.2() 1 & 6.2 & 6.2 \\
\hline $\begin{array}{c}\text { Signal Peak Mine Reservoir } \\
1\end{array}$ & Myev & 2016 & 4 & 16.4 (4.9) 37 & 5.7 & 23.9 \\
\hline
\end{tabular}




\begin{tabular}{|c|c|c|c|c|c|c|}
\hline $\begin{array}{c}\text { Signal Peak Mine Reservoir } \\
1\end{array}$ & Myev & 2016 & 5 & 16.3 (3.7) 137 & 6.9 & 25.1 \\
\hline $\begin{array}{c}\text { Signal Peak Mine Reservoir } \\
1\end{array}$ & Myev & 2016 & 6 & $20(3.8) 164$ & 12.2 & 29 \\
\hline $\begin{array}{c}\text { Signal Peak Mine Reservoir } \\
1\end{array}$ & Myev & 2016 & 7 & $23.6(2.8) 126$ & 17.9 & 29 \\
\hline $\begin{array}{c}\text { Signal Peak Mine Reservoir } \\
1\end{array}$ & Myev & 2016 & 8 & $22.8(4) 456$ & 9.8 & 30.7 \\
\hline $\begin{array}{c}\text { Signal Peak Mine Reservoir } \\
1\end{array}$ & Myev & 2016 & 9 & 17.7 (3.8) 119 & 9.8 & 28.5 \\
\hline $\begin{array}{c}\text { Signal Peak Mine Reservoir } \\
1\end{array}$ & Myev & 2016 & 10 & $15.4(2.3) 8$ & 11.2 & 17.4 \\
\hline $\begin{array}{c}\text { Signal Peak Mine Reservoir } \\
1\end{array}$ & Mylu & 2012 & 8 & $22(3.3) 21$ & 17.3 & 29.2 \\
\hline $\begin{array}{c}\text { Signal Peak Mine Reservoir } \\
1\end{array}$ & Mylu & 2012 & 9 & 18.4 (3.4) 52 & 6.5 & 25.2 \\
\hline $\begin{array}{c}\text { Signal Peak Mine Reservoir } \\
1\end{array}$ & Mylu & 2012 & 10 & 7.2 () 1 & 7.2 & 7.2 \\
\hline $\begin{array}{c}\text { Signal Peak Mine Reservoir } \\
1\end{array}$ & Mylu & 2012 & 11 & $9.6(0.6) 4$ & 9 & 10.2 \\
\hline $\begin{array}{c}\text { Signal Peak Mine Reservoir } \\
1\end{array}$ & Mylu & 2012 & 12 & 3.4 () 1 & 3.4 & 3.4 \\
\hline $\begin{array}{c}\text { Signal Peak Mine Reservoir } \\
1\end{array}$ & Mylu & 2013 & 1 & 4.4 () 1 & 4.4 & 4.4 \\
\hline $\begin{array}{c}\text { Signal Peak Mine Reservoir } \\
1\end{array}$ & Mylu & 2013 & 4 & $12.9(2.2) 10$ & 11.3 & 18.9 \\
\hline $\begin{array}{c}\text { Signal Peak Mine Reservoir } \\
1\end{array}$ & Mylu & 2013 & 5 & 15.5 (2.9) 47 & 7.9 & 25.7 \\
\hline $\begin{array}{c}\text { Signal Peak Mine Reservoir } \\
1\end{array}$ & Mylu & 2013 & 6 & $16.7(2.1) 62$ & 14 & 22.6 \\
\hline $\begin{array}{c}\text { Signal Peak Mine Reservoir } \\
1\end{array}$ & Mylu & 2013 & 7 & $20.3(2.8) 35$ & 15.8 & 27.5 \\
\hline $\begin{array}{c}\text { Signal Peak Mine Reservoir } \\
1\end{array}$ & Mylu & 2013 & 8 & $21.2(3) 53$ & 15.8 & 26.7 \\
\hline $\begin{array}{c}\text { Signal Peak Mine Reservoir } \\
1\end{array}$ & Mylu & 2013 & 9 & $20.6(4.2) 7$ & 13.6 & 27.2 \\
\hline $\begin{array}{c}\text { Signal Peak Mine Reservoir } \\
1\end{array}$ & Mylu & 2013 & 10 & $12.6(1.1) 11$ & 9.8 & 13.3 \\
\hline $\begin{array}{c}\text { Signal Peak Mine Reservoir } \\
1\end{array}$ & Mylu & 2014 & 4 & $10.1(2) 2$ & 8.7 & 11.5 \\
\hline $\begin{array}{c}\text { Signal Peak Mine Reservoir } \\
1\end{array}$ & Mylu & 2014 & 5 & 14.3 (2.7) 15 & 7.4 & 18.4 \\
\hline $\begin{array}{c}\text { Signal Peak Mine Reservoir } \\
1\end{array}$ & Mylu & 2014 & 6 & 16.8 (2.6) 25 & 9.5 & 21.2 \\
\hline $\begin{array}{c}\text { Signal Peak Mine Reservoir } \\
1\end{array}$ & Mylu & 2014 & 7 & 20 (3.6) 21 & 14 & 25.2 \\
\hline $\begin{array}{c}\text { Signal Peak Mine Reservoir } \\
1\end{array}$ & Mylu & 2014 & 8 & 22.5 (1.9) 32 & 19.1 & 27.7 \\
\hline
\end{tabular}




\begin{tabular}{|c|c|c|c|c|c|c|}
\hline $\begin{array}{c}\text { Signal Peak Mine Reservoir } \\
1\end{array}$ & Mylu & 2014 & 9 & 19.5 (3.5) 4 & 15.6 & 23.7 \\
\hline $\begin{array}{c}\text { Signal Peak Mine Reservoir } \\
1\end{array}$ & Mylu & 2014 & 11 & 15.5 () 1 & 15.5 & 15.5 \\
\hline $\begin{array}{c}\text { Signal Peak Mine Reservoir } \\
1\end{array}$ & Mylu & 2015 & 3 & $13.9(0.2) 2$ & 13.8 & 14.1 \\
\hline $\begin{array}{c}\text { Signal Peak Mine Reservoir } \\
1\end{array}$ & Mylu & 2015 & 4 & $11.1(1.2) 4$ & 9.5 & 12.2 \\
\hline $\begin{array}{c}\text { Signal Peak Mine Reservoir } \\
1\end{array}$ & Mylu & 2015 & 5 & 12.9 (2.6) 3 & 10.2 & 15.3 \\
\hline $\begin{array}{c}\text { Signal Peak Mine Reservoir } \\
1\end{array}$ & Mylu & 2015 & 6 & 21.4() 1 & 21.4 & 21.4 \\
\hline $\begin{array}{c}\text { Signal Peak Mine Reservoir } \\
1\end{array}$ & Mylu & 2015 & 6 & $22.5(3.4) 17$ & 15.1 & 25.9 \\
\hline $\begin{array}{c}\text { Signal Peak Mine Reservoir } \\
1\end{array}$ & Mylu & 2015 & 7 & $23(2.5) 108$ & 17 & 28 \\
\hline $\begin{array}{c}\text { Signal Peak Mine Reservoir } \\
1\end{array}$ & Mylu & 2015 & 8 & 21.7 (4.7) 116 & 10.8 & 30.3 \\
\hline $\begin{array}{c}\text { Signal Peak Mine Reservoir } \\
1\end{array}$ & Mylu & 2015 & 9 & $19.1(3.3) 162$ & 12 & 26.4 \\
\hline $\begin{array}{c}\text { Signal Peak Mine Reservoir } \\
1\end{array}$ & Mylu & 2015 & 10 & 19.3() 1 & 19.3 & 19.3 \\
\hline $\begin{array}{c}\text { Signal Peak Mine Reservoir } \\
1\end{array}$ & Mylu & 2016 & 2 & 9.4 () 1 & 9.4 & 9.4 \\
\hline $\begin{array}{c}\text { Signal Peak Mine Reservoir } \\
1\end{array}$ & Mylu & 2016 & 4 & $17.8(1.1) 4$ & 16.1 & 18.4 \\
\hline $\begin{array}{c}\text { Signal Peak Mine Reservoir } \\
1\end{array}$ & Mylu & 2016 & 5 & $21(4.2) 25$ & 12.2 & 24.9 \\
\hline $\begin{array}{c}\text { Signal Peak Mine Reservoir } \\
1\end{array}$ & Mylu & 2016 & 6 & $21.5(4.2) 24$ & 14.5 & 29.8 \\
\hline $\begin{array}{c}\text { Signal Peak Mine Reservoir } \\
1\end{array}$ & Mylu & 2016 & 7 & $23.2(2.3) 61$ & 16.6 & 28.9 \\
\hline $\begin{array}{c}\text { Signal Peak Mine Reservoir } \\
1\end{array}$ & Mylu & 2016 & 8 & 23.1 (3.7) 54 & 15.6 & 30.2 \\
\hline $\begin{array}{c}\text { Signal Peak Mine Reservoir } \\
1\end{array}$ & Mylu & 2016 & 9 & 17.3 (3.1) 61 & 13.3 & 24.1 \\
\hline $\begin{array}{c}\text { Signal Peak Mine Reservoir } \\
1\end{array}$ & Mylu & 2016 & 10 & 17.4 () 1 & 17.4 & 17.4 \\
\hline $\begin{array}{c}\text { Signal Peak Mine Reservoir } \\
1\end{array}$ & Mylu & 2016 & 11 & 17.8() 1 & 17.8 & 17.8 \\
\hline $\begin{array}{c}\text { Signal Peak Mine Reservoir } \\
1\end{array}$ & Myth & 2013 & 8 & 23.2() 1 & 23.2 & 23.2 \\
\hline $\begin{array}{c}\text { Signal Peak Mine Reservoir } \\
1\end{array}$ & Myth & 2015 & 6 & 21.3 (3.4) 15 & 15 & 27.4 \\
\hline $\begin{array}{c}\text { Signal Peak Mine Reservoir } \\
1\end{array}$ & Myth & 2015 & 7 & $21.7(2.1) 9$ & 19.3 & 26.5 \\
\hline $\begin{array}{c}\text { Signal Peak Mine Reservoir } \\
1\end{array}$ & Myth & 2015 & 8 & 23.8 (4.1) 20 & 19.8 & 31.3 \\
\hline
\end{tabular}




\begin{tabular}{|c|c|c|c|c|c|c|}
\hline $\begin{array}{c}\text { Signal Peak Mine Reservoir } \\
1\end{array}$ & Myth & 2015 & 9 & $24.5(5.2) 2$ & 20.8 & 28.2 \\
\hline $\begin{array}{c}\text { Signal Peak Mine Reservoir } \\
1\end{array}$ & Myth & 2015 & 10 & 17.4() 1 & 17.4 & 17.4 \\
\hline $\begin{array}{c}\text { Signal Peak Mine Reservoir } \\
1\end{array}$ & Myth & 2016 & 4 & $18(3.7) 6$ & 11.8 & 23.6 \\
\hline $\begin{array}{c}\text { Signal Peak Mine Reservoir } \\
1\end{array}$ & Myth & 2016 & 5 & 16.5 (3.9) 13 & 7 & 21.7 \\
\hline $\begin{array}{c}\text { Signal Peak Mine Reservoir } \\
1\end{array}$ & Myth & 2016 & 6 & $22.5(3.8) 14$ & 15.3 & 28 \\
\hline $\begin{array}{c}\text { Signal Peak Mine Reservoir } \\
1\end{array}$ & Myth & 2016 & 7 & $24(1.6) 5$ & 22.6 & 26.2 \\
\hline $\begin{array}{c}\text { Signal Peak Mine Reservoir } \\
1\end{array}$ & Myth & 2016 & 8 & $23.3(4.7) 23$ & 14.5 & 29.3 \\
\hline $\begin{array}{c}\text { Signal Peak Mine Reservoir } \\
1\end{array}$ & Myth & 2016 & 9 & $19.3(5.7) 10$ & 10 & 25.7 \\
\hline Spring Creek Mine & Epfu & 2012 & 8 & $24.9(2.8) 2083$ & 12.5 & 30.7 \\
\hline Spring Creek Mine & Epfu & 2012 & 9 & $21.2(2.5) 190$ & 13.5 & 25.7 \\
\hline Spring Creek Mine & Epfu & 2013 & 4 & 12.5 (1.9) 137 & 6.2 & 17.6 \\
\hline Spring Creek Mine & Epfu & 2013 & 5 & 17.5 (2.9) 989 & 6 & 26.2 \\
\hline Spring Creek Mine & Epfu & 2013 & 6 & 20.1 (3) 1123 & 9.5 & 28 \\
\hline Spring Creek Mine & Epfu & 2013 & 10 & 14 () 1 & 14 & 14 \\
\hline Spring Creek Mine & Epfu & 2014 & 3 & 6() 1 & 6 & 6 \\
\hline Spring Creek Mine & Epfu & 2014 & 4 & $15.6(4) 14$ & 9 & 19.6 \\
\hline Spring Creek Mine & Epfu & 2014 & 5 & $18.2(4.5) 89$ & 8 & 27.4 \\
\hline Spring Creek Mine & Epfu & 2014 & 6 & 18.4 (2.7) 243 & 7 & 25.5 \\
\hline Spring Creek Mine & Epfu & 2014 & 7 & 22.5 (3.3) 341 & 14.1 & 29.7 \\
\hline Spring Creek Mine & Epfu & 2014 & 8 & $21.1(2.4) 230$ & 15.5 & 26.4 \\
\hline Spring Creek Mine & Epfu & 2014 & 9 & $18(4.7) 6$ & 11.3 & 22.1 \\
\hline Spring Creek Mine & Epfu & 2014 & 11 & 3.6() 1 & 3.6 & 3.6 \\
\hline Spring Creek Mine & Epfu & 2014 & 12 & 6.7 () 1 & 6.7 & 6.7 \\
\hline Spring Creek Mine & Epfu & 2015 & 3 & 17.3() 1 & 17.3 & 17.3 \\
\hline Spring Creek Mine & Epfu & 2015 & 4 & $21.7(4.6) 21$ & 11.3 & 24.6 \\
\hline Spring Creek Mine & Epfu & 2015 & 5 & $16.4(3.4) 57$ & 8.2 & 21.7 \\
\hline Spring Creek Mine & Epfu & 2015 & 6 & $20.2(2) 39$ & 16.8 & 23.6 \\
\hline Spring Creek Mine & Epfu & 2015 & 6 & $23(2.5) 947$ & 16.8 & 29 \\
\hline Spring Creek Mine & Epfu & 2015 & 7 & $24.5(2.8) 1566$ & 15.5 & 30.8 \\
\hline Spring Creek Mine & Epfu & 2015 & 8 & $26.3(4.1) 1109$ & 12.8 & 32.8 \\
\hline Spring Creek Mine & Epfu & 2015 & 9 & $21.4(3.6) 134$ & 13.5 & 29.7 \\
\hline Spring Creek Mine & Epfu & 2015 & 10 & $17.3(3.4) 13$ & 10 & 23.6 \\
\hline Spring Creek Mine & Epfu & 2015 & 11 & $5.5(0) 2$ & 5.5 & 5.5 \\
\hline Spring Creek Mine & Epfu & 2015 & 12 & $0(0) 2$ & 0 & 0 \\
\hline Spring Creek Mine & Epfu & 2016 & 1 & $4.9(2.7) 5$ & 2.9 & 7.9 \\
\hline Spring Creek Mine & Epfu & 2016 & 2 & $7.4(0.8) 6$ & 6.9 & 8.4 \\
\hline Spring Creek Mine & Epfu & 2016 & 3 & $16(2) 5$ & 12.7 & 17.9 \\
\hline
\end{tabular}




\begin{tabular}{|c|c|c|c|c|c|c|}
\hline Spring Creek Mine & Epfu & 2016 & 4 & $16.4(3.7) 63$ & 3.6 & 21.4 \\
\hline Spring Creek Mine & Epfu & 2016 & 5 & 17.3 (3) 184 & 8.5 & 23.2 \\
\hline Spring Creek Mine & Epfu & 2016 & 6 & 24.4 (3.2) 1223 & 13.8 & 32.8 \\
\hline Spring Creek Mine & Epfu & 2016 & 7 & 28.5 (3.3) 2486 & 15.3 & 34.3 \\
\hline Spring Creek Mine & Epfu & 2016 & 8 & $28.5(3.2) 2723$ & 15.6 & 32.5 \\
\hline Spring Creek Mine & Epfu & 2016 & 9 & $22.3(4.7) 114$ & 14.1 & 29.7 \\
\hline Spring Creek Mine & Epfu & 2016 & 12 & 5.1() 1 & 5.1 & 5.1 \\
\hline Spring Creek Mine & Laci & 2012 & 8 & $21.1(3.9) 27$ & 10.2 & 27.4 \\
\hline Spring Creek Mine & Laci & 2012 & 9 & $16.6(2.3) 11$ & 13 & 21.4 \\
\hline Spring Creek Mine & Laci & 2013 & 4 & $12.2(1.7) 95$ & 10 & 17.6 \\
\hline Spring Creek Mine & Laci & 2013 & 5 & $15(3.5) 83$ & 6.9 & 22.2 \\
\hline Spring Creek Mine & Laci & 2013 & 6 & 19.9 (2.9) 177 & 12.7 & 27 \\
\hline Spring Creek Mine & Laci & 2013 & 10 & 11.2 () 1 & 11.2 & 11.2 \\
\hline Spring Creek Mine & Laci & 2014 & 4 & 11.7 () 1 & 11.7 & 11.7 \\
\hline Spring Creek Mine & Laci & 2014 & 5 & $15.6(1.6) 4$ & 13.5 & 16.8 \\
\hline Spring Creek Mine & Laci & 2014 & 6 & $17.1(3.7) 44$ & 8.5 & 24.6 \\
\hline Spring Creek Mine & Laci & 2014 & 7 & 22.2 (3.6) 103 & 13.6 & 29.7 \\
\hline Spring Creek Mine & Laci & 2014 & 8 & $20.2(2.4) 51$ & 15.5 & 25.4 \\
\hline Spring Creek Mine & Laci & 2014 & 9 & $10(2.5) 5$ & 8.7 & 14.5 \\
\hline Spring Creek Mine & Laci & 2015 & 5 & $19.1(1.8) 2$ & 17.8 & 20.4 \\
\hline Spring Creek Mine & Laci & 2015 & 6 & $18.8(1.1) 4$ & 17.6 & 20.3 \\
\hline Spring Creek Mine & Laci & 2015 & 6 & 22.6 (2.7) 351 & 15 & 28.7 \\
\hline Spring Creek Mine & Laci & 2015 & 7 & 22.8 (3.3) 361 & 15.3 & 30.8 \\
\hline Spring Creek Mine & Laci & 2015 & 8 & 23.5 (4.3) 197 & 12.5 & 32.5 \\
\hline Spring Creek Mine & Laci & 2015 & 9 & $22(2.8) 110$ & 13.8 & 28.7 \\
\hline Spring Creek Mine & Laci & 2015 & 10 & 17.6() 1 & 17.6 & 17.6 \\
\hline Spring Creek Mine & Laci & 2016 & 3 & 17.9 () 1 & 17.9 & 17.9 \\
\hline Spring Creek Mine & Laci & 2016 & 5 & $19.7(2.5) 12$ & 15.1 & 23.6 \\
\hline Spring Creek Mine & Laci & 2016 & 6 & 23.9 (3.7) 135 & 15 & 33.1 \\
\hline Spring Creek Mine & Laci & 2016 & 7 & 25.7 (3.5) 163 & 14.8 & 33.1 \\
\hline Spring Creek Mine & Laci & 2016 & 8 & $24.2(4.5) 142$ & 13.6 & 31.8 \\
\hline Spring Creek Mine & Laci & 2016 & 9 & $19.2(3.3) 13$ & 14 & 26 \\
\hline Spring Creek Mine & Lano & 2012 & 8 & 24.7 (4.1) 221 & 12.5 & 30.2 \\
\hline Spring Creek Mine & Lano & 2012 & 9 & $18.6(3.2) 66$ & 13.2 & 24.4 \\
\hline Spring Creek Mine & Lano & 2013 & 4 & $12.4(2.2) 161$ & 7 & 17.6 \\
\hline Spring Creek Mine & Lano & 2013 & 5 & 17.4 (2.9) 463 & 2.2 & 24.4 \\
\hline Spring Creek Mine & Lano & 2013 & 6 & 19.6 (3.5) 340 & 8.7 & 28 \\
\hline Spring Creek Mine & Lano & 2013 & 10 & $14.2(1.2) 6$ & 11.7 & 15 \\
\hline Spring Creek Mine & Lano & 2013 & 11 & $6.7(3.6) 4$ & 1.3 & 9 \\
\hline Spring Creek Mine & Lano & 2013 & 12 & $7.3(3.8) 2$ & 4.6 & 10 \\
\hline Spring Creek Mine & Lano & 2014 & 3 & 6() 1 & 6 & 6 \\
\hline Spring Creek Mine & Lano & 2014 & 4 & $16.4(3.1) 16$ & 10.8 & 19.6 \\
\hline Spring Creek Mine & Lano & 2014 & 5 & $17.7(4.5) 86$ & 10.5 & 27.2 \\
\hline Spring Creek Mine & Lano & 2014 & 6 & $18.2(2.7) 187$ & 8.5 & 24.6 \\
\hline
\end{tabular}




\begin{tabular}{|c|c|c|c|c|c|c|}
\hline Spring Creek Mine & Lano & 2014 & 7 & 22 (3.6) 176 & 12 & 29.7 \\
\hline Spring Creek Mine & Lano & 2014 & 8 & 20.5 (2.2) 119 & 16.5 & 25.1 \\
\hline Spring Creek Mine & Lano & 2014 & 9 & $17.5(2.4) 11$ & 12.7 & 22.1 \\
\hline Spring Creek Mine & Lano & 2014 & 11 & $13.5(0) 2$ & 13.5 & 13.5 \\
\hline Spring Creek Mine & Lano & 2015 & 3 & 16() 1 & 16 & 16 \\
\hline Spring Creek Mine & Lano & 2015 & 4 & $18.8(8.5) 3$ & 8.9 & 23.7 \\
\hline Spring Creek Mine & Lano & 2015 & 5 & 17.3 (1.6) 10 & 15.1 & 20.6 \\
\hline Spring Creek Mine & Lano & 2015 & 6 & $21.5(0.6) 6$ & 20.9 & 22.2 \\
\hline Spring Creek Mine & Lano & 2015 & 6 & $22.2(2.7) 599$ & 14.5 & 29.2 \\
\hline Spring Creek Mine & Lano & 2015 & 7 & $23.2(3) 654$ & 15.5 & 30.2 \\
\hline Spring Creek Mine & Lano & 2015 & 8 & $24.1(4.2) 502$ & 8 & 32.7 \\
\hline Spring Creek Mine & Lano & 2015 & 9 & 21 (3.7) 211 & 11.7 & 28.9 \\
\hline Spring Creek Mine & Lano & 2015 & 10 & $15.9(4) 12$ & 9 & 20.3 \\
\hline Spring Creek Mine & Lano & 2015 & 11 & $3.8(0.6) 10$ & 3.1 & 4.4 \\
\hline Spring Creek Mine & Lano & 2015 & 12 & $3.4(2.3) 6$ & 0 & 4.9 \\
\hline Spring Creek Mine & Lano & 2016 & 1 & $3(3.6) 13$ & 0.9 & 10.2 \\
\hline Spring Creek Mine & Lano & 2016 & 2 & $6.9(0) 3$ & 6.9 & 6.9 \\
\hline Spring Creek Mine & Lano & 2016 & 3 & 16.5 () 1 & 16.5 & 16.5 \\
\hline Spring Creek Mine & Lano & 2016 & 4 & 16.3 (4.9) 34 & 3.6 & 21.4 \\
\hline Spring Creek Mine & Lano & 2016 & 5 & $17.6(3.2) 137$ & 9.2 & 23.6 \\
\hline Spring Creek Mine & Lano & 2016 & 6 & $22.8(4) 462$ & 14.8 & 32.8 \\
\hline Spring Creek Mine & Lano & 2016 & 7 & 25.8 (3.4) 648 & 15.1 & 34.1 \\
\hline Spring Creek Mine & Lano & 2016 & 8 & 25.1 (3.6) 604 & 13.8 & 32.3 \\
\hline Spring Creek Mine & Lano & 2016 & 9 & $19.9(4.2) 96$ & 11.7 & 29.5 \\
\hline Spring Creek Mine & Lano & 2016 & 10 & 12.3() 1 & 12.3 & 12.3 \\
\hline Spring Creek Mine & Myci & 2012 & 8 & 23.1 (3.6) 209 & 14.1 & 30.2 \\
\hline Spring Creek Mine & Myci & 2012 & 9 & $21(2.6) 38$ & 14 & 24.7 \\
\hline Spring Creek Mine & Myci & 2013 & 4 & $14.5(3.2) 7$ & 7.5 & 16.3 \\
\hline Spring Creek Mine & Myci & 2013 & 5 & $15(6) 55$ & 2.1 & 22.4 \\
\hline Spring Creek Mine & Myci & 2013 & 6 & $20(3.3) 97$ & 11.5 & 26.5 \\
\hline Spring Creek Mine & Myci & 2013 & 10 & $13(1.1) 2$ & 12.2 & 13.8 \\
\hline Spring Creek Mine & Myci & 2014 & 4 & 16.5 (2.7) 18 & 10.8 & 19.3 \\
\hline Spring Creek Mine & Myci & 2014 & 5 & 17.5 (2.9) 285 & 7.5 & 26.9 \\
\hline Spring Creek Mine & Myci & 2014 & 6 & $16.3(2.4) 638$ & 8.5 & 22.4 \\
\hline Spring Creek Mine & Myci & 2014 & 7 & 20.2 (3.3) 704 & 11.3 & 29.5 \\
\hline Spring Creek Mine & Myci & 2014 & 8 & $20.2(2.2) 720$ & 15.6 & 26.5 \\
\hline Spring Creek Mine & Myci & 2014 & 9 & $20.4(3.4) 41$ & 11.7 & 25.4 \\
\hline Spring Creek Mine & Myci & 2014 & 11 & 3.4() 1 & 3.4 & 3.4 \\
\hline Spring Creek Mine & Myci & 2014 & 12 & $5.6(0.1) 2$ & 5.5 & 5.7 \\
\hline Spring Creek Mine & Myci & 2015 & 4 & $16.6(3.5) 2$ & 14.1 & 19.1 \\
\hline Spring Creek Mine & Myci & 2015 & 5 & $16.5(2.3) 75$ & 9.4 & 21.7 \\
\hline Spring Creek Mine & Myci & 2015 & 6 & $19.6(2.3) 27$ & 14.5 & 24.1 \\
\hline Spring Creek Mine & Myci & 2015 & 6 & 21.5 (3) 369 & 15.6 & 28.5 \\
\hline Spring Creek Mine & Myci & 2015 & 7 & 23.7 (3.5) 542 & 15.8 & 30.8 \\
\hline
\end{tabular}




\begin{tabular}{|c|c|c|c|c|c|c|}
\hline Spring Creek Mine & Myci & 2015 & 8 & 27 (3.9) 1324 & 12.8 & 32.8 \\
\hline Spring Creek Mine & Myci & 2015 & 9 & 21.7 (3.9) 789 & 10.8 & 29.7 \\
\hline Spring Creek Mine & Myci & 2015 & 10 & $19.8(5) 30$ & 7.9 & 24.2 \\
\hline Spring Creek Mine & Myci & 2015 & 11 & $7.1(3.1) 8$ & 3.7 & 11.2 \\
\hline Spring Creek Mine & Myci & 2015 & 12 & $4.9(2.2) 8$ & 3.2 & 10.2 \\
\hline Spring Creek Mine & Myci & 2016 & 1 & $6.6(4.7) 3$ & 1.1 & 9.4 \\
\hline Spring Creek Mine & Myci & 2016 & 2 & $9.2(2.5) 5$ & 5.1 & 11.3 \\
\hline Spring Creek Mine & Myci & 2016 & 4 & $14.1(4.1) 70$ & 4.6 & 21.2 \\
\hline Spring Creek Mine & Myсi & 2016 & 5 & $17.6(2.7) 186$ & 11.7 & 23.6 \\
\hline Spring Creek Mine & Myci & 2016 & 6 & $22.2(4.2) 474$ & 13.2 & 32.8 \\
\hline Spring Creek Mine & Myci & 2016 & 7 & 27.1 (4.3) 408 & 16.8 & 34.1 \\
\hline Spring Creek Mine & Myci & 2016 & 8 & $25.2(4) 504$ & 13.6 & 32.2 \\
\hline Spring Creek Mine & Myсi & 2016 & 9 & $23(3.9) 105$ & 12.2 & 29.5 \\
\hline Spring Creek Mine & Myсi & 2016 & 10 & $14.1(7.8) 4$ & 7.5 & 23.1 \\
\hline Spring Creek Mine & Myci & 2016 & 11 & $12.4(2.3) 2$ & 10.8 & 14.1 \\
\hline Spring Creek Mine & Myev & 2012 & 8 & 21.8 (3.7) 49 & 14.3 & 29.3 \\
\hline Spring Creek Mine & Myev & 2012 & 9 & $21(3.4) 5$ & 16.6 & 24.4 \\
\hline Spring Creek Mine & Myev & 2013 & 4 & 17 () 1 & 17 & 17 \\
\hline Spring Creek Mine & Myev & 2013 & 5 & $15.4(4) 36$ & 6 & 22.1 \\
\hline Spring Creek Mine & Myev & 2013 & 6 & $19.3(3.4) 60$ & 10.3 & 26.5 \\
\hline Spring Creek Mine & Myev & 2014 & 4 & $14.3(3.6) 2$ & 11.7 & 16.8 \\
\hline Spring Creek Mine & Myev & 2014 & 5 & $13.9(2.2) 11$ & 11 & 19.1 \\
\hline Spring Creek Mine & Myev & 2014 & 6 & 14.3 (3.2) 11 & 8.9 & 18.6 \\
\hline Spring Creek Mine & Myev & 2014 & 7 & 20.7 (1.7) 19 & 17.8 & 24.2 \\
\hline Spring Creek Mine & Myev & 2014 & 8 & $21.4(2.5) 26$ & 17.1 & 24.4 \\
\hline Spring Creek Mine & Myev & 2014 & 9 & $16.6(5.6) 4$ & 8.7 & 21.9 \\
\hline Spring Creek Mine & Myev & 2015 & 5 & $13.2(2.8) 4$ & 10.7 & 16.6 \\
\hline Spring Creek Mine & Myev & 2015 & 6 & 23.6 () 1 & 23.6 & 23.6 \\
\hline Spring Creek Mine & Myev & 2015 & 6 & 21.1 (2.9) 170 & 15.8 & 28.2 \\
\hline Spring Creek Mine & Myev & 2015 & 7 & 22.6 (3.1) 388 & 14.8 & 29.7 \\
\hline Spring Creek Mine & Myev & 2015 & 8 & $23.3(4.8) 396$ & 12.5 & 32.3 \\
\hline Spring Creek Mine & Myev & 2015 & 9 & 19.7 (3.6) 160 & 11 & 29.3 \\
\hline Spring Creek Mine & Myev & 2016 & 4 & 14.8 (3.1) 10 & 11.2 & 19.9 \\
\hline Spring Creek Mine & Myev & 2016 & 5 & 14.4 (3.6) 224 & 7 & 23.6 \\
\hline Spring Creek Mine & Myev & 2016 & 6 & 19.6 (4.2) 304 & 13 & 30.5 \\
\hline Spring Creek Mine & Myev & 2016 & 7 & $25.1(4.3) 278$ & 14.6 & 34 \\
\hline Spring Creek Mine & Myev & 2016 & 8 & 23.9 (4.3) 293 & 14.6 & 32.3 \\
\hline Spring Creek Mine & Myev & 2016 & 9 & 19.7 (3.7) 135 & 9.2 & 28.9 \\
\hline Spring Creek Mine & Mylu & 2012 & 8 & 24 (3.9) 209 & 12.7 & 29.8 \\
\hline Spring Creek Mine & Mylu & 2012 & 9 & 21.6 (3.1) 40 & 12.5 & 24.9 \\
\hline Spring Creek Mine & Mylu & 2013 & 4 & $14.7(1.3) 14$ & 12.2 & 16.8 \\
\hline Spring Creek Mine & Mylu & 2013 & 5 & $17.4(4.3) 365$ & 3.4 & 26.2 \\
\hline Spring Creek Mine & Mylu & 2013 & 6 & $21.3(3) 868$ & 8.4 & 28 \\
\hline Spring Creek Mine & Mylu & 2013 & 11 & 7.2() 1 & 7.2 & 7.2 \\
\hline
\end{tabular}




\begin{tabular}{|c|c|c|c|c|c|c|}
\hline Spring Creek Mine & Mylu & 2014 & 4 & $17.1(3) 6$ & 11.2 & 19.3 \\
\hline Spring Creek Mine & Mylu & 2014 & 5 & $17.8(5) 44$ & 7 & 26.9 \\
\hline Spring Creek Mine & Mylu & 2014 & 6 & $16.4(2.7) 512$ & 7 & 21.6 \\
\hline Spring Creek Mine & Mylu & 2014 & 7 & 20.9 (3.2) 1252 & 12.7 & 29.8 \\
\hline Spring Creek Mine & Mylu & 2014 & 8 & $21(2.5) 567$ & 14.8 & 26.4 \\
\hline Spring Creek Mine & Mylu & 2014 & 9 & $19.4(5.1) 9$ & 9.5 & 24.2 \\
\hline Spring Creek Mine & Mylu & 2015 & 4 & 8.7 () 1 & 8.7 & 8.7 \\
\hline Spring Creek Mine & Mylu & 2015 & 5 & $16.3(4.6) 52$ & 6 & 22.1 \\
\hline Spring Creek Mine & Mylu & 2015 & 6 & $19.1(2.4) 35$ & 14.6 & 24.2 \\
\hline Spring Creek Mine & Mylu & 2015 & 6 & 21.1 (3) 1168 & 14.5 & 29.2 \\
\hline Spring Creek Mine & Mylu & 2015 & 7 & $22(3.2) 947$ & 14.8 & 31 \\
\hline Spring Creek Mine & Mylu & 2015 & 8 & 24.7 (4.9) 573 & 8 & 32.7 \\
\hline Spring Creek Mine & Mylu & 2015 & 9 & 20.5 (4.1) 298 & 12.2 & 29.7 \\
\hline Spring Creek Mine & Mylu & 2015 & 10 & $22.4(1.6) 7$ & 19.1 & 23.9 \\
\hline Spring Creek Mine & Mylu & 2016 & 2 & 4.2 () 1 & 4.2 & 4.2 \\
\hline Spring Creek Mine & Mylu & 2016 & 4 & $13.1(4) 5$ & 9.2 & 18.6 \\
\hline Spring Creek Mine & Mylu & 2016 & 5 & 15.9 (3.8) 191 & 6.2 & 23.4 \\
\hline Spring Creek Mine & Mylu & 2016 & 6 & $22(4.2) 448$ & 13.6 & 33.3 \\
\hline Spring Creek Mine & Mylu & 2016 & 7 & $25.3(4.3) 534$ & 14.3 & 34.5 \\
\hline Spring Creek Mine & Mylu & 2016 & 8 & $24.4(4.4) 227$ & 15.5 & 32 \\
\hline Spring Creek Mine & Mylu & 2016 & 9 & $21.2(3.5) 57$ & 12.2 & 29.5 \\
\hline Spring Creek Mine & Mylu & 2016 & 10 & 22.9 () 1 & 22.9 & 22.9 \\
\hline Spring Creek Mine & Myth & 2013 & 6 & $17.7(0.8) 2$ & 17.1 & 18.3 \\
\hline Spring Creek Mine & Myth & 2015 & 6 & $21.7(2.5) 22$ & 17.4 & 26.4 \\
\hline Spring Creek Mine & Myth & 2015 & 7 & $22.8(3.4) 72$ & 14.8 & 28.5 \\
\hline Spring Creek Mine & Myth & 2015 & 8 & $21.8(5.2) 87$ & 12 & 32.5 \\
\hline Spring Creek Mine & Myth & 2015 & 9 & 19.9 (3) 14 & 15.1 & 25.4 \\
\hline Spring Creek Mine & Myth & 2016 & 4 & 16.8 () 1 & 16.8 & 16.8 \\
\hline Spring Creek Mine & Myth & 2016 & 5 & 14.9 (3.9) 42 & 6.9 & 23.1 \\
\hline Spring Creek Mine & Myth & 2016 & 6 & $21.4(5.1) 45$ & 14 & 31.2 \\
\hline Spring Creek Mine & Myth & 2016 & 7 & $24.1(4.8) 62$ & 16 & 34 \\
\hline Spring Creek Mine & Myth & 2016 & 8 & $24.9(3.7) 58$ & 17.9 & 31.8 \\
\hline Spring Creek Mine & Myth & 2016 & 9 & $20.2(3.4) 30$ & 15 & 29.7 \\
\hline West Decker Coal Mine & Epfu & 2012 & 8 & $16.9(4.3) 905$ & 5.9 & 24.7 \\
\hline West Decker Coal Mine & Epfu & 2012 & 9 & $16.3(2.7) 852$ & 6.2 & 22.4 \\
\hline West Decker Coal Mine & Epfu & 2012 & 10 & $9.7(6.2) 4$ & 3.7 & 17.3 \\
\hline West Decker Coal Mine & Epfu & 2013 & 4 & 12.9 (1.4) 97 & 8.5 & 15 \\
\hline West Decker Coal Mine & Epfu & 2013 & 5 & $14.7(3.2) 75$ & 6.5 & 21.4 \\
\hline West Decker Coal Mine & Epfu & 2013 & 6 & $16.2(2.5) 155$ & 11.3 & 23.6 \\
\hline West Decker Coal Mine & Epfu & 2013 & 7 & $17.8(3.3) 72$ & 13 & 23.2 \\
\hline West Decker Coal Mine & Epfu & 2014 & 4 & $10.5(2.7) 25$ & 8.5 & 16.3 \\
\hline West Decker Coal Mine & Epfu & 2014 & 5 & $13.9(2.8) 112$ & 5.9 & 19.9 \\
\hline West Decker Coal Mine & Epfu & 2014 & 6 & $15(2.1) 66$ & 11.5 & 18.4 \\
\hline West Decker Coal Mine & Epfu & 2014 & 7 & $20.6(0.6) 4$ & 20.1 & 21.4 \\
\hline
\end{tabular}




\begin{tabular}{|c|c|c|c|c|c|c|}
\hline West Decker Coal Mine & Epfu & 2014 & 8 & $14.6(2.3) 265$ & 11.5 & 20.1 \\
\hline West Decker Coal Mine & Epfu & 2014 & 9 & 14.4 (3.3) 174 & 3.4 & 21.1 \\
\hline West Decker Coal Mine & Epfu & 2014 & 10 & 10.5 () 1 & 10.5 & 10.5 \\
\hline West Decker Coal Mine & Epfu & 2014 & 12 & 3.4() 1 & 3.4 & 3.4 \\
\hline West Decker Coal Mine & Epfu & 2015 & 1 & $5(0.1) 30$ & 4.9 & 5.1 \\
\hline West Decker Coal Mine & Epfu & 2015 & 2 & $6(3.7) 5$ & 4.2 & 12.7 \\
\hline West Decker Coal Mine & Epfu & 2015 & 3 & $13.7(1.3) 14$ & 11.5 & 15.1 \\
\hline West Decker Coal Mine & Epfu & 2015 & 4 & 13.6 (3.9) 237 & 4.6 & 20.8 \\
\hline West Decker Coal Mine & Epfu & 2015 & 5 & $13.8(2) 288$ & 5.2 & 19.9 \\
\hline West Decker Coal Mine & Epfu & 2015 & 6 & $15.6(1.8) 143$ & 13 & 20.9 \\
\hline West Decker Coal Mine & Epfu & 2015 & 6 & $20.3(3.1) 1152$ & 13.8 & 27.4 \\
\hline West Decker Coal Mine & Epfu & 2015 & 7 & $17.8(2.8) 3000$ & 11 & 27.5 \\
\hline West Decker Coal Mine & Epfu & 2015 & 8 & $16.2(2.8) 953$ & 7.9 & 28.2 \\
\hline West Decker Coal Mine & Epfu & 2015 & 9 & 20.7 (3.1) 269 & 12.7 & 26.5 \\
\hline West Decker Coal Mine & Epfu & 2015 & 10 & $20.7(1.8) 11$ & 17.9 & 22.4 \\
\hline West Decker Coal Mine & Epfu & 2015 & 12 & $3.1(0) 8$ & 3.1 & 3.1 \\
\hline West Decker Coal Mine & Epfu & 2016 & 4 & 15 (1.4) 1283 & 10.5 & 19.3 \\
\hline West Decker Coal Mine & Epfu & 2016 & 5 & $13.5(2.9) 728$ & 9.5 & 21.1 \\
\hline West Decker Coal Mine & Epfu & 2016 & 6 & $19.7(3.5) 2143$ & 10.5 & 29.2 \\
\hline West Decker Coal Mine & Epfu & 2016 & 7 & $17.5(2.8) 4637$ & 11.2 & 29.8 \\
\hline West Decker Coal Mine & Laci & 2012 & 8 & 13.5 (3.5) 244 & 8.7 & 24.1 \\
\hline West Decker Coal Mine & Laci & 2012 & 9 & 14.7 (3.4) 152 & 4.4 & 20.8 \\
\hline West Decker Coal Mine & Laci & 2013 & 5 & 11 () 1 & 11 & 11 \\
\hline West Decker Coal Mine & Laci & 2013 & 6 & $17.6(2.2) 3$ & 15.1 & 18.9 \\
\hline West Decker Coal Mine & Laci & 2014 & 5 & $15.9(2.2) 19$ & 11.3 & 20.3 \\
\hline West Decker Coal Mine & Laci & 2014 & 6 & $14.8(2.8) 17$ & 9.4 & 18.4 \\
\hline West Decker Coal Mine & Laci & 2014 & 7 & $18.8(2.2) 4$ & 16.3 & 21.2 \\
\hline West Decker Coal Mine & Laci & 2014 & 8 & $13.9(1.8) 40$ & 11.8 & 19.6 \\
\hline West Decker Coal Mine & Laci & 2014 & 9 & $12.8(3.2) 94$ & 5.7 & 21.2 \\
\hline West Decker Coal Mine & Laci & 2014 & 10 & $11.3(2.6) 2$ & 9.5 & 13.2 \\
\hline West Decker Coal Mine & Laci & 2015 & 3 & $9.5(3.5) 8$ & 7 & 15.1 \\
\hline West Decker Coal Mine & Laci & 2015 & 4 & $14.3(3.4) 28$ & 7.5 & 19.4 \\
\hline West Decker Coal Mine & Laci & 2015 & 5 & $13.5(3) 61$ & 6.7 & 18.8 \\
\hline West Decker Coal Mine & Laci & 2015 & 6 & $16(1.3) 25$ & 13.6 & 20.3 \\
\hline West Decker Coal Mine & Laci & 2015 & 6 & 20.4 (2.9) 936 & 12.2 & 27 \\
\hline West Decker Coal Mine & Laci & 2015 & 7 & $18.2(2.6) 647$ & 12.3 & 27.4 \\
\hline West Decker Coal Mine & Laci & 2015 & 8 & $15.6(2.3) 250$ & 9.4 & 24.2 \\
\hline West Decker Coal Mine & Laci & 2015 & 9 & $16.7(3.6) 90$ & 9 & 27 \\
\hline West Decker Coal Mine & Laci & 2015 & 10 & $20.1(1.1) 2$ & 19.4 & 20.9 \\
\hline West Decker Coal Mine & Laci & 2016 & 1 & 0.9 () 1 & 0.9 & 0.9 \\
\hline West Decker Coal Mine & Laci & 2016 & 4 & $14.6(2.2) 36$ & 10.7 & 18.6 \\
\hline West Decker Coal Mine & Laci & 2016 & 5 & $16.4(2.9) 73$ & 7 & 21.1 \\
\hline West Decker Coal Mine & Laci & 2016 & 6 & 18.9 (3.7) 663 & 10.5 & 29.3 \\
\hline West Decker Coal Mine & Laci & 2016 & 7 & 18.9 (2.9) 778 & 11.8 & 29.5 \\
\hline
\end{tabular}




\begin{tabular}{|c|c|c|c|c|c|c|}
\hline West Decker Coal Mine & Lano & 2012 & 8 & 14.9 (3.3) 341 & 5.7 & 23.9 \\
\hline West Decker Coal Mine & Lano & 2012 & 9 & 12.4 (3.7) 765 & 3.1 & 20.9 \\
\hline West Decker Coal Mine & Lano & 2012 & 10 & $9.1(3.5) 6$ & 2.9 & 12 \\
\hline West Decker Coal Mine & Lano & 2013 & 4 & $12.6(2) 24$ & 8.4 & 15 \\
\hline West Decker Coal Mine & Lano & 2013 & 5 & $13.8(3.8) 37$ & 8 & 21 \\
\hline West Decker Coal Mine & Lano & 2013 & 6 & $16.1(2.7) 45$ & 8.5 & 21 \\
\hline West Decker Coal Mine & Lano & 2013 & 7 & $18.6(3.2) 22$ & 15 & 3.7 \\
\hline West Decker Coal Mine & Lano & 2014 & 4 & $13.3(3.2) 49$ & 8.9 & 18.3 \\
\hline West Decker Coal Mine & Lano & 2014 & 5 & $14.4(3.5) 388$ & 2.6 & 23.9 \\
\hline West Decker Coal Mine & Lano & 2014 & 6 & $15.1(2.2) 84$ & 9.2 & 18. \\
\hline West Decker Coal Mine & Lano & 2014 & 7 & $16.6(3.5) 6$ & 13.8 & 22.7 \\
\hline West Decker Coal Mine & Lano & 2014 & 8 & $13.7(1.7) 48$ & 11.3 & 17.4 \\
\hline West Decker Coal Mine & Lano & 2014 & 9 & $12.4(4.2) 55$ & 4.1 & 21. \\
\hline West Decker Coal Mine & Lano & 2014 & 10 & $15.5(0) 2$ & 15.5 & 15. \\
\hline West Decker Coal Mine & Lano & 2015 & 2 & $4.7(1.7) 23$ & 4.2 & 12.7 \\
\hline West Decker Coal Mine & Lano & 2015 & 3 & 13.2() 1 & 13.2 & 13.2 \\
\hline West Decker Coal Mine & Lano & 2015 & 4 & $8.9(3.2) 38$ & 4.9 & 20 \\
\hline West Decker Coal Mine & Lano & 2015 & 5 & $13.1(2.9) 177$ & 4.4 & 1 \\
\hline West Decker Coal Mine & Lano & 2015 & 6 & $15.4(1.8) 23$ & 12.2 & 18.6 \\
\hline West Decker Coal Mine & Lano & 2015 & 6 & $19.9(2.8) 1133$ & 12.2 & 27.4 \\
\hline West Decker Coal Mine & Lano & 2015 & 7 & 17.8 (3) 1637 & 11 & 27.7 \\
\hline West Decker Coal Mine & Lano & 2015 & 8 & $15.4(2.5) 874$ & 7.5 & 24 \\
\hline West Decker Coal Mine & Lano & 2015 & 9 & $17.6(3.2) 1109$ & 8.2 & 26.5 \\
\hline West Decker Coal Mine & Lano & 2015 & 10 & $17.9(2.6) 14$ & 14.8 & 20.8 \\
\hline West Decker Coal Mine & Lano & 2015 & 12 & $3.1(0) 2$ & 3.1 & 3.1 \\
\hline West Decker Coal Mine & Lano & 2016 & 4 & $14(2.4) 523$ & 5.9 & 19 \\
\hline West Decker Coal Mine & Lano & 2016 & 5 & $14.3(2.9) 768$ & 7.5 & 2 \\
\hline West Decker Coal Mine & Lano & 2016 & 6 & $18.5(3.5) 1192$ & 10.3 & 29.2 \\
\hline West Decker Coal Mine & Lano & 2016 & 7 & 19.1 (3) 1015 & 11.2 & 29. \\
\hline West Decker Coal Mine & Myci & 2012 & 8 & $17.7(3.1) 189$ & 7.4 & 24 \\
\hline West Decker Coal Mine & Myci & 2012 & 9 & $16.1(2.6) 170$ & 8.7 & 21.4 \\
\hline West Decker Coal Mine & Myci & 2013 & 4 & $12.4(1.8) 16$ & 8 & 14. \\
\hline West Decker Coal Mine & Myci & 2013 & 5 & $13.6(2.1) 20$ & 5.9 & 16 \\
\hline West Decker Coal Mine & Myci & 2013 & 6 & $16.4(2.3) 67$ & 7.7 & 20 \\
\hline West Decker Coal Mine & Myci & 2013 & 7 & $18.6(1.2) 35$ & 15.6 & 20 \\
\hline West Decker Coal Mine & Myci & 2014 & 4 & $11.4(3.5) 68$ & 4.7 & 17. \\
\hline West Decker Coal Mine & Myci & 2014 & 5 & $12.9(2.5) 409$ & 2.4 & 24 \\
\hline West Decker Coal Mine & Myci & 2014 & 6 & $13.1(2.2) 137$ & 8.9 & 17 \\
\hline West Decker Coal Mine & Myci & 2014 & 7 & $16.9(2.4) 55$ & 13.5 & 22.7 \\
\hline West Decker Coal Mine & Myci & 2014 & 8 & $15.5(1.7) 75$ & 11.8 & 19. \\
\hline West Decker Coal Mine & Myci & 2014 & 9 & $14.1(2.2) 1091$ & 4.9 & 21. \\
\hline West Decker Coal Mine & Myci & 2014 & 10 & $15(2.5) 13$ & 11.8 & 19 \\
\hline West Decker Coal Mine & Myci & 2015 & 3 & $11.3(1.4) 20$ & 8.7 & \\
\hline West Decker Coal Mine & Myci & 2015 & 4 & $9.9(2.2) 752$ & 0.3 & \\
\hline
\end{tabular}




\begin{tabular}{|c|c|c|c|c|c|c|}
\hline West Decker Coal Mine & Myci & 2015 & 5 & $11.9(2.3) 279$ & 3.4 & 19.8 \\
\hline West Decker Coal Mine & Myci & 2015 & 6 & $15.7(1.5) 126$ & 12.2 & 19.6 \\
\hline West Decker Coal Mine & Myci & 2015 & 6 & $17.8(2.1) 1134$ & 12.3 & 25.9 \\
\hline West Decker Coal Mine & Myci & 2015 & 7 & $18.3(2.6) 1096$ & 11 & 25.4 \\
\hline West Decker Coal Mine & Myci & 2015 & 8 & $16.5(2.8) 394$ & 7.9 & 28.2 \\
\hline West Decker Coal Mine & Myci & 2015 & 9 & 17.9 (3.4) 202 & 10.5 & 26 \\
\hline West Decker Coal Mine & Myci & 2015 & 10 & $17.4(2.5) 9$ & 13.3 & 20.3 \\
\hline West Decker Coal Mine & Myci & 2016 & 3 & 15.1() 1 & 15.1 & 15.1 \\
\hline West Decker Coal Mine & Myci & 2016 & 4 & 13.1 (1.6) 908 & 6.7 & 18.3 \\
\hline West Decker Coal Mine & Myci & 2016 & 5 & $13.5(2.7) 551$ & 4.1 & 20.8 \\
\hline West Decker Coal Mine & Myci & 2016 & 6 & $18.7(2.9) 1558$ & 10.3 & 29.2 \\
\hline West Decker Coal Mine & Myci & 2016 & 7 & $19.5(2.5) 986$ & 11.5 & 29.7 \\
\hline West Decker Coal Mine & Myev & 2012 & 8 & $14.1(4.7) 3$ & 9.7 & 19.1 \\
\hline West Decker Coal Mine & Myev & 2012 & 9 & $14.5(3) 6$ & 9.2 & 17.9 \\
\hline West Decker Coal Mine & Myev & 2013 & 4 & 7.2() 1 & 7.2 & 7.2 \\
\hline West Decker Coal Mine & Myev & 2013 & 5 & 15 () 1 & 15 & 15 \\
\hline West Decker Coal Mine & Myev & 2013 & 6 & $13.7(3.7) 2$ & 11 & 16.3 \\
\hline West Decker Coal Mine & Myev & 2014 & 5 & 13.6 () 1 & 13.6 & 13.6 \\
\hline West Decker Coal Mine & Myev & 2014 & 6 & 17.4 () 1 & 17.4 & 17.4 \\
\hline West Decker Coal Mine & Myev & 2014 & 8 & $16.1(1.2) 2$ & 15.3 & 17 \\
\hline West Decker Coal Mine & Myev & 2014 & 9 & $12.4(5.3) 12$ & 5.4 & 23.1 \\
\hline West Decker Coal Mine & Myev & 2015 & 5 & $8.4(1.6) 4$ & 7.5 & 10.8 \\
\hline West Decker Coal Mine & Myev & 2015 & 6 & $19(2.3) 50$ & 15.1 & 25.2 \\
\hline West Decker Coal Mine & Myev & 2015 & 7 & $17.7(3.1) 38$ & 12.8 & 24.2 \\
\hline West Decker Coal Mine & Myev & 2015 & 8 & $15.7(2.7) 36$ & 10.8 & 21.2 \\
\hline West Decker Coal Mine & Myev & 2015 & 9 & $14.6(4) 17$ & 8.4 & 22.1 \\
\hline West Decker Coal Mine & Myev & 2016 & 4 & 13.4 (1) 5 & 11.7 & 14.3 \\
\hline West Decker Coal Mine & Myev & 2016 & 5 & $12.4(3.3) 18$ & 4.7 & 15.8 \\
\hline West Decker Coal Mine & Myev & 2016 & 6 & $18(3.2) 37$ & 12.3 & 23.6 \\
\hline West Decker Coal Mine & Myev & 2016 & 7 & 19 (3.1) 23 & 12.3 & 24.2 \\
\hline West Decker Coal Mine & Mylu & 2012 & 8 & $17.1(2.8) 232$ & 9.8 & 23.4 \\
\hline West Decker Coal Mine & Mylu & 2012 & 9 & $13.1(3.7) 253$ & 0.4 & 21.4 \\
\hline West Decker Coal Mine & Mylu & 2012 & 10 & $9.5(4.8) 5$ & 2.4 & 15.8 \\
\hline West Decker Coal Mine & Mylu & 2013 & 4 & $10.6(3.1) 8$ & 3.7 & 14.5 \\
\hline West Decker Coal Mine & Mylu & 2013 & 5 & $11.6(2) 4$ & 8.7 & 12.7 \\
\hline West Decker Coal Mine & Mylu & 2013 & 6 & $17.6(2) 15$ & 12.7 & 21.4 \\
\hline West Decker Coal Mine & Mylu & 2013 & 7 & $18.1(1.3) 4$ & 17.1 & 19.9 \\
\hline West Decker Coal Mine & Mylu & 2014 & 4 & $11.4(5) 31$ & -0.3 & 18.1 \\
\hline West Decker Coal Mine & Mylu & 2014 & 5 & $13.2(2.2) 157$ & 1.1 & 18.1 \\
\hline West Decker Coal Mine & Mylu & 2014 & 6 & $13.9(2.2) 20$ & 10.8 & 17.6 \\
\hline West Decker Coal Mine & Mylu & 2014 & 7 & $18(2.1) 11$ & 13.2 & 20.3 \\
\hline West Decker Coal Mine & Mylu & 2014 & 8 & $15.5(1.9) 58$ & 12.8 & 19.8 \\
\hline West Decker Coal Mine & Mylu & 2014 & 9 & 13.6 (3.8) 207 & 2.4 & 21.1 \\
\hline West Decker Coal Mine & Mylu & 2014 & 10 & 12.7 (3) 2 & 10.5 & 14.8 \\
\hline
\end{tabular}




$\begin{array}{lcccccc}\text { West Decker Coal Mine } & \text { Mylu } & 2015 & 3 & 9.4() 1 & 9.4 & 9.4 \\ \text { West Decker Coal Mine } & \text { Mylu } & 2015 & 4 & 9.7(2.2) 233 & 4.6 & 16.5 \\ \text { West Decker Coal Mine } & \text { Mylu } & 2015 & 5 & 11.9(3.3) 131 & -0.8 & 19.3 \\ \text { West Decker Coal Mine } & \text { Mylu } & 2015 & 6 & 15.4(1.7) 87 & 12.2 & 20.3 \\ \text { West Decker Coal Mine } & \text { Mylu } & 2015 & 6 & 18.9(2.6) 3439 & 12.2 & 27.4 \\ \text { West Decker Coal Mine } & \text { Mylu } & 2015 & 7 & 18.2(3) 1196 & 10.8 & 27.5 \\ \text { West Decker Coal Mine } & \text { Mylu } & 2015 & 8 & 15(2.9) 3094 & 3.6 & 27.4 \\ \text { West Decker Coal Mine } & \text { Mylu } & 2015 & 9 & 17.2(3.2) 821 & 8.5 & 25.7 \\ \text { West Decker Coal Mine } & \text { Mylu } & 2015 & 10 & 14.4(4.8) 2 & 11 & 17.8 \\ \text { West Decker Coal Mine } & \text { Mylu } & 2016 & 4 & 12.9(2.7) 121 & 6.5 & 17.9 \\ \text { West Decker Coal Mine } & \text { Mylu } & 2016 & 5 & 11.3(3.3) 4165 & 2.7 & 21.2 \\ \text { West Decker Coal Mine } & \text { Mylu } & 2016 & 6 & 17.8(3.7) 2563 & 10.5 & 29.2 \\ \text { West Decker Coal Mine } & \text { Mylu } & 2016 & 7 & 19.5(3.6) 1937 & 11.3 & 31.2 \\ \text { West Decker Coal Mine } & \text { Myth } & 2015 & 6 & 18.1(0.7) 2 & 17.6 & 18.6 \\ \text { West Decker Coal Mine } & \text { Myth } & 2015 & 7 & 17.3() 1 & 17.3 & 17.3 \\ \text { West Decker Coal Mine } & \text { Myth } & 2016 & 5 & 11() 1 & 11 & 11 \\ \text { West Decker Coal Mine } & \text { Myth } & 2016 & 6 & 14.3(2.9) 2 & 12.2 & 16.3 \\ \text { West Decker Coal Mine } & \text { Myth } & 2016 & 7 & 18.6() 1 & 18.6 & 18.6\end{array}$




\section{Appendix C}

\section{Overview of Roosting Habitat and Home Range / Foraging Distance Documented for Montana Bats \\ Bryce A. Maxell, Montana Natural Heritage Program - 24 February 2015 \\ Updated 30-June-2017 by Dan Bachen}

The table, figures, and images below summarize and provide examples of what is known about winter, maternity, and day/night roost habitat use for Montana bat species in the state and/or elsewhere across their ranges. Protection of these cave, mine, cliff, rock outcrop, ground crevice, large tree, bridge, and building habitats with cracks and crevices ranging from $1 / 3$ to 1 inch in width and associated temperature and humidity regimes, is essential for protection and conservation of Montana's bats. Artificial bat roosts that provide summer maternity, night, and day roosts, can be deployed to serve as a surrogate for large diameter tree and other roosts that have been lost and/or to encourage roosting away from buildings where bats would be in close proximity to sleeping humans. Artificial winter roost habitat is not a viable management option at the present time.

\begin{tabular}{|c|c|c|c|c|}
\hline Species / Comments & Winter Roost & Summer Maternity Roost & Summer Day/Night Roost & Home Range/Foraging Distance \\
\hline $\begin{array}{l}\text { Pallid Bat } \\
\text { (Antrozous pallidus) } \\
\text { Low roost site fidelity with } 90 \% \\
\text { of inter-night movements of } 50- \\
600 \text { meters. }{ }^{3} \text { Highly social, } \\
\text { often using day and night roosts } \\
\text { in groups of } 20 \text { or more guided } \\
\text { by social vocalizations and } \\
\text { odors. }{ }^{2,4} \text { Yearling females } \\
\text { typically give birth to a single } \\
\text { pup, but older females typically } \\
\text { give birth to } 2 \text { pups. }{ }^{43}\end{array}$ & $\begin{array}{l}\text { Not documented in Montana, } \\
\text { but likely occurs in deep rock } \\
\text { crevices if the species is } \\
\text { present. }^{1,4}\end{array}$ & $\begin{array}{l}\text { Not documented in Montana. } \\
\text { Elsewhere in vertical and } \\
\text { horizontal rock crevices, } \\
\text { under rock slabs, in buildings, } \\
\text { and on taller and larger } \\
\text { diameter live trees and tree } \\
\text { snags with loose bark in } \\
\text { mature stands with southerly } \\
\text { aspects and lower } \\
\text { percentages of overstory. }{ }^{4,37,} \\
38,41,42,44\end{array}$ & $\begin{array}{l}\text { Under rock slabs, in horizontal } \\
\text { and vertical rock crevices, and } \\
\text { on farm equipment in } \\
\text { Montana. }^{1} \text { Elsewhere } \\
\text { occasionally on buildings, } \\
\text { bridges, caves, mines, vertical } \\
\text { and horizontal rock crevices } \\
\text { that are typically on east or } \\
\text { southeast aspects, and taller } \\
\text { and larger diameter live trees } \\
\text { and tree snags with loose bark } \\
\text { in mature stands with } \\
\text { southerly aspects and lower } \\
\text { percentages of overstory. }{ }^{2,4,21,} \\
22,23,30,37,38,39,40,41,44\end{array}$ & $\begin{array}{l}\text { Lactating females moved an average } \\
\text { of } 2,450 \text { meters }+/-845 \text { from roost } \\
\text { to foraging areas and had an average } \\
\text { foraging area size of } 1.56 \text { square km } \\
+/-0.88 \text { SE. Post-lactating females } \\
\text { moved an average of } 210 \text { meters } \\
\text { from roost to foraging areas and had } \\
\text { an average foraging area size of } 5.97 \\
\text { square km }+/-2.69 \text { SE in northern } \\
\text { California. }{ }^{37} \text { Individuals commuted } 1 \\
\text { to } 4 \text { km between day roosting and } \\
\text { foraging areas, } 0.5 \text { to } 1.5 \text { km } \\
\text { between day roosts and night roosts, } \\
\text { and switched day roosts often, } \\
\text { usually moving <200 meters } \\
\text { between roosts (range } 25 \text { to } 3,660 \\
\text { meters) in eastern Oregon. }{ }^{38,39} \\
\text { Individuals typically commuted } 1-2 \\
\text { km from day roosts to foraging } \\
\text { areas, but one male often used } \\
\text { different day roosts separated by } 10 \\
\text { km in California. } 42\end{array}$ \\
\hline $\begin{array}{l}\text { Townsend's Big-eared Bat } \\
\text { (Corynorhinus townsendii) }\end{array}$ & $\begin{array}{l}\text { Twilight areas of caves, mines, } \\
\text { and unused tunnels in } \\
\text { Montana. }{ }^{1,31,32,75,84} \text { Limestone }\end{array}$ & $\begin{array}{l}\text { Caves and mines, often in } \\
\text { twilight areas in Montana. }{ }^{1,75} \\
\text { Reported in caves, mines, }\end{array}$ & \begin{tabular}{|l|} 
In Montana, usually in caves \\
and mines, often in twilight \\
areas, but more rarely building
\end{tabular} & $\begin{array}{l}\text { Average one-way travel distances } \\
\text { between day roosts and foraging } \\
\text { areas was } 3.2 \mathrm{~km}+/-0.5 \text { SD for }\end{array}$ \\
\hline
\end{tabular}




\begin{tabular}{|c|c|c|c|c|}
\hline $\begin{array}{l}\text { High fidelity to maternity and } \\
\text { hibernacula roosts, lower } \\
\text { interseasonal roost site fidelity, } \\
\text { and travel up to } 24 \mathrm{~km} \text { from } \\
\text { hibernacula to summer foraging } \\
\text { areas. }^{73} \text { Forage and commute } \\
\text { adjacent to vegetation. }{ }^{72}\end{array}$ & $\begin{array}{l}\text { or lava tube caves and mines } \\
\text { are known to be used } \\
\text { elsewhere with arousal and } \\
\text { movement within or between } \\
\text { sites, possibly responding to } \\
\text { changing temperature. }{ }^{5,73,74,82}\end{array}$ & $\begin{array}{l}\text { buildings, and basal tree } \\
\text { hollows elsewhere. } .^{2,5,72,73,81,} \\
82,83 \text { Females prefer cooler } \\
\text { maternity roosts than other } \\
\text { vespertilionid bat species. }^{2}\end{array}$ & $\begin{array}{l}\text { attics, root cellars, and } \\
\text { pocket/daylight caves. }{ }^{1,21,31,32,} \\
75 \text { Reported in caves, mines, } \\
\text { buildings and large diameter } \\
\text { basal tree hollows elsewhere. } \\
5,72,81,82,83\end{array}$ & $\begin{array}{l}\text { males and } 1.3 \mathrm{~km}+/-0.2 \mathrm{SD} \text { for } \\
\text { females in coastal California; } \\
\text { maximum distance traveled from the } \\
\text { day roost was } 10.5 \mathrm{~km} .^{72}\end{array}$ \\
\hline Species / Comments & Winter Roost & Summer Maternity Roost & Summer Day/Night Roost & Home Range/Foraging Distance \\
\hline $\begin{array}{l}\text { Big Brown Bat } \\
\text { (Eptesicus fuscus) } \\
\text { Males often roost solitarily } \\
\text { during summer. Rarely move } \\
\text { more than } 80 \text { km between } \\
\text { summer and winter roosts. }{ }^{2,6} \\
\text { Roost switching is common at } \\
\text { natural roosts, but show high } \\
\text { fidelity to man-made roosts. }{ }^{64,65,} \\
71\end{array}$ & $\begin{array}{l}\text { Caves, mines, and some } \\
\text { evidence for rock crevices } \\
\text { which are probably the most } \\
\text { widespread winter roost in } \\
\text { Montana. }{ }^{1,31,84} \text { Known to use } \\
\text { narrow deep rock crevices or } \\
\text { erosion holes in steep valley } \\
\text { walls on the Canadian prairie } \\
\text { and buildings in Ohio. } 6 \text {, } 62\end{array}$ & $\begin{array}{l}\text { Buildings, bridges, large } \\
\text { diameter trees snags with } \\
\text { hollows or loose bark in } \\
\text { Montana. }{ }^{1,75} \text { Primarily large } \\
\text { diameter tree snag hollows } \\
\text { and crevices, but also live } \\
\text { aspen hollows, in more } \\
\text { sparsely spaced stands, deep } \\
\text { rock crevices, and older } \\
\text { human structures are known } \\
\text { to be used elsewhere. }{ }^{6,29,59,} \\
64,65,66,67,68,71\end{array}$ & 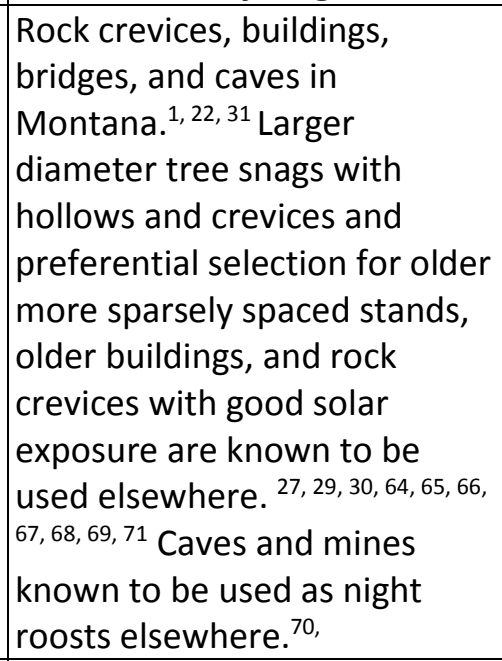 & $\begin{array}{l}\text { Average of } 1.5 \mathrm{~km}+/-0.9 \mathrm{SD} \text { (range } \\
0.4 \text { to } 1.8 \mathrm{~km} \text { ) from roosts to } \\
\text { capture locations with average } \\
\text { movement between successive } \\
\text { roosts of } 1.1 \mathrm{~km}+/-0.7 \mathrm{SD} \text { (range } 0.4 \\
\text { to } 2.0 \mathrm{~km} \text { ) in the Black Hills of South } \\
\text { Dakota. }{ }^{29} \text { Average one-way travel } \\
\text { distances between day roosts and } \\
\text { foraging areas of } 1.8 \mathrm{~km}+/-0.1 \mathrm{SE} \text { ) } \\
\text { range }(0.3 \text { to } 4.4 \mathrm{~km} \text { ) in southern } \\
\text { British Columbia. }{ }^{64}\end{array}$ \\
\hline $\begin{array}{l}\text { Spotted Bat } \\
\text { (Euderma maculatum) } \\
\text { High roost site fidelity with } \\
\text { multiple individuals following } \\
\text { the same nightly commuting } \\
\text { routes up side canyons to } \\
\text { foraging areas at speeds of up to } \\
53 \mathrm{~km} / \mathrm{hr}^{8,49} \text { Forage over } \\
\text { clearings and along cliff rims. }{ }^{49}, \\
50,51\end{array}$ & $\begin{array}{l}\text { Not documented in Montana. } \\
\text { Deep rock cracks and crevices } \\
\text { are commonly used elsewhere } \\
\text { and caves and human } \\
\text { structures are rarely used } \\
\text { elswhere. }{ }^{1,2,7,51}\end{array}$ & $\begin{array}{l}\text { Not documented in Montana. } \\
\text { Rock cracks and crevices in } \\
\text { upper portions of tall remote } \\
\text { south facing cliffs near } \\
\text { perennial waters are used } \\
\text { elsewhere. }{ }^{1,2,7,8,50}\end{array}$ & $\begin{array}{l}\text { Buildings and other human } \\
\text { structures in Montana. }{ }^{1,47} \\
\text { Rock cracks and crevices in } \\
\text { upper portions of tall remote } \\
\text { cliffs near perennial waters, } \\
\text { and, apparently more rarely, } \\
\text { cave entrances and buildings } \\
\text { elsewhere. } 2,7,8,45,46,47,48,49,50, \\
51\end{array}$ & $\begin{array}{l}50-60 \mathrm{~km} \text { round trip flight distances } \\
\text { nightly with average home range size } \\
\text { of } 297+/-25 \mathrm{SE} \text { (range }=242.5 \text { to } \\
363.8 \text { ) square } \mathrm{km} \text { in northern } \\
\text { Arizona. }{ }^{8} \text { Nightly round trip } \\
\text { commutes of }>77 \mathrm{~km} \text { between day } \text { roosts, foraging areas, and night } \\
\text { roosts that differed in elevation by } \\
\text { ca. } 2,000 \text { meters in northern } \\
\text { Arizona. }{ }^{49} \text { Nightly round trip foraging } \\
\text { flights of } 12 \text { to } 20 \mathrm{~km} \text { in British } \\
\text { Columbia. }\end{array}$ \\
\hline $\begin{array}{l}\text { Silver-haired Bat } \\
\text { (Lasionycteris noctivagans) }\end{array}$ & $\begin{array}{l}\text { Not documented in Montana. } \\
\text { Known to use loose bark, basal } \\
\text { tree cavities, cavities under } \\
\text { tree roots, and rock crevices } \\
\end{array}$ & $\begin{array}{l}\text { Large diameter tree snags } \\
\text { with loose bark or cavities in } \\
\text { Montana. }{ }^{1,9,26} \text { Hollows and } \\
\text { crevices in live aspen and }\end{array}$ & $\begin{array}{l}\text { Large diameter tree snags with } \\
\text { loose bark or cavities and a } \\
\text { building in Montana. }{ }^{1,26,78} \\
\text { Large diameter trees or tree }\end{array}$ & $\begin{array}{l}\text { Distance between capture locations } \\
\text { and roost snags ranged from } 0.1 \text { to } \\
3.4 \mathrm{~km} \text { (averages for juvenile males, } \\
\text { juvenile females, adult males, and }\end{array}$ \\
\hline
\end{tabular}


on more southerly aspects and large diameter and taller

in older stands of trees,

elsewhere with retreat to

more underground sites at

lower temperatures. 93 Use of

mines is also known. ${ }^{94}$

9, 59, 86, 90, 91, 92, 95, 96 snags in older stands with

hollows and crevices are

predominant summer roost

lower canopy closure stands

known to be used elsewhere.

elsewhere, but rock crevices,

buildings, bridges, and other

human structures also used. ${ }^{9}$

22, 86, 90, 91, 96 adult females were $1.3,1.5,1.8$, and

$0.5 \mathrm{~km}$, respectively) in northeastern Washington. ${ }^{96}$ 


\begin{tabular}{|c|c|c|c|c|}
\hline Species / Comments & Winter Roost & Summer Maternity Roost & Summer Day/Night Roost & Home Range/Foraging Distance \\
\hline $\begin{array}{l}\text { Eastern Red Bat } \\
\text { (Lasiurus borealis) } \\
\text { Species is a solitary rooster at } \\
\text { heights of } 1 \text { to } 6 \text { meters from } \\
\text { the ground, but forage and } \\
\text { migrate in groups. }{ }^{10}\end{array}$ & $\begin{array}{l}\text { Not documented in Montana } \\
\text { and thought to migrate far to } \\
\text { the south where they use tree } \\
\text { roosts on warmer days and } \\
\text { nights and retreat below leaf } \\
\text { litter when temperatures dip } \\
\text { below freezing. } 10,54\end{array}$ & $\begin{array}{l}\text { Maternity roosts or lactating } \\
\text { individuals have not been } \\
\text { detected in Montana. } \\
\text { Elsewhere, known to roost } \\
\text { mostly in dense foliage that } \\
\text { provides shade and } \\
\text { protection from the wind, but } \\
\text { also on trunks, of larger } \\
\text { diameter mature deciduous } \\
\text { and conifer trees, often in } \\
\text { riparian areas. }{ }^{10,52,53,55,56,57}\end{array}$ & $\begin{array}{l}\text { Not documented in Montana. } \\
\text { Elsewhere, known to roost } \\
\text { mostly in denser foliage, but } \\
\text { also on trunks, of larger } \\
\text { diameter mature deciduous } \\
\text { and conifer trees, often in } \\
\text { riparian areas. Also more } \\
\text { rarely in shrubs, under leaf } \\
\text { litter, and on human } \\
\text { structures. } 10,52,53,55,56,57\end{array}$ & $\begin{array}{l}\text { Maximum distances traveled to } \\
\text { foraging areas averaged } 1.24 \mathrm{~km} \\
\text { (range } 0.19 \text { to } 3.28 \text { ) and foraging } \\
\text { areas averaged } 94.4 \mathrm{Ha}+/-20.2 \mathrm{SE} \\
\text { with no significant differences } \\
\text { between sex and age classes in } \\
\text { Mississippi. }{ }^{52} \text { Maximum distances } \\
\text { traveled from diurnal roosts to } \\
\text { foraging areas ranged from } 1.2 \text { to } 5.5 \\
\text { km for females and } 1.4 \text { to } 7.4 \mathrm{~km} \text { for } \\
\text { males with average foraging area } \\
\text { size of } 334 \mathrm{Ha} \text { in Kentucky }{ }^{53}\end{array}$ \\
\hline $\begin{array}{l}\text { Hoary Bat } \\
\text { (Lasiurus cinereus) } \\
\text { Species is a solitary rooster at } \\
\text { heights of } 3 \text { to } 5 \text { meters from } \\
\text { the ground, but forage and } \\
\text { migrate in groups. }{ }^{11}\end{array}$ & $\begin{array}{l}\text { Not documented and thought } \\
\text { to migrate far to the south of } \\
\text { Montana in the winter. }{ }^{11}\end{array}$ & $\begin{array}{l}\text { Only a bridge roost } \\
\text { documented in Montana. }{ }^{1} \\
\text { Known to be a solitary rooster } \\
\text { in deciduous and conifer tree } \\
\text { foliage that offers shelter } \\
\text { from the wind and more } \\
\text { southern exposure to the sun } \\
\text { elsewhere. } 11,85,86,87,88,89\end{array}$ & $\begin{array}{l}\text { A bridge and cottonwood } \\
\text { foliage in Montana. }{ }^{1} \text { Known to } \\
\text { roost in deciduous and conifer } \\
\text { tree foliage elsewhere. }{ }^{1,11,85} \\
86,87\end{array}$ & $\begin{array}{l}\text { Females traveled one-way distances } \\
\text { up to } 20 \mathrm{~km} \text { from day roosts while } \\
\text { on first of up to five nightly foraging } \\
\text { bouts in Manitoba Canada. }{ }^{85}\end{array}$ \\
\hline $\begin{array}{l}\text { California Myotis } \\
\text { (Myotis californicus) } \\
\text { Roosts alone or in groups. }{ }^{12}\end{array}$ & $\begin{array}{l}\text { Recent acoustic and telemetry } \\
\text { data indicates species likely } \\
\text { overwinters in rock crevices in } \\
\text { Montana. } 1 \text {, Nate Schwab, personal } \\
\text { communication Rock crevices, } \\
\text { caves, mines, tunnels, and } \\
\text { buildings are used elsewhere. } \\
2,12,25,61\end{array}$ & $\begin{array}{l}\text { Not documented in Montana. } \\
\text { Elsewhere known to roost } \\
\text { under loose bark or in holes } \\
\text { or cracks in more isolated } \\
\text { larger diameter tree snags in } \\
\text { areas with lower canopy } \\
\text { closure. }{ }^{58,59} \text { More rarely, } \\
\text { known to use buildings } \\
\text { elsewhere. } 60\end{array}$ & $\begin{array}{l}\text { A house and a cellar in } \\
\text { Montana. }{ }^{32} \text { Elsewhere known } \\
\text { to roost under loose bark or in } \\
\text { holes or cracks in more } \\
\text { isolated larger diameter tree } \\
\text { snags in areas with lower } \\
\text { canopy closure. }{ }^{58,59} \text { Also } \\
\text { known to use rock crevices, } \\
\text { bridges, buildings, and other } \\
\text { human structures elsewhere. } \\
12,21,22,30,60\end{array}$ & *No documentation found. \\
\hline $\begin{array}{l}\text { Western Small-footed Myotis } \\
\text { (Myotis ciliolabrum) } \\
\text { Mostly a solitary rooster, but } \\
\text { sometimes aggregates in small } \\
\text { groups. Fidelity to roost areas is } \\
\text { shown, but roost switching }\end{array}$ & $\begin{array}{l}\text { Caves and mines documented } \\
\text { in Montana. }{ }^{1,76,84} \text { Known to } \\
\text { use lava tube caves, deep } \\
\text { cracks in ground, deep rock } \\
\text { crevices, tunnels, and drill } \\
\text { holes in rock elsewhere. } 2,13,77\end{array}$ & $\begin{array}{l}\text { Rock outcrop crevices with } \\
\text { good solar exposure in } \\
\text { Montana. }{ }^{1} \text { Known to rely } \\
\text { mostly on vertical and } \\
\text { horizontal crevices in cliffs } \\
\text { and rock outcrops, but also }\end{array}$ & $\begin{array}{l}\text { Rock outcrop crevices, bridges, } \\
\text { caves, mines, and buildings in } \\
\text { Montana. }{ }^{1,31,32} \text { Known to use } \\
\text { rock outcrops, cracks in } \\
\text { ground, tree hollows, and } \\
\text { trees with loose bark }\end{array}$ & $\begin{array}{l}6 \text { to } 24 \mathrm{~km} \text { round trip travel } \\
\text { distances from roosts to foraging } \\
\text { areas in north central Oregon. }{ }^{63}\end{array}$ \\
\hline
\end{tabular}


within those areas is frequent ${ }^{13}$

${ }^{63}$ Also show a high fidelity to

commuting corridors. ${ }^{63}$ documented using buildings elsewhere. ${ }^{13,63}$

elsewhere. ${ }^{13,63}$ No bats were detected using night roosts in a north central Oregon study. ${ }^{63}$ 


\begin{tabular}{|c|c|c|c|c|}
\hline Species / Comments & Winter Roost & Summer Maternity Roost & Summer Day/Night Roost & Home Range/Foraging Distance \\
\hline $\begin{array}{l}\text { Long-eared Myotis } \\
\text { (Myotis evotis) } \\
\text { Suspected of only traveling } \\
\text { short distances between } \\
\text { summer and winter roosts. }{ }^{14} \\
\text { Have low fidelity to individual } \\
\text { roosts, but high fidelity to roost } \\
\text { areas. }{ }^{97,98,99}\end{array}$ & $\begin{array}{l}\text { Caves and mines. }{ }^{1,75,84} \text { May } \\
\text { also use deeper rock crevices. } \\
14\end{array}$ & $\begin{array}{l}\text { Caves, cliff and rock outcrop } \\
\text { crevices, and large diameter } \\
\text { trees in Montana. }{ }^{1,26,76} \\
\text { Known to use sheltered } \\
\text { erosion cavities on stream } \\
\text { banks, crevices in basalt, } \\
\text { conifer stumps, conifer snags, } \\
\text { buildings, and mine tunnels } \\
\text { elsewhere. }{ }^{14,97,98,99}\end{array}$ & $\begin{array}{l}\text { Large diameter trees, rock } \\
\text { outcrops, buildings, and caves } \\
\text { in Montana. }{ }^{1,26,31,79} \text { Known to } \\
\text { use buildings, trees/snags with } \\
\text { loose bark, trestle bridges, } \\
\text { mines, rock crevices, stream } \\
\text { bank cavities, and sink holes } \\
\text { elsewhere. }{ }^{14,21,27,97,98,99}\end{array}$ & $\begin{array}{l}\text { Traveled an average of } 970 \text { meters } \\
\text { (range } 35-5,154 \text { meters) between } \\
\text { roosts in western Montana. }{ }^{26} \text { Moved } \\
1 \text { to } 812 \text { meters between day roosts } \\
\text { and had roosting home ranges that } \\
\text { ranged from } 0.08 \text { to } 1.93 \text { ha in } \\
\text { Alberta. }{ }^{97} \text { Traveled } 620 \text { meters from } \\
\text { capture sites to day roosts in } \\
\text { western Oregon } .^{98} \text { Traveled an } \\
\text { average distance between day roosts } \\
\text { of } 148.9 \text { m in northeastern } \\
\text { Washington. }\end{array}$ \\
\hline \begin{tabular}{|l|} 
Little Brown Myotis \\
(Myotis lucifugus) \\
Show high fidelity to summer \\
colonies and hibernacula across \\
years, but some individuals \\
relocated between years a \\
median distance of $315 \mathrm{~km}$ \\
between hibernacula (range 6 to \\
$563 \mathrm{~km}$ ) and 431 km between \\
summer roosts (range 25 to 464 \\
km). ${ }^{100}$ Males and \\
nonreproductive females \\
occupy cooler roosts than \\
pregnant or lactating females. ${ }^{15}$
\end{tabular} & \begin{tabular}{|l|} 
Caves and mines with high \\
humidities and temperatures \\
above freezing in Montana and \\
elswhere. ${ }^{1,31,36,75,84}$ May also \\
use deeper rock crevices. ${ }^{15}$ \\
Predominantly documented \\
using caves elsewhere. ${ }^{100}$
\end{tabular} & $\begin{array}{l}\text { Attics and roofs of buildings, } \\
\text { bridges, and bat houses in } \\
\text { Montana. }{ }^{1} \text { Known to use } \\
\text { cracks or hollows in larger } \\
\text { diameter tree snags in older } \\
\text { stands, rock crevices, and } \\
\text { buildings elsewhere. }{ }^{2,15,35,90,} \\
101,102,103\end{array}$ & $\begin{array}{l}\text { Large diameter tree, rock } \\
\text { crevices, buildings, bridges, } \\
\text { caves, and bat houses in } \\
\text { Montana. }{ }^{1,26,31,80} \text { Known to } \\
\text { use cracks or hollows in larger } \\
\text { diameter tree snags in older } \\
\text { stands, wood piles, and rock } \\
\text { crevices elsewhere. }{ }^{15,35,90} \\
\text { Caves and mines known to be } \\
\text { used as night roosts } \\
\text { elsewhere. }{ }^{70}\end{array}$ & $\begin{array}{l}\text { Average } 970 \text { meters (range } 35-5,154 \\
\text { meters) between roosts in western } \\
\text { Montana. }{ }^{26} \text { Traveled } 10 \text { to } 647 \mathrm{~km} \\
\text { from hibernacula to summer } \\
\text { colonies in Manitoba and } \\
\text { northwestern Ontario, Canada. }{ }^{100} \\
\text { Female home range averaged } 30.1 \\
\text { ha }+/-15.0 \text { SD during pregnancy and } \\
17.6 \text { ha }+/-9.1 \text { SD during lactation in } \\
\text { Quebec, Canada. }{ }^{101} \text { Males moved } \\
\text { and average of } 275 \text { m }+/-406 \text { SD } \\
\text { between successive roosts, had } \\
\text { mean minimum roosting areas of } 3.9 \\
\text { ha }+/-7.9 \text { SD, mean minimum } \\
\text { foraging areas of } 52.0 \text { ha }+/-57.4 \text { SD, } \\
\text { mean distance between roosting and } \\
\text { foraging areas of } 254 \text { m }+/-254.2 \text { SD, } \\
\text { and mean distances between } \\
\text { capture sites and first roosts of } 761 \\
m+/-623 \text { SD in New Brunswick. }{ }^{102} \\
\text { Mean home range area was } 143 \text { ha } \\
+/-71.0 \text { SE in New York. }{ }^{103}\end{array}$ \\
\hline
\end{tabular}




\begin{tabular}{|c|c|c|c|c|}
\hline Species / Comments & Winter Roost & Summer Maternity Roost & Summer Day/Night Roost & Home Range/Foraging Distance \\
\hline \begin{tabular}{|l|} 
Northern Myotis \\
(Myotis septentrionalis) \\
Low roost site fidelity, but often \\
stay in same general area within \\
a season. May travel up to 56 \\
km between summer and winter \\
roosts. 16
\end{tabular} & $\begin{array}{l}\text { Only known from a single } \\
\text { abandoned coal mine in } \\
\text { Montana. }{ }^{1,75} \text { Known from } \\
\text { caves, with a preference to } \\
\text { cluster in deep crevices and } \\
\text { possibly move between caves } \\
\text { within a winter elsewhere. }{ }^{16}\end{array}$ & $\begin{array}{l}\text { Not documented in Montana. } \\
\text { Known to use bark and } \\
\text { hollows of larger diameter } \\
\text { trees, usually in decay, and } \\
\text { building crevices and bat } \\
\text { houses elsewhere. } 16,29,35,69, \\
102\end{array}$ & $\begin{array}{l}\text { Not documented in Montana. } \\
\text { Known to use bark and } \\
\text { hollows of larger diameter } \\
\text { trees, usually in decay, and } \\
\text { building crevices and bat } \\
\text { houses elsewhere. }{ }^{16,29,35,69} \\
\text { Caves and mines known to be } \\
\text { used as night roosts } \\
\text { elsewhere. }\end{array}$ & $\begin{array}{l}\text { Average of } 2.2 \mathrm{~km}+/-1.4 \text { SD (range } 0.1 \\
\text { to } 5.9 \mathrm{~km} \text { ) from roosts to capture } \\
\text { locations with average movement } \\
\text { between successive roosts of } 0.6 \mathrm{~km}+/- \\
0.5 \mathrm{SD} \text { (range } 0.1 \text { to } 1.5 \mathrm{~km} \text { ) in the Black } \\
\text { Hills of South Dakota. }{ }^{29} \text { Females/males } \\
\text { moved and average of } 457 / 158 \mathrm{~m}+/- \\
329 / 127 \text { SD between successive roosts, } \\
\text { had mean minimum roosting areas of } \\
8.6 / 1.4 \text { ha }+/-9.2 / 1.4 \mathrm{SD} \text {, mean } \\
\text { minimum foraging areas of } 46.2 / 13.5 \text { ha } \\
+/-44.4 / 8.3 \mathrm{SD} \text {, mean distance between } \\
\text { roosting and foraging areas of } \\
584.6 / 293.0 \mathrm{~m}+/-405.8 / 282.8 \mathrm{SD} \text {, and } \\
\text { mean distances between capture sites } \\
\text { and first roosts of } 1001 / 402 \mathrm{~m}+/- \\
693 / 452 \text { SD in New Brunswick. }{ }^{102}\end{array}$ \\
\hline $\begin{array}{l}\text { Fringed Myotis } \\
\text { (Myotis thysanodes) } \\
\text { Very sensitive to roost site } \\
\text { disturbance. }{ }^{17} \text { Maintain at least } \\
\text { some level of group integrity } \\
\text { when switching roosts. }^{29}\end{array}$ & $\begin{array}{l}\text { Winter presence in Montana } \\
\text { known from a single individual } \\
\text { found roosting in a cave. Some } \\
\text { individuals may migrate south } \\
\text { of Montana. }^{1}\end{array}$ & $\begin{array}{l}\text { Caves. }{ }^{1} \text { Known to use cracks } \\
\text { and hollows of larger } \\
\text { diameter trees, usually in } \\
\text { decay, rock crevices on south- } \\
\text { facing slopes, and buildings } \\
\text { elsewhere. }{ }^{17,29}\end{array}$ & $\begin{array}{l}\text { Caves in Montana. }{ }^{1,32} \text { Known } \\
\text { to use cracks and hollows of } \\
\text { larger diameter trees, usually } \\
\text { in decay, rock crevices on } \\
\text { south-facing slopes, mines, } \\
\text { buildings, and bridges } \\
\text { elsewhere. }{ }^{17,21,22,29}\end{array}$ & $\begin{array}{l}\text { Average of } 1.0 \mathrm{~km}+/-0.6 \mathrm{SD} \text { (range } \\
0.1 \text { to } 2.0 \mathrm{~km} \text { ) from roosts to } \\
\text { capture locations with average } \\
\text { movement between successive } \\
\text { roosts of } 0.5 \mathrm{~km}+/-0.6 \mathrm{SD} \text { (range } 0.1 \\
\text { to } 2.0 \mathrm{~km} \text { ) in the Black Hills of South } \\
\text { Dakota. }\end{array}$ \\
\hline $\begin{array}{l}\text { Long-legged Myotis } \\
\text { (Myotis volans) }\end{array}$ & $\begin{array}{l}\text { Caves and mines in Montana } \\
\text { and elsewhere. } 1,19,31,36,75,84\end{array}$ & $\begin{array}{l}\text { Large diameter trees in } \\
\text { Montana. }{ }^{1,26} \text { Elsewhere in } \\
\text { taller, but random to normal } \\
\text { diameter tree snags with } \\
\text { loose bark or cracks, } \\
\text { especially in areas with less } \\
\text { habitat fragmentation, } \\
\text { greater snag density but with } \\
\text { greater tree spacing. } 28,33,34, \\
35 \text { Also in rock crevices, cracks } \\
\text { in the ground, and buildings } \\
\text { are known to be used } \\
\text { elsewhere with south-facing } \\
\text { roosts preferred. } 2,29\end{array}$ & $\begin{array}{l}\text { Buildings, mines, caves and } \\
\text { large diameter trees in } \\
\text { Montana. } 1,26,31,32,78,79 \\
\text { Elsewhere in taller but random } \\
\text { to larger diameter tree snags } \\
\text { with loose bark or cracks, } \\
\text { especially in areas with less } \\
\text { habitat fragmentation, greater } \\
\text { snag density but with greater } \\
\text { tree spacing, are known to be } \\
\text { used elsewhere with south- } \\
\text { facing roosts preferred. }{ }^{27,28,29,} \\
30,33,34,35 \text { Also in buildings, } \\
\text { cracks in the ground, rock } \\
\text { crevices, and caves. }{ }^{19,36}\end{array}$ & $\begin{array}{l}\text { Average of } 2.0 \mathrm{~km}+/-0.1 \mathrm{SE} \text { from } \\
\text { roosts to capture locations with } \\
\text { average movement between } \\
\text { successive roosts of } 1.4 \mathrm{~km}+/-0.1 \mathrm{SE} \\
\text { across four study areas in } \\
\text { Washington and Oregon. }{ }^{28} \text { Average } \\
\text { of } 1.9 \mathrm{~km}+/-1.6 \mathrm{SD} \text { (range } 0.4 \text { to } 3.7 \\
\mathrm{~km} \text { ) from roosts to capture locations } \\
\text { with average movement between } \\
\text { successive roosts of } 0.7 \mathrm{~km}+/-0.5 \\
\text { SD (range } 0.2 \text { to } 1.6 \mathrm{~km} \text { ) in the Black } \\
\text { Hills of South Dakota. }{ }^{29} \text { Average } \\
\text { home range size of } 647 \text { ha }+/-354 \mathrm{SE} \\
\text { (range } 16.5 \text { to } 3,029 \text { ha) for males, } \\
448 \text { ha }+/-78.7 \text { SE for pregnant }\end{array}$ \\
\hline
\end{tabular}




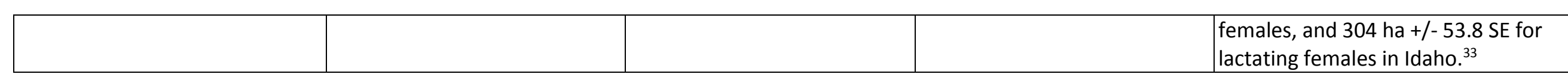




\begin{tabular}{|c|c|c|c|c|}
\hline Species / Comments & Winter Roost & Summer Maternity Roost & Summer Day/Night Roost & Home Range/Foraging Distance \\
\hline $\begin{array}{l}\text { Yuma Myotis } \\
\text { (Myotis yumanensis) } \\
\text { Sensitive to roost site } \\
\text { disturbance. }^{2}\end{array}$ & $\begin{array}{l}\text { Not documented in Montana, } \\
\text { but acoustic evidence indicates } \\
\text { overwintering in rock crevices } \\
\text { in cliffs. }{ }^{1}\end{array}$ & $\begin{array}{l}\text { Building, bridges, and bat } \\
\text { houses in Montana. }^{1} \\
\text { Buildings, bridges, caves, } \\
\text { mines, and abandoned cliff } \\
\text { swallow nests are known } \\
\text { elsewhere. } 2,20,21,22,25\end{array}$ & $\begin{array}{l}\text { Buildings, bridges, and bat } \\
\text { houses in Montana. }{ }^{1,79} \text { Large } \\
\text { diameter trees, buildings, } \\
\text { rock/cliff crevices and } \\
\text { abandoned cliff swallow nests } \\
\text { elsewhere. } 2,21,22,23,24,25,30\end{array}$ & $\begin{array}{l}\text { Average of } 2 \mathrm{~km} \text { (range } 0.59-3.5 \mathrm{~km} \text { ) } \\
\text { from roosts to capture locations in } \\
\text { California. }{ }^{24} 4 \mathrm{~km} \text { from maternity } \\
\text { roost to foraging areas in British } \\
\text { Columbia. }{ }^{25}\end{array}$ \\
\hline
\end{tabular}

${ }^{1}$ supported by observations in Montana's statewide point observation database.

${ }^{2}$ Adams, R.A. 2003. Bats of the Rocky Mountain West: natural history, ecology, and conservation. University Press of Colorado. Boulder, Colorado. 289 p.

${ }^{3}$ Lewis, S,E. 1996. Low roost-site fidelity in pallid bats: associated factors and effect on group stability. Behavioral Ecology and Sociobiology 39:335-344.

${ }^{4}$ Hermanson, J.W. and T.J. O'Shea. 1983. Antrozous pallidus. Mammalian Species Account 213:1-8.

${ }^{5}$ Kunz, T.H. and R.A. Martin. 1982. Plecotus townsendii. Mammalian Species Account 175:1-6.

${ }^{6}$ Kurta, A. and R.H. Baker. 1990. Eptesicus fuscus. Mammalian Species Account 356:1-10.

${ }^{7}$ Watkins, L.C. 1977. Euderma maculatum. Mammalian Species Account 77:1-4.

${ }^{8}$ Chambers, C.L., M.J. Herder, K. Yasuda, D.G. Mikesic, S.M. Dewhurst, W.M. Masters, and D. Vleck. 2011. Roosts and home ranges of spotted bats (Euderma maculatum) in northern Arizona. Canadian Journal of Zoology 89:1256-1267.

${ }^{9}$ Kunz, T.H. 1982. Lasionycteris noctivagans. Mammalian Species Account 172:1-5.

${ }^{10}$ Shump, K.A. Jr. and A.U. Shump. 1982. Lasiurus borealis. Mammalian Species Account 183:1-6.

${ }^{11}$ Shump, K.A. Jr. and A.U. Shump. 1982. Lasiurus cinereus. Mammalian Species Account 185:1-5.

${ }^{12}$ Simpson, M.R. 1993. Myotis californicus. Mammalian Species Account 428:1-4.

${ }^{13}$ Holloway, G.L. and R.M.R. Barclay. 2001. Myotis ciliolabrum. Mammalian Species Account 670:1-5.

${ }^{14}$ Manning, R.W. and J.K. Jones, Jr. 1989. Myotis evotis. Mammalian Species Account 329:1-5.

${ }^{15}$ Fenton, M.B. and R.M.R. Barclay. 1980. Myotis lucifugus. Mammalian Species Account 142:1-8.

${ }^{16}$ Caceres, M.C. and R.M.R. Barclay. 2000. Myotis septentrionalis. Mammalian Species Account 634:1-4.

${ }^{17}$ O'Farrell, M.J. and E.H. Studier. 1980. Myotis thysanodes. Mammalian Species Account 137:1-5.

${ }^{18}$ Keinath, D.A. 2004. Fringed Myotis (Myotis thysanodes): a technical conservation assessment. USDA Forest Service, Rocky Mountain Region. 64 pp. Available at: http://www.fs.fed.us/r2/projects/scp/assessments/fringedmyotis.pdf

19 Warner, R.M. and N.J. Czaplewski. 1984. Myotis volans. Mammalian Species Account 224:1-4.

${ }^{20}$ Betts, B.J. Microclimate in Hell's Canyon mines used by maternity colonies of Myotis yumanensis. Journal of Mammalogy 78(4):1240-1250.

${ }^{21}$ Dalquest, W.W. 1947. Notes on the natural history of the bat, Myotis yumanensis, in California, with a description of a new race. American Midland Naturalist 38:224-247.

${ }^{22}$ Geluso, K. and J.N. Mink. 2009. Use of bridges by bats (Mammalia: Chiroptera) in the Rio Grande Valley, New Mexico. The Southwestern Naturalist 54(4):421-429.

${ }^{23}$ Licht, P. and P. Leitner. 1967. Behavioral responses to high temperatures in three species of California bats. Journal of Mammalogy 48(1):52-61.

${ }^{24}$ Evelyn, M.J., D.A. Stiles, and R.A. Young. 2004. Conservation of bats in suburban landscapes: roost selection by Myotis yumanensis in a residential area in California. Biological Conservation 115:463-473.

${ }^{25}$ Nagorsen, D.W. and R.M. Brigham. 1993. The bats of British Columbia. University of British Columbia Press, Vancouver.

${ }^{26}$ Schwab, N. 2006. Roost-site selection and potential prey sources after wildland fire for two insectivorous bat species (Myotis evotis and Myotis lucifugus) in midelevation forests of western Montana. Master of Science Thesis. University of Montana. Missoula, MT. 89 pp.

${ }^{27}$ Arnett, E.B. and J.P. Hayes. 2009. Use of conifer snags as roosts by female bats in western Oregon. Journal of Wildlife Management 73(2):214-225. 
${ }^{28}$ Baker, M.D. and M.J. Lacki. 2006. Day-roosting habitat of female long-legged myotis in ponderosa pine forests. Journal of Wildlife Management 70(1):207-215.

${ }^{29}$ Cryan, P.M., M.A. Bogan, and G.M. Yanega. 2001. Roosting habits of four bat species in the Black Hills of South Dakota. Acta Chiropterologica 3(1):43-52.

${ }^{30}$ Dalquest, W.W. and M.C. Ramage. 1946. Notes on the Long-legged Bat (Myotis volans) at Old Fort Tejon and vicinity, California. Journal of Mammalogy 27(1):60-63.

${ }^{31}$ Hendricks, P., D.L. Genter, and S. Martinez. 2000. Bats of Azure Cave and the Little Rocky Mountains, Montana. The Canadian Field Naturalist 114:89-97.

${ }^{32}$ Hoffman, R.S., D.L. Pattie, and J.F. Bell. 1969. The distribution of some mammals in Montana. II. Bats. Journal of Mammalogy 50(4):737-741.

33 Johnson, J.S., M.J. Lacki, and M.D. Baker. 2007. Foraging ecology of Long-legged Myotis (Myotis volans) in north-central Idaho. Journal of Mammalogy 88(5):12611270.

${ }^{34}$ Lacki, M.J., M.D. Baker, and J.S. Johnson. 2010. Geographic variation in roost-site selection of Long-legged Myotis in the Pacific Northwest. Journal of Wildlife Management 74(6):1218-1228.

35 Psyllakis, J.M. and R.M. Brigham. 2005. Characteristics of diurnal roosts used by female Myotis bats in sub-boreal forests. Forest Ecology and Management 223:93102.

${ }^{36}$ Schowalter, D.B. 1980. Swarming, reproduction, and early hibernation of Myotis lucifugus and M. volans in Alberta, Canada. Journal of Mammalogy 61(2):350-354.

${ }^{37}$ Baker, M.D., M.J. Lacki, G.A. Falxa, P.L. Droppleman, R.A. Slack, and S.A. Slankard. 2008. Habitat use of Pallid Bats in coniferous forests of northern California. Northwest Science 82(4):269-275.

${ }^{38}$ Lewis, S.E. 1996. Low roost-site fidelity in Pallid Bats: associated factors and effect on group stability. Behavioral Ecology and Sociobiology 39(5):335-344.

${ }^{39}$ Lewis, S.E. 1994. Night roosting ecology of Pallid Bats (Antrozous pallidus) in Oregon. American Midland Naturalist 132(2):219-226.

${ }^{40}$ Schorr, R.A. and J.L. Siemers. 2013. Characteristics of roosts of male pallid bats (Antrozouz pallidus) in southeastern Colorado.

${ }^{41}$ Vaughan, T.A. and T.J. O'Shea. 1976. Roosting ecology of the Pallid Bat, Antrozous pallidus. Journal of Mammalogy 57(1):19-42.

42 Brown, P. 1982. Activity patterns and foraging behavior in Antrozous pallidus as determined by radiotelemetry. Bat Research News $23(4): 62$.

${ }^{43}$ Davis, R. 1969. Growth and development of young Pallid Bats, Antrozouz pallidus. Journal of Mammalogy 50(4):729-736.

${ }^{44}$ O'Shea, T.J. 1977. Nocturnal and seasonal activities of the Pallid Bat, Antrozous pallidus. Journal of Mammalogy 58(3):269-284.

${ }^{45}$ Geluso, K. 2000. Distribution of the Spotted Bat (Euderma maculatum) in Nevada, including notes on reproduction. The Southwestern Naturalist 45(3):347-352.

${ }^{46}$ Leonard, M.L. and M.B. Fenton. 1983. Habitat use by Spotted Bats (Euderma maculatum, Chiroptera: Vespertilionidae): roosting and foraging behavior. Canadian Journal of Zoology 61:1487-1491.

${ }^{47}$ Nicholson, A.J. 1950. A record of the Spotted Bat (Euderma maculata) for Montana. Journal of Mammalogy 32(1):197.

${ }^{48}$ Poche, R.M. and G.A. Ruffner. 1975. Roosting behavior of male Euderma maculatum from Utah. Great Basin Naturalist 35(1):121-122.

${ }^{49}$ Rabe, M.J., MS. Siders, C.R. Miller, and T.K. Snow. 1998. Long foraging distance for a Spotted Bat (Euderma maculatum) in northern Arizona. The Southwestern Naturalist 43(2):266-286.

${ }^{50}$ Wai-Ping, V. and M.B. Fenton. 1989. Ecology of Spotted Bat (Euderma maculatum) roosting and foraging behavior. Journal of Mammalogy 70(3):617-622.

${ }^{51}$ Sherwin, R.E. and W.L. Gannon. 2005. Documentation of an urban winter roost of the Spotted Bat (Euderma maculatum). The Southwestern Naturalist 50(3):402407.

${ }^{52}$ Elmore, L., D.A. Miller, and F.J. Vileella. 2005. Foraging area size and habitat use by red bats (Lasiurus borealis) in an intensively managed pine landscape in Mississippi. American Midland Naturalist 153:405-417.

${ }^{53}$ Hutchinson, J.T. and M.J. Lacki. 1991. Foraging behavior and habitat use of red bats in mixed mesophytic forests of the Cumberland Plateau, Kentucky. P. $171-177$ in J.W. Stringer and D.L. Loftis (eds.). $12^{\text {th }}$ Central Hardwood Forest Conference, U.S. Forest Service Southeast Experiment Station, Asheville, North Carolina.

${ }^{54}$ Mormann, B.M., M. Milam, and L. Robbins. 2004. Red bats do it in the dirt. Bats 22(2):6-9.

55 Perry, R.W., R.E. Thill, and S.A. Carter. 2007. Sex-specific roost selection by adult red bats in a diverse forested landscape. Forest Ecology and Management 253:4855.

${ }^{56}$ Mager, K.J. and T.A. Nelson. 2001. Roost-site selection by Eastern Red Bats (Lasiurus borealis). American Midland Naturalist 145:120-126. 
${ }^{57}$ Limpert, D.L., D.L. Birch, M.S. Scott, M. Andre, and E. Gillam. 2007. Tree selection and landscape analysis of Eastern Red Bat day roosts. Journal of Wildlife Management 71(2):478-486.

${ }^{58}$ Brigham, R.M., M.J. Vonhof, R.M.R. Barclay, and J.C. Gwilliam. 1997. Roosting behavior and roost-site preferences of forest-dwelling California bats (Myotis californicus). Journal of Mammalogy 78(4):1231-1239.

${ }^{59}$ Vonhof, M.J. and J.C. Gwilliam. 2007. Intra- and interspecific patterns of day roost selection by three species of forest-dwelling bats in southern British Columbia. Forest Ecology and Management 252:165-175.

${ }^{60}$ Krutzsch, P.H. Notes on the habits of the bat, Myotis californicus. Journal of Mammalogy 35(4):539-545.

${ }^{61}$ Young, D.B. and J.F. Scudday. 1975. An incidence of winter activity in Myotis californicus. The Southwestern Naturalist 19(4):452.

${ }^{62}$ Lausen, C.L. and R.M.R. Barclay. 2006. Winter bat activity in the Canadian prairies. Canadian Journal of Zoology 84:1079-1086.

${ }^{63}$ Rodhouse, T. and K.J. Hyde. 2014. Roost and forage site fidelity of Western Small-footed Myotis (Myotis ciliolabrum) in an Oregon desert canyon. Western North American Naturalist 74(2):241-248.

${ }^{64}$ Brigham, R.M. 1991. Flexibility in foraging and roosting behavior by the Big Brown Bat (Eptesicus fuscus). Canadian Journal of Zoology 69:117-121.

${ }^{65}$ Lausen, C.L. and R.M.R. Barclay. 2002. Roosting behavior and roost selection of female Big Brown Bats (Eptesicus fuscus) roosting in rock crevices in southeastern Alberta. Canadian Journal of Zoology 80: 1069-1076.

${ }^{66}$ Lausen, C.L. and R.M.R. Barclay. 2003. Thermoregulation and roost selection by reproductive female Big Brown Bats (Eptesicus fuscus) roosting in rock crevices. Journal of Zoology 260:235-244.

${ }^{67}$ Willis, C.K.R., C.M. Voss, and R.M. Brigham. 2006. Roost selection by forest-living female Big Brown Bats (Eptesicus fuscus). Journal of Mammalogy 87(2):345-350.

${ }^{68}$ Neubaum, D.J., K.R. Wilson, and T.J. O'Shea. 2007. Urban maternity-roost selection by Big Brown Bats in Colorado. Journal of Wildlife Management 71(3):728-736.

${ }^{69}$ Whitaker, J.O. Jr., D.W. Sparks, and V. Brack Jr. 2006. Use of artificial roost structures by bats at the Indianapolis International Airport. Environmental Management 38(1):28-36.

${ }^{70}$ Agosta, S.J., D. Morton, B.D. Marsh, and K.M. Kuhn. 2005. Nightly, seasonal, and yearly patterns of bat activity at night roosts in the central Appalachians. Journal of Mammalogy 86(6):1210-1219.

${ }^{71}$ Rancourt, S.J., M.I. Rule, and M.A. O'Connell. 2007. Maternity roost site selection of Big Brown Bats in ponderosa pine forests of the channeled scablands of northeastern Washington State, USA. Forest Ecology and Management 248:183-192.

72 Fellers, G.M. and E.D. Pierson. 2002. Habitat use and foraging behavior of Townsend's Big-eared Bat (Corynorhinus townsendii) in coastal California. Journal of Mammalogy 83(1):167-177.

${ }^{73}$ Dobkin, D.S., R.D. Gettinger, and M.G. Gerdes. 1995. Springtime movements, roost use, and foraging activity of Townsend's Big-eared Bat (Plecotus townsendii) in central Oregon. Great Basin Naturalist 55(4):315-321.

${ }^{74}$ Genter, D.L. 1986. Wintering bats of the upper Snake River plain: occurrence in lava tube caves. Great Basin Naturalist 46(2):241-244.

${ }^{75}$ Swenson, J.E. and G.F. Shanks Jr. 1979. Noteworthy records of bats from northeastern Montana. Journal of Mammalogy 60(3):650-652.

${ }^{76}$ Jones, J.K. Jr., R.P. Lampe, C.A. Spenrath, and T.H. Kunz. 1973. Notes on the distribution and natural history of bats in southeastern Montana. Occasional papers of the Museum of Texas Tech University 15:1-11.

77 Swenson, J.E. 1970. Notes on distribution of Myotis leibii in eastern Montana. Blue Jay 28:173-174.

${ }^{78}$ Swenson, J.E. and J.C. Bent. 1977. The bats of Yellowstone County, southcentral Montana. Proceedings of the Montana Academy of Sciences 37:82-84.

${ }^{79}$ Bell, J.F., G.J. Moore, G.H. Raymond, and C.E. Tibbs. 1962. Characteristics of rabies in bats in Montana. American Journal of Public Health 52(8):1293-1301.

${ }^{80}$ Bell, J.F. and L.A. Thomas. 1964. A new virus, "MML," enzootic in bats (Myotis lucifugus) of Montana. American Journal of Tropical Medicine and Hygiene 13(4): 607612.

${ }^{81}$ Sherwin, R.E., W.L. Gannon, and J.S. Altenbach. 2003. Managing complex systems simply: understanding inherent variation in the use of roosts by Townsend's Bigeared Bat. Wildlife Society Bulletin 31(1):62-72. 
82 Sherwin, R.E., D. Stricklan, and D.S. Rogers. 2000. Roosting affinities of Townsend's Big-eared Bat (Corynorhinus townsendii) in northern Utah. Journal of Mammalogy 81(4):939-947.

${ }^{83}$ Mazurek, M.J. 2004. A maternity roost of Townsend's Big-eared Bats (Corynorhinus towsendii) in coast redwood basal hollows in northwestern California. Northwestern Naturalist 85(2):60-62.

${ }^{84}$ Hendricks, P. 2012. Winter records of bats in Montana. Northwestern Naturalist 93(2):154-162.

${ }^{85}$ Barclay, R.M.R. 1989. The effect of reproductive condition on the foraging behavior of female Hoary Bats, Lasiurus cinereus. Behavioral Ecology and Sociobiology 24(1):31-37.

${ }^{86}$ Barclay, R.M.R. 1985. Long- versus short-range foraging strategies of Hoary (Lasiurus cinereus) and Silver-haired (Lasionycteris noctivagans) bats and the consequences for prey selection. Canadian Journal of Zoology 63:2507-2515.

${ }^{87}$ Veilleux, J.P., P.R. Moosman, Jr., D.S. Reynolds, K.E. LaGory, and L.J. Walston, Jr. 2009. Observations of summer roosting and foraging behavior of a Hoary Bat (Lasiurus cinereus) in Southern New Hampshire. Northeastern Naturalist 16(1):148-152

88 Klug, B.J., D.A. Goldsmith, and R.M.R. Barclay. 2012. Roost selection by the solitary, foliage-roosting Hoary Bat (Lasiurus cinereus) during lactation. Canadian Journal of Zoology 90:329-336.

${ }^{89}$ Willis, C.K.R. and R.M. Brigham. 2005. Physiological and ecological aspects of roost selection by reproductive female Hoary Bats (Lasiurus cinereus). Journal of Mammalogy 86(1):85-94.

${ }^{90}$ Crampton, L.H. and R.M.R. Barclay. 1998. Selection of roosting and foraging habitat by bats in different-aged aspen mixedwood stands. Conservation Biology 12(6):1347-1358.

${ }^{91}$ Mattson, T.A., S.W. Buskirk, and N.L. Stanton. 1996. Roost sites of the Silver-haired Bat (Lasionycteris noctivagans) in the Black Hills, South Dakota. Great Basin Naturalist 56(3):247-253.

${ }^{92}$ Betts, B.J. 1998. Roosts used by maternity colonies of Silver-haired Bats in northeastern Oregon. Journal of Mammalogy 79(2):643-650.

${ }^{93}$ Perry, R.W., D.A. Saugey, and B.G. Crump. 2010. Winter roosting ecology of Silver-haired Bats in an Arkansas Forest. Southeastern Naturalist 9(3):563-572.

${ }_{94}^{4}$ Pearson, E.W. 1962. Bats hibernating in silica mines in southern Illinois. Journal of Mammalogy 43(1):27-33.

${ }^{95}$ Parson, H.J., D.A. Smith, and R.F. Whittam. 1986. Maternity colonies of Silver-haired Bats, Lasionycteris noctivagans, in Ontario and Saskatchewan. Journal of Mammalogy 67(3):598-600.

${ }^{96}$ Campbell, L.A., J.G. Hallet, and M.A. O'Connell. 1996. Conservation of bats in managed forests: use of roosts by Lasionycteris noctivagans. Journal of Mammalogy 77(4):976-984.

${ }^{97}$ Nixon, A.E., J.C. Gruver, and R.M.R. Barclay. 2009. Spatial and temporal patterns of roost use by western long-eared bats (Myotis evotis). American Midland Naturalist 162:139-147.

${ }^{98}$ Waldien, D.L., J.P. Hayes, and E.B. Arnett. 2000. Day-roosts of female Long-eared Myotis in western Oregon. The Journal of Wildlife Management 64(3):785-796.

${ }_{99}^{9}$ Rancourt, S.J., M.I. Rule, and M.A. O'Connell. 2005. Maternity roost site selection of Long-eared Myotis, Myotis evotis. Journal of Mammalogy 86(1):77-84.

${ }^{100}$ Norquay, K.J.O., F. Martinez-Nunez, J.E. DuBois, K.M. Monson, and C.K.R. Wills. 2013. Long-distance movements of Little Brown Myotis (Myotis lucifugus). Journal of Mammalogy 94(2):506-515.

${ }^{101}$ Henry, M., D.W. Thomas, R. Vaudry, and M. Carrier. 2002. Foraging distances and home range of pregnant and lactating Little Brown Bats (Myotis lucifugus). Journal of Mammalogy 83(3):767-774.

${ }^{102}$ Broders, H.G., G.J.Forbes, S. Woodley, and I.D. Thompson. 2006. Range extent and stand selection for roosting and foraging in forest-dwelling Northern Long-eared Bats and Little Brown Bats in the Greater Fundy Ecosystem, New Brunswick. Journal of Wildlife Management 70(5):1174-1184.

${ }^{103}$ Coleman, L.S., W.M. Ford, C.A. Dobony, and E.R. Britzke. 2014. Comparison of radio-telemetric home-range analysis and acoustic detection for Little Brown Bat habitat evaluation. Northeastern Naturalist 21(3):431-445. 